
Public
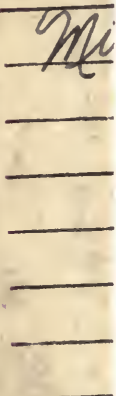

t

San Francisco, California

From the collection of the

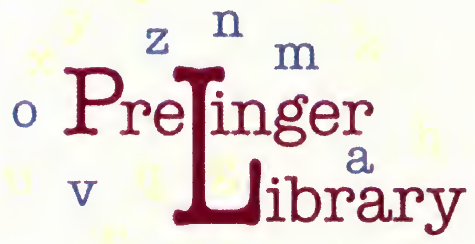

$\mathrm{p}$

$$
2006
$$

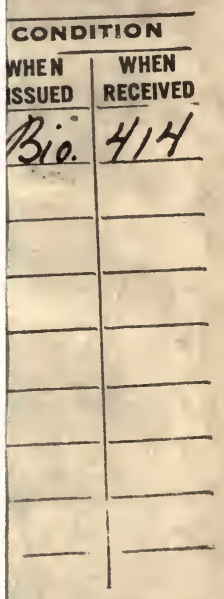


Dewertgman 


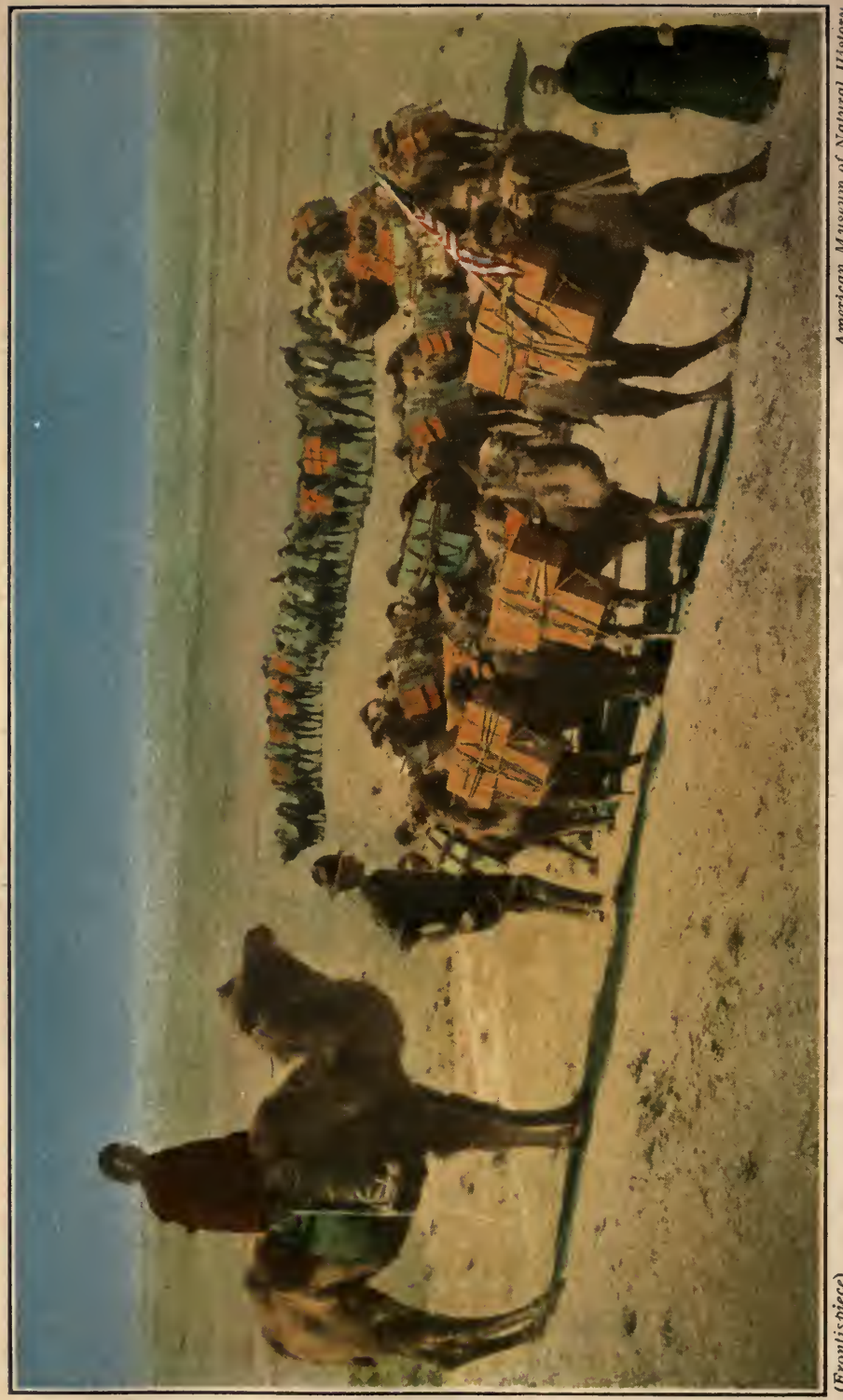

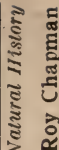

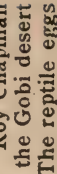

is 9

它

잉

ฐ

융워년엉

을

๙

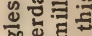

过

$D D_{0}$ 뎡 원

- 200

$+0$ ร5ํํ유 엉 온 을 ․․ 으 हु 究范 동ㅎㅀ

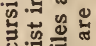
运递 엉 站范 का को 药 कृ ए 등 을 i i 덥형 t. ㄹ. 응 올 롱 눈 혼ㅎㄴ 령 웡 동휴 흔. $\Xi$ 당 3 둉 ธ. ํㅗㄴ. का के ह

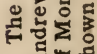




\section{ADVANCED BIOLOGY}

BY

FRANK M. WHEAT

Chairman, Department of Biology,

George Washington High School, New York

Instructor in Biology, New York University

AND

ELIZABETH T. FITZPATRICK

Chairman, Department of Health, Education,

George Washington High School, New York

ERST SIDE EVENIRB hoo SCHUEL
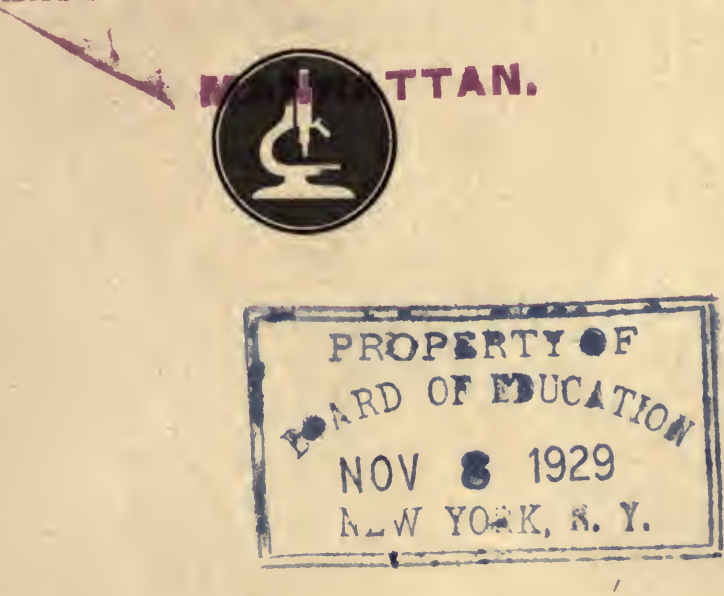

AMERICAN BOOK COMPANY

NEW YORK

CINCINNATI

CHICAGO

BOSTON

ATLANTA 
Copyright, 1929, By AMERICAN BOOK COMPANY

All rights reserved

AD. BIO, WH. AND EITZ.

W.P. 2 
DEDICATED TO TWO SUCCESSFUL PIONEERS

IN BIOLOGY TEXTBOOK MAKING

GEORGE W. HUNTER

AND

JAMES E. PEABODY

FROM WHOM THE AUTHORS OF THIS BOOK

RECEIVED THEIR EARLY TRAINING

AND INSPIRATION 



\section{PREFACE}

Most adolescent boys and girls are more interested in themselves than in abstract problems. Although colleges emphasize mathematics and languages in their entrance requirements and say little about science, there has been a rapid growth in the number of sciences elected in the high schools. This is primarily due to the realization that science is more a part of the lives of pupils than other school subjects and an answer to more of their questions. Youth is more interested in making direct observations and reasoning from them than in abstract thinking. There is a concerted effort to ascertain the truth about phenomena and to find out how and why things happen. Science teaches a valid method of interpreting evidence and helps one to arrive at logical conclusions.

In most secondary schools throughout the country elementary biology or general science is taught in the first or second year. There has been a growing demand for an advanced course in general biology to follow the elementary science course. This text has been written primarily to fill this need. The emphasis of the book is on problems relating to human welfare. The origin and principles of the developm nnt, structure, and functions of plants and lower animals are introduced mainly as a background for the proper understanding of human problems.

An interesting and novel feature of the text is the historical treatment of many of the subjects, which gives the pupil a bird'seye view of the entire subject without overwhelming him with unrelated facts. Teaching material is given at the end of each chapter, designed to help the pupil in organizing, in his own mind, the important principles discussed. The list of supplementary readings offers the pupil sources of information other than the text. All the laboratory problems necessary for a thorough 
understanding and mastery of the subject are included. These problems are usually given at the beginning of the chapters, so that the pupil may find out for himself many of the facts given later in the text. The pupil can exercise here the true scientific method: examination, observation, and confirmation of his findings.

The plan of presenting the subject matter is based on the practical experience in teaching this course for several years to high school pupils by means of mimeographed lesson sheets prepared by various members of the Biology Department of the George Washington High School, New York, N. Y. Changes in these sheets have been made, but much of the material has been elaborated into the present text. The enthusiasm of the Biology Department in the George Washington High School is due largely to the inspiration and support of Harold S. Campbell, Associate Superintendent of the New York high schools. In his annual report of 1928 he included the report of the District Superintendent of High Schools, Dr. John L. Tildsley. In this report, Dr. Tildsley summarized the objectives of science teaching and said :

"These objectives call for the creation of a more magnificent self. They call for the expanding of the element of appreciation, the kindling of imagination, the arousing of the sense of admiration and wonder, the excitation of the emotions, the development of the power of accurate observation, the desire for truth, courage to follow the truth, and above all, the setting forth of science as 'a 'way of life.'

The authors hope this text will open this broader "way of life" and inspire pupils to think and to act magnificently.

Thanks are due the Biological Supply Co., New York, for the use of photomicrographs prepared by Mr. Roy M. Allen, and also to Miss Marjorie Fitzpatrick, Mr. Charles Inman, and Mr. Paul B. Mann of New York city high schools, Prof. Ralph Cheney of Long Island University, and Miss Ada Weckel of Oak Park, Illinois, high school, for their critical reading of the manuscript. 


\section{CONTENTS}

CHAPTER

I. The Biology of To-Morrow . . . . . . . 1

II. The Growth of Science.$\quad$. . . . . 10

III. The Microscope '. • • • • • • • . 19

IV. The History of Cells . • . • . . . 27

V. Functions of a Green Cell . . . . . . . 34

VI. Typical Animal Cell • • . • . • • . 42

VII. The Resting and Dividing Cell . . . . . 53

VIII. Structure of Higher Plants . . . . . . 66

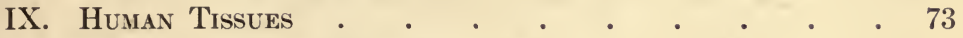

X. Human Tissues (Continued) . . . . . . . 83

XI. Food Nutrients . . . . . . . . . . . 91

XiI. The Teeth and Their Care . . . . . . . 107

XIII. The Digestive System . • . • • • . 114

XIV. Digestion and Absorption . . . . . . . . 125

XV. Blood and Its Importance . . . . . . . 143

XVI. Circulatory System • . . . . . . . 151

XVII. Lymphatic System . . . . . . . . 163

XVIII. The Skin and Kidneys . . . • . • . 170

XIX. Respiration • . . . . . . . . . . 177

XX. Metabolism . . . . . . . • . . 187

XXI. Ductless Glands . . . . . • • . . . 191

XXII. The Nervous Srstem • . . . . . . . 202

XXIII. Nervous Reactions . . . . • . • . . 215

XXIV. Mental Hygiene . . . . . . . . . 227

XXV. How Life Began . . • • • • • • . 239

XXVI. Asexual Reproduction . • • • • • . 246 
XXVII. Vegetative Propagation

XXVIII. Sexual Reproduction • • . • • • . 264

XXIX. Reproduction of Higher Plants . • • . . 273

XXX. Reproduction of Animals . . • • • . 281

XXXI. Protection of Young . • • • • . . 292

XXXII. Character of Offspring . • . • . . 306

XXXIII. Heredity . • . . • • • . . . 318

XXXIV. Mutations • • • • • • • • . 335

XXXV. Plant and Animal Breeding . • • • . 343

XXXVI. Eugenics • • • • • • • • • . 355

XXXVII. Progressive Development • • . • • . 371

XXXVIII. BACTERIA • . • • • • • . • . 391

XXXiX. Beneficial Activities of Bacteria . . • . 403

XL. Health . . . . . . . . • . 413

XlI. Smallpox and Its Control • • • • • 420

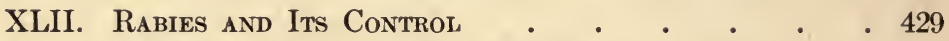

XliII. Tuberculosis and Its Prevention . • • . 437

XIIV. Diphtheria, Scarlet Fever, and Tetanus : • . 450

XLV. TYPhoId Fever . . . . . . . . . 464

XlVi. Certain Other Diseases . . • . . . 473

XlViI. The Control of Malaria and Yellow Fever . . 480

XlVIII. Defenses against Diseases . . • . . . 494

XLIX. Immunity . • . . . . . . . . 504

L. TAXONOMY . . . . . . . . 510

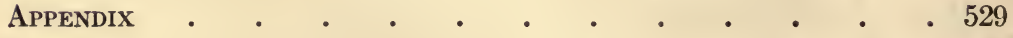

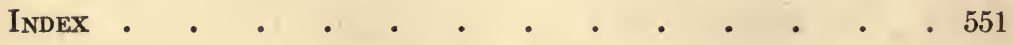




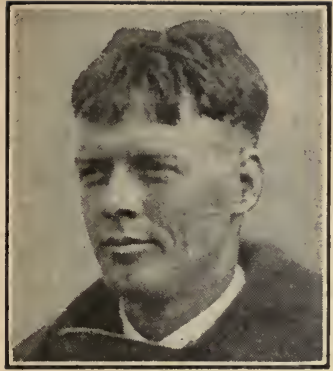

Underwood and Underwood

Col. Charles Lindbergh.

\section{CHAPTER I \\ THE BIOLOGY OF TO-MORROW}

At no time in the history of the world have the layman and the scholar turned to the field of science for inspiration and help as in the present age. We are all becoming more scientific-minded. Man is leaving behind him the era of superstition and romanticism and is seeking the truth in the light of science. Almost daily a miracle happens - a discovery that thrills the world with its tremendous import. Newspapers and periodicals devote column after column to scientific matters, and even the writers of drama and fiction go to science for plots. People discuss present-day science as they once discussed literature.

The age of science. Do you recall how interested and excited you were over the proposed New York to Paris flight of Charles Lindbergh? The entire nation followed the preparations and the performance of the fearless and skillful lone pilot. All marveled at the tiny monoplane with its speedy Wright whirlwind motor. Nations rejoiced in the success of the experiment, and almost overnight practically all people developed an interest in aviation. They became air-minded. Since that time great progress has been made in aviation, and travel by air over certain routes has become so safe that few hesitate to sponsor this method of transportation.

Not only in aviation, but in radio, color photography, and 
television, experiments have been made and are followed with intelligence and eagerness by great numbers of people. The radio

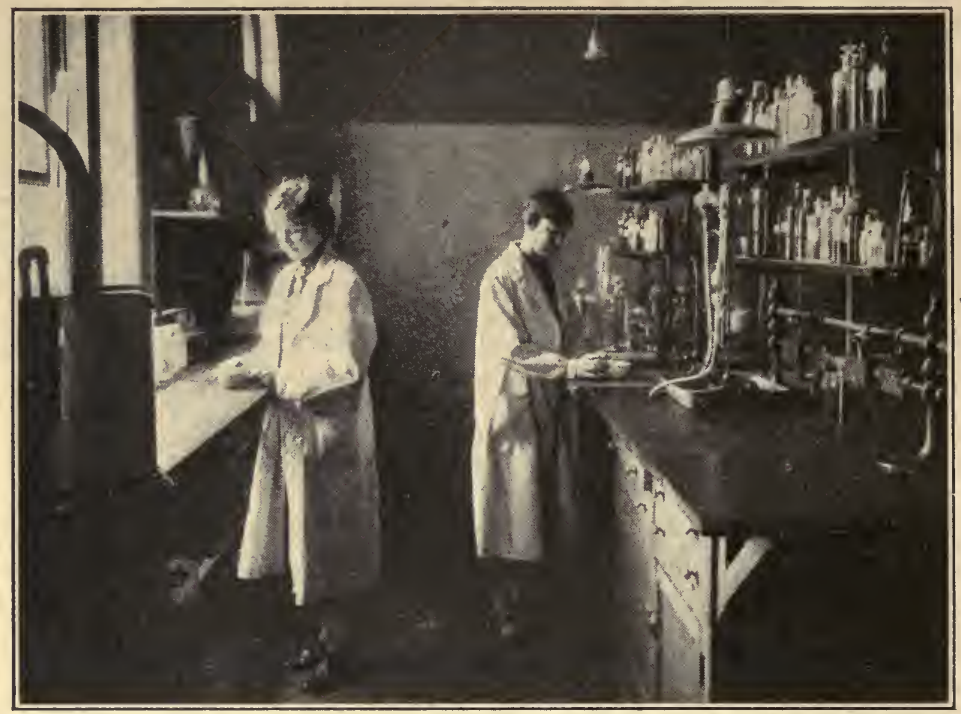

Ewing Galloway

Many of the problems of science are solved in the laboratory.

has brought the people of our nation together again and again. In our own homes, by a turn of the dial, we take part in great public gatherings miles away, enjoy a symphony, or listen to the World Series baseball games. The transmission of the human voice and of instrumental music through miles of space is now accomplished, with very little distortion. The reception of light waves, bringing movies into every home, is a development of to-morrow.

There is as wide an interest in the subject matter of biology as in other fields. The history of many scientific experiments and investigations has been so wide spread that there is hardly a school boy or girl who does not know the story of the control of malaria and smallpox. The dramatic death of Hideyo Noguchi, of the Rockefeller Institute, occurred at the culmination of years 
of work on yellow fever. Through his experiments, he revealed to the entire world the painstaking methods characteristic of a true scientist as well as the fearless persistence of a martyr.

Noguchi had solved the problem of yellow fever in South America, but the facts he found in that country did not seem to hold true for the African type of the disease. He, therefore, journeyed to Africa to make further studies. He contracted the disease and died before completing his work. He is one of the heroes of to-day.

What of yesterday? Consider the strides surgery has made since the early days of this science. For generations the chief medical and surgical treatments were sweating, bleeding, and amputations. In the Lewis and Clark expedition in 1804, one of the men became ill. Captain Clark described the treatment given him. A big hole was dug in the ground and a fire was built in it, in order to make the ground hot. Then the fire was removed and the man was laid on the hot earth and securely covered so that he would sweat. After this treatment he was bled. Not having any other knife, Captain Clark used his pocket penknife to open the blood vessel. Needless to say, the man died. Among primitive people of to-day similar methods are still in vogue. The medicine man in a certain primitive tribe still places people in holes heated to high temperatures, to sweat them. If a member of the tribe suffers from chronic headaches, the medicine man cuts a piece out of the sufferer's skull.

In civilized communities, surgery, as a scientific study, began with the hypothe-

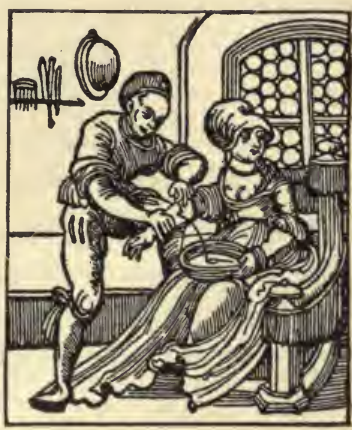

Physicians of yesterday practiced bleeding for many ailments. sis of Louis Pasteur, about 1860, that disease was usually due to the presence of microörganisms. Realizing that most of the surgical cases died from infections rather than the actual 
operation, Sir Joseph Lister (1827-1912) studied the work of Pasteur. Up to this time pus was always considered the necessary accompaniment of all wounds. Lister decided that germs must enter the wound from the air, from surgical instru-

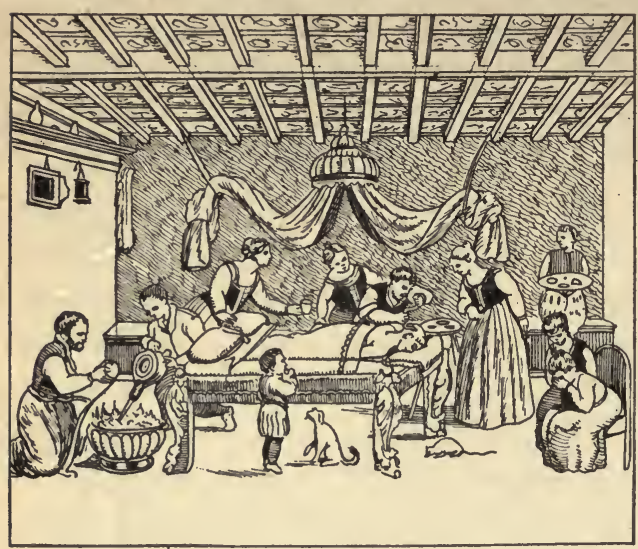

An old print shows that headache was treated by removing a portion of the skull. ments, or from other outside agencies. Thereafter, when operating, he used what he called antiseptics to kill the germs. He attempted to destroy the germs in the air by spraying the air of the operating room with a carbolic acid solution. He then protected the wound as much as possible from contact with the air. All his instruments were subjected to the most careful antiseptic treatments. He taught his principles of antiseptic surgery to the surgeons of France. The Franco-German War broke out in 1870 . It occurred to no one in France, in the first battles, to apply the new method of antiseptic surgery. In consequence, hundreds and thousands of wounded soldiers succumbed to gangrene and septicaemia, types of blood poisoning. Then doctors all over the world adopted antiseptic surgery. Infections which formerly followed many operations practically disappeared. Before Lister's time 70 per cent of all compound fractures resulted in death, and about 50 per cent of all major operations were fatal. After Lister's antiseptic methods were introduced these percentages were greatly reduced.

What of to-day? The most modern method of surgery is aseptic surgery. Germs are controlled by killing them with dry heat 
rather than antiseptics. It is now known that the entrance of germs into wounds is due to contaminated instruments or hands rather than to air. If the hands of the doctor and the body of the patient are washed with antiseptics and the instruments are thoroughly sterilized, there is very little chance of contamination from the air. The spraying of antiseptics in the air is no longer done. To-day, less than one per cent of the patients subjected to operation for compound fractures die.

Many of the mistakes of yesterday prevail even to-day. Along with pure science come the pseudo-sciences. For example, people were taught that definite areas of the brain controlled definite mental activities like motor control, vision, and judgment. Immediately the pseudo-science, phrenology, came into being. Phrenologists examined the various bumps on the head and claimed that these were related to the development of areas 'of the brain. They argued that success in selling, teaching, or executive ability could be predicted if the proper irregularities were present on the person's skull. But, through many years of observation and experimentation, it has been conclusively proved that the irregularities in the skull do not necessarily follow brain irregularities. With the invention of the radio and television came a revival in the belief in telepathy and spiritualism.

Interest in diets has led to fads in foods. People were told raw foods supplied vitamins. Some

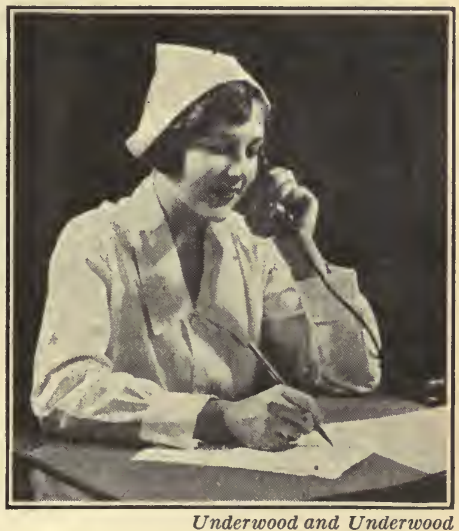

Nurses who use antiseptic measures saye as many lives as do doctors.

people began to eat nothing but raw foods such as nuts, fruits, and vegetables. Yeast was found beneficial in supplying a certain vitamin, and was at once accepted, by some, as panacea for all ills. 
What of to-morrow? The purpose of this book is to give a scientific biological background to pupils so that they may have

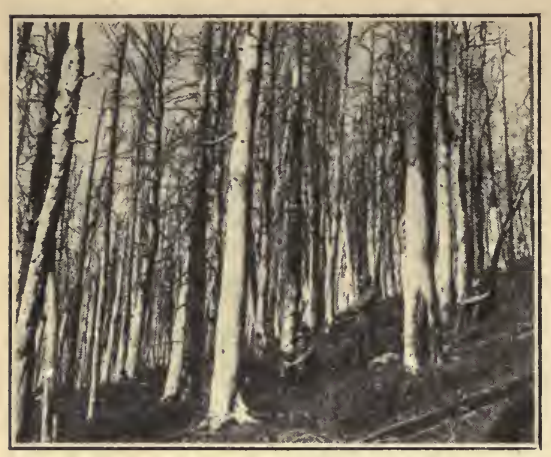

Underwood and Underwood

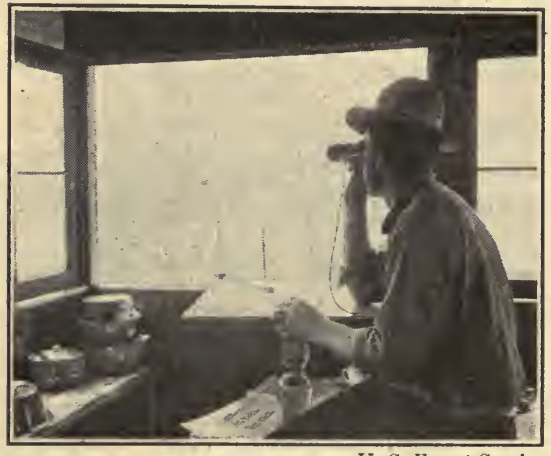

U. S. Forest Service

Forestry offers an opportunity for an out-of-door life of service.

a basis for discrimination between real and pseudo-science in connection with the human body. The aim of all science is to ascertain the truth from unbiased experiments without regard to emotional prejudices or statements based on speculation or imagination. In scientific experimentation one does not imagine or decide beforehand what is going to happen. Only actual observable facts are accepted. If experiments on disease were prejudiced, disease would never be controlled. Similarly, all life progresses more efficiently when it is lived scientifically. The scientific, unemotional attitude toward life's problems leads to a more exact solution.

It is hoped that the study of biology will be an inspiration to recreational activities. Everyone is happy in the pursuit of a hobby. It gives one a worthy use of leisure time. If this hobby takes one out of doors, the result is usually beneficial to his health. If certain facts about plants and animals are understood, an intelligent interest in their growth and habits is possible. Newspapers, magazines, books, and periodicals contain many scientific, articles and description of experiments. If a person has some 
knowledge of science, this wealth of material may be easily interpreted and understood by him. The present text aims to serve as a basis for the interpretation of future scientific readings. The more interests one can cultivate the more he gets out of life.

The relation of the biology of to-day to health cannot be overemphasized. One of the reasons why the people of yesterday had so many fears concerning disease was that they were ignorant of methods for preventing and controlling it. The prevention and control of disease can now be approached intelligently because there has been some education along these lines. Coöperation between the public and the Board of Health is made possible because of this health education. The general public understands what the health authorities are trying to accomplish. Because it knows that certain restrictions are formulated in the interests of health, the public readily accepts and carries them out. For example, there is not as much opposition to the Schick test and the subsequent immunization for diphtheria, as formerly, because the public has been intelligently instructed concerning the need of this scientific procedure to combat the disease.

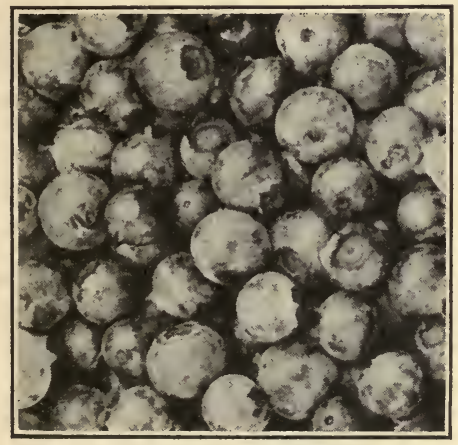

Wild blueberries.

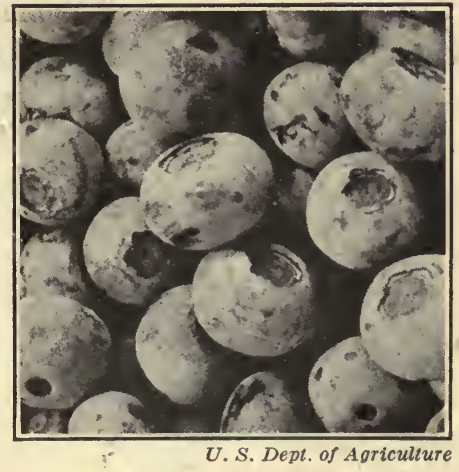

Cultivated blueberries.

Selection, cultivation and breeding have increased the size and flavor of many fruits.

When people cultivate proper personal habits and attitudes, and more intelligent interests in the home and community, they achieve 
greater social and civic success. Biology helps to free people of ignorant and useless racial customs. It is in school that proper standards of cleanliness, ventilation, feeding, and routine are frequently acquired. If hygienic habits of living are developed, and people persist in practicing them, better social customs will soon be established. For example, if students are taught the importance of buying bread, wrapped to prevent contamination, and if they insist on buying only wrapped bread, the storekeepers will soon supply it. Weighing evidence in studying scientific data, develops a control which makes the student approach family problems with greater wisdom. Every individual owes certain responsibilities to his community. Problems of sewerage, garbage disposal, street cleaning, water and milk supply, control of disease are all discussed in biology classes, and a better understanding of these will give each person a clearer conception of his obligation to himself and to the public.

The relation of biology to vocations. There are many vocational opportunities in scientific fields. Biology suggests and possibly lays the basis for many of these. The relation of biology to the study of dentistry and medicine is obvious. The achievements of Pasteur, Koch, and Noguchi are fine ideals to arouse enthusiasm and interest in research work. Bacteriology, chemical analysis, and oral hygiene are laboratory fields that are intensely interesting. Nursing is a vocation with a gripping human interest.

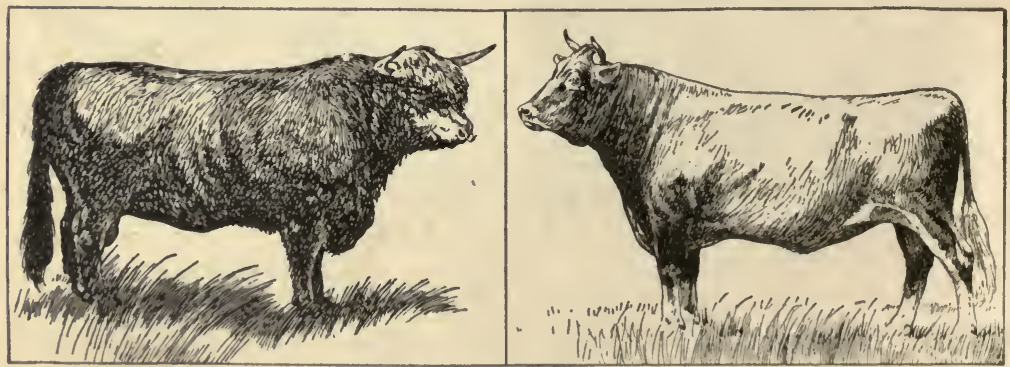

Science has improved the appearance, weight, and color of cattle. 


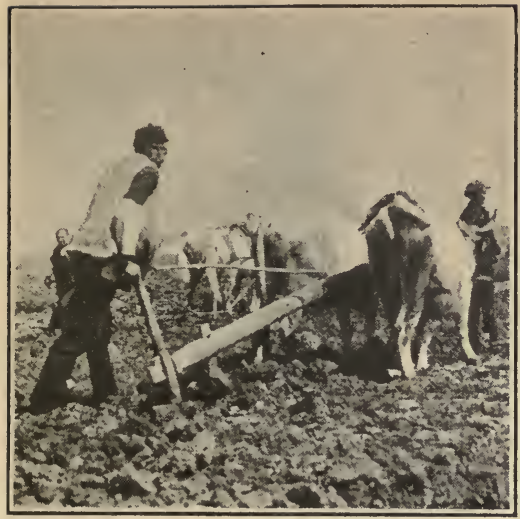

Underwood and Underwood

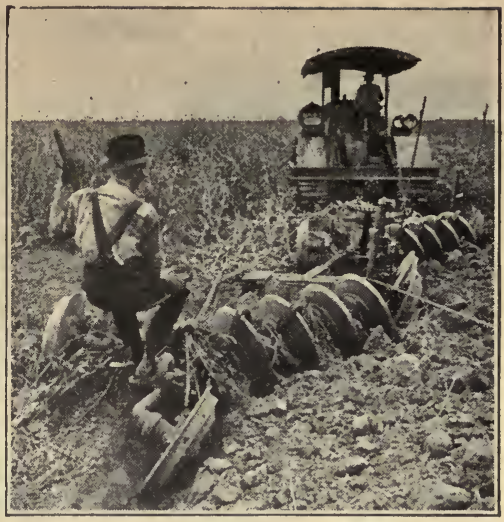

Ewing Galloway

Agricultural progress has been made possible largely through scientific discoveries and inventions.

Forestry, scientific farming, and animal and plant breeding are vocations that constantly need more recruits. Biology has contacts with all these vocations and is a means of giving students such information that will help them to decide whether they would like to pursue a scientific vocation.

To-morrow never comes. This book cannot give the latest information about living things. Before it leaves the printer's hands a new vitamin may be discovered, the cause and cure for cancer may be announced, a new and unexpected theory may necessitate a check and revision of much of the work that has been set forth in these pages. If you who read are careful in scrutinizing all data before you make a conclusion, you will have become more scientific. You may be one who will add a chapter to the biology of to-morrow; you may learn and tell the farmers of to-morrow how to grow two blades of grass where one formerly grew. This Advanced Biology includes the story of the biology of yesterday and of to-day. If it has been well told, it should make your to-morrow a healthier, happier, and more complete day.

WH. FITZ. AD. BIO. - 2 


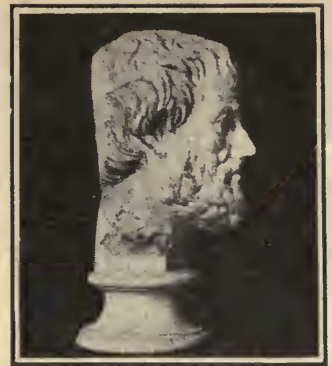

Aristotle, Father of Biology.

\section{CHAPTER II}

THE GROWTH OF SCIENCE

What changes have taken place in science? What is science? Into what branches may science be divided? What makes up biology? How is biology related to other sciences?

Science began when primitive man made an effort to explain certain phenomena that he could not understand. Sickness occasioned much speculation. Not knowing or understanding anything about the various organs of the body, he thought disease must be due to magic. Diseases were supposed to be caused by evil spirits whose wrath had to be appeased or whose favor had to be won. Ancient people burned sacrifices or beat tom-toms to drive away the evil spirits. Some thought that diseases could be transferred from man to animals. An example of this was the belief that a toothache could be cured if the afflicted person stood on the ground under an open sky, and spat in a frog's mouth, asking the frog to take the toothache away. Another remedy suggested, was to keep a hot, cooked, dried bean at the right elbow for three days if the tooth ached in the left side of the mouth. The order was reversed if the tooth was on the right side.

The advances of the Greeks. It was not until the time of Hippocrates (born about 460 B.C.) that the Greeks began to attribute disease to natural rather than to supernatural causes. Hippocrates taught that the body was in good health when the four juices, 
blood, phlegm, yellow bile, and black bile, were mixed in the proper proportions. Aristotle (384-322 B.c.) was one of the first men to realize that all phenomena should be investigated very carefully before a conclusion is made. $\mathrm{He}$ is called the Father of $\mathrm{Bi}$ ology because he made extensive studies of plants and animals and their development. Not only did he investigate, but he wrote down his investigations. He thus enabled the scientists who followed, to build on his work. Because so little dissection was permitted at this time, his work was full of errors; but, nevertheless, his studies were of tremendous value. This was the first step in breaking away from idle speculation. He started scientific investigation, reason based on observation.

\section{Early surgery and medi-} cine. Previous to the Middle Ages, many scientists talked and argued at great length about health and disease, but they were unable to make scientific irrvestigations because they were forbidden by law to dissect the human body. The only dissections permitted were those of lower animals such as dogs. Unskilled barbers usually made these dissections for the scientists. An instructor would

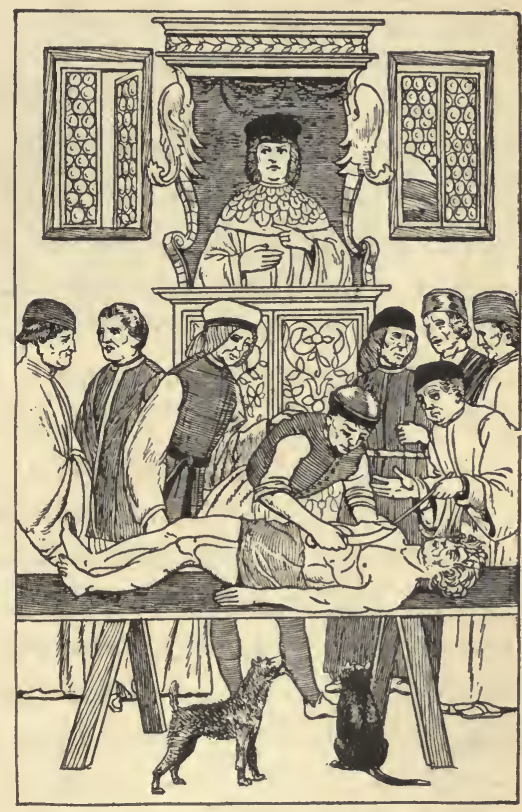

In the medical schools of the Middle Ages the lecturer stood in a pulpit. Barbers made the dissections and pointed out the structures as they were mentioned. A few favored animals received the discarded parts as they were cut off.

read to his class from an anatomy book written by a Greek physician, Galen. At the same time the barber pointed out the part 
of the structure mentioned in the text. It was not until the time of Vesalius (1514-1564) who lived in the Middle Ages, that surgery was put on a professional basis rather than a menial one performed by barbers and bath keepers. The study of the structure of the human body was now both permitted and encouraged. No longer was medical science purely speculative. It began to be based on observation. In lecturing, Vesalius pushed aside the clumsy surgeon barber and he himself demonstrated the parts of the dissected body in a proper way. He began to draw pictures of the dissections as he actually saw them. He disproved the old idea that man differed from woman by having one less rib on one side. He demonstrated by dissection that man and woman really have the same number of ribs on each side. Gradually, teaching came to be based on direct observation rather than opinion. As instructors acquired more and more knowledge of the human body, they insisted on making their own dissections, instead of depending upon the unscientific efforts of barbers. They realized the value of careful dissections in order to show the structure of the body.

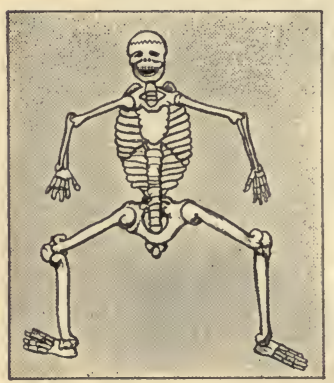

Before human dissections were made, skeletons were drawn very inaccurately.

The efforts of physicians to look for natural causes of disease rather than supernatural was the beginning of science, but they did not go far enough. While scientists were dissecting the human body and disproving fallacies in regard to it, they were not yet checking, experimentally, observations in the treatment of disease. For example, when a patient's cure seemingly followed the administration of some unusual remedy or drug, without further experimentation, doctors jumped to the conclusion that they had discovered the cure. During an epidemic of typhus fever, a Turkish upholsterer, having become ill with the disease, drank some liquid from a pail containing pickled cabbage, and recovered. Imme- 


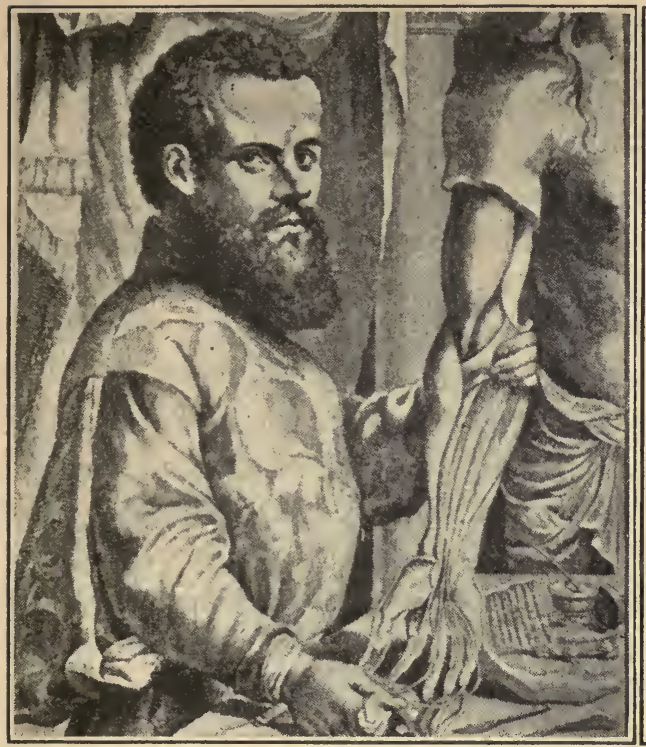

Vesalius:

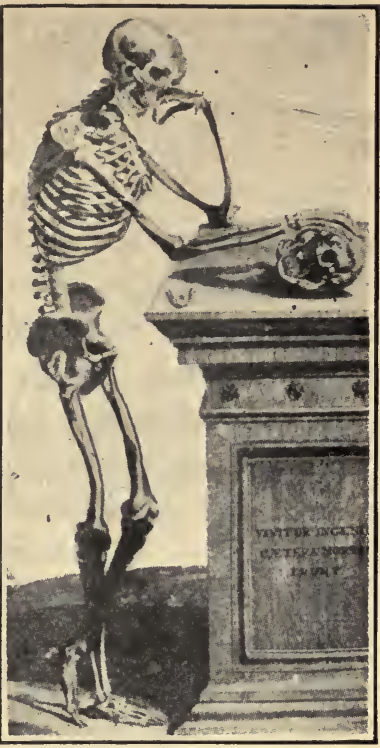

One of his drawings.

Vesalius made many careful and accurate human dissections. He observed with exactness and made records with rare skill.

diately, Turkish doctors declared cabbage juice was a cure for this disease. The next patient died in spite of this treatment, so they modified the prescription by saying that cabbage juice was a remedy for typhus provided the patient be an upholsterer.

The modern scientific method. Compare these methods with present-day methods in science. Imagination and speculation have their place, but they are not to be confused with reasonable judgment based upon experimentation and observation. A scientist must have imagination and be able to predict what the various causes of phenomena may be. Then he must test out each of his theories painstakingly under controlled conditions. This testing method must be performed repeatedly in order to allow for accidents. As a result of these experiments, observations are 
made and conclusions drawn. This is the modern scientific method; science based on experimentation and observation.

Consider the work of Paul Ehrlich. He wanted to find a chemical that had a deadly effect on certain microbes. First, he studied these particular disease germs by staining them different colors. Then he tried the effect of various chemicals on them. With great patience he persevered, rejecting those tests that were not satisfactory. Finally, his six hundred and sixth experiment was successful. He had found a substance which would kill the germ but would not injure healthy body tissue.

Dr. Thomas H. Morgan, University of California, is now working on the question of heredity. He has examined the size and various body differences of tens of thousands of tiny flies. One of the differences noted was a specimen with colorless instead of pink eyes. He has bred this and other different types through countless generations in his efforts to understand heredity. There is no wild jumping to conclusions in his work. It is based on definite, experimental evidence.

Think of the late Luther Burbank. Acres of ground were tilled, planted, cultivated, and the plants were closely observed by this experimenter. All but one or two plants grown were discarded in his search for specimens of the plant he wanted to breed. He, like other scientists, examined a wealth of data and then made a careful selection from this material.

Contrast the cabbage juice treatment of typhus fever mentioned before with the scientific investigation of malaria. It was first observed that people living near swamps contracted a fever ; therefore, it was thought that swampy air caused this malady. The disease was called malaria, meaning bad air. People were cautioned to close their windows, particularly at night, to keep out the bad air. Then, beginning with observations based on experimentation, doctors experimented with swampy air to see whether it would give malaria or not. They found that it had no direct relation to 
the disease. Then they hunted about for another probable cause. Due to carefully controlled experiments, doctors were able to demonstrate that malaria was caused by a definite microörganism which was carried by a certain species of mosquito. Once this had been demonstrated, the prevention, treatment, and cure of the disease followed. Certain towns along the Canal Zone, for many years infested with both malaria and yellow fever, have now become health resorts. The carrier of the germ has been exterminated and the disease is now under almost complete control.

Modern experimentation solves problems under controlled conditions. The aim of the problem must be kept in mind, the method of procedure must be decided upon, and all conditions carefully regulated. All possible data must be painstakingly collected and arranged in an orderly manner. Judgment must be suspended until all the evidence has been properly weighed. All observations should lead to a logical conclusion which will give information about the problem to be solved, if it does not actually solve it. Such scientific observation leads to straight thinking. Science is now becoming a philosophy of education rather than simply a method, and is applicable to all problems of life.

Viewpoints of science. Human knowledge may be divided into the arts and the sciences. Science is careful, exact, orderly arranged knowledge. Among the many sciences are chemistry, physics, and biology. Biology is that branch of science that deals with living things or things that have been alive. The word biology comes from two Greek words, bios - life; logos - study.

In studying this earth on which we live, and the living things that are found on our globe, the different scientists may each look at the things about them from different points of view and use different units upon which to build their sciences. In physics, the scientist may separate a board into small chips or into sawdust, and divide it further into smaller and smaller particles, finally, reducing it to the smallest possible particle and still have the 
material, wood. When the particles become so small that they can be seen only with an ultramicroscope, they are called molecules. The physicist calls everything that occupies space, matter;

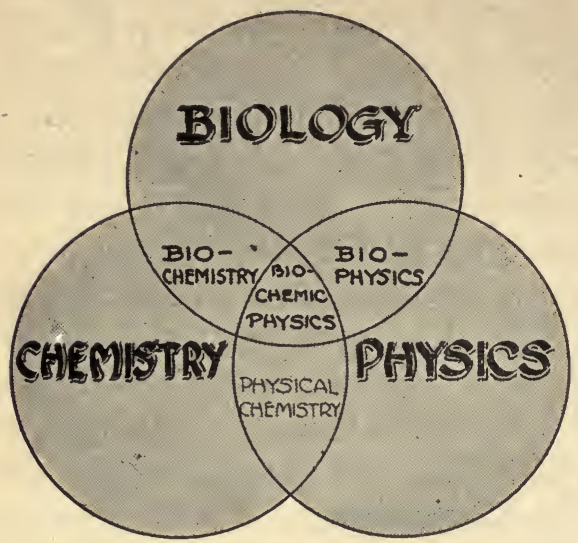

Biology is related to chemistry and physics. The topics embraced by both biology and chemistry have been organized as a special science, bio-chemistry. Note the other combinations shown in the diagram. and he thinks of matter as being made up of tiny molecules in motion.

The chemist takes substances or matter still further apart. Water, wood, rocks, air, our world in general, may be taken apart and separated into ninetytwo or more different substances called elements. Ninety of these have been separated from their compounds and studied. Most of the materials of the world, including living things, exist in the form of compounds, combinations of elements. The chemist has worked out methods for decomposing the compounds in order to study their constituents. If an electric current is passed through water, it will cause the liquid to be separated into two gases. If these two gases are collected in tubes we find that twice as much gas will collect in one tube as in the other. Both gases are colorless, odorless, and tasteless. If a glowing splint is thrust into the tube containing the smaller amount of gas, it will burn brightly - the gas supports combustion. It is oxygen. If a lighted splint is held near the tube holding the larger volume of gas, there is a slight explosion and the gas burns with a blue flame. It is hydrogen. Water has been separated into its elements, hydrogen and oxygen, and is shown to consist of twice as much hydrogen as oxygen. 'The molecules of these elements may further be divided into atoms. The chemist 
and physicist of to-day deal with matter in even smaller particles, as they have discovered that atoms are further divisible into protons and electrons, that is, particles that carry positive and negative electrical charges.

When a biologist looks at a living plant or animal he views it in the light of his particular training. To some biologists, the shape and form of the animal are of greatest interest. Others investigate the processes of the living organisms.

Biology a complex science. Many divisions of biology have been investigated, and there is so much known about each one that they are sometimes thought of as distinct subjects. The specialist who delves in a particular division is named in terms of the specialty he follows. The specialists who are interested in the study of bacteria (bacteriology) are known as bacteriologists. Protozoölogy, likewise, has protozoölogists who are interested in one-celled animals. Those who study snakes are interested in herpetology ; the specialist is a herpetologist. The entomologist studies insects; he is concerned with the science of entomology. Some scientists may specialize in only one branch of entomology. Bugs are classed as Hemiptera ; students interested in bugs are

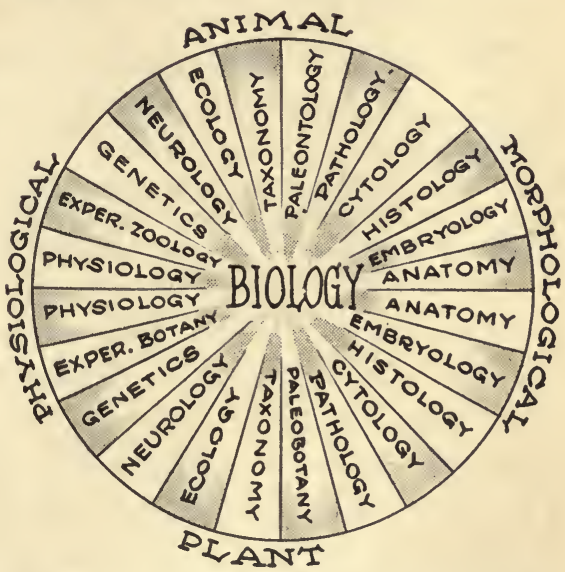

Biology is a complex science made of many ologies. hemipterists. Butterflies, the Lepidoptera, are the topic of study of the lepidopterists. In each of these branches of biology we have other subdivisions - morphology, the study of the forms and structures, and physiology, the study of the functions of the organs. 


\section{Questions And Suggestions}

1. Do you know of any superstitions that persist to-day in the treatment of disease? Is there any scientific foundation for such superstitions?

2. What was the importance of the work of Vesalius?

3. Who was Aristotle?

4. Contrast the ancient method in treating disease with the modern scientific way?

5. In your research notebook define the divisions of biology represented in the diagram. noted.

6. Give the proper names to the men who specialize in each division

7. a. List ten or more "ologies" not given in our outline.

$b$. Underline those "ologies" which might properly be included in the circle of biology.

c. Place an $m$ in front of the biological "ologies" just underlined, that are morphological, a $p$ in front of the ones that you think are physiological.

\section{Supplementary Reading}

Dana, Chas. L., Peaks of Medical History (Paul B. Hoeber [Medical Pub.]), chaps. i-vi.

Locy, W. A., Biology and its Makers (Henry Holt \& Co.).

Locy, W. A., Growth of Biology (Henry Holt \& Co.).

Osborn, H. F., From the Greeks to Darwin (Charles Scribner's Sons). 


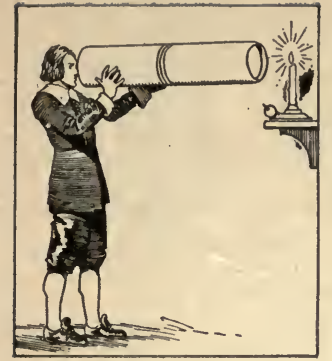

An early microscope.
CHAPTER III

THE

MICROSCOPE

How was the microscope invented? Was it much simpler at one time or is it still in its original form? What is the use of a microscope? Is there a definite technique to be observed in using it?

Biology has contacts with other sciences. Inventions and discoveries by physicists and chemists have made possible the explanations of many biological facts. It is almost impossible to discuss biology without including some physics and chemistry. The invention and improvement of the microscope by physicists have been largely responsible for the rapid progress of biology.

The history of the microscope. In Holland, in the seventeenth century, Anthony Von Leeuwenhoek (1632-1725) experimented 'with lenses, grinding hundreds and using them in various combinations to get different magnifications. He improved those that had formerly been used. He used dust, wood, and crystals to try the strength of his lenses. As he was interested in natural history, he investigated the minute structure of living things. He had little education and his researches were not conducted on an extremely scientific basis, and, therefore, his work was somewhat unsystematic and disconnected. However, his improved lenses opened up an entirely new field, namely, the investigation of minute living things. Scientists could now actually confirm certain of their speculations. A short time before the microscope was improved, William Harvey, an English physician, 
had said that blood moved through the body in a circuit and that the beating of the heart supplied the propelling force. He had no microscope, but reasoned this out from observations made from his dissections. Leeuwenhoek ground a lens to obtain proper magnification, placed a tadpole in a glass tube, and adjusted the tube in front of the fixed lens. When he looked through the lens, he saw the blood, in the tail of the tadpole, come down one blood vessel, cross to another, and return through still another. Thus, by means of his lenses, he proved Harvey's reasoning was correct in the specimen that he was studying. Leeuwenhoek did a great deal to stimulate interest in perfecting the microscope. In his day, for each new specimen studied a new lens had to be ground and the object permanently fixed in relation to this magnifier. To-day, microscopes are constructed quite differently. The lenses are permanently mounted in relation to each other, forming

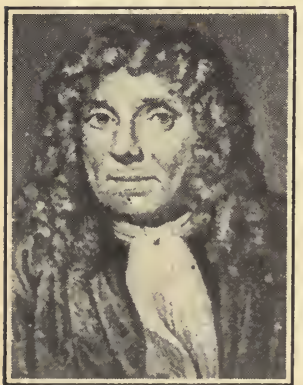

Von Leeuwenhoek.

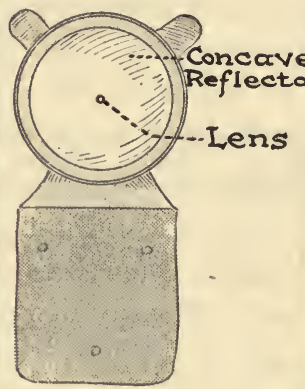

One of his many microscopes.

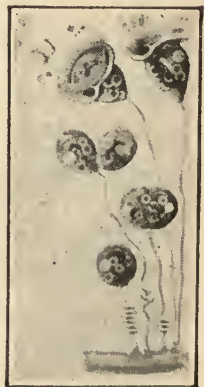

What he saw.

What he said:

"In the year I675 I discover'd living creatures in Rain water which had stood but a few days in a new earthen pot, glazed blew within. When these living Atoms did move they put forth two little horns, continually moving themselves. They had a tayl, near four times the length of the whole body, of the thickness (by my Microscope) of a Spider web; at the end of which appeared a globul."

a compound microscope. We simply change the slides on which the specimens are mounted. Many improvements have been made in microscopes since the time of Von Leeuwenhoek, and the com- 


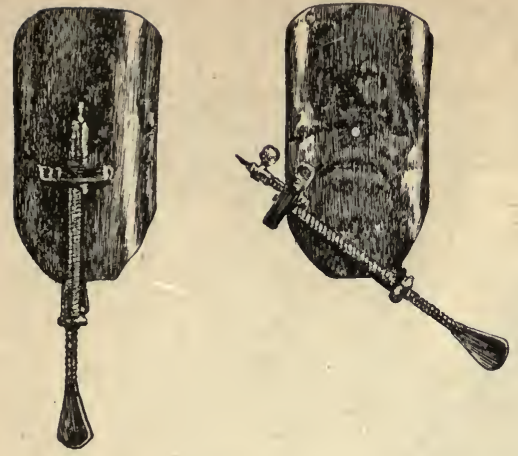

One of Von Leeuwenhoek's microscopes consisted of a lens, embedded in a large sheet of copper with a movable rod which acted as a mount for the specimen.

pound microscope of to-day is a very complicated and delicate instrument. It should always be handled with intelligence and care.

Problem. Place a compound microscope before you and find the various parts as they are mentioned.

I. 'There are three distinct sets of parts :

A. The mechanical part supports the other parts and makes possible their controlled movements.

$B$. The optical part does the actual magnifying which is made possible by the rays of light passing through the lenses.

C. The illuminating part is used to direct and regulate the light.

II. All of the mechanical parts together constitute the stand, which has a heavy base supporting a leg or pillar. Projecting horizontally from the top of the pillar, parallel with the base, is the stage. There is a hole in the middle of the stage through which the light passes. Above the stage is a continuation of the pillar, and extending from this over the stage is the arm, which carries a vertical tube. Projecting on both sides of the arm are disks with ridged edges. These are connected with a pinion that causes the tube to go up or down when it 
is turned. The larger disks with the rack and pinion make up the coarse adjustment, or coarse adjustment screws. By turning the disks gently, you can see the effect on the tube. The coarse adjustment is used to change the distance between the lenses and the object observed; that is, to focus. Below the coarse adjustment screws is a smaller pair of disks called the fine adjustment, or fine adjustment screws. The fine adjustment is also used for focusing, but is

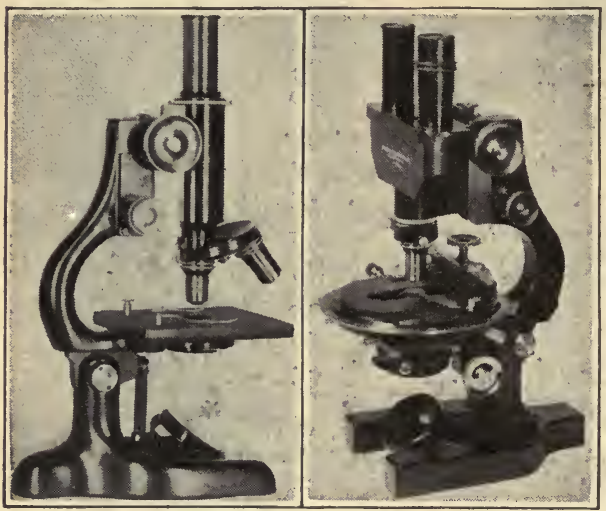

The microscopes of to-day vary from the kind we use in our school to those designed for elaborate research purposes. much more delicate than the coarse adjustment.

III. The optical system includes the part of the microscope containing the lenses. It consists of two sets of lenses in metal cases. The set placed at the lower end of the tube, near the object, is called the objective. On most microscopes there is a special attachment at the base of the tube for carrying two or more objectives conveniently; this is the nosepiece. With the use of the nosepiece it is possible to change from one objective to another with no loss of time. The objective that is in line with the tube is the one in use. In general, the longer the objective, the greater is the magnifying power. Most microscopes have low-power and high-power objectives. At the top of the tube, near the eye, is a set of lenses in a metal case called the eyepiece. This is easily taken out of the tube.

IV. The illuminating system makes possible the adjustment and focusing of the light on the object. It consists of the mirror, hung under the stage, and of the diaphragm, inserted in the opening of the stage. The mirror usually has two faces, one flat and one concave. The mirror can be turned in all directions and is used for throwing a beam of light from the window (or suitable lamp) upon the object and through the eyepiece, into the eye. The diaphragm is an arrangement for enlarging or diminishing the amount of light coming through the stage, by making the opening larger or smaller.

$\mathrm{V}$. Some microscopes have a joint in the pillar just below the stage, permitting the upper part of the stand to be tilted into a more convenient position. 
There are usually one or two clips fastened on the upper surface of the stage. These are slipped over the edges of the slide to hold it stationary.

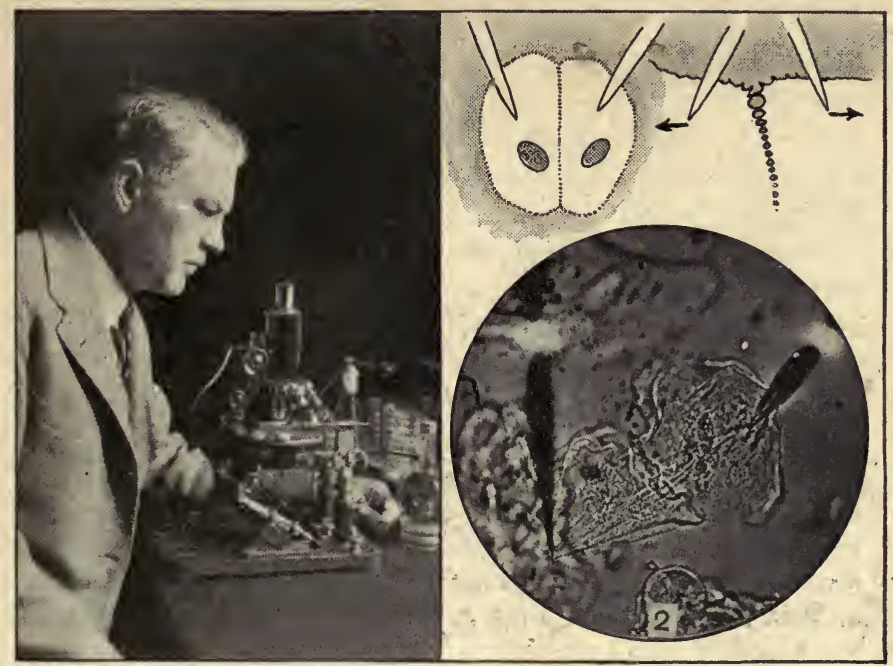

Professor R. H. Chambers has devised a microscope under which he can dissect out portions of a cell or inject fluids into the different parts of the cell. The drawings (upper right) show how he tears the cells apart. The other illustration is a photomicrograph showing a phase in the actual operation.

Directions for caring for a microscope. In lifting or carrying the microscope, grasp it firmly by the special handle above the stage, and carry it in a horizontal position. Then the removable parts cannot fall and break.

Allow nothing to touch any of the optical parts except especially prepared lens paper or a clean linen handkerchief, otherwise the ground surfaces will be scratched.

Before putting the microscope away, turn the nosepiece so that the objectives are not in a line with the tube. They are then protected by the stage, and the microscope is not apt to be damaged in placing it in the case.

Turn the clips in so they will not be broken.

Always follow the rules given below, for focusing. 


\section{Rules for' Focusing}

1. Revolve the nosepiece until the shorter objective is in a direct line with the tube. A click will be heard when this is accomplished.

2. Place your prepared slide on the stage so that the specimen is in the center of the opening of the stage.

3. Turn the mirror toward the nearest source of light. Manipulate it until a bright area appears on the slide.

4. While looking at the microscope from one side, turn the coarse screws clockwise until the low-power objective is one eighth of an inch from the slide.

5. Then look through the eyepiece and turn the coarse adjustment screws toward you (counterclockwise) until the specimen is clearly seen. If you wish to see more of the specimen than is shown in the field of vision, take hold of the slide with your thumbs and slowly move it in different directions.

6. Turn on your high-power objective. Listen for a click.

7. Use your fine adjustment screws. Turn them carefully (clockwise) until the object is clearly seen. The fine screws should be used constantly in focusing with high power in order to see all that can be seen.

Problem. How does an object appear when secn through the microscope?

Material : Compound microscope, slide, cover slip, pice of printed paper with very small type.

Method: Place a drop of water on the slide. Place a small piece of printed paper in the water and cover with a cover slip. The piece of paper is now mounted.

Lay the prepared slide on the stage, with the object as near as possible to the center of the hole in the stage. Follow your rules for focusing under low power, then under high power.

I. Look through the microscope at the printed letter.

II. Describe the texture of the paper as revealed.

III. Draw the single letter, preferably the letter " i," exactly as it appears under the low power. Note any apparent change in position.

IV. By means of a ruler measure your drawing; then measure the letter unmagnified. How many times does the low power appear to magnify? 
V. Turn on the high power. Again draw the letter, or as much as you can see of it, as large as it appears. Measure your drawing with your ruler and see how many times the high power appears to magnify the letter.

VI. Have your teacher tell you how much the microscope really enlarges the object with low and high power. Caution. Always find your object with the low-power objective, then turn on the high power. If you lose your highpower focus, go back to low power and refocus.

Problem. How do onion cells appear when viewed through the microscope?

Cut an onion into halves or quarters. Peel off one of the scalelike leaves. By means of a pair of forceps or knife strip the thin tissue from the inside of the scale. Mount a part of it on a glass slide in a drop of water and cover with a cover glass. Be sure to flatten your specimen before covering it. View the material under the microscope. Then remove cover glass, and stain the material by adding a small drop of dilute iodine solution. Again cover. Observe with low power, then high power.

I. The little divisions that make up the onion membrane are called cells.

$A$. The boundary of each cell is the cell wall.

$B$. Describe the color and shape of these cells.

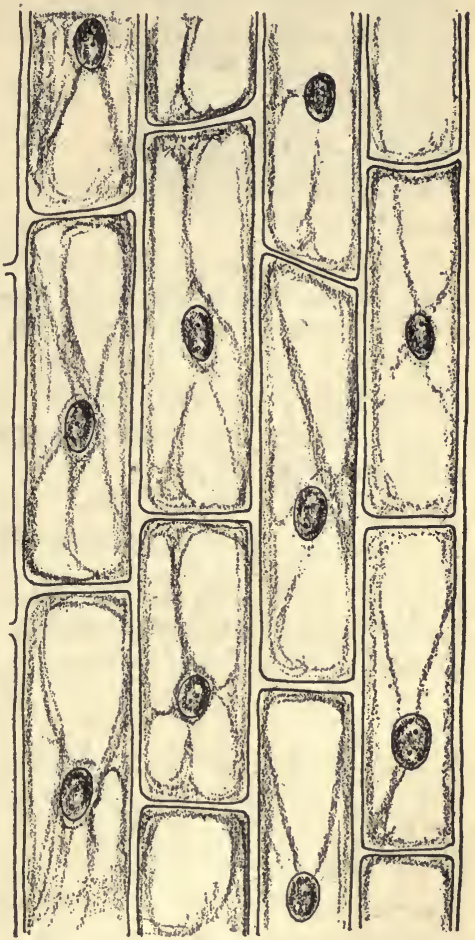

The cells of the onion tissue often show the heavy, woody walls and the large vacuoles that are characteristic of most plant cells.

II. Observe the position of the granular and clear areas. The granular areas make up the living part of the cell and are composed of a material called cytoplasm. The clear areas are the vacuoles or fluid-filled spaces in the cytoplasm.

III. Find a small dense area somewhere in the cytoplasm. This is the nucleus. A. Each nucleus has two disklike structures called nucleoli. Try to identify a nucleolus in a nucleus.

WH. FITZ. AD. BIO. -3 
IV. Make the following drawings in your research notebook :

$A$. A careful diagram of a group of cells five times as large as they appear under low power.

$B$. A group of cells twice as large as they appear under high power. Label cell wall, cytoplasm, vacuole, nucleus, nucleolus. Under the drawing, in small figures, state how many times enlarged your drawing has been made; for example, $\times 4$; $\times 5$; $\times 2$.

\section{Questions}

1. What is the difference between a simple and a compound microscope?

2. Classify the parts of a microscope into three divisions.

3. Name two effects a microscope has on objects.

4. Explain how to focus with the low power; with high power.

5. Why can we focus only thin material with our compound microscopes?

6. How do you mount a specimen, for study under the compound microscope?

\section{Supplementary Reading}

Locy, W. A., Biology and Its Makers (Henry Holt \& Co.). Locy, W. A., The Growth of Biology (Henry Holt \& Co.). Traeger, Alfred, The Microscope (E. Leitz Inc., 60 East 10th St., N. Y.). 


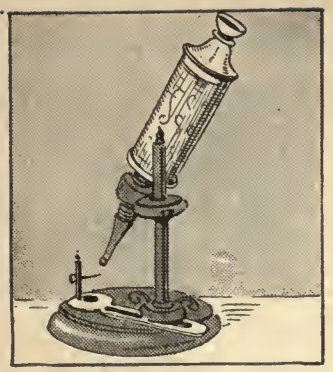

Robert Hooke's microscope.
CHAPTER IV

\section{THE HISTORY OF CELLS}

What he said:

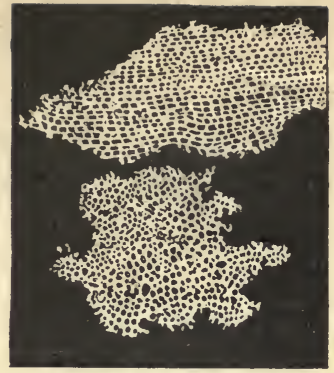

What he saw and drew.

"I took a good clear piece of Cork, and with a Pen-knife sharpened as keen as a Razor, I cut a piece of it off, and thereby left the surface of it exceedingly smooth, then examining it very diligently with a Microscope, me thought I could perceive it to appear a little porous, much like a Honey-comb."

How were living things first investigated? What were some of the results of these studies? What scientists contributed to this work?

The pages of history reveal how and by whom the terms used in describing the cell have been given to the science of biology. It was comparatively easy for us to find the structure of an onion cell under the compound microscope, but it has taken scientists three centuries to perfect the microscope and find out as much about cells as is known to-day.

The cell as first described. With yery crude lenses, arranged something like those in our compound microscope, Robert Hooke (1635-1703), an Englishman, examined a thin section of cork. He saw little boxlike structures which he called cells. He drew diagrams and published the results of his investigations. He saw only the cell wall after the living matter had disappeared, but the term cell, first used by him, has been retained.

The nucleus. Robert Brown (1773-1858) was a Scotchman. He studied medicine and became a surgeon's mate of a British regiment in Ireland. During his early years, and while connected with the army, he collected and studied plants and became known as a naturalist. In studying orchids, he discovered a structure in 
the cells which he called the nucleus; and later he found a like structure in the cells of many other plants. He wrote the following account of his discovery: "In each cell of a great part of the orchid family, a single circular area, generally somewhat more opaque than the membrane of the cell, is observable. The nucleus of the cell is equally manifest in many other families.

Theodore Schwann (1810-1882) found, as a result of careful work, that all the animals he investigated were made of cells. At the same time a friend of his, Matthias Schleiden, discovered that all of the plants he observed were made of cells. In the cells they studied, they both noted the nucleus that Brown had first discovered. They then made the supposition that all living things are made of cells. This became known as the cell theory, and this theory, having since been checked and rechecked by many scientists, and more facts added, is now accepted as a fact.

Protoplasm was discovered by a French naturalist, Felix Dujardin, in 1835, and named by Purkinje in 1840. But the scientist who made the name protoplasm best known was Hugo von Mohl (1805-1872). He was born in Stuttgart, Germany. He was graduated in medicine from Munich, became a professor of physiology at Berne, and later a professor of botany at Tubingen. He observed and analyzed the cell contents and described the movement in the cell of certain small bodies which later were termed chloroplasts. These were later shown to be the structures that contain green coloring matter. He brought into general use the term protoplasm and the fact that it is a living, streaming, growing, dividing substance.

Problem. How do the cells of Elodea appear when viewed through the microscope?

Examine the appearance of a single leaf of the Elodea. Mount it in water and heat by holding in the palm of the hand for a few minutes. The heating will activate the protoplasm. Focus with low, then high power. 
By slowly changing the focus you will be able to see that the Elodea leaf is made of more than one layer of cells.

I. What striking difference do you observe between Elodea and onion cells?

II. Describe the new structures that you observe in Elodea cells. These are called chloroplasts or green color bearers.

III. Observe two conspicuous differences between the chloroplasts and the rest of the cytoplasm.

IV. Trace the movement of the chloroplasts. Do they move around in the individual cells or do they pass from cell to' cell? Suggest a reason for your answer. The chloroplasts are carried by the movement of the streaming protoplasm.

V. Remove the cover glass, add a drop of dilute iodine, re-cover, and mount again.

VI. Describe the structures you now observe, that were also present in the onion cell.

VII. Make a diagram, times five, of a few of the cells of the Elodea. By means of arrows, trace the pathway of the moving chloroplasts. Label cell wall, cytoplasm, nucleus, vacuole, chloroplast.

Protoplasm, an active substance. The movement of protoplasm, observed by you in Elodea, was first observed by Von Mohl. He recognized that the living, streaming, moving protoplasm was, the cause of the motion that we observed in our Elodea cells.

Another name should be added to the list of those who devoted a large amount of their time to the study of the cell. Max Schultze, a German anatomist, in 1863, clearly brought out the fact that protoplasm (nucleoplasm and cytoplasm) is the unit of all physiological activities. Before his time, people thought that plant cells were made of one kind of living material and animal cells of another kind. Much has been added to the work of Schultze, but many of the facts discussed in the following paragraph are largely a result of his efforts.

The key to every biological problem is sought in the cell; for every living organism is, or at some time has been, a cell. As 


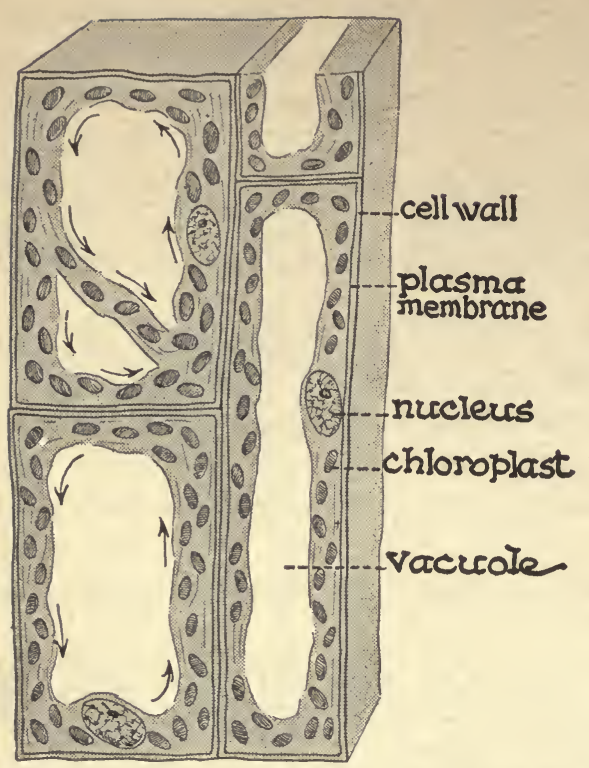

Compare the cells of Elodea shown in the diagram with those you studied under the microscope.

a result of the studies of the scientists previously named, the cell theory was further developed. It was now stated that all plants and animals are made of cells and cell products. The cell is the structural and physiological unit of the organism. The living organism can perform certain functions because its cells are adapted to perform these functions. Other scientists, using this theory as their basis, have been able to show the importance of the cell in the development of organisms and something of its importance in heredity. Many scientists are still investigating the structure and functions of cells and others are studying the cell in its relation to the development of the organism. This work, in spite of the fact that three centuries have passed, is still in a developmental stage.

Cellular nature of plants. The cells of green plants are not all alike in size and shape. A narcissus leaf is made of many layers of cells which vary in shape.

Problem. Study of the epidermis of the narcissus leaf.

I. Peel off a small strip of the thin lower surface (epidermis) of a narcissus leaf. Mount it for study, and focus it under the microscope.

$A$. Describe two different kinds of cells that are seen.

$B$. Note the small oval opening between paired cells. Suggest a use for these openings; for the cells that form them. 
II. Draw a few of the cells, making sure to include in your diagram, at least two differently shaped ones.

Higher plants are composed of many cells, but certain plants are very simple in structure. A green coating is frequently found on the north side of many buildings and of most trees. This is caused by the presence of thousands of tiny one-celled, green plants called Pleurococcus.

\section{Problem. Study of Pleurococcus.}

I. Scrape some of the green material on to a slide, mount in water, and study with low and high magnification. Describe the shape, color, and structure of the cells.

II. Draw a single cell and a group of cells. Label cell wall, the very large chloroplast which almost fills the cell, and the nucleus.

III. Write a description of what you have seen and tell how Pleurococcus differs from Elodea and narcissus cells.

A typical green cell that is easy to study is Spirogyra. As it floats on the surface of ponds, it looks like a tangled mat of green threads or hairs. A common name for it is pond scum or frog's "spittle." This latter name is given to it because it is slimy in texture and is found in the same habitat with frogs. Many people at one time thought that the frogs spit it out.

Problem. Study of the cells of Spirogyra.

By means of forceps and a pair of scissors detach a few strands of

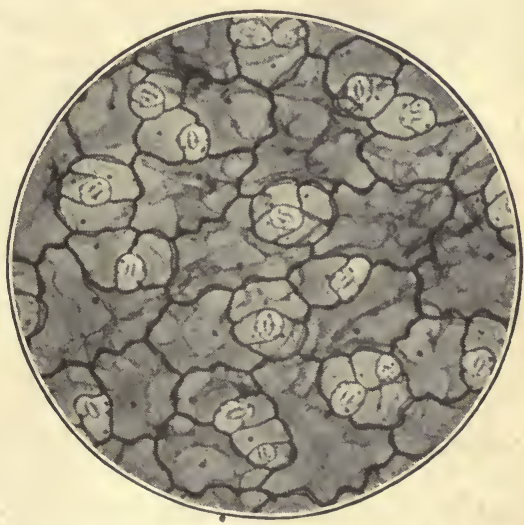

A photomicrograph of the lower surface of a leaf. Spirogyra. Mount them on a slide and cover with a cover slip. Focus the specimen under the low power. 
I. Describe the cellular structure of a single strand of Spirogyra. Whenever unicellular plants exist singly, end to end, the formation is called a filament.

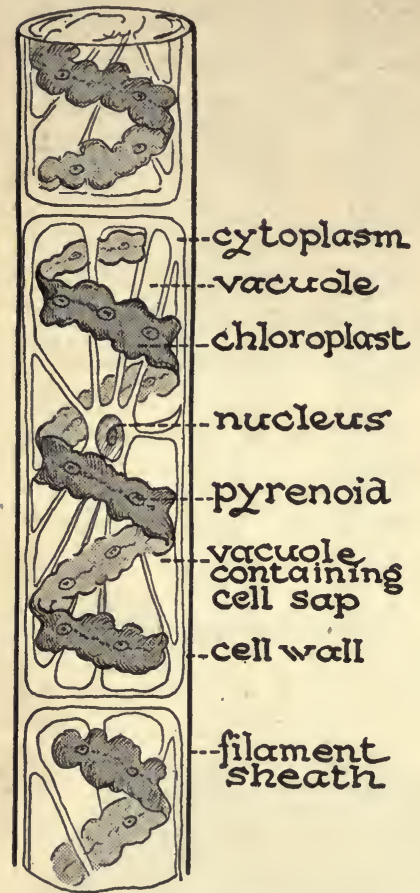

Spirogyra is a single-celled plant. These plants grow end to end and thus form strands called filaments.
In reality, each cell is a complete unit and in no way dependent on the adjoining cells.

II. Describe a structure in the cell that suggests the reason for the name Spirogyra. The name Spirogyra means coiling circles (spira-coil; gyros-circle). What resemblance is there between a chloroplast of Spirogyra and a chloroplast observed in the Elodea?

III. State the number of complete chloroplasts observed in each cell of your specimen.

$A$. State the number of complete turns found in each chloroplast.

$B$. Using the words wavy, irregular, or straight, describe the edge of the chloroplast.

IV. Identify the cell wall and the filament sheaths. This sheath is gelatinous and gives the strands their characteristic slimy texture.

V. Draw a cell five times larger than the one observed under the low power. Show in your drawing how the adjoining cells lie in relation to the one drawn. Label cell wall, filament sheath, chloroplast.

VI. Stain a cell with dilute iodine and focus under low power and then under high power. Locate and describe the nucleus, nucleolus, and strands of cytoplasm.

VII. Describe the location and suggest the nature of the contents of the vacuoles left among the strands of cytoplasm. The part of the cytoplasm inclosing the vacuoles is called the plasma membrane.

VIII. Locate the structures stained blue. These are specialized cytoplasmic structures called pyrenoids. What evidence is present that the pyrenoids store starch? 
IX. Draw a complete cell of Spirogyra as observed under the high-power objective. Label filament sheath, cell wall, strands of cytoplasm, plasma membrane, vacuole, nucleus, nucleolus, spiral chloroplast, and pyrenoid.

$\mathrm{X}$. Write a description of Spirogyra, mentioning all the parts that you saw.

The simplest plants have no root, stem, nor leaves. Such plants are called Thallophyta (thallus - shoot; phyton - plant). The green thallophytes are called algae. Their color is due to the presence of chloroplasts that vary in size and shape. The algae that we have already studied are Pleurococcus and Spirogyra. Other thallophytes lack chloroplasts and are usually
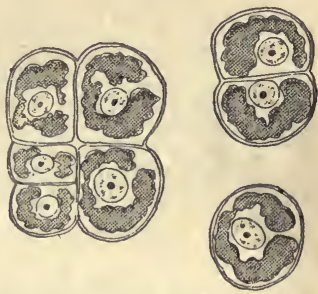

Pleurococcus is found as single plants or in groups. colorless. They are called fungi. Yeasts and molds are common examples of fungi.

\section{Questions}

1. Outline in tabular form, the names of the scientists, their nationalities, century in which they worked, and their contributions to the cell theory.

2. Draw as many labeled cells as are necessary to show cell wall, nucleus, cytoplasm, vacuole, pyrenoid, and chloroplast.

3. Compare Spirogyra with another alga that you have studied.

4. What makes Spirogyra slimy?

5. What is a reason for the name thallophytes?

\section{Supplementary Reading}

Gager, C. S., General Botany (P. Blakiston's Son \& Co.), chap. ii.

Haupt, A. W., Fundamentals of Biology, (McGraw-Hill Book Co. Inc.), chap. iv.

Holman, L. A., and Robins, W. W., Textbook of General Botany (John Wiley \& Sons).

Holmes, S. J., General Biology (Harcourt, Brace \& Co.), chap. iii. 


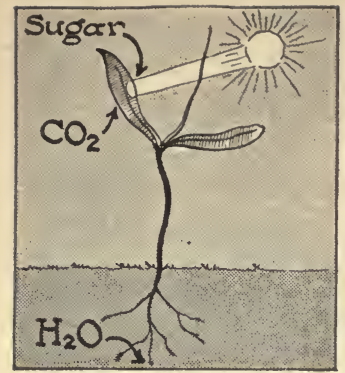

Nature's food factory.

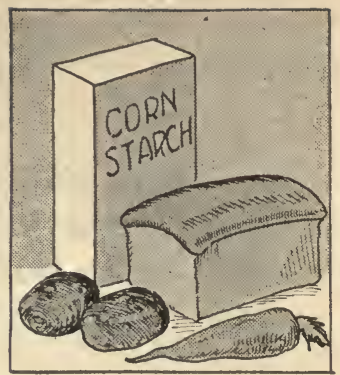

Some of the products.

What does a green cell use for food? Where does it get this food? What are the physiological activities of cells containing chlorophyll?

In order to live, a green cell must carry on certain functions. Because these functions are carried on within the organism they art called physiological functions. These functions may be classified as nutritive, adaptive, and reproductive. The nutritive processes can be further subdivided into absorption, food manufacture, food storage, digestion, assimilation, secretion, growth, respiration, and excretion. These have to do with the obtaining and the using of food. The adaptive processes have to do with the way the organism maintains itself and behaves in its environment. The reproductive processes have to do with the producing of new individuals.

Absorption. The Spirogyra takes in carbon dioxide, water, and mineral matter for the manufacture of food. These raw materials are in the form of gases and solutions and enter through the cell membrane. They remain in the vacuoles until they are needed. Oxygen, also, is taken in during the breathing process. The process by which material passes through a cell membrane is called osmosis or absorption. Many theories are advanced to explain this activity; but probably no-one theory holds under all conditions. According to one explanation there are minute openings in the membrane through which the liquid or gas passes. 
According to the molecular theory, all matter, whether solid, liquid, or gas, consists of molecules in constant motion. The molecules of solids are close together and there is very little space for them to move. There is more space between the molecules of liquids, and still more between those of gases. Consequently, molecules of liquids move more freely than those of solids, and molecules of gases move even more freely than those of liquids.

As the molecules in gases or solutions keep bounding up and down, knocking against each other and against the cell membrane, some of them move through the small openings. If the molecules are very active, as in gases or certain liquids, these molecules can pass readily through the membrane. The molecules of denser liquids move more slowly and therefore will take a longer time to go through the membrane. In general, the rate of osmosis of a substance depends upon the number of its molecules. During the process of osmosis the molecules of any substance pass through a permeable membrane more rapidly from the area of greater concentration to an area of lesser concentration. Osmosis never stops, although when there is a balance of materials on either side of the membrane, there may be so even an exchange of molecules that osmosis is not obvious. When the membrane is a living one, as in the cell membrane, the cytoplasm exercises a definite selective action, taking in and keeping in the materials needed by the cell and eliminating those not needed.

Demonstration. To illustrate the movement of molecules.

Make a very dilute solution of powdered carmine or India ink in water. Place a drop under the compound microscope and observe under both low and high magnification.

Describe the behavior of the particles observed in this experiment. According to the molecular theory, molecules are always in a state of motion somewhat similar to that of the particles observed by you.

Robert Brown, who was the first person to observe the nucleus in plant cells, first described the movement that you have just 
observed. This is called the Brownian motion. Modern physies teaches that molecules move not only in solution and during osmosis, but that the molecules of all matter are constantly bombarding each other. The desk on which you write is composed of tiny particles not inseparably cohering and sticking to each other, but in a slight, constant state of activity similar to that which you observed in the particles under the microscope.

Food manufacture. A green cell is one containing certain organized and specialized cytoplasm, called chloroplasts. There is found in these chloroplasts a complex, green material, chlorophyll, which is necessary for the manufacture of food. The green cell is independent because it does not rely on other organisms for its food; it manufactures its own. All cells, whether green or not, need carbohydrates (sugar and starches), fat, and protein. The cells manufacturing foods always absorb two compounds: a gas, carbon dioxide $\left(\mathrm{CO}_{2}\right)$, and water $\left(\mathrm{H}_{2} \mathrm{O}\right)$. These raw materials get through

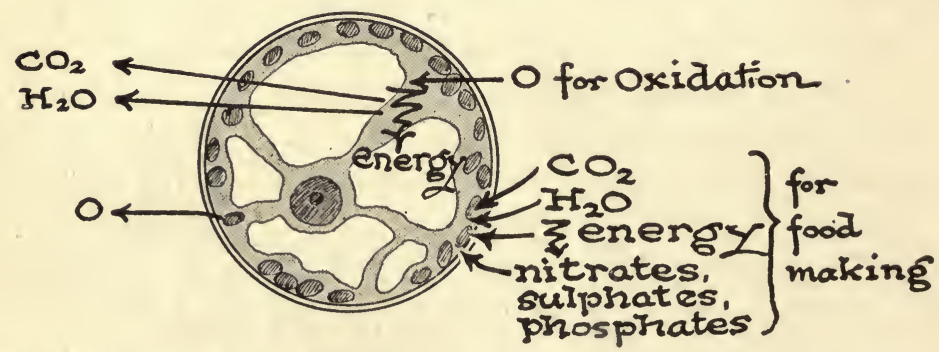

There is a continuous income and outgo of gases during respiration and photosynthesis.

the cell wall and plasma membrane by the process of osmosis. Then the chloroplasts by a number of complicated processes, with the aid of sunlight, chemically tear apart the elements of these compounds, water and carbon dioxide, and recombine them into simple carbohydrates. Since more oxygen is contained in the raw materials than in the sugar manufactured, oxygen is given off during the process. It is supposed by some that the elements 
carbon and oxygen, found in the compound carbon dioxide, chemically join with the elements hydrogen and oxygen, found in the compound water, and give rise to a more complex compound of carbon, hydrogen, and oxygen, probably formaldehyde. In this chemical process, oxygen is left over and is given off by the plant.

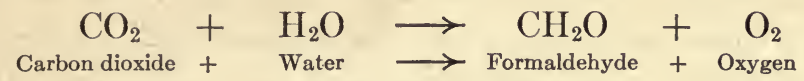

This process is one of the early steps in photosynthesis (phoso light; syn - together; tithemi - place: light places together). Photosynthesis is the combining of carbon dioxide and water by chlorophyll, in the presence of light, to make sugar.

The molecule of formaldehyde is then chemically joined with other molecules of formaldehyde to make a simple sugar. Further combining will build up more and more complex sugars such as monosaccharides, disaccharides, polysaccharides (starch is in this group). Sometimes other complex compounds such as oils and fats are produced. These are often feund in cells as storage products. They are again converted into simpler forms of sugar when they are to be used as fuel.

Sugar is also the basis for the formation of proteins. In order to manufacture protein a process known as protein-
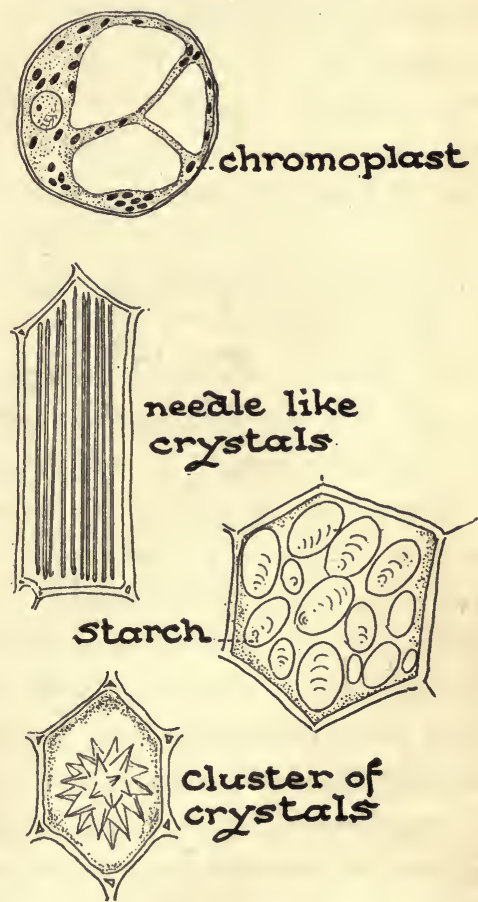

Cells may contain inclusions such as plastids (color-bearing particles), starch granules or crystals of various forms. 
synthesis - the plant absorbs minerals, in the form of nitrates, sulphates, and phosphates, which are dissolved in soil water. These substances furnish the plants with nitrogen, sulphur, and phosphorus which are thought to combine with some of the sugar to form protein, a substance containing carbon, hydrogen, oxygen, nitrogen, sulphur, phosphorus, and perhaps other minerals. Protein in protoplasm may be represented by the symbols $\mathrm{C}-\mathrm{H}-\mathrm{O}-\mathrm{N}-\mathrm{S}-\mathrm{P}$. Most protein is used for growth (protoplasm making), repair, and storage. Some is decomposed and the sugar (one of the products of decomposition) is burned as fuel, while the other products are thrown out as nitrogenous wastes or stored for further protein-synthesis.

Digestion. When the food substances that have been stored in the cells of the plant as fats, starches, and proteins are to be used, they must first be changed to a soluble form. The process of changing an insoluble nutrient into a soluble form that can be used by the organism is called digestion. Digestion is brought about by the presence and action of chemical substances called digestive enzymes. These act as catalytic agents, that is, they bring about a chemical change, but are themselves not affected by nor changed during the process. In the cell where digestion takes place, there is an amylase for the breaking down of starch, a protease for the digestion of proteins, a lipase for the digestion of fats. (The names of most of the enzymes end in ase.) After being digested, the liquid nutrients may be carried by means of the streaming, flowing motion of the protoplasm to that part of the cell where they are to be utilized. If there is an excess of food nutrients, they may again be combined into an insoluble form and stored in the cells until needed.

Respiration. Each living plant cell takes in oxygen through the cell membrane and gives off carbon dioxide by means of diffusion. The oxygen unites with the compounds of hydrogen, oxygen, and carbon and releases the energy, in the form of heat, stored in them. 
This union is a type of oxidation. The energy released from food was stored up at the time of the manufacture of food (photosynthesis and protein-formation) in a potential, latent or dormant form. The sun is the source of this energy. When oxidation of food occurs, the potential energy is converted and released as kinetic, an active form of energy. In plant cells, the kinetic energy takes the form of work energy or heat energy, resulting in the maintenance of a normal temperature. As a part of this oxidation process, wastes, carbon dioxide and water, are formed.

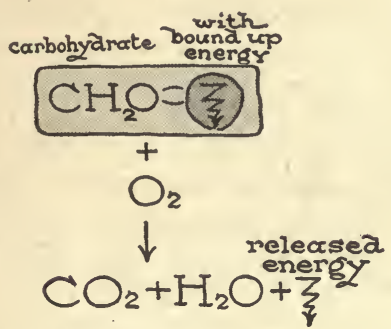

The green plant cell builds up carbohydrates which contain bound-up energy. This may later be set free as heat or work energy.

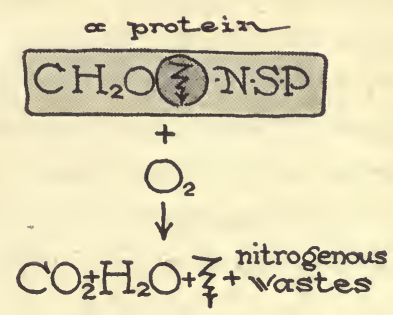

A protein may be broken down during oxidation and energy released. Among the resulting wastes are some containing nitrogen.

These the cell gives off. If respiration takes place at the time the plant is actively making sugar, the carbon dioxide and some of the water resulting from oxidation may be retained for further photosynthesis. Nitrogenous wastes are probably retained for further manufacture of protein. The oxygen released during photosynthesis may be retained for oxidation although it is usually given off. Thus a balance of materials may be maintained. Respiration, then, involves the taking in of oxygen, the oxidizing of certain food materials present, and réleasing energy and waste products. The respiration process is the means of releasing all of the energy needed by the plant.

Assimilation. The protoplasm of the cell is built of the elements $\mathrm{C}-\mathrm{H}-\mathrm{O}-\mathrm{S}-\mathrm{N}-\mathrm{P}$, and sometimes others. After protein manufacture is accomplished, the lifeless protein together with water and 
mineral materials may ultimately be transformed into living plant protoplasm. Scientists have not determined just how this trans-

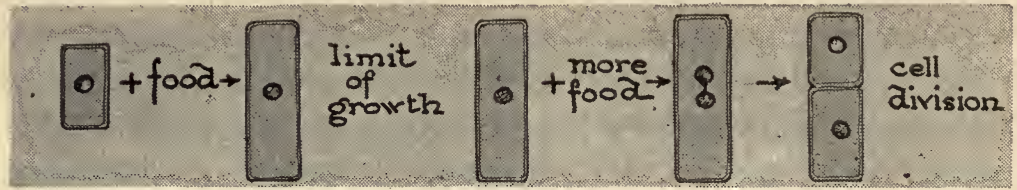

A green cell makes and assimilates food. Growth occurs. When no further growth is possible, the nucleus elongates, divides, and then the cell divides. Thus two cells are formed.

formation takes place. The name given to the change of a soluble protein into living protoplasm is called assimilation. True growth in an individual cell is due to the increase in the sum total of the amount of protoplasm resulting from assimilation. Cells may also increase in size, temporarily, when the cell vacuoles become filled with an abundance of watery sap. The entire plant organism grows by enlargement of the individual cells, until they attain a certain size. Then they divide, causing an increase in the total number of cells. This process of cell division will be discussed later.

Excretion. Any product given off through the cell wall as waste is called an excretion. If a product is built up and given off for a further use in the organism, it is called a secretion. Often, some oxygen formed during food manufacture is held and used for oxidation. The waste products from oxidation of sugars are carbon dioxide and water. When these wastes are not retained by the cell for further food making, they are excreted through the cell wall.

Irritability. There are many substances and forces, called stimuli, that act upon a green plant cell. A cell is spoken of as being sensitive or irritable to light, temperature, touch, chemicals, electricity, and many other stimuli. The real cause of the effects produced by these stimuli is to be found in the nature of protoplasm. It is the protoplasm that is irritable. Response is the name given to the reaction the organism makes to the stimulus. We have seen the effect on the protoplasm of the Elodea cell by 
holding it in a warm hand for a few seconds. Other stimuli have other effects depending upon the sensitiveness of the particular cell.

Cell division. A plant cell has a certain limit of size beyond which it does not grow. When this limit is reached, the nucleus may elongate and divide, producing two nuclei. There is a wall formed across the cell dividing the protoplasm into two parts, each containing one of the daughter nuclei.

\section{Questions}

1. Why may chlorophyll be considered the source of the food supply of the world?

2. Discuss the transformations of energy in a green plant.

3 . If green plants are eaten by animals, what additional transformations of energy may result?

4. Compare the oxidation of food in the plant cell with the oxidation of coal in the school furnace, giving points of similarity and difference.

5. Compare respiration in a green plant cell with photosynthesis, considering materials used, products formed, products excreted, and the purpose of the functions.

6. Why may carbon be considered the real fuel of a green cell ? In what form did it enter the cell? In what form did it ultimately leave the cell? Name all physiological functions involved in the use of carbon.

7. Trace the probable source of the carbon in coal.

8. Discuss a balanced aquarium, stating the give and take of materials between fish and green plants.

9. In the figure on page 36 the possible intake and outgo of materials in a green cell are shown. What are the actual interchanges of materials?

\section{Supplementary Reading}

Coulter, Barnes, and Cowles, A Textbook of Botany (American Book Co.), Vol. I.

Gager, C. S., General Botany (P. Blakiston's Son \& Co.).

Holmes, S. J., General Biology (Harcourt, Brace \& Co.), chap. vii.

WH. FITZ. AD. BIO. -4 


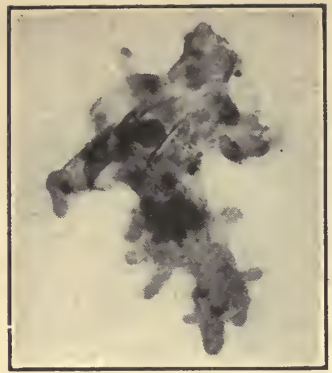

Photomicrographs of Amoeba
CHAPTER VI

TYPICAL ANIMAL CELL

Do animals ever consist of single cells? Can the simplest of animals be easily recognized as animals? Do they appear different from onecelled plants? Do they perform the same physiological activities?

Laboratory exercise. Study of an amoeba (amoibos - changing). The amoebas belong to the branch of animals called Protozoa (protos - first; zoonanimal : first or early animal). To locate amoebas, scrape the undersurface of the leaves of Elodea on to a glass slide. Add a drop of water and cover with a cover glass. Another method for finding amoebas is to crush some pond lilies or other water plants and let the mass remain undisturbed for a few weeks. Tiny animals will usually be found in the scum which forms. Mount some of the scum and locate an amoeba with low power, then study it with high power. Later stain with iodine and again observe.

I. Describe the amoeba, including shape, color, and number of cells.

II. How does its shape differ from any cell previously observed? The irregular projections of protoplasm are called pseudopodia or pseudopods (pseudo - false; podium - foot). What seems to be the function of the pseudopodia or false feet?

III. Describe the movement of the animal. Suggest a reason for the name amoeba.

IV. After staining it with iodine, find cytoplasm, nucleus, -and a space called the contractile vacuole. The clear outer protoplasm of the cell is called ectoplasm and the granular inner area is endoplasm.

V. State three structures found in the Spirogyra not found in the amoeba, and one structure found in the amoeba not found in the Spirogyra.

VI. State a difference in nutrition between Spirogyra and amoeba. 
VII. Name a function performed by the amoeba that the green cell cannot perform. What is the value of this function to an animal organism?

VIII. Make a drawing three inches in diameter, of an amoeba, and label cytoplasm, nucleus, plasma membrane, pseudopodia, and vacuole.

Life functions of an amoeba. Ingestion. When an amoeba comes in contact with a particle of food, it throws out pseudopodia which flow over and around the food. The food particle is then taken into the cytoplasm. This engulfing of food by the amoeba is its method of food getting, or ingestion. The amoeba shows little power of selection of food. It takes in almost anything small enough to be engulfed. The cytoplasm encloses the food and forms a vacuole or bubble encircled by plasma membrane. The food and some fluid deposited by the cytoplasm are inside the vacuole.

Digestion. The engulfed food is principally smaller microörganisms and particles of plants. These consist of combinations of the nutrients, proteins, fats, and carbohydrates. The particles circulate in the streaming cytoplasm in the form of a food vacuole. The cytoplasm secretes digestive enzymes to convert these nutrients into a soluble form which can then be assimilated by the protoplasm. As the circulating protoplasm carries the food around the cell, the digestive enzymes, protease, lipase, and amylase, gradually dissolve the nutrients in the food. These nutrients pass through the

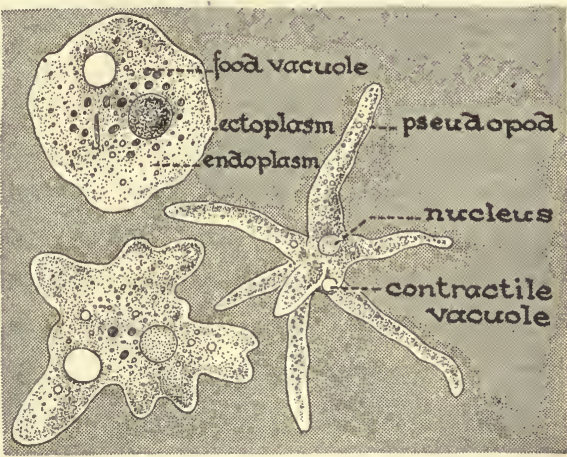

Amoeba may assume a variety of shapes. Compare the animals you studied under the microscope with these diagrams.

plasma membrane of the vacuole by osmosis and mingle with the cytoplasm. 'The indigestible materials that have entered with the food may be eliminated from any part of the organism. 
Assimilation. The digested protein together with water and absorbed mineral matter are converted into protoplasm by the ac-
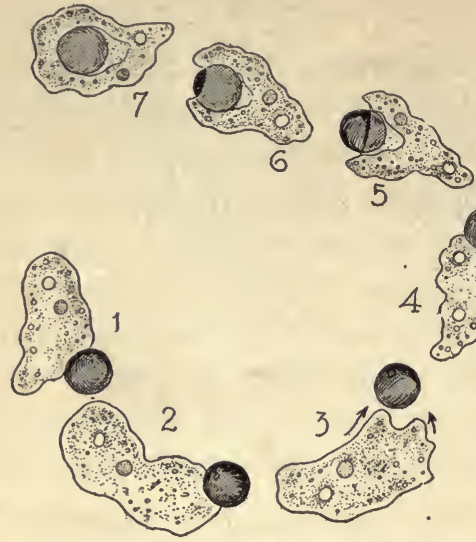

A patient investigator watched and described the behavior of an amoeba as it went after a spherical food particle which had rolled away. The drawing shows how the amoeba pursued and engulfed the particle of food. tivity of the cytoplasm of the living amoeba. This is assimilation. As a result of assimilation, new protoplasm is formed and the animal grows.

Respiration. Oxygen from the air in the water may enter through any part of the surface of the amoeba. It meets the digested nutrients in the cytoplasm and oxidation takes place, that is, oxygen combines with carbon and hydrogen compounds. As a result of oxidation, energy is released. This energy enables the amoeba to carry on its functions. Since protein, fat, and sugar contain carbon and hydrogen, carbon dioxide and water are oxidation products. Protein gives rise to nitrogen, sulphur, and phosphorus products, also, one of which, urea, is a nitrogenous waste. The wastes resulting from oxidation are collected in the contractile vacuole. This bursts and expels the wastes through the plasma membrane into the surrounding water.

Reproduction. When the amoeba reaches a certain maximum size natural to its species, the nucleus divides in two parts and then the entire cell cleaves in two equal parts. This results in the production of two distinct cells each with its own nucleus. This process is called reproduction by fission.

Irritability. The amoeba is very sensitive to outside stimuli. If it is touched with a pointed object, it draws away. It moves 
from the object by sending out tiny projections of cytoplasm, which are known as pseudopodia.

If an amoeba comes in contact with food, it surrounds the particles. If touched with wires carrying an electrical charge, it rolls itself up into a ball as if shocked. It shows no activity at freezing temperature, and it evidences the greatest activity at a temperature of about $85^{\circ} \mathrm{F}$. It will become spherical, hard, and lifeless in very hot water. If a beam of light is directed toward one side, the amoeba will move away from the light and toward the darkened portion. If a grain of sugar is placed in a drop of water, the amoeba will move toward the sugar; if a grain of salt is placed there, it will move away. If, with a very fine pipette, a chemical is injected into the amoeba's cytoplasm, the animal is able to sever off almost immediately that part of the cytoplasm and will move from the rejected part as quickly as possible. Recently, Dr. R. H. Chambers has developed methods of micro-dissection. By means of a microscope and a set of tiny dissection instruments and capillary pipettes he can dissect or inject chemicals in or near specimens while they are mounted under the microscope.

The responses or activities of the amoeba called forth by stimuli, are tropisms (tropos - a turning). These responses may be toward

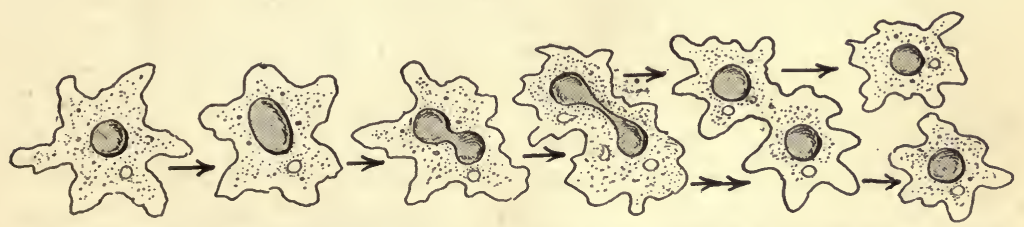

When the amoeba reaches its maximum size, the nucleus elongates and divides into two nuclei. The animal constricts and breaks apart into two daughter cells, each with one nucleus.

the stimulus, in which case the tropism is called a positive tropism and designated by a plus sign ( + tropism). If the resulting activity is a motion away from the stimulus, the tropism is con- 
sidered negative and is represented by a minus sign ( - tropism). The different tropisms are named in terms of the stimulus to which the organism is reacting. Listed below are the tropisms
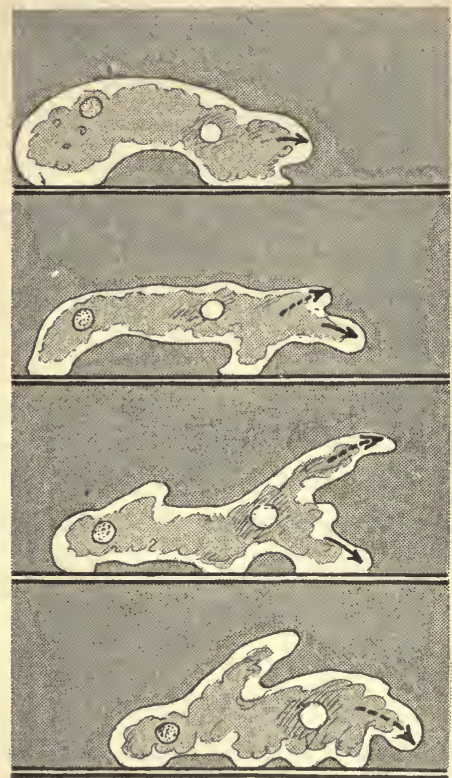

When the amoeba is viewed from the side, pseudopodia may be seen to extend which result in movements like walking.

direction. Gradually the rest of the cytoplasm carrying the nucleus flows into it; and again a thin stream is sent out. Thus the organism moves. By means of the function of locomotion it can get food and escape unfavorable conditions.

\section{Problem. Study of a Paramecium.}

Place some hay in a beaker of distilled water. Heat in order to soften the hay. Let the material stand two or three weeks. The addition of a little thyroid extract will usually promote the multiplication and growth of the organisms present. This is a Paramecium culture. 
I. Take a drop of the infusion, place it on a slide and cover with a cover glass. The microörganisms called Paramecia should be present.

$A$. Describe the cellular structure of a Paramecium.

$B$. How does its movement differ from that of the amoeba?

C. What proof have you that the Paramecium is cylindrical and not flat?

$D$. Describe the shape of a Paramecium. The end that goes forward most of the time is called the anterior end. The opposite end is the posterior end. Describe the shape of the anterior and posterior ends.

II. Put a few threads of cotton or some finely shredded lens paper on a glass slide. Place on this a drop of the Paramecium culture and focus under the low power.

A. Describe the appearance of the threads of cotton under the microscope. What effect do the threads of cotton seem to have on the movement of the Paramecium?

$B$. Note the furrow in one side of the animal. This is called the groove. At the base of the groove is a mouth, and the cylindrical structure below is the gullet.

C. Look closely at the plasma membrane. The structures that you should observe there, are projections of protoplasm and are called cilia. Describe any movements of the cilia. Suggest a function of the cilia.

$D$. The groove and gullet are also lined with cilia. Suggest a function for these cilia.

$E$. At either end of the Paramecium you will see a small clear bubble. Watch each bubble form, grow larger, and burst. These are the contractile vacuoles.

III. Stain the Paramecium with dilute iodine and mount under the low and high power. What evidence of ectoplasm and endoplasm can be seen? Describe a difference between these two kinds of protoplasm.

IV. Take a new culture and add a small amount of blue fountain pen ink to it, and again put a few threads of cotton on it.

$A$. Focus under the high power. Observe the long hairlike structures that are thrown out. These are protective structures of offense and defense called trichocysts.

$B$. Observe the two nuclei. The larger nucleus is called the macronucleus (makros - large) and the smaller, the micronucleus (mikros small). It may be necessary to stain the specimen with iodine to see these.

C. Draw a Paramecium three inches long and label all the structures observed.

D. Write a brief description of the Paramecium. 
The physiological functions of the Paramecium are somewhat more complex than those of the amoeba because of the increasing complexity of structure.

Nutrition. Bacteria and any other tiny plants or protozoa that are small enough are swept by the current created by the cilia

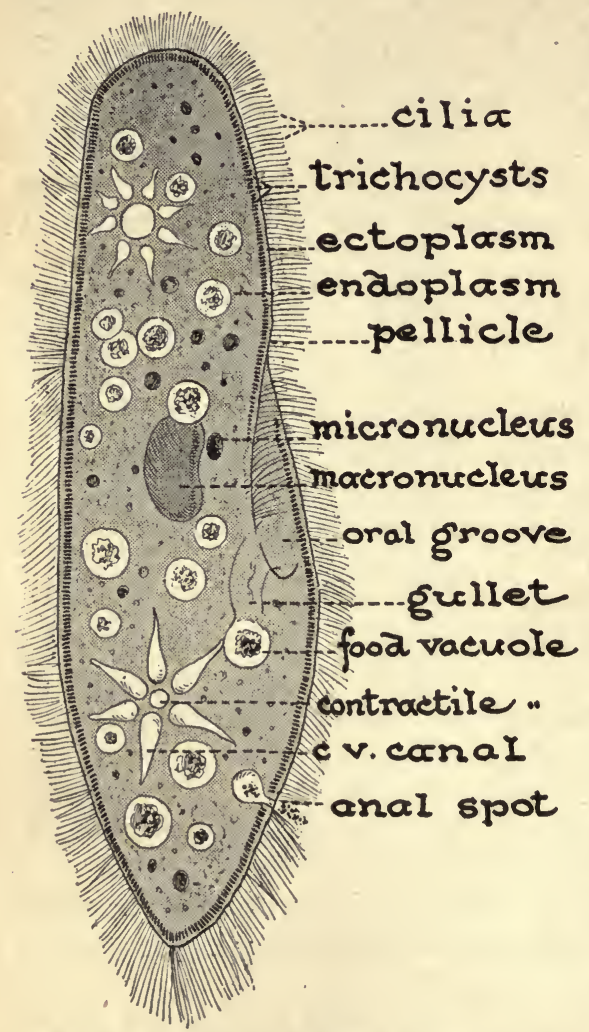

Note in this diagram the structures that you were unable to see in your microscopic study of the Paramecium. into the oral groove. These are driven through the gullet, where they are made into a food ball and discharged into the cytoplasm of the cell. The food ball is carried around as a vacuole by the streaming cytoplasm. The processes of digestion, circulation, assimilation, growth, and respiration in the animal are similar to those described for the amoeba. As the food is gradually digested, any material that cannot be digested is collected at a certain small area of the plasma membrane in the posterior end of the animal. The membrane breaks at regular intervals to throw off the solid food wastes. This mechanism is called the anal opening. Because the wall always breaks in the same region to let out the solid material, it is frequently called a weak spot. The wastes, carbon dioxide, water, and urea, formed 
in the oxidation process, are collected by tiny radiating canals and emptied into a central contractile vacuole at each end of the organism. When these vacuoles have attained a certain size, the cytoplasm. surrounding them contracts and discharges the wastes through the cell membrane. The contractile vacuole is constantly filling and discharging:

Locomotion. Cytoplasmic structures called cilia project through the cell membrane, and, by rapidly lashing back and forth, propel the animal through the water. The cilia are arranged in rows all over the body. All the cilia in a row beat at the same time. If, with a needle, the thread of cytoplasm which controls the beating of a row of cilia be cut, the entire row will cease to beat and remains paralyzed. Due to the more rapid beating of the cilia in the groove, the animal rotates and proceeds in a spiral rather than a straight path. It usually progresses with the blunt or anterior end forward, but can reverse its cilia and travel equally well with the pointed end forward. Such a reversion often takes place when it meets an obstacle. If confined in close quarters, the Paramecium can pass through small openings due to the elasticity of its body.

Reproduction. Reproduction occurs by fission, as in amoeba. Each of the two nuclei divides and each daughter cell contains a macronucleus and micronucleus. A new groove and gullet are formed in one of the cells and two new contractile vacu-

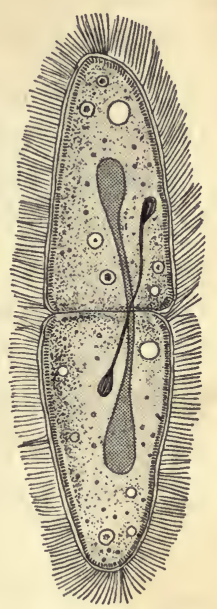

A Paramecium reaches a maximum size. The two nuclei elongate, a constriction appears, and the two daughter cells split apart. This is reproduction by fission. oles appear. When cell division or binary fission goes on indefinitely, the cells sometimes lose their vitality. When this happens they become smaller, and, in some cases, distorted. Under such conditions, two Paramecia come together. The cell membranes are dissolved at the point of contact and a bridge of cytoplasm 
is formed. The macronuclei disappear and the micronucleus goes through a series of complicated divisions. Finally one part of the nucleus of each cell passes over the cytoplasmic bridge and unites with a part of the micronucleus in the opposite cell. The animals then separate and there is a reorganization of parts to restore the structure to the normal state. This exchange and union of nuclei is called conjugation. The process seems to give the Paramecia renewed vigor and vitality. The cells may now go on dividing for hundreds of generations before conjugation again takes place.

It has been shown by one scientist that the lowered vitality of a colony of certain Paramecia may be caused by the surrounding media of their environment. If this becomes concentrated with wastes or materials that are unfavorable, conjugation may occur. On the other hand, if careful control is kept of this material in which the creatures live, and if abundant food is supplied and wastes removed, certain Paramecia will divide generation after generation without resorting to conjugation. L. L. Woodruff, a professor at Yale University, has kept a strain of Paramecia active for several years. Thousands of generations have been produced from the creature with which he started. By exercising great care to make

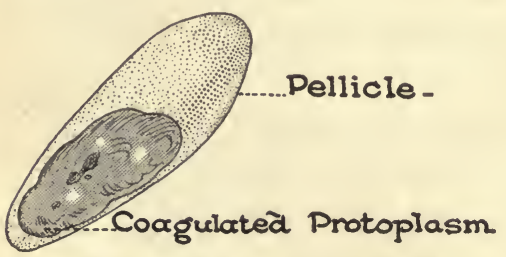

If alcohol is added to a Paramecium the protoplasm is dehydrated, the strands of cytoplasm, cilia, are pulled through the cell membrane or pellicle leaving the rows of openings in the pellicle. the surroundings most healthful for Paramecia, he has produced these thousands of generations without having them conjugate or die. This biologist believes that if conditions are carefully safeguarded and controlled, these tiny masses of protoplasm known as Paramecia will live forever.

Irritability. The reaction of Paramecia to external stimuli is much the same as in amoebas. Because of their cilia, they can respond more quickly toward or away from stimuli. They have a 
special set of protective structures called trichocysts. Each one of these structures consists of a pocket fitted with a long strand of cytoplasm which is thrown out when danger is near. This strand secretes a poisonous substance. The whole mechanism acts as a protection. A worm, much larger than a Paramecium, as it thrashes about, might injure the Paramecium. To protect itself, the Paramecium discharges trichocysts which will pierce or poison the enemy so that it will become inactive.

Protoplasm is specialized. The specialization of protoplasm in Paramecium is a step toward the specialized organs for definite functions found in higher animals. The protoplasm of

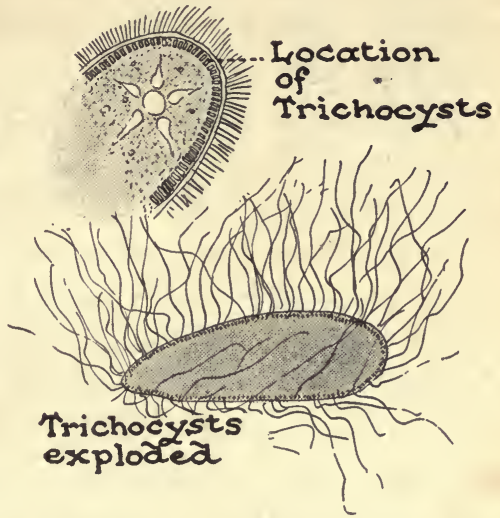

If a small drop of fountain pen ink is put on a Paramecium, the animal throws out long protoplasmic structures, trichocysts. These threads usually secrete a substance that stuns an enemy or the prey.

the amoeba shows very little differentiation. Pseudopodia may be thrown out from the body at any place. Food may be taken in and water given off from any part of the body. The only specialized protoplasm of the amoeba seems to be the nucleus and the plasma membrane around the contractile vacuole. In the Paramecium there are definite parts of cytoplasm specialized to form cilia. These are constant structures used for locomotion and for propelling food into the mouth. There is a definite part of the cytoplasm forming the groove, mouth, and gullet. Food is taken in through these structures. The two nuclei, the contractile vacuoles, cell membrane, and anal canal are all fitted or adapted to the functions they have to perform. The Paramecium is one of the simplest organisms to show definite adaptations to function. Physiological division of labor is accomplished by specialized protoplasm. 
Simple animals are very similar to simple plants in structure and function. The main differences are that animals have the function of locomotion, and are adapted to perform this function. Very few plants have the power of locomotion. Green plants manufacture their own food by means of their chlorophyll, and do not have to seek food as most animals do. In addition to the plasma membrane, there is a protective wall in the plant cells made of a nonliving material, cellulose. This is missing in animal cells. Amoebas are bounded by the plasma membrane only.

A cell, either plant or animal, has been shown to be a tiny mass of protoplasm, generally having a nucleus and having a boundary, a cell wall, or an animal membrane. The cell theory as first stated by Schleiden and Schwann has been checked by many scientists over a long period of years. To-day, it is no longer considered a theory but is accepted, somewhat modified, as a doctrine. Among other things this theory states that the cell is the unit of structure and of function and that all plants and animals are made up of cells and cell products.

\section{Questions}

1. Name the forms of energy needed by the amoeba or Paramecium.

2. Classify the physiological functions into nutritive, adaptive, and reproductive. Give the functions of each class.

3. Why is digestion necessary in animals; in plants?

4. What is the main difference in the nutrition of a plant and animal?

5. Discuss some positive and negative examples of tropisms.

6 . In outline form, compare the structure and function of a onecelled plant and a one-celled animal.

7. Discuss the importance of chlorophyll to animals.

8. What is meant by physiological division of labor and specialization of protoplasm?

9. Make a library report on the economic importance of protozoa. 


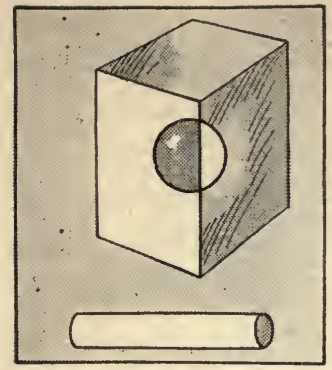

When cells divide
CHAPTER VII

\section{THE RESTING} AND

DIVIDING CELL

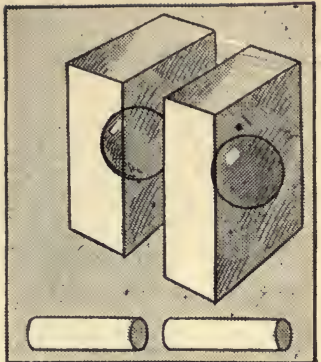

the surface area increases.

What is the nature of protoplasm? What progress has been made in the building of protoplasm in the laboratory? Are the functions of the cell related to the functions of the organism in any specific way?

All living things are made of cells. The cell is the unit of structure of the.Protozoa. Higher forms of organisms are composed of many cells. It is impossible to discuss the functions of higher organisms without referring to the cell in some detail, for it is really the cells of the organism that perform these functions. Cells vary in shape, size, and structure, but they are all alike in consisting of a mass of protoplasm usually containing a nucleus, and always surrounded by a plasma membrane. A plant cell is inclosed in a cell wall which usually consists of cellulose, a form of carbohydrate. Cells are either in a state of division, or, if not actively dividing, are said to be in a state of rest. When in the socalled state of rest, activities other than cell division are being carried on in the cell.

The nature of the cell and its make up. So complex is the cell and so much has been written about it that there is now a whole branch of biology, cytology, concerned with cell investigations.

Cytologists differ as to the detailed structure of protoplasm. Some think it is composed of an extremely minute network of fibers, similar in appearance to a sponge, inclosing a liquid. This is 
called the reticular theory. A second group of scientists believes that protoplasm is similar to a mass of bubbles, like soapsuds or a froth. This group thinks the so-called fibers are only the delicate
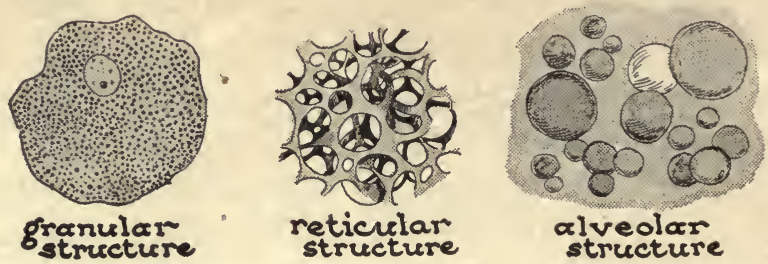

The structure of protoplasm has not been satisfactorily determined. It has been described in various ways and represented as in the above diagrams.

lines separating the bubbles from each other. This theory is called the alveolar or the foam theory. A third group thinks that protoplasm is an infinite number of very small, living, moving granules arranged in lines resembling fibers. These fibers, differently arranged, make various figures This is the granular theory. All biological problems are centered in the cell, including the manufacture and use of food, and an understanding of the nature of and the control of problems of heredity. Such problems shall never be satisfactorily settled until more knowledge of the work of the cell is gained.

Many investigators are now trying to solve cell problems. Consider the work of Alexis Carrell, the French surgeon. For years he and his assistants have been working at Rockefeller Institute, New York city, trying to develop various cells outside of a living organism. For sixteen years he has kept cells of a chicken embryo actively growing. He has also succeeded in getting other living cells to grow under controlled circumstances. He has been able to use some of these cultivated cells to try out the effects of various antiseptics. It is possible that cultivated tissues will be of great value in operations of skin grafting. Growing tissues for this purpose is only one small part of the work now being done by Carrell. He has transplanted whole organs from one animal to 
another and succeeded in having them grow and function in the second animal. His work on antiseptics, during the World War, was invaluable. It seemed almost impossible to keep wounds free from contaminating germs which destroyed the cells. He and Dakin prepared and introduced an antiseptic, Dakin solution, for treating wounds. This solution, which did not injure the tissue, saved many soldiers from gangrene and other infections.

The problem of heredity which involves an understanding of certain cells is now being studied by many scientists. It is a well known fact that certain physical and mental traits are transmitted from parent to offspring through a granular material, chromatin, found in the nucleus of the germ cells. This chromatin combines to form the structures, chromosomes, which carry and transmit certain character determiners. Experiments are now being made to find out exactly what characters are inherited, and the amount of the hereditary-bearing substance. Why has' a certain individual one ability, while another in the same family lacks this ability? Scientists, including T. H. Morgan, W. J. V. Osterhout, E. B. Wilson, and R. H. Chambers, are working on this problem at the present time.

Characteristics of protoplasm. Protoplasm is being studied by many biologists from four different viewpoints.

Physically - it is a constantly streaming, colorless, slimy, semiliquid substance similar to the white of an egg. It is a colloid. By that we mean it is a mass of tiny solid particles suspended in a liquid. It will not pass through a parchment membrane.

Chemically - it is a very complex unstable mass made of proteins and inorganic salts associated with a large amount of water and frequently containing carbohydrates and fats.

Structurally - it is variable. Sometimes it appears purely granular, other times fibrillar or threadlike, and again it may resemble a mass of foam or bubbles. It varies according to the activity and individual nature of the cell. 
Physiologically - it lives, moves, reproduces, and dies. If kept under proper conditions, such as Woodruff and Carrell kept it in

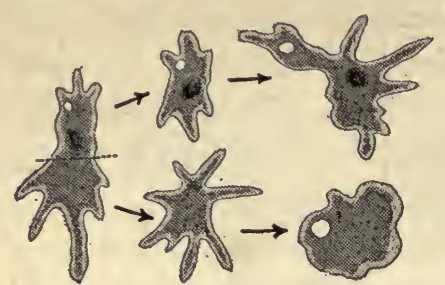

An amoeba was cut apart, one portion bearing the nucleus. Both fragments healed, both continued to move, but the one without a nucleus soon slowed down, came to rest, and died. The nucleated portion grew and became a normal amoeba. their experiments, protoplasm seems to be able to live indefinitely.

Dr. Calkins, of Columbia University, has performed many experiments on certain cells to show the importance of the presence of nuclear material. In one experiment he used Uronychia, a onecelled animal. Like its relative, Paramecium, this creature has two nuclei, a macronucleus and a micronucleus. The protozoölogist divided the tiny creature into two fragments, each having an equal part of the macronucleus and micronucleus. Both parts healed. Both grew and reproduced. Then he divided a second organism unequally so that the smaller

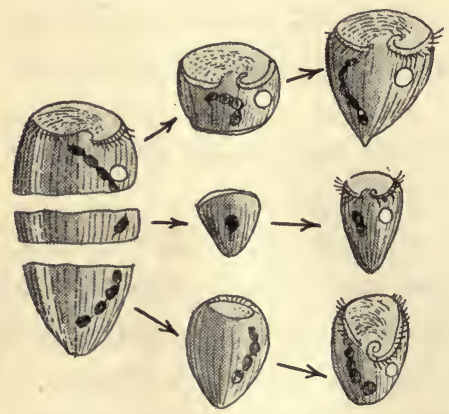

A Stentor, relative of Paramecium, was cut in three parts. Each fragment had a part of the nucleus. Each grew and regenerated missing parts, resulting in three new and complete animals.

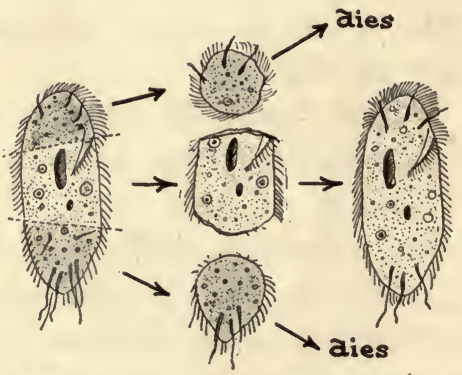

A Stylonychia was cut so that only one of three fragments contained nuclear material. All three swam about for a time but only the portion bearing the nuclear material regenerated lost parts, grew and reproduced.

part had little macronuclear material and no micronucleus. It took in little food, grew, but did not reproduce. It soon died. The larger portion of the animal, containing most of the macro- 
nucleus and the micronucleus, healed, took in food, grew, reproduced, and was soon a normal animal.

Many other similar experiments have been conducted, the results of which indicate that the macronucleus is an essential factor in the metabolism, growth, and regeneration of the organism. The micronucleus has to do with reproduction.

The protoplasm may be divided into cytoplasm and nucleoplasm.

Structures found in cytoplasm

Cell wall (plant cells only)

Plasma membrane

Vacuole

Cell sap

Vacuolar membrane

Chloroplasts (in some cells)

Starch grains

Crystals (protein, urea)

Centrosome (animal cells chiefly)
Structures found in nucleoplasm

Nuclear membrane

Nucleolus

Chromatin granules

Linin network

Nuclear sap

The functions of the different parts of protoplasm. The nucleus consists of very dense protoplasm bounded by a delicate membrane. It may lie anywhere in the cytoplasm of the cell. In fact, it is constantly being carried to different parts of the cell by the streaming movements of the cytoplasm. It generally contains a minute spherical body, the nucleolus. Sometimes there are several nucleoli in one cell. The exact function of the nucleolus is not known.

A typical resting nucleus shows many particles called chromatin granules. These are enmeshed in a network called linin. The chromatin granules are believed to be the carriers of hereditary character determiners; such as, the color of a person's eyes, his disposition, his height, or his artistic ability. These particles are able to absorb certain stains more readily than the rest of the materials found in the cell.

The nucleus controls the work of the cell. It is the center of all the physiological activities of the cell; such as, nutrition, respiration, WH. FITZ. AD. BIO. -5 
growth, and reproduction. A cell without a nucleus (the nucleus having been removed with a tiny knife) will not live. Without a nucleus, although food is present, the cell cannot take in food, nor

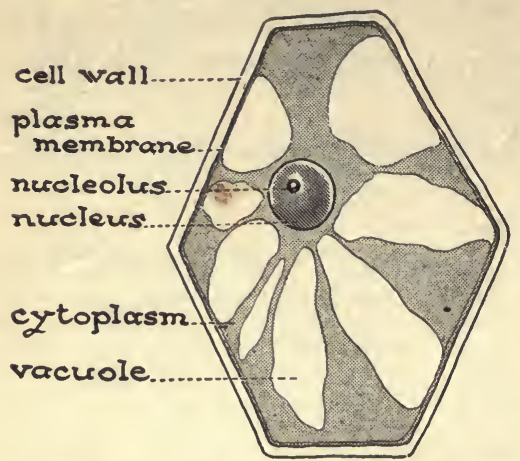

A plant cell has a cell wall. When viewed through a microscope large vacuoles are generally seen in the cytoplasm. use food, and its respiration becomes greatly reduced.

The cytoplasm is the living material of the cell, surrounding the nucleus. It is semifluid, less dense than the nucleoplasm, and is always in motion throughout the cell.

The cell wall is found as an additional bounding layer around plant cells. It is composed of a substance, cellulose, which has the elements found in starch; namely, carbon, hydrogen, and oxygen. Cytoplasmic threads penetrate into the cell wall, and cause it to grow in thickness by depositing woody material. As the cell gets'older, more and more woody material is deposited. Within this thickened wall of the plant cell is the plasma membrane. In the animal cell the plasma membrane is the only bounding layer; it lacks the material which makes up the plant cell wall. The plasma membrane is a very delicate layer of dense cytoplasm and has three functions in plants and animals. It regulates osmosis by permitting the entrance of needed materials. It determines the shape or form of the cell. It affords protection against loss of water.

A vacuole is a space in a plant cell which becomes filled with a liquid called the cell sap. The cell sap contains a little dissolved sugar, mineral salts, crystals (waste and storage products of the plant cell), and a great deal of water. The presence of a vacuole helps the plant cell to swell up and become very large even though only a small amount of cytoplasm is present. This cyto- 
plasm becomes stretched into a thin layer around the vacuole. The vacuole is bounded by a delicate layer of cytoplasm, the vacuolar membrane. Some animal cells possess vacuoles similar to the food and water vacuoles of the protozoans, and contractile vacuoles for the excretion of nitrogenous wastes.

A chloroplast is a small mass of cytoplasm, colored green by the chlorophyll pigment. The cell aided by this chlorophyll in the presence of sunlight is able to manufacture raw materials into the food necessary to build protoplasm.

The centrosomes are the foci for the starlike formations appearing like fibers of cytoplasm. These seem to be of great importance in the division of the animal cell.

The sum total of all the functions of the cell is called metabolism. It refers to the physiological activities of all living protoplasm. There are two kinds of metabolic activity; anabolism and katabolism. When the physiological activities tend to build up protoplasm, they are termed anabolic. Two examples of anabolic

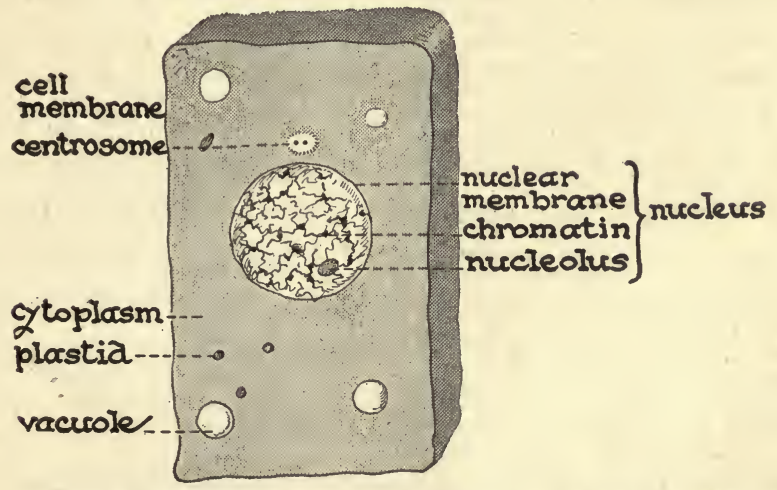

Compare this animal cell with the plant cell on the previous page.

activities are starch making and assimilation. When these processes tend to break down protoplasm, they are katabolic. An example of a katabolic process is the oxidation of food. Both types 
of processes are essential to the work of the cell and depend upon each other. The anabolic activities are concerned chiefly with nutrition and the katabolic, with energy release. For example, in order to perform the anabolic functions of assimilation; the cell is dependent on the energy derived from the katabolic function of respiration.

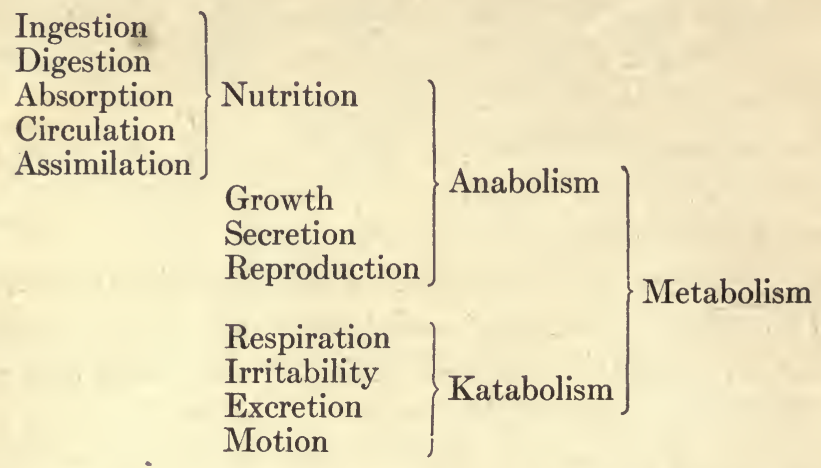

Cell division. When a cell is not at rest, it is in a state of division. There are two methods of division: 1. Direct division also called amitosis ( $a$ - without; mitos - thread), and 2. indirect division or mitosis. In amitosis, direct division, the nucleus simply pinches in half and becomes separated into two daughter nuclei. The cytoplasm sometimes cleaves and a part goes with each nucleus. This sort of division takes place only in cells which are very active and need very many nuclei in the work they are doing. Amitosis may also occur when cells have been very active, and the nucleus, becoming exhausted, multiplies rapidly by breaking into fragments.

Mitosis or indirect cell division. Mitosis is a very complicated process when compared with amitosis. The nucleus lives a very long time in an active and young cell, and will divide a great many times. Each time the nucleus divides, the entire cell divides, forming two new cells. Each cell will have exactly the 
same characteristics possessed by the original cell. This equal inheritance is made possible by the dividing of every chromatin
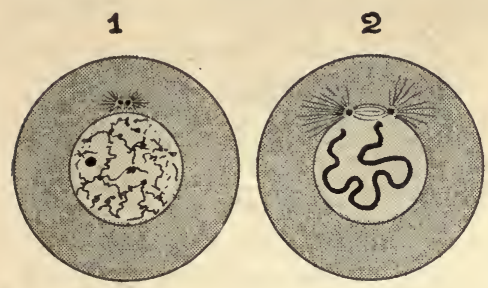

Prophase

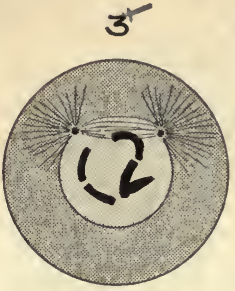

Metophase

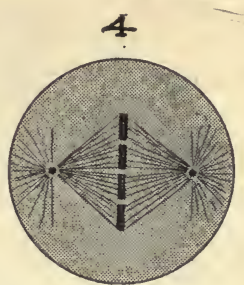

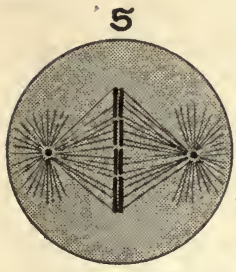

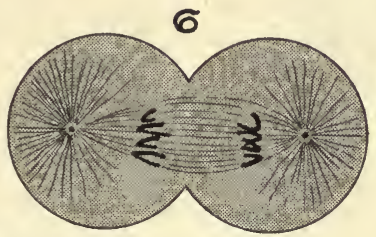

Anaphase

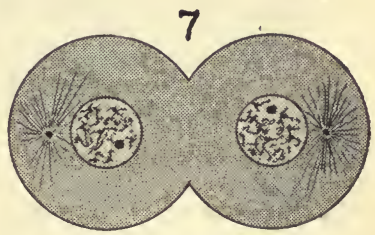

Telophase

Mitosis of an animal cell, Ascaris egg. Follow the stages shown in the diagram with the description given in the text.

granule into two parts, so that each cell will possess the same number. In order to bring about equal splitting, several distinct stages are gone through. This process takes place in all plant and animal cells, from the protozoans and algae to the cells of the largest trees and the most complex animal, man.

In many animal cells, the first sign of mitotic activity is the separation of the centrosome into two parts connected by delicate fibers. Around each center, groups of fibers radiate, causing the structures to look like stars. Hence, they are called asters and the radiating rays are known as astral rays.

The centrosomes move further apart around the nuclear membrane and the spindle threads become longer. At the same time the chromatin granules become arranged in the form of separate threads or a continuous coiled thread, the spireme. 
The spireme breaks up into a number of parts called chromosomes. Each plant or animal cell seems to have a definite number of these.

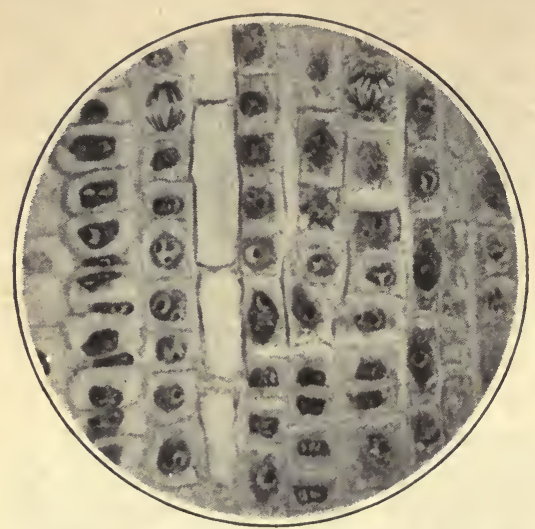

Photomicrograph of a section of a growing root tip of an onion. How many different stages of cell division can you identify?

In the cells of human beings, the spireme breaks up into forty-eight chromosomes. In the onion cell, there are sixteen. In a certain little fruit fly, there are only eight. These numbers are always constant for a particular species. Four chromosomes are shown in the diagrams (page 61) illustrating mitosis of an animal cell.

The asters separate more and more widely causing the fibers between them to become longer and to assume a spindlelike shape. Finally they reach the opposite sides of the nucleus. The chromosomes are now drawn into the central part of the spindle forming a ring around it, in a plane called the equatorial plate. All of these changes are a part of the prophase stage (beginning) of mitosis. The prophase ends with the chromosomes arranged on the spindle in the equatorial plate. Each chromosome now splits lengthwise into exactly two similar parts. The actual splitting of the chromosomes may occur before the arrangement at the equatorial plate. Division of the chromosomes has been accomplished. This is the metaphase (middle) stage of mitosis.

The split chromosomes begin to separate and move toward the centrosomes or the poles. As they approach their poles the chromosomes lose their regularity of outline, and upon reaching the poles each group becomes converted into two new nuclei. This is the anaphase (approaching the end) stage of mitosis. 
Mitosis ends with the telophase. A new cell wall forms on the spindle, midway between the two daughter nuclei. It divides the cell into two parts, in each of which a nucleolus now appears.

Problem. Study of mitosis. Study of prepared slides of Ascaris under the microscope.

I. Select a good example of the anaphase stage.

$A$. How does the slide differ from the diagrammatic drawings?

$B$. What causes you to identify it as an anaphase stage?

II. Identify any other stage of mitosis present in your specimen.

III. Make an outline drawing of three different cells seen through the microscope.

IV. Alongside of each drawing, copy and label the diagram of the phase which most nearly resembles the viewed cell.

A very similar process takes place in plant cells. Centrosomes are lacking. The spindle forms from material which is probably of

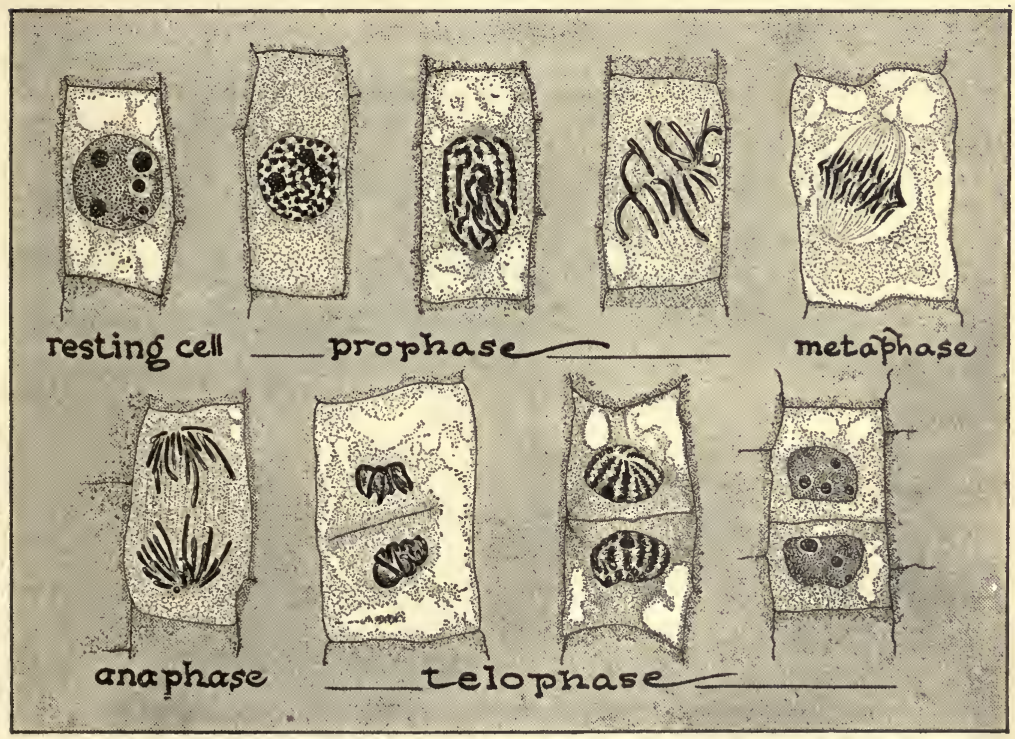

A group of cells of a plant showing various stages in the mitotic division of the cells. Compare and contrast this series with the diagrams showing mitosis of an animal cell. 
nuclear origin. A cleavage plate through the middle of the cell is secreted by the cytoplasm. This divides the cell into two parts. There is no constriction here as there was in animal cells.

Cell theory. The illustration (below) shows some of the men who have discovered and made known many interesting facts about animal and plant cells, so that we to-day have come to have four ideas concerning the cellular structure of all organisms.

1. The cell is the unit of structure of all living plants and animals.

2. The cell is the unit of all physiological functions of living plants and animals. It is the cell that breathes, digests food, excretes wastes, moves, and performs all the other physiological functions.

3. The cell embraces all the hereditary qualities of the organism within its nuclear membrane.

4. Plants and animals may consist of single cells. They always start with a single cell, and in their early stages of development (embryo) this cell divides and changes into many cells.

\section{Questions}

Hall of Fame for biologists who worked on cells
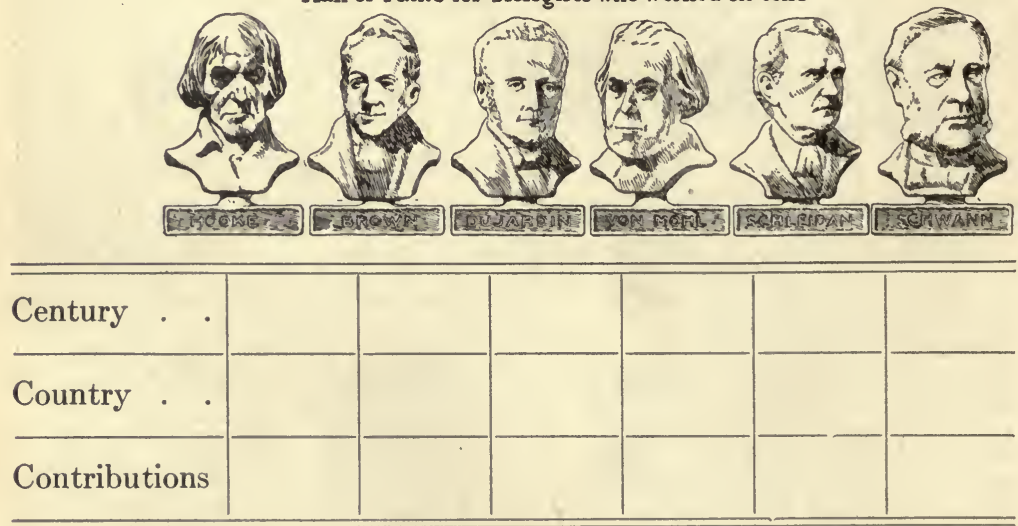

1. Copy the names and fill in the blank spaces in the above table. Check your results with your answer to question 1 on page 33 .

2. By means of paraffin, soap, clay, or plasticine try to make models of a resting cell and some of the stages of mitosis. 
3. Name the structures common to both plant and animal cells.

4. List the living and nonliving structures in a plant cell.

5. What parts of a cell are most active during mitosis ?

6. In what kinds of cells is amitosis carried on? Why?

7. Draw a characteristic stage of each phase of mitosis.

8. Why is it that every cell in an organism has chromosomes of the same nature found in every other cell?

9. Discuss the cell as the unit of structure; the unit of function.

10. Discuss the importance of the cell in the development of the individual.

\section{SuPPlementary Reading}

Gager, C. S., General Botany (P. Blakiston's Son \& Co.).

Locy, W. A., Biology and Its Makers (Henry Holt \& Co.).

Wilson, Edmund B., Cell in Development and Heredity (The Macmillan Co.). 


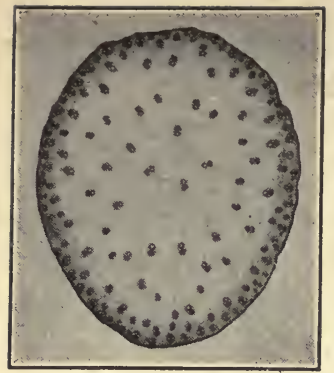

Photomicrograph of corn stem.

\section{CHAP'TER VIII \\ STRUCTURE OF HIGHER PLANTS}

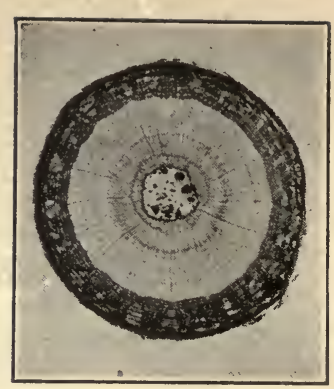

Photomicrograph of woody stem.

What is meant by physiological division of labor? Does each plant cell of a higher plant live independently or are the cells dependent on each other? Why can one type of cell perform a function better than another type? What advantages have higher plants over simpler?

Social division of labor. In any civilized community certain individuals perform one type of labor more efficiently than others. There are seamstresses to make clothes, shoemakers to make shoes, milliners to make hats, engineers to run trains, clerks to perform clerical work, typists to typewrite, and numerous other specialists, each performing a specific work. The people who are most successful are those who are particularly fitted or adapted for their positions, either through special training, natural talent, or size. More efficient work is accomplished in less time when it is divided among specialists than if each man had to do all the work himself. Similar to the division of labor among people in the industrial world, there are in our bodies numerous activities that are carried on by various structures especially adapted for that work.

Physiological division of labor. There is physiological division of labor in all plants and animals. The amoeba, Paramecium, Pleurococcus, Spirogyra, and countless other plants and animals are sufficient each in itself. Each with its one cell, by means of specialized protoplasm, performs all the activities and processes 
necessary for living. These organisms, however, live very simply. The many-celled plants and animals are much more complex. They consist of a variety of cells. Differentiation of cells and greater specialization of function are shown in these. Whenever there is a collection of similar cells in higher organisms, it is known as a tissue.

Problem. Study of the lower epidermis of a leaf.

Peal off the under surface of a geranium leaf. Mount it under the low power of the microscope.

I. Describe the shape, color, and structure of the different types of cells in the epidermis.

A. Epidermal cells have unusually thick walls. Remembering that the epidermis is the outer layer of the leaf, suggest a function of the thickwalled cells.

II. Note the small holes between the oval-shaped cells. Each of these is a stoma (from a Greek word meaning mouth or opening). Suggest a function for these openings or stomata.

$A$. The oval-shaped cells, called guard cells, are so constructed as to cause the opening and closing of the stomata.

$B$. Draw and label the lower epidermis of a leaf.

C. Describe briefly the functions of the cells and the adaptations of the cells to their functions.

Problem. Study of a cross section of the same leaf.

Place a leaf between two pieces of cork and cut through the cork with a sharp razor blade.

I. Describe the general arrangement of the cells in the upper epidermis, middle area, and lower epidermis of the leaf, as seen in the cross section.

II. Note the upper colorless epidermal cells bearing hair-like structures. Suggest a use for these cells.

III. Note the regular arrangement of the cells immediately under the upper epidermis. This layer is called the palisade layer or tissue. How are the cells in the palisade layer arranged so as to secure sunlight?

IV. Observe the loose arrangement of the cells under the palisade tissue. These cells form the spongy layer.

$A$. What is the advantage of the air spaces among the green cells?

$B$. State a function of the green cells of the leaves. 
V. Observe the side view of the cells in the lower epidermis. Describe the appearance of the epidermal cells, guard cells, and stomata.

VI. Discuss the importance of the translucent character of the epidermal cells.

VII. What specialization of structure for function is shown in a green leaf? Give at least five examples.

VIII. Secure two leaves from a rubber plant. Coat the upper epidermis of one and the lower epidermis of the other with vaseline. Seal the ends of the stems with vaseline. Keep them in a cool place for several days.

$A$. State your observations.

$B$. . Suggest a second use for the stomata.

\section{Problem. Study of the vascular and supporting tissues in a leaf.}

Break off a narcissus leaf and separate some tissues from the broken end. Mount on a glass slide.

I. Observe some of the cells with spiral thickenings on the walls. These are tracheids (xylem). In order to keep open the passageway of the tubes formed by these cells, spiral thickenings of wood form on the wall. Originally, there were cross walls where one cell ended and another began, but the partitions have been absorbed, leaving an uninterrupted canal. Water travels up through these tracheids.

II. There are other cells with sievelike plates at regular intervals. These are the sieve tubes (phloëm). Through these structures materials pass down the plant from the leaves to the roots.

$A$. Trace a material that travels from leaves to roots.

$B$. Why do materials tend to pass more quickly down than up?

$C$. What is a possible advantage of the sievelike cross walls in the sieve tubes? Each sieve tube is a single row of elongated cells placed end to end and the thickened end walls of these cells have numerous perforations.

III. There are also supporting tissues called wood fibers that have very thick walls of cellulose. Some of them consist entirely of cellulose and compose the supporting structure in a plant.

IV. Place the roots of a complete narcissus plant or any other young plant in red ink or eosin in order to show the continuous pathway from roots to leaves.

A. What evidence of the passage of fluids do you observe?

$B$. Trace the liquid that travels up in a plant from the point of entrance to the cells that use it.

V. Draw and label as many of the tissues as you have observed. 
VI. In a paragraph, discuss the functions of the vascular or conducting and supporting systems of a plant, including the adaptations or specializations for various functions.

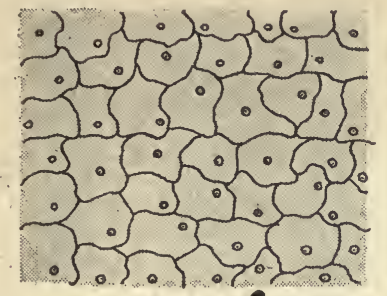

upper surface

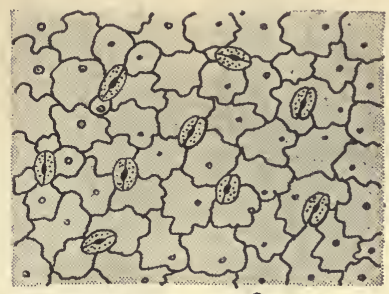

lower surface

Most leaves are covered with a layer or layers of thick-walled cells lacking chlorophyll. The lower surface of most leaves bears certain paired cells containing chloroplasts. These cells permit the passage of substances in and out the leaf. They are the guard cells.

Specialization in higher plants. Higher plants perform all the physiological functions necessary for life. Each individual cell performs many of these same functions, but at the same time each cell is adapted to perform one function better than others. Absorption of water and minerals is performed by special epidermal cells called root hairs, found on the roots. These cells are long and slender and grow among the particles of soil. The water and mineral matter pass through the root hairs, then through other root cells until they reach the ducts (xylem) near the center of the root. By means of capillary action, evaporation of water from the leaves, and the pressure of other fluids following, the water rises in the spirally-thickened

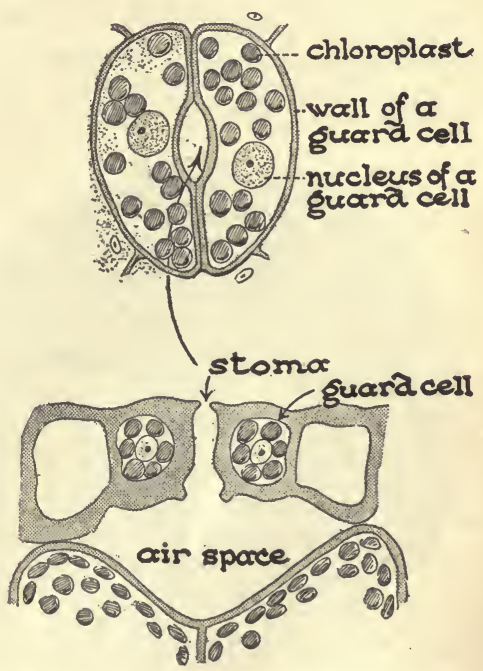

A surface view and cross section of the specialized epidermal cells which make a passageway to the air spaces within the leaf. 

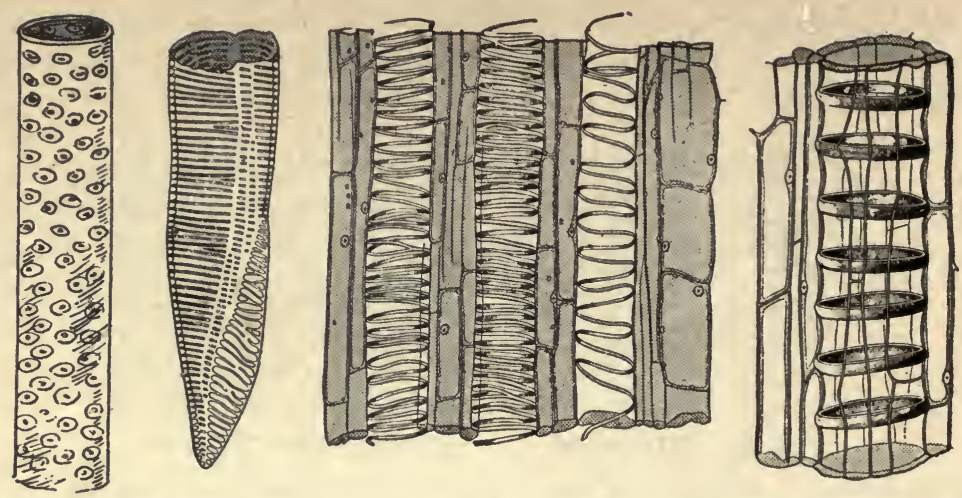

The fibrovascular bundles are made up of different types of woody cells, some pitted, others ribbed and still others strengthened by spiral or circular thickenings on the walls.

and pitted ducts until it reaches the chorophyll-bearing or green cells, particularly those in the middle part of the leaf. The green cells absorb the water from the tracheids. Here, again, absorption is a cell function. Oxygen for respiration and carbon dioxide for photosynthesis diffuse through the stomata in the lower epidermis of the leaves, through the air spaces in the middle layer

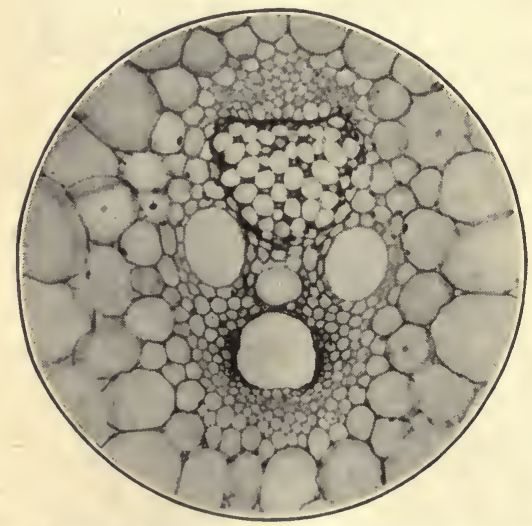

When greatly magnified, a woody bundle of the corn stem shows many of the tubes which are the passageways for the food of the plant. of the leaf, and, by means of osmosis, pass into the green cells. In these green cells, photosynthesis, fat manufacture, and protein manufacture take place. These processes are considered leaf functions because they take place in the green cells of the leaves. Again the function of the organism is really a cell function.

Excess food passes into the sieve tubes and is conveyed 
down to especially adapted layers of cells in the stem or in the cortex of the root, for storage in the form of starch granules, oil globules, and protein crystals. Turnips, parsnips, and radishes are used by people for food because of the extra plant food stored in them. In the case of the potato, the extra food is stored in an underground stem ; in asparagus, sugar cane, and rhubarb, it is stored in stems above the ground. When the plant needs food, a protease, lipase, and amylase secreted in the cytoplasm of the cells that store food, change the stored materials again to a soluble form. These dissolved foods are then distributed by means of the ducts.

Supporting cells are usually found in connection with the ducts and sieve tubes. These woody fibers support the plant.

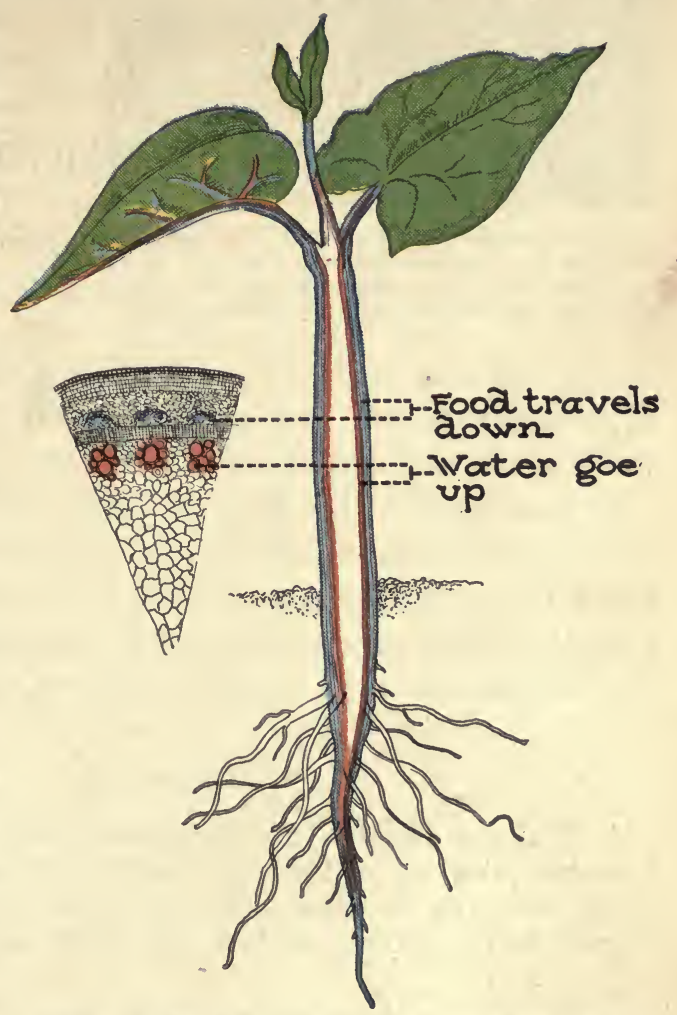

There are pathways through which fluids pass up and down in plants. Raw materials travel up the stem into the leaves. Food manufactured in the leaves travels down to the roots.

Those in the lower part of the plant strengthen the roots and help to anchor the plant in the ground. The woody fibers of the stems help hold up the leaves and enable them to get sunlight which is necessary for photosynthesis. 


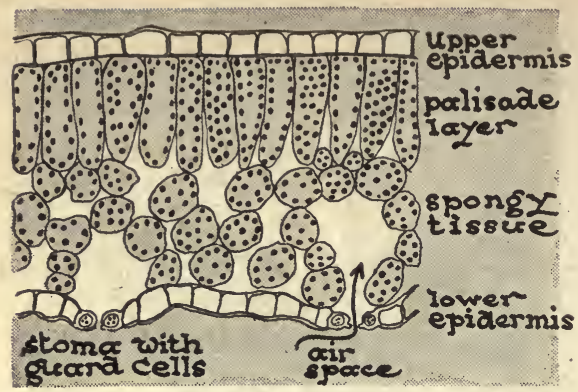

In cross section, a certain leaf will show cells arranged in an orderly fashion. One part of the leaf contains many air spaces through which gases are exchanged with the active surrounding cells.

All cells need energy to carry on their work. Each cell takes in oxygen, oxidizes the food distributed to it, and gives off carbon dioxide and some water. 'These materials may be distributed either through the vascular system or by passing from cell to cell. The entire plant grows by the growth and division of the individual cells. Reproduction of the plant is too complicated to discuss here, but it will be found later that it, too, is due to the division of certain cells.

In this chapter, the shape of plant cells has been considered. It is by their shape, organic nature, and arrangement that they are fitted to perform certain functions. Each cell of higher plants contains the structures discussed in a resting plant cell. It is by the coördination of all these cells that the entire plant functions.

\section{Questions}

1. Make an outline of the specializations in higher plants, using the headings $(a)$ name of tissue, $(b)$ function, $(c)$ adaptation to function.

2. Name the structure in a higher plant corresponding in function to each structure observed in a cell of Spirogyra.

\section{SupPlementary REadings}

Coulter, Barnes, and Cowles, A Textbook of Botany (American Book Co.), Vol. I.

Holman, R. M., and Robbins, W. W., A Textbook of General Botany (John Wiley \& Sons, Inc.).

Gager, C. S., General Botany (Blakiston's Son \& Co.). 


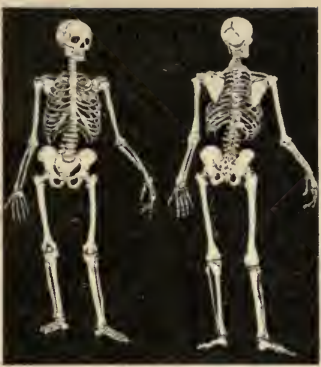

Bone cells build skeletons.

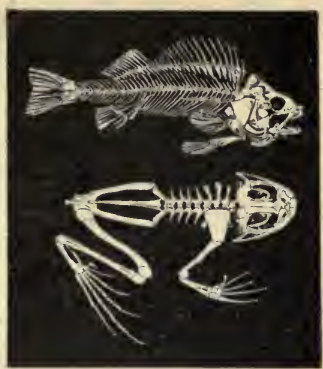

Skeletons of fish and frog.

How can the minute structure of the body be investigated? How is division of labor performed by the human body? What are bone and cartilage? How are the cells in a multicellular organism held together?

If a thin slice of any portion of the human body is examined microscopically, it will be found to consist of a mosaic of minute units called cells. It will be further found that no cell conforms to the diagram of the typical cell. There are masses of similar cells that build tissues, and these tissues build more complex structures called organs. Each organ has a special duty or function. In the human body there is greater specialization than in the higher plant. Each tissue or group of cells is adapted to perform a particular physiological function. At the same time, all the functions necessary for life are carried on within each cell.

The cell the unit of structure. Any function of an organism must be considered in relation to the function of tissues or of cells, because it is the individual cell that does the work. Fundamentally, each cell possesses the complete apparatus for life. Tissues can be compared to collections of single-celled organisms in that they perform most of the functions that the one-celled animals carry on ; but each group of tissue cells is fitted by shape, structure, location, or chemical powers to perform special functions necessary to the life of the complete organism of which it is a part. ThereWH. FITZ. AD. BIO. $-6 \quad 73$ 
fore, it may be said that all of the tissue cells work together to accomplish the life processes of the organism. This is brought

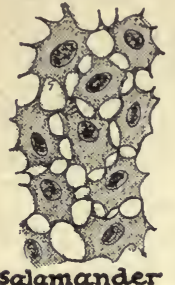

salamander
skir cells
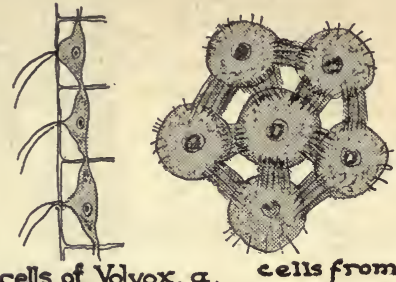

cells of Volvox, $\alpha_{2}$ colonial protozoan cells from

In most organisms, cells are not separate and distinct units, but are joined to one another by delicate strands of cytoplasm.

about by differentiation of structure for division of labor.

The cells of multicellular animals and plants are not isolated individuals, but are probably held together by some kind of cytoplasmic continuity.

Biologists have found in certain cases, and have reason to believe that in most cases, slender cytoplasmic bridges connect the various cells. This gives direct continuity to the cytoplasm throughout the entire organism, and probably enables the organism to act as a unit even though it is composed of millions of different cells.

Many cells of the body deposit intercellular materials, which give additional structural strength. The amount of the deposit varies. Bone cells deposit a large amount of such material; epithelium produces a very small amount. Protoplasm is the foundation substance of cells. Groups of similar cells with their intercellular materials make up tissues. When tissues are grouped to make a larger structure to perform a certain function, that structure is called an organ. Thus, the entire plant or animal organism is a combi-

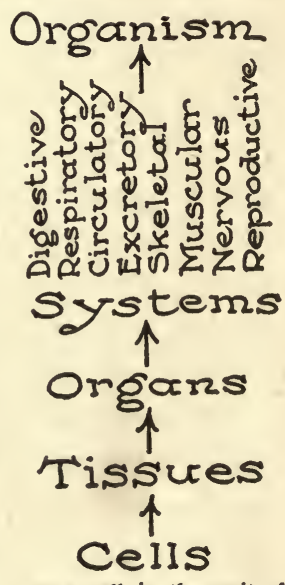

The cell is the unit of structure and function in every organism. nation of organs built of tissues, which in turn are built of cells. The tissues of the human body may be classified into epithelial, supporting or connective, muscular, blood, and nervous. 
Problem. Study of certain epithelial cells.

Take a toothpick and gently rub the inside of the cheek and gums. Mount the material from the toothpick in water and add just enough fountain pen ink to give a slightly-bluish tint to the water. Study with the low and high magnification.

I. Describe the shape, color, relative size, and structure of the cells.

II. Draw a group of epithelial cells at least five times larger than they appear.

III. Write a brief description of epithelial cells.

Epithelial tissue. Epithelial cells cover and line the organs of the body. They make very little intercellular material. Their function is protection and secretion. They may protect certain organs against invasion of foreign material, or, by means of their moist secretion, guard other organs against friction. The epithelial cells that line the nose, throat, digestive system, and air tubes or respiratory system make up the tissue called mucous membrane. (A membrane is a very thin structure consisting of a single layer or a few layers of cells.) The cells lining the windpipe have tiny projections of protoplasm called cilia. Germs are pushed or moved back into the throat by means of this brush-
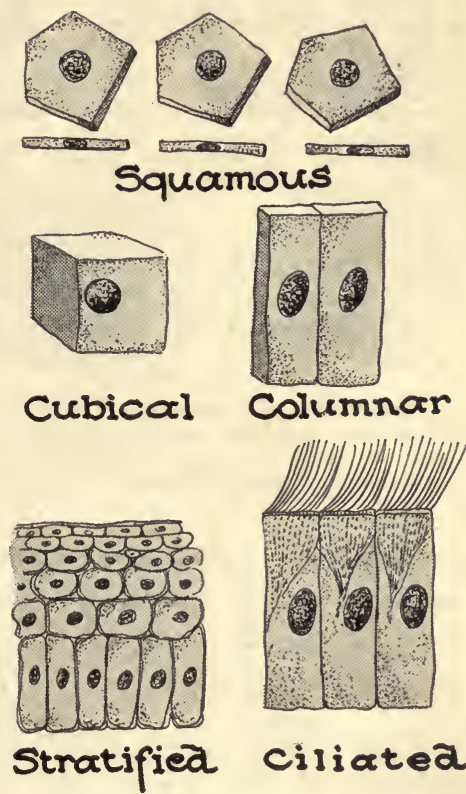

Stratified ciliatea

Epithelial cells vary in shape. They may appear as a single layer or groups of layers of flattened, cubelike, or columnar units.

The cheeks are lined with squamous epithelial cells; the respiratory tract with ciliated, epithelial cells.

like arrangement. They work very much like the cilia of the Paramecium. Because of the mucous epithelium in the alimentary canal, food slips easily through the tube with no friction. In addi- 
tion to mucous cells, there are other epithelial cells in the alimentary canal adapted for secreting digestive juices.

The epithelial cells lining the closed cavities of the body compose structures called serous membranes. The serous membranes line blood vessels, cover the lungs, line the chest, cover the heart, cover the abdominal organs and line the abdomen. The

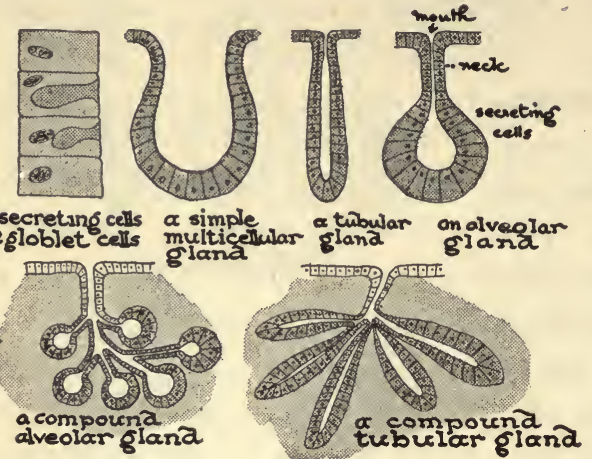

Epithelial cells may manufacture secretions. Certain cells, globlet cells, are found in the gullet. They give out mucin. Groups of epithelial cells often form simple or compound glands which give off certain secretions. serous membranes that cover the lungs are the pleurae (pleura-rib). In breathing, when the lungs become inflated or larger, and compressed or smaller, there would be friction between the lung and the wall of the chest, were it not for this moist epithelial tissue. If the pleurae become inflamed, the person is said to have pleurisy. If the secretion of fluid is interfered with, there is friction between the lungs and the. walls of the chest and breathing becomes painful.

The covering of the heart is the pericardium (peri-around; cardium - heart). This membrane prevents friction between the heart and lungs and other organs. The membrane lining the abdomen is the peritoneum. Infection or inflammation of the peritoneum is known as peritonitis. This condition is sometimes brought about when an infection extends from a ruptured appendix and attacks a part of the peritoneum. Friction among the abdominal organs such as stomach, liver, and intestines is avoided by the moist epithelial peritoneum. The abdominal organs do not float in the abdominal cavity. If they did, jumping or bending might throw them out of place. They completely fill the 
abdominal cavity, and are attached to each other and to the walls of the cavity by means of the peritoneum. A portion of the peritoneum, called the mesentery, holds the intestines to the backbone. If any organ of the abdomen is removed a part of the peritoneum must be cut to separate that organ from other organs.

The outside of the body may be compared with a body cavity in that it is covered with epithelial tissue called skin. The outer layers of the tissue become flat and horny, and the outermost ones are dead. The hair and nails develop from certain skin cells.

Supporting tissues. These tissues are all alike in that they connect and support the other tissues of the body. They, in contrast with the epithelial tissues, are noted for the abundance of intercellular material. The main supporting tissues are bone, cartilage, white fibrous, yellow elastic, and fat or adipose tissue.

Problem. Study of cross structure of bone.

Obtain some rib bones of a lamb from the butcher.

I. Let some of the rib bones stand in a ten-per cent solution of hydrochloric acid for at least two weeks.

$A$. How does this treated bone differ from ordinary bone in appearance and texture? The acid dissolves out the intercellular deposits of mineral matter, leaving only the organic material.

$B$. State the importance of mineral matter in the bone.

II. Burn some rib bones in a very hot flame.

A. How does the burned bone differ from ordinary bone? Since organic material burns, the bone cells were destroyed, leaving only the intercellular mineral matter. Bone consists of a combination of bone cells and intercellular material. This intercellular material is deposited by the bone cells. It is mineral matter, largely calcium phosphate and carbonate.

$B$. State the importance of the cells in the bone.

Secure some shank bones or other long bones from the butcher. Have him saw one lengthwise so that the interior of the long bone, including the enlarged head, may be studied. 
I. Describe the material covering the enlarged head of the bone.

$A$. Is this material (cartilage or gristle) shiny or dull; moist or dry; smooth or rough; tough or soft?

II. How does the texture of the bone under the cartilage differ from the bone of the shank?

III. Describe the material filling the space in the center of the bone. This is bone marrow and consists largely of fat cells. Compare the marrow in the head of the bone with that in the other parts.

IV. The outer membranous covering of bone is the periosteum (periaround; osteon - bone).

Problem. Study of microscopic structure of bone.

I. Mount a prepared slide of bone cells under the microscope. Note the spiderlike, irregular cells arranged around a central space. In living bone, this space is filled with blood vessels and nerves. Food passes through the blood vessels to the cells, and wastes from the cells are diffused into the blood vessels. The bone cells take calcium salts from the blood and deposit these minerals around their irregular projections.

A. Explain why bone cells with irregular projections of cytoplasm deposit greater amounts of intercellular mineral matter than they would if these cells were perfectly round.

$B$. Suggest a material that probably fills the spaces among the bone cells.

C. Draw a single bone cell five times larger than it appears under the microscope.

$D$. Draw a group of bone cells showing how they fit together and how they are arranged around the space through which the blood vessels run. Label cell, intercellular material, and canal.

II. Mount a prepared slide of hyaline cartilage under the microscope.

$A$. Describe the shape of the cells.

$B$. Describe the formation of the cells.

C. What evidence of intercellular material is there? The intercellular material of hyaline cartilage appears homogeneous throughout.

$D$. Draw a group of cartilage cells. Label cell, intercellular material, or matrix.

Cartilage and bone are closely related in their development, location, and function. In the embryo, the bones are first pre- 
ceded by cartilage. In infancy, the bones of the skull are soft and flexible, because they are largely cartilage. One can observe the soft texture of certain parts of an infant's skull until the child is about eighteen months old. As growth takes place, this cartilage becomes hard and rigid by the deposit of mineral matter. This compact substance is then called bone. A child's back should be carefully supported because its backbone is largely cartilage and is, therefore, very elastic, and

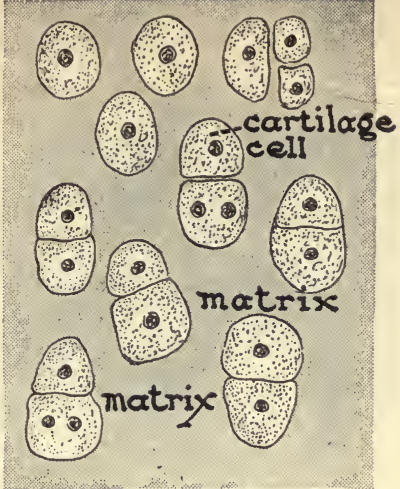

Certain cells, cartilage cells, occur in pairs. They deposit a thick, tough extracellular material called a matrix. may bend so much that the organs of the body will be injured.

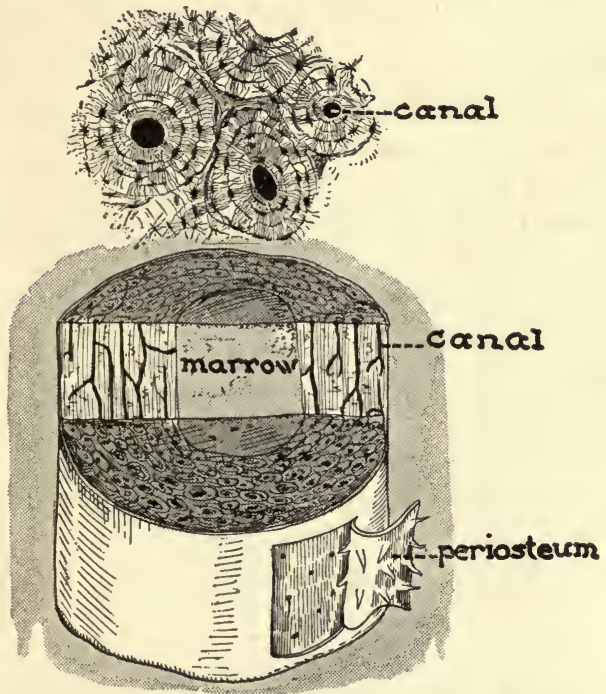

Magnified cross section of bone showing the arrangement of cells and canals through which blood vessels run. The outer covering, periosteum, and the center filled with marrow can be seen in the lower diagram.
When a bone is broken, the new part first develops as cartilage and is replaced, afterward, by true bone. The type of cartilage studied in the laboratory exercise is hyaline cartilage. It is found chiefly on the ends of bone. This is not the only kind of cartilage found in the body. There are other types with different functions. One function of cartilage is to give ease to the motion of joints, and by means of its tough elas- 
ticity build those parts of the body, such as the ear and voice box, that require strength combined with some elasticity. The intercellular substance of cartilage is a secretion of the cells.

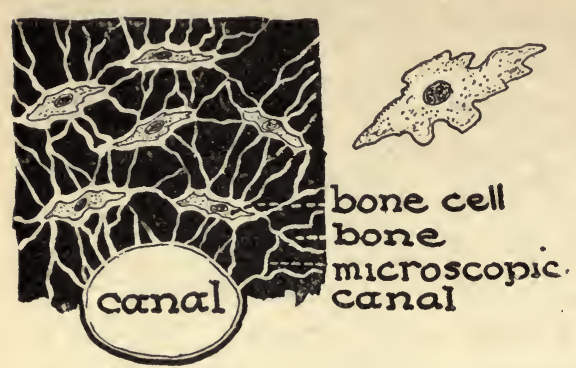

Bone cells, with their.irregular projections of protoplasm, deposit calcium compounds which they obtain from blood. An individual bone cell is also shown.

The proper growth of bone depends upon sufficient lime salts in the food and the presence of certain valuable growth promoters found in food, called vitamins, which stimulate the proper use of the lime salts by the bone cells. If bone does not develop properly, skeletal deformities, like bowlegs, may occur. This will later be discussed in connection with vitamins.

Problem. Study of other supporting tissues.

I. Place a prepared slide of fibrous tissue under the microscope.

$A$. Note the large deposits of wavy white fibers with cells scattered among them. The white fibers are the intercellular material which was secreted by the cells.

$B$. Draw a few cells with the surrounding fibers enlarged five times. Label the cells and intercellular fibers.

II. Place a prepared slide of fat or adipose tissue under the microscope. The large space filling the center of each cell is a vacuole filled with stored oil. The cytoplasm lies just within the wall, crowded there by the cnlarged vacuole. The nucleus can be noticed within the cytoplasm.

$A$. Describe the adaptation of adipose cells for fat storage.

$B$. Draw a group of adipose cells. Label cell membrane, nucleus, cytoplasm, and vacuole.

Fibrous, elastic, and adipose tissues. The white fibrous tissue is made strong and flexible by the intercellular fibers which are probably secreted by the cells. It builds ligaments, strong flexible bands, 
that hold the bones together at the joints. The tendons are also made of it. These attach muscles to the bones and are commonly called cords. In bending the wrist or stretching the neck, these cords are easily seen.

When elastic fibers predominate in connective tissue, it gives a yellowish color to the tissue and is known as elastic tissue. It is more elastic than fibrous tissue but not so strong. It is found between adjacent vertebrae, insuring elasticity to the vertebral column. It is also found in the walls of blood vessels.

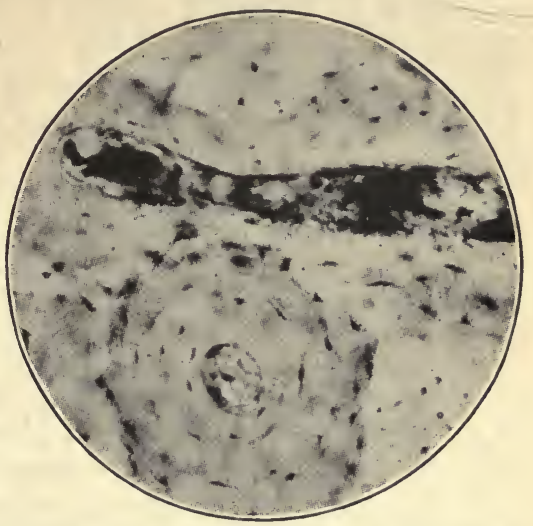

Compare this photomicrograph of a cross section of bone with the diagram shown on page 79 .

The adipose cells store excess supplies of fat. These cells compose, to a large extent, the yellow marrow of bone. Deposits

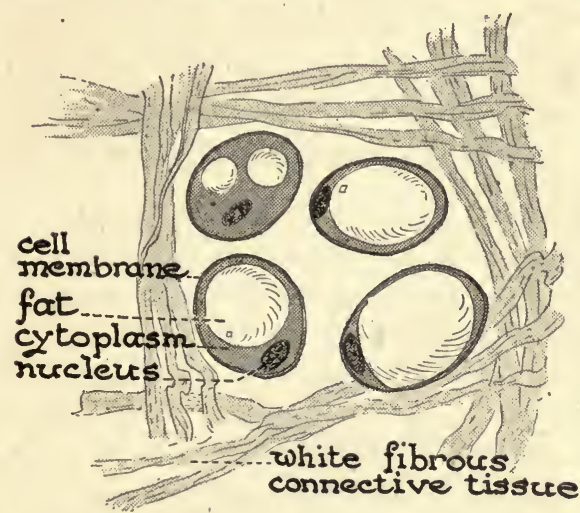

Droplets of oil enter certain cells. This oil increases in volume by additional particles entering the cells and crowding the protoplasmic contents of the cell into a small mass. The cell becomes distorted. The oil may be transformed to fat. Masses of these cells build the fat or adipose tissue of the body. of fatty cells are in the deeper layers of the skin and encase organs such as the kidneys and heart. When the fat is needed by the body for metabolism, certain enzymes in the cell enter the vacuole and digest the fat.

In general, the cells of connective tissue support the organs and make the framework of the body. The toughness of tendons, the extensile character of 

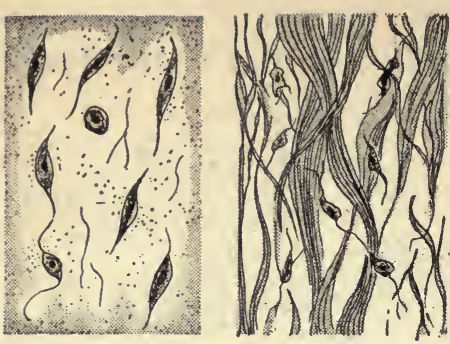

Certain connective tissue cells deposit long strands of extracellular material in the form of either yellow or white fibers.

elastic tissue and cartilage, and the hardness of bone are all due to the composition and distribution of the intercellular material of the connective tissue cells.

\section{Questions}

1. What is meant by secretion? $A$. Name four materials mentioned in this chapter secreted by cells. $B$. Give the purpose of each material.

2. Name three types of epithelial tissue. Tell the function of each.

3. What does the burning of bone show you?

4. What effect do some acids have on a bone?

5. Why should the food of an infant contain mineral matters?

6. Arrange in tabular form the names of the tissue cells discussed, the function of each, and an adaptation for each function. 


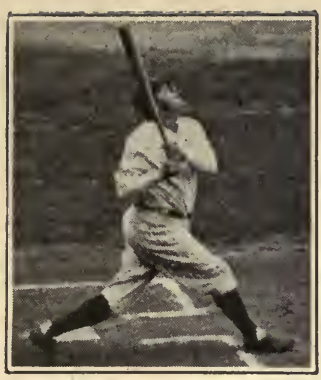

Babe Ruth, home run king.

\section{CHAPTER X \\ HUMAN TISSUES}

(Continued)

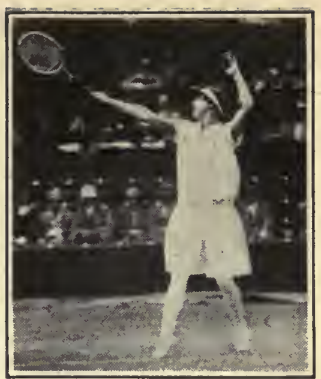

Helen Wills, queen of tennis.

Why is the body able to move? What tissue in the body makes movement possible? How are messages carried from one part of the body to another? In what way are the different tissues structurally related? What brings about coördination among the different parts of the body? Why may blood be considered a tissue?

Muscular tissues. All movement in the human body is brought about by muscular or contractile tissues. Whether it is the raising of an arm, the swallowing of food, the beating of the heart, contraction of the pupils of the eye in response to light, or the formation of so-called goose flesh in the skin when the body is cold, the movement is the result of changes in the muscular tissue.

\section{Problem. Gross structure of skeletal muscle (unmagnified).}

Secure some fore shank of meat from the butcher. Be sure to obtain a piece that shows the attachment of some of the meat to the bone. Have the butcher cut it up into enough sections to supply the class.

The meaty part of the shank is composed largely of a type of muscle called skeletal muscle because it moves the bones of the skeleton. This muscle is also called voluntary because it is under the control of the will.

I. Observe and describe the appearance of the muscle that is attached to the bone. The contracting or shortening of this type of muscle will move the bone by means of the strong inelastic tendons which are fastened to the bone.

II. Find a piece of beef showing the outside of the muscle. Describe the appearance of the covering or sheath. It is made of connective tissue. 
III. Tear or separate the muscle into parts. Notice that the muscle is divided into little bundles. Try to remove one of the little bundles from the others. Name and describe the tissue that separates one bundle from another.

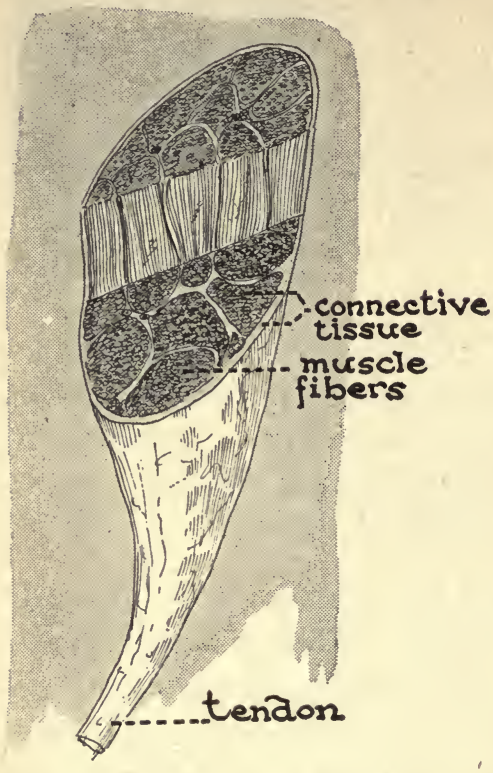

A muscle is made of bundles of muscle fibers, each bundle surrounded by connective tissue. A thick external layer of this connective tissue is continuous with the tendon which is composed entirely of connective tissue. The tendon joins the muscle to a bone.
Describe the shape of each little bundle. Describe the arrangement of the bundles in making up the entire muscle.

Problem. The structure of skeletal muscle.

- Select a tiny muscle bundle; place it flat on the glass slide and add a drop of dilute iodine. Cover with a cover glass and focus under low power, then high power.

I. Observe the long individual muscle fibers and find the tiny cross markings called striations. Skeletal muscle is often called striated muscle because of the presence of these characteristic cross markings.

II. Draw two or three muscle fibers five times larger than they appear under the microscope. Label fiber and striations.

III. Examine a prepared slide of striated muscle. Describe the appearance and location of the nuclei scattered along each fiber.

\section{Problem. The structure of smooth or involuntary muscle.}

Mount a prepared slide of smooth muscle under the microscope.

I. How does smooth muscle differ from striated muscle in appearance and shape of fiber and in nuclei?

II. Smooth muscle is called smooth or unstriated muscle because of the absence of cross-striations. It is also called involuntary because it is not under the control of the will. It is found in many internal organs such as the walls of the stomach and intestine, and is responsible for movement in these organs.

III. Draw and label two or three smooth muscle cells. 
Muscle tissue. It is impossible to discuss muscles without bringing in a discussion of other tissues. When a large muscle is dissected there is found not only the basic muscle cells, but several other related tissues. The muscle is completely covered by a sheath of connective tissue which extends in through the muscle, dividing it into large bundles, then smaller and smaller ones, until the bundles, or fasciculi, are so tiny that they are almost microscopical. This connective tissue furnishes support for the blood vessels and nerves which run through the muscles. Deposits of fat cells may sometimes be found within the muscular tissue.

Three kinds of muscles may be identified : cross-striated or skeletal, unstriated or smooth, and a specialized type which forms the substances of the heart, called cardiac muscle. The units in striated muscle are probably the fibers. They have many nuclei in contrast to the single nucleus in the elongated spindle-shaped cells of the smooth muscle fibers. Skeletal muscle is voluntary muscle. It is under the control of the will. Smooth muscle and cardiac muscle are involuntary or independent of the will. Cardiac muscle resembles voluntary muscle in having cross striations.

The special function of all muscles is the production of motion or the exertion of physical force. This is brought about by the shortening or contraction of the muscles which have the ability of returning to normal condition. These changes are known

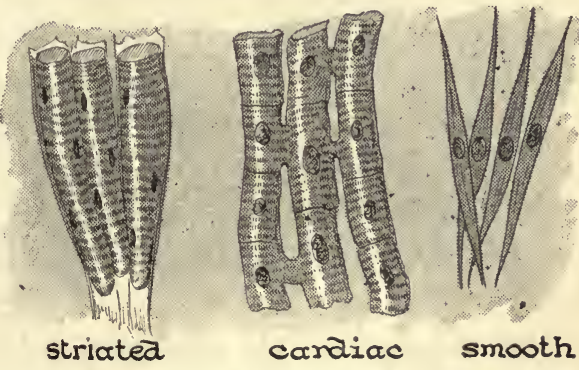

There are three types of muscle cells. Those having many nuclei and cross markings are called striated cells. The spindle-shaped ones with a single nucleus are smooth muscle cells. An intermediate form, crossstriated with a single nucleus, is found in the cardiac muscle cells. Compare the three types.

as contraction and relaxation. Normal muscle cells are always in a slight state of contraction known as muscular tone. This 
keeps the muscles in a condition ready for work. Muscles always exert a pull, not a push. Skeletal muscles usually occur in pairs, one of which opposes the other. For example, in front of the

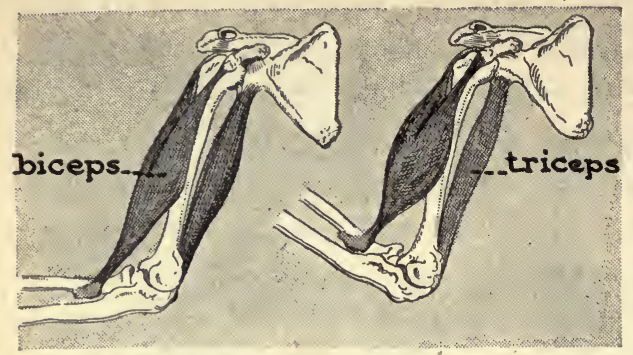

Muscles work in pairs. Motion is caused by the pull resulting from muscular contraction. If the biceps contracts, the triceps relaxes; the forearm is thus pulled up. upper arm there is a muscle called the flexor and on the opposite side, the antagonizing muscle called the extensor. The former causes the forearm to bend and the latter causes it to extend.

Nerve tissue. The organs of the body are composed of various tissues. These tissues and organs are interrelated and brought into communication by means of the nerve tissue. As telephone wires bring various homes of a community into communication, so nerve tissue brings the various organs into coördination. For example, we see a coin on the floor and pick it up. The eyes in seeing, the mind in deciding, the body in bending, and the fingers in picking up, all work in proper sequence due to regulation by the nerves. The muscles actually do the work but the nerves control the muscles. Muscles are kept in proper tone by repeated stimulation from the nerve cells.

If a nerve cell is examined microscopically, the cell body, cyton, with branching projections of protoplasm and with one long process is easily seen. The branching projections are the dendrites and the one long process is the axon. The nucleus lies near the center of the cell body. By means of

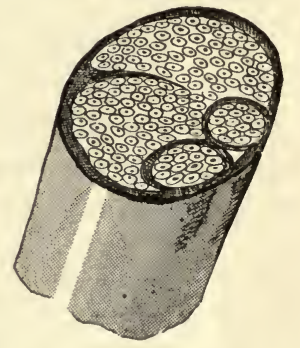

A nerve is a bundle of axons. Each bundle is surrounded by a fatty protective sheath. 
the dendrites, nerve cells connect with each other. The axon is the process which connects the nerve cell with a structure remote from the cell body.

If one hears a sound and walks toward it, an axon has carried the sound from the ear to a nerve cell in the brain. By means of dendrites, a connection was set up with another nerve cell which in turn carried the message to muscle cells. The muscle cells contracted so as to make walking possible. Axons vary in length : some are microscopic while others may be as long as two or three feet. The cell body is always of microscopic dimensions. Nerve cells attain the highest development of irritability. They are the highly sensitive cells of the body. They receive messages, transmit them, and regulate the responses to these messages. Nerve tissue will be studied in greater detail in a later chapter.

Blood tissue. Blood is so highly fluid

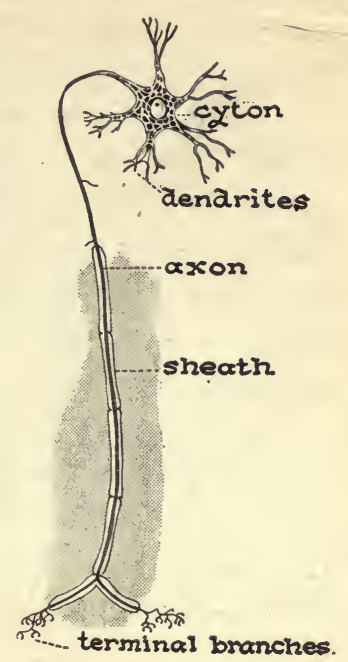

The unit of nerve structure is called a neuron. This cell has several processes, one sometimes very long, the axon. Although the cell body, cyton, is microscopic, the axon, also microscopic in diameter, may be two or three feet long. that it is not usually considered a tissue. A tissue has been defined as a group of similar cells, and blood, consequently, may be considered a tissue since it consists of groups of similar cells.

Problem. Study of blood tissue.

Press the finger near the tip until blood congests. Prick the end of the finger with a needle that has been passed through a flame to sterilize it. Touch a cover glass to the drop of blood on the finger; invert the cover slip on a glass slide. Mount under the low, then under the high power of the microscope.

I. Describe the shape, size, and color of the more numerous cells seen. These are the red corpuscles.

II. Examine all the substance under the microscope carefully and find an occasional irregularly shaped cell. This is a white or amoeboid corpuscle. 
Blood corpuscles. The red corpuscles are circular, biconcave disks lacking nuclei. There are approximately five million in a cubic millimeter of normal human blood. They have a yellowish red tinge when viewed singly, but in great numbers appear red. This color is due to an iron-bearing compound, haemoglobin, which is in the cytoplasm. This haemoglobin readily unites with oxygen and just as readily gives it up when oxygen is scarce. The red corpuscles, in spite of their extremely minute size, can carry large quantities of oxygen. Human tissue cells are not in direct contact with the oxygen of the air. Therefore, they depend upon the specialized red corpuscles for their supply of oxygen.

The white corpuscles are irregular masses of cytoplasm containing one nucleus or several nuclei. Normally, they number between eight thousand and nine thousand to a cubic millimeter. They move and feed in a manner similar to that of amoebas. They send out projections of protoplasm which engulf and digest

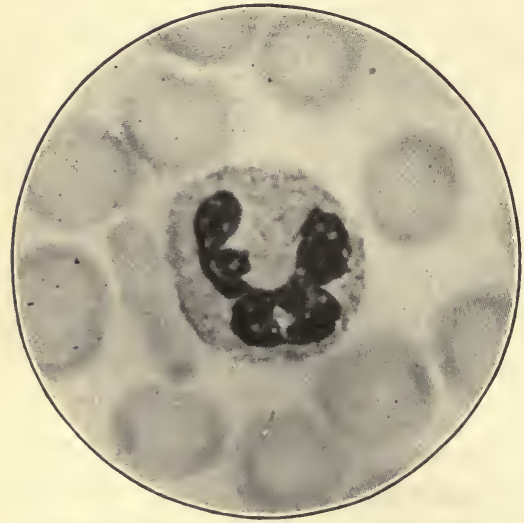

Part of a drop of blood that has been magnified 2500 diameters and then enlarged. The cell shown in the center with a large nucleus is a polynuclear white corpuscle.

foreign materials. They are sometimes called the scavengers of the body because they rid the body of germs and other foreign material. Unlike other body cells, they have the power of independent motion and can move in a direction opposite to the blood current. They can even make their way out of blood vessels and get into the surrounding tissues to destroy germs.

Specialization of human tissues. It is evident from the preceding chapters that the different tissues of the body are adapted to perform particular functions. Epithelial tissue is 
highly specialized for secretion, absorption, and protection; muscle tissue, for contraction; connective tissue, for binding together various parts of the body and for support; nerve tissue, for transmitting stimuli ; and blood tissue, for circulating materials. In general, the cells of all tissues can perform all the cell functions that are necessary for the life of the cell. When cells are highly specialized and are not in direct contact with the outside world, some of their functions become reduced and are practically lost. Then these cells

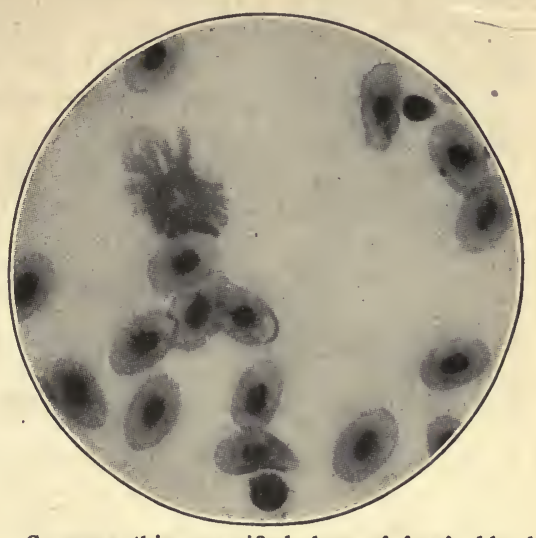

Compare this magnified drop of frog's blood with the human blood. This is not enlarged as many diameters as the preceding photograph. Notice the oval shape of the red corpuscles and the nucleus in each one.

become dependent upon each other to such an extent that life is impossible without this interdependence.

The tissue cells of higher animals do not have to seek food. They are supplied with food by the blood. Certain cells store small quantities of animal starch or glycogen and oil. These particles may be digested by the cells when the need arises. Assimilation and growth remain as functions of all cells. Cells obtain oxygen from the blood, oxidize the food for the release of energy, and use this energy for their work. The muscle cells and white corpuscles release energy, in the form of mechanical energy, particularly for motion. The epithelial cells use chemical energy, and nerve cells use nervous energy. The connective tissues and red corpuscles need only sufficient energy to perform their general cell functions. Most of the tissue cells lose their power to divide mitotically. The epithelial cells and the white corpuscles are the only ones, thus far studied, which retain this power throughout life. 


\section{Questions and Suggestions}

1. Name all the tissues of the hand and give the function of each.

2. Compare muscle, nerve, and blood tissues in function and in adaptations of their structure for the function mentioned.

3. Compare a Paramecium and a tissue cell with respect to functions and adaptations for function.

4. Compare a Spirogyra cell and a tissue cell as to functions and adaptations for function.

\section{SuPPLEMENTARY READINGS}

Kimber and Gray, Textbook of Anatomy and Physiology (The Macmillan Co.). Stöhr, Textbook of Histology (P. Blakiston's Son \& Co.).

Williams, Jesse F., Healthful Living (The Macmillan Co.). 


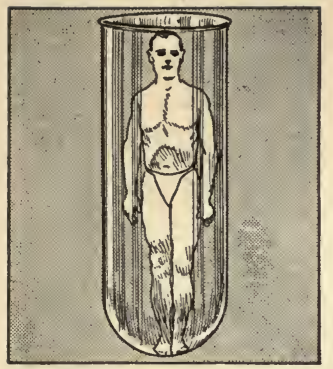

When broken into elements

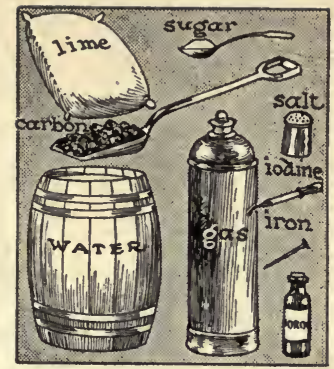

man is worth eighty-two cents.

What is a food? What is the relation of a nutrient to a food? What is meant by dietary requirements and dietary deficiencies?

Since the cell is the basis of our make-up, and since these cells live, grow, and work, each must have food and oxygen to carry on the various life processes. The cells of the brain must have' food and fuel to do the part each does in the thinking process. Muscle cells in the thumb and fingers must be nourished and given oxygen if they are to do their work.

What is a food? A beefsteak includes lean meat, fat, and bone. Only a part of this can be used by the body. This part is nutritious. Potatoes contain about 70 per cent of water and varying amounts of starch, protein, and mineral salts. As a matter of fact, they contain only about 25 per cent of nutritive material. The small per cent of cellulose which makes up the cell walls has no more real food value than the wood of a lead pencil. It passes through the food tube, unaffected chemically. Such parts of the potato are called waste. Thus we see that every food may contain both nutrients and wastes. Certain of the waste materials that pass through the food tract undigested, serve the real and desirable purpose of giving bulk to the diet.

What is a nutrient? Any substance, such as carbohydrate, protein, or fat, that yields material for growth and repair of tissues or, 
when oxidized, can be used as fuel for the release of energy, is a nutrient. The term nutrient is frequently used to denote the

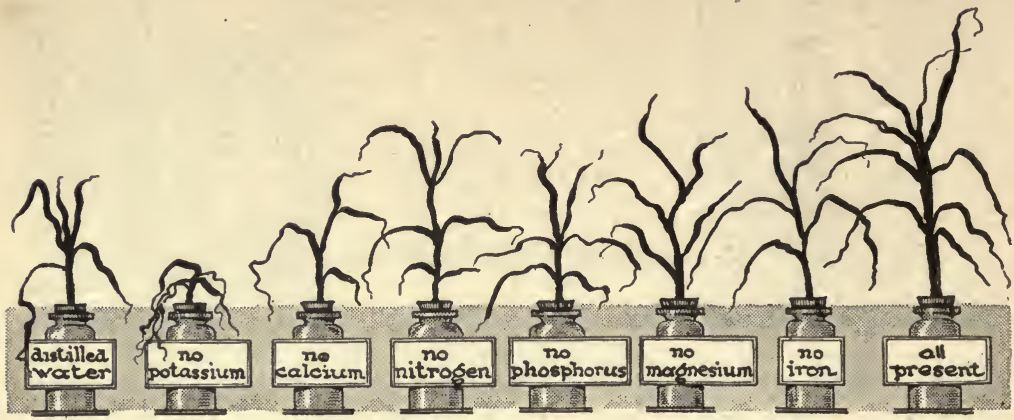

Malnutrition exists among plants as well as animals. Many different minerals are essential for the best growth and development of plants. When soil is properly fertilized, it contains all the essential mineral matters. Note the relation of minerals to plant growth.

whole food, but foods are really made of combinations of nutrients. The energy which was originally stored in the food by the plant during the process of photosynthesis, is released from the food during the oxidation process in the body. This stored energy is set free from the food in some active form; such as, muscular energy, chemical energy, heat energy, or nervous energy. When sugars and fats are burned, either over a fire or in the body, waste products, carbon dioxide and water, are formed; when protein is burned in the body, nitrogenous wastes, containing some urea and uric acid, are formed in addition to carbon dioxide and water. All foods do not yield the same amounts of energy. The oxidation of those foods in which sugars and fats are concentrated in the greatest amounts, yields the most energy with the least amount of waste. The best fuel nutrients are sugars and fats, and the growth or building nutrients are proteins, salts, and water.

When the diet contains an insufficient amount of sugars and fats, some of the proteins may be oxidized for the release of energy. But proteins give off great amount of wastes which the body usually has difficulty in disposing of effectively. 
The energy in food is measured in terms of heat units called calories. A calorie represents the amount of heat required to raise the temperature of one gram of water one degree centigrade. When we deal with food values, we use the large calorie, which is 1000 times the small calorie. In other words, the large calorie is the amount of heat required to raise one kilogram (1000 grams) one degree centigrade. When we speak of the caloric value of a food we mean the power of food to yield heat units. Food chemists group our food nutrients into the five classes which were studied in general science or elementary biology. The table on page 94 will help you recall certain facts concerning these classes of nutrients.

Complete and insufficient diets. Even if a person ate some of all the nutrients, it would not necessarily mean that he was getting a sufficient or a complete diet. If the diet is composed entirely of the nutrients from the same food, it is usually insufficient, and the person using this diet may suffer from malnutrition. Careful investigations have disclosed that 15 to 25 per cent of the children in the United States are suffering from malnutrition.

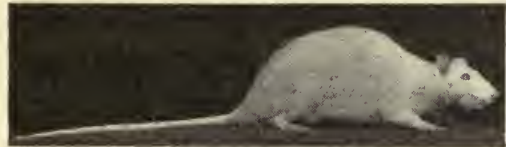

Gliaden and egg diet

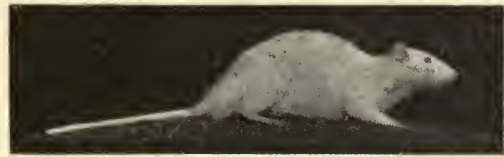

Gliaden and milk diet

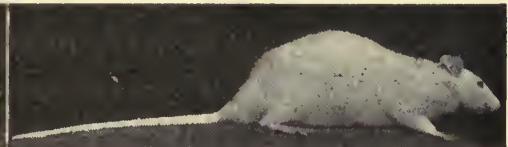

Gliaden and meat diet

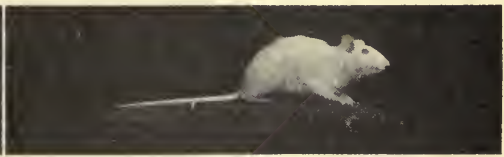

Gliaden diet

Rats need a variety of food materials. These four-month-old rats are from the same litter and have been fed the same quantity of food, but of different variety. Note the differences in size.

Protein insufficiency. An investigation was made, by McCollum and others, in a certain institution for children to discover the 


\begin{tabular}{|c|c|c|c|c|c|c|}
\hline 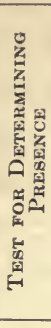 & 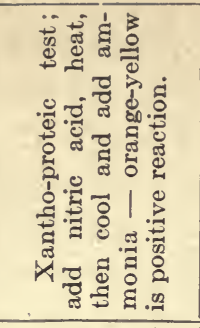 & 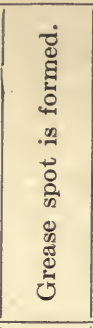 & 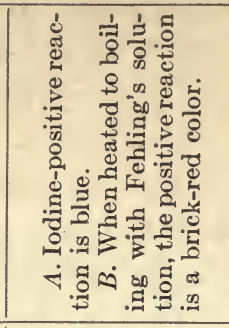 & 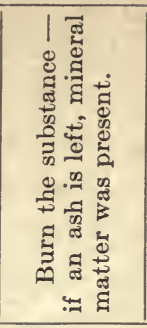 & 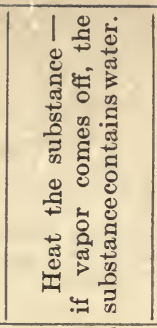 & 㐘 \\
\hline 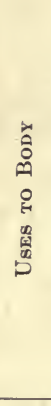 & 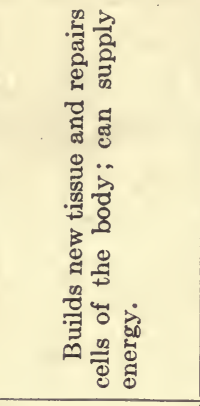 & 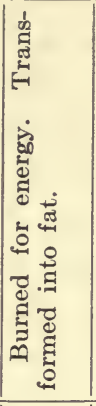 & 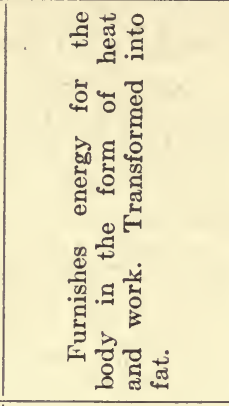 & 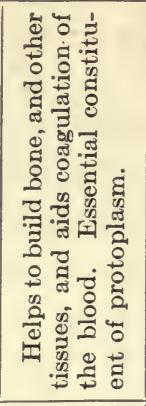 & 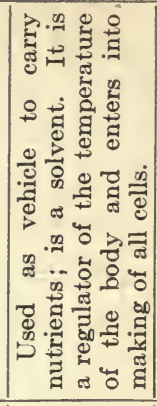 & : \\
\hline 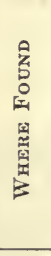 & 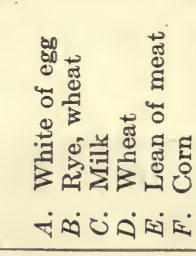 & 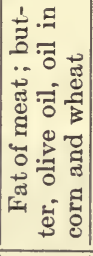 & 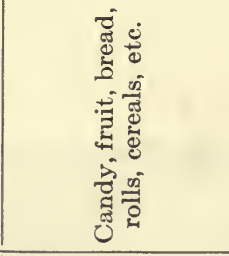 & 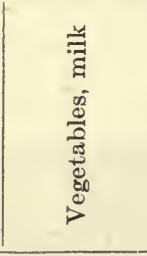 & 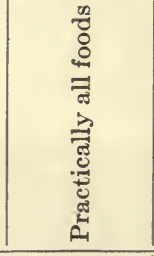 & $\frac{7}{8}$ \\
\hline 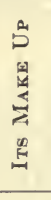 & 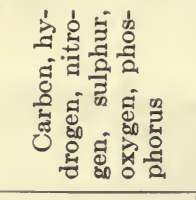 & 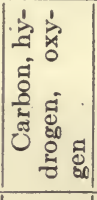 & 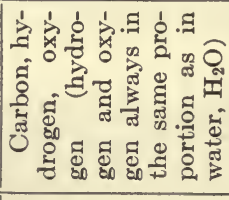 & 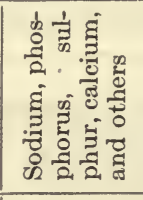 & 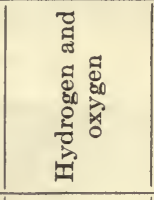 & $\begin{array}{l}0 \\
\end{array}$ \\
\hline & 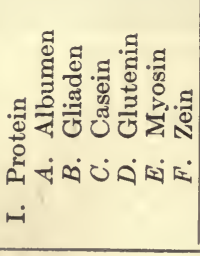 & 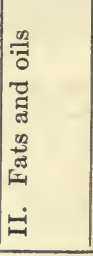 & 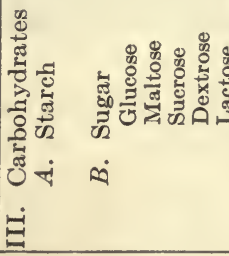 & $\mid$ & 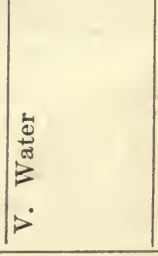 & \\
\hline
\end{tabular}


cause of so many cases of malnutrition. The institution was in excellent condition as far as ventilation, sanitary facilities, sun porches, playgrounds, and careful supervision were concerned, but severe malnutrition existed among the children. A careful study of the food of the children showed that there were sufficient nutrients in the foods given in the diet, but that the proteins were supplied chiefly by lean meat. The children were then divided into two groups. In one group, a quart of milk was added to the daily diet of each child. In the other group, the children continued on the original diet without the milk. Within a short time there was a marked increase in body weight among the children in the milk-fed group. The increased weight was maintained, and there was a noticeable change in behavior. The milk-fed group became much more active than the group of children not receiving milk. From this and similar experiments it was concluded that a diet composed largely of cereals, vegetables, meat, and bread does not prove satisfactory for the physical development of the young children and that the addition of milk furnishes the supplementary food for the type of diet that is lacking in sufficiently varied animal proteins. It has also been found that the proteins in peas, beans, and other vegetables are of less anabolic (pertaining to the building of protoplasm) value than those in milk, egg, and meat.

Mineral insufficiency. There seems to be a marked tendency in the average American, to-day, to have proportionately too little calcium in his diet in relation to the amount of phosphorus. It may be partly in consequence of this, that millions of the school children of the United States have been found to have defective teeth. The calcium must not only be present in the diet, but must be in such a form that the body is able to use it. The utilization of calcium will be more fully discussed in a later chapter. The list on the following page gives some of the common foods that contain various minerals. 
Sodium - common salt.

Potassium - meat and vegetables.

Magnesium - meat and vegetables.

Calcium -milk and leafy vegetables.

Sulphur - meat and vegetables.

Phosphorus - cheese, cod, haddock, celery, spinach, and lettuce.

Iron

- meat, milk, eggs, whole wheat, spinach, and beans.

Iodine - milk, leafy vegetables, fruits, and water.

If the diet is widely varied to include a great many different foods, sufficient minerals are obtained. When the diet is not properly varied, specific minerals are sometimes prescribed by doctors. For example, limewater is put in the milk of infants, or medicines containing iron are given children. The body utilizes to some extent the minerals in this form. However, it is cheaper and better to get the minerals from food. Here, they are bound chemically with organic substances, in which form they are easily assimilated by the body.

Vitamins. Certain of our foods are of great use to the body in that they are health regulating. It is known that such foods

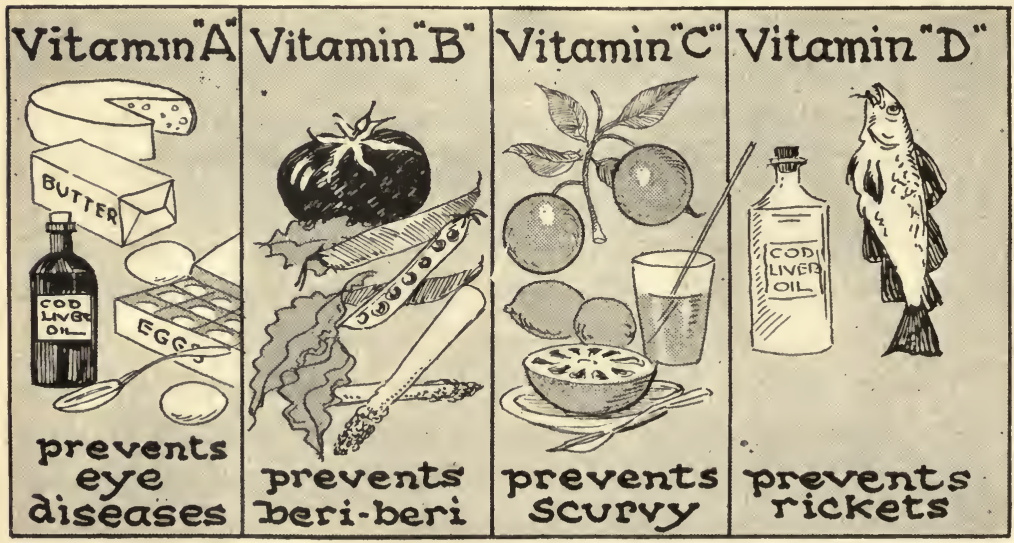


contain certain substances known as vitamins. Little is known of the physical and chemical properties of vitamins, although a great deal of research is being done in this field of nutrition. The amount of vitamins in food has not yet been

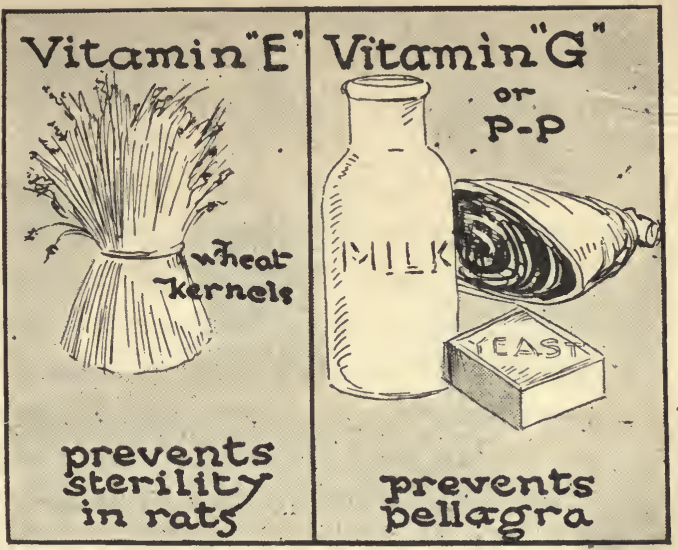
measured in definite units, but is expressed by relative terms such as abundant, rich, fair, poor, and deficient.

Vitamins, a long-known need. Writings of the ancient Greeks show that scurvy, now attributed to a diet deficiency, was then known, but not understood. The person suffering from this disease loses weight, is anaemic, pale, and weak. During the Middle Ages, the records of the Crusaders show that this disease occurred frequently. The Crusaders attributed it to something injurious in the diet rather than to the lack of some requirement. Indeed, armies of nearly all countries have suffered from scurvy during wars. It has appeared whenever extended campaigning or other conditions have limited the opportunity to get fresh and varied foods.

At one time it was impossible to supply sailors on long voyages with fresh foods. Captain Cook, the English explorer and trader, was one of the first to recognize the value of fresh food as a protection against scurvy. His extended voyage, which was begun in 1772 , was much discussed because no scurvy appeared among the crew. This was true even though the voyage lasted over three years and covered vast stretches of the South Pacific and South 
Atlantic Oceans. Such a conquest of the ancient scourge was unknown up to that time. Captain Cook attributed his success to the liberal use of such fresh fruits and vegetables as could be obtained, and to the frequent use of sauerkraut and barley. Partly on account of this experience and partly because of similar observations and controlled experiments, regulations for the British navy have required, since 1795 , that all ships' crews be supplied with fruit juices (usually limes) and vegetables which were thought to prevent scurvy. (Because English sailors eat limes for the vitamin in lime juice they are often called " limeys.") Not only armies and navies, but exploring expeditions, camps of lumbermen, and other isolated communities of persons have suffered from scurvy. Although this disease had indicated, during all centuries of recorded history, that some peculiar food substance was required, recognition of the vitamin-character of the substance which prevents scurvy came only in recent years (1919-1920).

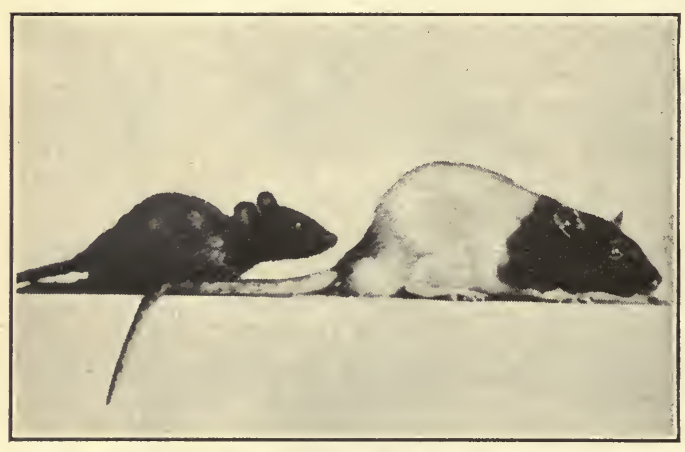

The diets of these two rats of the same age were alike in every respect except in the kind of fat. The rat on the right received butter which contains vitamin $A$; the rat on the left received lard which lacks vitamin $A$. The stunted rat developed an eye disease.

A similar story is found in the history of the peculiar disease called beriberi. This has also been one of the ancient scourges of certain races. Definite records, dating back to an extremely remote period, appear in Chinese writings, telling of the ravages of beriberi.

Beriberi is characterized by inflammatory changes in the nerves and it usually involves distortion and even paralysis of the limbs. Like scurvy, this disease may prove fatal if it is not counteracted 
by appropriate foods. Beriberi occurs frequently in Asiatic countries where rice is the staple article of food. The unquestioned prevalence of the disease among rice-eating people led to the theory that a fungus or bacterial growth in rice might be the cause of this disease. However, a deficiency in the vitamin diet is now gènerally held to be the cause of this disease.

Present-day knowledge of vitamins. The experiments performed in the

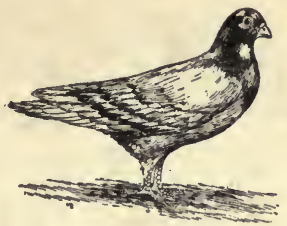

Funk fed pigeons of the same age the same amounts of rice. The one on the left ate whole rice. The one on the right was fed polished rice which lacks vitamin B. The second pigeon is showing symptoms of polyneuritis which can be cured by feeding it foods rich in vitamin B. Polyneuritis is also found in people.

last few years have given us considerable knowledge of vitamins. Unless we include them in the diet, deficiency diseases may result.

An interesting experiment was carried on to determine the diet essential for the normal development of rats. Baby rats were fed equal amounts of food with proper kinds and proportions of proteins, carbohydrates, and mineral matters, but one group was given fat in the form of lard and the other given butter instead of the lard. The rats fed on lard failed to grow normally and they soon developed sore eyes. The butter-fed rats grew into normal healthy rats. Lard lacks the vitamin known as the fat-soluble vitamin $A$. This vitamin occurs in milk, butter, cod-liver oil, egg yolk, and in the leafy green parts of vegetables such as spinach, lettuce, and cabbage; and in the yellow pigmented parts of plants such as carrots and sweet potatoes. The lack of vitamin A causes stunted growth, and a certain eye disease, xerophthalmia, which may result. in permanent blindness. Taken in its early stages, however, this disease can be cured by including in the diet some foods containing vitamin $\mathrm{A}$.

In another experiment, two groups of pigeons were fed, one on 
polished rice and the other on whole grains of rice. In a short time, the first group developed a peculiar nervous disease, polyneu-

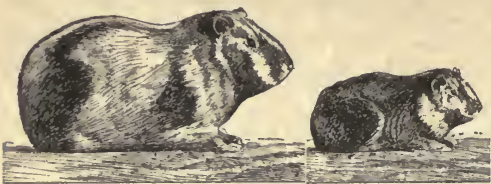

Two guinea pigs of the same litter were fed standard diets. The one on the right did not get any vitamin C. It lost weight, and developed scurvy which is a type of malnutrition. ritis or animal beriberi. The group fed on whole rice remained healthy. Polished rice lacks a vitamin known as vita$\min B$. When the polyneuritic sufferers were given food or water solutions made from rice polishings, they recovered.

Vitamin B occurs in yeast, milk, and in the hulls or outer coverings of grains and fruit. In fact, this vitamin is found in so many common foods that it is probably only an extreme diet of some kind that will induce beriberi.

Two sets of guinea pigs were fed a diet containing evaporated powdered milk in addition to the basal foods required. In the case of one set, the milk was heated for a long period; the other set of pigs was fed the unheated milk. The first group developed scurvy; the second group remained healthy. Tests show that vitamin $C$, which is present in milk and the lack of which causes scurvy, is, in a large measure, destroyed by heat. Pas-

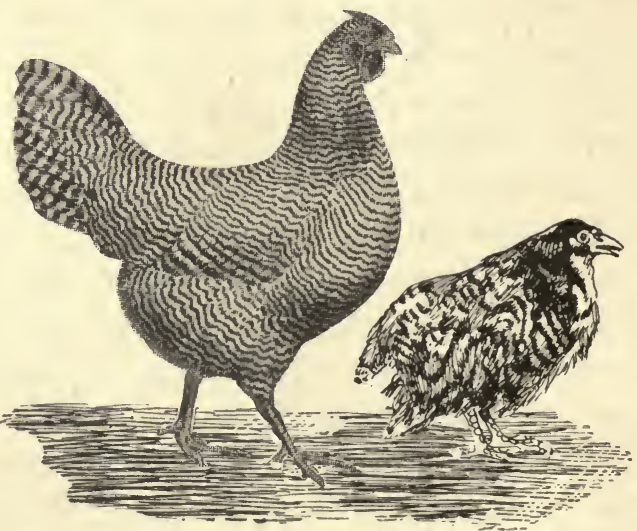

Leg disease, a type of rickets, may be due to lack of vita$\min \mathrm{D}$. These chickens are the same age and received the same amounts of different foods. Food containing vitamin D or even sunlight will help to prevent and cure rickets. teurization of milk, therefore, may destroy this vitamin. For this reason, babies that are fed on pasteurized milk should be given 


\begin{tabular}{|c|c|c|c|c|c|c|c|c|c|c|c|c|c|c|c|}
\hline & "A" & " & "C" & "D" & "E" & "F" & "G" & & "A"s & "B" & "C" & "D" & "E" & " F" & $" G "$ \\
\hline $\begin{array}{l}\text { VEGETABLES } \\
\text { Spinach }\end{array}$ & $* * *$ & $* * *$ & $* *$ & & $* *$ & & $* *$ & $\begin{array}{l}\text { MEAT, FISH, ETC. } \\
\text { Lean muscle }\end{array}$ & * & * & $*$ & & * & & $* * *$ \\
\hline Lettuce & $* * *$ & $* *$ & $* * *$ & & ** & $* *$ & $* *$ & Pork, lean & * & $* *$ & * & & $*$ & & $* * *$ \\
\hline Cabbage & $* *$ & $* * *$ & $k * * *$ & * & $* *$ & & $* *$ & " fat & ** & & & & & & $* *$ \\
\hline Potatoes, white & * & $* *$ & $* *$ & & & & $*$ & Chickens & & & & & * & & $* *$ \\
\hline Beans, kidney & & $* *$ & & & & & $*$ & Turkeys & & & & & $*$ & & $* *$ \\
\hline Beans, navy & & $* *$ & & & & & * & Bains & ** & $* *$ & & & $*$ & & $*$ \\
\hline Beets & & $*$ & & & & & $*$ & Liver & $* * *$ & $* * *$ & $* * *$ & & $*$ & $* * * *$ & $* * * *$ \\
\hline Carrots & $* * *$ & $* *$ & $* *$ & & & & $*$ & Heart & $* * *$ & $k * * *$ & $*$ & & $*$ & & $* *$ \\
\hline Onions & & * & $* *$ & & & & $* *$ & Kidneys & $* *$ & $* *$ & * & & $*$ & & \\
\hline Peas, fresh & $* * *$ & $* *$ & $* *$ & & $* *$ & & $* *$ & Fish & & $*$ & & & $*$ & & $* *$ \\
\hline Asparagus & & $* * *$ & & & & & $*$ & Fish, roe & $* * *$ & $* *$ & & & $*$ & & $* *$ \\
\hline Parsley & & $* * *$ & & * & * & & $*$ & Oysters & & & $* * *$ & & $*$ & & $*$ \\
\hline Parsnips & $* *$ & $* *$ & & & & & $* *$ & "NUTS & & & & & * & & \\
\hline Celery & & $* *$ & & & & & * & Almonds & * & $* *$ & & & & & \\
\hline Cauliflower & $*$ & $* *$ & * & & & & * & Brazil nuts & & $* *$ & & & & & \\
\hline Cucumbers & & $*$ & & & & & $* *$ & Chestnuts & & $* *$ & & & & & \\
\hline Mushrooms . & & $* *$ & & & & & $*$ & Cocoanuts & ** & $* *$ & & * & & & \\
\hline Squash & $* *$ & $* *$ & & & & & $* *$ & Filberts & & $* *$ & & & & & \\
\hline FATS AND OILS & & & & & & & & Hickory nuts & $*$ & $* *$ & $*$ & & & & 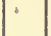 \\
\hline Cod Liver Oil & $* * *$ & & & **** & & & & Peanuts & $*$ & $* *$ & & & & & \\
\hline Butter & $* * *$ & & & & & & & Pecans & & $* *$ & & & & & \\
\hline Corn Oil & * & & & & & & & Walnuts, English & & $* *$ & & & & & \\
\hline Margarine, oleo & * & & & & & & & " black & & $* *$ & & & & & \\
\hline Mutton fat - & $* *$ & & & & & & & DAIRY PRODUCTS & & & & & & & \\
\hline Beef fat & $* *$ & & & & & & & Milk, whole & $* *$ & $* *$ & * & $* *$ & * & & $* * *$ \\
\hline Cottonseed & & & & & * & & & “ skim & $*$ & $* *$ & $*$ & $*$ & & & \\
\hline Olive & & & & & * & & & "' condensed & $*$ & $* *$ & $*$ & & & & \\
\hline FRUITS & & & & & & & & “ “ sweetened & d) $* *$ & $* *$ & $*$ & & & & \\
\hline Prunes & & * & & * & & & & " evaporated & $* *$ & $* *$ & & & & & \\
\hline Apples & $*$ & $* *$ & $* *$ & & & & $*$ & "powdered & $* *$ & $* *$ & & & & & \\
\hline Bananas & * & $* *$ & $* *$ & & * & & * & Butter & $* * *$ & & & * & * & & \\
\hline Grapefruit & & $* *$ & $* * * *$ & & & & $* *$ & Cheese, whole milk & $* *$ & * & & & & & \\
\hline Lemon juice & & $* *$ & $* * * *$ & & & & * & MISCELLANEOUS & & & & & & & \\
\hline Pineapples & $* *$ & $* *$ & $* *$ & & & & * & Yeast & & $* * *$ & & & & & ***** \\
\hline Limes. & & *** & * & & & & * & Molasses, beet & & $* *$ & & & & & \\
\hline Orange juice & $* *$ & $* *$ & $* * *$ & & & & $* *$ & " sorghum & & $* *$ & & & & & \\
\hline Tomatoes, raw & $* * *$ & $* * *$ & $* * * *$ & & & & $*$ & " cane & & $* *$ & & & & & \\
\hline “ canned & $* * *$ & $* *$ & $* * *$ & & & & * & Eggs & $* *$ & $* *$ & & & & & $* *$ \\
\hline Raisins & & $* *$ & $*$ & & & & $*$ & Eggs, yolk & $* * *$ & $* * *$ & ** & $*$ & * & & $* *$ \\
\hline GRAINS AND SEEDS & & & & & & & & Alfalfa & $* * *$ & $* * *$ & $* *$ & & * & & \\
\hline Wheat, whole & * & $* *$ & & $*$ & $* * *$ & & & Clovers & $* * *$ & $* * * *$ & ** & & $*$ & & \\
\hline " germ & $* *$ & $* * *$ & & & $* * *$ & & & Green grass & $* * *$ & $* *$ & $* *$ & & & & \\
\hline "bran & $* *$ & $*$ & & & & & & Timothy & $* * *$ & $* *$ & $* *$ & & & & \\
\hline Corn, white & & $* *$ & & & $* *$ & $* * *$ & & Tea & & & & & $* *$ & & \\
\hline "yellow & $* *$ & $* *$ & & & $* *$ & & & & & & & & & & \\
\hline Oats & & $* *$ & & & $* *$ & & & & $* * *$ & abun & ndant & & & & \\
\hline Barley & $*$ & $* *$ & & & $* *$ & & & & $* *$ & ampl & & & & & \\
\hline Rice, bran & & $* * *$ & & & & & & & $*$ & insuf & ficien & & & & \\
\hline Cottonseed & * & ** & & & $*$ & & & & & defi & ient & & & & \\
\hline
\end{tabular}


other foods to supply this vitamin. Vitamin C occurs largely in citrus fruits such as lemons and oranges, and in tomatoes and

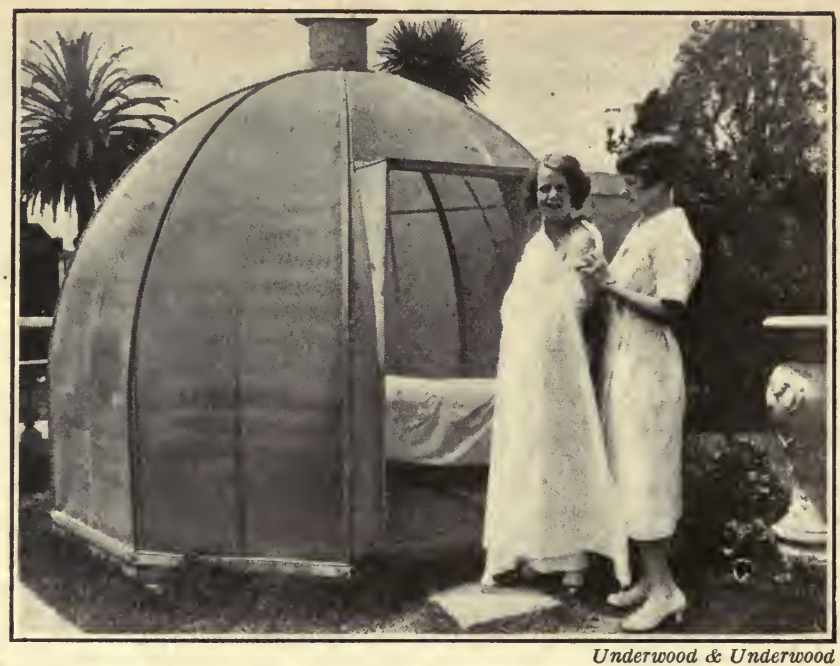

Sunbaths are valuable for insuring the proper functioning of the body. Irradiation guarantees an optimum storage of calcium in the body.

cabbage. During the early days of the great World War, before the United States had entered the fight, a foreign ship entered Hampton Roads with hardly enough healthy sailors to man the vessel. The ship had been transformed into a commerce raider, a gun had been mounted both forward and aft, and the hold was filled with enough canned and concentrated foods to provision the crew of sailors and marines for many months. But fresh foods were lacking and the crew was repeating the experience of many sailors; it was suffering from scurvy. Orange juice, tomato juice, milk, raw cabbage, celery, carrots, and water cress, which are known to contain vitamin $\mathrm{C}$, were, at once, included in the diet of the sailors and they soon recovered.

Many animals suffer from a disease known as rickets, which is an improper formation of bones. This seems to be caused by the 
non-deposit of the mineral calcium which is present in certain foods. The head of a rachitic animal usually becomes bulky, and the bones at the knees and ankles, become enlarged. For experimentation, a set of rats was fed a diet which contained vitamin A, but lacked another substance known as vitamin $D$; another set of rats was given vitamin $\mathrm{D}$ in the form of cod-liver oil. The first group developed rickets ; the second group grew normally: Many physicians and scientists have observed and reported that the exposure of rachitic children to the rays of the sun has brought about an improvement and cure of rickets. In these cases there were plenty of calcium and phosphorus already present in the blood, and the sunlight is thought to have influenced, in some way, the activities of certain cells so that they could make efficient use of . these minerals. Other scientists have found that it is the ultra-violet rays in sunlight, which are instrumental in preventing and curing rickets. These curative rays cannot pass through ordinary window glass, but will pass through quartz glass.

If babies are given food deficient in vitamin $\mathrm{D}$, but which has been exposed to these light rays, the food can be activated so as to cause it to have the same effects as the ultra-violet rays. Cotton-seed oil, which ordinarily contains no vitamin D, becomes antirachitic when exposed to sunlight.

Two scientists, Evans and Bishop, have added another member to the

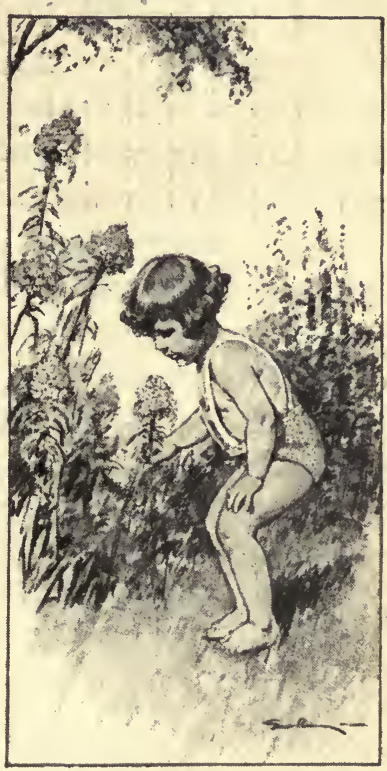

Sunsuits give children the benefit of the direct rays of the sun. vitamin family. They found that this vitamin, which they named vitamin $E$, has a marked influence upon the fertility of rats. 
When a diet lacking certain food stuffs which contain vitamin $\mathrm{E}$ is fed to rats, the reproductive organs of both the male and female will be affected. Investigators have found that a deficiency of this vitamin in the mother rat will lead to the resorption of the embryos even after development has proceeded in a normal manner for a week or more. Other research workers have found that this vitamin is present in meat, wheat germ, rolled oats, yolk of egg, milk fat, and lettuce.

In the biology of to-morrow, there will probably be other vitamins to add to the group already listed. There has been some work with what has been called vitamin $G$ or vitamin P-P (pellagrapreventive). Lack of it is known to induce pellagra, a disease afflicting many people in the southern states. The late Dr. Joseph Goldberger, of the United States Public Health Service, found that certain foods contain this pellagra-preventive, but as yet the amount of the preventive substance in various foods has not been conclusively determined.

Diets. Malnutrition was at one time attributed to poverty, but scientists have found that there is almost as much malnutrition among the wealthy as the poor. Proper dieting is a matter of selecting different foods that give all the elements essential to the health of the individual. Many dietary studies have been made in various countries and among people doing different types of work. From these studies and from experiments, certain foods have been recommended that are thought to make for physical fitness.

All diets should include proteins, fats, and carbohydrates, probably in the proportions of one, three, and six. They should include, among other foods, a quart of milk and some leafy or raw vegetable so that a sufficient supply of vitamins can be guaranteed.

It should be kept in mind that people of different ages, sizes, weights, and occupations naturally require different amounts of calories of foods. The following table is suggested for determining the number of calories of food needed by boys and girls : 
Boys

Girls

*From 12-13. . . . . . . . . . 2300-2700. . . . . . . . . . 1850-2150

From $13-14 \ldots . . \ldots \ldots$. . 2500-2900. . . . . . . . . 1950-2250

From $14-15 \ldots . . . . .2600-3100 \ldots$. . . . . . . 2050-2350

From $15-16 \ldots . . . . .2700-3300 \ldots . . . .2150-2450$

From $16-17 \ldots . . . .2$. 2700-3400. . . . . . . 2250-2550

Problem. Study of personal diets.

I. Consult the age and weight tables in the Appendix and find out whether you are normal, underweight, or overweight.

II. From the table given above, determine the number of calories you need per day.

III. Make out the following outline and list the foods eaten by you on a definite day.

\begin{tabular}{|c|c|c|c|}
\hline & Proteins & - Fats & CARBOHYDRATES \\
\hline 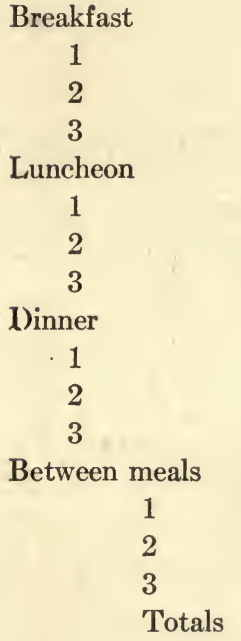 & 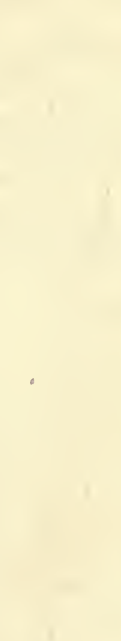 & & 1 \\
\hline
\end{tabular}

* New York Association for Improvement of the Condition of the Poor. WH. FITZ. AD. BIO. -8 
A. Consult the tables of caloric values of foods in the index.

1. Fill in the number of calories of protein, fat, and carbohydrates for each food you have listed. Total the columns.

2. Add the totals. Compare your actual totals with the number of calories you really need according to the given table.

a. Are you eating enough, too little, or too much food?

$b$. Is the ratio of protein, fat, and carbohydrates correct?

c. Consult the suggestions given in the discussion of foods and see whether all the types of food needed are in your diet.

3. What changes, if any, should be made in your diet?

a. Give reasons in each case. The age-weight tables are not authentic opinions as to malnutrition. They are, however, somewhat indicative of the condition of nutrition.

\section{Questions}

1. Define a food and a nutrient.

2. What elements in foods are fuel for oxidation?

3. What are vitamins? What are their value in the diet?

4. What are deficiency diseases? Name several.

\section{Supplementary Reading}

Harrow, Benjamin, Vitamins (E. P. Dutton \& Co. Inc.).

McCollum \& Simmonds, The Newer Knowledge of Nutrition (The Macmillan Co.).

Sherman, Chemistry of Food and Nutrition (The Macmillan Co.).

Stieglitz, J. O., and others, Chemistry in Medicine (Chemical Foundation Inc.). 


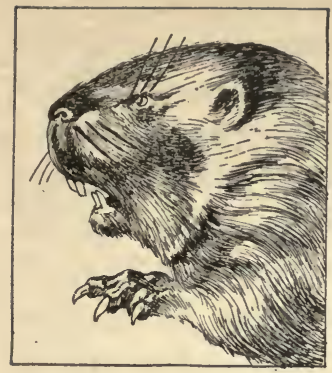

Continuous growth of teeth.

\section{CHAPTER XII \\ THE TEETH AND THEIR CARE}

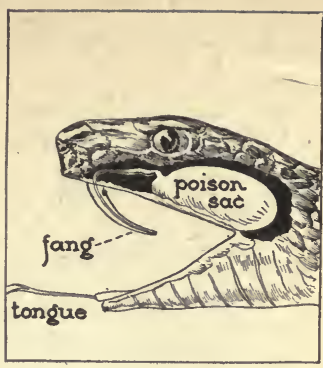

Fangs of snake inject venom.

What are some causes of bad teeth? What is meant by the hygiene of the teeth? Why are the teeth of the average American probably inferior to those of his great-grandparent?

Detailed structure of teeth. Not only are teeth important in helping to prepare food for use in the body, but they are invaluable in maintaining the health of the body. The teeth, embedded in sockets in the upper and lower jawbones, are especially fitted for the work that they have to do. Each tooth has a root which consists of one or more divisions contained in the socket; a crown which projects above the gums; and a neck which is the narrow portion at the edge of the gum.

Each tooth is composed principally of dentine. In the center of the dentine is the pulp cavity which tapers into the root canal and ends in a small opening at the extremity of the root. The pulp cavity is filled with loose connective tissue containing blood vessels and nerves which enter through the root canal. The dentine is crowned with a hard layer of enamel composed mostly of calcium. The dentine of the root is covered with cement which has practically the same composition and structure as bone, but is much harder. Every normal person has two sets of teeth during his life, the first or temporary, and the permanent. There are twenty temporary teeth, ten in each jaw: four sharp-edged in- 
cisors, two sharp canines, and four molars especially adapted for crushing and grinding. The first teeth of the temporary set usu-

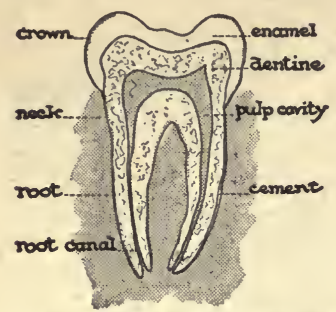

Blood vessels and nerves enter the tooth cavity, which consists of connective tissue, through the root canal. This cavity is well protected by the enamel, dentine, and cement. ally begin to appear when the child is six months old and the last appear when he is about two years old. The teeth of the lower jaw usually develop before the corresponding ones of the upper jaw.

The permanent teeth push out the temporary teeth. The milk molars are followed by the permanent premolars, but there are no predecessors for the permanent molars.

The first permanent teeth usually appear about the sixth year while the last ones may not appear until the twenty-fifth year. The molars are the first to appear, then the two central incisors about the seventh year, the two lateral incisors at eight, the bicuspids at nine and ten, the canine at eleven or twelve, the second molars at twelve or thirteen, and last are the third molars or wisdom teeth.

The first permanent teeth to appear are the upper first molars which do not replace any of the milk teeth, but come down behind the second temporary molars. When the temporary teeth of children are neglected these six-year molars are often neglected, and consequently have to be extracted early in life.

Arrangement of teeth. When the mouth is closed the upper front teeth protrude slightly over the lower front teeth. If the teeth are irregular or out of position, this arrangement is changed. Adenoids are sometimes responsible for narrowing the jaw and throwing the teeth out of position. People with poor formations of teeth can not chew their food properly and digestion is therefore impaired. When teeth are crowded and out of place, the food may remain lodged between the teeth and cause them to decay. The normal contours of the face are changed by malformations of the 
teeth. In order to insure good health, teeth should be straightened and adjusted. Dentists are usually able to obtain better results in correcting these malformations if the teeth are not fully grown.

Care of the teeth. When the teeth are not cleaned frequently, a substance known as tartar often forms upon them, usually near the gums, and prevents the bacteria from being rubbed off by the action of the tongue. For this reason artificial means must be employed to keep the teeth free from this gummy tartar which later becomes hard. The teeth should be brushed at least twice a day with a tooth paste or powder which contains some substance, such as chalk, which is hard enough to rub off all deposits but not hard enough to injure the enamel of the teeth. The

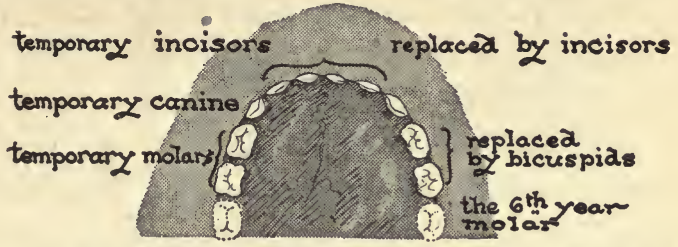

The teeth of a six-year-old child are temporary teeth with the exception of the six-year molar. This is the first permanent tooth to cut through the gum. Its position is just back of the milk molars.

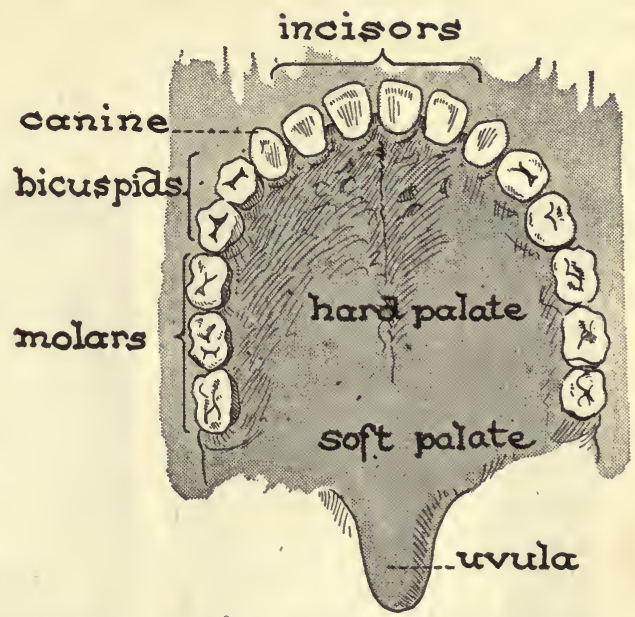

Permanent teeth are for biting, chewing, and grinding. teeth should be brushed with a fairly stiff brush after each meal, in order to remove all particles of foods, and to stimulate the circulation of blood in the gums. The mouth cavity should then be thoroughly rinsed out with a mouth wash or a solution made 
by dissolving a half teaspoonful of salt in a glass of warm water. Fruit acids such as dilute lemon juice or vinegar should be used occasionally. If food lodges between teeth, it should be removed by dental floss. A metallic toothpick or pin should never be used as it may chip or crack the enamel. Wooden toothpicks should also be avoided as they tend to cut and irritate the gums. Silk thread is not an adequate substitute for dental floss because it is not sterile and it may cut the gums.

Periodical visits to the dentist. In spite of the best care, the tartar cannot be entirely removed from the teeth with a brush. The bacteria which are held in the soft tartar will probably decay the food left in the teeth. Sometimes the fermenting of sugar between the teeth and the acids formed by bacteria will dissolve the enamel, causing soft spots or tiny cavities in the teeth. The soft dentine is then exposed to the decay action of the bacteria. Once a year, at least, a visit should be made to the dentist for the purpose of having the teeth cleaned and any small cavi-
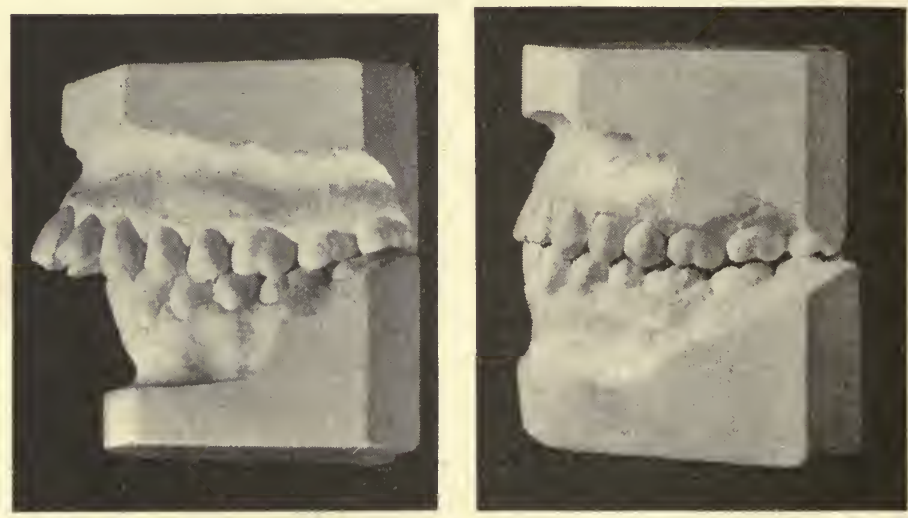

By skillful dental attention, projecting incisors of the upper jaw can be made to articulate, that is, meet the lower jaw.

ties detected and filled before they seriously weaken the teeth. When accumulated tartar remains on the teeth, the gums may 
recede, become swollen and inflamed, and the teeth may loosen in their sockets. This is one type of pyorrhea which a dentist may be able to prevent.

When the teeth decay, pus is frequently formed and absorbed by the blood. The pus may travel through the body and may settle in the valves of the heart, causing a heart defect, or in the joints, causing rheumatism. Besides this, decayed teeth give the breath an objectionable odor and the mouth an
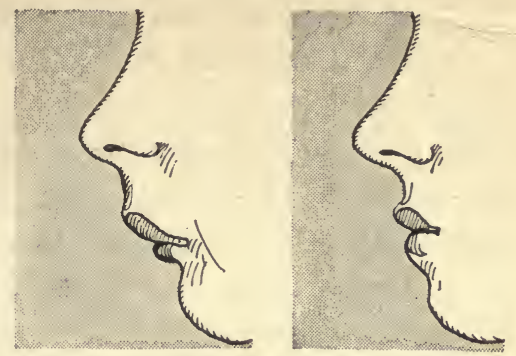

The contours of a profile may be changed by straightening the teeth. These outlines are the profile of a boy before and after he had received dental treatment.

unsightly appearance. Physicians often insist that patients have the teeth examined and treated before they will treat them for other ailments. The disorder sometimes disappears when the teeth are put in good condition. Defective teeth do not have as great grinding power as have sound teeth, and, therefore, hinder digestion.

Relation of diets to teeth. More important than repairing unsound teeth is the building of sound teeth. Foods containing calcium should be eaten so that the bone cells can deposit sufficient calcium to build strong teeth. Every diet should include some foods containing vitamin $\mathrm{D}$, so that the body will be stimulated to make the greatest possible use of the calcium. Foods that demand chewing, such as breads made of whole cereals, toast, apples, and celery, are also essential for the development of good teeth. Hard foods cause the teeth to move slightly in their sockets. This has a massaging effect upon the gums and tends to promote circulation through the pulp cavity and the part of the gum holding the teeth in the bony sockets. One of the reasons why former generations had better teeth than many of the people to-day, is because they ate coarser foods which supplied minerals 
and vitamins in greater quantity than our present refined and rich foods. The coarse foods act like tiny toothbrushes in scratching food particles off the teeth and polishing their surfaces.

Causes of teeth decay. Other conditions besides lack of individual care may cause defective teeth. A low resistance of the teeth to decay is frequently caused by some defect in the development of the individual. For example, some babies have a thin, faulty skeletal development which naturally affects the forming of the teeth. This may be caused by a deficient diet - both of the expectant mother and later of the child. The calcification of the temporary teeth and the permanent molars takes place during the child's prenatal life and if the mother does not get the kind of food that is essential for the development of the teeth of the unborn child, the chances are that its teeth will be defective. Again, if a child's diet lacks vitamin D, it is possible that the proper amount of calcium for forming teeth will not be deposited. Dental attention to the first teeth is often neglected because parents fail to realize the necessity of it or cannot afford the high cost of such care. Frequently, the failure on the part of children to tell their parents of cavities in their teeth, either because they are too young or because they are afraid of being hurt by the dentist, is the cause of poor teeth. 'The lack of competent dental facilities in many communities is by no means an infrequent reason for bad teeth.

\section{Problem. Survey of the condition of the teeth.}

Use a mirror or mirrors to assist you in your survey.

I. Note your bite by closing the teeth and drawing back the lips.

$A$. Describe any malformations such as teeth out of place, upper teeth protruding over lower teeth, or lower teeth extending over upper teeth.

$B$. Discuss the importance of having the teeth straightened.

$C$. What is the advantage of having an orthodontist (a dentist specializing in treating irregularities of the teeth) straighten your teeth rather than a regular family dentist? 
II. By means of a small mirror, observe the front surfaces of your upper teeth, the inner surfaces of the lower front teeth, and the inner surfaces of the back teeth. Use the tongue to explore the surfaces of the teeth for tartar.

$A$. Describe the appearance and location of any tartar.

$B$. Diseuss the origin of tartar.

III. Look carefully at the gum line of the teeth. Describe the appearance of any tooth from which the gum has receded, exposing the neck.

$A$.' Why will such teeth decay quickly?

$B$. What effect will the removal of tartar and proper massaging of the gums probably have on such teeth?

IV. By comparing your teeth with the diagram on page 109, name and describe the condition of any tooth showing a cavity. In each case describe the apparent extent of the cavity. Explain why your examination is not as complete as a dentist's?

V. If you have any roots of broken teeth left in your gums, give, briefly, the history of each. By referring to the diagram determine the name of each.

$A$. Discuss whether the loss of the tooth could have been prevented.

$B$. Discuss dangers from the presence of roots left to decay in the mouth.

VI. If you have any spaces left by extracted teeth, what are your plans in regard to these spaces? Again refer to the diagram to name the missing tooth.

VII. When did you last visit a dentist? When will you again visit one?

VIII. If you are unable to detect any defects in your teeth, tell why you cannot conclude that your teeth are in good condition?

IX. By means of red and blue litmus, test whether the reaction of your mouth is acid or alkaline. Note. When blue litmus turns pink, an acid is present. When pink litmus turns blue, an alkali is present.

$A$. State one possible source of acid-formation. What effect has acids on enamel?

$\mathrm{X}$. What advice can you give your parents in regard to the care of the teeth of your small brothers and sisters?

XI. Discuss the value of X-rays in dentistry.

\section{Questions}

1. Describe the arrangement, number, and kinds of temporary teeth.

2. Describe the arrangement, number, kinds, and structure of permanent teeth.

3. Explain the hygienic methods of cleaning teeth.

4. What is the relation of proper diets to good teeth?

5. What are some of the causes of bad teeth? 


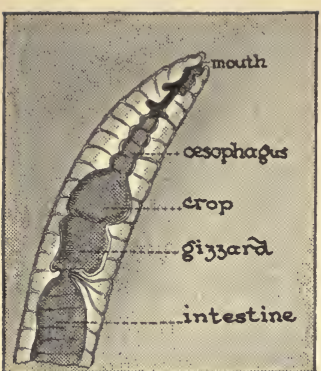

Part of food tube of worm.

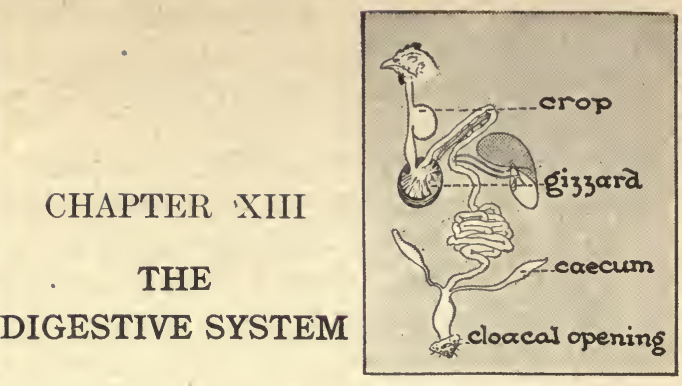

Alimentary canal of a bird.

What similarities and differences are found in the digestive systems of a frog and of a man. How is food prepared for digestion?

We shall find in dissecting a frog and studying its digestive organs that its digestive processes are in many ways similar to those of man.

Problem. Study of the internal organs of a frog.

Place the frog to be studied in a covered jar. Pour a little ether into the jar or put in the jar a sponge saturated with ether. When the animal is dead, remove and place it, with the ventral side up and the head away from you, in shallow water in an individual dissection pan. Pin the legs out so that they will not interfere with the dissection. By means of forceps, lift up the skin in the center of the body and cut a very small opening near the posterior end. Insert the point of the scissors into the opening and carefully cut along a median line to the mouth. Again insert the scissors near the legs and cut through the muscles under the skin. Be sure to cut through the pectoral girdle of bones near the forelegs. Insert the scissors at the anterior and posterior ends of the median slit and cut at right angles on either side. Turn back and cut off the loosened flaps of skin and the underlying muscles, in order to expose the internal organs. If the specimen is a female, remove nearly all the eggs so that the organs may be more readily seen.

I. Make an outline drawing, natural size, of the shape of the frog.

II. Observe the cone-shaped heart midway between the fore limbs. If the heart is still beating, it does not indicate that the animal is alive. It means 
that all of the cells of the body are not yet dead, especially the cardiac muscle cells. Draw in the outline, the heart in its proper position.

III. The lungs lie on either side of the heart partly covered by the liver. By means of a blowpipe passing from the mouth to the windpipe of the frog, gently inflate the lungs.

$A$. Account for the color of the lungs. Press them with the wooden end of the dissecting needle. Are they spongy or compact?

$B$. What is the relation of the texture of the lungs to the amount of air present?

$C$. By means of a hand lens determine and describe the external and internal structure of the lungs. Draw the lungs in position in your outline.

IV. The large, lobed, reddish-brown organ that lies to the right and behind the heart, and covers part of the lungs, is the liver. It is one of the digestive glands. Note the greenish bile sac (or gall bladder) attached to the liver. Sketch in your outline the liver and gall bladder.

V. On the left side of the body note the long, tubular, pouchlike stomach. Push the handle of the dissecting needle down the gullet into the stomach. Draw as much of the stomach as can be seen.

VI. The tubular structure leading from the stomach and filling the lower part of the body cavity forms the intestines. At the lower end of the small intestine the tube becomes larger and disappears between the two thighs. This large tube is the large intestine, the last part of which is the cloaca. The large intestine is similar to the large intestine in higher animals in that it excretes the solid wastes of the body, but it is also a reservoir for nitrogenous wastes and reproductive cells. In this respect it is different from a true large intestine and consequently is called a cloaca. Draw the small intestine, latge intestine, and cloaca.

VII. In the U-shaped loop made by the stomach and the small intestine is a small, light-colored, pear-shaped organ. This is an important digestive gland called the pancreas. Draw the pancreas.

VIII. Describe how the internal organs of the frog are held in place. This structure is termed the mesentery.

IX. Label heart, lungs, liver, gall bladder, stomach, small intestine, large intestine, cloaca, and pancreas.

$\mathrm{X}$. After all the required drawings are made, take your specimen from the dissection pan and wrap it in mimeograph paper which has been moistened 
in a salt solution. Keep it in a cold place so that you may continue your investigation the next day.

XI. Write a paragraph describing the internal organs of the frog, as seen from the ventral view.

Problem. Study of the food tube or alimentary canal of the frog.

By means of your forceps, lift up the heart and carefully cut it out of the body. Lift up each lung, and carefully cut it out. In a similar manner remove the liver.

I. Describe the organ leading into the stomach. This is the gullet or esophagus. Place the blunt end of the dissecting needle in the anterior end of the gullet. Carefully force it through the gullet. Where does it lead? Is the passageway a continuous one or did the needle meet any obstructions?

II. Make another outline drawing of the frog and sketch in it the alimentary canal. Label gullet, stomach, small intestine, large intestine, and cloaca.

III. Remove the alimentary canal. The two small brownish red structures near the backbone in the center of the body cavity are the kidneys. The kidneys remove the nitrogenous wastes (urea) from the blood. Each one is connected to the cloaca by a small duct.

IV. Write a paragraph describing the alimentary canal.

Problem. Study of the internal organs of man.

Remove the front wall from the mannikin.

I. Contrast the chest and abdominal cavities of man with the body cavity of the frog. Note the presence of a partition, the diaphragm.

II. Locate and describe the organs in the chest or thoracic cavity.

III. Name all the abdominal organs observed in the frog, which can be identified in man.

IV. State four easily recognized differences in structure or position between the organs of the frog and man. The large intestine takes care of solid wastes only; therefore, it is a true large intestine and not a cloaca.

V. Make an outline drawing of the mannikin and sketch the organs in position. Label chest cavity, diaphragm, abdominal cavity, heart, lungs, liver, stomach, small intestine, and large intestine. 
Problem. Study of the digestive system of man.

Remove the heart and lungs from the mannikin.

I. Locate the gullet and describe its exact position, including origin and end.

II. Remove the intestines and locate the pancreas and the posterior end of the large intestine called the rectum.

III. Describe the connection of the pancreas and liver with the small intestine.

IV. Compare the alimentary canal of the frog and man as to relative size of organs and as to complexity in structure.

V. Make a diagram of the digestive system and label throat, gullet, stomach, small intestine, large intestine, rectum, pancreas, and liver.

The meaning of digestion. Before a cell in the brain, in the tip of the toe, or any other part of the body can use the meat or the vegetable that is eaten, many changes, physical and chemical, must take place in the food. The organs that are particularly adapted for carrying the food particles, for preparing it for absorption, and making it ready for use by the various body cells, make up the digestive sys-

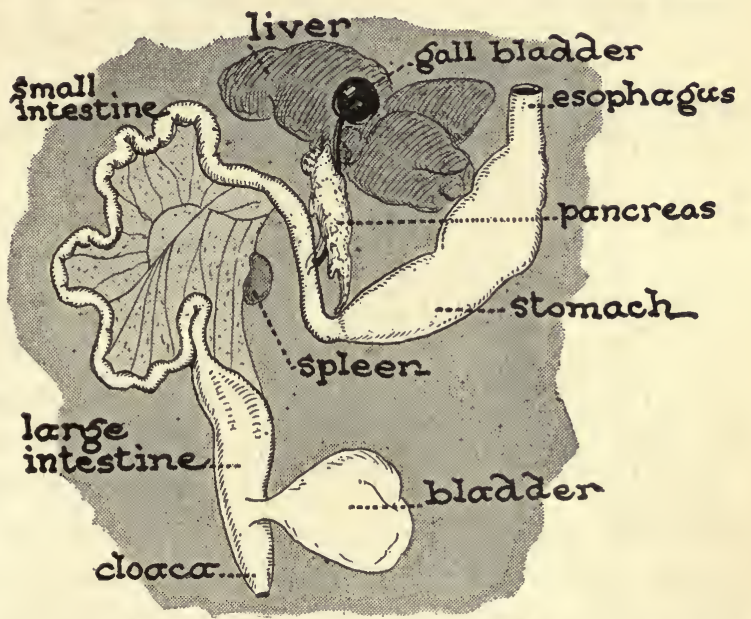

The digestive organs of the frog have been removed from the body to show some of the digestive glands and the continuous alimentary canal. The bladder is one of the excretory organs which drains into the cloaca. The latter is the receptacle for materials from the alimentary canal, reproductive organs, and the bladder.

tem. In the thrashing of grain, much of the useless is separated from the useful parts of the cereal. So, in the digestive 
system, the part of the food that cannot be used is separated from the nutrients. This process may be called a refining pro-

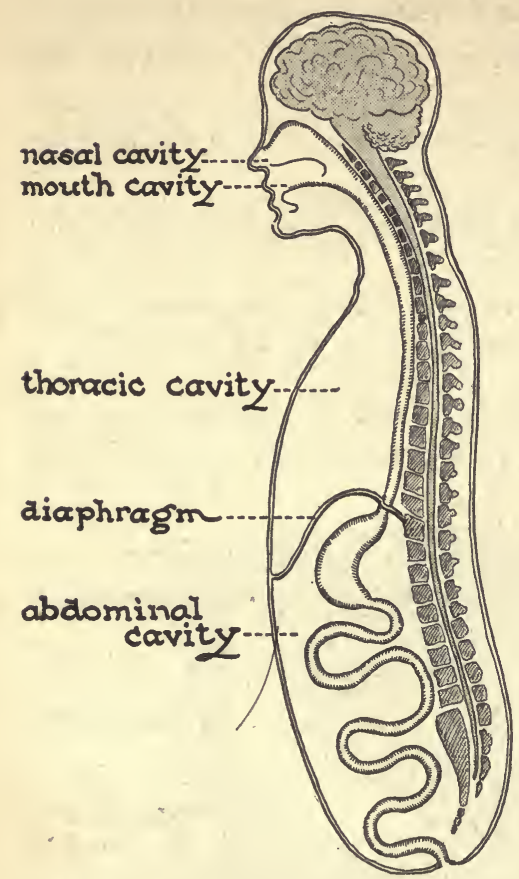

Man is a collection of tubes and cavities. The spinal column is a bony tube, the nerve cord a solid rod made of delicate tissue, and the food tube a long, continuous, pipelike canal. cess. The waste or indigestible part of the food is ultimately expelled from the body. The nutrients are made ready for absorption by the action of various juices. In this digestive process the nutrients are reduced to simpler and simpler organization or certain standard forms to enable them to pass through the walls of the blood vessels. For example, the proteins in eggs, milk, and meat are reduced to standard protein products; the starch in bread and potatoes and the sugar in fruits, candies, and carrots are all reduced to a standard carbohydrate product. The process of digestion really consists of refining, digesting, and standardizing processes.

Digestive organs. The digestive system may be said to consist of two groups of digestive organs, those making up the alimentary canal or food tube in which foods are actually digested, and the accessory organs, the glands, which make or secrete the juices for digestion. A gland is a collection of epithelial cells that secretes a juice. Some of the digestive glands are large organs outside the food tube, such as the pancreas and liver, and others are minute structures. in the lining of the food tube, as the gastric, peptic, and intestinal glands. 
When the digestive glands are located some distance from the food tube, they have a tube or duct which effects the discharge of the secretion into the alimentary canal.

Alimentary canal of man. The alimentary canal is a tube passing through the body, in which food is made ready for the use of the organism. While food remains in the canal, it is actually separated from the body because it can pass out of the body at the posterior end of the tube without having affected the other organs of the body. Consider the alimentary canal of the worm. It is a straight tube passing from the anterior to the posterior end of the animal's body. It is a tube within a tube. Before food is actually in the body, it must get from that inner tube into the surrounding structures. The alimentary canal of man is a continuous tube from

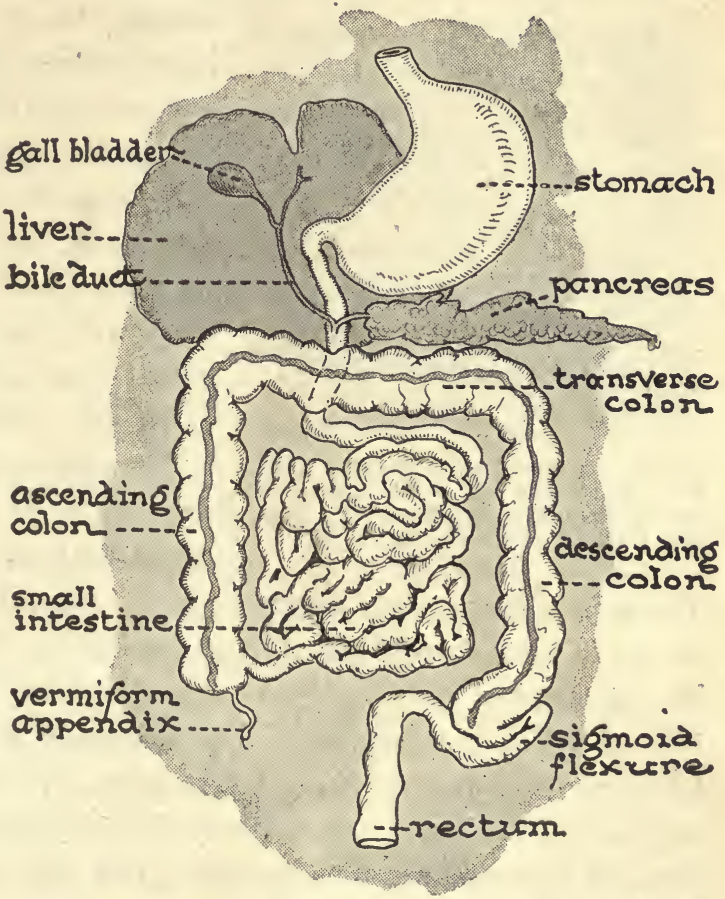

The digestive organs of man are displaced to some extent in this illustration, in order to show clearly their relations to each other. They are somewhat similar to those of the frog. Note the absence of a cloaca, however. Higher animals have a large intestine but no cloaca.

the mouth to the anus or end of the large intestine. There is much variation in width in various sections, and it is twisted and coiled 
to a great extent in some parts, but is just as truly a tube as the simple straight alimentary canal in the worm's body. The alimentary canal consists of the mouth cavity with its accessory organs: the teeth, tongue, glands, throat or pharynx, esophagus, stomach, the small intestine, and large intestine.

Mouth. The mouth cavity, nearly oval in shape, is lined with a soft membrane which is kept moist by saliva secreted from glands. The palate or roof of the mouth consists of a hard portion in front,

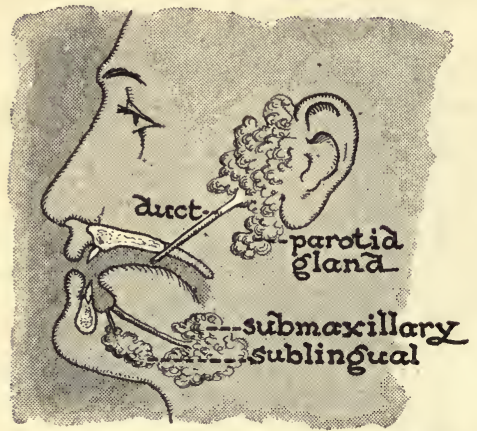

There are three pairs of salivary glands which empty into the mouth. The teeth may become coated with tartar which is partially a deposit from the saliva. formed by a bone covered by mucous membrane, and of a soft portion farther back containing no bone. The hard palate forms the partition between the mouth and nose; the soft palate arches backward, and from the middle of its lower border there hangs a pointed portion of the soft palate called the uvula (little grape).

The tongue is a muscular organ, and has on its upper surface many small projections called papillae. These papillae contain certain nerve cells that make it possible for us to taste sweet, bitter, sour, and salt. When these specialized structures (taste-buds) are stimulated by the food, the secretion of saliva is increased and gastric fluid in the stomach starts to flow. The tongue helps mastication by pushing and rubbing portions of the food against the roof of the mouth, and also guides the food into position for chewing. The tongue assists in swallowing by manipulating the food into small masses and pushing them back to the throat cavity.

Due to the complex movement of the lower jaw, up and down, forward and back, and from side to side, the teeth shave, slice, crush, and grind the food. The broad surfaces of the molars 
help in the grinding process. During this process the food is thoroughly mixed with secretions from the glands of the mouth.

The chief secretion of the mouth is supplied by the salivary glands. These are three pairs of compound saclike glands, the parotid, sublingual, and submaxillary. A parotid gland is placed just under and in front of each ear; the ducts from the glands pass forward along the cheek and open opposite the second molar. The submaxillary glands are situated below the jaw and under the tongue, and open into the mouth cavity underneath the tongue. Several of the small ducts from the sublingual glands also open in the floor of the mouth beneath the tongue. The secretion of these salivary glands, mixed with the secretion of the small glands of the mucus epithelium, is called saliva.

Everyone has probably experienced an unusually large flow of secretion from the salivary glands when appetizing food is seen or smelled. The stimulation of the salivary glands may also be brought about by the thought of food that is liked, especially when one is hungry. This is known as psychical or mental stimulation. Flavoring substances and extractives that stimulate the taste buds furnish chemical stimuli, and the pressure of food in the mouth and the action of irritating substances cause a mechanical stimulation.

Saliva. Saliva consists of water, inorganic salts, some mucin, and the enzyme ptyalin or salivary diastase. Saliva is usually slightly alkaline in its reaction and has four distinct functions: (1) it assists in mastication and swallowing by moistening and softening the food; (2) it lubricates the food and enables it to slide smoothly down the esophagus; (3) it dissolves dry and solid food such as salt and sugar, thus enabling us to taste them, and thereby stimulating a further flow of salivary and gastric juices; (4) the enzyme ptyalin acts upon starch, converting it into sugars, dextrin and maltose. These are intermediate WH. FITZ. AD. BIO. -9 
products in the digestion of starch. They are simpler products than starch, but not simple enough to be used by the body. The change, due to ptyalin, takes place when there is a slightly alkaline condition. Saliva that is distinctly acidulous hinders or arrests the digestive process. Boiled starch is changed more rapidly and completely than raw, but food is rarely retained in the mouth long enough for the saliva to more than begin the transformation of starch.

Throat and esophagus. The mouth cavity narrows to form the pharynx or throat cavity. It is shaped somewhat like a funnel, with its narrow or constricted end turned downward to form the beginnings of the esophagus and the windpipe. There are seven openings in the throat cavity, leading to the nose, ears, mouth, larynx, and gullet. Two, in front above, lead into the back of the nose and are known as the internal nasal openings. Two, one on either side above, lead into the ears and form the openings to the Eustachian tubes. One midway in front connects with the mouth. Two below, one opening into the windpipe through the glottis and the other behind the glottis into the gullet.

The pharynx is a passageway for air from the nose to the glottis, and for food from the mouth to the gullet. When the food is ready to be swallowed, it is brought together on the upper surface of the tongue and pressed backward into the pharynx. Then the muscles in the pharynx contract, drawing the pharynx upward and causing it to dilate to receive the food. The muscles then relax, causing the pharynx to sink and forcing the food into the esophagus. At this instant, breathing is temporarily suspended and the air passages closed against the possible entrance of food. The soft palate is drawn back, thus closing and protecting the nasal passages. The entrance to the windpipe is shielded by being pulled forward under the base of the tongue. There is an additional safeguard through the folding down of the epiglottis, a specialized cover. When food is once within the esophagus, breathing 
may be resumed. Practically no digestion takes place in the pharynx due to the facts that food is swallowed quickly and no enzymes are secreted here.

The esophagus is a comparatively straight tube, about nine or ten inches long. It descends in front of the spine, passing through the diaphragm and terminating in the stomach. It has one set of muscles extending around it circularly and another layer of muscles longitudinally arranged. By a series of contractions of the circular and the longitudinal muscles, food is passed by a series of wavelike movements into the stomach. Once the top ring of muscles is stimulated by the swallowing of the food, a wave of contraction goes through the full length of the gullet, causing the food to enter the stomach. This series of rhythmic, wavelike contractions of circular and longitudinal muscle fibers which affect successive portions of the tube downward is called peristalsis. The constricted portion is always preceded by an area of relaxation which renders the contraction more effective in forcing the contents onward. The direction is normally the same, and the action is under the control of the nervous system. This movement might be compared with placing a large marble in a narrow rubber tube and forcing it through by successively pinching the tube.

During the processes of mastication, moistening, and swallowing, the food is reduced to a soft, pulpy condition. Any starch it may contain begins to change into sugar in the mouth, but it remains in the mouth, throat, and esophagus so short a time that digestion cannot be completed in them.

\section{Questions AND SUggestions}

1. How does the body cavity of the frog differ from the cavities in man's body?

2. Name three purposes of the digestive process.

3. Compare the alimentary canals of the worm, frog, and man, stating all similarities and differences. 
4. If saliva were acid in reaction instead of alkaline, what effect would it have on the teeth?

5 . What are the functions of the soft and hard palates?

6. State the uses of the tongue.

7. What is the importance of saliva in digestion?

8. Describe the protection of the various openings from the throat during the action of swallowing.

9. Define peristalsis. Discuss the importance of peristalsis in the digestive process.

\section{SupPlementary Readings}

Howell, Wm. H., Textbook of Physiology (W. B. Saunders Co.).

Kimber, D. C., and Gray, C. E., Textbook of Anatomy and Physiology (The Macmillan Co.).

Martin and Weymouth, Elements of Physiology (Lea \& Febiger). 


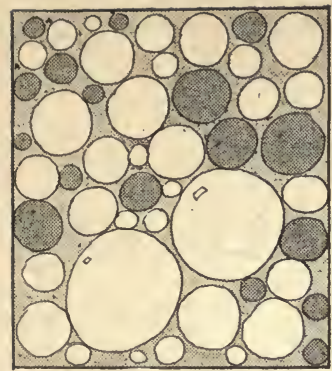

Large globules of fat.

\section{CHAPTER XIV \\ DIGESTION AND ABSORPTION}

What changes does the food undergo in the stomach and intestines? What is the importance of gastric juice in digestion? How is the small intestine adapted for carrying on digestive activities? What is the importance of absorption?

The stomach. The esophagus ends in the stomach, a collapsible, saclike enlargement of the alimentary canal, which serves as a temporary receptacle for food. When contracted, the shape of the stomach is comparable to a sickle blade or a sausage. When distended with food, it is more pouchlike. The stomach has two openings ; one where the esophagus joins it, known as the cardiac opening, the other which communicates with the small intestine and is known as the pylorus (gateway). Both the cardiac and pyloric entrances are guarded by ringlike muscles known as sphincters. These muscles contract and keep the openings closed, except when food is passing through them. Food is kept in the stomach until it is ready for intestinal digestion; then the circular fibers guarding the pyloric valve relax.

The walls of the stomach are made of muscle and other tissues. The inner coat (mucous membrane) of the stomach is honeycombed by tiny, shallow pits, which are the openings or mouths of the gastric glands from which the gastric juice is discharged. The gastric or digestive juice is composed of water, hydrochloric acid, 
and the enzyme, pepsin. The meat known as tripe is the lining of a cow's stomach and somewhat resembles the lining of the human stomach.

\section{Problem. What is the effect of gastric juice on protein?}

I. Prepare some very thin slices of hard-boiled white of egg. Put a slice of this into each of four test tubes. Have the pieces alike in size. Add to the four tubes the following liquids :

$A$. Water, to tube number one.

$B$. Water in which pepsin has been dissolved, to tube number two.

C. Water and very dilute hydrochloric acid, to tube number three.

$D$. Water, very dilute hydrochloric acid, and pepsin (artificial gastric juice), to tube number four. Place all four test tubes and contents in a warm place or in an incubator.

II. Compare the appearance of the egg in the four tubes at the end of one, two, four, eight, and twenty-four hours. What effect have the various constituents of gastric juice on protein?

III. Give your explanation of the following :

$A$. When people are suffering from indigestion, why are pepsin and hydrochloric acid sometimes prescribed?

$B$. If there is too much hydrochloric acid in a person's stomach, why is bicarbonate of soda sometimes prescribed?

$C$. Why is it unwise for a person to take artificial gastric juice or bicarbonate of soda unless a doctor definitely prescribes it?

Problem. What is the effect of the size of protein food on the time needed for digestion?

I. Place in a test tube some finely chopped white of egg. Put into another test tube some larger pieces of white of egg, and into a third test tube, some very large pieces of white of egg. Cover the egg white in each tube with artificial gastric juice.

II. Compare the appearance of the egg in the three tubes, hour by hour, and note the results.

III. What do you find to be the relation of the size of food to the time needed for digestion? Of what value is thorough mastication of food? 
Problem. What animal proteins are digested most quickly and most thoroughly?

I. Put into separate tubes equal-sized, thin slices of the following proteins:
$A$. Well-boiled beef.
$D$. Roast pork.
$B$. Raw beef.
E. Roast lamb.
C. Roast beef.
$F$. Fried fish.

Pour an equal amount of the artificial gastric juice into each tube. Place the tubes in a warm place.

II. Compare at regular intervals the amounts of protein in each tube.

III. State your conclusion from your observations.

Secretion of gastric juice. The secretion of gastric juice is continuous. Even when one is not eating there is a small amount of secretion, but during the act of eating and throughout the entire period of digestion, the rate of secretion is greatly increased. This rate is regulated by several factors : (1) Psychical stimulations are brought about by the sensations of eating. The taste and odor of food stimulate the beginnings or receptors of certain sensory nerves situated in the mouth and nose. This stimulation

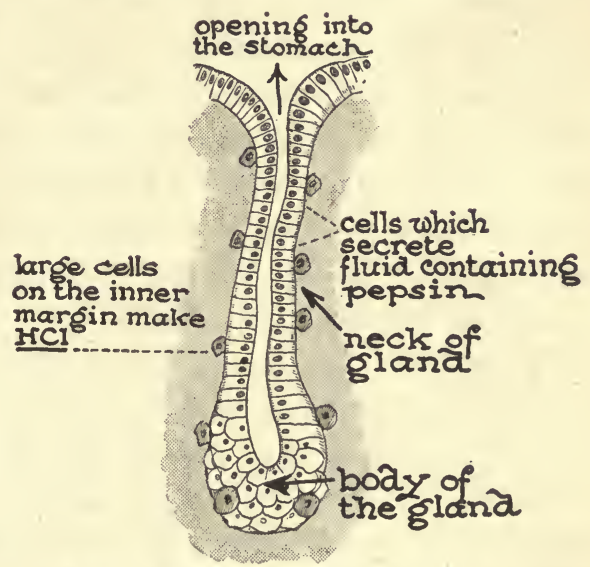

In a gastric gland certain cells secrete pepsin, while certain others secrete hydrochloric acid. With water, these substances are the main constituents of gastric juice which is emptied from the gland into the stomach.

results in activating the gastric glands. It has been demonstrated, experimentally, that as soon as a dog tastes, smells, or sees food, the flow of gastric juice increases. A psychic stimulation results in a copious flow of gastric juice. Such a stimulation is always brought about when one eats appetizing food. 
(2) Chemical stimulation is brought about by the materials contained in certain foods and by the stimulating materials contained in the products of digestion. Certain foods such as meat juices or extractives, by their actions upon the nerves of the stomach, stimulate the gastric glands to pour forth their secretion. Such substances are called secretagogues. Other foods such as milk and bread do not seem to contain these substances. When these foods are eaten, the secretion of juices is probably due to a mechanical stimulus resulting from the presence of food in the stomach. When certain digestive products are formed, they, in turn, stimulate a further secretion of gastric fluid. The amount of secretion depends upon the quantity and nature of the food to be digested.

Gastric juice is a thin, nearly colorless liquid with an acid reaction. It contains some inorganic salts, but the essential constituents are hydrochloric acid and two enzymes, pepsin and rennin. Hydrochloric acid in normal gastric fluid is found in the proportion of 0.2 to .5 per cent. Experiments have shown that a higher concentration of hydrochloric acid is not favorable for the digestive action of pepsin. It has the following functions: (1) it activates the pepsinogen (an inactive form of pepsin) of gastric juice to form pepsin; (2) it provides an acid medium which is necessary for the pepsin to carry on its work; (3) it swells the protein fibers, thus providing a larger surface for the action of pepsin; (4) it kills many bacteria that enter the stomach; and (5) it helps to regulate the opening and closing of the pylorus.

Pepsin, which may be called gastric protease, is first formed by the gastric glands as pepsinogen and is changed into pepsin when it comes into contact with the hydrochloric acid. It has the power of changing proteins into the intermediate products called proteoses and peptones. These are a simpler form of proteins, but not yet simple enough to be absorbed. Therefore, they are called intermediate products of digestion.

Rennin, another enzyme found in gastric juice, acts upon casein, 
the protein of milk. It converts this substance into a clotted mass, the curd. The pepsin carries on the digestion of curd more efficiently in this form than it could in the original form.

Food is kept in constant motion in the stomach. By means of the action of the stomach muscles, food is churned back and forth and up and down until it is reduced to much finer

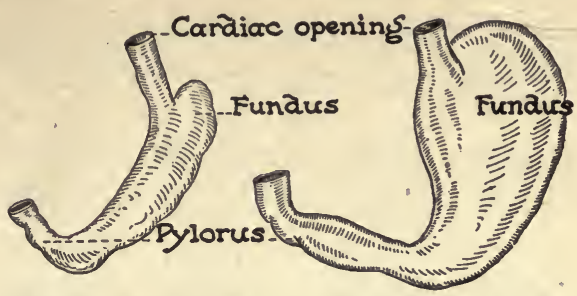

The diagram on the left shows the shape of the stomach when empty; and the one on the right shows the approximate shape after a hearty meal.

particles than were formed in the mouth. The partially digested food is in liquid form and is called chyme. The reduction of the food into these very fine particles is invaluable in increasing the amount of food surface to be exposed to the action of digestive juices in the small intestines. The cardiac sphincter prevents the return of food to the gullet during the churning process. At intervals the pyloric sphincter opens and some of the chyme is forced into the small intestine by a wave of contraction.

The stomach acts as a reservoir, holding the food and feeding it at regular intervals to the small intestine. The time required for gastric digestion of a meal depends upon the quantity and kind of food eaten. An average meal requires about five hours for gastric digestion. Solid particles tend either to keep the pyloric valve closed, or to force it to relax, because of fatigue, before the food has reached a semi-fluid condition. It is largely the acidity of the chyme that causes the relaxation of this valve, but in the small intestine the acid has just the opposite effect. When the acidulous chyme passes into the intestine, it causes the sphincter to contract. The pylorus then remains closed until the acid has been neutralized by the alkalinity of the intestinal juice. Since few enzymes are produced in the stomach and their digestive action is incomplete, the digestion of the nutrients continues in the small intestine. 
The salivary digestion of starch continues in the stomach until the acid permeates all parts of the food mass. Proteins are the
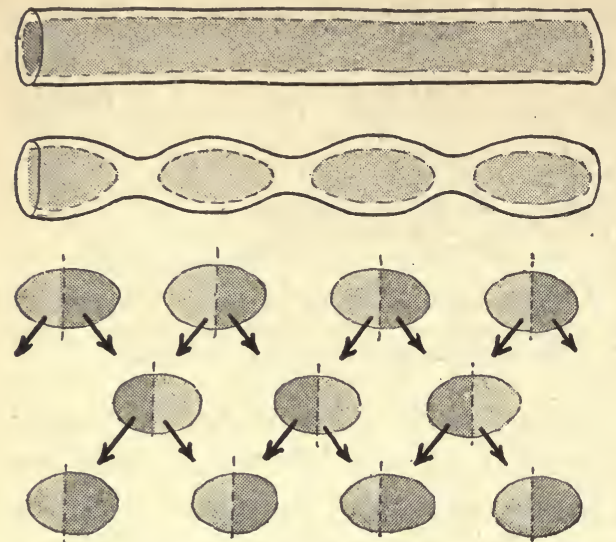

Rhythmic constrictions of the walls of the small intestine break the food into equal segments. Each of these segments is halved and unites with the adjacent half of the next segment. Segmentation continues, insuring the complete mixture of digestive juices and food. nutrients chiefly affected in gastric digestion, but even their digestion is incomplete. Many bacteria that enter with the food are killed by the germicidal action of the hydrochloric acid.

The small intestine. The small intestine is a narrow, tubular organ. about one inch in diameter and from twenty to thirty feet in length. It is so coiled that it easily fits into the central part of the abdominal cavity. It is continuous with the pyloric end of the stomach. A membrane, the mesentery, attaches the coils of the intestine to each other and to the backbone.

The small intestine has muscles which permit two kinds of movement, one called rhythmic segmentation and the other peristalsis. The rhythmic segmentation is caused by local constrictions of the intestinal wall at regular intervals. These constrictions divide the chyme into a number of equal parts. Within a few seconds, each of these portions is halved and unites with the corresponding halves of the adjacent segments. In the next constriction the segments are restored to their original position. This enables the mass to be thoroughly mixed with the digestive juices. Then a peristaltic movement, a succession of waves of contraction and relaxation, beginning at the anterior end of the small intestine, sweeps along, forcing the food onward through the tube. 
Certain glands in the lining of the intestine secrete a juice called succus entericus or intestinal juice. Near the region where the small intestine leads from the stomach is the opening of a duct formed by the union of ducts from the liver and pancreas. Through this duct, juices from the liver and pancreas are emptied into the small intestine, where they mingle with the secretions of the intestinal glands.

The pancreas. The pancreas is a flat, pear-shaped gland, about five inches in length, that lies slightly back of the stomach, between the lower part of the stomach and a fold of the intestine. It secretes an alkaline digestive juice called pancreatic juice. This juice contains three enzymes: (1) An amylase, amylopsin, similar to the ptyalin of the saliva, continues the conversion of starch into simple sugars. (2) A protease, formed as trypsinogen, is converted into trypsin by the action of a substance, enterokinase, secreted by the mucous membrane of the small intestine. Trypsin, similar to, but more powerful than pepsin, changes the proteins into the simpler products, peptones and amino-acids. lipase, steapsin, breaks down fats into fatty acids and glycerol. Some of these fatty acids then combine with the alkaline salts, present in the juices of the intestine, to form soaps. Fats are insoluble, but these soaps are readily soluble in water. Soapmaking, saponification, is part of the digestive process. The breaking down of the fats and the saponification that follows are made easier by emulsification, that is, the breaking up of the fat into very tiny particles.

Problem. What is the effect of an alkali on a fat?

Place in each of two test tubes a small amount of olive oil. Add an equal amount of sodium hydroxide (an alkaline material) to the oil in one of the tubes. Let it stand for forty-eight hours, shaking occasionally.

I. What difference do you observe between the oils in the two test tubes? The change you have brought about in the one is called an emulsion.

II. Mount a drop of oil under the microscope. 
III. Mount a drop of the emulsified oil under the microscope.

$A$. Describe the difference in the appearance of the two drops of oil.

$B$. Why would you expect emulsification to hasten the digestion of fats?

IV. Bile and succus entericus are alkaline liquids. What effect would you expect them to have on the digestion of fat?

The liver. The liver is the largest gland in the body. It is situated on the right side of the body, and covers part of the

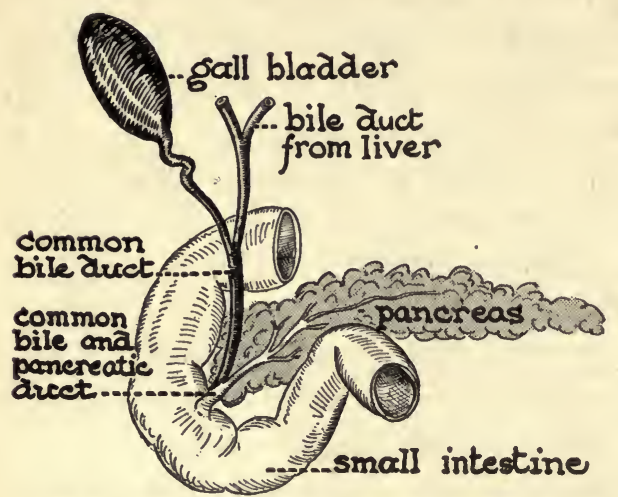

Not far below the stomach, a tube empties into the intestine. This tube leads from the pancreas, the liver, and the gall bladder, and drains juices into the intestine. stomach, small intestine, and large intestine. The upper surface fits closely into the under surface of the diaphragm. The liver secretes an alkaline juice, yellowish-green or brown in color, called bile. This pours, by means of the bile duct, into the small intestine only during the period of digestion. The alkali in the bile activates the digestion of fats and helps in the absorption of digested fats. The excess bile passes through another duct into the bile sac or gall bladder, where it is stored until needed. Sometimes a part of the bile substance crystallizes in this duct, forming gallstones. In such a case, the bile duct is closed and the excess bile passes into the blood, causing jaundice.

Intestinal digestion. The presence of food in the small intestine stimulates the flow of intestinal juice which contains a number of enzymes. An intestinal protease, erepsin, helps to convert the proteoses and peptones formed in the stomach into the end products of digestion, amino-acids. Several inverting enzymes in the intestinal juice convert the double sugars into single or 
simple sugars. Some of these enzymes complete the digestion of starch and sugar which was started in the mouth. Another constituent, enterokinase, causes the trypsinogen of pancreatic juice to form trypsin. There is also an active hormone, secretin, in the intestinal juice. It has no digestive action but passes into the blood stream and is carried to the liver and the pancreas, which it activates. (A hormone is a chemical substance formed in one part of the body and activating another part.) It has been found that if a dog is fed and some of its blood in the vein leading from the intestine is introduced into the blood of another $\mathrm{dog}$, the liver in the second $\mathrm{dog}$ immediately secretes a large amount of bile, and the pancreas secretes pancreatic juice, which shows that a hormone must have passed through the blood and was carried to the pancreas and the liver. In the human body, most of the food is digested in the small intestine because the food stays there longest. It has been estimated, from observations, that the last food of a meal passes out of the small intestine about ten hours after eating. There are more enzymes in this part of the digestive tract than in any part of the canal, which, also, accounts for the large amount of digestion that occurs here.

The large intestine leads from the small intestine. It is about five feet long, and about two and one half inches in its broadest part. A little

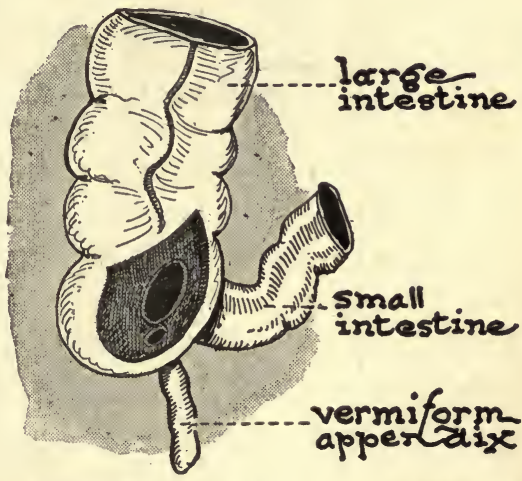

Enough of the small intestine and large intestine are shown to make clear the position of the appendix. Note the opening of the appendix.

pouch is formed where the large intestine connects with the small intestine. Leading from this pouch is a short, narrow, wormlike tube, usually less than the diameter of an ordinary lead pencil, 
CHART OF CHEMICAL DIGESTION

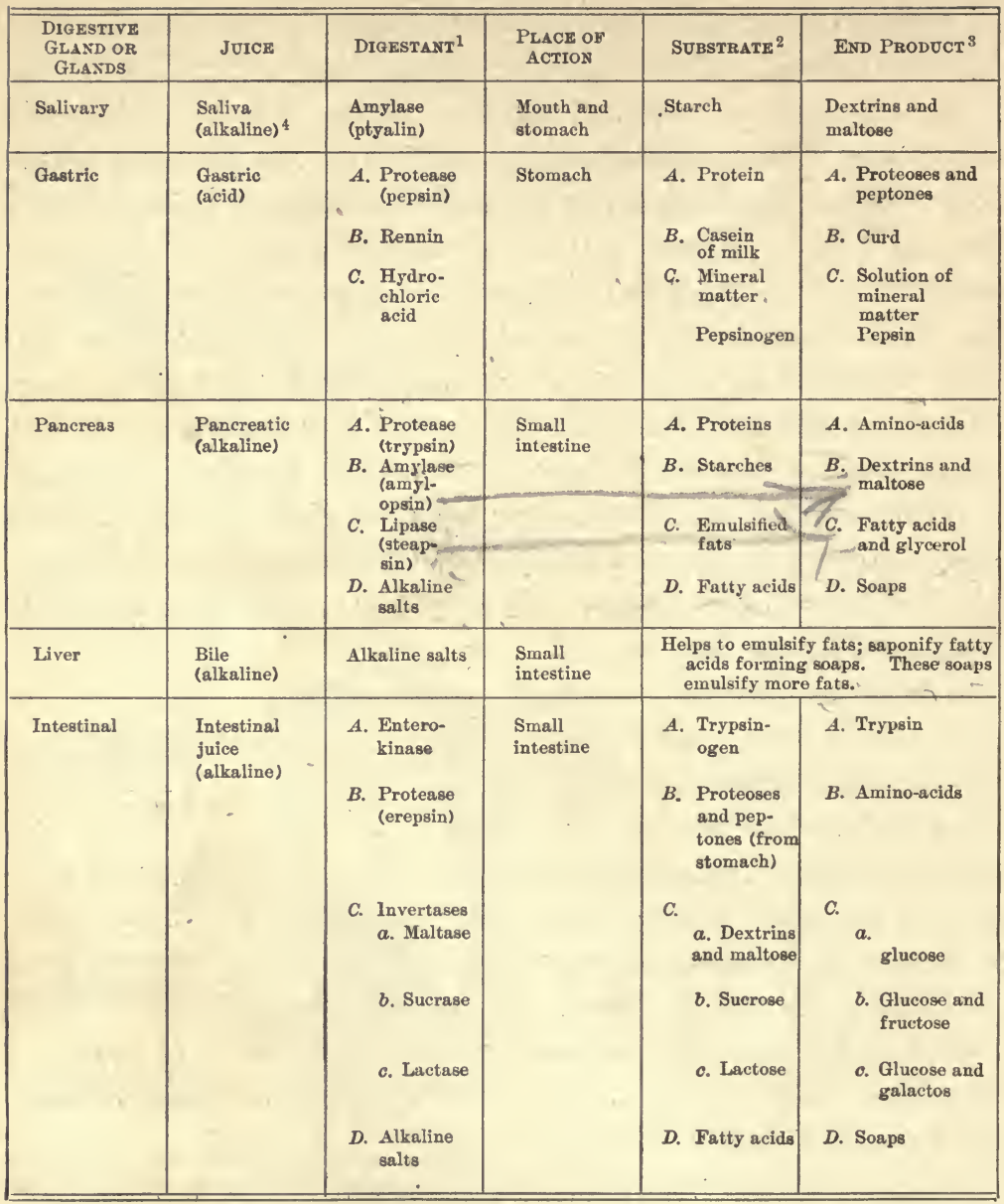

${ }^{1}$ Digestant is a term used to designate a chemical material which brings about a change in the digestive process.

2 Substrate is a term used to designate the material changed in the process of digestion.

${ }^{8}$ End product denotes the prodnct at the termination of the particular process considered. This product may or may not be ready for absorption.

4 There is a maltase in saliva which converts small quantities of maltose into glucose. 
called the vermiform appendix. It has no useful function. If food collects in the appendix, it is not easily drained out. This food may decay, which causes an inflammation commonly known as appendicitis.

The large intestine is divided into the ascending colon, transverse colon, descending colon, and sigmoid flexure (see page 119). The colons and the sigmoid flexure inclose the folds of the small intestine. The sigmoid flexure ends in the rectum. The rectum is six to eight inches long and leads into the anal canal, having an external aperture, the anus. This opening has an internal sphincter muscle of the involuntary type, and an external sphincter that is voluntary. These sphincters control the passage of solid waste from the body.

The process of digestion is continued to a slight degree in the large intestine, due to the presence of the digestive fluids with which the food became mixed in the small intestine. The indigestible waste materials associated with all foods, are removed from the body through the large intestine by means of peristaltic movements. The chief waste product is the cellulose of vegetable food and the fiber of meat.

Bacteria are abundant in the large intestine. They cause the putrefaction of unabsorbed proteins. Some of these products of putrefaction are absorbed from the intestine by the blood, while the others are eliminated from the body. If an excessive amount of toxic products from this putrefaction is absorbed into the blood, a headache or feeling of lassitude will usually result. Normally, the rectum is empty until just before defecation or the elimination of solid waste.

\section{ABSORPTION}

After foods have been refined, split, and standardized in the digestive process into the simplest forms; namely, amino-acids from proteins, glucose and galactos from carbohydrates, and fatty 
acids, glycerol, and soaps from fat, they are in a diffusible form and may enter the blood where they will be utilized. The process of food passing through the lining of the alimentary canal and through the walls of the blood vessels is called absorption. Absorption is a process of osmosis. Very little absorption takes place in the mouth, throat, gullet, and stomach because the food is in

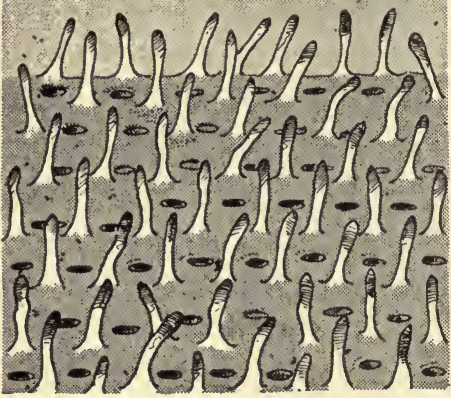

Hairlike microscopic structures known as villi are found in great numbers on the lining of the small intestine. They increase the absorbing surface. The mouths of intestinal glands show among them. constant motion, and because very little of it has reached the end point of digestion.

Most absorption takes place in the small intestine for the following reasons: (1) The digestion of most of the food has been completed. Many of the end products of the digestive process are formed in the small intestine.

(2) The great length of the small intestine gives a larger absorbing surface. (3) The small intestine is narrow and the food is pressed against all its surfaces. Consequently, not only the lower surface but all its surfaces are used in the absorbing process.

(4) Muscular activity helps the food to mix with the digestive juices so that complete digestion takes place. At the same time, the food is pressed against the absorbing surface. (5) There are special adaptations, folds, and villi, for increasing the absorbing surface. The length of the small intestine is at least twenty feet in an adult. The number of epithelial cells used in absorption is tremendously increased by the presence of circular folds or ridges around the circumference of the lining of the intestine. On these ridges are little structures called villi, so numerous and so close together that they resemble the nap on carpet. Each villus consists of an outer layer of epithelial cells inclosing a network of capillaries and a central lymph- 
channel called a lacteal. The spaces among the capillaries, lacteals, and epithelial cells are filled with fluid known as lymph. The digested food is absorbed by osmosis through the epithelial cells of the villi. These villi cells exert a selective action, permitting only the passage of certain materials. The process of osmosis here is an active not a passive one. Some soluble salts are readily absorbed, while others like tartrates, citrates, and calcium salts cannot penetrate the epithelial cells. Fatty acids, glycerol, and soaps enter the epithelial cells, and during the process of passing through them are change back into fat particles. This fluid or lymph then passes into the lacteals and through other lymphatics, leading from the lacteals, and eventually drain into the blood system. The amino-acids, simple sugars, salts, and water pass directly from the epithelial cells of the intestine into the capillaries and thus become part of the liquid portion of the blood.

The adaptations of the

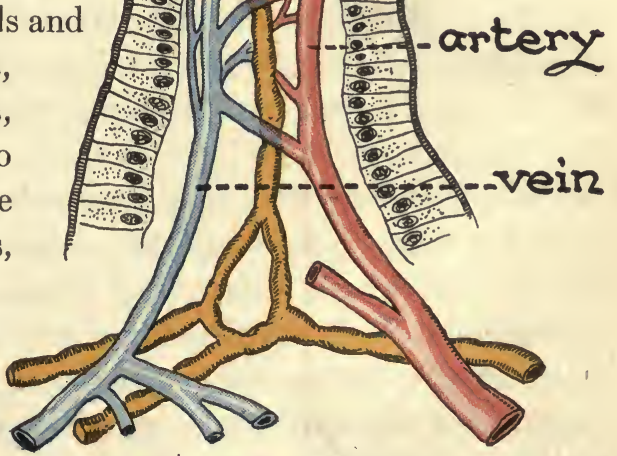

The villus is adapted for absorption. A network of microscopic blood vessels absorbs digested food from the small intestine. A lymphatic, known as a lacteal, runs through the center and takes in the digested fat.

small intestine are so adequate that practically everything difWH. FITZ. AD. BIO. -10 
fusible has been absorbed by the time the residual material enters the large intestine. This material consists chiefly of indigestible substances and some water. The water is gradually absorbed during the progress of the chyme along the large intestine. 'This results in the waste material becoming more and more pasty as it approaches the rectum for elimination.

Hygiene of digestion. The body is normally in a state of health and not disease. It is abuse of some kind that induces digestive disturbances commonly called indigestion. Hygienic habits of living make for good digestion. Activity in the open air and sunlight, good food, relaxation, and rest are of prime importance. Proper nutritional habits should be established. Meals should be taken at regular intervals. If the digestive organs are worked periodically, more regular digestive habits are established. As a rule, food should not be eaten between meals, for it disturbs the physiological routine and, also, interferes with the appetite.

Since the alimentary canal is composed largely of muscles, exercise is invaluable in keeping these muscles in proper tone. By tone we mean the constant and unconscious tendency of the muscles to contract under normal conditions. However, strenuous exercise immediately after a meal is bad, as it withdraws much of the blood from the digestive organs where it is needed, and sends it to the active skeletal muscles that are working. This may result in indigestion and possibly muscular cramps. Consequently, neither maximum digestion nor proper muscular activity is obtained. This is one reason why persons are subject to digestive or muscular cramp if they swim too soon after eating a heavy meal. For a similar reason, undue excitement or mental stress should be avoided during or after meals. Since the stimulation of digestive glands is partly psychic (mental), violent emotions interfere with the proper flow of digestive juices and withdraw the blood from the digestive organs and send it to the brain and nervous system. Pavlov, a famous Russian physiologist, learned 
through scientific experimentation, that, in dogs, fear, anger, and rage interfered with proper digestion and altered the character of the digestive juices.

Food should be thoroughly chewed to be properly mixed with the saliva. As much water as possible should be taken each day. There is no objection to drinking water with the meals, providing that it is not so cold that the organs might be chilled, and that the food is thoroughly masticated and not washed down by the water.

As the undigested part of the food passes through the colon, it gradually loses its water through the process of absorption. This waste becomes semisolid. It should be removed daily at a regular time. If this defecation does not take place because of haste, or inconvenience, or some irregularity in the routine, the water in the solid waste becomes absorbed and the waste becomes so compact that it is difficult and in some cases impossible to eliminate. A cathartic or laxative must then be taken to stimulate the activity of the intestinal movement. It is a well-known fact that improper foods may cause constipation. This condition may be avoided by eating bulky foods which will stimulate the work of the muscles of the canal. Such bulky foods are vegetables, salads, and fruits. Proper routine, including regularity of meals and regular times for elimination of wastes, must, also, be established.

Laxatives are useful in removing an acute condition of constipation but should not be taken regularly. Some laxatives contain drugs that stimulate the nerves controlling the muscles used in defecation. Other laxatives called the salines contain the salts of sodium, potassium, and magnesium. They cannot be absorbed by the intestine. They hold a great deal of water in solution and increase and soften the bulk of the material, favoring its movement along the canal. Epsom salts, magnesium citrate, and sodium phosphate are examples of saline cathartics. A third type of laxatives is heavy oil, mineral or castor oil. These line the food 
tract with a film of oil. The oil tends to soften and increase the bulk of the intestinal content.

The best ways of preventing and overcoming constipation are : to act on the desire for defecation, to have a regular time for

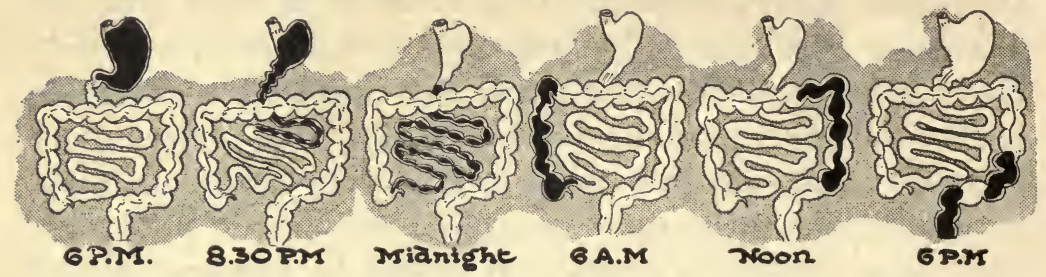

As digestion takes place, food is gradually absorbed until the wastes only enter the large intestine. When these wastes reach the rectum, they are ready for elimination. It takes about twenty-four hours for digestion to be completed and for the wastes to reach the rectum.

doing this, and to eat plenty of fruit and vegetables, as these tend to promote peristalsis.

Some investigators think that one of the contributing factors to old age is the toxins absorbed from putrefaction in the large intestine. The toxins affect the arteries causing the hardening of the arterial walls. The bacteria of putrefaction work best in an alkaline medium. A Russian physiologist, Metchnikoff, conceived the idea of introducing an acid into the large intestine. He thought this would check putrefaction and possibly postpone old age. He observed that Bulgarians who used a great deal of sour milk, live to a greater age than other people. He knew that the acids from most foods are neutralized before they reach the large intestine, but that unlike ordinary sour milk the acid in Bulgarian milk does reach the large intestine. Different strains of bacteria in milk cause the two different reactions. Bacillus bulgaricus and bacillus acidopholus which is a related strain of microörganisms, have been cultivated in America. Experiments are now being made with acidopholus milk to determine whether it does have a beneficial effect although there is as yet no definite proof.

People should not become faddists in eating. Nutritional 
rules established by proper authorities should be followed. If one suffers from overweight or underweight, his diet should be regulated professionally and not by quack methods. All kinds of diets are in vogue; namely, milk diets, raw-food diets, cookedfood diets, vegetable diets, and fruit and nut diets. As a rule, most, if not all of these, should be questioned and avoided by the average person unless a competent physician has done the prescribing. Otherwise, a diet might be selected that would be detrimental rather than beneficial in many instances. The use of patent medicines of all kinds should usually be avoided. Some people decide they have too much acid in their systems and take an alkali like bicarbonate of soda to counteract it. In many cases, bicarbonate of soda stimulates the production of more acid. Others decide they lack gastric juice and buy artificial gastric juice or pepsin and hydrochloric acid to aid digestion. This may interfere with the proper production of natural gastric juice. Still others believe a physic taken once a week is invaluable. This may get the system so used to drugs that in time it will not be able to function without artificial stimulation. Headaches, due to digestive disorders, are cured by getting rid of the cause of the disturbance, not by headache powders.

Briefly stated, normal digestion is our heritage. If habits of eating the proper foods and defecation are regular, digestion will usually be normal. The best way to clear up indigestion is to find out the cause of the irregularity and correct it.

\section{Questions and Suggestions}

1. If the kinds or classes of enzymes are amylases (starch-splitting), proteases (protein-splitting), and lipases (fat-splitting), classify, in their right classes, all the enzymes that you have studied.

2. Discuss the different causes for the stimulation of the flow of digestive juices.

3. Discuss the importance of peristalsis in eliminating the solid wastes of the body. 
4. Locate four sphincters and discuss the importance of each.

5. Plan an experiment which will illustrate the digestion of starch.

6. Name three intermediate and three end products in the digestion of nutrients.

7. Discuss the importance of the villi in absorption.

8. Give two classes of cathartics or laxatives. After reading the labels of two or three common cathartics, state to which class each belongs.

9. Name an unhygienic condition that is frequently found among high school students and which may lead to digestive disorders.

10. What is the objection to reading and eating at the same time?

11. Name two scientists who have investigated food habits. Discuss their contributions to the science of nutrition.

12. Review and report on the digestion and osmosis experiments set up in elementary science.

\section{Supplementary Readings}

Haggard, H. W., Science of Health and Disease (Harper \& Brothers). Kimber and Gray, Textbook of Anatomy and Physiology (The Macmillan Co.). 


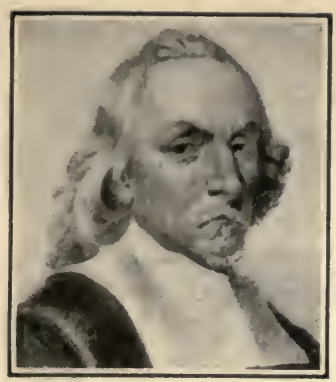

William Harvey.

\section{CHAPTER XV}

BLOOD AND ITS IMPORTANCE

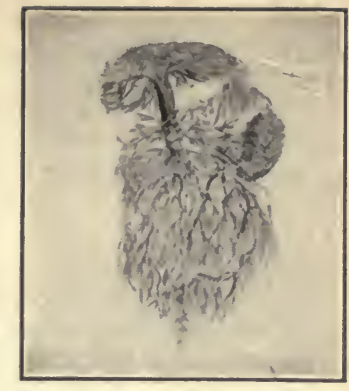

Vesalius' idea of blood vessels.

What is the relation of blood to the other tissues of the body? Where is blood made and where are its various parts destroyed? What is meant by different types of blood? What is the importance of blood tests?

Many cells are far from the source of supply of food and oxygen and far from the organs which will excrete their wastes. The blood, therefore, acts as a medium for the distribution of food materials and oxygen to the cells, and for the collection of wastes from the cells.

Circulation is the ceaseless movement of blood through the body in a system of closed tubes called blood vessels which branch to all parts of the body. When blood flows from any. part of the body, blood vessels have been broken.

\section{Problem. Study of the blood.}

Secure some freshly drawn blood from a butcher or slaughterhouse. Keep in a tightly corked bottle when not in use.

I. Pour two or three ounces of the blood into an open dish and beat it vigorously with a few broom straws.

$A$. Describe the nature of the material removed by this beating. This material is called fibrin. As the beating exposed the blood to the air, the liquid blood protein, fibrinogen, was converted into the solid form, fibrin.

B. Describe the material left in the dish. This is defibrinated blood. 
II. Continue beating until no more fibrin can be removed.

III. After beating, remove the fibrin, and pour the remainder of blood into a bottle. Label it defibrinated blood.

IV. Pour a like quantity of unbeaten blood into a second bottle and let both volumes of blood stand, corked, for several days. Compare the appearance of the materials in the two bottles.

$A$. Describe any solid mass, a clot, which may have formed in one or both bottles.

B. Describe the liquid around the clot. This liquid is called serum. Compare blood serum with defibrinated blood.

V. Place an ounce of blood in a third bottle and, by means of a delivery tube, add oxygen to it.

$A$. What effect has oxygen on the color of blood? This is now oxygenated blood. It may be compared to arterial blood.

VI. Place an ounce of blood in a fourth bottle and, by means of a delivery tube, add carbon dioxide to it.

$A$. What effect has carbon dioxide on the color of blood? This is deoxygenated blood. It may be compared to venous blood.

VII. Look at the veins in your wrist.

$A$. Do veins appear to have oxygenated or deoxygenated blood in them?

B. Explain why blood which flows from a cut vein looks like oxygenated blood.

\section{Problem. Study of blood serum.}

Test the serum for protein, starch, sugar, fat, water, and mineral matter. Account for the presence or absence of each.

Problem. Study of blood corpuscles.

Mount a tiny drop of blood on a glass slide. By means of the edge of a square cover glass, smear the drop across the slide, making a thin film. Cover with Wrights' Blood Stain for three minutes; then wash off the stain. Examine with low power and then with high power of the microscope. Observe the regular, disklike, yellowish cells which cling together; when in masses of great numbers they appear red. These are the red corpuscles. The larger, irregular, blue cells are the white corpuscles. When unstained, they are colorless.

I. Estimate roughly, the proportionate number of the two types of corpuscles. 
II. Suggest a reason for calling the white corpuscles the amoeboid corpuscles?

III. State three structural differences between the red and white corpuscles.

IV. What functions did the Amoeba perform with its pseudopodia? Certain white corpuscles can, in a similar manner, engulf bacteria. These bacteria are then digested by the white corpuscles and are thus eliminated from the blood.

Composition of the blood. Blood may be called a tissue. It consists of a yellowish liquid, the plasma, and cellular particles, the red and white corpuscles and blood platelets. The plasma is a clear fluid containing fibrinogen and other blood proteins, nutrients, and wastes. Some of the proteins in the plasma are peculiar only to the blood. Little is known of the use of these, with the exception of fibrinogen. The use of fibrinogen, which was transformed to fibrin in our laboratory exercises, will be discussed later. Amino-acids are also a part of the blood plasma. These amino-acids are digested proteins which are called building stones of the body. They are distributed by the blood to the cells, and they build protoplasm. Fat, glucose, water, and mineral matters are also taken in by the plasma from the alimentary canal. More than 80 per cent of the plasma is water. There is from 0.07 to 0.15 per cent each of fat and glucose. The proteins constitute from 6 to 8 per cent of the plasma.

All of the food that is digested and absorbed does not circulate. It is either used or stored in cells and gradually returned to the blood as tissue cells require it. Animal cells can store fat and starch, but very little protein. Therefore, any extra protein eaten tends to be broken down and eliminated. Secretions from ductless glands are absorbed directly into the blood. These liquids circulate through the body, activating, inhibiting, or regulating the more remote parts.

Oxygen is taken from the lungs by the red corpuscles of the blood. The wastes, carbon dioxide, water, and urea, collected from 
the different cells as the blood circulates, are dissolved in the plasma and carried to the kidneys, lungs, or skin, where they are thrown out.
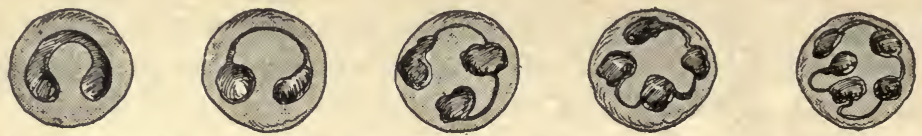

There are various kinds of white corpuscles. When stained, one type, the polymorphonuclear, shows nuclei with one, two, three, four, or five enlargements. In health there is a definite relationship of the numbers of each of the above; in starvation or disease the fours and fives are seldom found. Blood tests usually indicate the general condition of the body.

Chemical substances in blood, known as antibodies, help to combat germs directly, or neutralize the toxins which the bacteria secrete in the blood. These substances are produced by the cells during a disease or infection. Immunity to disease, which will be discussed later, is brought about in the body by these antibodies.

Blood a tissue. Red corpuscles were mentioned when tissues were considered. They are five to seven hundred times as numerous as the white corpuscles. Present investigations show that they differ from other cells in that they lack a nucleus. They are made from living cells located in the blood-forming tissue of the marrow of bones. Millions of these corpuscles are formed and given off into the blood each minute. They are thought to gradually disintegrate as they move about. The final destruction of the small fragments probably takes place chiefly in the spleen and the liver, but may occur in any part of the blood system. The correct functioning of the red corpuscles depends upon the haemoglobin which is the oxygen-carrying pigment of the blood.

A serious decrease in the number of red corpuscles or a deficiency in haemoglobin in the corpuscles causes a condition known as anaemia. Certain types of anaemia in people have been treated successfully by a liver diet. The liver which is used as food probably contains iron gotten from destroyed corpuscles. This form of iron seems to be more valuable than the iron compounds in medicines used for treating anaemia. The body seems 
to be able to assimilate it better. Whether the liver diet is remedial because of the iron present, or whether there is a vitamin present that stimulates the body to make more red corpuscles, has not yet been conclusively proved. In order to determine whether a person has anaemia, a drop of blood is put on a slide which is divided into sections by marks etched on the surface. These spaces are called counting chambers. The number of red corpuscles are estimated in relation to the white corpuscles present. This is called a blood count. If there are too few red corpuscles in relation to the white corpuscles, the person is said to be anaemic. There is also a color scale used to test for anaemia.

The haemoglobin in the red corpuscles readily unites with oxygen and forms an unstable compound, oxyhaemoglobin. When the oxygen-carriers pass cells deficient in oxygen, the oxyhaemoglobin will give up its oxygen supply. The red corpuscles of man are smaller than the red corpuscles of nearly every other animal. This allows a greater number per given volume and gives a greater absorbing surface. Thus these numerous small corpuscles carry a greater supply of oxygen than if they were larger.

The white corpuscles are larger and less numerous than the red corpuscles. Because they are capable of independent motion, they can force their way among the cells, which make up the walls of capillaries, and escape into surrounding tissues. Certain of the white corpuscles, by means of their protoplasmic processes, en-

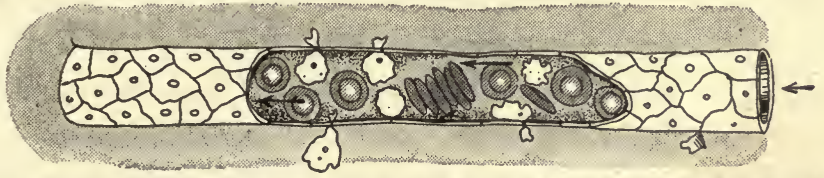

White corpuscles can make their way among the cells in the walls of capillaries by their independent motion. They may be found in tissues where they can attack invading bacteria.

gulf the bacteria in blood and other tissues, and digest them. These are the phagocytes and the process is phagocytosis. It is thought that white corpuscles originate in lymph nodes and in the 
spleen through mitotic division. When an infection occurs in the body, they multiply in great numbers and later disintegrate or probably escape from the blood upon mucous surfaces, especially

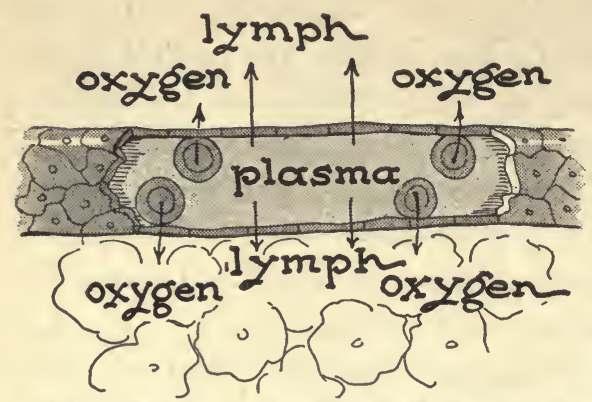

The cells take oxygen carried by the red corpuscles and use it to burn food for the release of energy. in the intestine. When white corpuscles are destroyed by bacteria, the dead corpuscles are called pus. An abnormal increase of white corpuscles in the blood usually indicates the seriousness of the infection and the amount ' of resistance offered by the body.

Blood clotting. Blood platelets are tiny bits of protoplasm found in blood. They are small cells, but lack a nucleus. Their origin is probably similar to the red corpuscles. They are concerned with blood clotting which takes place when blood is exposed to air because of wounds or hemorrhages. Blood platelets disintegrate and give rise to a substance which plays an important part in the change of the liquid protein, fibrinogen, always present in blood, to a solid form, strands of fibrin. Calcium, also present in blood, is involved in the precipitation of the fibrinogen. The strands of the fibrin entangle the blood cells and form a jellylike blood clot. Bleeding may thus be stopped. The body then repairs, by building new cells, the blood vessels from which the blood is flowing. People whose blood does not clot in the normal length of time or who bleed profusely even from the slightest wounds are called bleeders. The disease is haemophilia and it is usually hereditary.

Blood grouping. The composition of human blood is constant with the exception of the oxygen and carbon diaxide content. But since the serum of one person may be injurious to that of 
another; four different groups of blood are now recognized. Under ordinary conditions the body can renew blood as fast as it is needed. However, in certain diseases and in cases of severe hemorrhages it is necessary to introduce a quantity of blood into the veins of the patients. In such cases, blood of a similar group must be transfused, or results may be fatal through the dissolving of the corpuscles, and a consequent release of a foreign haemoglobin. Therefore, doctors always test a donor's blood to make sure it is of the same group as the patient's before making a transfusion.

Necessity for circulation. Each cell in the body has work to do. This work is performed through energy released in the cell. We have already learned that glandular cells use chemical energy, muscular cells use mechanical energy, and nerve cells need nervous energy. All of these forms of energy are obtained from the food. When the food is oxidized in the body, the energy stored in the food is released. Each cell must receive a supply of oxygen and food in order to carry on the oxidation process with its accompanying release of energy. When the food is oxidized in the cells, carbon dioxide, water, and nitrogenous wastes are formed, and must be removed. The blood carries the oxygen and food to the cells and removes the wastes from them. Since the white corpuscles combat germs, the blood must carry a continuous supply of these to whatever part of the body that

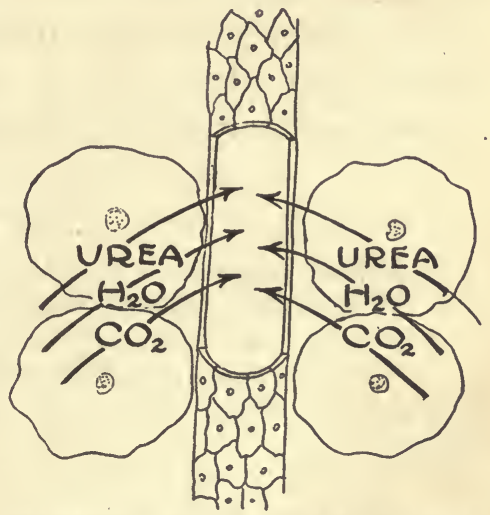

Wastes formed during oxidation reach the blood by means of the process of osmosis. needs them. At the same time other protective chemical substances, antibodies, must be carried to the places needing them. As food is oxidized in various tissues, heat is released. A great 
deal of heat is generated in active tissues such as muscular tissue and the liver, and the surplus heat must be distributed to passive tissues. Very little heat is generated in passive tissues such as bone, so heat must be sent to them by the blood when needed. The blood system may be compared to a hot-water system. The muscles are like little furnaces in which burning takes place. This heats the surrounding blood. As the blood circulates it radiates heat to the tissues that need it, and thus keeps the temperature of the body constant at ninety-eight and six tenths degrees Fahrenheit $\left(98.6^{\circ} \mathrm{F}\right.$.). When glands give off secretions and have no ducts for draining the secretions, the blood absorbs them.

In brief, the functions of blood are nutritive, protective, and excretory. When scientists can supply the same conditions to tissues outside of the body as those provided by the blood, tissues can be kept alive under experimental conditions outside of the body. This was one of Carrell's big achievements. He experimented for many years before he was able to duplicate the conditions outside of the body that were found in the blood. When he succeeded in doing this, he was able to grow tissues outside of the body. His work has been previously discussed on page 54 .

\section{Questions}

1. Definé oxygenated and deoxygenated blood.

2. What is defibrinated blood?

3. Compare arterial and venous blood.

4. Compare the red and white corpuscles in appearance, size, number, and function. Where are they made and where are they destroyed?

5. Give six functions of blood.

6. Why does blood clot; why is blood clotting necessary? 


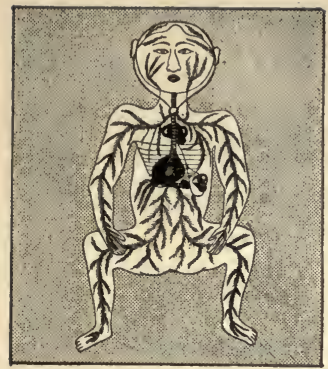

An early drawing showing the circulation of the blood.
CHAPTER XVI

CIRCULATORY

SYSTEM

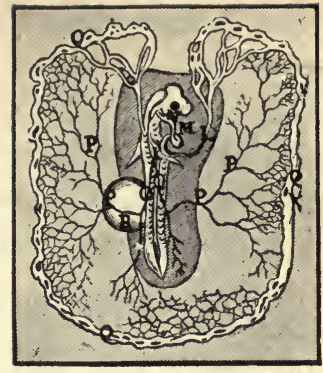

Malpighi's drawing of circulation of a chick embryo.

What are the paths of blood through the body? What canses the circulation of blood? What is the relation of circulation to the cells?

Problem. The study of blood vessels.

Anaesthetize a tadpole with a little ether. Place the tadpole in a Petri dish in a little water so that the tail can be mounted under the low power of the microscope. Do not keep the tadpole out of water more than ten or fifteen minutes at one time.

I. Note the blood flowing up some blood vessels and down others.

$A$. Suggest a reason for the parallel arrangement of the blood vessels.

$B$. Suggest a function of the connecting blood vessels.

II. The large elliptical cells passing through the vessels are the red corpuscles.

A. What is the advantage of having corpuscles in the smallest blood vessels pass cells in a single line?

$B$. Compare the red corpuscles of the frog with those of man.

III. Look at the sun or at a very bright light. Note tiny particles passing through a network of tubes in your eyes. These are the red corpuscles passing through tiny blood vessels.

In the plant, a system of tubes called ducts and sieve tubes distributes cell sap. In the human body, a network of tubes makes up the circulatory system for the distribution of blood throughout the body. Materials must enter and leave the blood by means 
of osmosis, since the blood vessels are closed tubes. Blood supplies the cells with building and fuel materials and collects wastes from them; therefore, some blood vessels must be tiny enough to get near to all the cells. These microscopic blood vessels, the capillaries, connect with larger and larger vessels, the arteries and veins, until the largest arteries and veins attain a size of one half inch in diameter.

\section{Problem. Study of the heart.}

Secure from the butcher, the heart of an ox or of any other large animal. If the school is near a slaughterhouse, it may be possible to secure enough specimens so that each group of four students may have one. Cut the hearts longitudinally. (These hearts may be kept in formaldehyde for further study.)

I. Describe the covering of the heart (the pericardium).

$A$. What is its purpose?

$B$. Explain all adaptations for this purpose.

II. Count the number of chambers or spaces in the heart.

$A$. The upper spaces are the right and left auricles; the lower spaces, the right and left ventricles. 'Describe the structure, location, and size of auricles and ventricles.

$B$. Describe the structure that separates the left side of the heart from the right side.

C. Find the movable flaps, known as valves, that separate the auricles from the ventricles.

1. Describe the valves in the left side of the heart, including in your description the number of flaps, method of attachment, and function. Observe and describe the valves in the right side.

$a$. When blood passes from the auricles to the ventricles, what effect would you expect it to have on the valves?

$b$. If blood attempts to pass from the ventricles to the auricles, what effect would it have on the valves?

c. What must be the normal direction of the flow of blood?

III. Name the tissues that largely comprise the walls of the heart.

$A$. Compare the thickness of auricle walls with those of the ventricle.

$B$. Compare the thickness of the wall of the left ventricle with other walls of the heart.

C. What is the relation of thickness of heart muscle to its activity? 
IV. Find the large vessel coming up through the center of the heart. If enough of it is still attached, it will be seen to curve around in back of the heart. Put the wooden end of your needle through it.

$A$. Into what chamber does the needle penetrate? This vessel is an artery known as the aorta. It sends blood by means of branches to every part of the body except the lungs.

$B$. Describe the walls of the aorta.

1. Suggest a reason why arteries hold their shape instead of collapsing.

2. Suggest a reason why they were originally named arteries (aer - air; terin - to hold).

V. Locate two large vessels leading into the right auricle. These are two veins called the upper or superior vena cava and the lower or inferior vena cava. They bring blood from all parts of the body, except the lungs, to the heart.

$A$. Compare the walls of these veins with the walls of the aorta. Which are capable of greater movement? Why?

$B$. What is a probable difference in function between the inferior and superior vena cava?

$C$. Suggest a reason why veins do not hold their shape when empty of blood.

VI. Try to locate the pulmonary artery originating in the right ventricle and the four pulmonary veins leading to the left auricle. The pulmonary artery takes blood from the heart to the lungs; the pulmonary veins convey blood from the lungs to the heart.

$A$. Suggest a reason for the blood going to the lungs.

$B$. Suggest a reason for the blood returning from the lungs.

VII. Make a simple diagram of the heart and the blood vessels connecting with it. Label right auricle, left auricle, right ventricle, left ventricle, septum or partition, valves, aorta, superior vena cava, inferior vena cava, pulmonary artery, and pulmonary veins.

VIII. Write a brief paragraph describing the structure of the heart.

The organs of circulation. The arteries, veins, capillaries, and heart make up the circulatory system. Approximately in the center of the chest cavity, with its apex pointing toward the left, is the conical-shaped heart. It is a highly muscular organ proWH. FITZ. AD. BIO. -11 
tected from friction by the moist membranous pericardium which covers it. The ribs and breast bone furnish protection against mechanical injury. Large blood vessels collect blood from all parts of the body and return it to the heart; others take blood from the heart to all parts of the body. The heart tissue, itself, has an independent blood supply called the coronary circulation. When the heart is cut longitudinally, it is found to consist of four chambers, a left auricle, a right auricle, a left ventricle, and a right ventricle. A thick muscular partition through the center of the heart separates the left side from the right side. The auricles receive blood from the veins and give it to the ventricles. The act of receiving blood does not require much work, so the auricle walls have comparatively little muscle in them. The ventricles force the blood into the arteries to all parts of the body. In order to accomplish this pumping, the ventricles have very thick muscular walls. The walls of the left ventricle are much thicker than the walls of the right ventricle. They have to send blood to all parts of the body while those of the right ventricle merely send blood to the lungs. Separating the ventricles from the auricles are trapdoor arrangements called valves. These are made of very strong connective tissue attached by cords of the same tissue to the ventricles. On the left side, there is a valve with two flaps, and on the right side, a valve with three flaps. The blood passes from the auricles to the ventricles, by merely pushing against the valves. If the blood backs up during the contracting of the ventricles, the valves fill up, close, and prevent the return of the blood into the auricles. Thus the blood is kept moving in only one direction.

Arteries. The tubelike blood vessels which carry the blood from the heart to all parts of the body are called arteries. The largest ones branch from the ventricles, and subdivide into smaller vessels until a network of very fine tubes, practically microscopic, is formed. These connect with the capillaries. The arteries can 


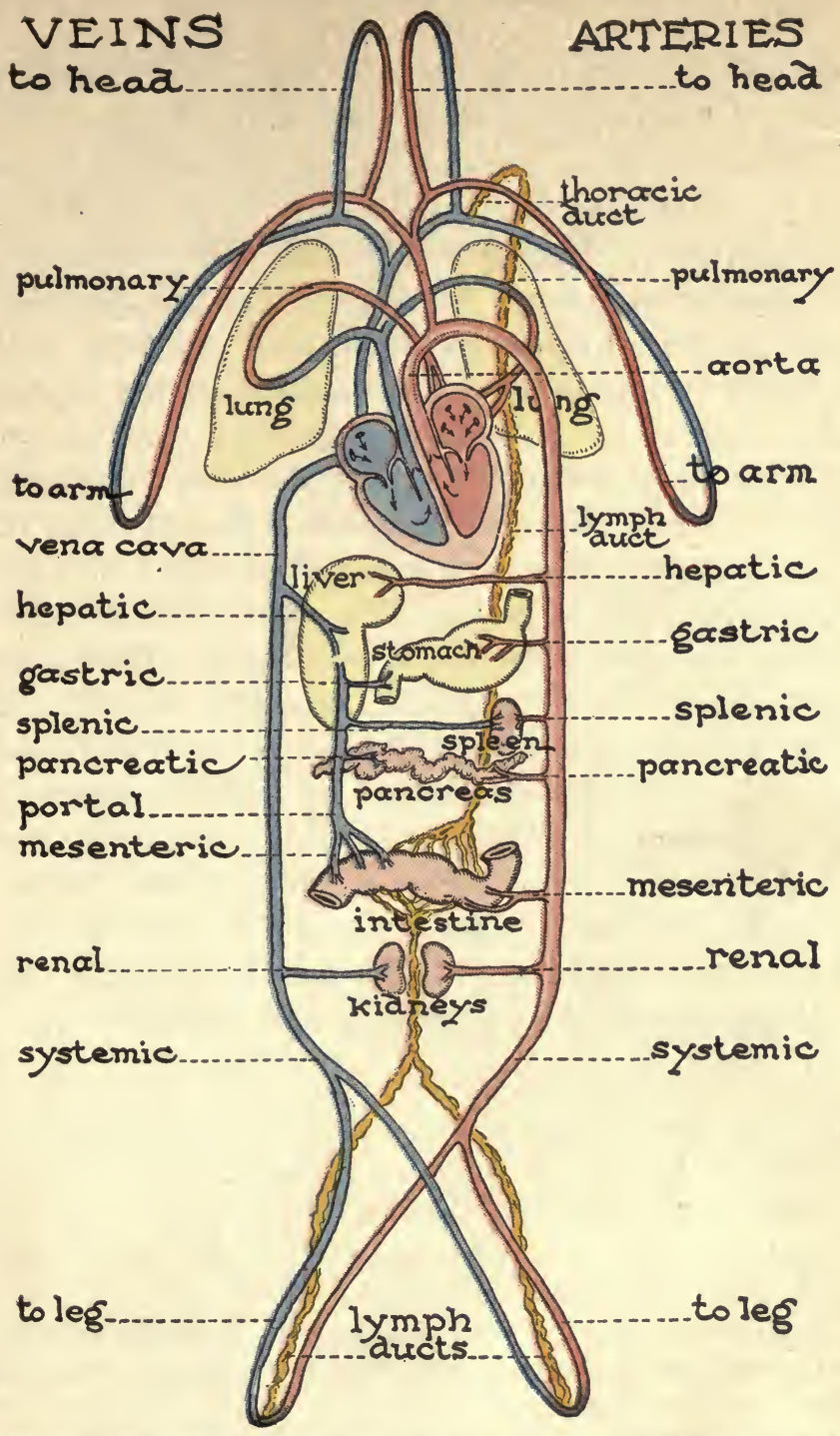

A diagram of the circulatory system in which the blood vessels containing arterial blood are colored red, those containing venous blood are colored blue, and the lymphatics are yellow. Note that, in general, only the arteries contain arterial blood, but the veins coming from the lungs have received a new supply of oxygen and hence also contain oxygenated blood. 
usually be distinguished from veins and capillaries by the presence of considerable muscular tissue in their walls. Early anatomists, when dissecting bodies, found these tubes empty and thought that they were supposed to carry air and therefore called them arteries (air-tubes). Even the smaller arteries have muscular walls and can contract and relax. The aorta and its branches are full of blood all the time. When the left ventricle contracts, the blood cannot move forward into the comparatively narrow arteries fast enough to make room for the new supply sent out by the ventricle. But, due to the elasticity of its walls, the aorta can expand and receive the incoming blood. The impulse of the blood sent into the arteries by the ventricular contraction causes a wave of distention to travel along the blood vessel. This wave of distention is called the pulse. The pulse is the passive stretching and contracting of the elastic tissue. If an increased amount of blood is sent to a particular part of the body, at any time, the arteries can always accommodate it because of the elasticity and muscularity of their walls. The pulse may be felt wherever an artery comes near the surface of the body. Most arteries are deeply embedded in other tissues so that the pulse is not easily noted. Arteries run near the surface in the wrists, neck, and temples. Probably the most convenient place for taking a pulse is in the wrist.

\section{Problem. Study of the pulse rate.}

I. Place the middle finger of the right hand about two inches from the ball of the thumb of the left hand, thus locating a pulse. Count the number of beats felt per minute. This is called taking the pulse rate.

II. Run up and down stairs two or three times. Again take the pulse rate. $A$. What effect has exercise on the pulse rate?

$B$. Keeping in mind the fact that the walls of the heart and arteries have large deposits of muscle and elastic fibers, give the reason for the effect of exercise on the pulse rate.

C. Discuss the beneficial effects of exercise on the arteries.

$D$. If you get insufficient exercise, what effect would it eventually have on the heart and arteries? 
III. Lie down or sit quietly for two or three minutes. Again record the pulse rate.

$A$. What is the effect of rest on the heart and arterial muscles?

IV. What is the value of the arterial pulse?

$\mathrm{V}$. If more blood is needed in one part of the body than in another, how can it be properly distributed?

The normal pulse rate in men is seventy-two beats per minute, for women seventy-six. The pulse rate is still higher in children. An abnormal pulse indicates some sort of unusual physiological condition.

Capillaries. The capillaries are microscopic tubes found among the cells of the tissues. These vessels are characterized by a lack of muscular tissue. Their walls are membranous. They receive the blood from the arteries and send it to the veins. Materials pass from the blood through the thin walls of the capillaries to the cells in surrounding tissues. The waste products in the lungs and other parts of the body pass into the blood from which they are later discharged. This interchange of products is possible because of the membranous walls of the

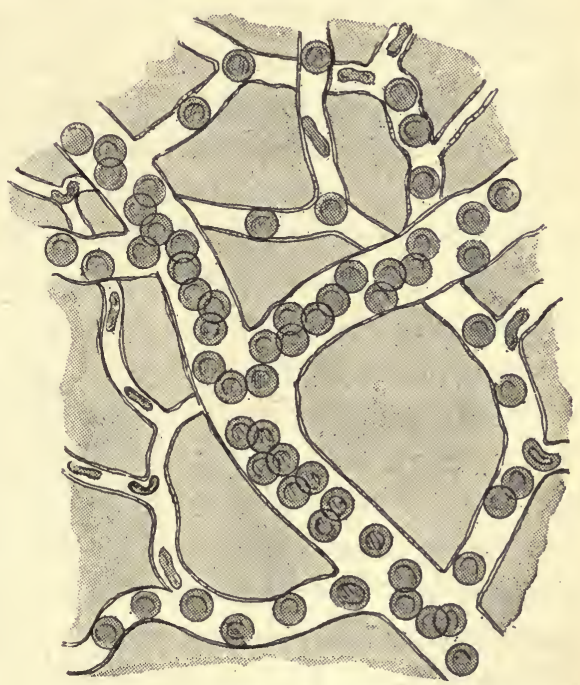

Capillaries are so tiny and numerous that they traverse the most minute parts of the body. The red corpuscles pass through in practically single file. capillaries. The blood receives enough of an impulse from the heart to keep it moving through the capillaries.

Veins. The veins receive the blood from the capillaries and return it to the heart. The veins connected directly with the capillaries 
are very tiny in size. They get larger and larger as numbers of them join together. The largest ones empty into the heart.
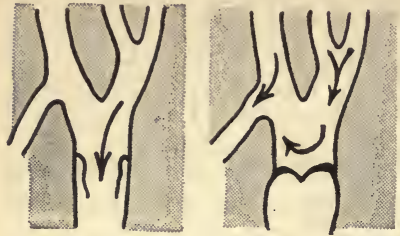

Blood flowing through veins passes valves. When these valves are opened due to back pressure, the blood finds other vessels, sometimes smaller, to traverse. Their walls are composed of elastic, connective, and some muscular tissue. There might be a back-flow of blood in the veins were it not for the valves that are found along the walls of the veins. These open in the direction of the blood flow which is toward the heart. If blood tends to flow back, they open, and prevent the blood from moving in the opposite direction. These valves may be seen if you let your hand hang down, and shut off the blood supply by holding the wrist tightly. The blood will back up against the mouth of the valves and open them. As blood fills these valves, a slight distension of the vein will be noticeable. In some older people, these valves are likely to thicken and show as swellings on the body. Since so small an amount of muscular tissue is present in the walls, very little muscular contraction is possible. The movement of blood in the veins is caused by the heart which is aided greatly by the valves in the veins, by muscles, and by the lungs. The network of capillaries feeding the veins increases the flow just as tributaries to a river cause the flow of water in the river. As breathing takes place, pressure on the large

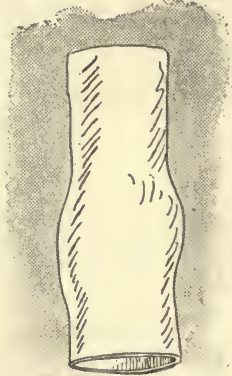

The valves in veins are like tiny watch-pockets. If blood tends to flow back, the valves fill up and close the passage; thus blood is kept moving in one direction. veins in the chest is released. This causes the blood to flow up through the veins. Skeletal muscles in all parts of the body 
squeeze the veins as they contract. This, too, forces the blood onward. If a person is inactive for a long time, the blood in the veins becomes sluggish. When we sit still for too long a time, we say a foot has " gone to sleep." Other signs of discomfort may, also, be evident because the skeletal muscles are not propelling the blood through the veins.

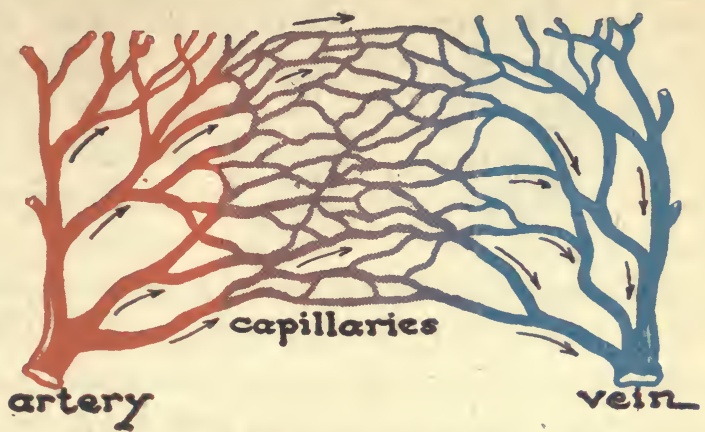

Course of the blood. The systemic circulation.

One blood vessel leads directly into another. The structure of their walls varies, however. The small, museular-walled arteries lead into the membranous capillaries which, in turn, lead into the smallest of the veins.

The circulation of the blood through the body is easily understood and remembered if we keep in mind that the sequence of organs is from the left ventricle, arteries, capillaries, veins, and right auricle. Blood never returns directly to the same side of the heart it left and the auricles do not connect with each other, nor do the ventricles connect with each other.

The left ventricle contracts and sends the blood past the valves into the large artery called the aorta. This, in turn, contracts and sends blood through smaller and smaller arteries to all parts of the body except the lungs. As the arteries get in among the tissues of the body, the muscular tissue decreases until the walls of the blood vessels consist of a single layer of cells. These vessels are the arterioles which terminate in the capillaries. After giving up the needed materials to the cells through the walls of the capillaries and collecting excretions from the cells, the blood passes into tiny veins. As the small veins lead from the tissues they join and increase in size until they form the two largest 
veins in the body. The veins from the organs below the heart drain into the lower or inferior vena cava; the veins from the organs above the heart, with the exception of the lungs, lead into the upper or superior vena cava. Both of these large veins empty into the right auricle. From there, blood passes to the right ventricle. This completes the systemic circulation. The systemic circulation enables the blood to supply tissues with needed materials and collect excreted materials from them.

The pulmonary circulation. The right ventricle contracts and sends the blood into the pulmonary artery which divides into two sets of branches, one set going to each lung. In the lungs, these arteries get smaller and smaller until they lead into the capillaries of the lungs. The carbon dioxide, collected from the cells, is given to the lungs and a new supply of oxygen is absorbed through the capillary walls. The blood then travels through veins of increasing size until it reaches the large pulmonary veins, which take the blood to the left auricle. This is called the pulmonary or lung circulation. The pulmonary circulation enables the blood to give up the carbon dioxide and take in a new supply of oxygen.

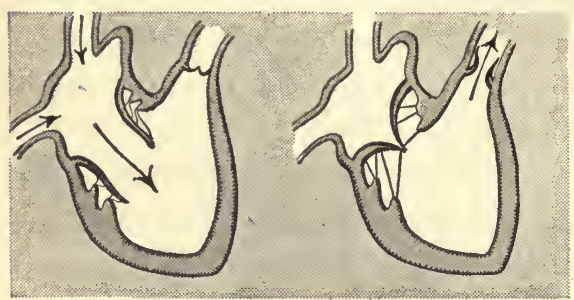

The passageway between the left auricle and left ventricle is guarded by a mitral, two-cupped valve. The passageway between the left ventricle and the aorta is also guarded by a valve. By their action, valves allow the blood to flow in only one direction.

The portal circulation. As the blood passes through capillaries in the stomach and small intestine it absorbs food that has been digested and standardized into endproducts. Were all this collected food to circulate until utilized the amounts of nutrients in the blood would vary considerably. The nutrients in the blood are kept constant to a large extent by the activity of the liver. The 
stomach, small intestine, spleen, and pancreas receive blood from the capillaries in those organs and send it to a large vein called the
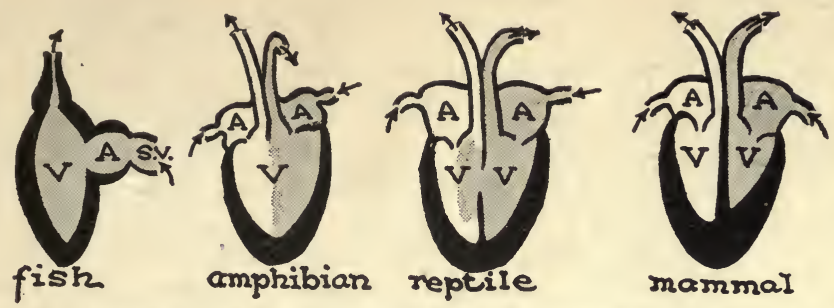

Among the vertebrates, animals are found with different numbers of chambers in the heart. The heart of mammals is really two separate hearts, each composed of an auricle and a ventricle.

portal vein. This vein breaks up into capillaries in the liver. By means of osmosis the liver cells either take out excess nutrients or give needed sugar to the blood. Another vein, the hepatic vein, then carries the blood with its normal content of nutrients to the inferior vena cava. It takes, approximately, thirty seconds for the blood to make a complete circulation.

Functions of the liver. The liver, like all other organs, consists of cells. These cells use oxygen and food and give up carbon dioxide, water, and urea. The partially disintegrated red corpuscles are completely destroyed in the liver. The liver uses some of these corpuscles and other substances as a basis for the manufacture of bile. The function of bile was discussed in a former chapter (page 132). Excess glucose is stored in the liver in the form of glycogen or animal starch. When the cells of the body need sugar, this glycogen is converted back into glucose and absorbed again by the blood. Protein is not stored in animal cells; therefore, excess proteins must be destroyed. This destruction is accomplished mainly by the liver. The carbon, hydrogen, and oxygen are taken from the protein or amino-acid and stored as glycogen. Through an oxidation process, urea is formed in the cells from the nitrogen, sulphates from sulphur, and phosphates from phosphorus. These are given into the blood and are extracted by the kidneys. 
SPECIAL FUNCTIONS OF BLOOD

\begin{tabular}{|c|c|c|}
\hline & Blood Gives & BLOOD TAKES \\
\hline Skin . . & $\begin{array}{l}\text { Water, organic salts, urea, excess } \\
\text { heat }\end{array}$ & \\
\hline $\begin{array}{l}\text { Lungs. . } \\
\text { Kidneys . }\end{array}$ & $\begin{array}{l}\text { Carbon dioxide, water } \\
\text { Water, urea }\end{array}$ & \\
\hline $\begin{array}{l}\text { Small } \\
\text { intestine }\end{array}$ & $\begin{array}{l}\text { Materials for making digestive } \\
\text { juices }\end{array}$ & $\begin{array}{l}\text { Secretin, digested nutri- } \\
\text { ents, water }\end{array}$ \\
\hline Liver . • & $\begin{array}{l}\text { Excess protein, excess sugar, } \\
\text { secretin, worn-out red cor- } \\
\text { puscles, materials for manu- } \\
\text { facture of bile }\end{array}$ & $\begin{array}{l}\text { Needed sugar, urea, excess } \\
\text { sugar, and organic sub- } \\
\text { stances }\end{array}$ \\
\hline
\end{tabular}

Blood functions. As the blood circulates through the various organs of the body, it gives up certain materials and takes in others. Those functions that take place in all cells are called general functions; those peculiar to any organ are called specific functions. Assimilation and oxidation are activities performed in all cells; therefore, they all need food and oxygen and all give up carbon dioxide, water, and urea to the blood. These are known as general functions. Since each organ hảs a special work, there are certain activities performed by the blood in relation to these particular functions. In the above table a list is shown of special work carried on by the blood in some of the organs.

\section{Questions and Suggestions}

1. Compare arteries, veins, and capillaries as to $(a)$ position, $(b)$ size, (c) structure, and (d) function.

2. Explain how to take a pulse.

3. Trace a complete circulation, naming, in order; all the blood vessels traversed from the time the blood leaves the left ventricle until it returns.

4. What is the function of each of the systems of circulation: $(a)$ systemic, (b) pulmonary, and (c) portal. 


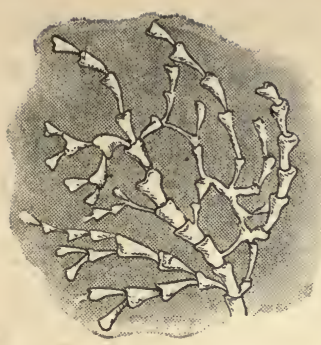

Lymph tubes.

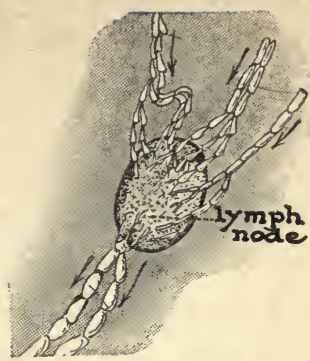

Enlarged lymph gland.

How was the circulation of blood first demonstrated? What is the relation of lymph to blood? What effect has alcohol on the heart?

Historical survey of the circulation. The study of the circulation was one of the first biological investigations that was conducted experimentally. In the early seventeenth century, Galileo, the Italian physicist, had performed and observed experiments on falling bodies, and William Gilbert of England had experimented with electric and magnetic attractions. Due to their work and influence William Harvey (1578-1657) began to make some experimental investigation in biology. The Greek anatomist, Galen (131-201 A.D.), had taught that there was an ebb and flow of blood within both veins and arteries throughout the body. He thought that the left side of the heart contained blood which was vitalized by a mixture of animal spirits in the lungs. The veins were supposed to contain crude blood. He believed that the blood passed from the right to the left side of the heart through very minute pores in the partition. It was also supposed that one kind of blood flowed from the liver, to the right ventricle, to the lungs, and then through the veins, while another kind of blood flowed from the left ventricle to the lungs and then through the arteries. In 1510, Leonardo da Vinci wrote a manuscript which included notes and drawings of the heart and the blood vessels. He had studied 
the movement of the heart in living pigs and he explained how the aorta led from the heart and branched and ramified through all parts of the body. Sometime later, Vesalius attacked one of Galen's beliefs by doubting the existence of pores in the partition in the center of the heart. But people were still under the influence of traditional teaching, and would not willingly discard too much of that which had been previously taught. In his dissections, Vesalius had noted the parallel arrangement of the arteries and veins, but he still did not observe that the blood moved in a circuit. Another investigator, Cesalpino, in 1571, ventured the opinion that some of the blood left the heart through arteries and returned by the veins. He seems to have reasoned this out without experimentation. In 1616, Harvey stated that he not only failed to find pores in the heart through which blood passed from one side to the other, but that the partition of the heart was unusually solid and compact. $\mathrm{He}$ examined the heart action of some forty different animals and described the pulsations. He examined the circulatory system of a dead man by making an injection of warm water from the pulmonary artery through the lungs into the left ventricle. He finally concluded as a result of his observations and experimentations that the blood moves in a circuit, and that the beating of the heart supplies the propelling force. Although he understood that the blood moved in a " kind of circle," he did not know about the capillary network connecting the arteries and veins. Malpighi, in 1660 , was the first to observe, with the aid of lenses, that the blood moved through the capillaries from the arteries to the veins.

Harvey's work was the result of reasoning based on the observations of the structure and pulsations of the heart. He explained how the contraction of the heart forced blood into the arteries and how this movement produced the pulse. He pointed out that the amount of blood which left the left ventricle of the heart in a given time must return and be sent out again, because, in a half 
hour or less, the heart, by successive pulsations, forces into the aorta more blood than that found in the entire body. Harvey's discovery of the circulation of the blood opened up the field of physiology for later investigators.

Source of tissue fluid. The liquid from the blood plasma is continuously being diffused through the walls of the blood vessels, into the tissue spaces where it is known as tissue fluid. This fluid differs from blood in that it lacks red corpuscles and may have a higher content of waste materials due to the collection of wastes from the cells. White corpuscles can make their way through the cells in the walls of the capillaries and escape into the tissues; therefore, they may be found in tissue fluid.

Lymph vessels. Special vessels, similar to capillaries and veins, drain the excess fluid as it collects in the tissues, and return it to the blood. These are called lymph vessels or lymphatics and the fluid in them is now called lymph. The fluid that collects in a blister is lymph or tissue fluid. Even the smallest lymphatics are closed off from the tissue spaces by very thin membranes. These minute vessels lead to vessels of increasing size until they finally unite into two very large ones above the heart. The one on the left, called the thoracic duct, carries the lymph flow from the left side of the head and chest, also from the left arm, abdomen, and the two

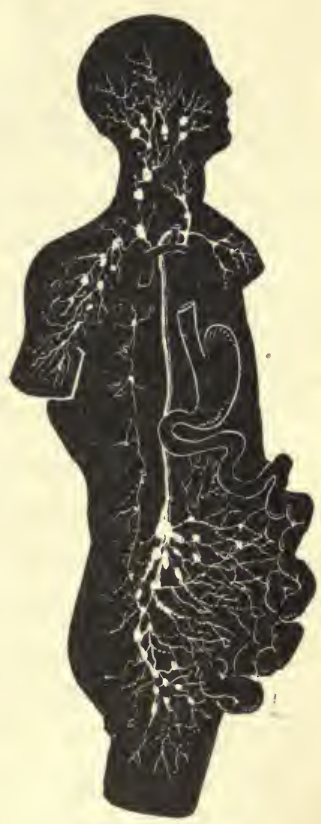

Lymph vessels, known as lymphatics, drain the fluid from all the tissues of the body. Ultimately, they lead into a vein of the blood system. legs. The one on the right drains the lymph from the right side of the head and chest, and the right arm. They both lead into the venous system from an outlet in the superior vena cava 
near the heart. Valves similar to those found in the veins are along the larger lymphatics and prevent the back flow of the lymph. In addition to the lymphatics that drain the tissue fluid, there are

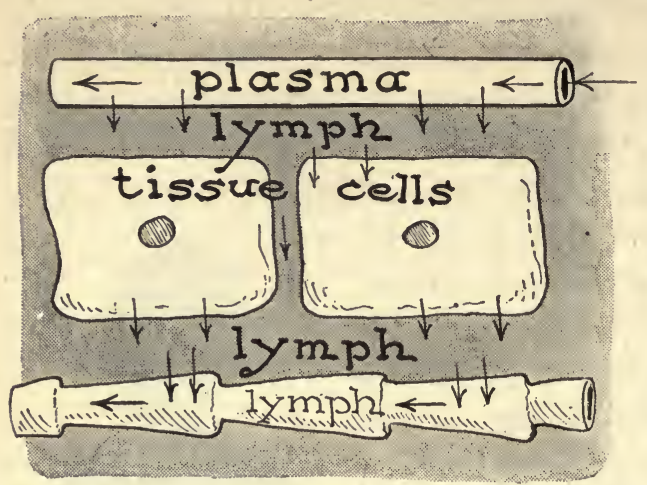

Liquid materials soak through the walls of capillaries and bathe the tissue cells. Spaces among the cells are called lymph spaces. The excess lymph is gathered up by special tubes, lymph tubes. A lymph tube is shown in the diagram to be parallel to a blood vessel (this representation is diagrammatic). some called lacteals that are in the villi of the small intestine. These lymphatics aid in the absorption of digested nutrients, particularly fats. After a meal, the lacteals are filled with a white fatty substance. At other times, the fluid filling the lacteals is very similar to the lymph in the other lymphatics. Lymph is kept moving by the contraction of skeletal muscles squeezing the lymphatics. This is similar to the effect of the skeletal muscles on the veins. The flow of lymph is also controlled by the muscular movements of the body, and by the release of pressure on the thoracic duct during each inspiration. This release causes the lymph in vessels where the pressure is greater, to flow into the thoracic duct.

The spaces formed between the membranes and the various organs of the body which they cover are similar in structure to lymphatics, and may be thought of as expanded lymph spaces. The fluid found in the pleural, the pericardial, and the peritoneal sacs is similar to the lymph in the lymphatics.

Lymph nodes are expanded portions of the lymphatics found in all parts of the body. Large collections of them are located under the arms and in the neck. Others, in fewer numbers, occur 
in many other parts of the body. They are made of fibrous tissues interspersed with numerous spaces. In these spaces, white corpuscles collect and multiply by division. Bacteria are frequently brought to the lymph nodes, where they are destroyed by the phagocytes, germ-killing white corpuscles, which are found in such numbers in the lymph nodes that they have little difficulty in destroying bacteria. Occasionally, bacteria escape from the nodes and travel through the lymph into the blood and thus spread infections. If bacteria multiply rapidly and cause an infection in a lymph gland, a wall of calcium is sometimes deposited around the germs. This prevents the bacteria from escaping into the system. A gland of this kind can be cut out and thus the infection is removed. A lymph gland acts as a sieve to remove bacterial and foreign materials and thus prevents many injurious substancesfrom getting into the blood and returning to the heart. The lymph glands of miners frequently become clogged with dirt and, therefore, become greatly enlarged.

Hygiene of the circulatory system. Blood vessels are kept in tone and the blood is kept circulating normally by regular exercise, fresh air, good food, hot and cold baths, and sufficient sleep. A large part of the repair of the body takes place during sleep. Many red corpuscles are made at this time. If one does not get enough sleep, pallor may result, which is due to an insufficient number of red corpuscles. This may cause anaemia. Regular exercise is better than spasmodic exercise. When a person does not exercise sufficiently, the heart and arterial muscles become weak and the muscles all through the body lose their tone. Any unusual exercise will then cause palpitation of the heart. Violent emotions overstimulate the heart and blood vessels. Occasionally, this causes the rupture of a blood vessel. A violent fit of anger has been known to cause a condition of this kind. Drinking water is invaluable in aiding the blood to give off waste materials collected from the cells, into the organs of excretion; namely, the lungs, skin, and kidneys. 
The cells of the body depend upon the circulatory system for the distribution of building and fuel materials. The importance of the circulatory system is, therefore, apparent. If violent physical exercise takes place shortly after a meal, a greater supply of blood is sent to the working muscles and withdrawn from the digestive organs where energy is needed. Indigestion may result.

When blood congests in veins, it causes the veins to become enlarged and these vessels are known as varicose veins. This condition frequently occurs in people who do not exercise or have to stand for long periods of time. Tight garters will retard the circulation of the blood and cause congestion. The arteries of many elderly people lose their elasticity. This may be caused by too much physical work, too much mental work, or by poor digestion. Calcium salts may accumulate in the walls and cause them to harden. This condition is known as hardening of the arteries, or arteriosclerosis.

When capillaries are broken, but the skin is unbroken, the injury is a bruise. Alternate hot and cold applications will usually have a stimulating effect. When the skin as well as the capillaries are broken, the injury is an abrasion. It should be washed with an antiseptic and bandaged to prevent infection. If arteries are cut, the blood flows in spurts; pressure must be applied between the cut and the heart in order to prevent loss of blood. This pressure is best applied by means of a tourniquet which should be released every few minutes in order to keep the blood circulating in the limb. Stopping the flow of blood will help to clot the blood. If a vein is cut, the blood flows smoothly. In this case, the tourniquet should be applied on the side of the cut away from the heart. These are first-aid measures only, and in case of an injury to a vein or artery of considerable size, a physician should be called to treat the cut.

Alcohol is believed to injure the white corpuscles to such an extent that they lose their ability to destroy germs. It also dilates 
blood vessels, causing them to lose their ability of contracting and relaxing. Tobacco is likely to cause palpitation of the heart. Both alcohol and tobacco seemingly interfere with good physique. For that reason, part of an athlete's training is abstinence from them. Good health habits exclude alcohol, tobacco, and drugs.

\section{Questions and Suggestions}

1. How was it possible for early scientists to study the circulatory system scientifically?

2. What three erroneous ideas in regard to circulation were exploded through the scientific method?

3. Draw a diagram of a liver cell, a lymphatic, and a capillary. By means of labeled arrows, indicate the possible exchanges of materials.

4. In what cells in the body can blood leave an unusual supply of $(a)$ calcium, $(b)$ fat, $(c)$ worn-out red corpuscles, $(d)$ protein for destruction? Where can it collect $(a)$ oxygen, $(b)$ urea, $(c)$ white corpuscles, (d) glucose?

5. What is the relation of the lymph system to the blood system?

6 . What rules of health should be followed if the organs of circulation are to be kept in the best possible condition?

7. In terms of circulation, discuss a possible cause of each of the following diseases: anaemia, varicose veins, and hardening of the arteries.

\section{SuPPLEMENTARY REAdings}

Haggard, H. W., The Science of Health and Disease (Harper \& Bros.).

Kimber, D. C., and Gray, C. E. Textbook of Anatomy and Physiology (The Macmillan Co.). 


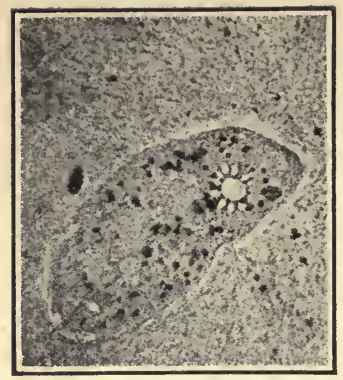

Excretory organ of Paramecium.

\section{CHAPTER XVIII}

THE SKIN

AND

KIDNEYS

What is the necessity for excretion? How are wastes eliminated from the body? What is the relation of the skin and kidneys to excretion?

Importance of excretion. The wastes formed in the various tissues as a result of oxidation are carbon dioxide, water, organic salts, and urea (nitrogenous compound). These wastes would interfere with the normal functions of the cells unless removed as quickly as formed. The removal of these wastes is called excretion, and the organs that eliminate the wastes are called the excretory organs. The importance of an efficient system of elimination can not be sufficiently emphasized. Carbon dioxide is eliminated chiefly by the lungs, although some is dissolved in water and is excreted by the skin and kidneys. Water and some soluble salts are eliminated by the skin as perspiration, and by the kidneys as urine. Some water, however, passes out of the lungs in the form of vapor. Most of the urea is excreted through the kidneys as part of the urine, and a small amount through the skin , as part of the perspiration.

\section{Problem. Study of the skin.}

I. Touch the skin and describe how it feels.

$A$. Explain the source of the heat in the skin.

$B$. When one is exercising violently, why does the skin become much warmer than it does at other times? 
II. Describe the skin of the hand as it appears to the eye. Tiny structures in the deeper layers of the skin push up the outer layer into little ridges.

$A$. Note the arrangement of the lines on the tips of the fingers, particularly the thumb. Describe the arrangement.

$B$. Moisten the finger slightly and, if convenient, touch powdered carbon with it. Then press it on clean blank paper.

1. What is the impression called?

2. What use may finger prints serve?

$C$. Examine your skin and observe whether it normally has breaks or abrasions on it. What is the value of an unbroken skin?

III. Hold the hand out straight and then clinch the fist. Does the skin appear dry and brittle or soft and flexible?

A. This characteristic is due to oil secreted in oil glands in the skin.'

$B$. Describe what may have happened to cause dry skin.

IV. Touch your tongue to the back of your hand. Describe the taste. This is due to perspiration secreted by sweat glands in the skin.

$A$. Why is the perspiration not always seen on the skin?

$B$. What condition favors the evaporation of the perspiration?

C. What condition hinders the evaporation of the perspiration?

$D$. Under what conditions do we perspire excessively? Why?

V. If wet-and-dry-bulb thermometers are available, pour a little water on the bulb.

A. After a few minutes, read and record the temperature of each thermometer.

$B$. What effect has the evaporation of moisture on temperature?

C. What effect would you expect the evaporation of perspiration to have on the temperature of the body?

VI. Hold the hand so that the back of it is on a level with the eyes. Note the hairs covering it. These hairs probably help to absorb perspiration from the skin.

VII. Cite some evidences of the skin gradually peeling from your body. This is the outer dead skin called the epidermis.

VIII. Prick the skin gently with a pin. As a result of the pin prick tell :

$A$. What two sensations may be experienced by the nerve endings in the skin.

$B$. What other sensation may be experienced by skin?

IX. Summarize your answers to the above questions by stating four functions of skin. 
Structure of the skin. The skin is a smooth, moist, flexible organ varying from one one hundredth to one tenth of an inch in

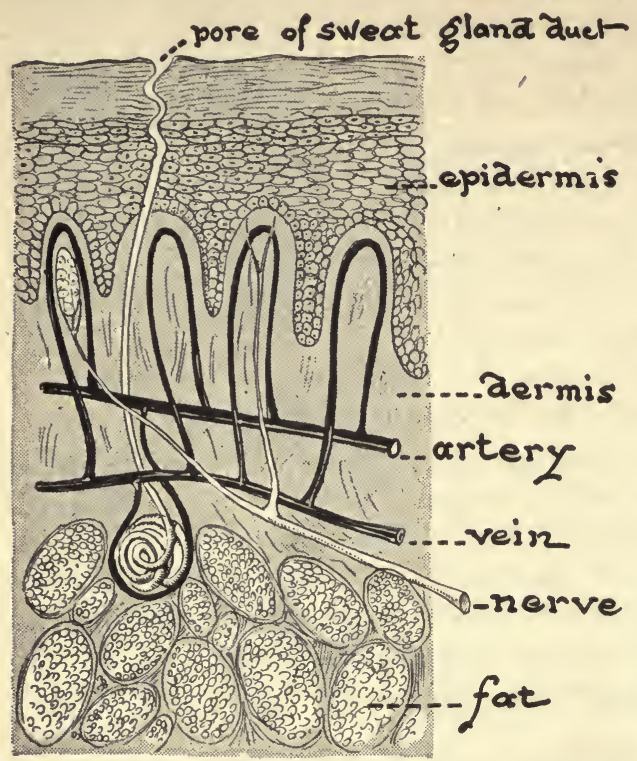

The surface cells of the skin are constantly wearing or washing off and are replaced by cells underneath. Blood vessels nourish the skin; and the various nerve endings receive different stimuli from the environment. thickness. It consists of an outer layer called the epidermis, and an inner layer called the dermis. As the cells grow out in the deepest part of the epidermis layer, they are gradually transformed into flat scales. The outermost layers are dead and are being constantly rubbed off the surface of the skin. The deepest layer of the epidermis contains pigment cells, and is called the Malpighian layer after the scientist who first observed

it. There are normal pigments in this layer, which are the basis for white, yellow, red, or black skin colors of various people. In this layer may develop deposits of pigment, which are known as freckles.

The dermis is the true skin. It is composed largely of connective tissue. Tiny muscles may run through the dermis. These, by contracting, will cause the tiny hairs in the epidermis to stand erect. They also cause the " goose flesh" when a person is cold. The contraction of millions of tiny muscles give a little heat and, at the same time, a warning that the body is cold. . The dermis is richly supplied with blood vessels, lymph vessels, nerves, and hair follicles. The blood vessels carry water in which urea and carbon 
dioxide are dissolved. Some of the wastes are eliminated by sweat glands. Each sweat gland ends in an opening in the epidermis called a pore. The perspiration leaves the body through the pores. . Specialized epithelial cells form roots of hairs in the deeper layers of the true skin. These hairs grow out through hair follicles which are formed by the downward extension of the epidermis. Only the lower part of the hair lives and grows. Associated with the hair follicle is an oil gland. This supplies nourishment to the hair since it opens either into a hair follicle or upon the surface of the skin. The oil keeps the skin flexible and soft. Within the tiny papillae or end-organs in the dermis are specialized nerves for receiving the sensations of pressure, pain, and temperature. Fat cells are also found in the dermis.

Functions of the skin. The skin is an organ of excretion. By means of the millions of blood vessels, sweat glands, and pores, it

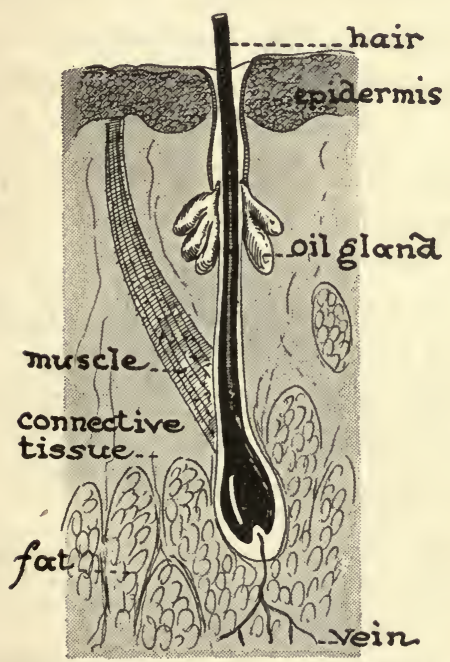

Hairs grow from hair follicles formed by the downward extension of the epidermis into the true skin. The hair in the bottom of the follicle enlarges to form a bulb which is well supplied with blood vessels.

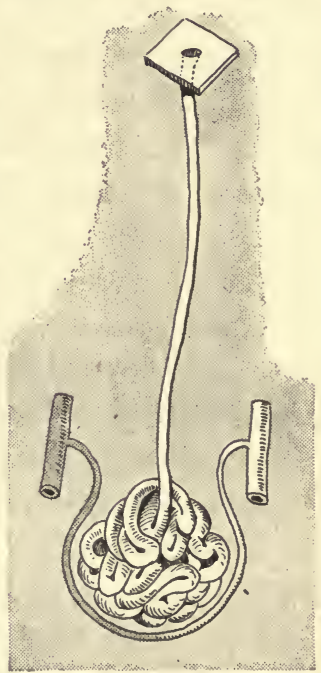

A sweat gland is composed of a duct and a coiled portion surrounded by capillaries. Wastes gathered from the blood by the glands are eliminated through a pore in the skin. 
excretes on an average of a quart of perspiration daily. In cases of kidney trouble, it may relieve the kidneys by secreting more wastes. The skin is an organ of protection. By means of its un-

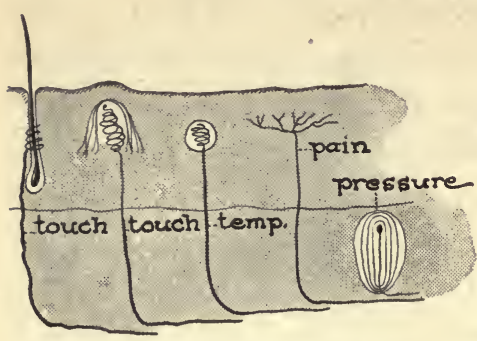

The skin is an organ of feeling. The papillae put us in touch with our environment by sensing pressure, pain, and temperature.

broken outer dead layer of epidermis, it protects the underlying living dermis from an excessive loss of water and from the invasion of germs and dirt. The skin is an organ of sensation. By means of the papillae in the dermis, it receives sensations of pressure, pain, and temperature. The skin is invaluable in equalizing and maintaining the proper body temperature. If the body is too warm, a greater supply of blood is sent to the skin so that the heat will radiate. If the body is not sufficiently warm, the blood vessels in the skin contract and very little heat radiates out. The evaporation of the perspiration on the skin cools the body.

Hygiene of the skin. When the water of the perspiration evaporates, organic salts and urea clog the pores. These wastes can best be removed by warm, soapy baths. If left in the pores, the wastes, resulting from perspiration, give the body an objectionable odor. A tepid bath should be taken at least twice a week. Cold showers are stimulating to the skin, muscles, and the blood vessels, and, if possible, should be taken daily. Dirt may clog the pores in the skin and cause blackheads. If the skin is kept clean, blackheads will not form.

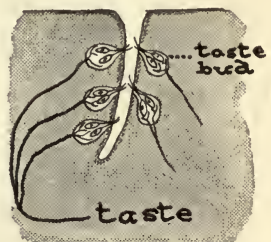

Papillae on the tongue contain nerve endings which transmit taste stimuli to the brain. Sometimes bacteria clog the pores, and cause infections, pimples, or possibly boils. Pimples should not be pinched or scratched with the fingers as bruises may result and cause more serious 
infections. Let them heal naturally or have a physician care for them. When pimples become chronic, the condition is called acne. If acne is treated in time, it can be cleared up completely. If not, it leaves scars that are permanent. Any unhygienic condition of the body is likely to affect the skin. In order to keep the skin in good condition, hygienic suggestions concerning food, water, rest, exercise, and sunlight should be followed.

The kidneys. The or-

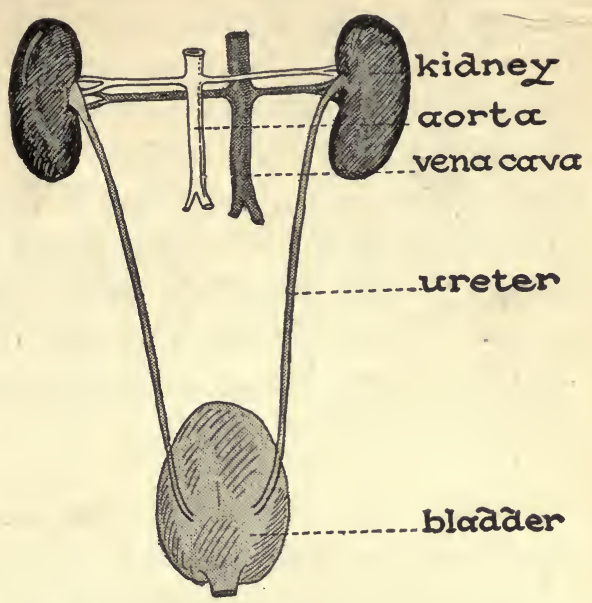

The kidneys gather liquid wastes from the blood. These materials are passed through the ureters to the bladder and held in this reservoir ready for excretion. gans that excrete most of the water and urea from the body are the kidneys. They lie in the abdominal cavity in the small of the back above the waist line. They are bean-shaped with the concave side turned toward the spine. The bulk of the kidney is made up of small coiled

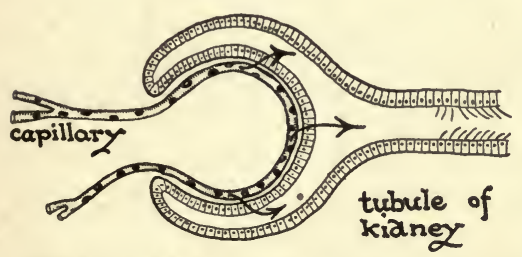

The kidneys are made up of numerous minute tubules. Their intimate relation with blood vessels makes possible the absorption of wastes. tubes, tubules, closely packed together and containing a large number of blood vessels, and some nerves and lymphatics. Water, urea, and organic salts pass by means of osmosis into the tubules. These tubules lead into two tubes, one from either kidney, the ureters, which are connected to a hollow muscular sac called the bladder. The kidneys secrete urine which passes down the ureters to the bladder. The bladder serves as a 
reservoir for the urine until it is expelled from the body. When the bladder is moderately distended, it holds about one pint. The average quantity of urine secreted in twenty-four hours by the average adult is about forty ounces, or 1.2 liters. The kidneys eliminate toxic materials formed in the body during illness. In ridding the body of its poisons, the kidneys are frequently damaged. In order to keep them in as good condition as possible, about seven glasses of water should be drunk daily. This will dilute any toxic materials present and keep the kidneys well washed. Too much animal protein food should not be eaten as the kidneys may become overworked, in eliminating the urea, an oxidation product of proteins.

\section{Questions And Suggestions}

1. What is excretion?

2. Name the organs of excretion and discuss how each one eliminates waste.

3. Name four functions of the skin and explain the ways in which the skin is adapted to perform each function.

4. Discuss the hygiene of the skin.

5. What unhygienic habits may injure the skin?

6. What is the relation of the work of the skin to the work of the kidneys?

7. Discuss the value of water to the skin and kidneys.

\section{Supplementary Reading}

Kimber, D. C., and Gray, C. E., Textbook of Anatomy and Physiology (The Macmillan Co.). 


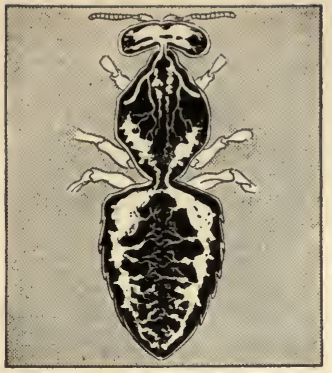

Tracheae of insects.
CHAPTER XIX

RESPIRATION

What is the relation of respiration to excretion? How do the lungs perform their function?

Most of the carbon dioxide and some of the water, formed by the body cells during the oxidation of food materials, are eliminated through the lungs. There are no organs in a higher plant similar to the lungs of man, although the stomata, openings for the intake and outgo of air, in leaves are concerned with the function of respiration in the plants. The abundance of these stomata make up for their microscopic size. They are found in the epidermal tissues of a leaf and they lead to air spaces among cells between the two layers of epidermis. These openings are not adapted for securing large quantities of air at one time. They may be compared to the nostrils of man rather than to the lungs.

Lower animals have various devices for securing air. An insect has small openings in the abdomen and thorax which lead into branching tubes. These tubes subdivide until they can reach the smallest cell in the body. By compressing and releasing their body regions, somewhat like a bellows, the animals are able to take in oxygen and give out carbon dioxide. Watch the abdomen of a fly or a bee and note the breathing movements. A fish has gills which are branched structures with such thin walls that air can pass directly from the water into the blood stream. The air 
is taken from the water which is drawn into the mouth and forced out through the gills. Respiration takes place in every living cell, but breathing is only possible in the higher animals that have lungs.

The air tubes. The outer openings of the air tubes of man are the nostrils. These lead into two nasal passages terminating in the throat cavity. The nasal passages are specially adapted for pre-

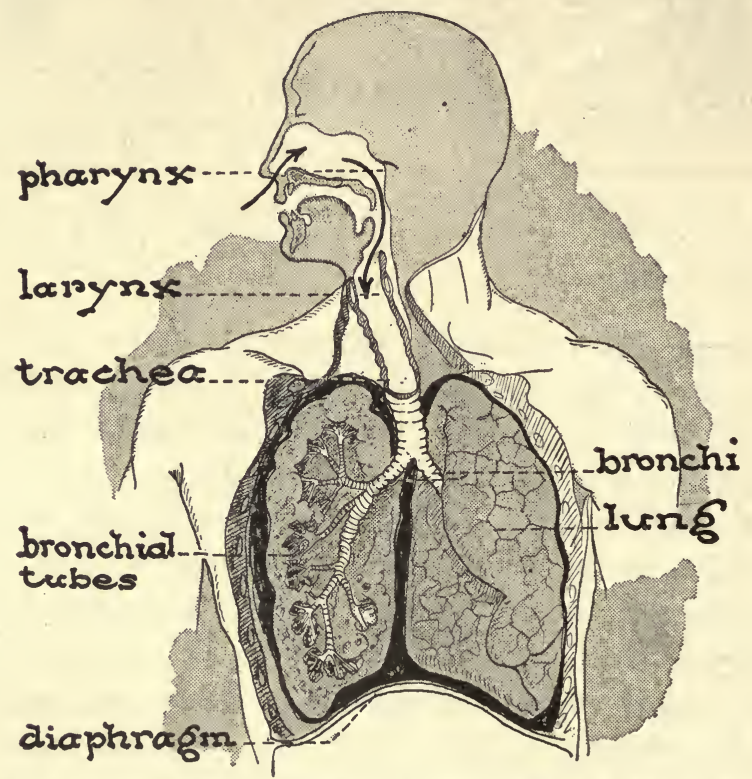

The breathing organs of man are arranged in a continuous passageway from the nostrils to the ends of the bronchial tubes. These tubes subdivide and terminate in air sacs.

paring air for the use of the body. The hairs in the nose act as a filter, to keep dust and other foreign particles from entering the lungs. The mucous lining absorbs the fine foreign particles and inhibits the development of germs. The mucous lining also moistens the air. Very dry air is irritating to the lungs.

The air is warmed in the nasal passages by coming in contact with the blood vessels in the linings of the narrow passageways. Air then passes through the glottis into the larynx or voice box. 
The larynx contains some thickened cartilage, popularly called Adam's apple, which projects slightly on the front of the neck. The air passes down the trachea or windpipe into the two branches, the bronchi, which subdivide again and again into the bronchial tubes, which in turn end in small pouchlike sacs called the air sacs. All of the air tubes are lined with mucous membrane which warms and moistens the air. The trachea is lined with specialized epithelial cells bearing hairlike processes or cilia. These cilia are in constant motion. Their function is to push or move any solid particles, in the air, into the throat, where they are expelled.

There are numerous capillaries in the air sacs. The walls of the capillaries are membranous and air easily diffuses through them into the blood. The red corpuscles take the oxygen from the air. Thus blood becomes oxygenated. At the same time, the blood gives out its carbon dioxide. The air sacs may be compared with tiny balloons. The fact that they are so numerous and are capable of such inflation affords a tremendous surface for the absorption of oxygen even though the lungs themselves are fairly small. The large absorbing surface of the air sacs makes possible the presence of a tremendous number of capillaries running through them. Therefore, a great deal of oxygen quickly passes into the blood and carbon dioxide passes out. The lungs consist of millions of air sacs and bronchial tubes held together by connective tissue. The moist membranous pleurae cover the lungs, and prevent friction during the breathing movements.

Diseases of the respiratory tract. The mucous membrane lining the pharynx contains many lymph glands and at the back and upper part of the cavity there is a large mass of this lymphoid tissue. During infancy and childhood this tissue may increase greatly, and the child is then said to have adenoids. Adenoids are a menace to health. They may obstruct the openings to the Eustachian tubes, and in some cases cause deafness.- They usually interfere with the passage of air through the nose and 
necessitate mouth breathing. This not only allows dust and other foreign particles to pass into the lungs, but tends to alter the shape of the jaws and facial features. Malformations of teeth are fre-

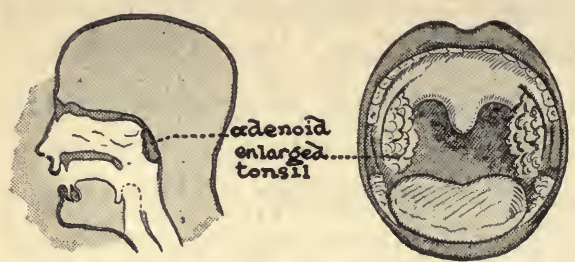

Thickened mucous membranes may form abnormal growths, known as adenoids, in the back of the nasal passageways. Tonsils are the normal growth of lymphoid tissue in the throat. quently caused by adenoids. Mouth breathing is usually a forced breathing and frequently alters the shape of the chest.

In the lower part of the throat, on either side, are small masses of lymphoid tissue called tonsils. The function of the tonsils is not understood exactly. They are thought, when in a normal condition, to help protect the body from infection by acting as filters and preventing the entrance of bacteria. If they become infected with bacterial growths, they serve only as centers of infection. When they are infected, the pus in them passes directly into the lymph and then into the blood. This may cause such conditions as rheumatism, anaemia, or produce an undesirable heart condition. If the tonsils become much enlarged, they fill the throat cavity and interfere with the passage of air to the lungs and of food to the gullet. Inflammation of the tonsils is called tonsillitis.

Problem. Study of the lungs.

Secure the lungs and windpipe of a sheep or lamb from the butcher.

I. Try to cut the windpipe or trachea.

$A$. Describe the structure.

$B$. What is the value of the presence of cartilage in the trachea?

$C$. Describe the arrangement of the cartilage in the trachea.

II. Observe the appearance of the lungs.

$A$. Describe the outer shiny covering. Discuss the functions of the covering.

$B$. Describe the structure of the lungs, including the shape, texture, color, and tissues present. 
$C$. Insert a glass tube in the trachea and breathe into it.

1. What effect has this on the lungs?

2. When you blow into the lungs, do you find any structures that might account for the texture of the lungs?

III. Cut out a small piece of the lung tissue where the bronchus enters it.

$A$. Describe the branches of the bronchus in the lungs.

$B$. Try to trace one of the tubes until it ends. How does it end?

Problem. Study of the mechanics of breathing.

Place a cork, through which runs a glass rod, in the mouth of a small-sized bell jar. Attach a balloon to the end of the rod inside the jar. Cover the bottom of the jar with a piece of rubber sheeting in the center of which a string is attached.

I. To what structures in our bodies are the glass tube, balloon, bell jar, and rubber sheeting comparable?

II. Pull the string gently, so that the rubber sheeting moves down.

$A$. What effect has the lowering of the rubber sheeting on the balloon?

$B$. Is the balloon pressed on by air in this position of the rubber sheeting as much as in the former position?

$C$. When the pressure on the outside of the balloon is released, what effect has it on the air in the balloon?

$D$. Why, then, does air enter the balloon?

$E$. To what is this comparable in human breathing?

III. Push the rubber sheeting into the battery jar to form an arch.

$A$. What effect has the arching of the rubber sheeting on the balloon?

$B$. In this position, is the balloon pressed on by air as much as in the lowered position?

$C$. When the pressure on the outside of the balloon is increased, what effect has it on the air in the balloon?

$D$. Why, then, does air go out of the balloon?

$E$. To what is this comparable in human breathing?

IV. To what is the inflation and deflation of air in the balloon due?

V. What is one of the reasons for inspiration and expiration in man?

Mechanism of breathing. Air with its oxygen is taken in, and air with increased amounts of carbon dioxide is given off as a result of muscular activity. The floor of the chest cavity is formed by the muscular diaphragm. The upper surface of the diaphragm 
is arched around the lower part of the heart and lungs. When its muscular portion contracts, the diaphragm flattens and moves downward, while the ribs are elevated. The spaces between the ribs are filled by muscles. When these muscles relax the ribs return to their original position. This reduces the chest cavity from side to side. The diaphragm now returns to its original position and the abdominal walls contract and push the liver and stomach against the diaphragm, which contracts, presses against the lungs, and in so doing, pushes out the walls of the chest cavity, front and back. Thus the chest cavity is increased in size from top to bottom by the contraction of the diaphragm, and from back to front and side to side by the activity of the muscles between the ribs.

The lungs are as large as the cavity they occupy. When the chest cavity increases in size, the lungs are no longer pressed upon, and, since they are somewhat elastic, they fill the space made by the expanding chest. The air in the lungs now spreads out to fill the space formed by the enlarging of the lungs. Movement of air always occurs when there are differences in pressure. As the air spreads out, its density becomes lower. Air rushes in from the outside to equalize the pressure. This is inspiration or the taking in of air. The blood vessels in the air sacs are thus supplied with air from which the haemoglobin extracts oxygen and to which it gives up carbon dioxide.

Expiration is the forcing out of air. It is due to the relaxation of muscles which crowd the lungs into a smaller space. Air in the lungs is then denser than air outside and it is expelled in order to equalize the pressure. This is expiration. The taking in of the air, the exchange of gases in the air sacs, and the giving out of air is breathing or respiration.

Cell respiration. When the oxygen of the air gets into the blood, it is carried to the cells. It passes from the blood through the walls of the capillaries, through the lymph spaces and into the cells. 
Oxidation of the food then takes place, releasing energy for cell work and forming the wastes, carbon dioxide, water, and urea. The carbon dioxide is given off to the blood. Respiration is of two types, external respiration and internal respiration. External respiration is concerned with inspiration and expiration. Internal respiration involves the exchange of air between the blood and tissues.

The rate of respiration. The average rate of respiration for an adult is twelve to sixteen per minute. This rate is partly determined by the amount of carbon dioxide in blood. If this amount rises above a certain percentage, the nerve centers controlling respiration are stimulated and this results in deeper breathing at an increased rate. Thus more oxygen is obtained and the carbon dioxide is removed more rapidly. This explains the second wind of athletes. Due to violent exercise, a great deal of oxidation takes place, causing the accumulation of carbon dioxide in the blood. Then the nervous response follows, increasing the respiratory rate and giving the athlete more oxygen for releasing more energy. The rate of respiration may, also, be influenced by strong emotion and by old age. In respiration, the lungs are never emptied of air; only about one tenth of the air is normally changed with each respiratory movement. This is called tidal air.

\section{Problem. Modifications of breathing.}

I. Analyze the type of respiration, and next to each write whether it is an inspiration or an expiration in each of the following: yawning, sighing, sneezing, coughing, hiccuping, sobbing.

II. Explain how the control of the diaphragm will probably suppress any one of them.

The air we breathe. Inspired air contains about 20.96 per cent oxygen, 79 per cent nitrogen, and 0.04 per cent carbon dioxide: Expired air contains about 16.4 per cent oxygen, 79 per cent nitrogen, and 4.1 per cent carbon dioxide. The same air can be breathed many times before the oxygen is entirely exhausted. 
Through experimentation, scientists have discovered that people are most uncomfortable in still air. Under the clothing, in close contact with the skin, is a blanket of air. This air absorbs perspiration, body odors, and the excess body heat. If this air cannot circulate, it becomes moist, and the perspiration does not evaporate as it should. If air in a room is set in motion by an electric fan, the contaminated and moist air moves, and cooler and drier air takes its place. This brings relief to the body. For years, people thought ventilation consisted largely of getting rid

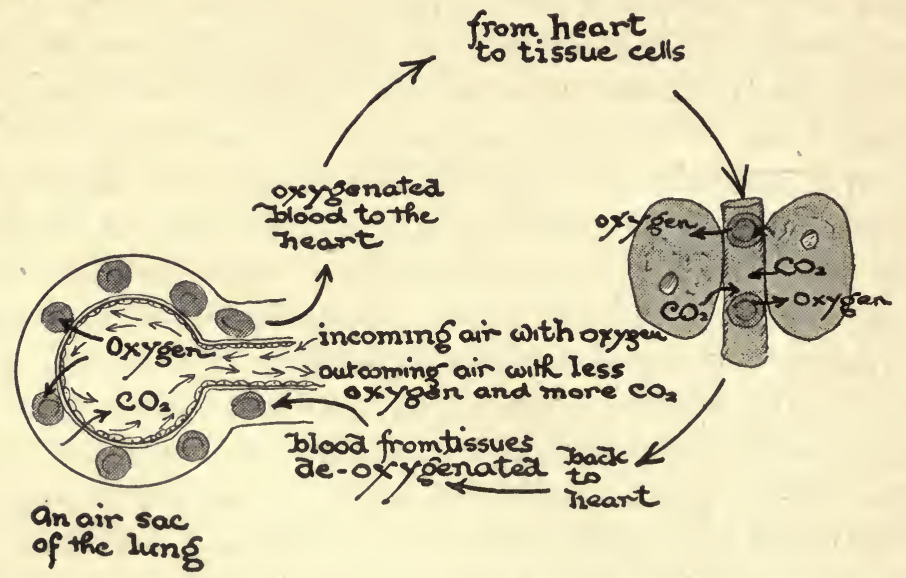

The exchange of carbon dioxide and oxygen between the air sacs and blood vessels is external respiration; between the cells and blood vessels it is known as internal respiration.

of carbon dioxide. Now it is known that ventilation problems also involve the control of body odors, heat, and moisture.

When air is set in motion, the stagnant air is removed and fresh air is brought in contact with the body. Air that is best for breathing should be at a temperature from about 65 degrees to 70 degrees. It must be slightly moist, must be moving, and must he free from dust and impurities. The problem of ventilation is to treat the atmosphere of a room so that air will have at all times the four characteristics mentioned. 
Methods of ventilation. Many systems of ventilation have been devised, but probably in many places none works as efficiently as properly regulated windows. Windows should be opened at the top and bottom during both day and night. Air comes in the lower opening, becomes heated, rises, and goes out through the upper openings. Care should be taken that drafts are kept out of the room since these are likely to chill the body. A board placed at the bottom of an open window can be arranged so as to drive the air upward and will thus prevent drafts. In winter, care must be taken to keep the air moist. If the air is too dry, the mucous membrane lining of the nose and throat will become dry and irritated, probably resulting in inflammation in the respiratory tract. The various methods of heating homes are likely to dry the air. The evaporation of water in pans placed under or on radiators will aid greatly in moistening the air. To-day, we find that ventilating systems are included in many of the modern heating plants. In these systems the same air is circulated repeatedly. It is washed, heated or cooled, and freed of moisture in each circuit.

Hygiene of respiration. A great many deaths are caused annually by diseases of the respiratory tract. Many of these could be prevented if the respiratory tract were kept in good condition. The nose is adapted for preparing the air for the use of the body, and, therefore, it should be used for breathing. Any interference with nasal breathing should be corrected.

\section{Questions AND Suggestions}

1. Suggest two experiments that may be used to show that oxidation takes place in the body.

2. Name the adaptations of the respiratory system for $(a)$ purifying air, (b) warming air, (c) moistening air.

3. Make a labeled diagram of the air passages. Trace the path of air from the time it first enters the body until it reaches the cells.

WH. FITZ. AD. BIO. -13 
4. Discuss the mechanics of breathing.

5. State the aims of ventilation.

6. How would you ventilate a room?

7. What causes discomfort in a poorly ventilated room?

8. In a diagram containing a cell, lymph space, and a capillary indicate, by means of arrows, how respiration takes place.

9. Build a box to.illustrate a room with two windows. Manipulate the windows to illustrate proper ventilation. Candles may be used to show the best methods of ventilation.

\section{Supplementary Readings}

Howell, W. H., Textbook of Physiology (W. B. Saunders Co.).

Williams, S. F., Healthful Living (The Macmillan Co.). 


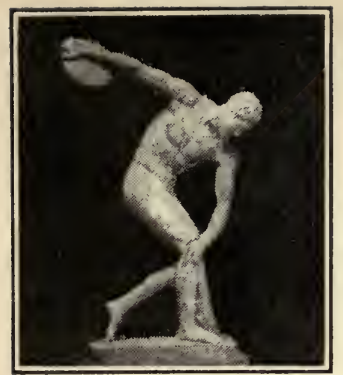

Exercise promotes

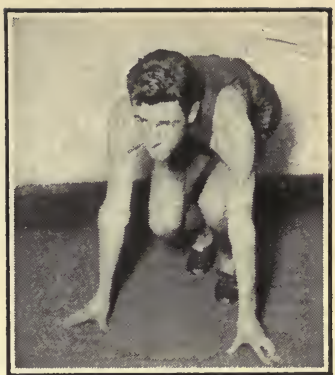

good metabolism.

What is metabolism? How are the food nutrients used in the body?

Metabolism is the term usually applied to all the processes which are concerned in the building up and breaking down of the protoplasm in an organism. In short, it is the sum total of all the physical and chemical changes by which the protoplasm utilizes food, releases energy, and eliminates waste products in order to maintain, repair, and produce more protoplasm. The processes of absorption, assimilation, respiration, and excretion are phases of metabolism, while digestion may be called a secondary process which makes possible the primary activity of absorption. If the process of metabolism is that of synthesis or building up, it is called anabolism, but if the process is one of tearing down, it is katabolism. When the building up of protoplasm is greater than the breaking down, growth takes place.

No one function of the body can be discussed without referring to one or all of the others because many of the functions take place at the same time. During the anabolic process of assimilation, the katabolic process of oxidation is also being carried on in order to release enough energy for the assimilation and growth processes. Before oxidation is possible, the digestion of the food must take place. In fact, the process of metabolism involves all the physiological functions of the body as well as the special activities that 
take place within the cells themselves. No matter what kind of food is taken into the body, it must first be broken down into a soluble form, and assimilated by the cell, before it can be converted into the kind of tissue needed.

Metabolism of carbohydrates. All carbohydrates are reduced to simple sugars, glucose or galactose, in the digestive process, by means of enzymes. They are then absorbed by the capillaries in the villi and sent to the liver by way of the portal vein. The excess sugar is taken in by the liver and stored there as an insoluble form of carbohydrate, known as glycogen or animal starch. Muscle cells, too, store up small quantities of sugars in the form

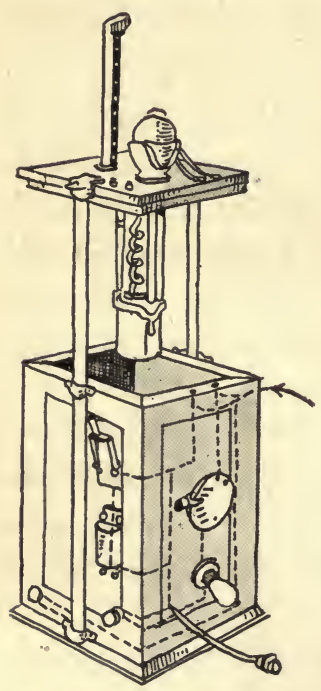

The food burned in a bomb calorimeter and the energy released is carefully determined. The number of calories shown in the food tables is found in this manner. of glycogen. This glycogen is then converted, as it is needed, into glucose and given to the blood. Thus the percentage of sugar in the blood is kept constant at 0.07 to 0.15 per cent. If more carbohydrates are eaten than can be used or stored, the surplus tends to be converted inito body fat. As the sugar circulates, it is absorbed in the tissue fluid and cells by means of osmosis. Here it is oxidized to release energy in the form of muscular work or heat to keep the temperature of the body constant. In the oxidation process, sugar is converted into carbon dioxide and water. These wastes are carried by the blood to the lungs, skin, and kidneys for elimination from the body.

Metabolism of protein. Proteins are decomposed by enzymes in the digestive tract into the simple compounds, amino-acids. These are absorbed into the blood and from there into the tissue fluid and cells. The body uses the various amino-acids for build- 


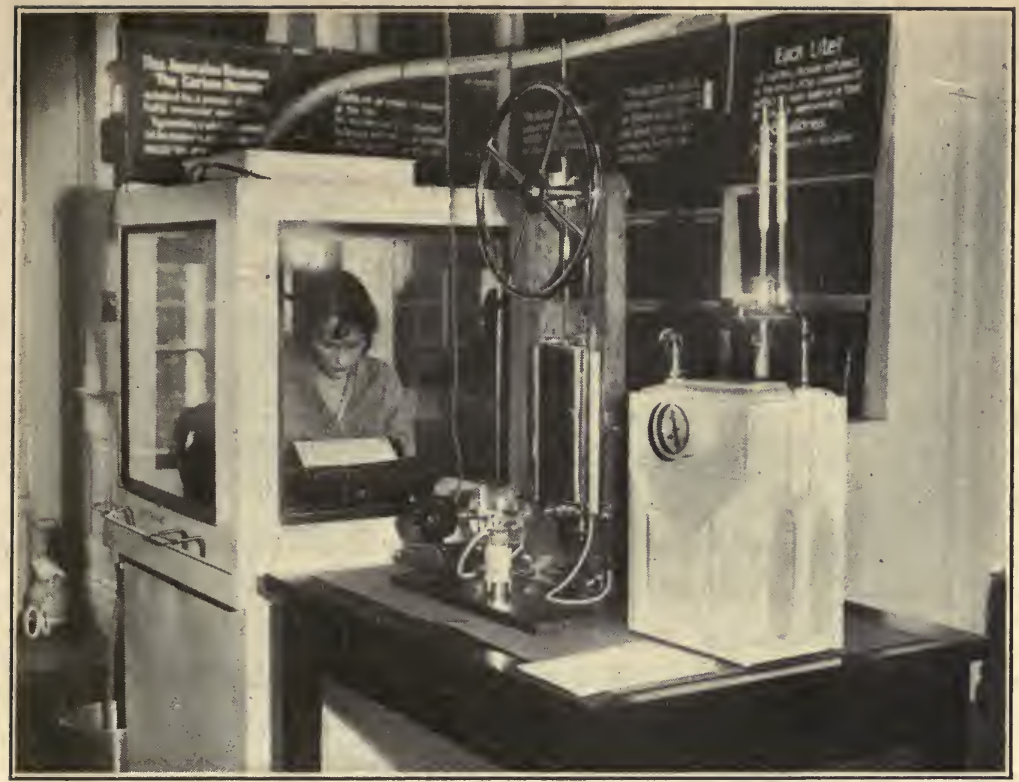

Underwood and Underwood

The amounts of human energy expended in various types of work can be measured by a calorimeter. This is done by devices which measure the amount of oxygen absorbed and the amount of carbon dioxide given off by the subject doing a particular kind of work.

ing new cells and for repairing any wastage. If there is a surplus of protein materials it is thought that a portion is held as a sort of storage supply in the liquids or tissues of the body. This surplus is then utilized when the loss of protein from the cell is greater than the supply needed by the body for repair, as in time of illness.

If there are more proteins (in the form of amino-acids) than the cells can utilize, some of the excess is eliminated by the kidneys, while others are taken care of by cells in the liver. These cells in the liver are capable of separating the amino-acids into ammonia and non-nitrogenous products (oxygen, hydrogen, carbon, sulphur, and phosphorus). The ammonia combines with other elements to form urea which is eliminated by the kidneys as 
fast as it is formed. The sulphur and phosphorus are given off by the excretory organs, and the carbon, hydrogen, and oxygen combine and are stored in the liver in the form of glycogen. This substance is then released as the body needs it.

Metabolism of fats. Fats are absorbed as glycerol, fatty acids, and soaps by the lacteals of the villi. In the absorption process, they are reassembled as fats, probably by the epithelial cells in the villi. The absorbed fats travel through the lymphatics to the thoracic duct which empties them into the venous system just before draining into the superior vena cava. In this way, they become a part of the blood. These fats are not in the original form of the fat; they are made into a fat which is characteristic of the species in which the metabolism is taking place. They are carried to the cells, where they are burned as fuel which serves as a source of energy for muscular work and other activities. The resulting wastes, carbon dioxide and water, are eliminated by the organs of excretion. Some fat is used to build fatty tissue and the extra fat is then stored in the vacuoles of cells in the form of drops of oil.

\section{Questions}

1. Give a definition of metabolism.

2. Classify the functions of the body into anabolic and katabolic functions.

3. Discuss the metabolism of sugar; of protein; of fat.

\section{Supplementary Readings}

Burton-Opitz, Textbook of Physiology (W. B. Saunders Co.). Howell, W. H., Textbook of Physiology (W. B. Saunders Co.). Martin and Weymouth, Elements of Physiology (I ea \& Febiger). Mitchell, P. H., General Physiology (McGraw-Hill Book Co.). 


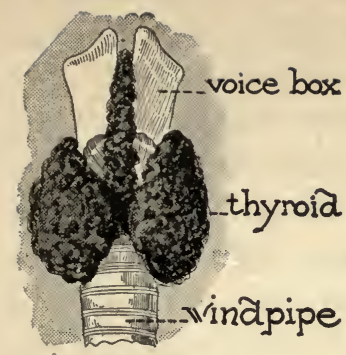

The thyroid gland.
CHAPTER XXI

\section{DUCTLESS}

GLANDS

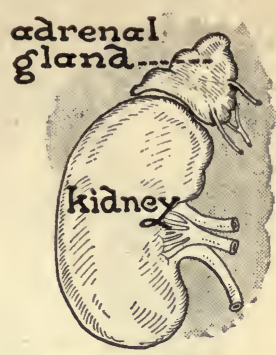

An adrenal gland.

What are ductless glands? How do they affect the development and the activity of the body? What is the scientific status of gland grafting?

The glands of the body, that have already been discussed, had ducts to convey their secretions to an outer surface, such as the mouth, stomach, or skin. Certain glands in the body have no ducts, but pass their secretions directly into the blood or lymph. These glands are called endocrine glands (endocrine is from a Greek word which means " to separate within "). These glands manufacture substances which go into the blood stream, and activate or influence another organ or organs in the body. These manufactured substances are called hormones (from the Greek word meaning to " excite" or " arouse").

Internal secretions. The hormone secretin was mentioned when the digestive system was studied. It is liberated from the wall of the small intestine when the acid food enters from the stomach. It is absorbed directly into the blood and is carried to the pancreas and liver. It stimulates these glands to activity so that the bile and pancreatic juice immediately start to flow. Carefully checked experiments have proven that this activity always takes place. For example, an experiment was given in the chapter on digestion to show that secretin travels through the blood from the intestine to the pancreas. Another interesting experiment has been performed to show how digestive juices 
are produced. Some of the mucous membrane of the small intestine was scraped off, treated with acid, and injected into the

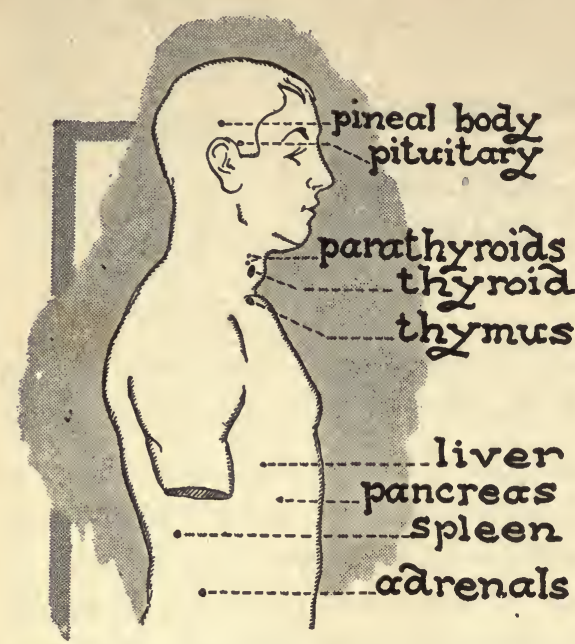

Most of the ductless glands of man are indicated in their approximate positions. blood. Immediately, pancreatic juice flowed. The flow is thought to have been effected by the stimulation of the digestive glands by secretin which was probably formed from an inactive hormone in the mucous membrane.

The internal secretions are of great importance in bringing about coördination among the various organs. It is thought by some investigators that every kind of tissue may give rise to individual substances or principles which pass directly into the blood, and affect the general working or metabolism of the body. Not many studies have been published concerning tissue secretion. To date, investigations have been confined largely to the hormones of the ductless glands.

It is difficult to secure the internal glandular secretions of human beings in a pure state. Therefore, our knowledge concerning them is based primarily on experimentation on lower animals, chemical examination of the glands and their extracts, and of the blood near the glands, observations of the effects resulting from disturbances of the ductless glands, and effect of direct injections of extracted or synthesized internal secretions.

The thyroid. The thyroid is one of the ductless glands. It is situated in the neck and consists of two divisions or lobes, one on 
either side of the voice box or larynx, and usually connected by a narrow strip of tissue. The entire structure weighs between one and two ounces. The substance secreted by the thyroid is called thyroxin and it contains about 65 per cent of iodine. It passes directly into the blood since there is no duct to convey it from the gland. Thyroxin influences the rate of oxidation in the body. The method of testing this rate is by determining the person's basal metabolism. Basal metabolism means the heat or energy expended by the body when there is almost complete absence of absorption from the digestive tract, and almost complete muscular and mental repose. A normal person who has fasted for fifteen hours preceding the test would use a definite amount of oxygen, depending on his weight, height, and age. This amount has been determined, and is a measure of normal basal metabolism. Doctors can tell whether or not a thyroid gland in any person is overactive by performing this basal metabolism test. A large amount of thyroid secretion increases the metabolism in the body, which is noted by an increase in the amount of oxygen used, as compared with the amount used by a normal person. A decrease in thyroid secretion results in a lower rate of basal metabolism; that is, less oxygen is used than that usually required by a normal person.

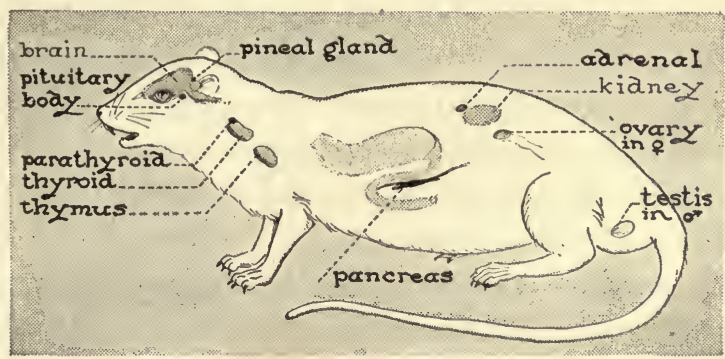

The location of the ductless glands in the rat.

Thyroids have been removed from animals, and harmful effects were observed. Other thyroids were grafted in another section 
of the body, and the aforementioned results disappeared, although the effect of the grafted thyroids were only temporary. Extracts of the thyroid tissue are now given to persons whose glands do not function properly. In most cases the treatment is successful.

Thyroxin probably has some effect upon growth and development of organisms. Gudernatch, while teaching at Columbia University, has carried on a series of interesting experiments on glandular feeding. He fed thyroid glands (of different animals) to very young tadpoles and they promptly went through metamorphosis and became frogs. In some cases the frogs were no larger than a beetle. When he removed the thyroid gland from other tadpoles, they never became frogs, although they grew larger than the usual size of a tadpole.

Thyroid deficiency, due to degeneration or an operative removal of the glands, in an adult gives rise to dullness and general apathy or sluggishness. The person gets very stout, although his appetite may be diminished. The heart beats slower, the nervous system becomes sluggish, and the general intelligence is lowered. This condition is easily explained by the slow rate of oxidation. The disease is called myxedema. If in a young child the thyroid glands waste away, his head and face usually become enlarged, and look deformed, and his abdomen becomes swollen. The mental faculties as well as the physical character of these sufferers show lack of development and often lead to a condition of idiocy. This disease is called cretinism, and children suffering from it are called cretins. When thyroid extract is fed to persons suffering from myxedema or cretinism, they usually improve and sometimes are completely cured, provided no essential organs have been affected. This thyroid treatment must, however, be kept up indefinitely because the patients' glands are atrophied and will remain inactive.

In some communities, a large percentage of the population shows an enlargement of the thyroid glands; this condition is known as endemic goiter. It is common in certain sections of our country. 
One might think that the thyroid is over active in endemic goiter, since it is enlarged. However, the reverse is true as the general symptoms are the same as in myxedema. Thyroid tissue is rich in iodine. This is a constituent always present in drinking water of localities near the sea, in sea weed, and in sea foods 'such as oysters and crabs. When people live in areas remote from the sea, they drink glacial water which is practically lacking in iodine. Endemic goiter is usually prevalent in such districts. An example is our own Great Lake district. Studies have been made in many schools in these localities. Inorganic iodides have been administered to the children and iodine has been put in their drinking water. Within a short time the number of cases of goiter showed a remarkable decrease. However, it is probably unwise to feed iodine as a treatment to all people suffering from endemic goiter. In case the gland is already enlarged, the iodine may stimulate the gland to produce too much thyroxin. This would increase the metabolism of the body. When people suffer from excessive thyroid activity, foods containing iodine are often removed from their diets. In all cases iodine should be used only upon the advice of a physician.

An excessive secretion of thyroxin gives rise to a disease called exophthalmic goiter. The symptoms are just the opposite of those in underactivity of the gland. Instead of a stupid, apathetic condition there is a restless, nervous one. There is a wasting away of tissues in spite of an enormous increase in food consumption. The pulse and heart action are very rapid and often irregular. The thyroid usually increases in size accompanied by an abnormal protrusion of the eyeballs. There are various treatments, the most successful being complete rest in order to slow up metabolism. Sometimes, X-rays are used to check the activity of secretion and sometimes the gland is partially removed. A more common operation is the tying off, temporarily, of one of the blood vessels in order to lessen the amount of the secretion reaching the blood. 
The parathyroid. There are four small structures attached to the thyroid glands, and weighing in all about two grains. They are the parathyroids (para means "near"). These, also, produce an internal secretion which is related to the calcium metabolism. Their removal, or atrophy, gives rise to tetany, a sudden convulsive contraction of the muscles, which may result in death. The injection of small doses of parathyroid extract usually results in temporary relief. The effects of too great activity of the

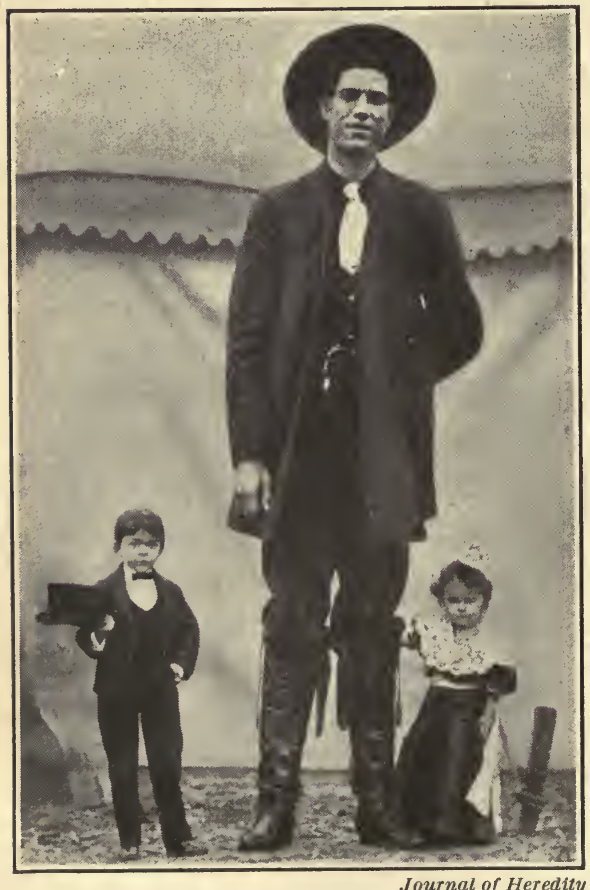

Giantism and dwarfism are probably due to defective pituitary glands. parathyroids have not been fully ascertained, although it has been shown by Collip that the injection of parathyroid extract into the blood of an animal will increase the calcium content of the blood while the removal of a part of the parathyroid tissue will give rise to a calcium deficiency.

Pituitary gland. The pituitary gland weighs about one sixteenth of an ounce and is located at the base of the skull. Galen and Vesalius knew that there was a pituitary gland. They thought it had something to do with the secretion of the nose. (Pituitary is from the Latin word pituita, meaning phlegm.) Modern experimentation has proved that there is no such connection or relationship. There seems to be sufficient evidence to justify the acceptance of 
the opinion that the anterior lobe or part of the gland affects the growth of the skeleton and the posterior part causes several important bodily changes. Although the hormone or hormones of the posterior lobe have not been obtained in pure form, injections of extracts from the lobe will cause a rise in blood pressure, an active contraction of smooth muscles, and a conversion of glycogen into sugar. Any irregularity in the gland affects growth, especially that of the skeleton, and the tone of the muscle cells of the blood vessels. Undersecretion in children causes them to remain small and fat. Some dwarfs are known to have very small pituitary glands. These dwarfs are well-proportioned, unlike the cretins with their overdeveloped heads and abdomens. Too active a pituitary gland in a young person causes an enlargement of the bones. The individual assumes giantlike proportions. If the gland is affected in an older person, acromegaly, a gradual enlargement of the bones of the head, hands, and feet usually results. A rise in blood pressure and the slowing of the heart beat usually accompanies the condition.

The adrenal glands. The adrenal glands ( $a d-$ on; renes kidneys), called suprarenal bodies by some investigators, are two small glands situated just above the kidneys. They weigh about one seventh of an ounce. The inner part of the glands produces a secretion called adrenin. The secretion, if there is any, of the outer or cortical region has never been isolated and its function is obscure. Adrenin, like thyroxin, can be obtained from the gland in a pure form. It has also been built up or synthesized by chemists in the laboratory. It is known commercially as adrenaline. Both the natural and the synthetic product tend to contract the arteries, thus causing an increase in blood pressure. Visceral arteries are affected to a greater extent than the arteries leading to the muscles. Adrenin also hastens blood clotting and strengthens the heart beat. It stimulates the liver to release the stored sugar. This 
sugar is then available for oxidation in the muscles. Thus the muscles may receive an additional source of energy. The amount of secretion from the adrenal glands is greatly increased during strong emotional excitement such as fear and anger. If a person is badly frightened, he seems to have unusual ability to escape from the danger. This is due to the activity of the adrenals which are stimulated by the emotion, and to the fact that adrenin is absorbed into the blood stream in unusually large quantities. The pouring out of the hormone by this gland increases the blood pressure, strengthens the heart action, and contracts the visceral arteries so that the blood supply to the muscles is increased. At the same time, the liver is stimulated to release more sugar than it would normally. In consequence, the blood supply brings more food to the muscles which are better able to respond to the emergency. Fatigue, too, is postponed. This often explains the great strength people have during an emotional crisis. The physical endurance of the dancing dervish is an example of adrenal activity. If physical activity does not result from stimulation of the adrenal gland, a violent nervous reaction takes place. People should avoid, as far as possible, situations that will arouse violent emotions, unless resulting activity is desirable. Since

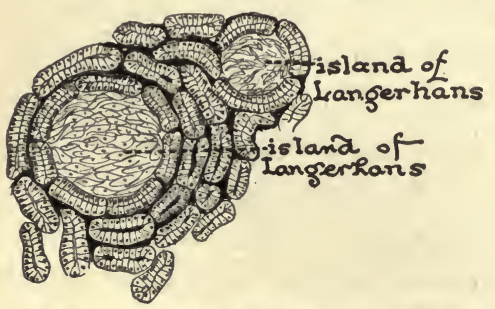

Certain groups of cells, known as the islands of Langerhans, are a part of the pancreas. They secrete insulin.

blood is withdrawn from the viscera during violent emotions, grave digestive disturbances may follow. It has been demonstrated that mental activity may be seriously interfered with, causing extreme nervousness.

Addison's disease, characterized by great muscular weakness, darkening of the skin, low blood pressure, feeble heart action, and intestinal disturbances, is attributed, by many investigators, to degeneration or injury of 
the adrenal cortex. It is almost always fatal. Removal of the adrenals in lower animals always results in death.

The commercial product, adrenaline, is used to prevent or check bleeding. It causes a temporary constriction of blood vessels in the area, which results in checking the flow of blood. In operations for the removal of tonsils, surgeons often spray the patient's throat with an adrenaline solution before the operation, in order to prevent a great loss of blood. This is known as the bloodless operation.

The pancreas. The pancreas is a digestive gland secreting pancreatic juice which passes through a duct to the small intestines, where it acts upon the food particles coming from the stomach. The pancreas also acts as a ductless gland. Certain cells embedded in the pancreas, called the islands of Langerhans, produce a secretion called insulin which contains a hormone that is absorbed directly into the blood. This hormone stimulates the liver to give up its glycogen. At the same time, it accelerates the oxidation of sugar in the tissue cells. Thus sugar is removed from the blood and the body. If the islands of Langerhans lose the ability to produce this secretion, the sugar is not used and some of the extra sugar remains in the blood and some is excreted with the urine. The individual develops a disease known as diabetes. Dr. F. G. Banting and his associates discovered (in 1922) that the insulin obtained from the normal pancreas of animals would produce a marked decrease in diabetes symptoms. Much suffering has already been lessened through this discovery. Insulin is available for treatment of diabetes although it is not considered a cure. Its use must be continual, since as yet no method has been discovered for stimulating the defective condition of the organ so that it can make its own insulin.

Other glands. The thymus is a small gland in the neck below the thyroid. It probably has a close relation to growth and possibly to sexual development. Experiments seem to prove 
that it checks for a time the development of the reproductive organs. It is very large in a young child, but gradually reduces in size during adolescence until it is very small in adults. The pincal

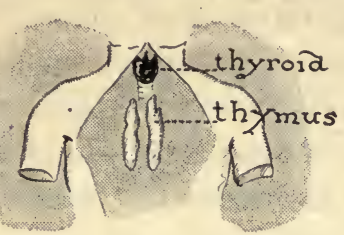

In a very young infant the thymus is exceedingly large. body is at the base of the brain behind and above the pituitary. Extracts from this gland do not have any observable effect. There is some evidence, however, that the injury or destruction of this gland in young children is usually followed by abnormal development. Beneath the diaphragm, behind and to the left of the stomach, is the spleen. It increases in size after a meal and reaches its maximum about five hours after digestion. 'Then it slowly decreases to its former size. The cause of this activity is not known. This gland possibly plays a part in the formation and destruction of red corpuscles, because quantities of them are found in it. If the spleen is removed from animals suffering from one type of anaemia, splenic anaemia, beneficial results follow.

The reproductive glands, also called the gonads, produce a secretion that passes through a duct, and another secretion that is absorbed directly into the blood. Certain of the cells of these organs make the sex cells which leave the glands through ducts. This secretion is dealt with in a later chapter on reproduction. The normal development of the body depends upon the internal secretion of the sex glands. If the male sex glands or testes are removed from young animals, it modifies the normal course of their development. Thus we have the normal bull contrasted with the modified ox and the normal stallion with the modified gelding. Modified animals never acquire complete secondary sexual characters.

The secondary sexual characters in man include the beard, and the large larynx which accounts for the deep voice. After maturity has been attained, the changes that follow the loss of 
the internal secretions are less striking. As old age approaches, these glands become less active. Great prominence has been given experiments along the lines of postponing old age by treatment of these glands. These experiments are supposed to affect senescense, old age, and bring about rejuvenescence, youth and vigor. Various methods of rejuvenation have been tried; such as, grafting glands of young monkeys on human beings or feeding glandular extracts. All of these methods are still in the experimental stage. Old age is the breaking down of many of the systems in the body, and it is extremely doubtful whether glandular extracts will rejuvenate the entire body. 'To date, the persons who received the grafted glands showed improvement for a short time only. The grafted gland was, ultimately, absorbed by the surrounding tissues. Some scientists attribute the temporary youthful effects to the optimism and enthusiasm of the subject rather than a definite physiological effect. The experiments seem to have some effects, but they are too experimental to discuss as facts.

\section{Questions and Suggestions}

1. Name four diseases related to the thyroid activity. State the amount of thyroid secretion in each disease.

2. Discuss a diet deficiency affecting the thyroid glands. How has the resulting disease been controlled?

3. Account for giantism and dwarfism by glandular activity.

4. Discuss all the physiological activities involved in a dancing dervish.

5. Name two hormones used commercially. Give the use of each.

6. Make out an outline of the ductless glands and fill in the following headings as far as possible: (a) Name of gland, (b) Endocrine or hormone, (c) Use to the body, (d) Disease resulting from oversecretion, (e) Disease resulting from undersecretion.

7. During a fire, the farmer who owned the house carried a cook stove from the burning building. After the fire he found he could not move the stove without help. It took three men to carry the stove. How can you explain his unusual strength?

8. What effect does a large checring section probably have on the players of a football team?

WH. FITZ. AD. BIO. -14 


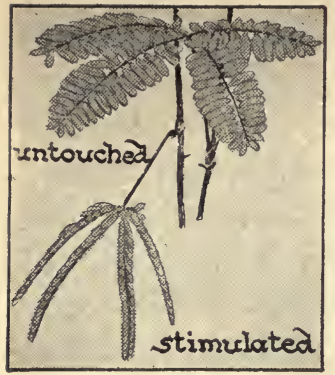

Mimosa, a sensitive plant.
CHAPTER XXII

THE NERVOUS SYSTEM

How are plants and animals adjusted to their environments? What is the structure of the brain? What are the functions of the brain? Is phrenology a science? What scientific studies have been made of the nervous system?

Irritability. Plants and animals must adjust themselves to their environment in order to survive. Different conditions in the environment provoke responses in organisms. Certain of the responses of the amoeba were considered in the discussion on the functions of the amoeba. If the response is very definite and without exception for a given stimulus, that response is known as a tropism. Such responses are characteristic of plants and of animals without a nervous system. In animals with a nervous system, if the response is definite, mechanical, and without exception, the reaction may still be called a tropism. For example, the swarming of the bees, the fluttering of moths around a light, and the burrowing of worms into the earth are frequently called tropisms. When an animal has a well-developed nervous system, the responses are more varied and individual. The nervous system governs and regulates the responses to stimuli. In this latter case the response is called a nervous reaction instead of a tropism. The response of the organism, whether it is a nervous reaction or a tropism, is due to the activity of the protoplasm in the 
individual cells of the active organism. This property of protoplasm is called irritability. The possession of this property enables an organism to make the adjustments necessary for living in certain environments. Some simple experiments with plants will demonstrate tropisms.

\section{Problem. What response does a plant make to sunlight?}

Prepare a box with a series of shelves arranged alternately on opposite sides so that they overlap each other. The shelves should be several inches apart and extend into the box about three fourths of the distance. Cut a small window in the side of the box near the top. Place a plant in a small flower pot in the bottom of the box. Keep the plant well watered. Place the box so that the window faces the sunlight and leave it for two or three weeks.

I. $A$. Describe the growth of the plant, telling how it differs from the normal or usual method of growth of a plant.

$B$. What was the value of the shelves in the experiment?

C. The response of an organism to light is called phototropism (a turning to light). Is the response of the shoot of the plant toward or away from sunlight?

$D$. What is the value of phototropism to a plant?

Problem. What is the response of different parts of a plant to gravity?

Prepare and fill a pocket garden or Petri dish with moist cotton, and place mustard seeds in a row across the middle of it. Keep the same edge or side of the garden up until the seedlings have grown to be three fourths of an inch. After making definite observations, turn the garden to an angle of forty-five degrees and keep in this position until the plants have grown another three fourths of an inch. Then make a second examination. Repeat this three or four times, in each case waiting the number of days required for three fourths of an inch additional growth before making a definite conclusion and before turning again. Be sure to keep the moisture evenly distributed throughout the cotton.

I. A. Describe the direction of growth of the root; of the shoot.

$B$. After your first turning what was the result? after the second, third, and fourth? This response of the plant to gravity is called geotropism.

C. How does gravity affect the growth of the root and of the shoot?

$D$. What is the value of geotropism in a plant? 
Problem. What is the response of plants to water?

Prepare a pocket garden filled with cotton. Plant mustard seeds in a vertical line across the middle of it. Water the seeds by moistening the cotton on one side of the garden. Try to keep one part of the cotton always moist and the other part dry.

I. A. Describe the growth of the root and of the shoot. A response to water is called hydrotropism.

$B$. What is the value of hydrotropism to the plant?

$C$. Describe any evidence in the experiment that shows whether gravity or water is the stronger stimulus.

$D$. If you have ever seen willow trees growing along the bank of a river, describe how hydrotropism tends to affect their growth?

The uses of tropisms. Plants make responses to other stimuli. The response to chemicals is chemotropism, response to heat, thermotropism, and to touch or contact, thigmotropism.

All the activities of living plants and animals involve a series of responses. As we have already learned, the responses of plants are definite for given stimuli. Leaves always grow toward the light,

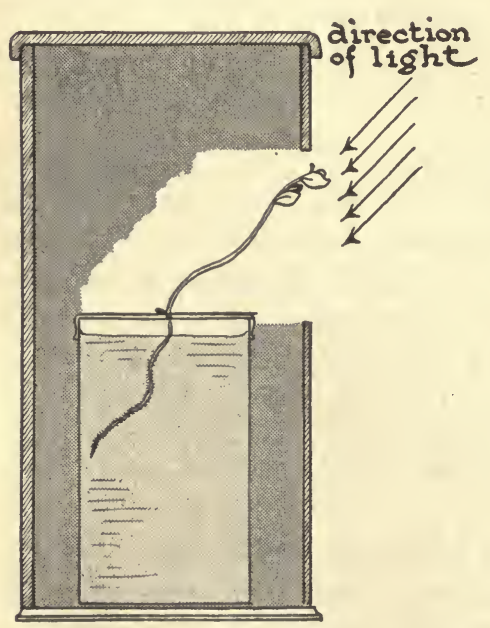

Light influences the growth of plants. Most stems and leaves turn toward the light and a large area of leaf surface is exposed. roots grow toward gravity, and stems grow away from gravity. Responses are divided into two kinds of reactions, one toward the force or stimulus, positive tropisms, and the other reactions away from the stimulus, negative tropisms. In general, tropisms are protective. Without sunlight, the leaves would not be able to make starch; without the pull of gravity, roots would be unable to anchor the plant in the ground. Tropisms help the organism make the best possible adjustments to its environment. 
Irritability in man. In order to understand the reactions of man, the mechanism that brings about the reactions must first be studied. This mechanism is called the nervous system. It is often compared to a telephone system. An expert operator at a switchboard quickly brings different rooms in a building, different homes in a city, different cities, and even

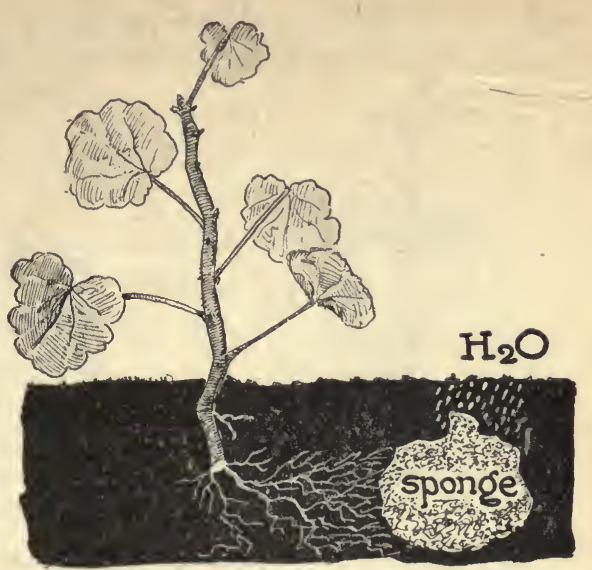

Water, also, acts as a stimulus; sometimes it is stronger than gravity and causes a turning of roots from their normal direction toward the water supply.

different nations into communication by means of messages sent over various connecting wires. In a similar way, the various organs of our body are made to work together by means of nerves that are brought into connection by means of nerve centers. There are two groups of structures composing the nervous system: (1) the central nervous system and (2) the sympathetic or autonomic nervous system. These two systems are intimately connected with each other.

Protection of the central nervous system. The central nervous system consists of the brain and the spinal cord. The brain is covered and well protected by three membranes which separate the skull from the skull cavity. These membranes secrete the cerebro-spinal fluid and contain blood vessels which transmit blood to all parts of the nervous system. If glancing blows strike the skull, the movable mat of hair and skin tend to weaken the force of the blow. In infants, the skull bones are not completely joined together and, consequently, their brains are not as well protected as the brains of adults. However, by the time the child is 
eighteen months old these bones have grown together, forming a comparatively firm, hard structure. The spinal cord is protected

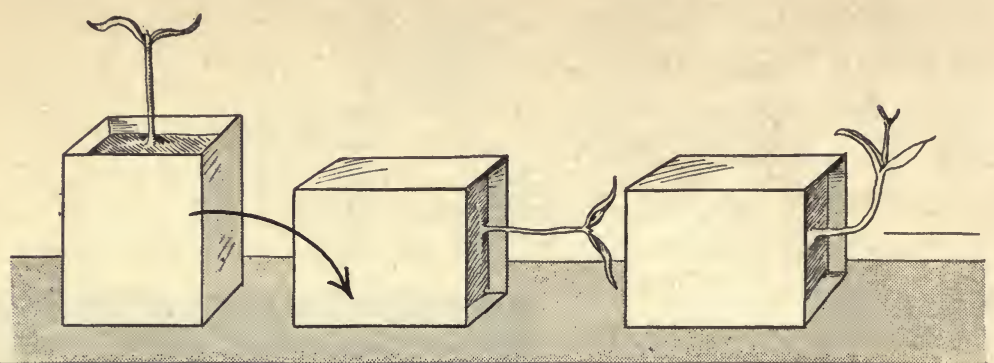

When the position of the growing plant is changed, gravity again determines the direction of the growth of the root and of the stem.

by the spinal or vertebral column and moist lining membranes similar to those in the skull. The vertebral column in an adult, made up of twenty-six movable bones called vertebrae, permits flexibility, and gives protection to the delicate spinal cord inclosed. From both the brain and spinal cord, nerves extend to all parts of the body. These nerves are embedded in other tissues which afford them protection.

The structure of the brain. The cerebrum. The larger part of the brain is called the cerebrum. It is divided into two hemispheres. The outer surface, the cortex, is composed of gray matter

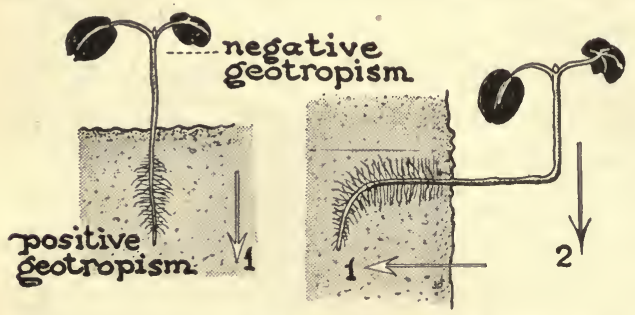

This plant responds to gravity: stems, negatively; roots, positively.

(cell bodies and synapses), and shows many irregular convolutions. Underlying the cortex are found nerve fibers. These are axons of the neurons. They make up the white matter.

Certain definite areas of the cerebrum are concerned with definite functions. These include motor areas which control movements, 
and sensory areas which are concerned with sensations. Centers of sensation, motor activities, speech, judgment, reasoning, memory, and many other activities requiring thought are located in the cerebrum. Association units link these together and make possible all kinds of connections. Later, in considering the activity of the nervous system, the cerebrum will be called the third level.

The cerebellum. Below the cerebrum and partially covered by it lies a smaller portion of the brain, the cerebellum. This is the center of muscular coördination and maintenance of body equilibrium. It may be called the second level of the central nervous system.

The medulla oblongata. The brain is connected to the spinal cord by means of the medulla oblongata. This is really the enlarged beginning of the spinal cord but is considered a part of the brain. It is the crossing place for most of the impulses to and from the nerves of the brain. It contains the nerve centers which govern breathing, regulate circulation, and maintain normal tone of the muscles in the blood vessels. It controls certain acts such as sneezing, swallowing, vomiting, and blinking.

The spinal cord. The spinal cord, nearly
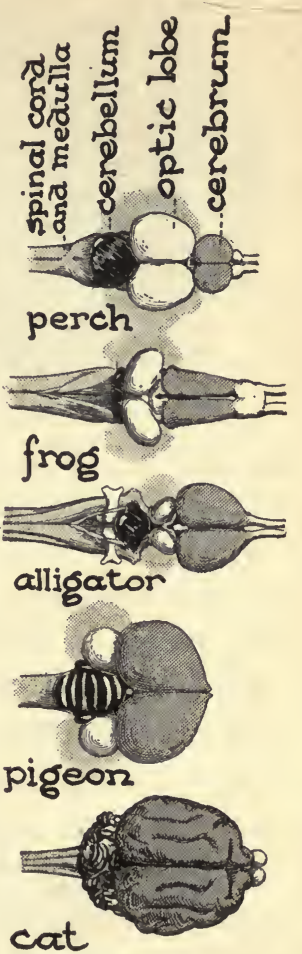

Note the relative size of the cerebrum in each of the brains. There is a close relation between size of the cerebrum and intelligence. The lower down in the animal scale, the smaller the cerebrum and the lower the intelligence.

cylindrical in form, runs through the hollow vertebral column. It is connected with the brain by the medulla oblongata. The spinal cord serves as a pathway for nervous impulses from various parts of the body to and from the brain, and has centers of simple 


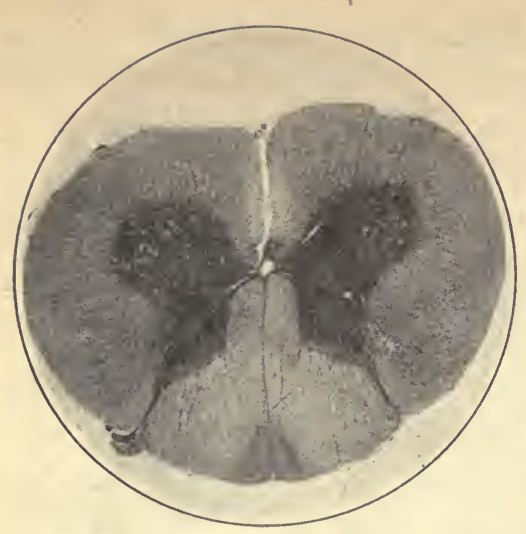

A photomicrograph of the spinal cord shows the white matter on the outside, and the gray matter in the form of anterior and posterior horns, on the inside.

nervous activities which will be discussed later. Nerve cells (gray matter) are found on the inside of the cord and nerve fibers (white matter) are found on the outside. The spinal cord is called the first level of the central nervous system.

Cellular structure of the central nervous system. The unit of structure of nervous tissue is a highly specialized cell called a neuron. It contains the cell body proper, the cyton, from which fine protoplasmic processes (dendrites) extend. One of the processes may extend a great length and is known as the axon. The dendrites divide and with branches from other' nerve cells form a mass of extremely fine fibers. The gray matter of the brain and spinal cord consists of nerve cells or neurons. Collections of these are nerve centers. A number of axons banded together constitute a nerve. Nerves transmit messages from sense organs such as the eye, to the cell bodies of the neurons; or from the cell bodies of the neurons to muscles or glands. In order

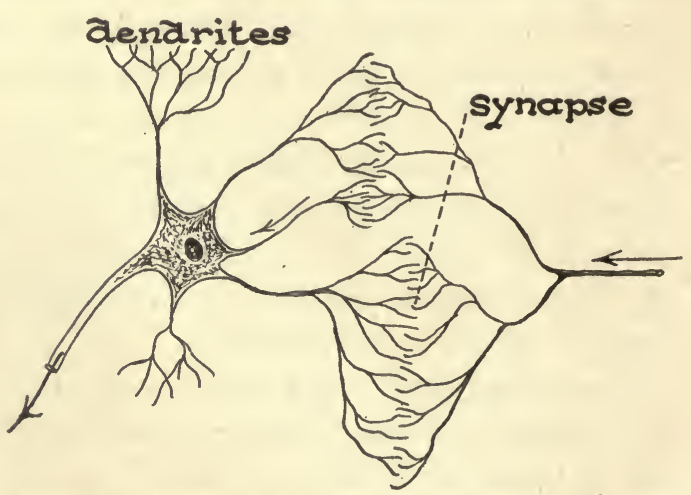

When the dendrites of two neurons come into contact with each other, they form synapses. Nerve impulses travel from axon to dendrite across one of the many junctions or synapses. 
that the nervous system may function as a whole, impulses or disturbance must readily pass from neuron to neuron. The point of junction between two neurons is commonly known as a synapse. The sense organs, known as receptors, receive the stimulus which starts the nerve current. The ends of the nerve fibers of these organs are on the outside (skin) of the body. The muscles or glands are called effectors because they bring about activity. An axon that connects with a sense organ is called an in-going, sensory, or afferent axon; one that connects with a muscle or gland is an outgoing motor, or efferent axon. The neurons possessing these axons may be either sensory or motor neurons. For example, when the finger is placed on a hot object and immediately withdrawn, a series of actions has taken

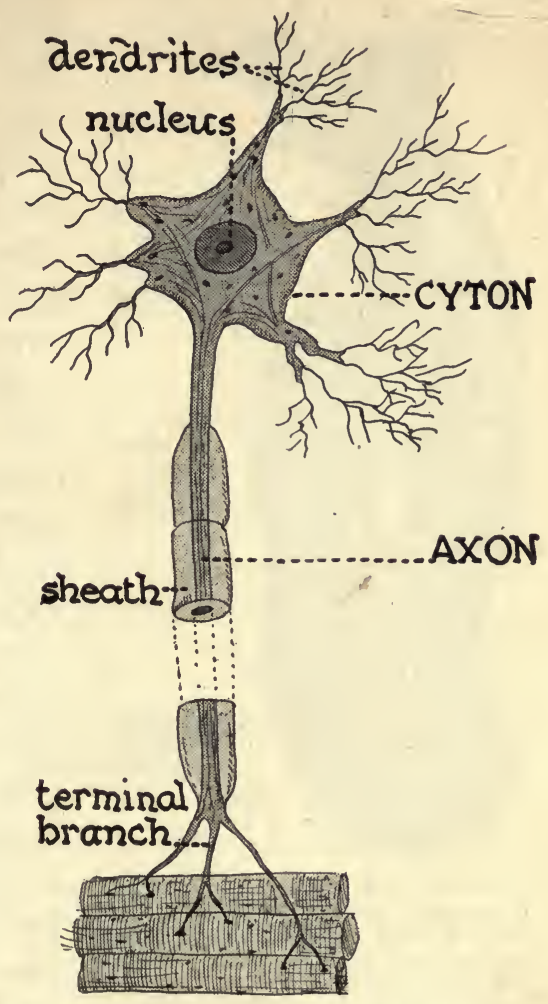

The unit of structure of the nervous system is the nerve cell or neuron. The control of all activities in the entire body is maintained by these cells. place in the nervous system. The nerve fiber in the finger received a stimulus which it carried to the brain. The brain in turn sent out a message to the muscles in the arm and hand, so that the finger was immediately removed from the object.

When neurons connect sensory with motor neurons, they are called associative neurons. Groups of nerve cells situated outside 
of the brain or spinal cord are known as ganglia. Neurons are linked together by means of irregular projections of protoplasm,

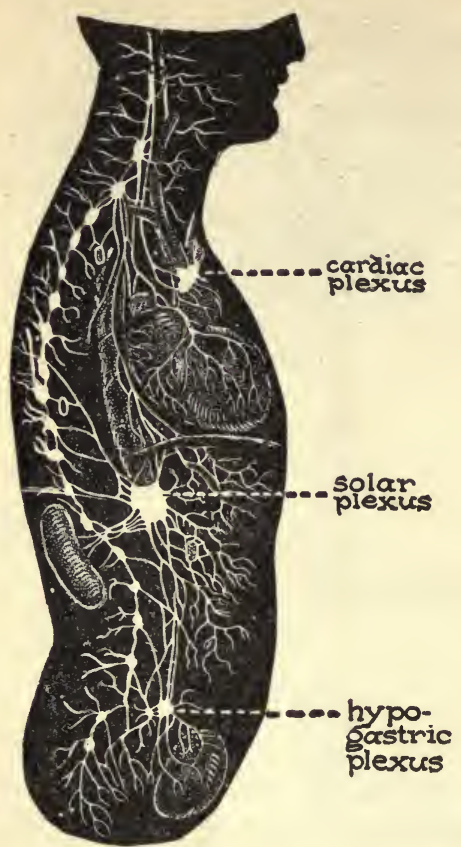

The autonomic nervous system consists of a series of ganglia, most of which are included in two chains that lie parallel to the spinal column. These are connected with nerve centers in the spinal cord.

A very large and unpaired ganglion is called a plexus. dendrites, establishing synapses. The nature of these contacts is not known. They may be compared with contacts made by two electrically charged wires. The type of energy establishing the contacts in a synapse is nervous energy.

\section{Autonomic nervous system.} This system is also called the sympathetic system or self-acting system. In front of the spinal cord, and lying parallel to it on either side of the vertebral column, are two rows of ganglia, connected with one another by nerve fibers. Certain large ganglia, called plexus, are also part of this autonomic system. Three of the plexus are the cardiac plexus in the thoracic cavity, the solar plexus in the abdominal cavity, and the hypogastric plexus in the pelvic cavity, but they connect with many other small ganglia in the thoracic and abdominal regions. The autonomic system transmits some of the disturbances of the central nervous system to the heart, glands, and involuntary or plain muscles. Since the viscera (internal organs) in general are made up of smooth muscle, it follows that the so-called automatic mechanisms of the body, such as, the beating of the heart, the contraction and dilation of the muscles, and 
the secretions of various glands, are largely under the control of the autonomic system. Emotions affect the autonomic system, and consequently circulatory and glandular disturbances follow or accompany emotions. If a plexus is injured, a violent reaction in certain internal organs would naturally follow.

Studies of the nervous system. Scientists have been able to show that there are many similarities in

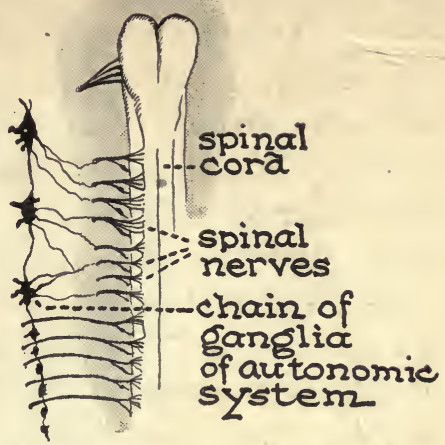

The ganglia of the autonomic nervous system are connected with the central nervous system by nerve fibers. the nervous systems of man and other vertebrates. Probably one of the outstanding differences is the fact that man's cerebrum is generally larger and heavier, proportionately, than that part 'of the brain of any lower animal. The lower down the

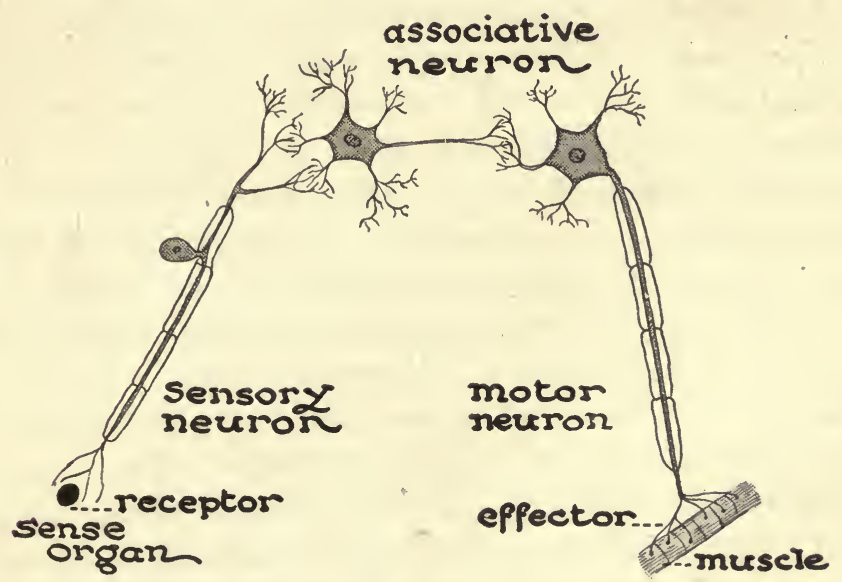

There are three types of neurons. Some take sensory stimulations into the body, others take motor or glandular stimulations out to muscles or glands to bring about a response. A third type, known as the associative neuron, may lie between the other two types of neurons and set up connections between them. 
animal is in the scale of classification the smaller is the cerebrum. The convolutions or creases in man's brain are more intricate and deeper than those found in lower animals. 'These convolutions give greater brain surface and contain more functioning neurons. Scientists once thought that one man was more intelligent than another because one had a heavier brain than the other. But this does not seem to hold true. The brain of Cuvier, a great scientist, weighed about four pounds, while that of Gambetta, a French statesman, weighed only two and a half pounds. Since mental defectives have been found with brains weighing more than four pounds, weight alone does not mean everything. The size and weight of a person must be taken into account when considering weight of brains.

Neurons never increase in number. The only growth possible is the setting up of connections or synapses among the different neurons. The more connections or synapses that are made, the greater will be the number of mental processes, which is a factor in determining the intelligence of animals. The ability to make connections easily seems to be an inherited character. This may account for the fact that some families have members more intelligent than those of other families.

One of the methods of studying the nervous system is through experimentation with lower animals. Flourens and others have observed that if the cerebrum of a pigeon is removed, the pigeon loses all voluntary or conscious action. It will not move toward food nor away from danger. However, if food is put into its mouth, it will swallow. When the cerebellum alone is removed, balance and coördination become disturbed. The pigeon, if placed on the edge of a table, will fall. It has difficulty flying because of a lack of balance and muscular coördination. Its voluntary activities, however, are intact. It experiences desire for food, fear of danger, and other sensations. If the medulla is removed, respiratory, circulatory, and heart actions cease and the bird dies. 
A second method of investigation is by clinical observations and examination, and autopsies. Doctors observe and examine the

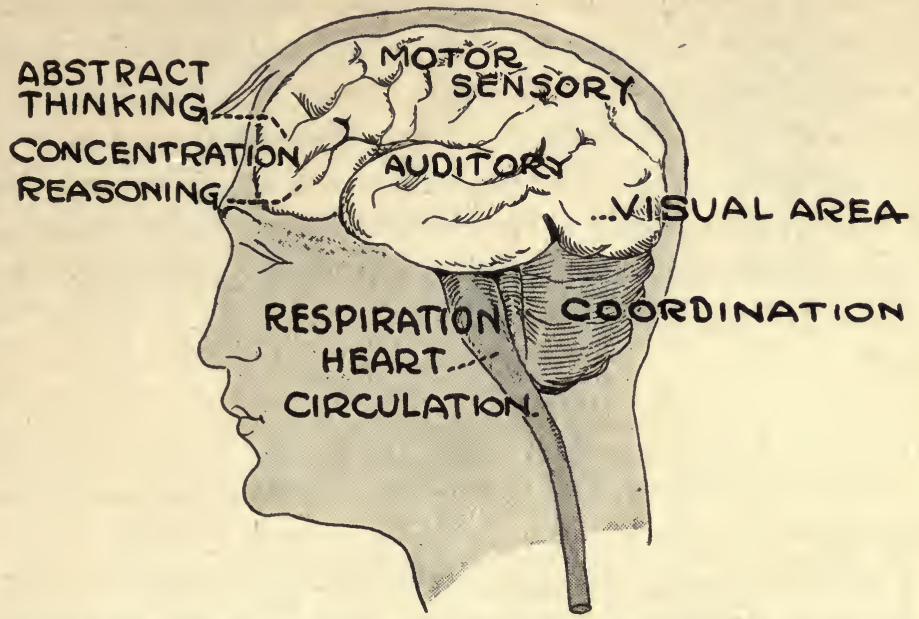

According to clinical investigation and animal experimentation, the control of certain physical functions is localized in definite areas of the brain. There is considerable investigation at present as to whether there is general control of these functions as well as definite control. The localization of intelligence, including thinking, association, and memory, is still open to discussion.

symptoms of people that show nervous disorders, and in many cases are able to determine the cause of the trouble. When such patients die and autopsies are performed on the bodies, the doctors can, in some cases, link up the nervous disorder with the part of the nervous system showing disease. For example, if a person had been paralyzed on the left side of the body, the autopsy will show whether an obstruction, probably in the form of a blood clot, is on or near the part of the brain controlling muscular movements. If a person were blind, the autopsy may show injury to the area of the brain controlling vision.

Certain scientists, through experimentation and study of normal and of diseased brains, have localized certain areas in the brain. For a time people carried the idea of localization to an extreme. 
They thought that a person's aptitudes and traits of character could be told from the swellings over these various areas. If one man had a swelling over the vision area, they assumed that he could see better than another. If he had a high forehead, they reasoned that his powers of judgment must be better. Thus the pseudo-science of phrenology arose. Phrenology, as such, has been disproved. Careful investigations have shown that the brain does not conform exactly to the shape of the skull and that bumps or enlargements on different heads are usually malformations of the skull and not brain enlargements.

\section{Questions ANd Suggestions}

1. What is a stimulus? What is a response?

2. Discuss an experiment illustrating phototropism; geotropism; hydrotropism.

3. What is the value of tropisms to the plant?

4. State the difference between a tropism and a nervous reaction.

5. Name a function of the central nervous system.

6. Discuss the protection of the brain and spinal cord.

7. Describe the structure and the function of the cerebrum.

8. Discuss the function of the cerebellum; the medulla oblongata ; the spinal cord.

9. Describe in detail the unit of structure of the nervous system.

10. Draw and label a neuron. Name and define three types of neurons.

11. What are nerve centers and ganglia?

12. Compare the brain of man with the brain of lower animals.

13. Discuss two ways of studying the nervous system.

14. Describe the structure and function of the autonomic nervous system.

\section{SuPPlementary REAdings}

Gates, A. I., Elementary Psychology (The Macmillan Co.).

McDougall, W., Outline of Psychology (Charles Scribner's Sons). 


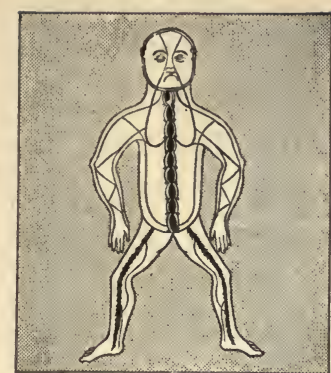

Ancient idea of the human nervous system.
CHAPTER XXIII

\section{NERVOUS REACTIONS}

What mental activities are learned and what are instinctive? What is a habit? What is the best method for learning facts?

When tropisms were discussed, the importance of stimuli was stressed. As in plants and lower animals, it is a stimulus in a higher animal that starts the nervous impulse which results in a typical reaction. This impulse may be any one of three types of mental activities: (1) inborn automatic, (2) acquired automatic, and (3) voluntary.

Reflex activities. There are certain motions and acts that a baby performs soon after it is born. These responses are simple reflexes or inborn automatic activities. The child does not have to learn or acquire them. Some of these responses may involve the brain, while others are controlled only by the spinal cord. The child comes into the world equipped with pre-formed connections or tendencies to connections in his nervous system. In other words, nervous pathways or patterns are already set up. A new-born baby that has not learned to think will pull his foot away if pricked with a pin. He will sneeze if his nostrils are tickled with a feather, and will grasp a finger if it is placed on the palm of his tiny hand. Each of these responses to the given stimulus is a reflex. If a person swings one leg freely over the other and taps it just 
below the knee cap, the tap will cause the knee to jerk. In this experiment, the stimulus is received by a nerve ending in the skin. The stimulus starts an impulse which travels along a sensory neuron into the spinal cord. There, this sensory neuron links up with an outgoing motor neuron by means of a synapse. The outgoing neuron conveys the impulse to a muscle in the leg, which causes the knee to jerk. In some way, the in-going sensory stimulus is changed into an outgoing muscular reaction. The sense organs, in this case " touch spots" in the skin, receive the stimulus and are the receptors. The muscle effects the reaction and is the effector. The pathway of the receptor, composed of the afferent axon, afferent cyton, synapse, efferent cyton, efferent axon, and the effector make up the reflex arc. Any reflex arc involving the central nervous mechanism begins and ends in the outer part of the body.

Types of responses. The simplest response activities involve at least two neurons. Experiments have been made with frogs whose brains have been severed from their spinal cords. Such frogs lose all conscious activities, but are still capable of making certain reflexes. If the toe is pinched, the leg is withdrawn; violent pinching causes a distinct jump. If a paper wet with dilute acetic acid is placed on the skin of the leg, the frog makes movements to brush off the paper.

If the foot of a sleeping baby is tickled, the foot is withdrawn. This is a reaction or reflex of the first level. The impulse, caused by the stimulus, passes into the spinal cord and out again without involving any other connections. The knee jerk is another example of a reflex of the first level. But, if the blow on the knee is severe enough, the person may gasp or scream or balance himself to preserve his equilibrium. He may even show an increased heart beat. The centers of respiration and circulation are stimulated, and balance and coördination are brought into the action. This response is of the second level. A number of neurons enter into this activity. Some of them are sensory and a great many 
are motor, which result in a number of reactions. Reactions of the second level are more complex than those of the first level and will involve parts of the body somewhat distant from the point of stimulation.

When the knee jerk arouses thought or deliberation, it is classified as a third level response. For example, if the blow is so severe that one would rub the in-

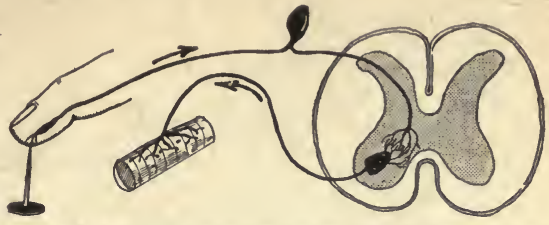

A reflex arc is traversed when the finger touches a nail. The sense organ in the skin is stimulated, the stimulus is carried over the afferent or sensory neuron through a synapse to a motor neuron. This ends in a muscle which contracts and causes the finger to be pulled away.

jured spot or examine it deliberately, certain neural connections would be made in the brain. Such consequent thoughtful activities attend the reflex so closely that they are sometimes considered a part of the reflex. Such reaction is called an activity of the third level.

Conditioned reflex. A baby is born with the pathways for a certain number of reflexes already established. When certain sense organs are stimulated, the impulses travel along these pathways until they reach muscles or other mechanisms, which carry on the processes essential for maintaining life; such as, sucking, digesting of food, crying, coughing, and moving. When these responses are caused by other than the original stimuli, they are said to be modified or conditioned. For example, Pavlov, a Russian physiologist, observed that the secretion of saliva in a dog is a reflex act, resulting from nerve pathways established in the dog at birth. The appearance of food or the taste of food in the mouth acts as the stimulus, and the flow of saliva is the response. If the same person always feeds the dog, the saliva of the dog will, after a certain number of times, flow at the sight of that person even though no food is given. The stimulus in this case is the sight of the person, not the food, and the response is the flow of the saliva. The response occurs in the hungry animal even when it can neither see WH. FITZ. AD. BIO. -15 
nor smell any food. The animal has evidently acquired a new reflex path. The acquired reaction is conditioned upon a memory

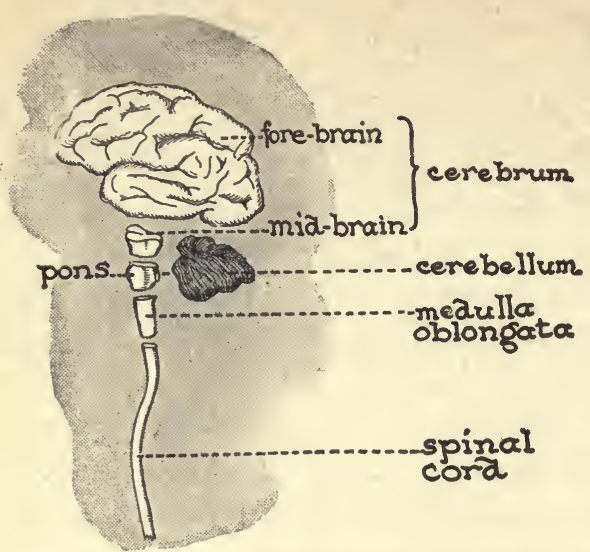

The three levels of the nervous system are shown here. The first level is in the spinal cord, the second level is in the cerebellum, and the third level is in the cerebrum. association which connects the presence of a certain person with the eating of food.

In another experiment, a bell was rung, and exactly two minutes later food was given a dog. Ultimately, after the experiment had been continued a long time, the saliva flowed in the dog's mouth exactly two minutes after the ringing of the bell even though no food was given. This could be carefully measured because of a little tube that was injected into the duct leading from the salivary gland of the dog. Saliva could be seen flowing from the duct exactly two minutes after the ringing of the bell. Sparrows usually build nests in the gutters and holes in barns and houses. Building the nest is inborn, but buildings have not existed as long as nest-building, so nesting in houses must have become conditioned by buildings. Conditioned responses depend upon associations formed in the cerebrum. Injury to a certain part of the cerebrum of a dog with a con-

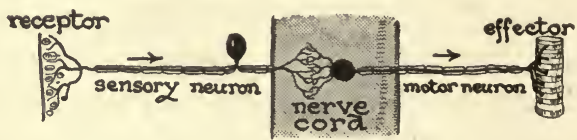

In a reflex of the first level, the center of control is the spinal cord. This is the simplest type of reflex. The response is usually very simple.

ditioned response has caused the ability of the animal to respond to the stimulus to be entirely destroyed.

The original reflexes that are a part of the organism at birth are 
evidently not fixed and invariable but are flexible and modifiable and may become changed or conditioned by factors in the environment. The organism begins to relate factors in the environment to his activity. This results in the changing of the original reflex. Certain acts may be consciously inhibited, that is, diverted or blocked. For example, a child instinctively makes known his wants by crying. He has learned that when he cries, he has always been picked up. One day, he finds that crying does not bring the desired attention. He

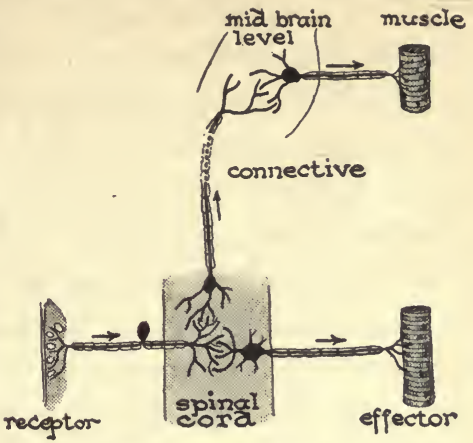

In response of the second level, centers in the spinal cord and mid-brain exercise control. A number of activities will result. stops after a while, and, in time, learns to express his wants in another way. The changing is due to consciousness. It probably explains the beginning of the learning process or conscious acts.

Voluntary activities. Any act involving will or thought is a voluntary act. The name "voluntary" refers to the will. All activities, excepting the so-called inherited reactions, performed for the first time are voluntary acts of the will. When the activity requires attention, memory, judgment, or association, a great many associative neurons are used. For example, the hand is put in water and is held there while a decision is made as to whether the water is of the desired warmth. Then the hand is withdrawn. The sensation is received by the skin as the receptor. The impulse travels along an afferent axon to the spinal cord, up to the brain. A number of neurons located in the brain are stimulated, bringing about a condition of consciousness, and resulting in attention. Meaning becomes attached to the sensation that has been received and a mental decision is possible. A comparison may be made with previous water used in washing; the thought may 
occur that injury will result in case it is too hot; a consideration of the fact that cold water will not cleanse may be involved. Finally a decision is made. Then a connection is made with a motor neuron. The impulse goes down the efferent axon to the muscles in the hand and the arm, which are the effectors of the activity and the hand is withdrawn from the water. In many instances, the learning of facts such as rules of grammar is impossible without attention. Attention is partly dependent upon inborn tendencies and partly upon acquired habits. The more closely the activity is related to the child's life and the more associations he can make, the easier it will be for him to remember the facts. For instance, if a child lives in a city, it will be easier for him to understand the problems of city government and traffic conditions, than for the child who has always lived in a rural

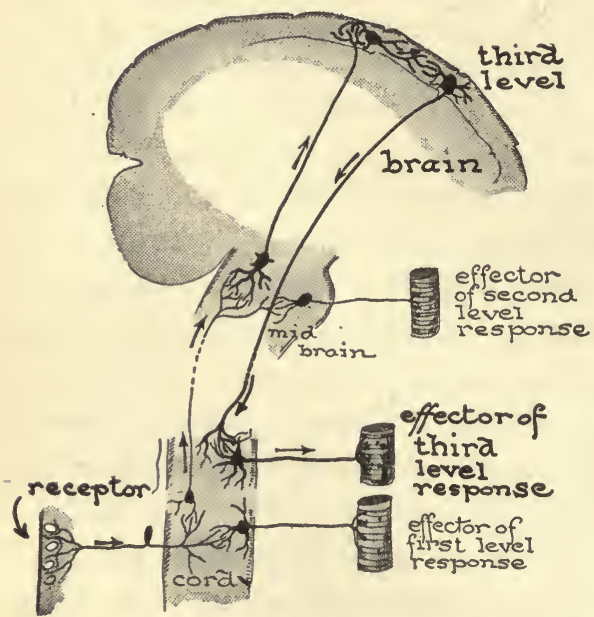

A third-level response uses neural connections in the brain. Psychologists think that all learned reactions are dependent upon connection established in the brain level. was the gums of the mouth. When the toothbrush was placed in the mouth, a sensory axon took the impulse into a sensory community.

Acquired automatic activities. Activities which are learned in a person's lifetime, but have become automatic" through repetition, are acquired automatic activities. For example, brushing the teeth is an act that had to be learned. By directing attention and thought on the action the first few times the teeth were brushed, definite technique and skill were soon gained. The receptor 
neuron of the cerebrum. As the child thinks whether he shall brush the teeth up and down or across, whether his mouth should be open or closed, whether he should lean over the basin or not; connections are set up among association neurons. Finally, he comes to a decision. An impulse is sent along an efferent axon down the spinal cord and into the muscles of the arm which are the effectors. The actual brushing of the teeth is the motor response. The correct response was consciously made. This is an act of the third level involving the cerebrum. After the teeth have been brushed a number of times, the decisions and the associations are no longer necessary. While the act was conscious, inhibitions, in the nervous system blocked certain paths of conduction between the sensory stimulus and the motor response. As the act was repeated, time after time, these inhibitions disappeared, and the act was easier to perform. The more times the impulse passed over the pathway of discharge, the less was the resistance to it. After this reaction was learned a synapse was set up between the afferent and efferent pathways, leaving out some of the association neurons. In some habits the pathway is essentially the same, but consciousness is omitted. It has become automatic and has descended to the second or possibly the first level. It is now an acquired automatic activity involving no thought and is commonly called a habit. Habits are frequently defined as acts which were first done in a typically voluntary way, but after sufficient repetition they are done in a comparatively reflex way. Memory is most important in changing conscious acts into habits.

Importance of the autonomic system in nerve activities. The internal organs, in general, are under the control of the autonomic nervous system. The autonomic system controls the contractions and relaxations of the smooth muscles. It regulates glandular activities and heart action. Emotions activate the autonomic system. If an emotion such as fear or anger accompanies a reflex act, habit, or a conscious act, the autonomic system enters into the 
activity. There will then be attending respiratory, circulatory, and glandular activities. For example, a rabbit sees a cat and tries to escape. The sensory stimulus is the sight of the cat while the reaction is the motor act of escaping. Because fear attends this voluntary act, the autonomic centers of the rabbit are stimulated and their resulting activities favor the response of outwitting the cat. The bronchial tubes of the frightened rabbit are relaxed and rapid breathing is made easier. The contraction of blood vessels in the viscera and the increased heart action forces more blood into the skeletal muscles. Thus, extra oxygen and fuel are supplied to the muscles. The nerves stimulate the adrenal glands which pour out their secretion into the blood, causing the liver to give up more sugar into the blood, as well as increasing the endurance and strength of the muscles and reducing the activities of digestion. The rabbit has more energy than normally and is able to run faster from the cat which is activated purely by the instinct of hunting. But, if the cat is aroused by a strong feeling of hunger, we would find in her activities very similar to those in the rabbit. In this case, the probability of escape by the rabbit would be lessened.

The nature of the nerve impulse. The nature of the nerve impulse is not fully understood. The speed with which it travels along a motor nerve fiber is about three hundred and ninety feet per second. The time elapsing between the application of a stimulus and the response varies in different individuals and in the same individual under different conditions. It depends upon the strength of the stimulus. If the stimulus is very strong the response will be prompt. If the stimulus is weak the response is not made or is made very slowly. The time of reacting is dependent upon the nature of the stimulus. For example, the response to a person walking toward you is quite different from the response given when an automobile comes toward you. The time is also affected by the number of synapses through which the 
stimulus has to pass. If the nervous pathway is long, a proportional length of time is required for a response. Removing your hand from a hot stove requires less time than deciding which way to jump when you find yourself in the path of a fast moving car.

The importance of reactions. The patterns of behavior formed almost immediately after birth are called instinctive or innate acts. These include the avoiding reactions such as struggling when held, and withdrawing from or rejecting anything that is causing discomfort, as moving the leg if it is being pinched; and the approaching reactions or movements caused by hunger and by the stimulation of certain sensitive parts of the body, such as, tickling the bottom of the feet or rubbing the back. These reactions or established patterns are frequently modified to meet changing conditions. For instance, a small child will usually push away or strike at a person who annoys him, but later he will modify his tendency because the group he lives in demands a different method of reaction. He will learn to respond to reason and not follow his instinctive desire to fight.

Habit formation. When voluntary activities are made habitual, they are performed more easily and quickly. When completely established, they act the same as instincts. When activities become habits the brain is not needed and it is then set free to make new responses or activities. This results in the growth and development of the mind or consciousness. If the attention were concentrated on the daily performance of brushing the teeth, dressing, walking, and other necessary activities, the mind would be occupied continuously on acts that are necessary for mere existence. The possession of useful habits sets the mind free to attend to the gaining of new knowledge. Individual progress may be said to be dependent upon the ability to acquire habits.

There are three rules for making conscious activities, such as combing the hair, or starting an automobile, habitual. First, there must be concentration on the performance of the act. 
When there is a real desire to build the habit, it is more easily formed. Second, the activity must be repeated a number of times under exactly the same conditions, permitting no exceptions to the performance. Third, the act becomes automatic more quickly if feelings of satisfaction attend the performance. If any annoyance accompanies the performance, it delays the forming of the habit. If exceptions are made in the type of reaction, judgment again enters into the performance and it continues as a thoughtful act rather than a habitual one. In performing the act each time in exactly the same way, the same nerve path is traversed. Then when the stimulus is received, the impulse goes more quickly over the pathway, and synapses connect up with greater facility.

In order to break a bad habit, there must first be a sincere desire to get rid of it. The activity must be brought back to consciousness so that the will may be directed on breaking it. For example, in order to break the habit of biting the nails, red pepper may be put under the nails. The sharp biting effect on the tongue will bring to consciousness the fact that the nail is in the mouth. Each time the person realizes that the nail is in the mouth, he must take it away from the teeth and there must be no exception to this reaction. The person who says he cannot break himself of a bad habit means he does not want to break it. If the growth of the nail and the improved appearance of the hand brings satisfaction, the habit of refraining from biting the nails will be more speedily established. The most effective way of breaking a bad habit is to substitute a good habit which will be more satisfying than the bad habit. For example, if a boy has formed the habit of standing on a street corner in the evening, he may find that joining an athletic club will be so satisfying to him, because of its activities and congenial companions, that in a short time his old habit has lost its influence altogether. It is not wise to ever perform an idle or vicious voluntary act, for if a synapse has once been established between two neurons in performing an act, it is easier 
for the nerve impulse to go over this pathway a second time. There is always the danger of an undesirable voluntary act becoming habitual. The years of childhood are the critical ones in habit formation and, therefore, in character building; childhood habits form the basis for later conduct. If the acquired habits are later found to be undesirable, it is necessary to make substitutions and this is a waste of mental energy. Consequently, it is of the utmost importance to build correct habits by the proper conditioning of reflexes in the beginning.

To a large extent, mental growth results from acquiring a large number of useful voluntary activities. Probably every one has the same number of neurons. There is an infinite number of possible connections among them. The same neuron may link up with several others and this may result in a great many different activities. As each new act is performed, a new combination of neurons is connected by means of synapses. The more synapses are made, the greater will be the growth in experience, judgment, memory, and reasoning. If any voluntary act which is useful can be relegated to the realm of habit, it will give greater opportunity for acquisition of new voluntary acts.

\section{Problem. The study of nervous activities.}

List twenty simple activities performed by you in one day. Next to each activity put the class in which it belongs : inborn automatic, acquired automatic, or voluntary.

I. Which of the voluntary activities listed would be desirable as habitual ones? Why?

$A$. What prevents them from becoming habits?

B. How may they be changed into habits?

II. How can you improve the performance of any act in your life?

\section{Questions ANd Suggestions}

1. What is a reflex arc? Make a labeled diagram of the reflex arc.

2. Name three types of nervous activities.

3. What is the value of reflexes to the organism? 
4. To which activity is a tropism comparable?

5. Draw and label a diagram of a reflex activity.

6. Explain and give an example of the conditioned reflex. What scientist has been investigating it?

7. How could you make learning to swim a habit?

8. Make a labeled diagram of an acquired automatic activity.

9. Explain how the habit of biting the nails may be broken through (a) inhibition, (b) substitution. Explain which method is the more desirable method.

10. Why is it possible for people to break themselves of bad habits, if they have an earnest desire to do so?

11. Discuss the advantages and disadvantages of adopting a child of six months; of five years; and of fourteen years of age.

12. Give examples, from your own experience, of habit formation, and discuss the importance of attitude as an aid and as an interference.

13. What is the value of a habit of regularity? Of concentration? Of thoroughness?

14. What is the relation between habit formation and vocational success?

15. What is the importance of voluntary activities?

16. What is the function of the autonomic system?

17. Discuss the value of basing education on what a child wants to know rather than on what an adult thinks he ought to know? Should a child study only those things he wants to know?

18. What is the relation of interest and attention to understanding?

\section{Supplementary Reading}

See Chapter xxii. 


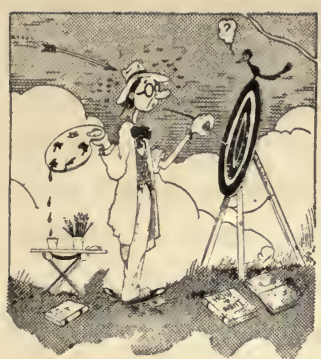

Some like to paint -

\section{CHAPTER XXIV}

MENTAL

HYGIENE

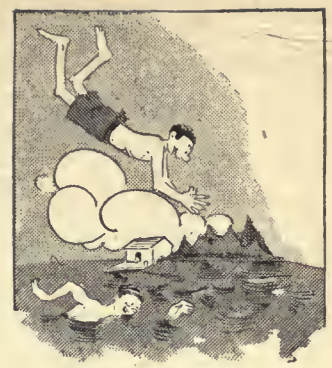

Others enjoy a swim.

What relation have psychologists found between intelligence and success? How can the nervous system be kept in good condition? What may be the effects of worry, fear, temper, and introspection?

The ability to meet and solve the problems of one's life without needless worry, leads to serenity, contentment, and happiness. The solution of problems brings a joy of achievement that is conducive to greater success. The boy whose problems are decided for him does not find the contentment nor achieve the poise that is attained by the boy who faces and solves his own problems. Poise is usually considered one of the constituents of. success in the varied and complex life of the present day.

Intelligence. Intelligence may be defined as the native capacities of a person to learn, to reason, to exercise mental control, and to solve his life's problems. Can a child be apparently dull or backward for a number of years and suddenly become highly intelligent? Scientific experimentation seems to prove that certain mental capacities are native or inborn. If a child of six years of age is found, experimentally, to be of average intelligence, when measured by certain tests, he will, with very rare exceptions, be found to have average intelligence at seven years; eight years, and, in fact, all through his life. If a child of six years tests below average, the chances are that he will remain at that level all through life. 
The scientific measurement of intelligence, most frequently used as individual tests, is a series of performance tests arranged in the

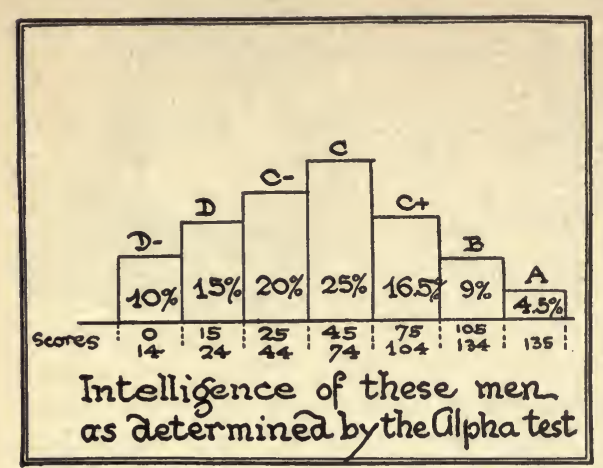

The Army Alpha test was given to $1,700,000$ men in the United States army in the World War. It consisted of 212 questions. Grade A was given to those answering correctly $\mathbf{1 3 5}$ or more of the $\mathbf{2 1 2}$ questions; grade B for 105 to 134 correct answers. A was earned by only $\mathbf{4 . 5} \%$ of the men. Compare the results attained by the other groups of men as shown in the table. order of difficulty. 'The first of such tests was worked out by two French psychologists, Alfred Binet and Theodore Simon. These tests have been, revised by different persons in America, and among these revisions-is one by Terman, known as The Stanford Revision of the Binet-Simon Scale. This scale includes tests of memory, language comprehension, size of vocabulary, knowledge of familiar things, judgment, and many other mental tasks that are a part of every child's experience. Standards, to show what children of certain ages should know, have been established by comparisons of the results or ratings made by children of definite ages in all sections of the country. For instance, there are a certain number of questions that a six-year-old child is supposed to answer correctly. If a particular child of six answers less than this score, he is said to have a mental age, M.A., of four or five, or whatever age that score is supposed to measure. If he answers more than the required number, he may have a mental age of seven, eight, or even more. Thus there are tests ranging in difficulty from those for a three-year-old child to those given to an adult. For the practical purpose of measuring progress in school the intelligence quotient, I.Q., is used. This is obtained by dividing a child's mental age by his age in years. This quotient will usually remain fairly constant from year to year, for his men- 
tal age will grow or increase as he becomes older. The average intelligence quotient for any given age is 100 . For example, a pupil has a mental age of six, according to the Stanford Revision Scale, and his age in years is six. Divide his M.A. 6 by his real age 6 and the intelligence quotient will be 1.00 . (This I.Q. is usually expressed without a decimal, as 100:) Another pupil of ' 6 has a mental age of five and a half and his real age is six. Dividing his mental age, five and a half, by his age in years, six, we find that he has an intelligence quotient of 92 . If a pupil's mental age is eight and his age in years (chronological age) is six, his intelligence quotient is 133 . The I.Q. of the average high school graduate probably ranges from 90 to 105 .

Relation of intelligence to progress in school. In general, pupils who have low intelligence quotients have difficulty in making progress through school. When pupils with high intelligence quotients have difficulties with their studies, it is usually due to physical defects, irregular attendance, late entrance, refusal to study, or some other remediable condition. If a child is retarded in school, even though he puts forth sincere efforts, and there is no remediable condition interfering with his school work, then he should be given a different type of schooling. Various voca-

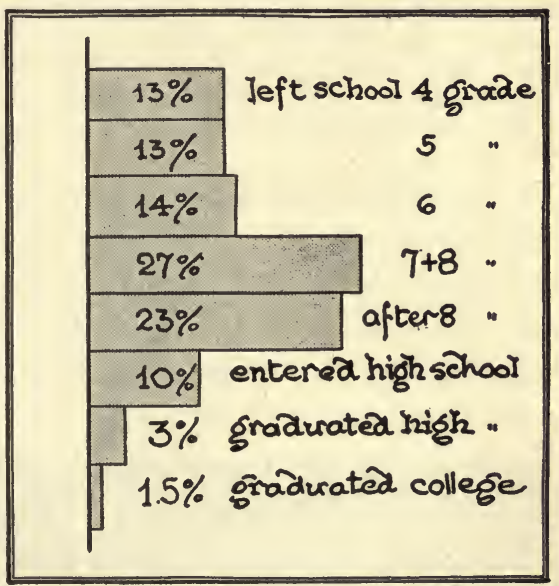

The schooling of the American population has been graphically described in the above table. This is based on a report of the Department of Labor. Compare the distribution of schooling in this table with the distribution of intelligence in the preceding table.

tional schools are endeavoring to provide courses for such children, so that they may take subjects which they can understand and in 


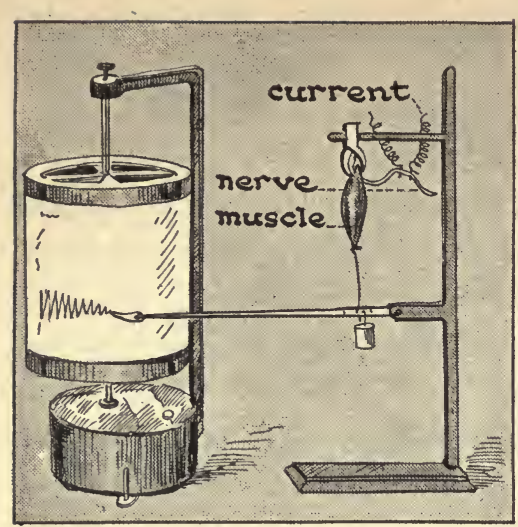

The leg muscle of a freshly killed frog is attached to a lever. It is stimulated through the nerve by means of a make and break electric current. A tracing of the contractions is made on a revolving drum. At first the contraction is very decided and regular. Slowly the response decreases until the muscle ceases to react. Rest or washing the muscle will start the response anew, but in a short time fatigue again is apparent.

which they can achieve success. If a child is permitted to fail term after term, he falls into an attitude of mind which destroys his confidence and he makes only a half-hearted effort to succeed. He gets the habit of failing. The pupils who reach high school are, to a certain extent, a selected group, because the dullest pupils have become discouraged and dropped out. Education results in the growth of experience or mental age, but not native ability.

Relation of intelligence to vocations. Individuals who rate low in the intelligence tests are not, necessarily, undesirable members of society. There are relatively few children so dull that they cannot succeed in some line of work. Many failures and much discontent are due to the fact that boys and girls sometimes enter vocations that require too much or too little intelligence in relation to their mentality.

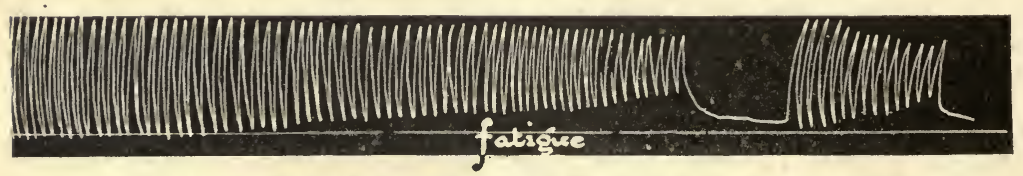

Record made on a revolving drum by a stimulated frog's muscle.

In an experiment conducted by J. K. Flanders and reported by Terman, the intelligence of a group of employees of an express company was tested. These people were employed to do work that required about the same level of mental ability. It was 
found that their intelligence, that is, their I.Q., ranged from sixtytwo to one hundred and four. This work could have been done efficiently by persons with scores of ninety. C. W. Waugh gave intelligence tests to eighty-two street-car motormen and conductors. The investigation showed a range of intelligence from sixty-five to one hundred and ten. A score of eighty to ninety was probably sufficient for a person to do this type of work well. Those with higher intelligence did not do more work nor do it more efficiently than those of lower intelligence. One of the men scoring low had a serious accident on his car. It is a fairly well-established fact that a motorman or conductor with an I.Q. of less than seventy-five is, as a rule, an unsafe risk. There is a big economic loss, to the company as well as the individual, in employing men of high intelligence to do work that could be done as well by men with less intelligence. Educators should direct those students of highest I.Q. ratings into lines of work which will require superior intelligence, and those of mediocre intelligence, low I.Q., into lines of work for which they are fitted. At present, there is too great a waste of mental ability by men and women filling positions that could be competently filled by persons of less ability. Students studying professional subjects should be those of fairly high intelligence rather than merely those with money enough to pay for such training.

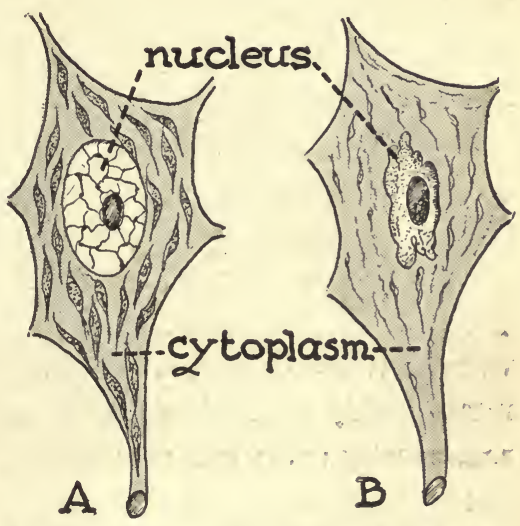

A tired nerve cell (B) shows fewer chromatic granules than a rested nerve cell (A).

A healthy mind. The nervous system, like the other systems of the body, is kept in the best possible condition if the body is 


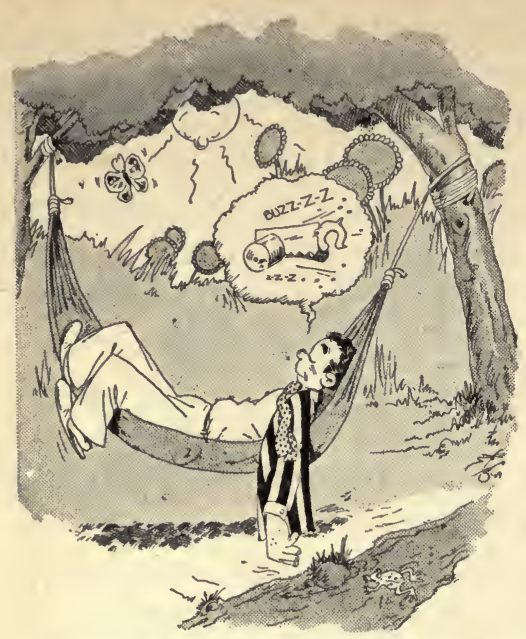

Idleness does not bring satisfaction for any great length of time. It is, frequently, the result of bad habits and usually, if not always, leads to inefficiency.

him indoors. People with hobbies are usually able to make use of their leisure time in a way that is enjoyable as well as beneficial to them.

Fatigue. Prolonged or continued contractions of muscles in any kind of work result in fatigue. This condition is almost always followed by a steady decrease in efficiency. The feeling of fatigue is very complex and is often associated with such mental states as lack of interest, lack of will power, and distaste for the work. Work done under compulsion usually results in fatigue more readily than when interest is a part of the work. This is one reason why every one should do the kind of work which he really likes.

Experiments have shown that if some blood of a fatigued animal is injected into a rested one, signs of fatigue are promptly produced in the second animal. Fatigue is probably due to an accumulation of waste substances in the cells and in the blood, resulting from 
the oxidation process. If these accumulate faster than the organs of excretion can eliminate them, they are likely to act as poisons. At the same time, the food material in the cell becomes exhausted. Because all bodily activities are slowed up during sleep, the sleeping organism has a chance to eliminate the accumulated wastes. The repair of all tissues goes on during sleep. It is the muscle cells and brain cells, particularly, that become fatigued. In monotonous work the same neurons are constantly being used. This work should be counterbalanced with some kind of recreation. Rest and play balance mental work because different pathways are traveled. Fatigued synapses offer resistance to impulses. This naturally results in inefficiency or lack of activity. A high school boy or girl should sleep, approximately, nine hours a night in order to efficiently restore fatigued nerves and muscles.

Mental poise. Mental hygiene has for its object the promotion of mental poise and serenity, and the prevention of mental disorders. Nervous instability is shown by a predisposition to strong emotions that are easily aroused and are only controlled with difficulty. Worthless nervous activities should be eliminated as far as possible by cultivating proper attitudes. Worry and fear stimulate certain parts of the autonomic nervous system and may have injurious physiological effects. Strong emotions tend to stimulate the adrenal glands, causing the withdrawal of blood from the viscera. This may result in serious digestive

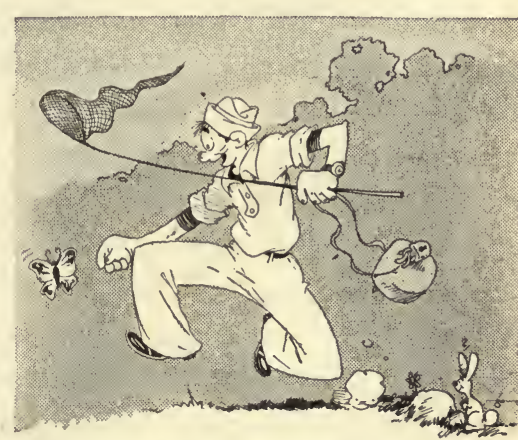

A hobby gives one a satisfying way of spending leisure time. The more interesting and novel is the hobby the greater will be the resulting satisfaction.

disorders. Worries exercise neurons with no worthwhile gain. Frequently, the worry is over an act that is beyond control. For WH. FITZ. AD. BIO. -16 
example, people worry whether or not it will rain; whether or not they will be struck by lightning; whether or not they will be hit
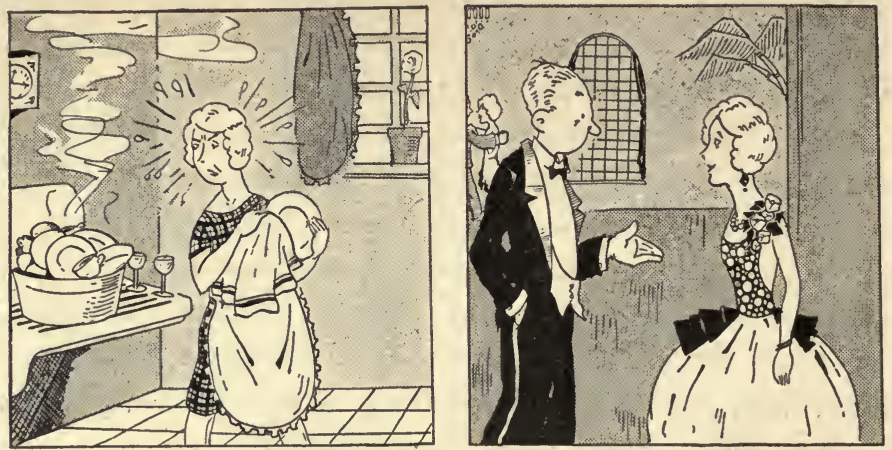

The frame of mind with which one enters into an activity directly affects the results. Cheerfulness towards duty is a desirable attitude to cultivate as it tends to bring about greater efficiency and mental poise.

by an automobile. If a condition is recognized as beyond control, thought of it should be put out of mind. Nothing can be done about it. Mental energy should not be wasted.

Another type of worry is closely akin to fear. Some persons are afraid they will not pass an examination; others are afraid of the dark, or of a neighbor's criticisms. The only way to control these worries is to face them fairly and think them through to a conclusion. In practically every case, the fear or worry would not exist if the problem had not been avoided in the beginning. For example, the refusal to study will, in most cases, result in a fear of failure. When children face the painful consequences of conduct, accept failure or blame at face value, decide about problems rather than evade issues, face their difficulties squarely, and make a decision, they achieve mental poise. If the nervous energy consumed in fear were put into solving the problem, it would be used to a better advantage and the person would be happier. If one is afraid of failing in an examination, he should find out the cause of his fear. Possibly, the solution is to work harder. Possibly, the pupil is 
beyond his grade. If so, he should recognize the conditions and ask to be demoted. The effect of the failure is worse than the demotion. If afraid of the dark, one should investigate the dark place and see how unreasonable is the fear. If the mind is kept occupied with useful and cheerful thoughts, fears and worries disappear. Fears in children as well as in older persons may be overcome by a quiet reassurance and a reasonable, sympathetic investigation.

Bad temper is frequently an excuse for inefficiency. It is a way out of an annoying situation. If, instead of giving way to moodi-

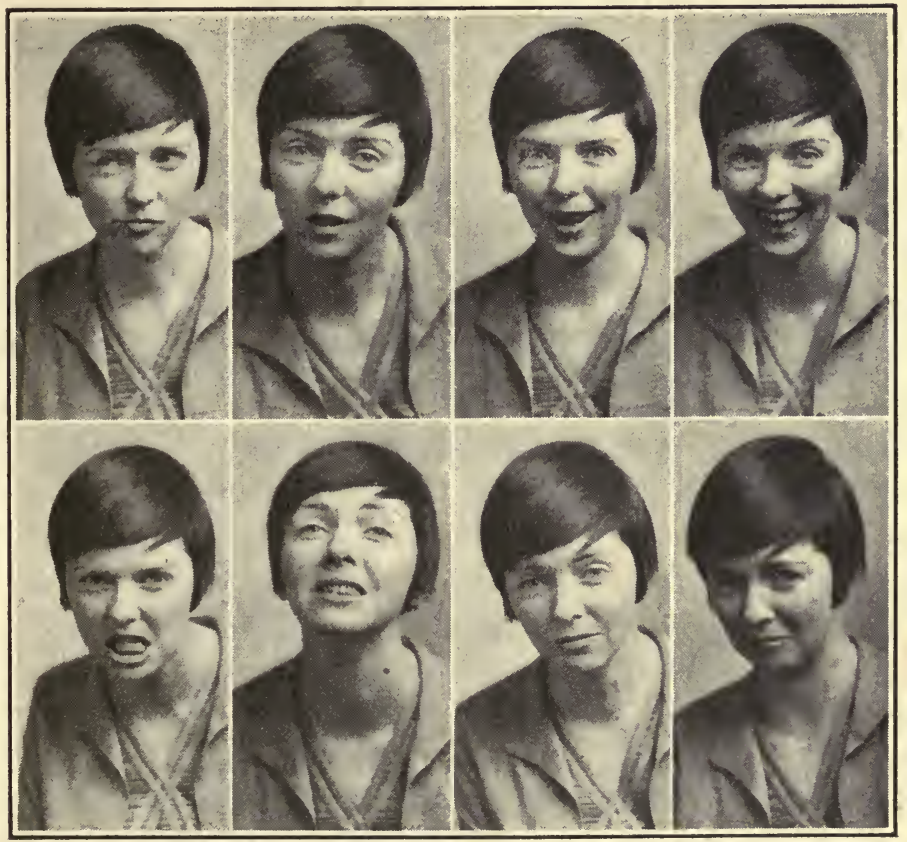

Strong emotions are frequently shown in facial expressions. Note the facial expressions in the picture and try to determine what emotion is registered in each.

ness or temperament, the person tries to understand the difficulty confronting him and tries to think out a solution, a normal re- 
sponse will follow. These normal responses help to develop proper attitudes. If one once recognizes that bad temper is futile, that it is wasteful of emotion, and that it interferes with sane reasoning, he will overcome it. It is particularly valuable to think things through to a conclusion and to keep a sense of humor. These are invaluable in overcoming nervous difficulties and building up an optimistic view of life.

Introspections are usually to be avoided in adolescence. When one thinks about himself, it is from so emotional and prejudiced a viewpoint that a fair judgment cannot be made. Day dreams may be conducive to success. They may make one ambitious and spur him on, if he dreams of achievements. But, if imaginary slights are exaggerated and dwelt upon, such thinking leads to mental disturbances. Social intercourse with other children, particularly in free play, is especially desirable in order to help a child adjust himself in the social group in which he must live.

A feeling of inferiority is a symptom common to a disturbed nervous system. When children are laughed at, or spurned, or too severely criticized, they lose their self-confidence and their selfrespect. This feeling of inferiority inhibits effort and causes dissatisfaction, unhappiness, and possibly failure. Responsibilities should be given such children. Confidence, respect, and wholesome consideration should be shown their efforts, so that they will make proper efforts toward success. Children should be able to take effective action when necessary.

Self-pity and lack of confidence are also undesirable. They may come from trying to accomplish things that are too difficult or are beyond the mental ability. Talking over problems with a person whose judgment is valuable and worthwhile, often helps to direct a person's activities along the proper lines. It reduces strain and worry and prevents the repression of thought.

Effects of Tobacco and Alcohol on the Nervous System. There is a distinction to be made between the effects of tobaceo upon 
young people and adults. Many school records show that, in general, smokers have lower scholastic standing than non-smokers. 'The use of tobacco in early youth forms a habit that becomes increasingly hard to break and in many instances has harmful effects upon the general ability of the person.

Tests have been made to see the effects of alcohol on mental and motor achievements. One of these tests showed that after a person takes alcohol in any form the rate of his pulse is increased, steadiness is decreased, coördination is lessened, and his ability to add is decreased. In all the tests that have been made, alcohol appears to reduce functional capacity. Recent tests show that alcohol is not a stimulant but a narcotic. The apparent stimulation following the taking of alcohol is due to the deadening of the nerve centers of control. The higher nerve centers of judgment and decision become dulled, resulting in irresponsibility. The memory tests which were conducted showed that seventy per cent less work was done when the subject was using alcohol. Many industrial organizations, railroads, and mercantile houses require their men to be total abstainers. The experience of these corporations proves that alcohol interferes with efficiency and is directly responsible for many accidents. The investigation of these two drugs is still largely experimental.

\section{Questions}

1. What is the relation of intelligence to school progress?

2. What remediable conditions may cause failure?

3. What can be done with dull children?

4. How do you think that it is possible for psychologists to determine the intelligence necessary for efficiency in various positions?

5 . What precautions are necessary for keeping the nervous system in good condition?

6. What is fatigue? How can it be overcome?

7. What are the objections to worries, fears, bad temper, and introspection?

8. What can be done for a person with an inferiority complex? 
9. What is the value of play, leadership, and success to a child?

10. Why do some people dislike their work? Is it possible to remedy this condition?

11. Criticize the habit of debating with oneself without coming to a decision.

\section{Supplementary Readings}

Burnham, Wm. Henry, The Normal Mind (D. Appleton and Co.).

Gates, Arthur I., Psychology for Students of Education, Chap. xviii (The Macmillan Company).

Robinson, James Harvey, Mind in the Making (Harper Bros.).

Terman, L. M., Intelligence of School Children (Houghton, Mifflin Co.).

Wiggam, Albert Edward, Exploring Your Mind (D. Appleton and Co.). 


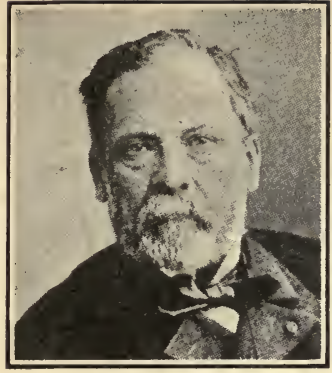

Louis Pasteur.
CHAPTER XXV

HOW

\section{LIFE BEGAN}

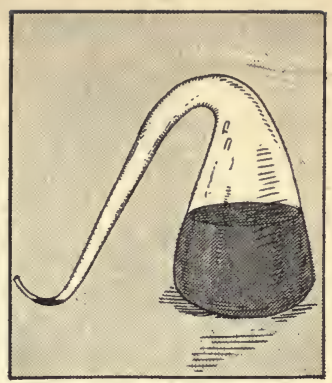

His flask of nutritive fluid.

What was one of the ancient ideas of the origin of life? How and by whom was the theory of spontaneous generation disproved? How do some scientists of to-day explain the origin of life?

The ancient idea of the origin of life. At the time of Aristotle, and for many centuries following the work of this Greek teacher and philosopher, there was a theory, supported by scientific men, concerning the origin of life, and known as spontaneous generation. According to this theory, living things rose spontaneously from non-living materials. Bees were supposed to come from the dead bodies of young bulls; young rats could be made to appear in a box containing soiled rags and wheat grains; horsehairs in water would turn to worms; and mice and other animals came from the mud of the river Nile. Dew was supposed to give rise to insects, and rain would bring frogs. Instead of investigating whether this was the way life originated, people speculated that it must be true and scorned anyone who doubted it. The unbelievers were told to go to the Nile and see the fields swarming with mice begot from the mud which was deposited when the Nile overflowed its banks.

Experimental evidence. Francesco Redi, an Italian physician, (1629-1694), was one of the first persons to conduct a series of experiments to test, scientifically, the theory of spontaneous 
generation. He had noticed that flies were usually seen about meat, and thought that there might be some connection between

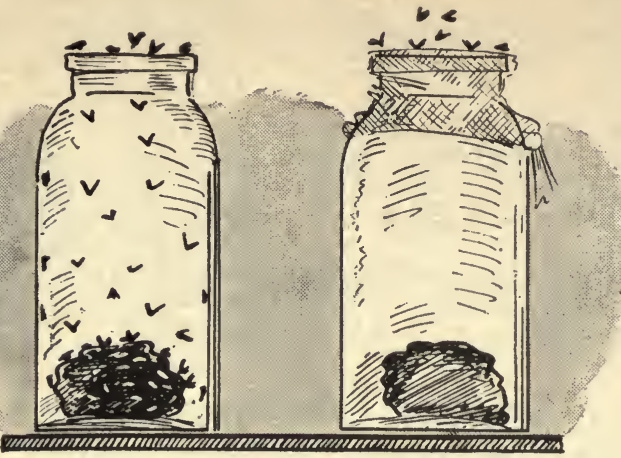

Using this simple experiment, Redi started a discussion which ended with the overthrow of the belief of spontaneous generation.

flies and the maggots which were supposed to rise spontaneously from the meat. Is a result of this observation, he put some meat in three different jars. One of these jars was left uncovered, one was covered with parchment, and the third was covered with a fine gauze. In the first jar, the meat spoiled; maggots and, later, flies appeared in the mass. In the parchment-covered jar, the meat putrefied, but no maggots appeared. In the gauze-covered jar, the meat putrefied, and flies laid eggs upon the gauze. These eggs produced maggots which later developed into flies. Therefore, Redi concluded that maggots of flies could not have originated from decayed meat alone, but from fertile eggs laid there by other flies that were attracted by the odor. No eggs were found near the second jar as the parchment kept the odor from escaping. Redi decided that life must come from preëxisting life. He performed other experiments and concluded that in cases when life seemed to have been produced from dead matter there had always been the introduction of material from living organisms.

Leeuwenhoek's contribution. After Redi's experiments, the question of the origin of life was again discussed. Many people were willing to accept the explanation that the living things they could see, such as rats and frogs, must originate from other living things. In 1687, Leeuwenhoek perfected the microscope and dis- 
covered an entirely new world of living microörganisms. He discovered bacteria and protozoa, which many investigators said were the organisms from which more complex organisms originated. By means of the microscope he was able to prove that the weevils found in granaries were hatched from minute eggs deposited on the wheat grains by winged insects.

Needham and Spallanzani. About 1770, Needham, an Englishman. became interested in these experiments. He boiled meat extract in glass flasks which he closed securely with corks. He thought he had killed all the life present with the boiling process. In every case, he found that great numbers of microörganisms appeared sooner or later. He decided that if life appeared, it must originate spontaneously. He started the spontaneous generation controversy anew. The Abbé Spallanzani, an Italian, suspected that Needham had not been very careful in conducting his experiments and that germs in the air might have entered the flask. He repeated the same experiments, but used glass flasks that could be hermetically sealed in a flame while the infusion was still hot. No organisms appeared. Needham objected to Spallanzani's experiment, saying that the prolonged heating had destroyed the nutritive value of the substance. Spallanzani then permitted some air to enter the glass and almost at once microscopic organisms appeared, showing that the nutritive qualities of the material were still there.

The importance of oxygen. About this time a scientist named Priestley discovered oxygen and its importance to life. Scientists now asked whether the boiling of the closed flasks had not changed the oxygen so that it had lost its life-giving properties. But no more scientific experiments were performed until 1836, when an experiment was devised that permitted clean air to enter the culture medium continuously. Organisms in the air were removed by passing the air through a series of tubes containing substance which would kill all living matter. No organisms appeared in the flask. 
Many scientists were now willing to accept the fact that living organisms did not originate spontaneously.

The revival of the discussion. After some years of practically no discussion on the subject, Pouchet, in 1859, suddenly revived the controversy. He believed that spontaneous generation was one of the means employed by nature for the production of living things, so he set out to prove it. He filled a bottle with boiling water and inverted it with the mouth of the bottle under mercury. Then, by means of a delivery tube, he introduced oxygen through the mercury into the bottle of water. The oxygen gradually displaced some of the water. By means of a pair of forceps, which he had first heated, he thrust some hay through the mercury into the bottle. The hay, too, had been carefully heated to a very high temperature. The hay floated in the water in the bottle, in the oxygen atmosphere. Microörganisms appeared in great numbers.

Louis Pasteur enters the controversy. A French scientist, Louis Pasteur, thought that Pouchet had not set up his experiment carefully enough and that germs must have entered with the oxygen or hay. Pouchet asked how it was possible for air to contain so many germs that they developed in every organic material. He said the air would be misty with them. Pasteur began to wonder whether germs might not be more numerous in some air than in other air.

In order to investigate this, Pasteur filled a number of glass flasks with a liquid that would easily spoil. He boiled the liquid and sealed the flasks while the liquid was still boiling. He opened some of the bottles in different places where there were people and dust. He then sealed the flasks again, and in all cases organisms appeared. He next went to the Alps to investigate air at an altitude so high that it would be free from dust. He went to the Mer de Glace, high up in the Alps. He opened twenty of the flasks that had been carefully prepared, and immediately sealed them again. Subsequently, microörganisms appeared in only one 
flask. Pasteur concluded that the amount of dust and germs in different localities must vary. In reporting this experiment to the French Academy of Sciences, he stated that the study of the germs which accompanied dust might lead to a knowledge of the origin of various diseases. But the conception of germs and disease was so vague that no attention was paid to this statement.

While Pasteur was conducting his experiments in the Alps, Pouchet was testing air in Sicily, on Mt. Etna, and on the sea. He found microörganisms in all his air tests. Many people believed in the validity of Pouchet's work rather than that of Pasteur's. One scientific journalist wrote that Pasteur's work was too fantastic to be exact.

Then Pouchet decided to repeat Pasteur's experiment. Accompanied by two other scientists, he departed for the Alps with a number of narrow-necked flasks filled with hay infusion. At the foot of a glacier of the Maladetta, 3000 meters above the sea level, he opened four of his flasks. Then the tubes were carefully sealed. Microörganisms soon appeared in the flasks. Pouchet then concluded, since there was no dust at the place where he had opened the flasks, that air did not bring in the germs, but that they must arise by themselves from the organic material.

The debate became so heated that the Academy of Sciences appointed a commission to examine the experiments of Pasteur and Pouchet. Both scientists were invited to present their experiments, but Pouchet said the weather was so cold that it might compromise his results. Some time later, Pasteur was invited to give a lecture on spontaneous generation at a scientific meeting at the Sorbonne. A theatrical performance could not have drawn a larger crowd. Every seat in the room was taken and many scientists and students were there. Pasteur simply and carefully performed his experiments, explained them, and presented his conclusions. He explained to the audience that boiling destroyed the germs, but if air entered after the boiling, it carried 
in more germs and the organic material would then decompose and show numerous microörganisms. If care were taken that no air entered, no organisms appeared. He repeated Pouchet's first experiment and showed a source of probable error. In a darkened room, he directed a beam of light upon the apparatus and the audience saw that the surface of the mercury was covered with dust particles. Pasteur showed that when the forceps were plunged through the mercury, they took some of the dust particles with them. He then explained that the floating particles of dust contained living germs. Later, the Commission decided that the contest should be settled by one experiment. Pouchet wanted more. The Commission refused and gave its decision in favor of Pasteur.

John Tyndall. In 1876, John Tyndall, an English physicist, published the results of his experiments. He had devised a very elaborate box or chamber which enclosed a volume of air. This

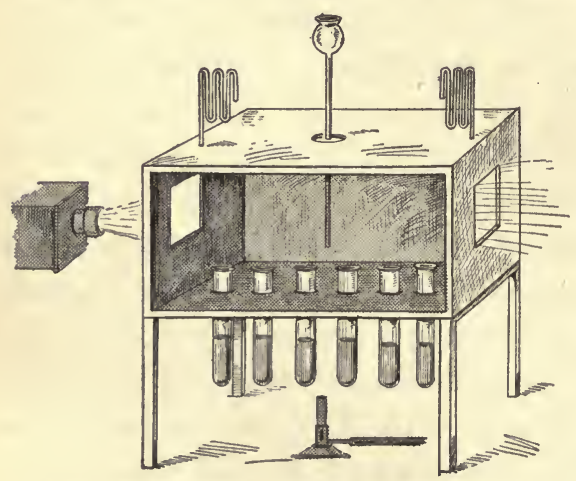

Tyndall's apparatus added further 'data which helped disprove the theory of spontaneous generation. Identify the different parts of the apparatus from the description given in the text. was so regulated that any particles floating in the air would settle and be held on a sticky substance, such as glycerine, spread over the sides of the box. Tyndall passed a powerful beam of light through an opening in the box to make sure that no free dust particles were present in the air. If dust particles were present in the air, the beam of light would illuminate them. Then he applied heat to the test tubes of nutritive fluids that were suspended in the box. These fluids consisted of mutton broth, turnip broth, and fluids from other plants and animals. 
The mouths of the test tubes were freely exposed to the air in the box. The fluids remained free from microörganisms for an indefinite period of time. Later, in order to check his results and to demonstrate that the fluids contained nutritive value, he removed them from the box and exposed them to the outside air. Microörganisms appeared. The importance of this work and the work of Pasteur was far reaching. Keeping dust out was the basis for the sterilization of wounds and surgical instruments, which later led to antiseptic surgery. It was, also, the basis of canning by heating.

Origin of life. If life comes from life, where did the original life come from? Science has been unable to solve this riddle. One theory, popularly accepted by some scientists, is that when the earth cooled down, there were unusual conditions that made possible the combining of certain elements in the right proportion to form the simplest one-celled plants. From these plants, gradually the entire plant and animal kingdoms have originated. There are no means of knowing whether this actually took place or not. The origin of living matter still remains unsolved by scientists.

\section{Questions And Suggestions}

1. State the ancient idea of the origin of life. What evidence have you for thinking that some people still have this idea about some forms of life?

2. Describe the first experiment that was made to prove the theory of spontaneous generation.

3 . Discuss the importance played by the microscope in the controversy.

4. Discuss the experiments of Needham and Spallanzani.

5. Of what value to scientists of this time was the discovery of oxygen?

6. Discuss the controversy of Pouchet and Pasteur.

7. How do present-day scientists explain the origin of life? 


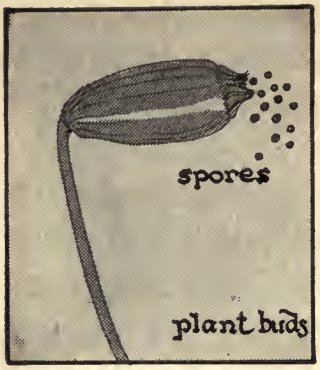

A moss capsule.
CHAPTER XXVI

ASEXUAL REPRODUCTION

What is asexual reproduction? What kinds of organisms reproduce asexually? What are some of the asexual methods of reproduction?

Only living things have the power to produce new organisms similar to themselves. Schleiden, Schwann, and other scientists showed that cells, living units of life, came only from preëxisting cells. This process of a cell or cells producing other cells is called reproduction. It is a process peculiar to living organisms. Icicles, crystals, stalactites, and stalagmites may grow by tiny additions to the outside. These additions are known as accretions. After accretion has gone on for some time, parts may break off. This type of growth and division is fundamentally different from the growth and division that come from within a living cell. A given organism cannot live forever; therefore, nature has provided, by reproduction, a means of continuing the species.

The necessity for division. In living cells, particles of food material are taken into the cell body and made into cell material, protoplasm, by the process of assimilation. This additional protoplasm causes the cell to grow. The cell grows until it becomes so large that there is not enough surface to take in sufficient food for the increased mass of protoplasm. By the process of dividing in half, two more surfaces may be obtained, through which food may be absorbed from the outside. After dividing, each cell again 
takes in food from the outside, grows to its maximum size, and then divides again. In the simpler animals and plants, after reproduction, the new cells may separate and go about as single individuals or they may cling together. If they cling together, each acts as a separate individual independent of the other.

Reproduction by binary fission. The simplest of all plant organisms belong to the Thallophyta. As previously mentioned, they are distinguished by the character of having no division into roots, stems, and leaves. The thallophytes are divided into two sub-groups: the fungi - thallophytes lacking chlorophyll, such as bacteria, yeasts, and molds; and the algaethose having chlorophyll, such as Pleurococcus and Spirogyra.

Bacteria show little differentiation of structure. They consist of a mass of protoplasm with nuclear material scattered through the cytoplasm. They are surrounded by a thin cell wall. Bacteria reproduce by splitting in half, thus forming two new individuals. During reproduction, the nucleus elongates and divides. A cell wall is formed between the two nuclei, cutting the cell in half. Probably the division of the nucleus is always a mitotic division. Sometimes the newly formed cells remain attached; often they separate. This type of reproduction is binary fission.

Many of the algae reproduce much like bacteria. Pleurococcus is found on the shady, moist sides of trees, rocks, and stumps everywhere. Each plant is a single cell consisting of a cell wall, cytoplasm and nucleus. It makes its own food, reaches a maximum size, and divides. The resulting cells either separate or remain in clusters. The cells of Spirogyra divide by

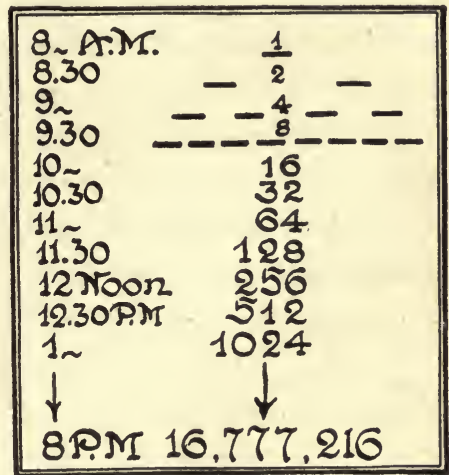

Germs multiply rapidly when conditions are favorable. 
binary fission. In Spirogyra, the mitotic figure is clearly seen when properly stained. As the cells of Spirogyra divide, they
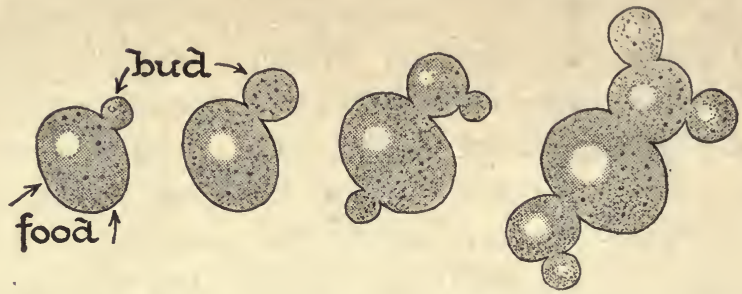

A yeast cell takes in food and grows a bud which enlarges and produces other buds. This results in a chain or group of yeast cells.

cling together end to end and form a long filament. Each cell is an independent organism. Amitosis, division without a spireme, is very infrequent. There is doubt as to whether amitosis is ever a method of reproduction in any normal and healthy cell.

Many one-celled animals such as the Protozoa reproduce by fission. In the amoeba, the nucleus divides mitotically, the cytoplasm constricts between the two nuclei, and two new cells are formed. The Paramecium, too, reproduces by a like method. The micronucleus elongates, constricts, and divides. The macronucleus resembles a degenerative nucleus in that it divides amitotically. It frequently disintegrates and dies. Then the micronucleus builds a new macronucleus. As the cytoplasm constricts, a new mouth, groove, and gullet appear in one side of the organism. A new vacuole appears at the end of each new cell. The cytoplasm divides completely and the two new Paramecia swim away from each other.

Reproduction by budding. Another type of reproduction found among simple organisms is budding.

Problem. Study of budding in yeast cells.

Break a small part of a compressed yeast cake into a dilute solution of molasses. Stir and then let the mixture stand in a warm place over night. Mount a drop of the material under the low power and then the high power of the microscope. 
I. Describe the shape, color, and size of yeast cells.

$A$. Note and describe the large vacuole in each cell.

$B$. Describe any other structures that you see.

II. Notice tiny protuberances on some of the cells. These are called buds. This process of reproducing is called budding.

$A$. Compare the buds with the original cells as to size and appearance.

$B$. Describe a difference between budding and binary fission.

$C$. Compare the different buds in size. Account for the difference.

$D$. Examine the field of the microscope carefully and see whether the buds always cling to the original cell or whether they separate from it.

$E$. If the original cell is called the mother cell and the bud is the daughter cell, do you find any granddaughter cells? Explain how you recognize them.

$F$. There is a nucleus in the yeast cell although it may be difficult to see it without staining. The nucleus divides mitotically in forming the bud.

$G$. Draw : 1. Yeast cells ten times larger than seen under the microscope. Label cytoplasm, vacuole, and cell wall. 2. Yeast cells budding. Label cell and bud. 3. A group of yeast cells with successive buds.

$H$. Compare your sketch with the diagram on page 248 .

Budding of hydra. Hydra, a small, many-celled animal, is often seen growing on the side of an aquarium jar. Hydra has a mouth, several armlike structures called tentacles, a thick wall of outside body cells, and a delicate inner layer of cells inclosing a body cavity. The tentacles wave about and capture food which they convey to the mouth. When hydras are mounted under the

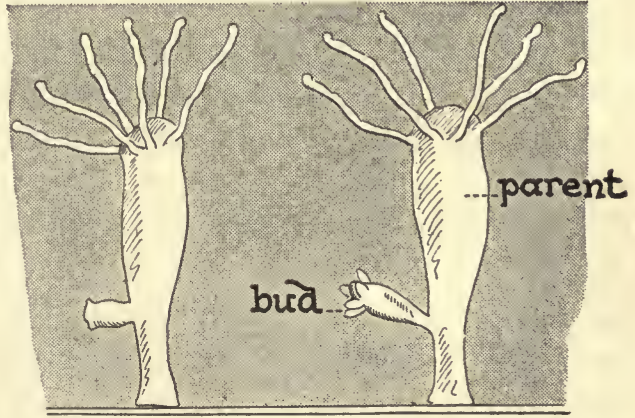

The hydra often grows a bud and occasionally an animal is found with a daughter bud bearing active tentacles. microscope, one or more buds may be seen on the body walls. Each bud is a new animal in some stage of development. 
Budding is not as common a form of reproduction as binary fission. Budding and binary fission are types of reproduction

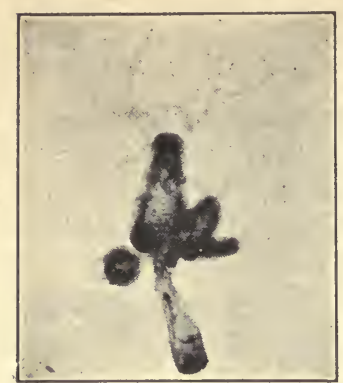

Photomicrograph of a hydra. Note the buds still attached to the original animal. found only among the simplest of plants and animals.

\section{Reproduction by means of spore forma-} tion. A third type of reproduction found among simple organisms is spore formation. If yeast cells are subjected to an unfavorable condition of heat, food, or water, they sometimes go through another type of reproduction. Each yeast cell will develop an unusually thick wall. The nucleus and cytoplasm break up into three to eight parts. Very often only four parts are formed. Each of these parts is known as a spore. The spores will remain in the spore case, the thickened cell wall, until the surrounding conditions become favorable for them to live alone. Then the spore case will absorb water, burst, and the four yeast cells will come out. Each will feed, grow, and reproduce. This type of propagation accomplishes protection as well as reproduction.

Due to lack of chlorophyll, yeast cells are unable to make their own food. They depend upon food already made. Their food consists of fruit juices and other sugar solutions. If molasses solution, preserved fruit, grape juice, or any other sweet food is left exposed to the air, wild yeast plants, in the form of spores, will settle on the surface and multiply rapidly. The yeast cells give off enzymes which attack the sugar, breaking it down into alcohol and carbon dioxide. This process of yeast attacking sugar and converting it into alcohol and carbon
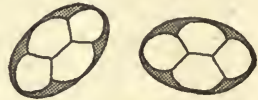

Yeast cells often form a group of four spores. These last over an unfavorable period and at the proper time break from the spore case. Each spore becomes a yeast plant. dioxide is known as fermentation. Bubbles of carbon dioxide may be observed in fermented solutions. The test performed in 
elementary science may be used to show that these bubbles are carbon dioxide. The alcohol may be recognized by the odor. The process of fermentation always accompanies the reproduction of yeast plants. Since the yeasts are dependent upon nonliving organic material for food, they are called saprophytes.

\section{Problem. Spore formation in mold.}

Place a piece of bread on several thicknesses of well-moistened filter paper or blotting paper on a plate: Expose the bread to the air, sprinkle it with dust, or infect it with mold spores. Cover with an inverted glass dish that is raised up on one side to admit a little air. Be sure the bread does not dry. Moisture, a limited amount of air, warmth, and organic material are necessary for the growth of molds. The hairy growth that appears on the bread is one type of mold, the bread mold. It takes from one to four days to grow these plants.

Mount a minute amount of the hairy structure under the low power of the microscope.

I. Describe the shape and color of the cells of the mold.

$A$. Are there any cross walls to the cells?

$B$. Do the cells branch or not? These cells are known as hyphae.

C. The mat of cells is known as the mycelium. The threads that are actively feeding and growing are called the vegetative hyphae.

II. Try to mount a piece of mold that was actually embedded in the bread.

$A$. The anchoring rootlike structures are called rhizoids. By means of these, the mold gives out enzymes that dissolve some of the bread. The dissolved bread is then absorbed as food by the mold.

I. Observe the structure of the mold and explain why it is probably a saprophyte.

II. Discuss the difference between the mold and Spirogyra in types of nutrition?

III. When the mold is three or four days old, note the tiny dark bodies that appear. Mount a bit of the mold structure, including several of these bodies, under the low power of the microscope. Be sure not to take too great a mass of material.

$A$. Describe the structure of one of the small black bodies. This is a sporangium.

$B$. Describe the structure that holds the sporangium. This is a reproductive hypha.

C. Locate a broken sporangium. Describe the structures escaping 
from the sporangium. These are known as spores. Each little spore is capable of producing a new mold plant.

$D$. Draw a complete and a broken sporangium.

I. Label sporangium, spore, and reproductive hypha.

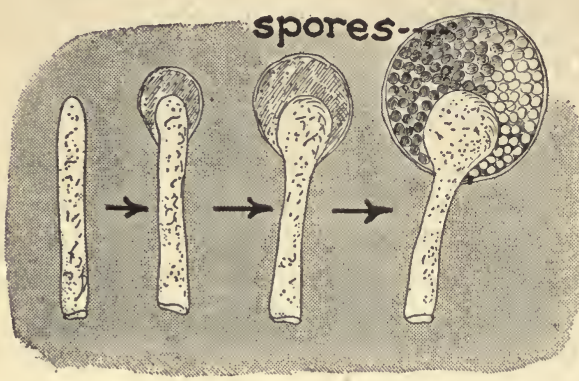

An aërial hypha develops a sporangium or fruiting body. This structure produces and disperses the spores.

A sporangium sometimes forms at one end of the hypha. The protoplasm within this end breaks into a great many tiny structures, spores. When ripe, this fruiting body or spore case, the sporangium, breaks open and the spores scatter. The spores can outlast unfavorable conditions. During unfavorable conditions, the mold is in a dormant or resting stage in the spore. If it settles on food and there are proper conditions of moisture and warmth, then the spores will develop into new mold plants. The name for some molds is mildew. Certain of the molds, as the powdery mildew that grows on the lilac, live and secure their nourishment from living organisms. An organism that gets nourishment from a living plant or animal, and gives nothing in return; is a parasite.

Problem. Study of sporangia in various types of molds.

I. Let a peeled banana stand in a covered dish for forty-eight hours. When it shows a mold growth, examine it with the microscope for fruiting bodies or sporangia.

II. Bring to class samples of various kinds of molds or mildews. Examine them under the microscope for the fruiting bodies or sporangia.

$A$. Draw as many different types of sporangia as you have observed.

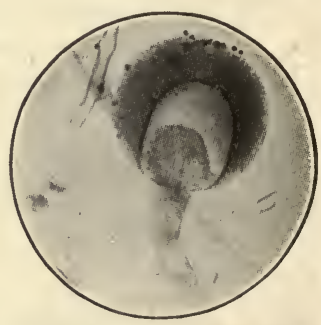

This photomicrograph shows not only a large sporangium, but certain of the spores are also in focus. Compare this illustration with the diagram at the top of the page. 


\section{Amoebas and certain} other protozoans may go into a resting stage similar to spore formation, when conditions are unfavorable. This resting stage in a protozoan is called encystment. Spore formation is not, necessarily, a method of reproduction. It may be a method of protection only. But since such a cell can germinate, when brought into favorable conditions, it is usually classed with

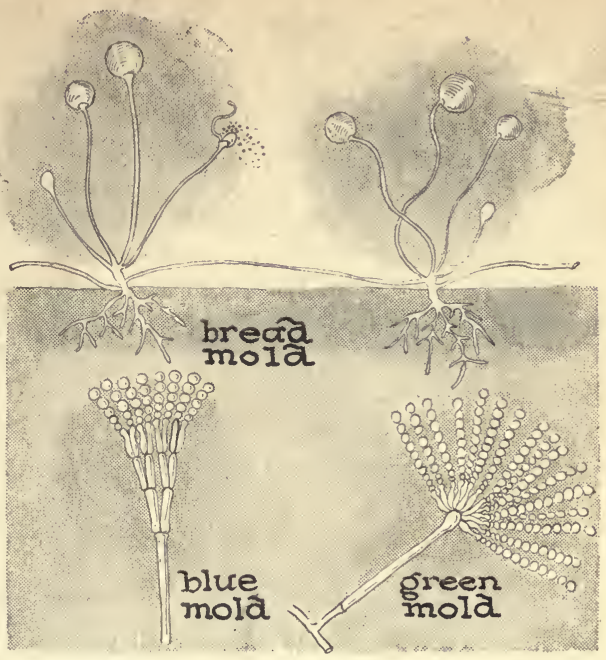

Different species of molds form different types of sporangia. Compare the different sporangia in the picture. reproduction. Spallanzani, Pasteur, and Tyndall discovered in their investigations of spontaneous generation that certain microorganisms were harder to kill than others. It was later found that some are more resistant to a high temperature than others, and that some are spore formers and can resist unusual conditions, while others are incapable of forming spores.

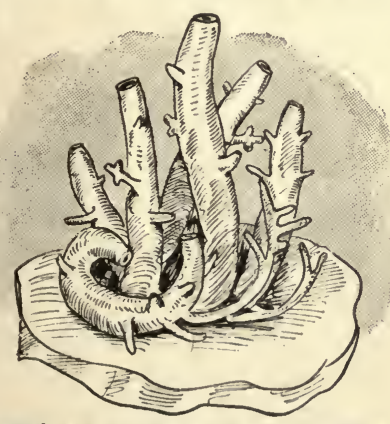

Certain sponges reproduce by budding.

Each of the types of reproduction described, namely binary fission, budding, and spore formation, is really a type of cell division. Each of the new cells formed is produced by a single organism. Therefore, it is called asexual (without sex) reproduction, in contrast to sexual reproduction in which two organisms take part. 


\section{Questions AND Suggestions}

1. What is the purpose of reproduction?

2. Discuss binary fission.

3. Give examples of organisms that reproduce by binary fission.

4. Discuss reproduction by budding. Illustrate in two organisms.

5. Set up an experiment to illustrate fermentation.

6. Discuss spore-formation in yeast; in molds.

7. Besides reproduction, what is another purpose of spore formation?

8. Compare and contrast binary fission, budding, and spore formation.

9. Compare a saprophyte and a parasite with an organism that makes its own food. Give examples.

\section{SUPPLEMENTARY REAding}

Atkinson, G. F., College Textbook of Botany (Henry Holt \& Co.). Gager, C. S., General Botany (P. Blakiston's Son), chaps. xv-xxv. Greaves, J. E. and E. O., Elementary Bacteriology (W. B. Saunders), chap. vi. Holmes, S. J., General Biology (The Macmillan Co.), chap. x. 


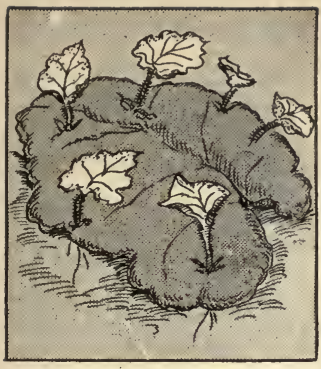

Leaf with little plants.
CHAPTER XXVII

\section{VEGETATIVE PROPAGATION}

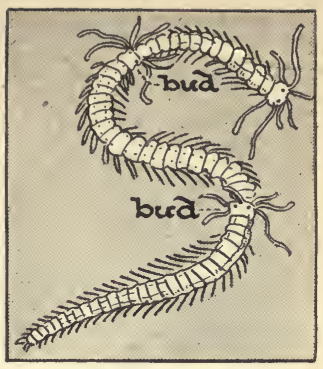

A sandworm with buds.

What is vegetative propagation? What processes in animals may be comparable to vegetative propagation of plants?

An individual organism begins its independent life when it becomes separated from a preëxisting individual. Roots, stems, and even leaves sometimes develop into new plants, although ordinarily their functions are for purposes of nutrition and not reproduction. When a portion of a plant, ordinarily used for nutrition, is separated and used for reproduction, the process is called vegetative propagation. Vegetative propagation is an asexual method of reproduction. Usually, only one organism contributes to the propagation or continuance of the life of the organism. Man makes use of vegetative reproduction to produce new species rapidly. When the method of reproduction of a plant is devised by man, it is called an artificial method of propagation; when it is found in nature, it is called a natural method.

Artificial methods of vegetative propagation. Cuttings. Higher plants may be propagated by cutting pieces of stems from the plant and planting these cuttings in moist sand or water. If a twig is cut from a willow tree, and the cut end placed in water or moist soil, roots will usually develop from that end, while buds will develop from the other parts of the cutting. Each cutting will produce the missing parts and grow into a complete, independent 
plant. Geraniums and other house plants are usually propagated in this way since they may be produced much more quickly from

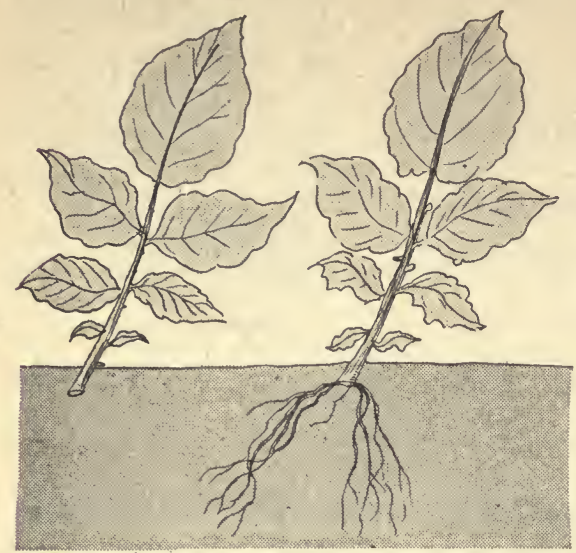

A piece of stem cut from certain plants and placed in water or moist sand will grow roots.

cuttings or slips than from the germination of seeds. Another advantage of slipplanting is that one is usually sure to grow a plant true to the type from which the slip was taken. The seeds of plants do not always develop into plants exactly like the organisms from which they were gathered.

Regeneration. There is a type of propagation found among certain animals that is somewhat similar to cuttings of plants. It is possible for many organisms to reproduce lost parts and grow into complete individuals. The common earthworm is a good example. If a worm is cut through the middle, each half may develop the missing parts and each part may become a complete worm. Certain flat worms may be divided into several parts and each part will become a complete individual. Sponges are propagated by cutting a sponge into many sections and sowing the ocean floor with them. Each section will develop into a complete organism. The regrowth or reproduction of the parts of an organism which have been lost or destroyed is called regeneration. If some animals such as crustaceans (lobsters, crabs, shrimps) and echinoderms (starfishes, sea urchins, sea cucumbers) lose one of their appendages or rays, they are able to regenerate these parts. Sometimes a lobster is seen with one claw much larger than the other. This probably indicates that the smaller is a second growth, the first having been lost in a fight or through an accident. 
In the higher animals, including man, whole organs are not restored. A process similar to regeneration is evidenced, though, in the healing of a wound and in the knitting of a broken bone. An ordinary scratch or even an extensive cut often heals without so much as a scar, because the destroyed cells are quickly replaced by new cells. The capacity of lower animals to regenerate an entire individual from a portion of another individual is an extreme example of the same ability as is manifested in the healing of a wound. The type of regeneration shown in the regrowth of destroyed tissues is known as physiological regeneration in contrast with the regeneration that is a method of propagation.

Grafting. If, under certain conditions, the freshly cut surfaces of two plants are brought into contact with each other, the plants will grow together as one. People for many ages have been using this knowledge for the propagation of fruit trees. A stem called a scion is cut from a tree and is so attached to a rooted stem called the stock that the cambium or growing layers of each are brought into close contact. The actively growing cambium cells of each unite the two stems. In time, food from the stock will pass through the ducts and nourish the scion. This is called a stem

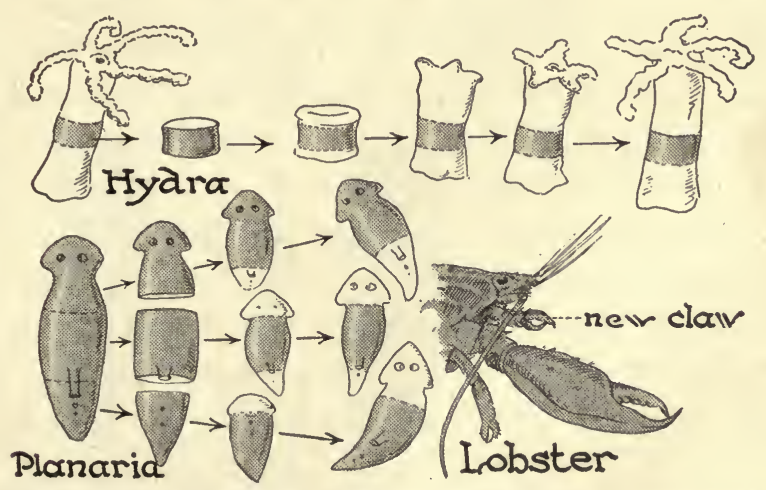

Animals often regenerate lost parts. The lower animals, when injured, may grow back few or many lost organs. The simpler the animal, the greater is the power of regeneration. In the human body only limited regeneration takes place. 


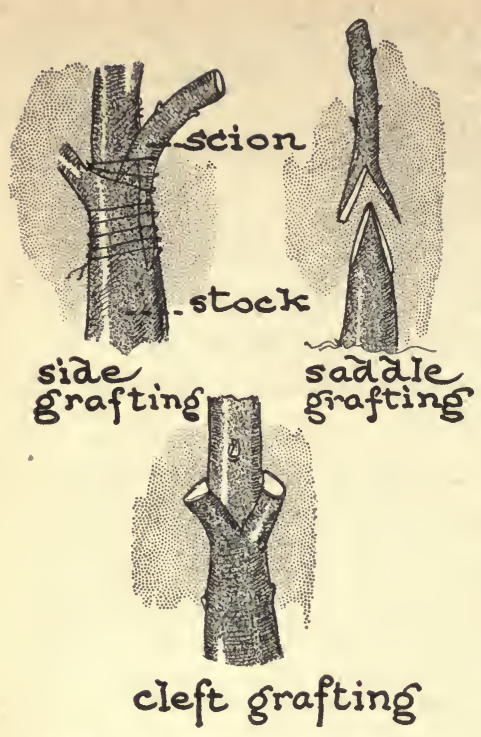

Twigs may be grafted together in various ways.

graft. During the process of uniting, the two stems must be closely bound to prevent them from breaking apart. A coat of grafting wax protects the region of contact from an excessive loss of sap and evaporation of water, and from contact with spores of fungi. Successful grafting is accomplished only between members of the same or closely related species. Stem grafting is commonly used in propagating fruit trees.

Grafting is, also, done by means of buds. A bud on a strip of bark with its underlying cambium layer is cut from a branch and is inserted into the slit in the bark of the stock so that the two cambium tissues are in close contact. The process of binding and waxing is similar to that used in stem grafts. Fruits, nuts, and flowers are obtained more rapidly by grafting than by the planting of seeds. The graft will probably always breed true to the scion type. Consequently, as in the case of propagation by cuttings, a horticulturist is sure of the result of the graft, if it is successful. Grafting is also a method of propagating seedless fruits. If a seedless fruit has been produced by plant breeding, it is propagated vegeta-
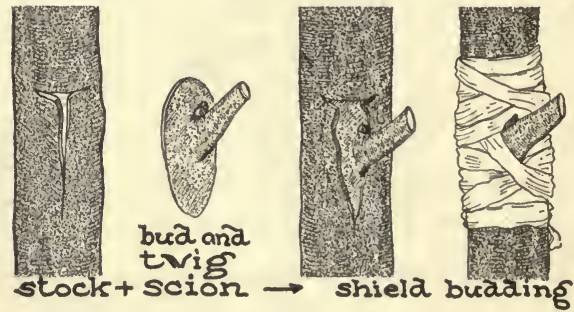

Shield budding is a type of grafting. The bud of the desired variety is inserted into a slit in the bark of the stock. It is then bound tightly to keep in place until healing and growth take place. 
tively by grafting. Every possible tree of the same or closely related species may be made use of in grafting. If a tree is found to produce inferior fruit, instead of uprooting it and planting new seeds, a scion from a tree that produces good fruit may be grafted on the inferior tree. If the grafting is successful, the original tree will, in the future, produce the improved variety. Varieties of the Old World grape have been grafted on the stock of the wild grape, and from this combination we have obtained the different types of grapes found in our country to-day. The roots of the Old World grape are easily injured by a root louse, but the roots of the wild grape are not affected by this insect. Besides, the wild grapes are hardy and are able to grow well in this climate. By grafting, it has been possible to produce grape vines that are immune from destruction by the louse, and yet yield the desired type of grape.

A type of grafting is fre-

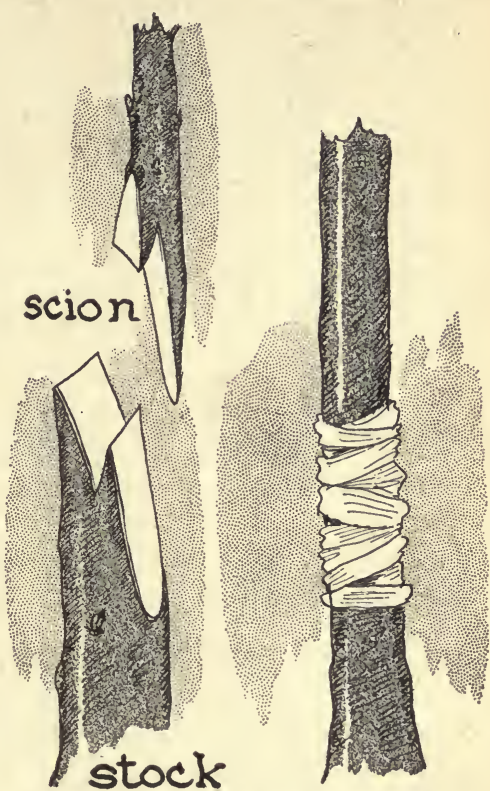

Tongue grafting is a type commonly used. The important feature here, as in all grafting, is to bring the cambium layers of stock and scion together. quently used by surgeons in animals. But, here, grafting is a type of regeneration and not of reproduction. In most cases, grafting is only used when tissues are severely injured. For example, if considerable skin has been destroyed by a burn, small sections of skin are removed from other parts of the body and grafted on the injured area. This grafted skin grows over the injured area and thus repairs the tissue. If a bone in the body is tubercular, sections of healthy bone from another 


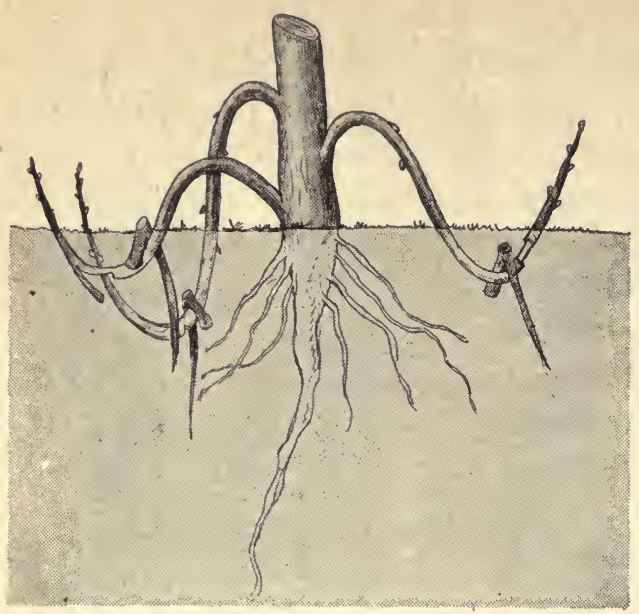

Layering is a modification of cutting. It consists in bending down a stem and covering it with earth. Deepnotching or ringing the bark of the part buried usually hastens the rooting of the stem. part of the organism may be grafted on to the diseased bone. But, to a large extent, grafting of tissues, bones, and even organs is still in an experimental stage and has not proven successful in all instances.

Natural methods of vegetative propagation. Layering, another method of propagation, often occurs without the assistance of man. In many cases the branches of a tree or brush may bend down until they come in contact with the soil. Sometimes they become covered with soil. If there is sufficient warmth and moisture in the soil, the branches will develop roots. Frequently, after developing roots, they break off from the tree and form an entirely new plant. The raspberry, with its arching stems, illustrates this type of propagation.

Runners are branches that trail along the ground. Sometimes, the ends or joints of these branches come in direct contact with moist soil. Roots and, finally, a shoot develop at this point, forming a new plant. Strawberries, as

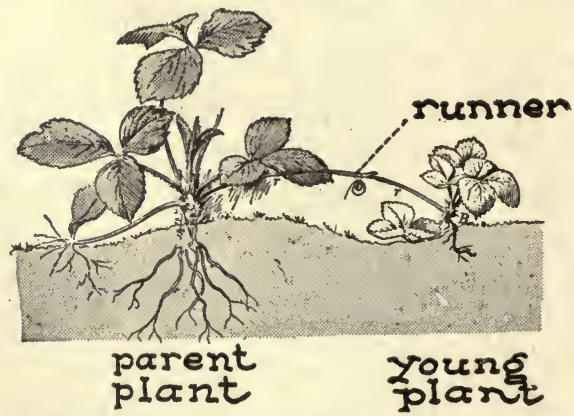

When runners are found in plants, they may be used as an easy and rapid method of propagation. 
well as many grasses and weeds, are usually propagated in this way. Seeds of strawberries are so very difficult to cultivate that horticulturists depend entirely upon the runners for propagation.

Rhizome. A common form of subterranean stem is the rhizome or rootstock. It is a horizontal stem running

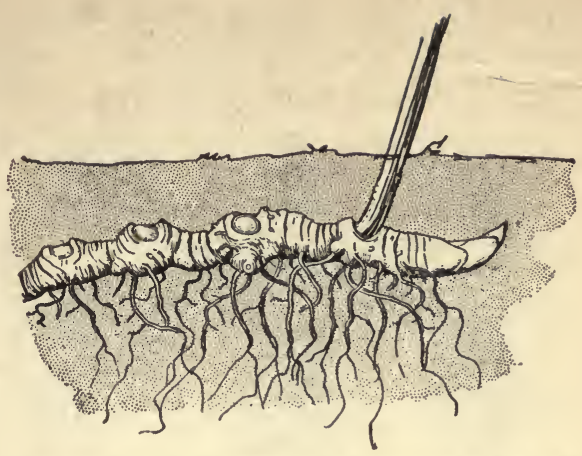

Underground rootlike stems or rhizomes are characteristic of some plants. As they run under the ground, they give off buds which form new plants.

under the ground. As it grows beneath the soil, it sends off roots from its under surface, and leaf-bearing branches from its upper surface. The rhizome is usually somewhat thickened with food. Growth takes place year after year from the same rootstock which

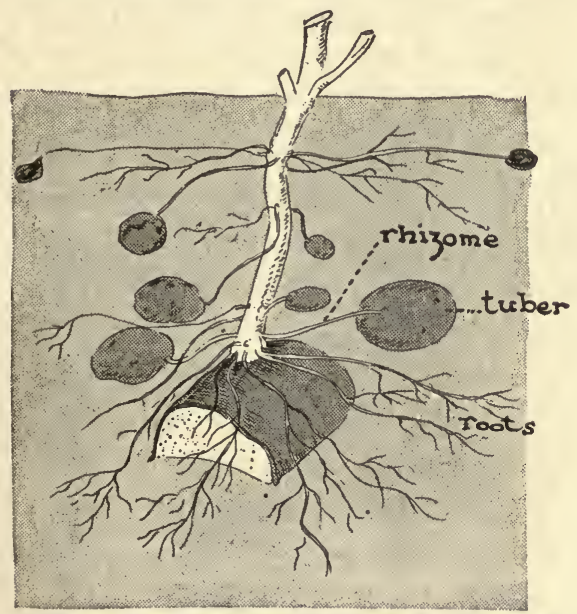

Potatoes can be raised from seeds. The usual method of propagating potatoes, however, is to use a part of the tuber with an eye. The eye is a bud from which the stems and roots grow. bears the annual scars of the ground stems. In the common ferns, the so-called fronds are simply large leaves developed directly from the rhizome. Bloodroot, Solomon's-seal, wakerobin, lily of the valley, and many other spring flowers are propagated from rootstocks.

Tubers. The potato plants have slender, underground stems. Certain regions of these stems enlarge to form tubers. The 
"eyes" in the tubers are really buds. When used for propagation, the potato is cut so that each section has at least one "eye." These sections can be planted and each bud or "eye" will develop a

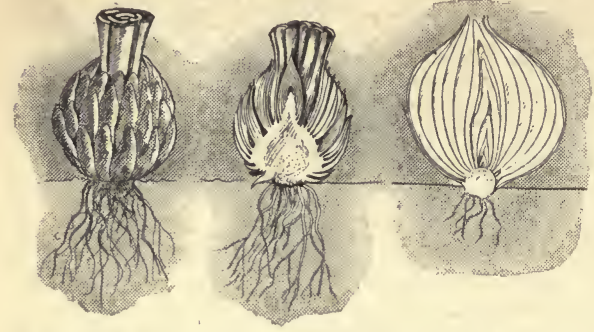

A whole bulb and the cut section of two bulbs are shown. Note the short stem and the thick fleshy leaves arranged around the stem. root system and an aërial stem. Each tuber can form as many plants as it has buds or "eyes." Nourishment in the form of starch is stored in the tuber and feeds the growing plant until its leaves are produced. Sometimes when potatoes are kept in a damp cellar, the eyes absorb moisture and begin to develop into stems.

Bulbs. A bulb consists of a modified under-ground stem. Leaves, thickened with stored foods, grow from this stem and closely overlap each other to form the scales of the bulb. A terminal bud growing from the tip of the stem is in the center of the scalelike leaves. If a bulb is cut, the parts of an entire plant may be seen. When planted, the embryo stem absorbs food from the inclosing thickened leaves, sprouts, and develops into an elongated stem bearing true leaves. As the stem elongates, it sends up the leaves and flower blossom above the ground. The true leaves manufacture more food than is needed and the surplus is sent down to the under-ground stem, where it is again stored to form another bulb. Sometimes, more than one bulb will be formed. Onions, tulips, and some lilies are examples of plants that may be propagated by bulbs. A collection of bulbs may be seen in the narcissus. Bulbs are frequently dug up from the soil so they will not freeze in winter. Before they are again planted, they are separated from each other and planted singly. 
In all the forms of propagation discussed, the continuance of the species is insured. Only one organism is involved in the process. A part of one organism propagates an entire new organism. Consequently they are all forms of asexual reproduction.

\section{Questions And Suggestions}

1. What is vegetative propagation? Why is it a method of reproduction?

2. Name three artificial methods of vegetative propagation.

3. Set up an experiment to illustrate propagation by cutting.

4. What is the value of cuttings?

5. Discuss regeneration.

6. Devise an experiment to show how grafting is done.

7. Name and describe five natural methods of vegetative propagation.

8. Discuss two methods of vegetative propagation by over-ground stems.

9. Discuss three methods of vegetative propagation by underground stems.

10. Secure, and bring to class, some plants that propagate by layering, by runners, and by rhizomes.

11. Plant a tuber and a bulb and observe their growth. Draw them in various stages of growth.

\section{SuPPLEMENTARY Readings}

Gager, C. S., General Botany (P. Blakiston's Son \& Co.).

Transeau, E. N., General Botany (World Book Co.). 


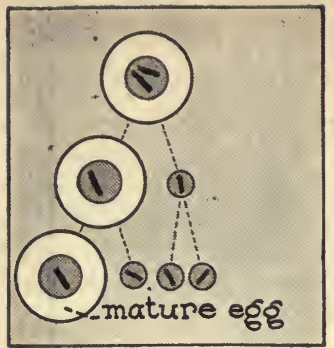

Maturation of an egg.
CHAPTER XXVIII

SEXUAL REPRODUCTION

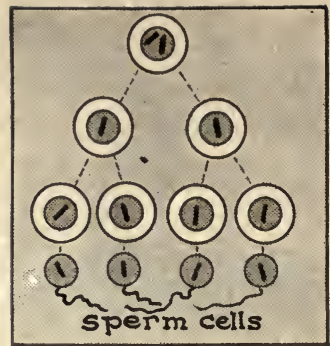

Maturation of a sperm.

What is sex? What are some different types of sexual reproduction?

Sexual reproduction in mold. In many organisms, instead of a single organism developing directly from a half or a part of another one, two special cells from different organisms unite to form one cell. The cell resulting from this union develops into a new organism. The two cells that unite are called gametes, and the cell formed as a result of the union is called a zygote. The formation of a zygote is called sexual reproduction. Certain lower plants reproduce both asexually and sexually. Normally, the bread mold reproduces by asexual spores. When growth conditions are unfavorable, a sexual method of reproduction may occur in this organism. Two threads or hyphae of mold plants will grow toward each other until the tips meet. A cell wall forms near the end of each tip, cutting off a part of the protoplasm at the end of each hypha. These are similar in size and appearance, and are known as the gametes. The intervening walls between the gametes are dissolved and the contents of the two cells intermingle. A thick wall develops around the fused material, the zygote. As the zygote grows the outer wall becomes black. The structure in this stage is called a zygospore. Under favorable conditions the zygote germinates, and develops into a new plant.

When the uniting gametes of an organism are.very similar in size and activity, their union is called conjugation. Therefore, a 
zygospore may be defined as a structure resulting from the conjugation of similar gametes. Although the hyphae of a mold all look somewhat alike, there must be a physiological or chemical difference between them. It has been observed that there are two types of hyphae, which have been named the plus and the minus strains. If a plus strain meets a minus strain, conjugation will occur. It is now possible to isolate or to purchase plus and minus strains of mold spores. When these are planted on opposite sides of a slice of moistened bread, hyphae will grow out from each strain and when they meet, zygospores are formed.

Sexual reproduction in Spirogyra. During sexual reproduction in the Spirogyra, portions of the cell walls between the two filaments grow perpendicular projections and form a bridge. The cell walls in the middle of the bridge are dissolved, probably by the action of enzymes. Then through the
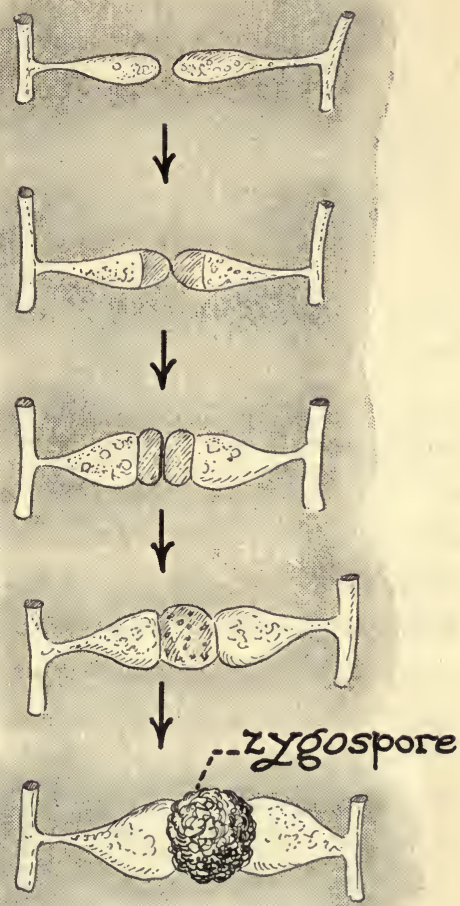

Hyphae of mold plants grow toward each other and meet. In the area of contact, material from each intermingle to form a zygospore. work of vacuoles the entire contents of the cell in one filament are moved across the bridge and fused with the protoplasm of the other cell. After conjugation, a thick cell wall develops around the fused protoplasm and the structure is known as a zygospore. If one cell in a filament is an active cell, that is, its contents pass 


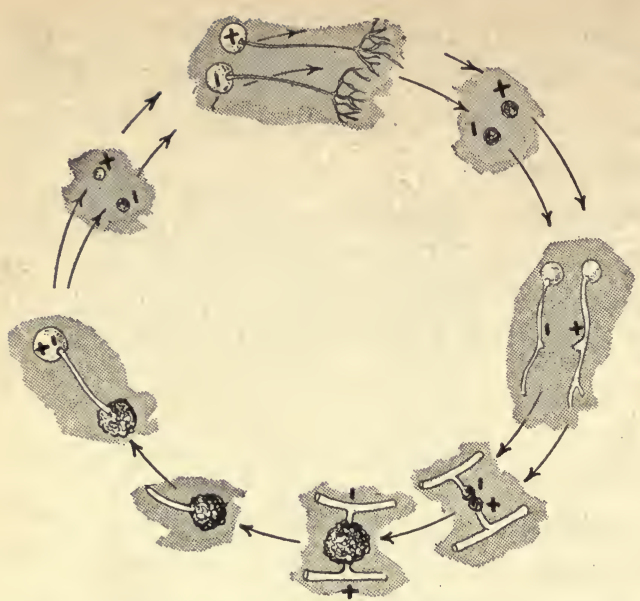

There seems to be two different strains of bread mold called plus and minus. The hypha of unlike strains attract each other and zygospores are formed.

through the bridge, all the cells in that filament are active cells. If one individual in a filament is a passive cell, all other cells of that filament are passive or receiving cells. There is more physiological differentiation in the Spirogyra than in the mold, in that the gametes behave differently. The active gametes may be compared to male gametes of higher plants and animals, the passive, or receiving gametes, to female gametes. After the zygospores are formed, the filament sheath breaks down, the cell walls disintegrate, and the zygospores fall to the bottom of the pond. They stay in a dormant condition until there is sufficient water and warmth to pro-
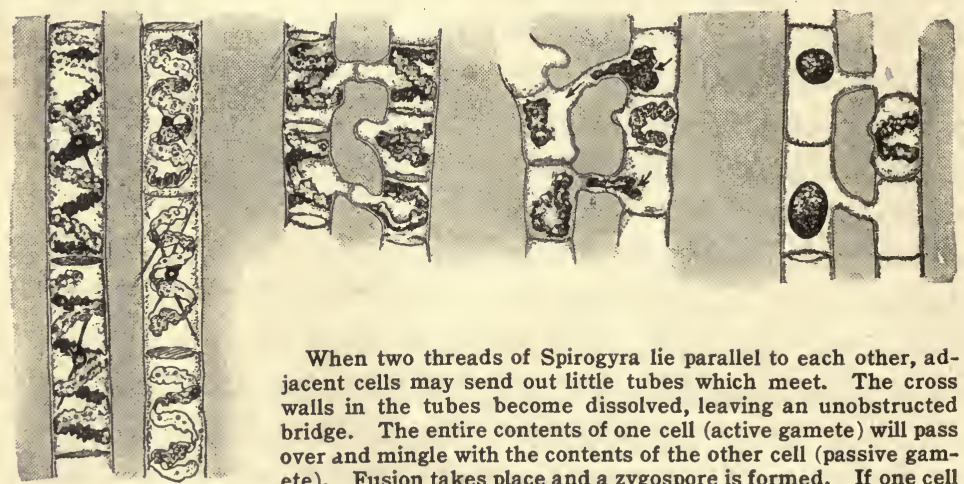

When two threads of Spirogyra lie parallel to each other, adjacent cells may send out little tubes which meet. The cross walls in the tubes become dissolved, leaving an unobstructed bridge. The entire contents of one cell (active gamete) will pass over and mingle with the contents of the other cell (passive gamete). Fusion takes place and a zygospore is formed. If one cell in a filament has a moving gamete, the contents of all the cells of that filament behave in a similar way. 
mote their germination. Then each zygospore absorbs water, breaks the zygospore case, and forms a new filament as a result of binary fission. There like or isogometes

is enough stored food in the zygospore to start the germination and sustain life until the filament can make its own food.

Sexual reproduction in Paramecium. Paramecia

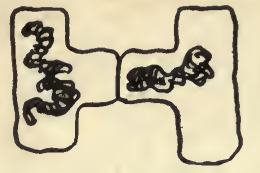

Each of the cells which fuse during conjugation is known as a gamete. When very nearly alike they are called isogametes. Conjugation is the result of the fusion of part or all of the cell contents of isogametes. The result of the union is a zygospore. ordinarily reproduce asexually by binary fission. Occasionally, sexual reproduction takes place. Two cells lie next to and in contact with each other. At the point of contact the cell membranes dissolve. Various complicated changes take place in the nuclei of the two animals. The macronucleus in each breaks up and disintegrates. The micronuclei go through a number of divisions and finally one fragment of each micronucleus passes over and unites with a fragment of the micronucleus in the opposite cell. After this mutual exchange and fusion of micronuclear material, the two

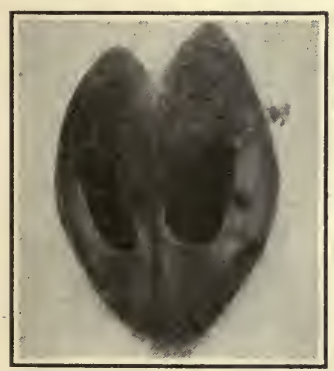

A photomicrograph showing the conjugation of Paramecia.
Paramecia separate, and the micronucleus of each goes through further complicated divisions, which result in the formation of both a micronucleus and a macronucleus. The conjugation of the Paramecia seems to bring about a renewed vigor. In certain species conjugation may occur once in every two or three hundred generations, although these same species may live a very long time, providing conditions are favorable, without ever reproducing sexually.

Certain recent experiments have shown that the environment affects the vitality of the Paramecium. By removing wastes and 
by keeping proper food present, thousands of generations have been produced from a single healthy individual of a certain species.

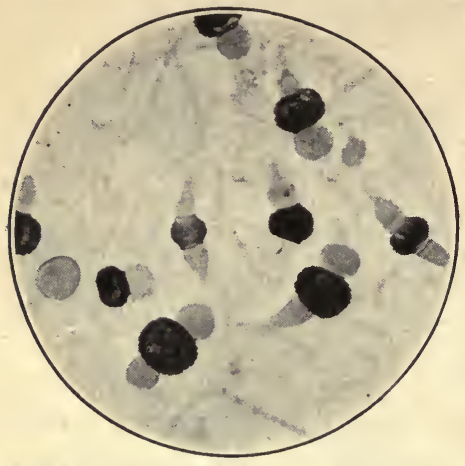

The photomicrograph of a mold culture which shows several zygospores.

Under these conditions, conjugation has not taken place between any two animals, but certain internal changes occurred which were observed and described.

Sexual reproduction in higher plants and animals. In the sexual method of reproduction of the Spirogyra, the entire cell acts as the gamete. In the Paramecium, simply a part of the micronucleus acts as the gamete, but like-sized portions of the micronucleus unite. When gametes are similar they are known as isogametes or like gametes. In most higher plants and animals, certain specialized organs produce cells that form gametes. There are two kinds of gametes. One, a very small cell usually equipped with a motile tail, is called the microgamete or the sperm cell. The other type of reproductive cell is the receiving or passive cell. It is larger and is called the macrogamete or egg. It usually stores food particles called yolk granules. When the egg is ripe, a sperm may penetrate the egg. The nucleus of the sperm fuses with the nucleus of the egg. This process is called fertilization. The cell formed by the union of the egg and sperm cell is known as a zygote.

Maturation. Every species of an organism has a constant number of chromosomes.

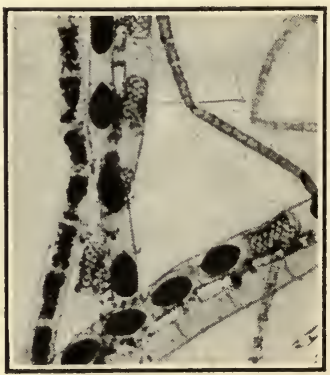

Photomicrograph of filaments of Spirogyra showing conjugation and zygospores. The number is always the same for that species. During fertilization, when two cells unite, the number of chromosomes would 
double were it not for certain changes which occur in the development of the egg and sperm. These changes are called maturation or the ripening of the gametes. The cells that are to produce the germ cells or gametes are the primary sex cells. They have the same number of chromosomes as the somatic or body cells. When ready to ma-

unlike gametes

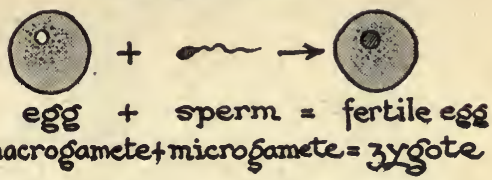

Fertilization is the union of dissimilar gametes; the union results in a zygote or a fertilized egg. ture, the sex cells split by a process much like mitosis, but the chromosomes do not split lengthwise. Instead of splitting evenly, one half the total number of unsplit chromosomes goes into one daughter cell and one half goes into the other. This is the part of the ripening or maturation process called reduction division.

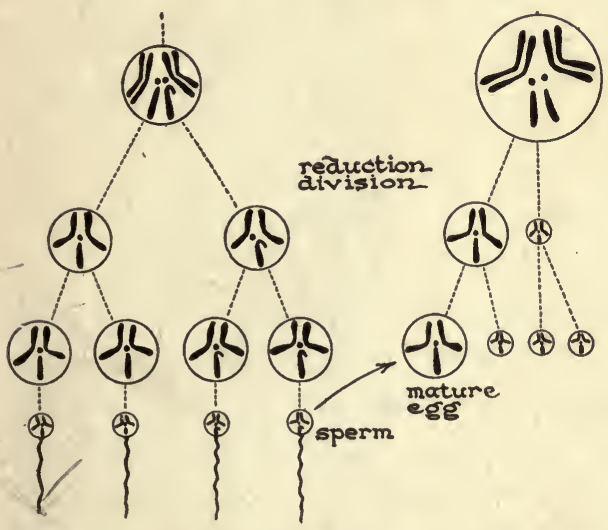

The primary sex cells of the fruit fly have eight chromosomes. During the ripening process, reduction division takes place; the resulting cells have one half the original number, in this case four chromosomes. Each of these cells then divides, mitotically forming the sex cells. From each primary male sex cell there forms four sperms, all of which can function. Each primary female sex cell gives rise to one egg and three reduced cells known as polar cells. The egg has most of the yolk and is generally the only one of the female sex cells to function as a gamete. One sperm unites with a mature egg in the process of fertilization.
The number of chromosomes is reduced one half. After this, each of these cells again divides, mitotically. Thus from every primary sex cell, cells are produced, each of which has half the original number of chromosomes. If the primary sex cell had eight chromosomes, each gamete would have half this number or four chromosomes. The production of the sperms by maturation of the pri. mary sex cells is called spermatogenesis; the 
production of the mature eggs is called oögenesis. All of the spermatozoöns or sperms of a species are of the same size and structure. All may function. Each of them has a tail-like structure of cytoplasm called the flagellum. By means of the fla-

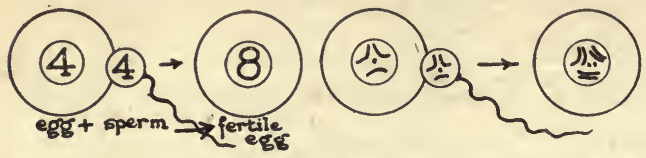

Many sperms of animals may swim about the mature egg. One sperm penetrates the cell membrane of the egg and carries, in this case, four chromosomes. These are added to the reduced number in the nucleus of the mature egg. The union of sperm and egg with the restoring of the original number of eight chromosomes is fertilization. gellum the sperms are able to move about. Oögenesis is essentially the same as spermatogenesis. One large cell is the real functioning gamete, called the mature egg. It contains the food supply or yolk. In the process of maturation of the egg, three minute cells known as polar bodies are thrown off.

Fertilization. After the maturation process, the gametes formed from the primary sex cells are ready for union or fertilization. The sperm is always in a moist environment and by means of its flagellum swims to the egg. In some eggs there is a small opening in the membrane called the micropyle. Through this opening one sperm enters. When an egg lacks the micropyle, a sperm penetrates the plasma membrane. The head of the sperm contains the nucleus. The flagellum or tail of the sperm is usually left outside of the egg. After a sperm nucleus enters the egg, a chemical change takes place within the cell membrane. This permanently seals the egg so that'no more sperms can enter. The nucleus of the egg and of the sperm fuse. The entrance of the sperm restores the original number of chromosomes in the egg cell. Mitosis of the fertilized egg then takes place. It differs from typical mitosis in that half the number of chromosomes came from the sperm and half came from the egg. The chromosomes are said to be paternal and maternal in origin. Immediately, mitotic division or cleavage takes place and two cells are formed, each with the same number of chromosomes, as the species 
to which the parents belonged. From here on the cells cleave or split again and again by mitosis until enough cells are formed to take on the shape of the plant or animal embryo. The yolk in the egg supplies food for the rapidly dividing cells. Fertilization produces a variety of characters by combining chromosomes from different individuals. This re-

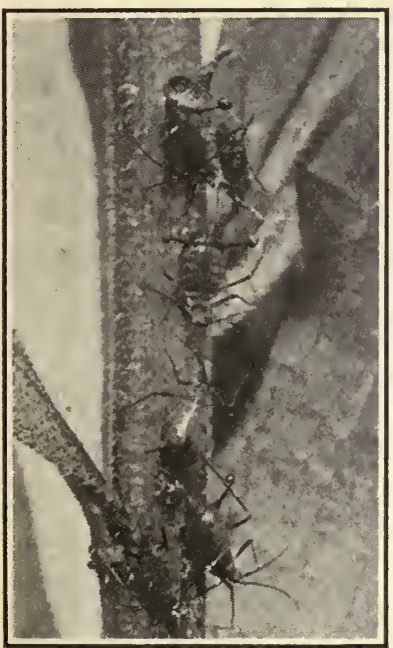

Spring and summer aphids or plant lice are wingless females which produce young, parthenogenetically, e very 10 to 20 days. In the autumn, males are produced, mating occurs, and fertilized eggs are laid which last over the winter and hatch into females the following spring.
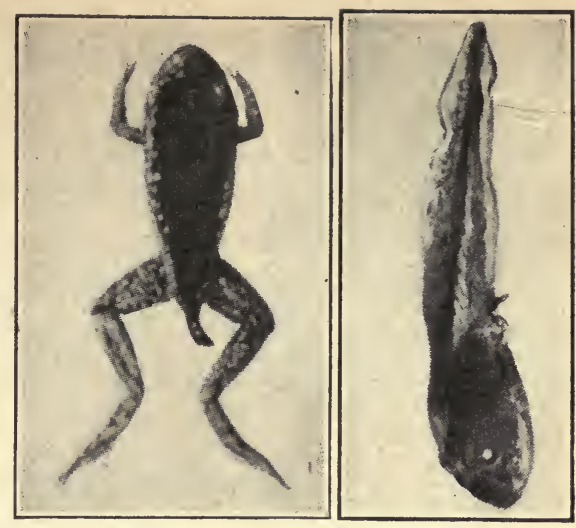

The parthenogenetic frogs produced experimentally by Jacques Loeb resembled normal frogs in their appearance.

sults in the possibility of slight or great differences among offspring.

Parthenogenesis. It has been stated that the entrance of. a sperm into an egg stimulates a chemical change in the membrane which seals the micropyle, and stimulates the egg to go through repeated divisions. The late Jacques Loeb, formerly at the Rockefeller Insti.tute, carried on a series of experiments to investigate the nature of the fertilization process. He pricked the eggs of sea urchins with an electric needle and found that this brought about a membrane activity and the egg began to cleave. He next found that certain chemicals caused the same results. This process of the development of the egg without the entrance of a sperm 
is known as parthenogenesis. Loeb succeeded in developing, parthenogenetically, frogs' eggs. Several reached the tadpole stage. A few grew to be nearly adult in appearance and then died. Their development was incomplete. Parthenogenetically developed organisms may have only half the number of chromosomes common to the species, since no sperm chromosomes are present. The process has never been performed, experimentally, in any organism higher than the frog. It sometimes occurs in nature in certain of the insects and certain worms.

\section{Questions AND Suggestions}

1. What is the difference between sexual and asexual reproduction?

2. Discuss sexual reproduction in the mold; in the Spirogyra.

3. State a difference between conjugation in mold and Spirogyra.

4. Discuss sexual reproduction in Paramecium.

5. What is the value of sexual reproduction in the life history of the Paramecium.

6. Compare fertilization with conjugation.

7. Discuss spermatogenesis; oögenesis.

8. What is the purpose of maturation?

9. State two cellular activities of the egg that always follow the entrance of sperm.

10. State two differences between an unfertilized and a fertilized egg.

11. What is parthenogenesis? Discuss some experiments that have been carried on in parthenogenesis.

\section{SUPPLEMENTARY READing}

Atkinson, G. F., A College Textbook of Botany (Henry Holt \& Co.).

Gager, C. S., General Botany (P. Blakiston's Son \& Co.).

Holmes, S. J., An Introduction to General Biology (Harcourt, Brace \& Co.). 


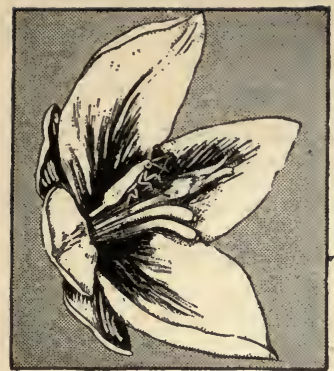

Insect pollination.
CHAPTER XXIX

REPRODUCTION

OF HIGHER

PLANTS

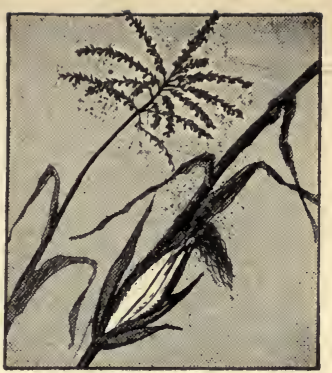

Wind pollination.

How are new plants formed? What is the function of a seed and of the fruit? What are some different types of fruits?

A flowering plant consists of the nutritive organs, the roots, stems and leaves, and the reproductive organs, which are found in the flower. The reproductive organs in the flower produce specialized cells, gametes, which function in sexual reproduction.

Problem.' Study of the tulip.

If the tulip is not in season, the gladiolus, sedum, or any perfect flower may be used. (The flower was probably studied in elementary science, so certain facts learned at that time are not emphasized in this exercise.)

I. Remove the three outer sepals and the three petals found within them.

$A$. What is the relation of the position of these parts to the organs found in the center of the flower?

$B$. What are the functions of the sepals and petals?

II. Locate the organs in the center of the flower. Why are they called essential organs?

$A$. The single enlarged structure found in the center of the flower is the pistil. The upper end comprises the stigma. The lower part of the swollen stalk is the ovary.

$B$. The structures with spear-shaped heads arranged around the pistil are the stamens. The heads of the stamens, the anthers, produce the pollen. How does the pollen escape from the anthers?

C. Draw a pistil. Label stigma and ovary. Draw a stamen. Label anther and pollen. 
III. Shake some pollen from the anther on to a glass slide. Mount under the microscope.

A. How many cells make up each pollen grain?

$B$. Describe the shape, color, and structure of pollen.

$C$. Draw and label several pollen grains enlarged ten times.

IV. Cut a thin cross section of the ovary of the pistil. Examine it with a hand lens.

A. The seedlike structures are the ovules. Note that the ovules are attached to the ovary wall and are not loose in the ovary. The part of the ovary wall to which the ovules are attached is called the placenta.

V. Place an ovule on a glass slide. Cover it with a cover glass and crush it by carefully pressing down with the cover glass.

A. Does an ovule consist of one or more cells?

B. Note a central structure. This is the embryo sac containing the egg cell.

$C$. Draw the outline of an ovule showing the position of the embryo sac.

Structure of the flower. The flower consists of a calyx made up of sepals and a corolla made up of petals. While the essential

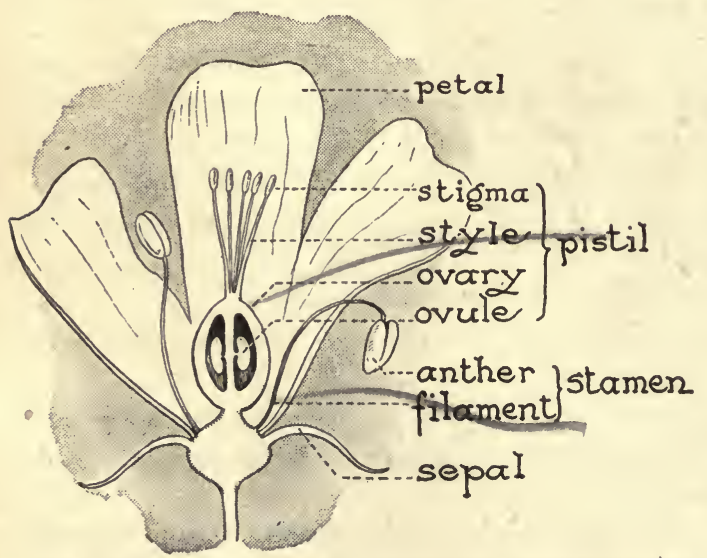

The essential organs for the reproduction of higher plants are the stamens and pistils. Accessory organs are the sepals and petals. The sepals enfold the entire flower, forming a bud. Thus the organs are protected while they are ripening. The petals are frequently showy and attract insects. This may result in pollination. The stamens produce pollen and the pistil produces ovules. organs are ripen. ing, they are protected from rain, insects, and certain mechanical injuries by the tightly enfolding calyx and corolla. The whole structure com. prises the bud. As the essential organs ripen, the calyx and corolla unfold and expose the matured pistils and stamens. 
Problem. What effect has a sugar solution on pollen grains?

Make up cane sugar solutions of thirty-five per cent, ten per cent, and three per cent. Take three hangingdrop glass slides and on each one lay a cover glass from the center of which hangs a drop of sugar solution containing some pollen. Use a different sugar solution for each slide. Seal the cover slip air tight by means of vaseline. (Petri dishes or Syracuse watch crystals may, also, be used.) Different pollen requires different concentrations of sugar solution in order to grow. A three-per-cent sugar solution is usually needed for tulip, narcissus, and onion; fifteen per cent for sweet pea and nasturtium. Let the preparations stand for one or two days. Then mount under the microscope.

I. Describe the shape of the pollen grains when first produced by the anther.

II. Describe what has happened to the grains.

A. These outgrowths are called pollen tubes.

$B$. Describe the food for the pollen tubes which you have grown.

III. Carefully crush the tip of a ripe stigma on a glass slide and mount under the microscope.

$A$. Do you find any pollen grains here?

1. How did they get on the stigma?

$B$. Do any pollen grains show pollen tubes?

1. In which direction are they growing?

2. Where must the germinating pollen tubes obtain their food?

IV. Draw and label a germinating pollen grain.

Production of the male gamete. Pollen is produced in the anther of the stamen. Certain cells in the anther go through a maturing process in which reduction division takes place.

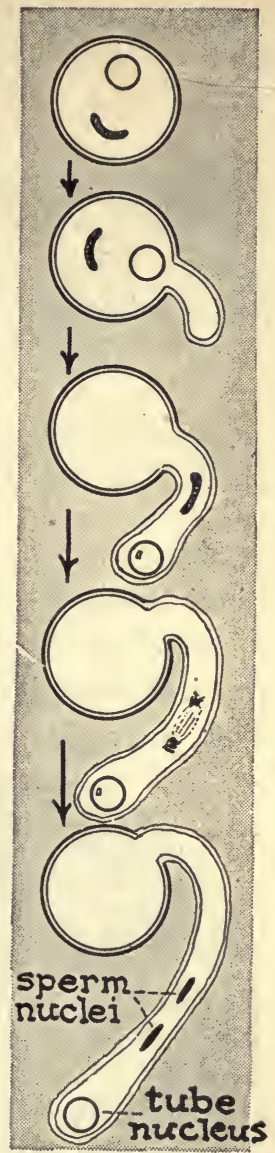

A pollen grain has two nuclei, one a tube nucleus, the other a generative nucleus. As the pollen grain forms the pollen tube, male gametes (the sperm nuclei) are produced from the generative nucleus. Mature pollen nuclei contain one half the number of chromosomes characteristic of the other cells of that particular plant. When 
mature, the ripe anther of the stamen splits and scatters the pollen. Agencies, such as wind, insects, or water, may assist in

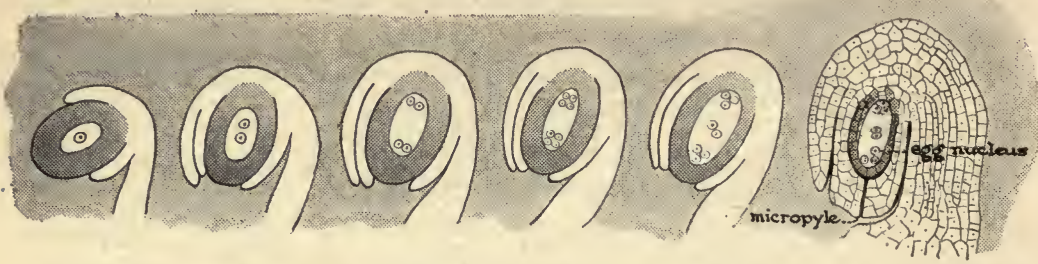

Complicated nuclear divisions in the embryo sac of the ovule of a plant result in the formation of eight nuclei, of which one is an egg nucleus and two are polar nuclei. The two polar nuclei lie near the center of the cell. The egg nucleus is the female gamete.

scattering the pollen. When pollen reaches the stigma of the pistil, it may be caught and held by hairy outgrowths particularly adapted for that purpose. The stigma then secretes certain nutrient materials which the pollen absorbs. After this pollination; the pollen grain germinates by sending out a slender thread-

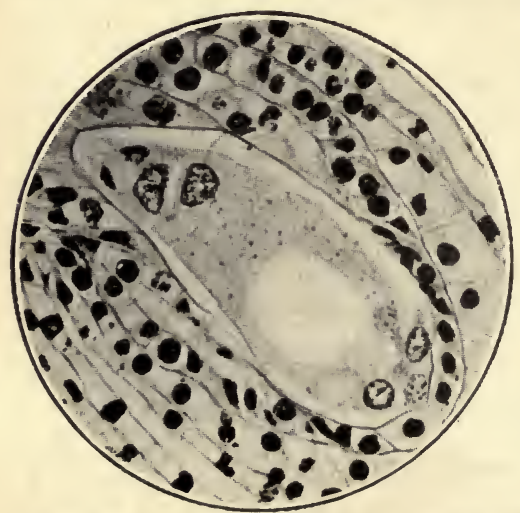

A photomicrograph of a part of an ovule showing the nuclei in the embryo sac. Compare it with the diagram at the top of the page. like tube. The pollen tube grows down through the pistil and penetrates the ovule through an opening called the micropyle. As it grows, certain nuclear divisions take place, which produce two sperm nuclei. The sperm nuclei with their reduced number of chromosomes are the male gametes of the plant.

- Production of the female gamete. Each ovule in the ovary is formed as the outgrowth of a few cells from the ovary wall. One of the cells in the interior of the young ovule appears larger and richer in 
protoplasm than the other cells. It divides, and, in the division, reduces the number of chromosomes by one half. There are a number of complicated divisions that follow. Ultimately, an egg

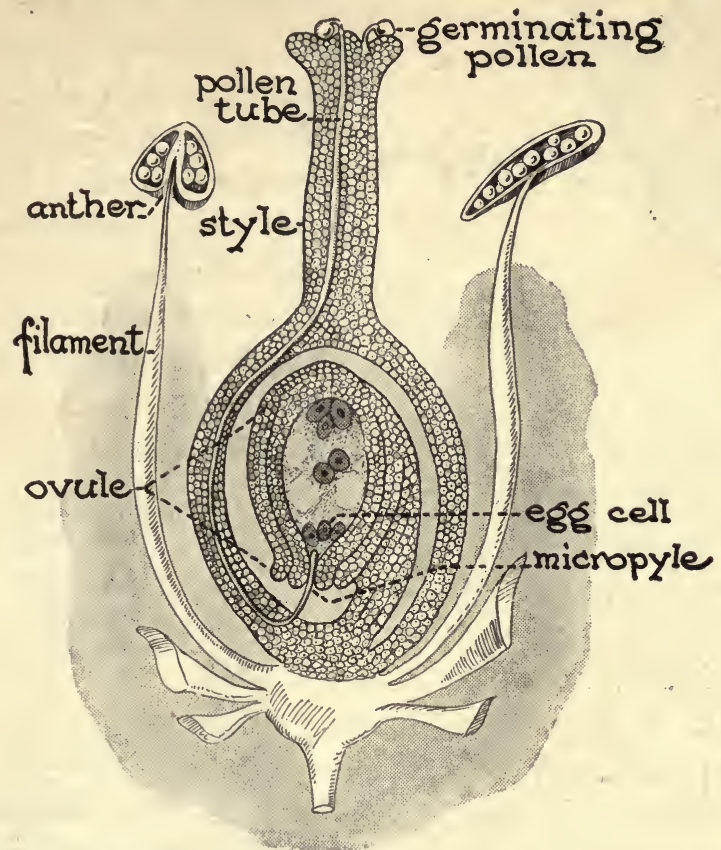

A longitudinal section of a pistil shows pollen grains germinating through the pistil in the form of tubes. One tube is shown penetrating the micropyle of the ovule. The pollen tubes carries the sperm cells to the embryo sac of the ovule.

nucleus and two polar nuclei are produced among other nuclei found in the embryo sac. The egg nucleus with its reduced number of chromosomes is the female gamete of the plant.

Fertilization. When the end of the pollen tube gets to the embryo sac, the wall of the pollen tube and the wall of the embryo sac are dissolved and one of the two sperm nuclei unites with the egg cell nucleus. The two polar nuclei fuse and the second sperm nucleus sometimes unites with them. This completes 


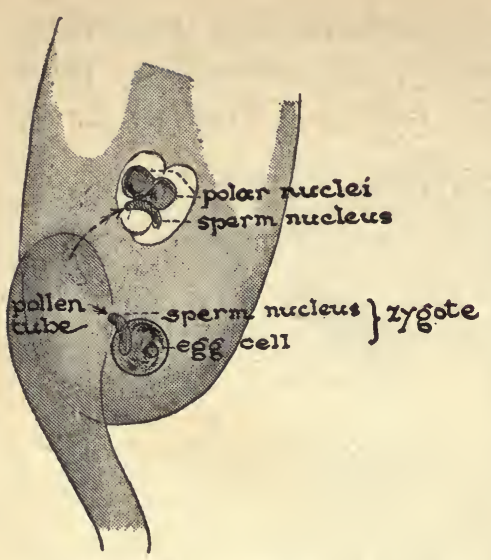

In the process of fertilization in a flower a sperm nucleus unites with an egg cell nucleus to form the embryo of the future plant. The other sperm nucleus unites with a pair of nuclei, the polar nuclei. This latter union results in the eadosperm nucleus which divides and, in time, forms the food supply for the embryo. Double fertilization is characteristic of flowering plants.

placenta of the ovary to the ovule. Food passes through these ducts, which nourishes and effects the rapid division or cleavage of cells in the tiny embryo in the ovule. At the same time, the endosperm tissue of the ovule grows rapidly and stores the future food supply for the embryo. As the embryo develops into a many-celled structure, differentiation of the cells sets in and the first root or hypocotyl, the first bud or plumule, and the seed leaves or cotyledons are formed. The endosperm develops at the same time. the process of fertilization. The union of the sperm and egg nuclei forms the one-celled embryo which develops into a tiny plant. The union of the other sperm nucleus and the polar nuclei forms the cell that starts the endosperm which is the food supply of the tiny plant. This double fertilization results in the future plant and its food supply.

Formation of the seed and fruit. There are ducts going from the plant through the

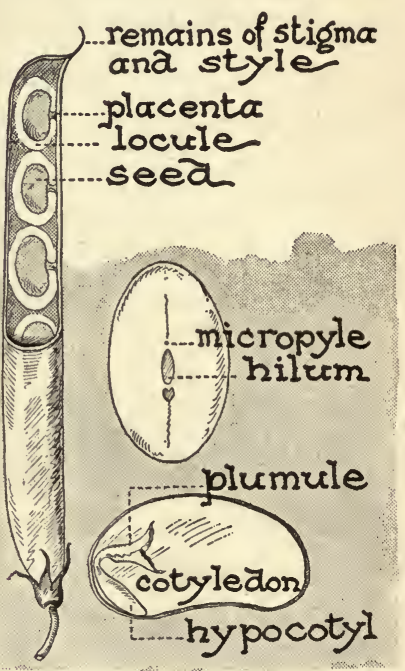

After fertilization takes place in a flower, the petals, stamens, and frequently the stigmas of the pistil dry and generally fall off. The embryo and endosperm develon and the ovule coats grow to accommodate the increased size of the seeds. The pod with its contents is the fruit. 
In the grains, the endosperm is a well-developed, localized, and easily identified structure; in seeds such as beans, peas, and many nuts, the cotyledons possess the food supply for the developing plant.

While the embryo and endosperm are developing, the ovule coats absorb food from the ducts and develop into seed coats. The ripened ovule and its contents constitute the seed. The ovary wall grows to accommodate the developing seeds and forms the fruit. The fruit protects the seeds until they are completely developed. Fruits are frequently adapted to disperse the ripe seeds. These seeds escape from the fruit, and the embryos they contain will develop into new plants, if they fall on moist soil of proper temperature.

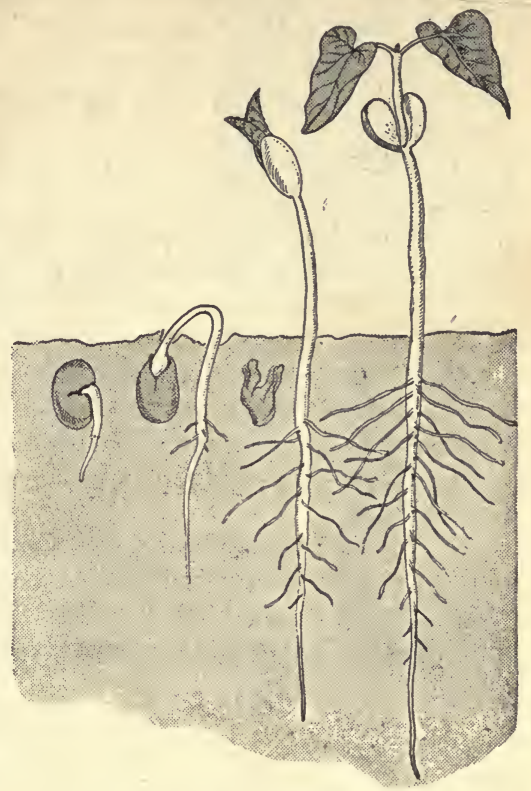

Given the proper conditions of moisture and warmth, a new plant will develop from the embryo in the seed. Which of the organs of the plants is developed from the hypocotyl?

The fruit is a ripened ovary and its contents, together with any other part of the plant that has ripened with it.

When the bean seed is planted, it absorbs water, and sends a little arched shoot, the hypocotyl, into the ground. The lower seed or radicle forms the root system. As the upper part or true hypocotyl straightens out, it brings the plumule above the ground. This forms the stem and leaves. The cotyledons feed the tiny plant until its leaves are able to make sufficient food to carry on the life process. The cotyledons may either remain underground or be lifted into the air by the growth of the hypocotyl. 


\section{Questions $\Lambda$ nd Suggestions}

1. Name the parts of a flower and give the function of each.

2. What do we mean by self- and cross-pollination? What are some agents of cross-pollination?

3. Will the pollen of a daisy germinate on a rose? How do you know?

4. Give the history of the pollen grain from the time it is produced until it functions.

5. Give the history of an ovule until fertilization takes place.

6. Discuss the fertilization of an ovule.

7. How many seeds can possibly be produced in any plant?

8. What three processes are necessary for the formation of a seed?

9. Discuss the development of a seed and a fruit.

10. Discuss the adaptations of the following plants for dispersing their seeds : maple, elm, thistle, Bidens, pea, apple.

11. Give examples of seeds or fruits dispersed by (a) wind; (b) animals.

12. Cut a longitudinal section of some fruit (apple, cucumber, watermelon, pea). Draw and label seed, seed stalk, placenta, ovary wall.

13. Plant a bean seed. When it appears above ground, uproot it and draw. Label hypocotyl, plumule, and cotyledons. 


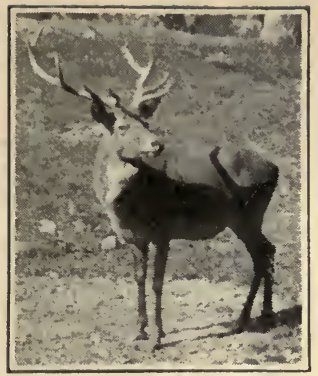

N. Y. Zoölogical Soc.

The buck has antlers.

\section{CHAPTER XXX}

REPRODUCTION

OF ANIMALS

Is the reproduction of an animal similar to that of a plant? Do all animals reproduce in the same way? How does an animal develop from the fertilized egg? How long does the development take?

Seeds and eggs. The common form of propagation for higher plants is through the formation of seeds, and for higher animals, through the production of fertile eggs. Most seeds are capable of sprouting even after remaining at rest for a long time, sometimes after many years. The germs in the seeds are destroyed, however, if the seeds become very wet and then dry again.

The eggs of chickens and other birds are well known. The eggs of fish and frogs are somewhat similar to those of birds, although they are found in the water. Eggs of butterflies may frequently be seen on plants. Those of water animals usually die if they become dry. Birds' eggs have to be kept warm if they are to incubate and to hatch. Those of insects may lie dormant over the winter and develop in the spring. In general, eggs need more care than seeds. In the case of many animals, the eggs must develop soon after they are formed or they will die. They cannot live in a dormant form for an indeterminate period of time as seeds can.

Secondary sexual characters. In the lowest animals, it is often quite impossible to distinguish the individuals that produce male WH. FITZ. AD. BIO. -19281 
gametes from those that produce female gametes. Among the frogs, fishes, and other lower animals, there is little external morphological difference in sex, but the higher species of animals show external differences. In addition to the structures that are directly related to producing and discharging gametes, distinct characteristics are found in other parts of the body. The males of many species of birds can be easily recognized by the crests on the heads and spurs on the legs. The male birds are usually more gayly colored and have sweeter songs than the female birds.

Among many mammals (animals that are warm-blooded, covered with hair, and suckle their young), there are usually differences in horns, size of body, habits, voice, temperament, and interests. Contrast the male reindeer with the doe. The male is a huge animal with tremendously large antlers. He is a vicious fighter and protects the doe. She lacks antlers, is proportionately small and slender, and usually depends upon the male for protection. The differences just noted are called the secondary sexual characters of reindeers.

Spawning of frogs. Fertilization takes place in animals in a way very similar to plants. The gametes mature and are then brought together in a way peculiar to the organism. Different
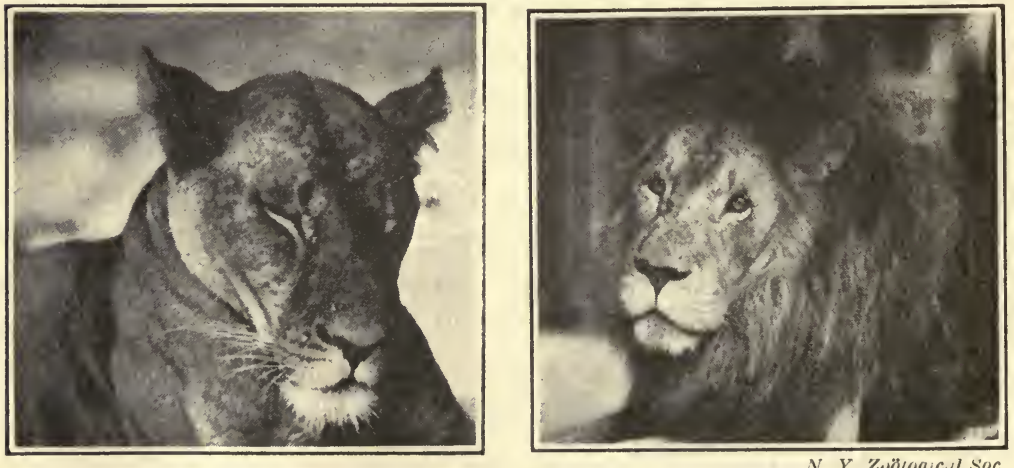

The lioness and lion show interesting secondary sexual characters. Note the huge head and shaggy mane of the lion. 
species of frogs reach maturity in different periods of time. At maturity, the female frog lays fifty or a hundred eggs, ova, in the water. Each ovum consists of a tiny cell surrounded by yolk or food which is covered with a gelatinous coating. The adult male frog discharges sperms into the water. These sperms are motile, swimming by means of tiny tails. If a sperm comes in contact with an egg, it penetrates the egg membrane, and the
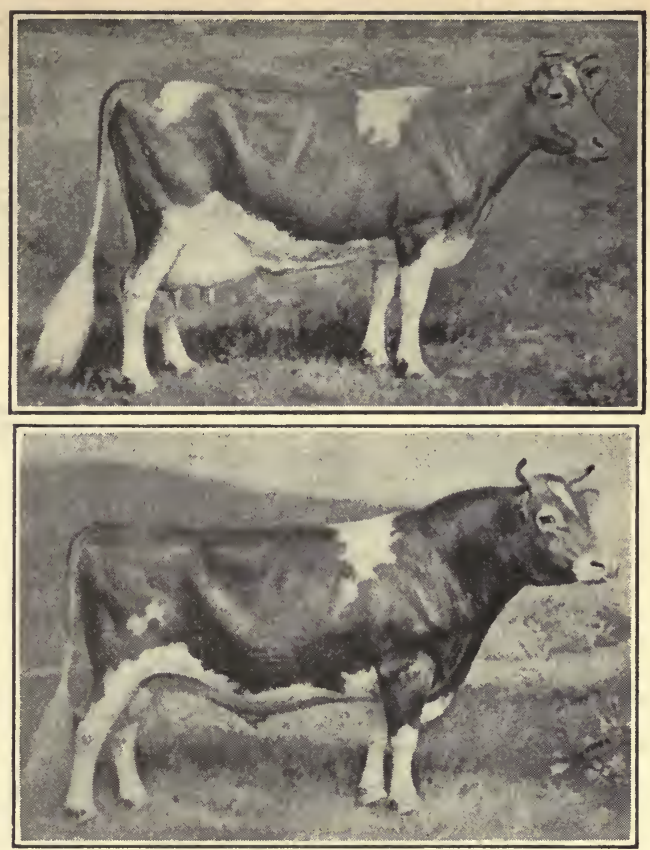

The cow, placid in disposition, is easily distinguished from the excitable and frequently dangerous bull.

nuclei of the egg and sperm unite in the fertilization process. If the eggs are not fertilized, they soon die and disintegrate. If sperms do not reach eggs, they, too, are wasted. The fertilization of the frog's. eggs is external to the body and takes place in the water. This differs from the plant where fertilization was internal, taking place in the ovary of the pistil. After fertilization, the gelatinous sheaths of the frog's eggs absorb water and swell. This mass of foamy material insures some degree of protection against enemies. Fish or other frogs cannot readily swallow this huge mass of gelatinous eggs.

Fish spawn in a way very similar to frogs. They usually go to quiet, shallow waters for spawning, although a few species of 

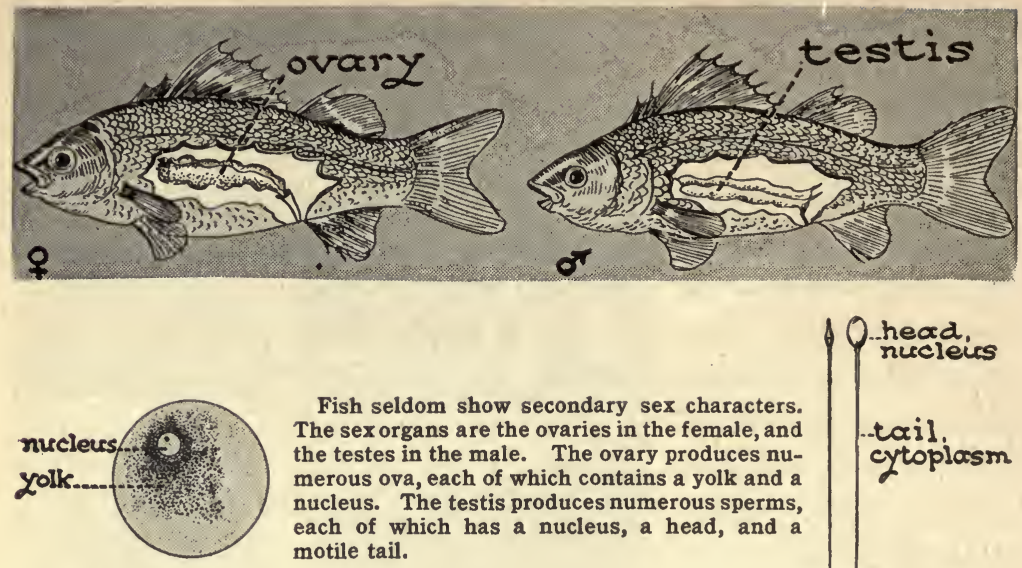

Fish seldom show secondary sex characters. The sex organs are the ovaries in the female, and the testes in the male. The ovary produces numerous ova, each of which contains a yolk and a nucleus. The testis produces numerous sperms, each of which has a nucleus, a head, and a motile tail.

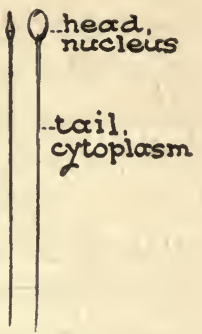

river fish spawn in the ocean. So powerful is the urge to spawn in natural spawning grounds, that the salmons will leap falls or artificial barriers in their efforts to reach quiet water on their way from the deep sea. Many die of exhaustion on their way if they meet too many obstacles. Those that surmount the barriers usually die soon after spawning.

Production of eggs. Certain organs of the frog are specialized for the production of gametes. In the female, the two ovaries secrete hundreds of tiny ova. The ovaries may be compared to the ovaries or pistils of flowers which produce egg cells. Ovaries are common to all females in the animal kingdom. The ova of the frog go through a maturation process in which the number of chromosomes is reduced one half. This process results in the production of mature gametes. The mature ova reach the body cavity of the frog and then pass through the tubes called oviducts into the cloaca. They leave the cloaca by means of an opening at the posterior end of the female frog's body and pass into the surrounding water. Ova are characterized by their compara- 
tively large size, due to the yolk in them, and the fact that they are incapable of independent motion.

Production of sperms. The pair of organs that secretes the male gametes or sperms is the testes. The sperms, too, in the maturation process lose half their chromosomes. By means of tubes the mature sperms of the frog pass from the testes into the cloaca and out of the body. These sperms are known as milt. Each sperm is a single cell consisting largely of a nucleus. The cytoplasm is drawn out to form a tail. By means of the tail lashing back and forward, the sperm swims in the water. (Probably a chemical attraction draws the sperm to the egg.) The nuclei of the sperm and egg fuse in the fertilization process, forming a fertilized egg cell, the one-celled embryo. In this process the original number of chromosomes is restored.

The entrance of the sperm into the egg effects the initial development of the egg as well as brings about variation by combining the chromosomes from two parents. Each fertilized egg contains one-half maternal and one-half paternal chromosomes.

Development of the frog. The fertilized egg or embryo absorbs food from the yolk. It grows and divides mitotically to form a two-celled stage. These two cells feed, grow, and divide to form a four-celled stage. Mitotic divisions continue, forming various many-celled stages called cleavage stages. Finally, the solid mass of cells, the morula, becomes arranged in the form of a singlelayered hollow ball of

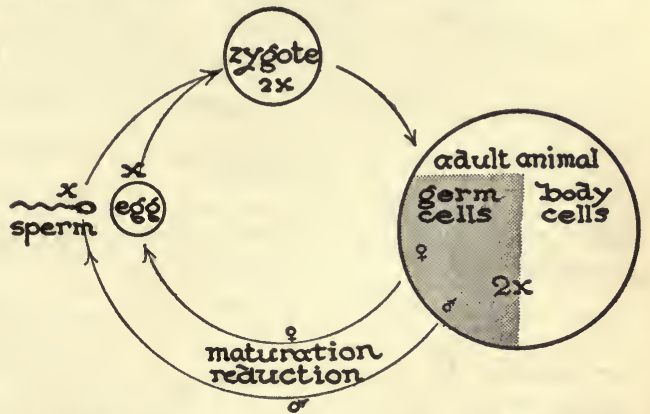

Primary sex cells are formed early in the life of an organism. They contain the same number of chromosomes, $2 x$, as body cells. In the maturing of the sex cells to form sperms and eggs, the chromosomes become reduced to half the number, $x$. When the sperm unites with the egg to form the zygote, the $2 x$ number is again restored. 
cells known as the blastula. The blastula pushes in just as a rubber ball may be dented in by pushing on one side. A double-layered,

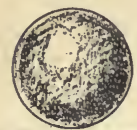

fertile egg

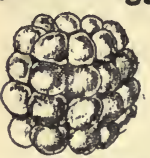

32 cellea embryo

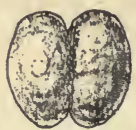

2-cellea

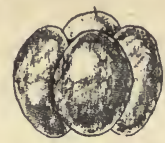

4.cellea

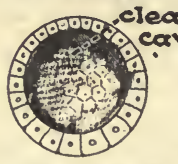

hollow ball. blastula

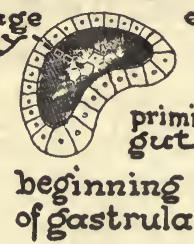

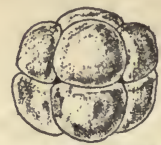

8-ellea

ectoaerm.

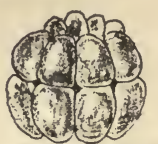

16-cellea enaoaerm.
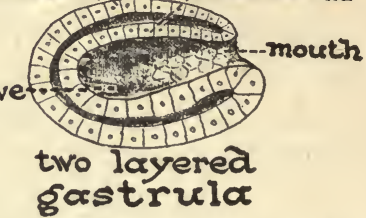

The embryo begins as a single fertilized egg. This divides to form two, then four, and clea vage continues until a great many cells are formed. Gradually differentiation sets in and specialized structures may be recognized. Thus the various organs of the organism develop.

cup-shaped structure known as the gastrula is thus formed. The embryo is now two-layered. The outer layer is the ectoderm, the in-

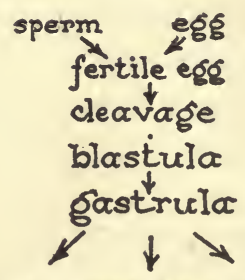

ectoderm mesoderm endoderm. epracrmis muscles

central nervous system receptors

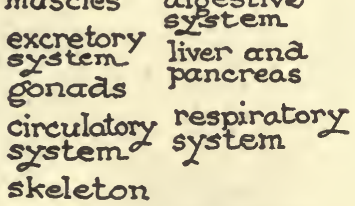

The fertile egg, byrepeated divisions and certain changes, becomes the gastrula with its three primary germ layers. Further differentiation and development goes on and the organ systems arise. ner layer the endoderm. A middle layer, the mesoderm, soon appears between the ectoderm and the endoderm. The folded edges of the gastrula grow toward each other, forming a tiny mouth. Marked differentiation now starts and forms the characteristic tadpole. The skin and nervous system are formed from the ectoderm. The digestive and respiratory systems are formed from the endoderm. The excretory, reproductive, muscular, skeletal, and blood systems form from the mesoderm. During this development the yolk is entirely absorbed by the actively dividing cells. 
Life history of the frog. The little tadpole then hatches from the gelatinous egg. For the first two or three days, it remains attached to grass by a sucker-like mouth. Then it begins to feed on algae and other vegetable matter. When first hatched, it has $e x$ ternal gills, which grow out into long, branching tufts. Later, four pairs of internal gills are formed and the external gills are absorbed. The hind limbs soon appear; later, the fore limbs develop. The tail then decreases in size and is gradually absorbed. The gills, too, are absorbed, and lungs are formed to take their place. The two-chambered

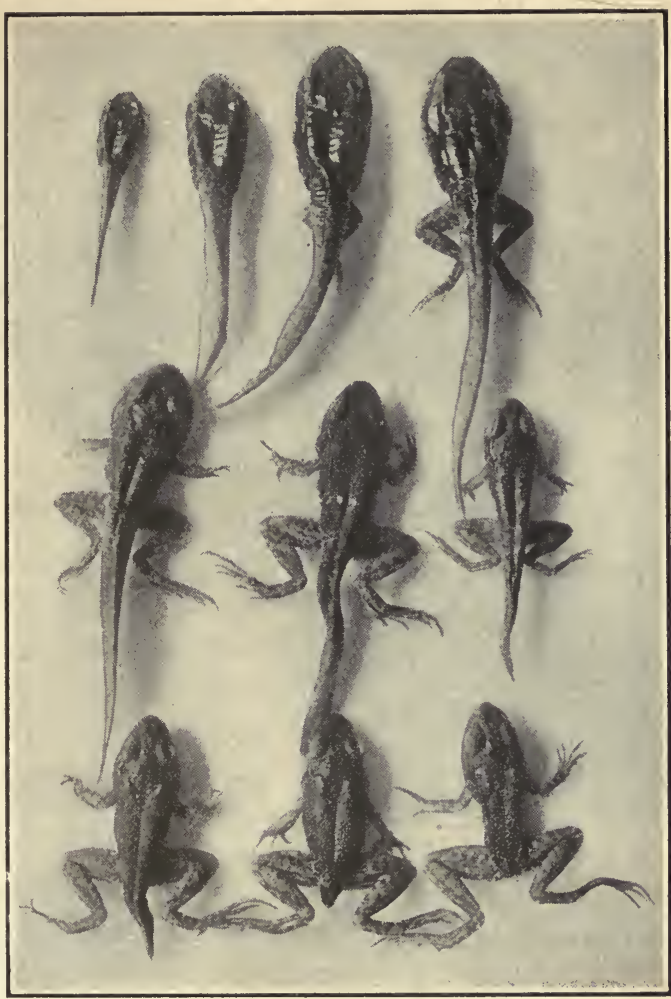

N. Y. Zoölogical Soc.

The frog first hatches from the egg in the form of a tadpole. Gradual changes take place which transform it into the adult frog. This change of body form is called metamorphosis.

heart of the tadpole becomes the three-chambered heart of the frog. Finally the form resembling that of the adult frog is acquired.

Reproduction of other animals. The reproduction of all higher animals is similar to the reproduction of the frog. All females have ovaries producing eggs, and males have spermaries or testes producing sperms. The sperms fertilize the eggs and thereby 
cause the formation of embryos. Sperm cells must be kept moist in order to function. In the case of frogs and fish, fertilization is

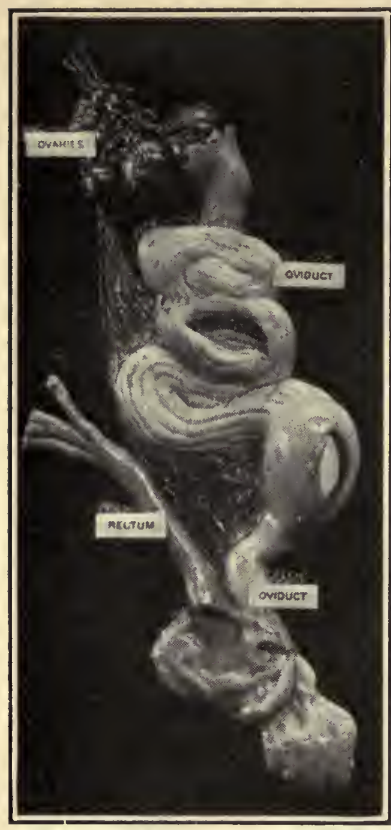

Amer. Museum of Natural History

In the birds, the original egg cell and yolk become surrounded by other materials during the passage down the oviduct. It becomes surroundeci by a coating of albumen, the white of the egg, which is secreted by the glands of the oviduct. A lime coating which forms the shell is then secreted around the whole mass.

In the case of birds, the embryo starts an internal development. The fertilized egg receives a huge deposit of yolk, then albumen is spread around it, and finally it is enveloped in a hard shell of lime. The yolk external and the water keeps the cells moist. In the case of animals that do not live in the water, such as insects, birds, or mammals, the egg cell is retained within the body of the female, and the union of the egg and sperm is internal. Thus the danger of the gametes drying is averted. The embryo develops outside of the body when the fertilization is external. The water also keeps the developing embryo moist. When the fertilization is internal, the embryo may partially develop or may completely develop within the body of the female. For example, the eggs of the insects start developing within the female, but later they are deposited in the ground or on plants and the development continues there.

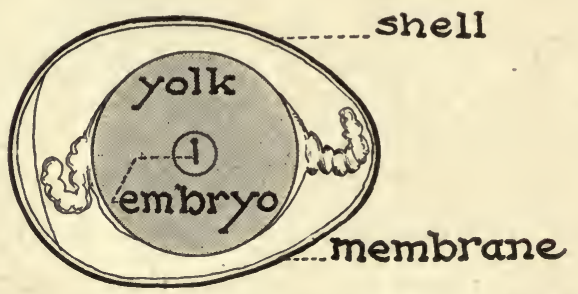

A section through a chicken's egg shows the tiny embryo attached to the yolk. Surrounding the yolk is the albumen which protects the embryo from shock. Two thin membranes within the shell form an air chamber at one end. 
and albumen serve as food for the developing embryo and at the same time keep it moist. The shell permits air to enter, but prevents the evaporation of moisture. Then the mother bird lays the egg, and the development of the embryo continues in the egg, but external to the mother's body.

In mammals, for example the rabbit, the entire development is internal.

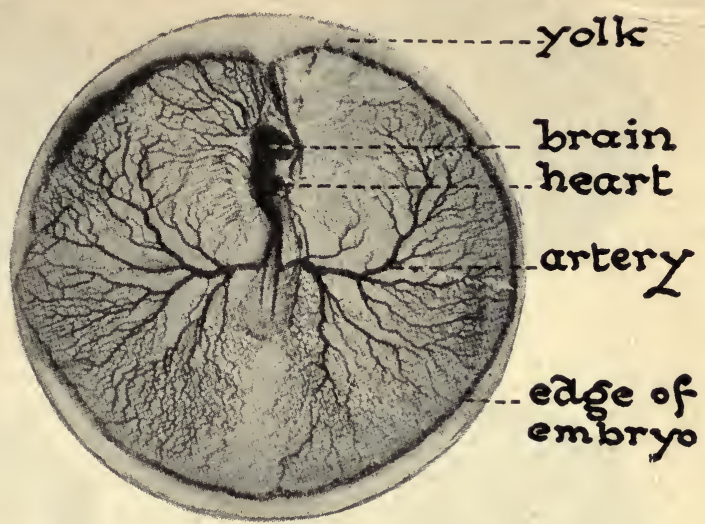

A photomicrograph of a fertile egg that has been incubated for two days. Cell differentiation and specialization have taken place to the extent that the brain, heart, and blood vessels can be recognized.

When the embryo

is completely developed into a young animal, very much like the adult, it is born. The word "birth" is used to describe the process

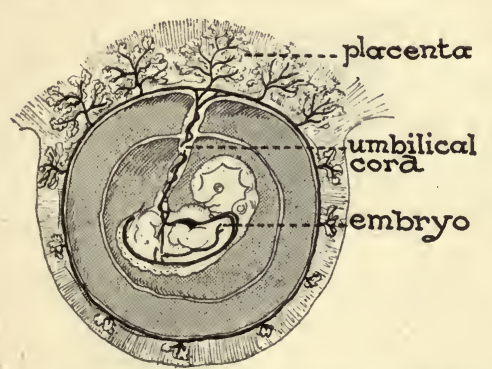

The unborn mammal is suspended in the uterus by the umbilical cord. Surrounding fluids serve as protection against shock. Food for development is absorbed by osmosis through the placenta.

There is a similarity in function between the placenta of flowering plants and the placenta of mammals. They both are the of the little individual coming forth after complete internal development. This is in contrast with hatching, which is the coming forth of an animal from an egg. During its internal development, the mammalian embryo is kept moist and warm within the body of the mother. It is fed by absorbing food from blood vessels which pass through the placenta of the mother organism. 


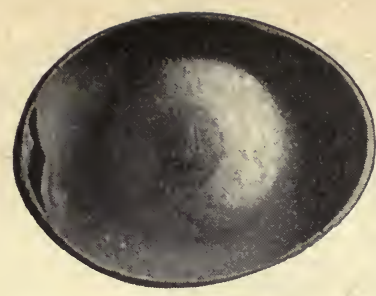

2 days

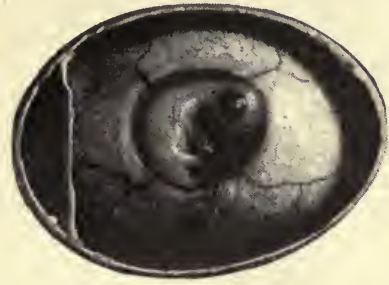

10 days

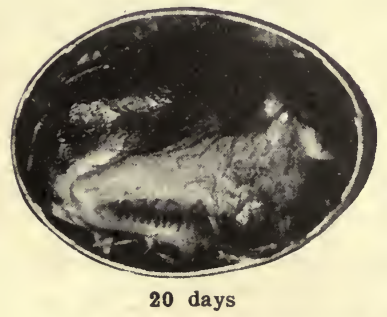

As development of the egg takes place, more and more differentiation occurs. The yolk serves as the food for the developing organism. First the circulating system may be recognized, later the head, limbs, and other organs. When the animal is completely developed, it pecks its way out of its shell.
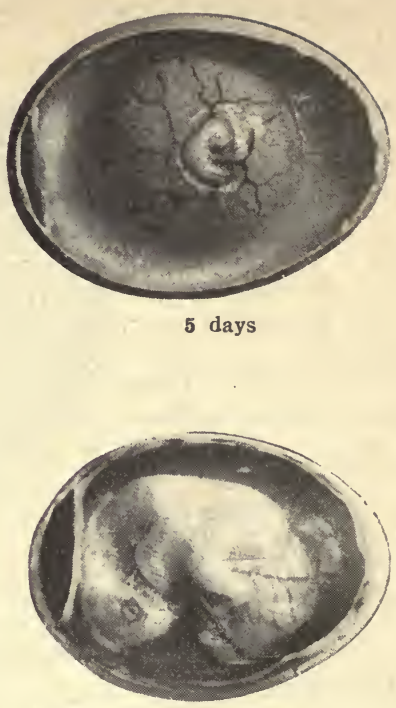

15 days

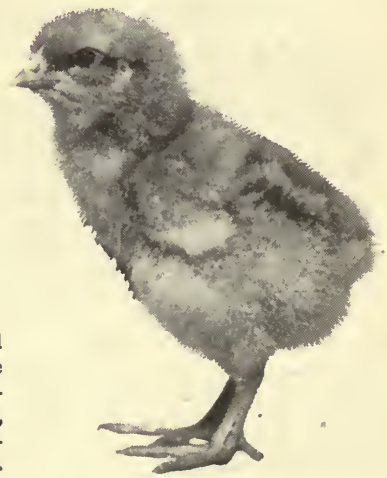


parts of the mother organism, through which the developing embryos are fed. In the case of many plants, ducts and sieve tubes pass through the placenta and through the seed stalk and thus carry nourishment to the seed. In the case of mammals, the blood vessels of the embryo extend into the placenta through a cordlike structure somewhat similar to the seed stalk.

\section{Questions And Suggestions}

1. Name three differences between seeds and eggs.

2. Describe the spawning of frogs.

3. Give the following facts concerning the reproduction of the frog: reproductive organs, the names of reproductive gametes, where fertilization takes place (use the word external or internal), where the organism develops, and the food of the developing embryo.

4. Discuss the complete development of the frog embryo.

5 . Name the germ layers of the embryo and state the organs developing from each layer.

6. Using the outline headings given in question 3 , fill in the outline for a flower, bird, and mammal.

\section{Supplementary Readings}

Atwood, Wm. H., and Heiss, Elwood D., Educational Biology (P. Blakiston's Son \& Co.).

Haupt, Arthur W., Fundamentals of Biology (McGraw-Hill Book Co.).

Holmes, S. J., General Biology (Harcourt, Brace \& Co.).

Jewett, F. G., The Next Generation (Ginn and Co.).

Scott, George G., The Science of Biology (T. Y. Crowell Co.).

Wiggam, A. E., Fruit of the Family Tree (Bobbs-Merrill Co.). 


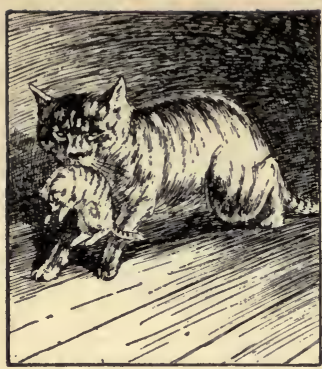

The cat takes care of its kitten.
CHAPTER XXXI

PROTECTION OF YOUNG

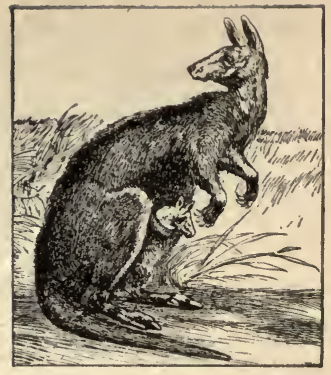

The young kangaroo in its mother's pouch.

What proportion of offspring lives to maturity?

What is the relation of the period of adolescence to length of life? Do all animals take care of their young? What animals show the greatest parental instinct?

We have already learned that all plants and animals start life as single cells. In some species this single cell is a spore, in others a zygospore, and in still others a fertile egg. Whether the creature is a Paramecium or an elephant, it started life as a single cell.

The offspring of simple organisms. When an amoeba, a bacterium, or any other one-celled organism divides into two new cells, the production of the new individuals marks the disappearance of the parent. It is impossible for the daughter cell and the mother cell to exist at the same time. Yet, it would not be true to say that the mother cell dies, for the protoplasm of which it consisted continues to live in the two new daughter cells. Each of the young amoebas is quite as capable of taking care of itself as is the older individual from which each came. Except for the growth in the comparatively short time between one cell division and the next, which in the case of some organisms would be only about twenty minutes, the young individual is in every way like the full-grown individual. There is, probably, some difference in size, however. 
Among the lowest plants and animals that produce spores or encysted cells, the new individual usually has a cell wall or coat that is somewhat thickened. This is a means of protection against drought, mechanical injury, or perhaps against the digestive juices of some animal that might ingest it. The cell also contains a tiny drop of oil or some other excess of food material that will sustain life until the protoplasm is able to obtain food through its own activities. Such cells are usually produced in large numbers and are deposited in every current of water and air. Only a very small proportion of such reproductive cells ever starts a new life. It is very largely a matter of chance which one will and which one will not mature. For example, mold spores are scattered by the bursting of the sporangium. They are found practically everywhere; but only those that fall upon food in a favorable environment will develop.

Infancy among seed plants. In the seed-bearing plants, each generation receives from the preceding one a great deal more than a quantity of protoplasm. The gametes are produced in proportionately small numbers, compared to the reproductive elements of seedless plants. The female gametes especially are very few, only one to each ovule. Then there is a great variety of structures whose function is to make fertilization possible and probable. For example, the display of the flower by means of the showy corolla and fragrance will attract the insects that are in search of nectar. In taking the nectar, the insects will become covered with pollen which they will later, accidentally, transfer to other flowers. The position and character of the stigma of each flower show special adaptations to catch pollen, and to aid and effect the formation of the pollen tube. All these are parts of the equipment of the parent plants, which aid in the process of fertilization.

The fertilized ovum or zygote is retained within the ovule. It is supplied with nourishment which the young embryo uses up in developing. The growth of the embryo goes on to a certain 


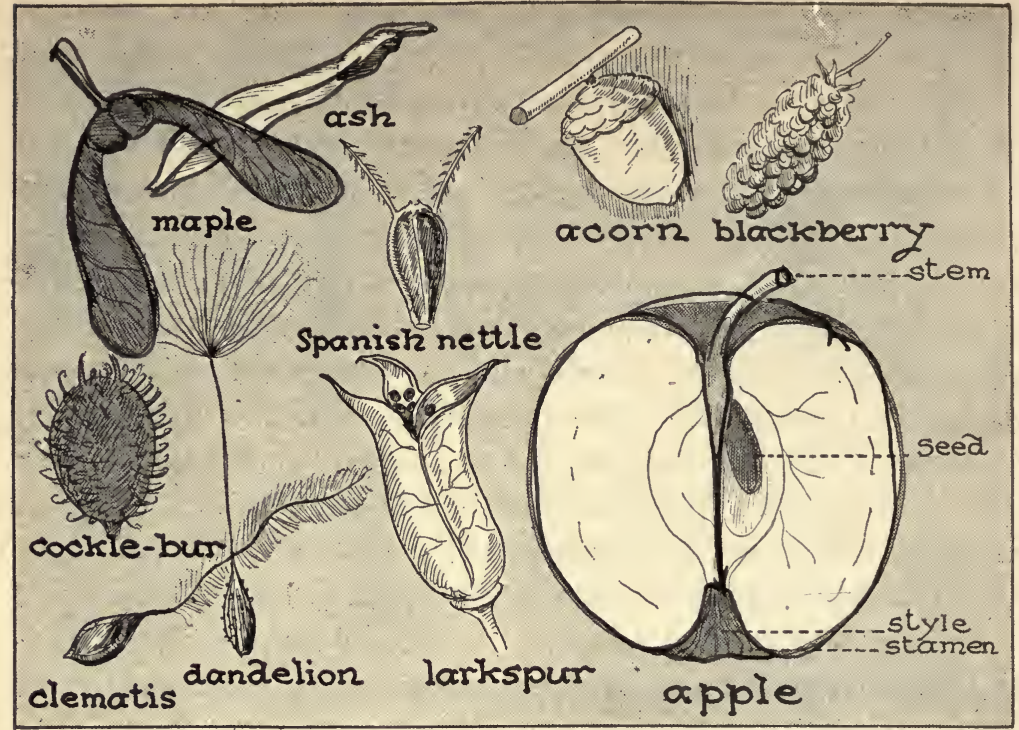

Fruits and seeds are frequently adapted for dispersal from the parent plant. Winglike and feathery structures are adaptations for wind dispersal ; barbs and sweet fleshy parts are adaptations for dispersal by animals.

point and then stops. A surplus of nourishment, in the form of endosperm or cotyledons, may be stored about the young embryo. Additional protective covers generally grow about the seed. All of these processes and structures are clearly relatéd to protecting the young plant and supplying it with nourishment that it can use until it develops the leaves which make its own food.

The seed cover, or the fruit, often possesses a variety of structures such as wings, stickers, fleshy pulp, or down, which aid the young seed to travel some distance from the parent plant. These structures are also the result of parental activity, and they contribute to conditions that make for the favorable growth of the young plant. If the young plants were to grow too close to the parent plant, they might not secure sufficient sunlight for starch manufacture nor enough minerals from the soil for protein man- 
ufacture. The seed is protected while within the fruit. It has been supplied with food which it can use long after it has left the ovary, and it has been supplied by the parent plant with some adaptation in order to get away to a more favorable environment.

Infancy among lower animals. Frogs, fish, and other water animals usually produce large numbers of eggs and sperms and discharge these into the surrounding water. Probably many thousand more sperms than eggs are produced. Even though a large proportion of the eggs may become fertilized, most of the sperms die as they greatly outnumber the eggs. Probably only a small proportion of the fertilized eggs ever get far beyond the earliest stages of development They are frequently devoured by animals in the water. Because fertilization takes place in the water and the embryos develop externally, a great majority of the young die or are destroyed.

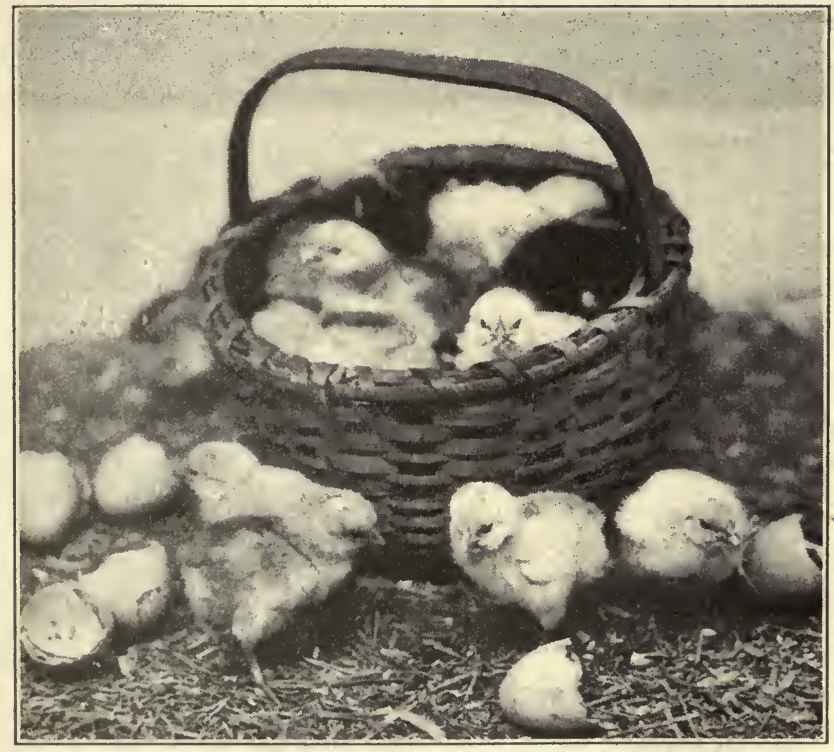

H. Armstrong Roberts

The mother hen watches over, shelters, and helps the chicks gather food. 
There is very little care of the young among the lower animals such as frogs and fishes. In many cases the parent dies before

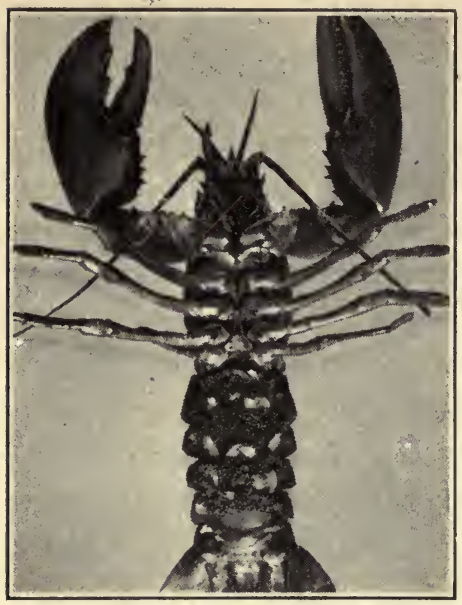

Museum of Natural History

The female lobster carries the eggs while they are developing. Special hairs attached to the appendages of the abdomen secrete a sticky substance which holds the eggs in place. This secures protection. the egg has had time to hatch. The fertilized eggs are usually left unprotected. In an occasional species there is some evidence of what may be called parental care. For example, in some fishes, the parent may find a protected spot, as under a rock, and lay a mass of eggs. The salmon and other fish that spend part of their lives in salt water and part in fresh water, migrate many miles up large rivers and deposit eggs in shallow water far from their natural enemies. Yet, of the one or two million eggs laid by each female, only a very few will ever reach maturity. Among the crustaceans (lobsters, crabs, shrimps) the female sometimes produces a sticky fluid about the eggs. As the eggs come out of the body and become fertilized, they are attached to the swimmerets on the abdomen of the female and remain there until the embryos are ready to hatch. Among the mollusks (oysters and clams) the fertilized eggs remain within the cavity of

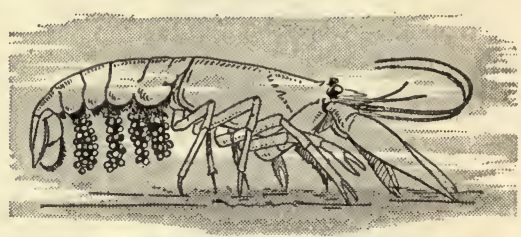

The, female crayfish not only carries her eggs attached to the swimmerets, but by straightening her abdomen and waving the swimmerets brings the eggs in contact with a supply of oxygen. the mother's mantle and so are protected by the shell until the young hatch and are able to swim. 
Infancy among insects. There is a wide range of egg-laying habits among insects. Some kinds of insects leave the eggs almost anywhere. Others lay the eggs in a material that is likely to furnish food for the young as soon as the eggs hatch. The grasshopper lays her eggs in the ground and never sees them again. The butterfly lays her eggs on a leaf and, shortly afterwards, dies. The fly deposits eggs in decaying meat or other organic matter so that food is present for the fly larvae which hatch out of the eggs.

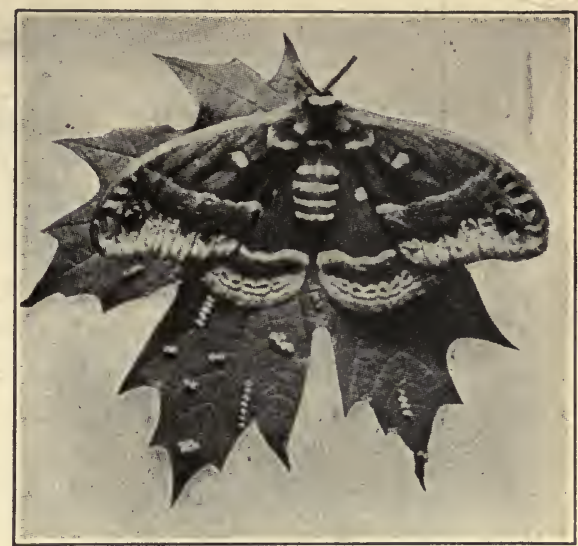

The butterfly usually lays her eggs on the food that will be used by the tiny larvae. When the little caterpillars emerge from the eggs, they begin to eat the leaves upon which they hatched.

The elaborate preparation for the laying of eggs of ants, bees,

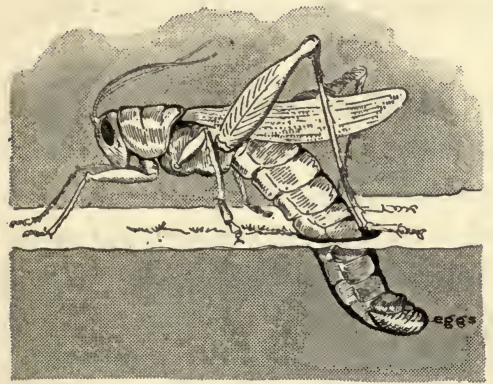

In the autumn, the female grasshopper lays fertilized eggs in a hole she digs in the ground. These eggs stay in the ground during the winter. The rays from the sun of the late spring warm the earth and incubate the eggs. Small newly hatched grasshoppers crawl from the earth. wasps, and termites is remarkable. The solitary wasps show great industry and ingenuity in building their nests, in catching caterpillars or spiders, in treating the prey to prevent decay, and in packing these victims into a nest with the eggs. The adults die or fly away shortly after completing such a nest, and they never have a chance to see their offspring. In the bee colony, the young are remarkably protected. The eggs are laid in certain cells of the hive. When the egg hatches into a minute, footless grub or larva, it is fed for the WH. FITZ. AD. BIO. -20 
first few days on rich food produced in the stomach of certain of the worker bees that act as nurses. Later, they are fed with a

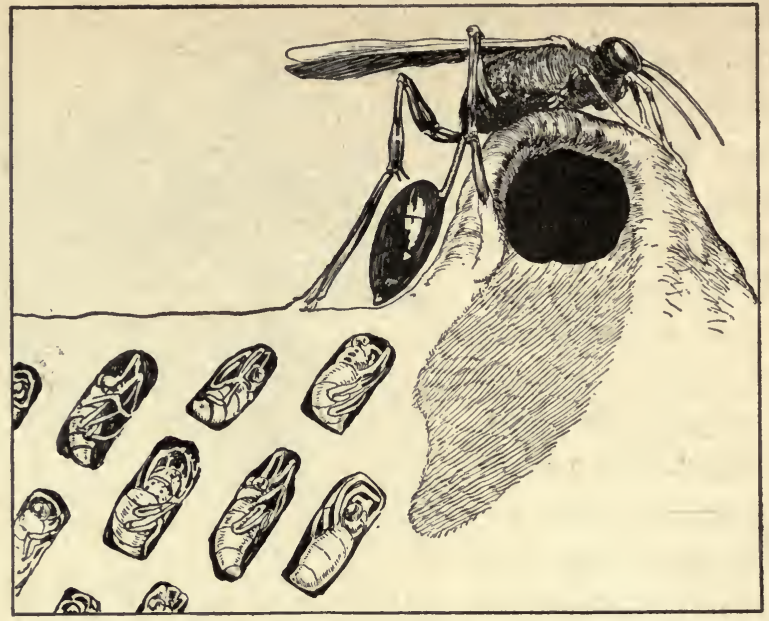

The digger wasp fills her nest with spiders stung in such a way as to paralyze and preserve them. An egg is laid on each spider; when the larva hatches it is well supplied with food.

mixture of pollen and honey. Certain of the worker bees act as soldiers to guard the hive.

Infancy among birds. Among the birds, the new individual receives a large amount of protection and food for a very long period. After fertilization, which takes place inside the mother, the egg immediately starts to develop. The yolk and albumen represent large food supplies, and the shell is an effective protection. After the egg is laid in a specially made nest, the developing embryo is kept warm by the parent's body. This is known as incubation. It makes possible a safe and rapid development.

Both the parents of many species of birds take part in the nest-building, in protecting the nest, and in incubating the eggs. When the young of some species come out of the eggs, they are fully developed and very much like the adult. The young chick, for example, begins to walk and to pick up food particles almost 
immediately. Among other birds, such as robins and eagles, the young birds are quite helpless. The feathers have not developed, the eyes are closed, and the nestlings are not able to feed themselves. The parents fly about gathering insects, fish, lambs, or whatever it is that constitutes their food. They bring this food home to the nestlings. They protect them from other animals and teach them, in due time, how to fly and get food for themselves. Only when the young are strong enough to fly and forage for themselves are they put out of the nest. Birds lay comparatively few eggs because, due to internal fertilization and parental care, each egg produced is almost sure to develop into a young animal. Birds usually lay one egg at a time, and when the number that is usual for the particular variety of bird is reached, the parents start to incubate the eggs. Compare this number, one to sixteen, with the millions of eggs produced by the salmon and the lack of parental care after the salmon eggs are laid and sprayed with milt.

Infancy among mammals. The embryo of a mammal is protected, by the mother's body, a longer time than that of any other animal. During this period, development goes on, and at birth, the young is easily recognizable as belonging to a particular species. The embryos of different species take varying lengths of time to develop. The rabbit develops

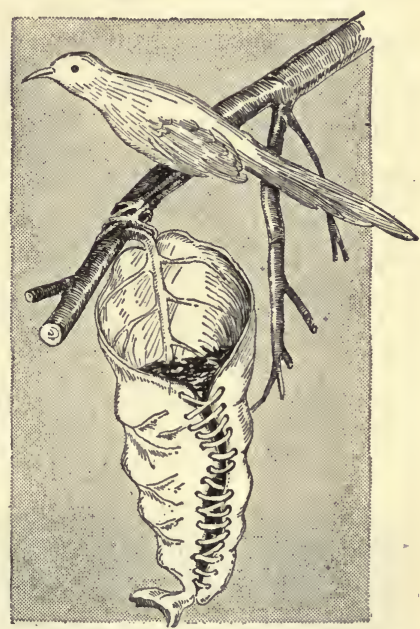

Care of young is often shown by birds. The tailor bird weaves an elaborate hanging nest in which the eggs are hatched and the young cared for. Both the mother and father birds brood over them. One forages for food while the other keeps the nestlings warm. from the fertile egg in three weeks and then is born; the human baby needs about nine months; and the elephant about two years. This shows remarkably rapid develop- 


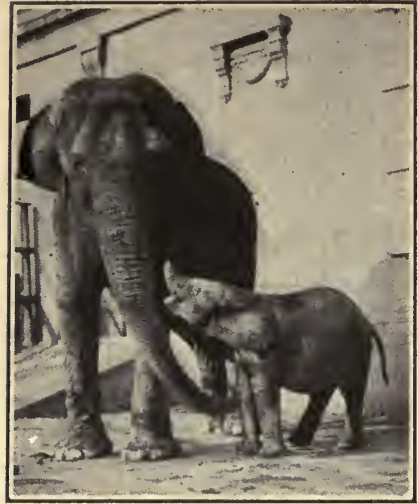

N. Y. Zoological Soc.

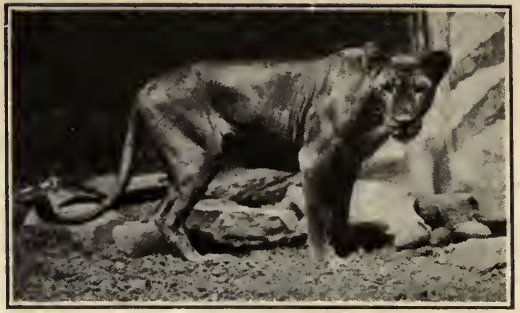

N. Y. Zoölogical Soc.

ment of cells considering the fact that each animal originated as a single fertilized cell.

The kangaroo and the other marsupials, pouch animals, give birth to the young while these are still poorly developed. The babies crawl into the pouch on the mother's abdomen. The skin lining this pouch contains glands that secrete a milky fluid by means of which the young kangaroos feed. All female mammals have well-developed milk glands and normally suckle their young for a long or short period of time.

Some mammals are able to stand or walk immediately after birth. This is true of the calf and of grazing animals generally. Among other species, the new-born young is completely helpless. The kitten does not open its eyes for several days. The human
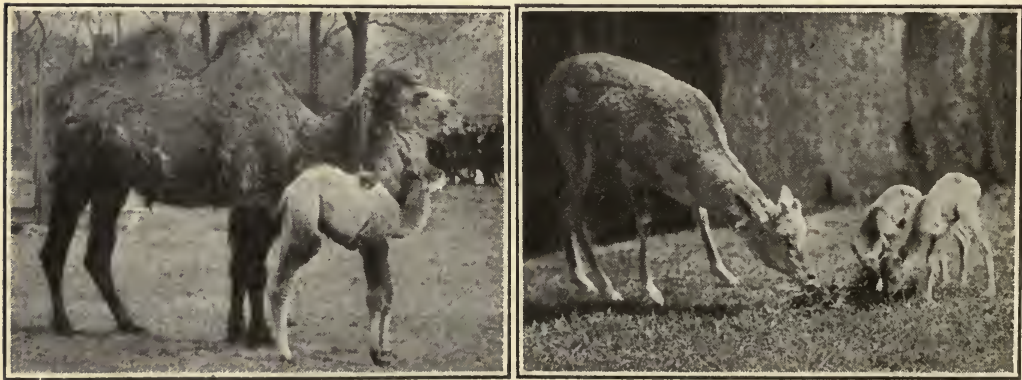

N. Y. Zoßlogical Soc.

Care of young reaches its heights among the mammals. 


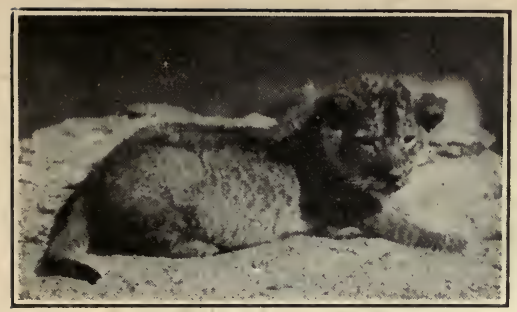

N. Y. Zoölogical Soc.

baby does not begin to walk for a year or more. -Generally, we .find that the higher the species the longer the infant depends on its parents for protection and nourishment.

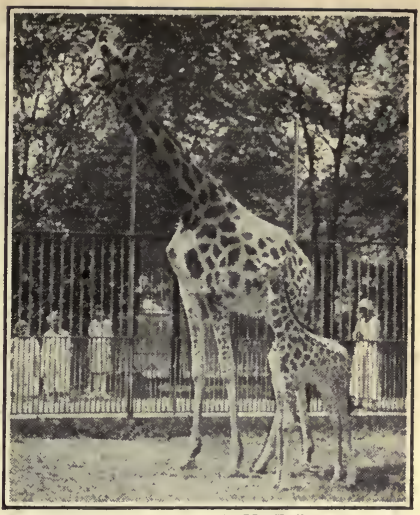

N. Y. Zoölogical Soc.

Infancy in man. In man, protection of the young is shown in the highest degree. Not only is there a long period of dependence of the baby upon the nourishment obtained from the mother's body before birth, but there is a long period of suckling after birth. The greatest care and protection are given the young during its infancy and childhood. As communities become more prosperous, there is a tendency on the part of the parent to postpone for each child the assumption of individual responsibilities.

Among the different races of mankind, and even among different peoples of the same race, there is a great deal of variation with
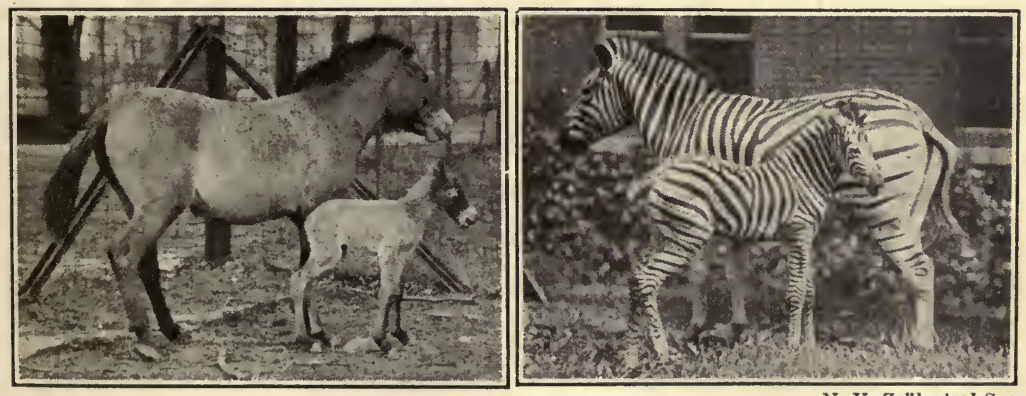

N. Y. Zoß̈logical Soc

Note the length of infancy given in the table on the following page. 


\section{TABLE OF ADOLESCENCE}

\begin{tabular}{|c|c|c|}
\hline Animal & Growing Period & Length of Life \\
\hline $\begin{array}{l}\text { 1. Elephant (Asiatic or } \\
\text { Indian) . }\end{array}$ & $\begin{array}{l}\text { Full grown but not fully } \\
\text { mature at } 25 \text { years. } \\
\text { Full vigor and strength } \\
\text { at } 35 \text {. }\end{array}$ & 70 to 80 years. \\
\hline 2. Camel : . . . & 4 to 6 years. & 20 , very rarely 35 to 40 . \\
\hline 3. Horse . . . & 3 to 4 years. & $\begin{array}{l}18 \text { to } 30 \text { years; very } \\
\text { rarely to } 40 \text { years. }\end{array}$ \\
\hline 4. Cattle . . . & 3 to 4 years. & 14 to 20 years. \\
\hline 5. Sheep . . . . . & 1 to 2 years. & 6 to 10 years. \\
\hline 6. Pig . . . . & 1 to 2 years. & 6 to 12 years. \\
\hline 7. Dog. . . . . & , 2 years. & 12 years. \\
\hline $\begin{array}{r}\text { 8. Norway Rat (Mus } \\
\text { norvegicus) }\end{array}$ & 14 months. & 3 years. \\
\hline 9. Cat.... & 1 to 2 years. & 12 years. \\
\hline 10. Lion . . . . & 3 to 4 years. & 12 to 20 years. \\
\hline 11. Man . . . . & 20 to 25 years. & 65 to 75 years. \\
\hline
\end{tabular}

Compiled by Dr. C. V. Noback, New York Zoölogical Park.

regard to the prolongation of infancy. In general, development is found to be more rapid in warm countries than it is in colder regions. For this reason maturity, in practically all forms, is reached at an earlier age among peoples in the tropics and semitropics than among those who live in temperate and colder countries. This, however, cannot be accepted without admitting an 
exception. The Eskimos, for example, mature, in general, at an earlier age than the English. There are, no doubt, other factors

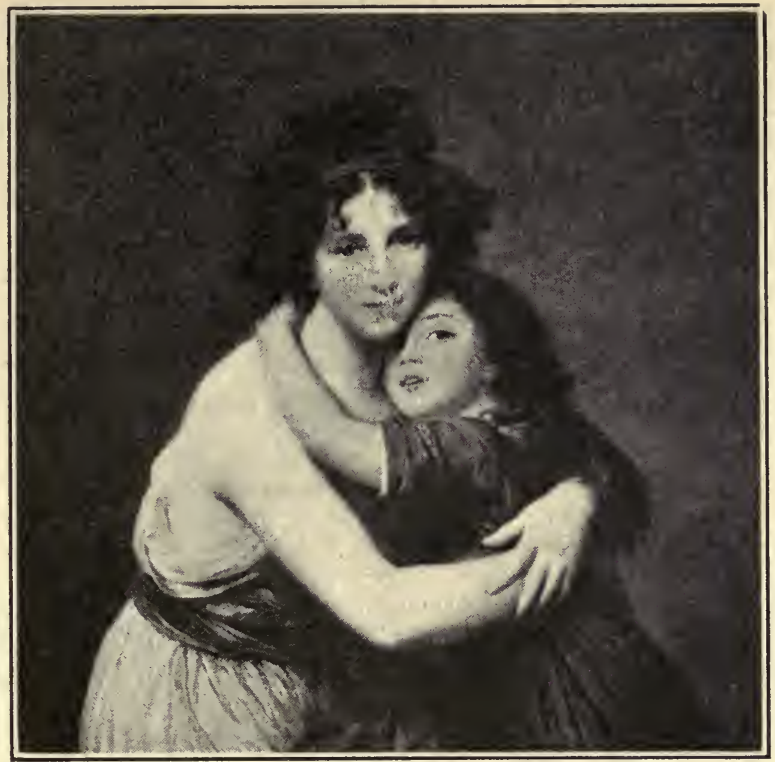

(Madame Le Brun and her daughter.)

All through the ages, artists, in their works of art, have expressed mother love and the dependence of children.

than climate that influence development and maturity. It is probable that nutrition has an influence. There is also another side to be considered, for rapid development or early maturing is, generally, connected with relatively shortened duration of life. The average length of life and the proportional number of people of advanced age are usually greater in those communities that give the children a longer period in which to develop, protect them more completely from various dangers, and look after their needs more thoroughly.

Development of parenthood. Among the simplest organisms, there is no period of life corresponding to parenthood. At a certain 
phase in the organism's growth, it divides into two parts. In the act of reproduction, it ends its own existence. Among the higher organisms, especially birds and mammals, reproduction of new individuals is generally repeated for a long or short period of years. A considerable portion of the individual's activities has to do with preparing for and caring for the young. In mankind, parenthood often makes a greater demand upon the individual than just making a living. On the other hand, the increased activities of adults for the young make possible for each generation a better preparation and a richer equipment for life. So much that concerns and so much that is of value to humans lie beyond the problems of making a living in the material sense.

\section{Questions AND Suggestions}

1. What are the advantages to a species in producing eggs or seeds? What disadvantages?

2. Explain the necessity for such a tremendously large egg production among fish as compared with the small production among birds.

3. How can an organism make provision for offspring that it can never see or know?

4. What are some of the advantages of prolonged infancy? What are some disadvantages?

5. How may animals that look after their young benefit from such activities?

6. How can plants be said to take care of their young?

7. What are some of the dangers from which the young of plants must be protected?

8. What are some of the dangers from which the young of animals must be protected?

9. Discuss whether or not a kitten's development can be hurried by forcing its eyes open?

10. Discuss whether there is any way of hastening or slowing up the development of a plant or an animal?

11. Make a special report on the breeding habits of the stickleback or some other nest-building fish.

12. Give a report on the migrations and breeding habits of the salmon or of the eel.

13. Give a report on the breeding habits of the "obstetrical toad" and compare these with the breeding habits of common toads. 
14. Report on the migrations and nest-building of the egret, cowbird, or some particular species of bird to show the relation of these activities to the protection and welfare of the young.

15. Give a library report on the care of their young by ants.

16. Give a library report on the care of their young by spiders.

17. Describe the domestic life of some carnivorous mammals.

18. Report on the community life of the beaver with special regard to the care of the young.

19. Report on the social life of some anthropoid.

\section{SuPPLEMENTARY REAdings}

Guyer, Michael F., Being Well Born; An Introduction to Heredity and Eugenics (Bobbs-Merrill Co.).

Haupt, A. W., Fundamentals of Biology (McGraw-Hill Book Co.).

Holmes, S. J., General Biology (Harcourt, Brace and Co.).

Jewett, Mrs. F., The Next Generation (Ginn and Co.). 


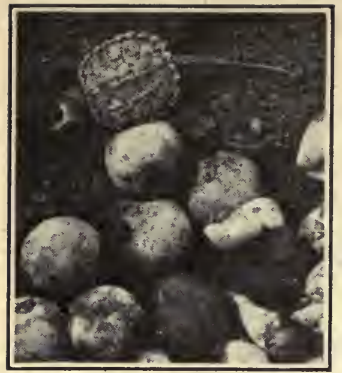

Museum of Natural History Turtle Eggs.
CHAPTER XXXII

\section{CHARACTER OF OFFSPRING}

What characters can be inherited? What is the relation of environment to heredity? Can a person consciously influence his inheritance? What constitutes good environment?

Variety in protoplasm. The fact that the living matter of all plants and animals is protoplasm, has already been discussed. All protoplasm is alike in certain of the elements that compose it; it is alike in its growth by assimilation, its sensitiveness to stimulations, and its response to these stimulations. Yet protoplasm of one species is different from that of another. The protoplasm of a squash seed, for example, develops only into a squash vine and never into a rosebush, never into a canary. The protoplasm of a wren's egg develops into a wren, never into a willow tree nor a wolf. These differences are thought to be located in certain parts of the chromosomes called genes. These genes are the real carriers of hereditary traits. By a gene is meant the part or unit factor of the chromosome, which is supposed to represent an hereditary character. The development of the organism depends largely upon the character-determiners of the genes.

A many-celled organism grows by the individual cells dividing mitotically. In this mitotic division, each chromosome splits into two parts exactly alike. Hence, the chromosomes in all cells 
derived from one cell will be alike. The squash seed contains protoplasm that is a combination of the protoplasm of the parent plants. The protoplasm in the fertile egg of a frog is a part and a continuation of the protoplasm of the parents of the frog. Each kind of protoplasm continues to be much the same, generation after generation.

There is no difficulty in recognizing maple trees, because all maple trees resemble each other. Bean plants resemble bean plants. Elephants resemble elephants. All the individuals of a species are much alike. Yet no two individuals are ever exactly alike. We might call by name and know several hundreds of the

\section{* EXAMPLES OF CHROMOSOME NUMBERS}

(in Animals and Plants)

\begin{tabular}{|c|c|c|c|c|}
\hline Common Name & Scientific Name & Group & $\begin{array}{c}\text { In Body } \\
\text { Cells }\end{array}$ & $\underset{\text { ETES }}{\text { IN GAM- }}$ \\
\hline Sponge & Sycandra raphanus & Porifera & 16 & 8 \\
\hline Hydra & Hydra fusca & Coelenterata & 12 & 6 \\
\hline Earthworm & Lumbricus herculeus & Annelida & 32 & 16 \\
\hline Snail, fresh water & Paludina vivipara & Mollusca & 14 & 7 \\
\hline Crayfish & Cambarus virilis & Crustacea & 200 & 100 \\
\hline Malarial mosquito & Anopheles punctipennis & Insecta & 6 & 3 \\
\hline House mosquito & Culex pipiens & Insecta & 6 & 3 \\
\hline House fly & Musca domestica & Insecta & 12 & 6 \\
\hline Starfish & Asterias vulgaris & Echinodermata & 18 & 9 \\
\hline Frog & Rana catesbiana & Vertebrata & 26 & 13 \\
\hline Chicken & Gallus domesticus & Vertebrata & 18 & 9 \\
\hline Cat & Felis catus & Vertebrata & 35 and 36 & 17 and 18 \\
\hline Dog & Canis familiaris & Vertebrata & 21 and 22 & 10 and 11 \\
\hline Horse & Equus caballus & Vertebrata & 60 & 30 \\
\hline Monkey & Macacus rhesus & Vertebrata & 48 & 24 \\
\hline Man & Homo sapiens & Vertebrata & 48 & 24 \\
\hline Spirogyra & Spirogyra neglecta & Thallophyta & 24 & 12 \\
\hline Moss & Sphagnum squarrosium & Bryophyta & 40 & 20 \\
\hline Bracken fern & Pteris aquilina & Pteridophyta & 64 & 32 \\
\hline Pine & Pinus sylvestris & Gymnospermae & 24 & 12 \\
\hline Pea & Pisum sativum & Angiospermae & 14 & 7 \\
\hline Wheat & Triticum vulgare & Angiospermae & 42 & 21 \\
\hline Corn & Zea mays & Angiospermae & 20 & 10 \\
\hline
\end{tabular}

* Reprinted by permission from Wilson: The Cell in Development and Heredity (1925) by The Macmillan Co. 
pupils of our school. Scientists distinguish, in a like manner, thousands of individuals of one species of a plant or animal. These
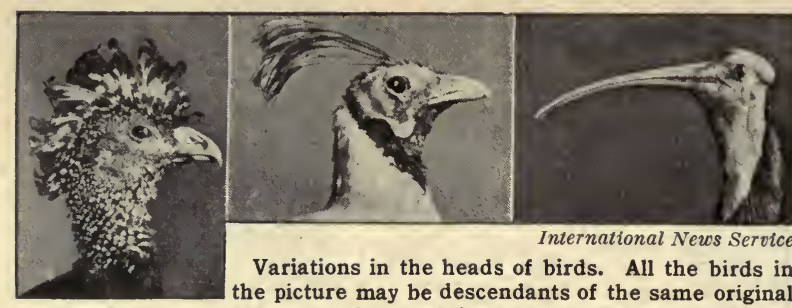

Variations in the heads of birds. All the birds in the picture may be descendants of the same original ancestor. Compare these with others that you know.

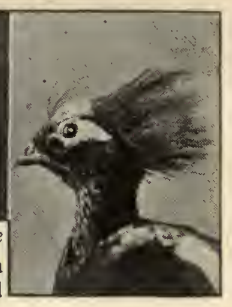

are all enough alike to be classed as insects, pigeons, or dahlia plants; but each is different in some way from all the others.

The problems of these likenesses and differences are the problems of heredity. The study of heredity includes a study of all the various characteristics in the offspring that are more or less similar to those characters of the ancestors.

Environment influencing development. Individuals of the same species may differ because all do not have exactly the same conditions during their early development. Differences in temperature, in the relative amount of moisture, in the character of weather, or of food may cause plants to be stunted or slow to flower in one region when compared with the similar plants of another region. A more abundant supply of mineral salts, or more sunshine at certain periods, may produce plants that are somewhat better developed than others grown from the seeds of the same parent plant. Every farm, every roadside, and every city lot furnish examples of plants that have thrived better or worse than their neighbors, because of variations in their environments.

Temperature, moisture, light, and food are some of the factors that influence the development of animals as well as of plants. The male of the European bullfinch has a bright red breast; the female is entirely brown. If the male bird is fed on hempseed, its plumage changes to a dull color similar to the female. Among honey- 
bees, a given egg may develop either into a worker or into a queen. This development depends upon the nourishment that the larva receives. It is thought by some investigators that the kind of food (honey or pollen) may cause the differentiation in the growing larva. Others think that when a larva is given large amounts of foods, it becomes a queen; if a small amount of food, it becomes a worker.

Many abnormal forms result from a variety of unfavorable conditions during development. This is true in human beings as well as in other species. Even where the results are not abnormal, inequalities of environment bring about differences among individuals of the same species. For example, lack of Vitamin D in the diet results in bone deformities. Lack of iodine may give rise to cretins. Too much food is likely to make people fat.

Trees may become distorted by the wind. Plants grown in poorly-lighted places show an irregular development as the result of a one-sided illumination. Well-fed cows give more milk than poorly-fed cows. The lizards and spiders of Mammoth Cave, Kentucky, are colorless. Exposure to sunlight is thought to increase the amount of pigment in the human skin. One who has learned to swim behaves differently in the water from one who has not learned to swim. A trained horse runs faster than an untrained horse of the same parentage. A child who has recovered from measles does not get the disease a second time when exposed to the infection. One can become accustomed to cold weather or to rainy climate. One can become skillful in some art or game as a result of practice; or his muscles can become weak after a period of disease or disuse.

Where the differences between two individuals result from differences in outward conditions, or from environment, the practical problem is to find out how to treat plants or animals so as to get the desired results. Will one kind of fertilizer or mineral salts result in more cotton per acre? Will one kind of food produce more milk per cow? If so, an adjustment can be made so that the 


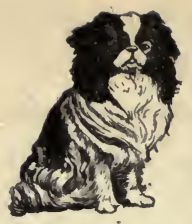

Japanese spaniel

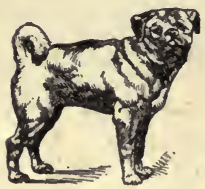

Pug

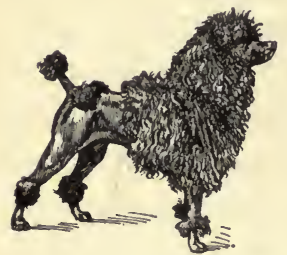

French poodle

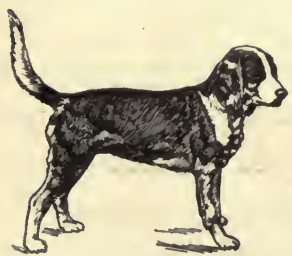

Beagle

organism in question will benefit. Will one system of training make all individuals musical, or quick at figures, or skillful in athletics? If so, and if it is desirable, the system should be investigated and applied to all children.

All of these examples, and others which they may suggest, are proof that plants and animals are influenced by what happens to them. These differences that depend upon the environment are known as variations. Because they. affect the body cells of the organisms and not the germ cells they are also called somatic (body) variations. They affect the individual without affecting, to any extent, the offspring of that individual.

Ancestry influences development. The careful farmer or animal raiser has learned to select the seeds for his planting, or the animals which he is to use for breeding. Large seeds are more valuable than small seeds, especially when a seed crop, such as beans or peas, is desired. A healthy cow that gives a relatively large amount of milk is a more promising mother for good calves than a cow that yields only a small quantity

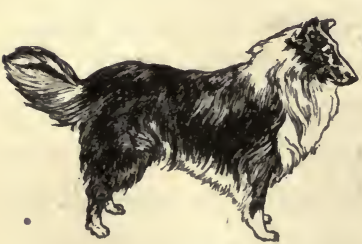

Collie

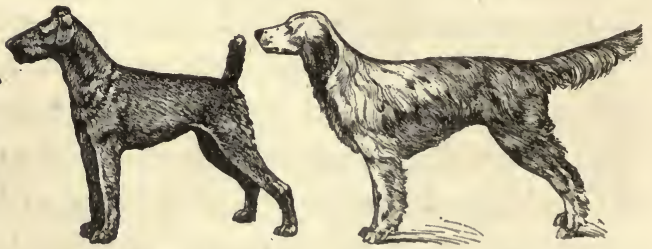

Airedale
English setter

All dogs probably originated 
of milk. The ability to produce a large quantity of milk is a trait transmitted from parent to offspring. If the farmer is interested in eggs for market, he selects hens that lay over 200 eggs a year, and uses these eggs for hatching purposes. These offspring, with a few exceptions, are good egg-layers. This trait, too, seems to be an hereditary one. For centuries, those engaged in plant and animal industries have selected the best individuals and the best seeds for continuing or improving their stock.

Certain differences are found among cotton plants and corn plants, among cows and horses, among human beings, and, in fact, among all organisms that seem to have nothing to do with the kind of treatment they get. Some cotton plants produce long fibers and others short fibers, because they come from certain stock. They belong to a particular breed. Some calves become Holstein cows and others become Jersey cows, no matter how they are fed.

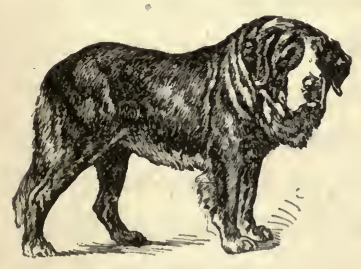

St. Bernard

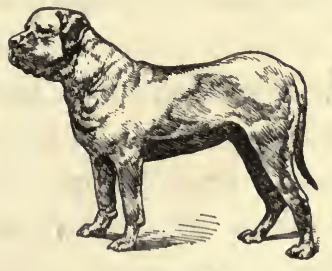

Mastiff

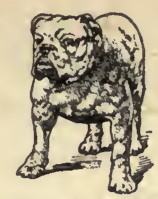

English bulldog

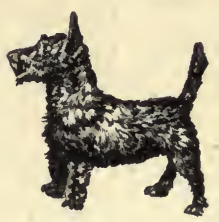

Scotch terrier

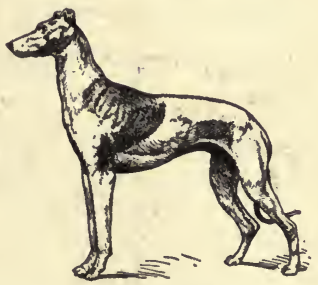

Greyhound

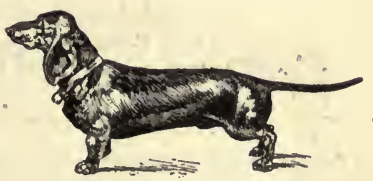

Dachshund

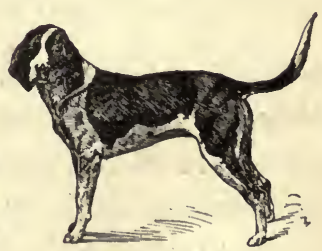

American fox hound

from the same ancestor. 
In the same incubator, under like conditions of moisture and temperature, some eggs hatch into Rhode Island Reds and some

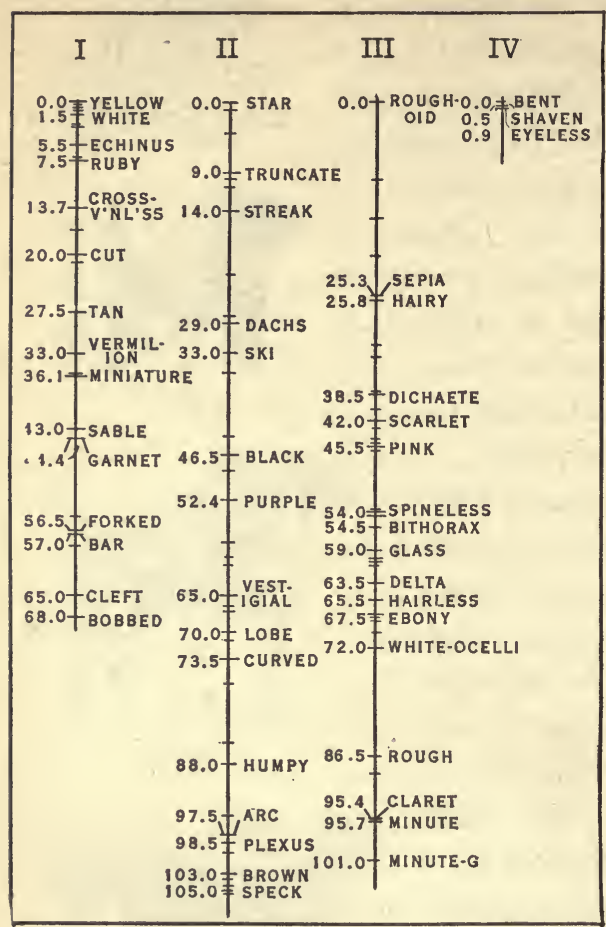

After Morgan

Through careful experimentation, definite parts of chromosomes in the fruit fly have been found to be related to definite structures. Scientists have mapped on the chromosomes of the fruit fly, the location of the genes which carry certain definite characters. into Plymouth Rocks. One horse is said to inherit a definite coat color, a certain shape, or the ability to run rapidly or to pull heavy loads. These characters are part of his breed. The Johnson children are known to be tall and the Brown children are short, although they all seem to be given good food and care. They inherit their stature. In addition to anything in the environment, which may influence the qualities or characteristics of individuals, there is something with which the individual is born. Something that influences his development and makes him different from others that live in the same environment. The characters that are inherent in the individual are known as germinal variations. They are a part of the ancestry because they are in the genes of the chromosomes in the fertilized cell. They can be transmitted from parent to offspring because they are a part of the germ plasm in the egg and sperm. 
How the genes behave is a problem of heredity. Experience has shown, that as a result of selection it is possible not only to maintain the quality of the stock, but also to improve it, up to a certain point. Wherever selection is neglected, the cultivated plants or domestic animals deteriorate in quality. They deteriorate whenever the food or any living condition is neglected. The rule of the successful breeder is to select animals or plants showing the best ancestry. Then he should provide the best conditions for their healthy growth and development.

Effect of environment on ancestral traits: An unborn child may be influenced by conditions prevailing in either parent. Malnutrition or serious ill-health on the part of the mother has an injurious effect on the offspring. Severe shock or grief, worry, nervous exhaustion, the influence of a very few diseases, lead, mercury, or alcohol poisons in the blood or tissues may act detrimentally on the unborn offspring. The effect of these conditions or materials is to interrupt the proper nutrition of the offspring, directly poison it, or, by generating toxins in the mother, poison the developing embryo. Disease-toxins affect unborn children to such an extent as to sometimes cause malformations, arrested development, instabilities of the nervous system, general physical or mental weaknesses, or even death before birth. The children of inebriates comprise a striking proportion of criminals, imbeciles, and those with predispositions toward certain diseases. All of these effects are environmental effects, not inherited effects. The character of the environment of the child before and after birth is a factor of great importance in the development of the child.

Many prospective mothers think they can develop musical ability in a child by studying and playing music before the child is born. Or they hope they may produce beauty in the child by long contemplation of a picture of a beautiful child. The explanation frequently given for birthmarks is that the mother ate many strawberries or tomatoes before the child was born or she was WH. FITZ. AD. BIO. -21 


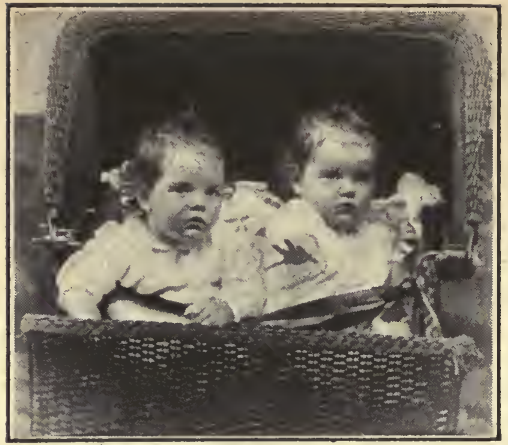

Very similar twin girls.

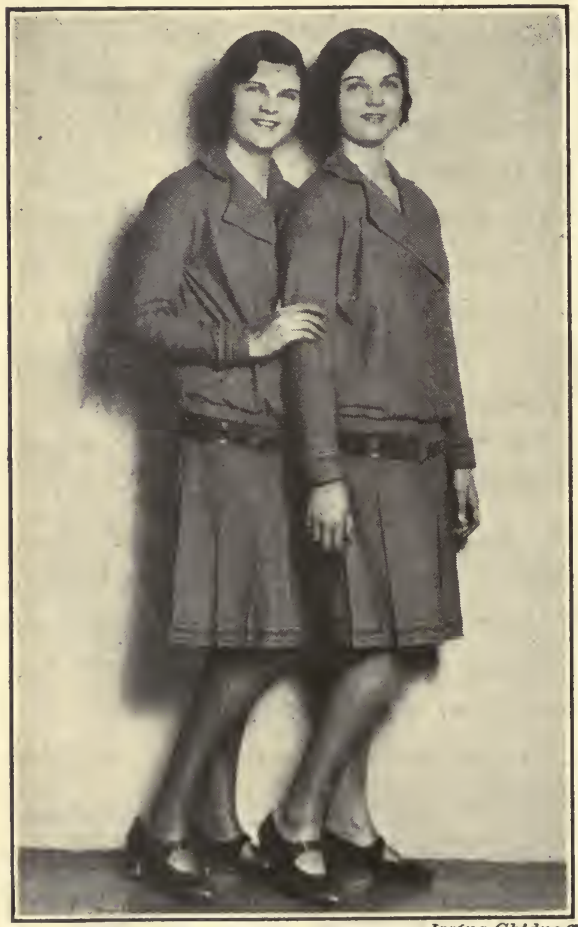

Iroing Chidnot

A recent photograph of the same identical twins. It is very difficult to distinguish one from the other. frightened by a fire. If the birthmark has hair growing on it, the explanation given is that a mouse frightened the mother before the child was born. Structural changes cannot be produced in a child by maternal impressions or mental experiences of the mother. - The physiological explanation of birthmarks is that a number of small blood vessels of the skin of a new-born infant have remained dilated in a particular spot. This is a somatic, not a germinal variation.

The characters combined in the chromosomes during the union of the sperm and egg are the characters that will develop in the child. If musical ability is in one of the genes, it will be present in that of the offspring. If not, no amount of practice on the part of the parent will develop the talent. The only structural changes made in the offspring are those that can be affected by good 
or poor nutrition or toxic materials of one sort or another. Only by affecting germ cells can the character of the offspring be controlled or changed. Recently, it has been demonstrated that internal secretions circulating in the blood of the parent may affect the germ cells. Thus, if a mother's thyroid is overactive and too much thyroxin circulates in the blood, the germ cell may be affected in such a way that abnormalities in the developing offspring are frequently brought about.

Differences among offspring. It has long been known that certain characteristics or qualities reappear in successive generations. Why do not all the characteristics of the parents reappear? If each new individual were merely a portion of an older individual, it could readily be seen how the characters would continue from one generation to the next. When plants are propagated by cuttings or by grafts, the plants thus produced remain rather uniform. Where reproduction is sexual, two germ cells, the gametes, come from different sources. Each germ cell carries many qualities that are characteristic of the protoplasm of each parent. These qualities reappear with a certain degree of probability and in relationship after the two germ cells have united. Each individual resembles both parents, but, necessarily, also differs from both
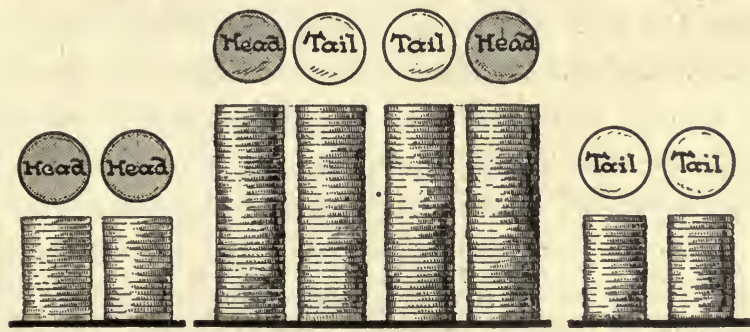

When two coins are tossed a great number of times, two tails turn up $\mathbf{2 5} \%$ of the times, two heads turn up $25 \%$ of the times and one head and one tail turn up $\mathbf{5 0} \%$ of the time. This occurrence is called the law of chance. In the few matchings shown in the diagram, the result would probably not be as near the 25, 50, 25 ratio as is pictured. This illustrates the law of chance (page 317 ).

parents. A child may have brown eyes like the mother's and curly hair like the father's. Offspring of the same parents also differ 
from each other. Some are tall and some are short, some are blue-eyed and some are brown-eyed. This will be explained later.

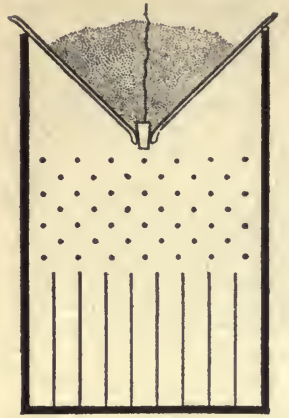

$\propto$

When peas are rolled down an inclined plane through a small opening against nails, they line up in pens in a very definite order. When this experiment is performed, a sorting out, known as a normal distribution, occurs. Few peas are found in the end pens, many in the center. This illustrates the law of chance, discussed on p. 317.

We have learned that a process called maturation or ripening occurs in the development of mature germ cells in the parent organism. This maturation of the germ cells accounts for the differences among offspring. Chromosomes are thought to possess combinations of character-determiners called genes. These genes are found to be in pairs in the primary sex cells. During the maturation process, reduction takes place. In reduction, one member of each pair goes to a given germ cell. Consequently, each germ cell has one half the number of chromosomes found in the primary sex cell. If the primary sex cell had a pair of characterdeterminers for eye color, one of this pair carries blue and the other brown, the chromosome containing the gene for blue eyes would go to one germ cell and the chromosome containing the gene for brown eyes would go to another germ cell. A primary sex cell may have two characters for a particular trait, but a germ cell only has one and is pure in regard to any particular trait. Consequently, the same organism may produce germ cells with unlike characters. The sperm cells of the male are not identical; eggs, too, have different character-determiners or genes in them. When the sperm and egg meet in the process of fertilization, the characters of the fertilized egg depend upon the characters in the genes in the combined chromosomes of both the sperm and egg. 
These combine according to the law of chance. Combinations of a pair of characters follow a definite proportion.

\section{Problem. How may the law of chance be demonstrated?}

I. Put in a jar one hundred black beans and one hundred white beans of the same size. Mix the beans thoroughly. Without looking, take out two beans at a time.

$A$. Score the number of times two black beans, two white beans, and one black and one white bean are taken out.

$B$. At least one hundred pairs should be taken out before the scores are summarized.

C. State the proportion of two black to a combination of one white and one black, and to two white.

II. Toss two coins at least one hundred times before totaling your scores.

$A$. Score the number of times two tails appear.

$B$. Score the number of times two heads appear.

$C$. Score the number of times one tail and one head appear.

$D$. State the proportion of two heads to a combination of one head and one tail, and to two tails.

III. When it is purely chance that controls the meeting of members of a pair of characters, the proportion resembles that obtained with the beans and coins. In order to get a proportion, the experiment should be carried on a large number of times.

\section{Questions AND Suggestions}

1. In what structures of germ cells are characters of organisms located?

2. What is meant by heredity?

3. What effect has environment on development? Give examples.

4. What effect has ancestry on development? Give examples.

5 . What effect has environment on ancestral traits?

6. Account for differences among offspring.

7. How can the best offspring be obtained?

8. Discuss the law of chance. Describe an experiment to illustrate it.

9. How does the law of chance operate in the fertilization of an egg by sperm? 


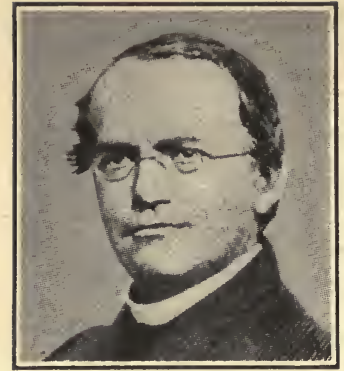

Gregor Mendel
CHAPTER XXXIII

\section{HEREDITY}

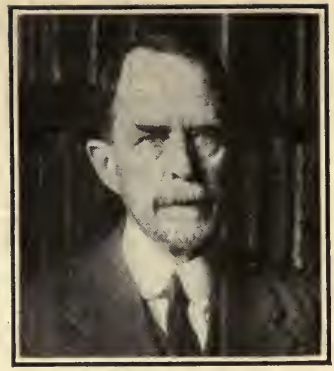

Thomas Hunt Morgan

What are Mendel's laws of inheritance? How were they formulated? Do these laws of inheritance hold true for all plants and animals?

It has been found that truly heritable traits or characteristics of an individual are comparatively independent of each other and may be inherited independently. These characters are called unit characters. If the members of a pair of unit characters meet in an offspring, each member retains its identity and each character of the pair may be separated in subsequent generations. The following illustration is an example of the behavior of a pair of unit characters.

Incomplete dominance. There are two distinct kinds of Andalusian fowls, one having white feathers splashed with black; and the other, black feathers. The black fowls are known as pure black because, for generations, their ancestors have been black, have mated, and have given rise to only black descendants. The splashed-white fowls are known as pure white; their ancestors were white, they bred with white, and have always produced white offspring. A fowl from each group was selected as a parent. They were mated, fertile eggs were laid and incubated, and the outcome of the experiment awaited. Would the chickens be black, or white, or a mixture of the two colors? Although the experiment was repeated several times, the results were always the same. 
All the young chicks were a queer mixture of mottled black and white which is known as blue. They were mixtures and not pure bred like their parents. When the members of a pair of widely different unit characters, in this example, black and white feathers, meet in an offspring, the offspring is called a hybrid. Evidently, neither the black nor the white character could mask or dominate the other, and a blending took place. The blending of a pair of characters is called incomplete dominance.

Next, the investigators wished to discover what the descendants of the hybrids would be like. Only blue Andalusian fowls, hybrids, were chosen for this part of the experiment. This hybrid generation is known as the $F_{1}$ generation or the first filial generation. It was found that the offspring of hybrids varied in color. Some were blue like their parents, some were white like one grandparent and some were black like the other grandparent. When a large number of them were examined, it was found that the different colors of the chicks always occurred in the same proportion. For every two chickens that were blue like their parents, there was one black and one white chicken. In other words, 25 per cent were black, 25 per cent were white, and 50 per cent were blue. This generation, in which there was a reversal to ancestral type by the segregation of the original unit characters, is known as the second filial or the $F_{2}$ generation. Evidently, the original characters must have retained their identity since some of the offspring showed one character and others showed the opposite character.

The next step in the experiment was to find what would happen when the different members of this mixed group became parents. A black fowl was mated with another black and all the offspring were black. In other words, the black chickens of the $\mathrm{F}_{2}$ generation not only looked black like their black grandparents, but were pure as shown by the fact that they had only black descendants. They possessed only genes or character-determiners for black.

A white of the $\mathrm{F}_{2}$ generation was mated with another white and 
all their offspring were pure white. But when blue fowls were mated with others like themselves, some of their offspring were
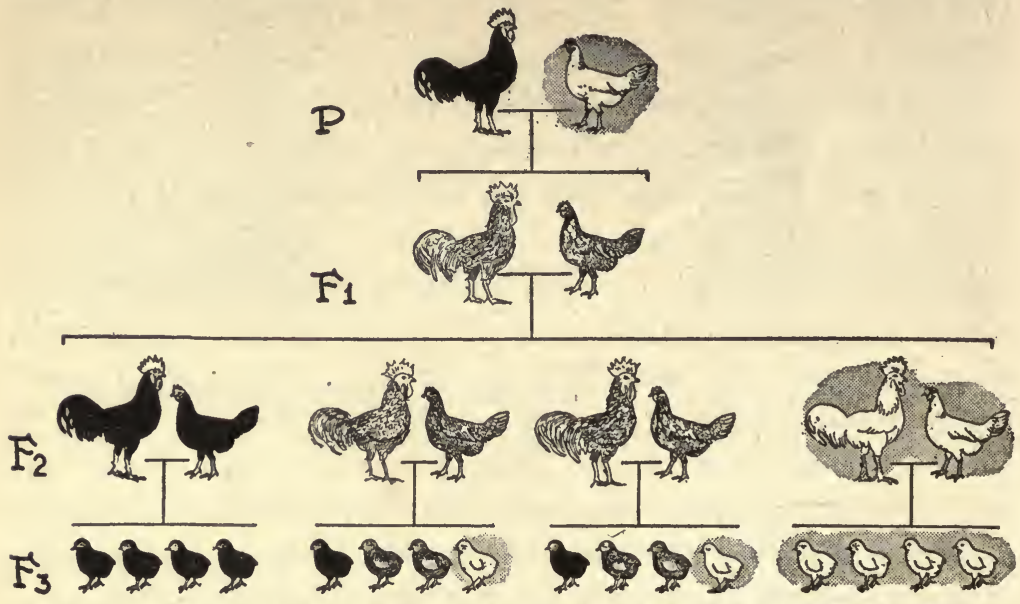

If a black Andalusian cock is mated with a white Andalusian hen, the offspring is a hybrid showing a mottled blending of the black and white called blue. This is an example of incomplete dominance. If a blue cock is mated with a blue hen, 25 per cent of the offspring will probably be black, 50 per cent may be blue, and 25 per cent may be white. Black pairs will breed only black; the white pairs will breed only white; but the offspring of a blue pair will segregate, sort out, into black, blue, and white.

black, some were white, and some blue. The blue chickens, then, of the $\mathrm{F}_{2}$ generation were hybrids.

The inheritance of color in the Andalusian fowl is :

(1) Pure black mated with pure black will always produce pure black.

(2) Pure white mated with pure white will always produce pure white.

(3) Pure black mated with pure white will always produce 100 per cent blue hybrids.

(4) Blue hybrids, when mated with blue hybrids, tend to produce 25 per cent black, 25 per cent white, and 50 per cent blue.

The four-o'clock is another example of an organism in which a hybrid, produced by two distinct species, is unlike either, but is a blend of the two. The pure lines or types of four-o'clocks have 
either red flowers or white flowers. If red flowers are pollinated with pollen from red flowers, their seeds will produce red flowers. If white flowers are pollinated with pollen from white flowers, their seeds will produce plants with white flowers. But if red flowers are pollinated with pollen from white flowers, or white flowers with pollen from red flowers, the seeds will produce plants bearing pink flowers. This pink is a blend of the white and red colors. A flower of this type and the seed that produces it is called a hybrid. If these hybrids are allowed to pollinate themselves, their seeds

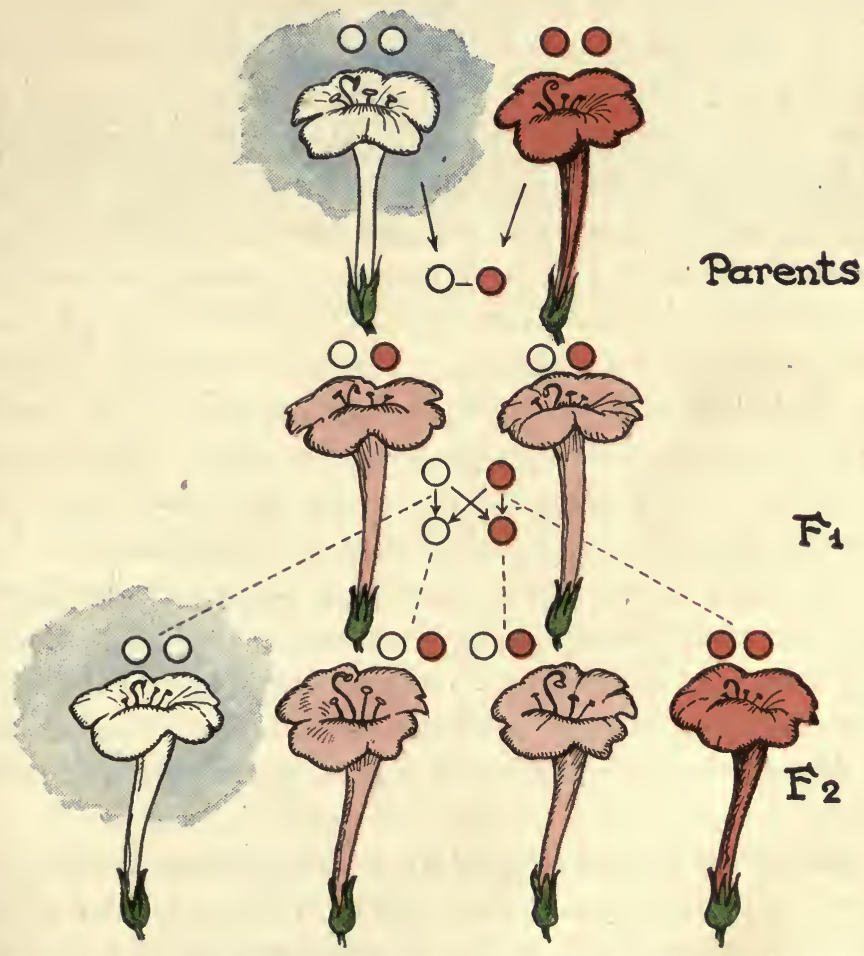

The hybrids of the Japanese four-o'clock show incomplete dominance. The pure generation is usually called the parental, $P$; the hybrid generation is the $F_{1}$; and the next generation showing segregation is the $F_{2}$. The little circles represent gametes and are colored to show genes or the color determiners which each flower carries. 
will produce plants which will bear flowers of three colors. Of every 1000 blossoms, approximately 250 will be red, 250 will be white, and 500 will be pink. The colors will be in the proportion of $1: 1: 2$. The various colors of the four-o'clocks of succeeding generations will vary in the same way as those of the Andalusian fowl.

Experiments in plant breeding. Long before the foregoing experiments were carried on, Gregor Johann Mendel (1822-1884), an Austrian priest and head of a monastery, was especially interested in cultivating garden peas. He found that his pea plants differed from each other in very many characters. Tallness, coloration of seeds petals, and pods, and the hairiness, roughness, or smoothness of skin on the seeds and stems were some of the differences that he observed. Then he asked, "How does a particular quality carry over generation after generation?" He proceeded to seek the answer to his problem by experimentation. He selected tall plants and kept them in a certain garden plot. Away from these he raised a plot of plants that were always short. He called each group a pure-type. The unit character of height was one particular trait in which he was interested. He crossed pairs of plants which differed in this single character. He repeated his experiments to investigate other out-standing characters. Tall plants were crossed with short; plants with yellow seed coats with those of green seed coats; plants having smooth seeds with those having rough seeds; hairy stemmed plants with hairless stemmed ones. In each of these crosses he watched the behavior of the variations of a single character only. After many experiments, Mendel learned and made known some very important principles.

The Mendelian laws of inheritance. - The Law of Dominance. When two of the pea plants that differed from each other in one characteristic were crossed, all the offspring resembled one of the two parents in regard to that characteristic. If one parent were tall and the other short, all of the next generation would be tall. 


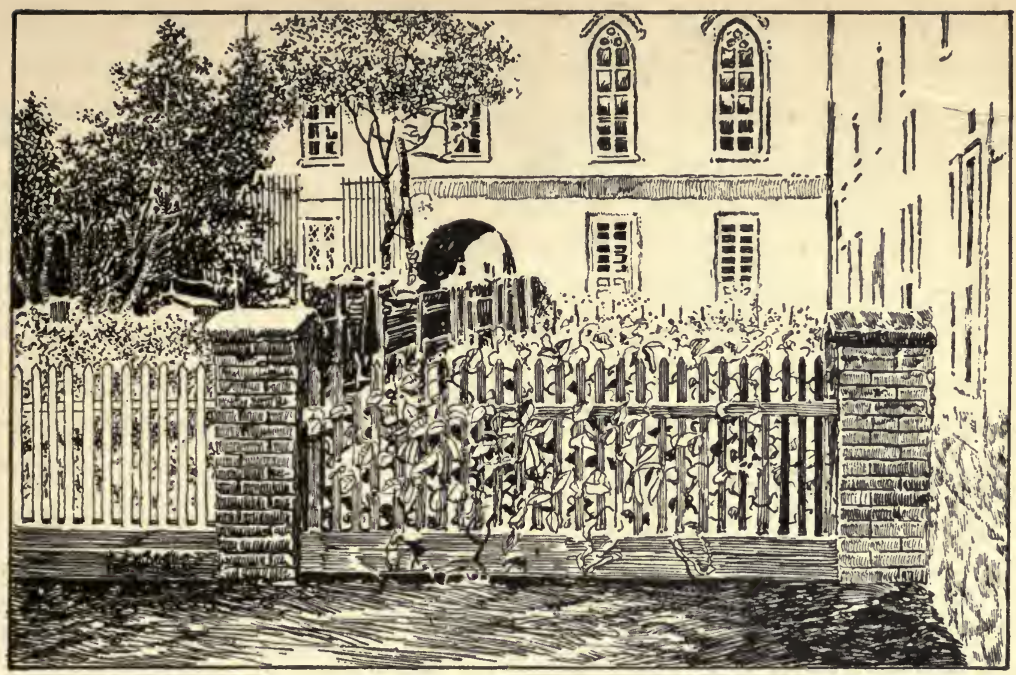

Gregor Johann Mendel planted peas in his garden in Brïn, Austria. He crossed plants showing different characters and produced a variety of hybrids.

If one of the parents were of a wrinkled-seed variety and one of a smooth-seed variety, all of the next generation would have smooth seeds. If one of the parents had a yellow seed coat and the other had a green seed coat, all of their offspring had yellow seed coats. To get more accurate results, he pollinated artificially the flowers of two varieties and kept them protected from all insects. The seeds from these plants were gathered, planted, and the new plants were carefully watched. Mendel found that the characters did not blend, as was later noted in the Andalusian fowl. One character completely dominated the other character and concealed it. This is called complete dominance. It has been found to occur in many species of plants and animals, and is true for many different characters. This is known as Mendel's Law of Dominance.

Dominance, as used in studies of heredity, is a condition in which one of a pair of characters will appear in the offspring and hide or mask the other. The character that shows in the offspring is 
called the dominant character. The second quality, the one that does not show, although it is actually present, is known as

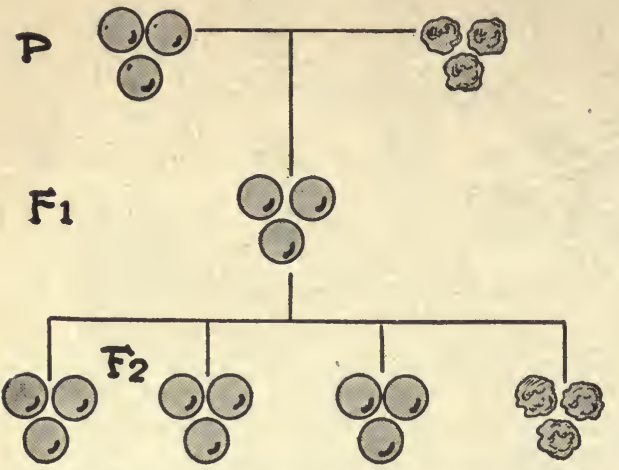

When pollen from a pea plant that produces smooth seeds is transferred to a plant that-produces wrinkled seeds the resulting plants will produce smooth seeds. In peas, smooth seed coat dominates a wrinkled seed coat. The resulting offspring are hybrid, for when they are selfpollinated their offspring show 75 per cent smooth seeds and 25 per cent wrinkled seeds; of the 75 per cent smooth seeds, 25 per cent is pure bred smooth, and 50 per cent is hybrid smooth. This illustrates complete dominance, because smooth seed coat completely dominates wrinkled seed coat and does not blend with it. the recessive character.

Whatever there is in the protoplasm that produces the recessive quality, it is not destroyed in the crossing, for, as shall be seen, the quality may reappear in later generations. In the example given, the dominant traits were tall plants, smooth seed, and yellow seed coats. The recessive traits were short plants, wrinkled seeds, and green seed coats. The individual resulting from a cross of opposite characters is called a hybrid. For example, when plants with yellow seed coats are crossed with those with green seed coats, the offspring appear yellow. Because one of the parents had green seed coats the protoplasm of the offspring must be different. These yellow offspring are hybrids since they conceal the character determined for green. In all external appearances, hybrids resemble pure dominants.

Mendelian Law of Segregation. Although the tall descendants of a mixed parentage, that is, a tall and a short, may appear tall like one of the parents, its protoplasm or part of its protoplasm is really different. This must be true since it has been built up in part from the protoplasm of the short parent. In the same way, the plant with the yellow seed coat of mixed parentage may resemble, in seed coat color, one of the parents, but it is still a 
descendant of the green seed coat stock, and its protoplasm is probably different. Mendel made further experiments with his peas to find out what happened in later generations. He made three kinds of crosses: (1) he cross-pollinated a hybrid with the dominant parental type; (2) he crossed a hybrid with the recessive parental type; and (3) he crossed a hybrid with a hybrid.

Consider the third experiment first. When hybrids were self pollinated, their seeds always produced species of both ancestral types. For example, hybrid peas with yellow seed coats produced seeds with both yellow and green coats. The hybrids must have carried greenness even though it did not show. There was a splitting up, so to speak, of the combined inheritance into its two components, yellowness and greenness in the seed, or into dominance and recessiveness. This sorting out of members of a pair of factors is called segregation. This has been found to occur not only in hybrids but also when pure-breeding varieties are crossed. When large numbers of offspring of hybrids are considered and tabulated, the segregation is always in the proportion of one recessive to three dominants, of which one is pure and two are hybrids.

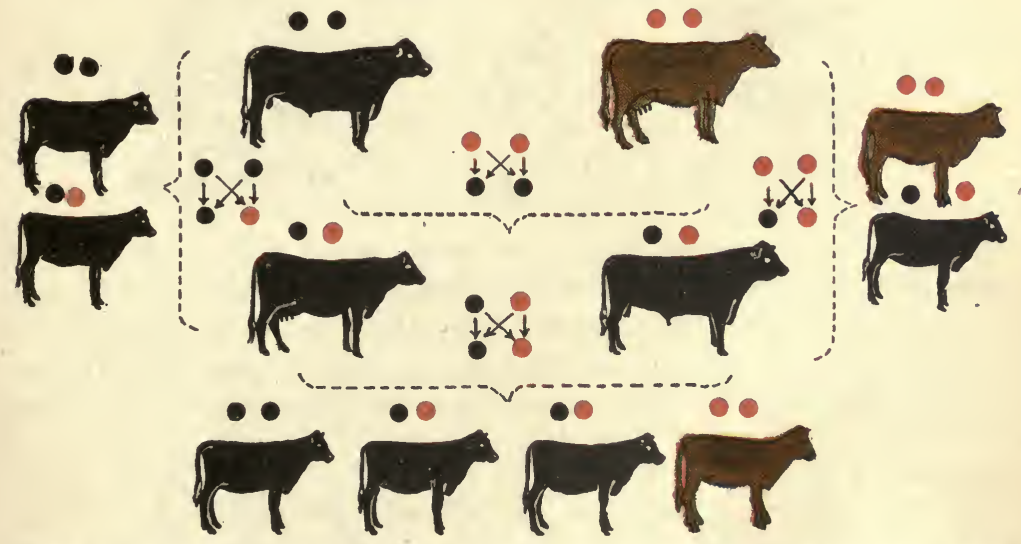

Black dominates red in cattle. Name the three generations shown in the diagram. Note. also on the sides of the diagram the result of what the animal breeder calls a back cross; i.e., hybrid with black and hybrid with red. 
When hybrids are crossed with pure dominants, the offspring will still retain the dominant appearance, but the recessiveness is not destroyed, for it may be made to reappear in later generations. These offspring are found to be, approximately, one half pure dominant and one half hybrid, although they may all look dominant.

When hybrids are crossed with pure recessives, segregation again takes place. One half are recessive and one half appear to be dominants, but are hybrids. When hybrids are crossed with hybrids, or with pure dominants, or with pure recessives, the original parental characters tend to sort out. This, Law of Segregation, is perhaps the most important discovery that Mendel made, although it grew out of his discovery of dominance.

Problem. What combinations of chromosomes result from maturation and fertilization?

I. Place two black beans (to indicate chromösomes carrying black color) in the circle representing the egg mother cell, and two white beans (indicating

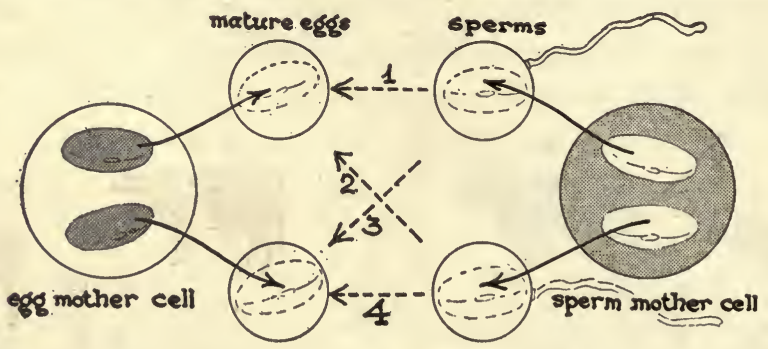

the chromosomes carrying white color) in the sperm mother cell. Assume that the chromosomes have the character-determiners or genes for color only.

$A$. Move the chromosomes from the primary sex cell to the gametes to show the change that takes place during maturation (reduction division).

$B$. Move the beans in the circles representing the sperms, so as to indicate
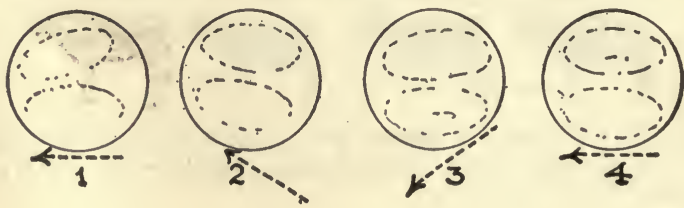
fertilization of the eggs. Show the combinations of chromosomes in the eggs.

$C$. Draw in the representations of the chromosomes (beans). Color the black and leave the white uncolored. How will you represent the hybrids?

II. Repeat the above experiment, using the hybrid combination of chromosomes in the primary sex cells. Keep in mind that primary sex cells can be hybrid in regard to a particular character, but germ cells must be pure.

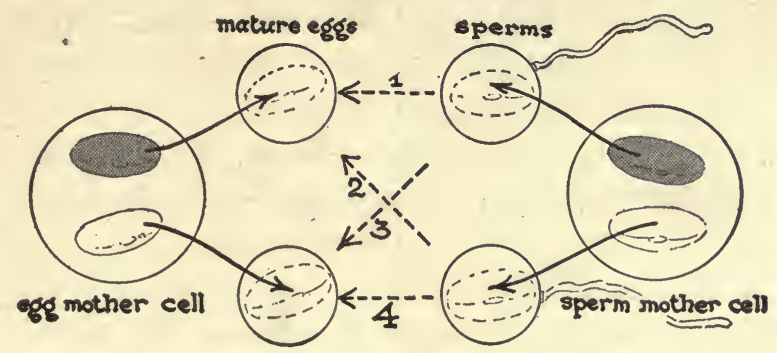

A. Record combinations resulting from fertilizations.
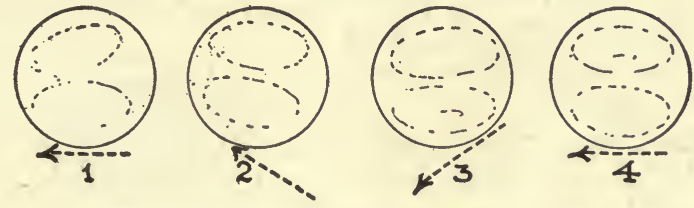

III. Repeat the experiment, using in the male primary sex cell the hybrid combination, and in the female primary sex cell two recessive white chromosomes. Record by means of diagrams, the various possible combinations resulting from fertilizations.

IV. Repeat the experiment, using hybrid in one cell and dominant black in the other.

V. What law of heredity did experiment I illustrate? What law of heredity did experiments II, III, and IV illustrate?

Mendelian Law of Unit Characters. Another important contribution that Mendel made was his emphasis upon the study of single characters. In speaking of organisms, the general type is referred to rather than the individual traits that make up that type. Fox terrier, for example, or crimson rambler, describes a complete picture without the need of hundreds of details in which 
that particular variety differs from others. It is known, however, that the individual organism is a combination of thousands of small

\section{sperm carries}

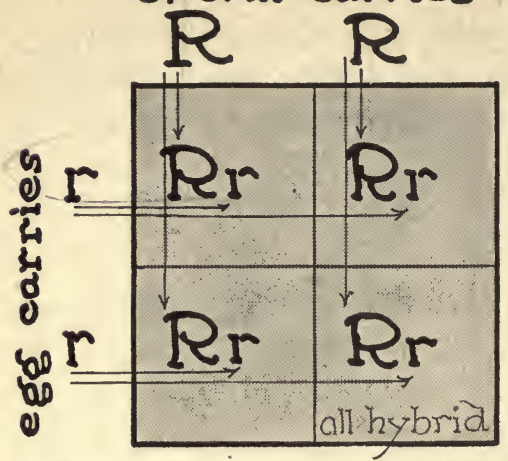

An experimenter, Punnett, devised a method of predicting how genes combine after crossing. He used boxes known as Punnett squares.

Turn to diagram on page 321. The gametes of the parent flowers carry only red, $R$, or no color, $r$. Note in the above diagram, all the possible combinations of gametes and observe the identical result $\mathrm{Rr}-\mathrm{a}$ hybrid.

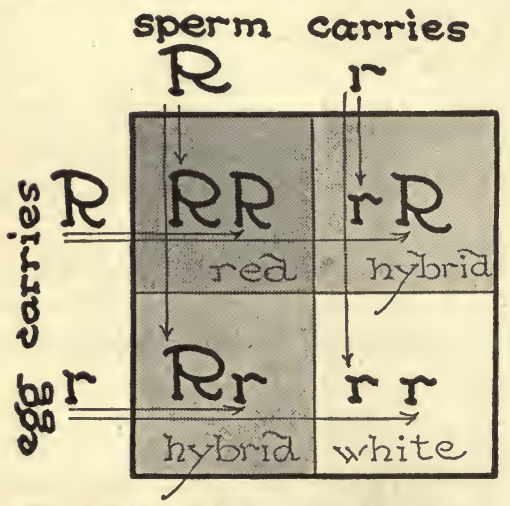

The gametes of hybrid, pink four-o'clocks, carry either the dominant $R$ or the recessive $r$. When fertilization occurs, the law of chance determines whether a pure dominant, a hybrid or a pure recessive is formed. Notice how the combinations are described with the Punnett squares. differences, some structural, and some physiological or the result of physiological activity. The problem of heredity has to do with studies in respect to each of these individual characters.

After Mendel had assured himself that there was dominance and, later, segregation for each of several pairs of characters, he took up the problem of organisms that differed in two characters. For example, some tall plants were hairy and some were smooth; some hairy plants had yellow seed coats and some had green seed coats; some of the yellow seed coats were wrinkled and some were smooth. By experiments in crossing for several generations, Mendel found that dominance and segregation occurred for each character independently of the other characters, and so he formulated the Law of Unit Characters.

Simply stated, it means that a pair of characters behaves independently of any other pair 
of characters. This makes it possible to secure various combinations of characters not associated in the original pure stocks. For example, tallness is dominant to shortness, and yellow seed coat is dominant to green seed coat in peas. If a tall, green seed plant is crossed with a short, yellow seed plant, the offspring will all be tall with yellow seeds. Dominants conceal recessives. The fact that each parent had a dominant and a recessive trait does not interfere with the laws of heredity which state that the dominant traits conceal the recessive traits and each pair of characters behaves independently. New combinations of characters are thus set up in the offspring. Offspring do not resemble either parent in all characters.

Mendel worked eight years on his garden peas and was able, in 1859 , to present his results. At that time everybody was excited about Darwin's work. Few, in fact, none, had time for Mendel.
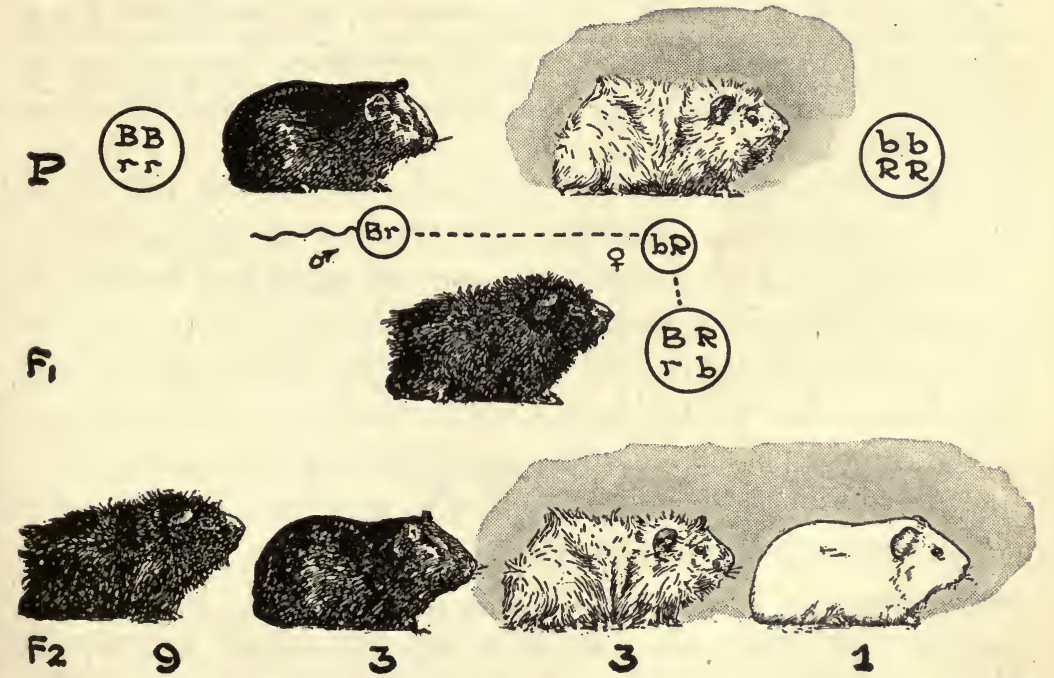

In this breeding experiment, pigmentation and quality of hair of guinea pigs are the factors involved. $\mathbf{R}$ represents rough hair, $r$ smooth hair; $B$ denotes black color and $b$ white color or absence of black. The circles show the genes carried by body cells and matured germ cells. An animal of the $F_{1}$ generation carries two pairs of characters and is known as a dihybrid. When dihybrids are bred, the $F_{2}$ generation segregate in the ratio of $9,3,3,1$.

WH. FITZ. AD. BIO. -22 
His paper was received in silence and soon forgotten. In 1900, sixteen years after his death and thirty-five years after his dis-

\begin{tabular}{|c|c|c|c|c||}
\hline 0 & $B R$ & $B r$ & $R b$ & $b r$ \\
\hline$q$ & $B R$ & $B r$ & $R b$ & $b r$ \\
$B R$ & $B R$ & $B R$ & $B R$ & $B R$ \\
\hline$B r$ & $B R$ & $B r$ & $R b$ & $b r$ \\
\hline$B r$ & $B r$ & $B r$ & $B r$ \\
\hline$R b$ & $B R$ & $B r$ & $R b$ & $b r$ \\
\hline & $R b$ & $R b$ & $R b$ \\
\hline$b r$ & $B R$ & $B r$ & Db & $b r$ \\
\hline
\end{tabular}

The Punnett square may be used to show the possible sorting out during maturation of germ cells of dihybrids and the various combinations resulting after fertilization. Each square represents an individual. This square shows the possible combinations of genes in the nature of guinea pigs of different colors and types. The dominant characters are, large $\mathbf{B}$, representing a pigmented coat, and $R$, rough hair. The recessive characters are, $b$, denoting white coat, and r, smooth hair.

coveries, three other scientists working independently arrived at the same conclusion. Mendel's work was then given full credit.

Since 1900, it has been proven again and again that heredity is frequently shown in animals exactly as in Mendel's peas. If pure black guinea pigs are crossed with pure white guinea pigs, all the resulting hybrids will be black, showing black to be dominant.

The guinea pig and garden peas show complete dominance. The Andalusian fowls and four-o'clocks, in which the characters blend, show incomplete dominance.

Summary. 1. A pure character is one that gives rise only to a character like itself. The plant or animal possessing it carries pairs of like factors; for example, two factors for height, one paternal and one maternal.

2. A plant or animal is a hybrid when it carries a pair of contrasting factors. For example, hybrid tall carries both tallness and shortness. 
3. Mendelian Laws of Heredity.

a. Law of Dominance. When an individual, which is pure as to a particular factor, is crossed with another individual which is pure in the contrasting factor, only one of these factors will appear in the offspring. The character which appears is called dominant; the other which is inherited, but is concealed, is called recessive.

b. Law of Segregation.

(1) When hybrids are mated, the contrasting factors are segregated out in the proportion of 25 per cent pure dominant, 50 per cent hybrid, and 25 per cent pure recessive.

(2) When hybrids are mated with pure dominants, there is a segregation of 50 per cent hybrid and 50 per cent pure dominant.

(3) When hybrids are mated with recessives, there results a segregation in the proportion of 50 per cent hybrids, 50 per cent recessives.

c. Law of Unit Characters. Every organism contains many pairs of factors. Each factor, as tallness or shortness, is inherited as a unit, that is, independently of the other and of any other pair of characters.

These laws were formulated by the study of the external appearance of the results of different matings. What caused it all? A difference in the protoplasms of the germ cells has been mentioned. This is true. Recent investigators have formulated a theory that has stood the test of much careful checking. The chromosome is concerned with heredity. It is thought that a certain part of the chromosome called the gene or character-determiner actually directs and controls the development of the individual characters.

Chromosome theory of inheritance. If both parents are pure as regards a particular character, both the egg and sperm will contain the determiner for that character and the offspring will receive a double contribution of the determiner or "gene." For example, the genes of a pure tall pea plant will carry only tallness ; the genes in a pure dwarf pea plant will carry only dwarfness. The offspring of such pure breds, whether dominant or recessive, cannot help but be pure. The offspring of the tall plants will be 
tall and those of the short will be short. Diagrams similar to Punnett's (on page 328) may be worked out. Use a capital " $T$ " for tallness and small " $t$ " (small letter indicates the lack of dominant character) for dwarfness. The gametes at the top of the squares are the sperms and those at the side are the eggs. The combinations in the squares will show the meeting of the chromosomes in the fertilized eggs.

Formulae.

(1) $T \times T=T T$.

(2) $t \times t=t t$.

When the dominant character is present in one parent only, then the offspring will have only a single contribution of the gene. The offspring will appear dominant but will be a hybrid.

Formula.

$T \times t=T t$.

What happens when hybrids are mated? It is believed that during maturation the contrasting factors of hybrids go into the separate eggs or sperms. Thus, the male primary sex cell will be hybrid, but one half of the sperm cells will carry the recessive factor and one half the dominant. Likewise, half the eggs will carry a dominant factor and half the recessive. In other words, the mature germ cells are always pure as regards any one character. The individual may be a hybrid but the gametes are never hybrid.

When these gametes of hybrid parents join, the law of probability shows that the following combinations are possible.

Formula.

$$
\stackrel{T}{\underset{t}{\longrightarrow}} \underset{t}{\longrightarrow}=T T, T t, t T, t t
$$

When a hybrid is crossed with a dominant, the gametes of the hybrid will be half dominant and half recessive, while the gametes of the dominant parent will all carry the dominant gene. The following combinations are possible.

Formula.

$$
\stackrel{T}{\longrightarrow_{t}} \underset{T}{\longrightarrow}=T T, T T, t T, t T
$$


When a hybrid is crossed with a recessive, the gametes of the hybrid will be half dominant and half recessive, while the gametes of the recessive will all carry the recessive gene.

Formula. $\quad{ }_{t}^{T \underset{X}{\longrightarrow} t}=T t, T t, t t, t t$

Results are half recessive and half hybrid.

\section{HEREDITY IN ORGANISMS}

Kind of Organism Dominant Character Recessive Character

Wheat.......... Late ripening..... Early ripening

Wheat........... Susceptibility to rust Bearded

Barley........... Beardless......... Immunity to rust

Maize........... Round, starchy

Cotton........ Colored lint......... White lint

Sweet pea....... Colored flower...... White flower

Cattle......... Hornlessness...... Horns

Rabbits......... Short fur......... Angora fur

Mice........... Pigmented coat.... White coat

Leghorn chickens.. White plumage..... Pigmented plumage

Canary.......... Crested head...... Plain head

Land snail....... Plain shell........ Banded shell

Horses......... Black.......... Chestnut

Bay............. Black or chestnut

Gray............ To all colors

Man........... Curly hair......... Straight hair

Dark hair......... Light; red

Brown eyes....... Blue eyes

Normal pigmentation Albinism

Broad fingers (lacking one joint).... Normal length

\section{Questions And Suggestions}

1. Contrast somatic variations with germinal characters.

2. Diagram, using the Punnett square, the Andalusian fowls through two generations.

3. Diagram, using the Punnett square, the Japanese four-o'clocks through two generations. 
4. State and illustrate the three Mendelian Laws.

5. Using a pair of characters in wheat, show how it may breed through two generations, showing all possible combinations in the offspring. At the beginning of your work, be sure to explain the representations you use for characters.

6. Using a pair of characters in cattle, show how it may breed through two generations, showing all possible combinations in the offspring.

7. Give a library report on the life and work of Gregor Mendel.

\section{Supplementary Reading}

Conklin, Edwin Grant, Heredity and Environment in the Development of Men. (Princeton University Press).

Guyer, Michael F., Being Well Born; an Introduction to Heredity and Eugenics. (Bobbs-Merrill Co.).

Journal of Heredity, American Genetic Association. Washington, D. C.

Morgan, Thos. Hunt, The Physical Basis of Heredity. (J. B. Lippincott Co.). Shull, A. F., Heredity. (McGraw Hill and Co.).

Sinnott, E. W., and Dunn, L. C., Principles of Genetics. (McGraw Hill Book Co.).

Walter, H. E., Genetics. (The Macmillan Co.). 


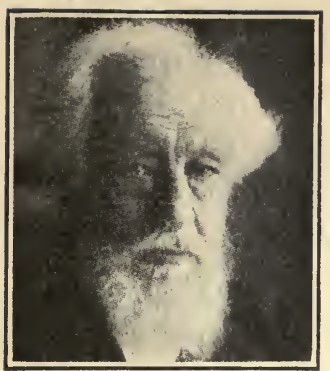

Hugo de Vries.

\section{CHAPTER XXXIV} MUTATIONS

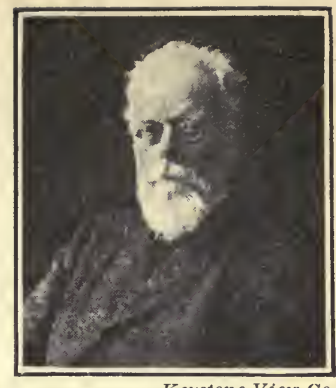

Keystone View Co.

August Weismann.

What are mutations? What is the importance of mutations?

The theory of germ plasm. August Weismann (1834-1914), a German biologist, tried to produce a race of tailless mice. He cut off the tails of tiny baby mice as soon as they were born. When these grew to adult mice, they mated and had offspring. These baby mice had long tails. He repeated this experiment generation after generation. Cutting the tails of the parent mice did not affect the germ cells of either males or females. He then selected mice with the shortest of stubby tails, mated them, and continued to select the shortest-tailed mice for parents. After several generations of this selecting and mating, a short-tailed race of mice was produced. He had selected mice whose germ cells carried the gene for short tails.

As a result of this second experiment, Weismann formulated a theory explaining why offspring resemble their parents. He believed that the germ plasm does not arise anew in each organism, but is actually received from its parents. Explained briefly, his theory is this: Each new organism begins as a single cell, which is pure germ plasm. In higher plants and animals this cell is formed from the union of an egg and sperm. The cell, called a fertile egg, begins to divide. Very early in this division, and before differ- 
entiation begins, one cell or more will be set aside to produce future germ cells. The other cells go on multiplying and form the soma,

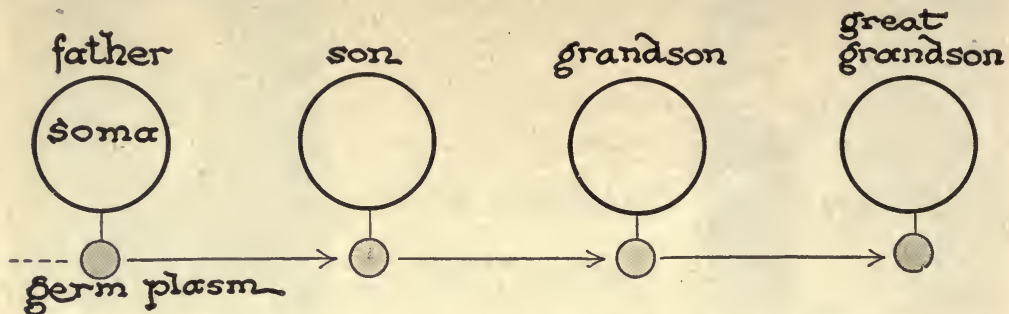

According to August Weismann germ plasm is derived from the germ plasm of previous generations. Thus it is continuous from one generation to another. Successive generations must resemble each other since they are all derived from the same germ plasm. Somatoplasm develops from germ plasm.

or body cells. From the former cells, there develop all the germ cells of the organism. Germ cells consist of germ plasm, body cells of somatoplasm. When some of the germ cells of one individual meet the germ cells of another individual, they will unite, and, while still in the form of germ plasm, a small portion of it will be set aside to form the germ cells of the embryo. Thus the germ plasm is continuous from one generation to the next.

This continuity of germ plasm has actually been demonstrated. One observer records that when, in a certain worm, the sixteenth cell stage of the embryo is reached, one cell is set aside to form the germ plasm and the other fifteen of the cells form somatoplasm.

Mutations. It has already been stated that variations of the body plasm, which occur during the life of the individual, such as dwarfing of trees by wind and the acquiring of muscular skill, are generally considered not inheritable. These are often called acquired characters. Acquired characters are somatic variations which are due to environment and are not as a rule transmitted from parent to offspring. Some experimentation has recently been undertaken to show that, under certain conditions, acquired characters may be transmitted from parent to offspring. But, since these abilities were acquired by the soma cells long after the germ 
cell became separated from the body cells, investigators are having difficulty in explaining how the germ cells can transmit the acquired characters.

However, a plant or animal will often appear, which, from the first, is so unlike its parents that it is called a freak or sport. For example, albino animals which have no pigmentation in fur, skin, or eyes are not infrequent. White rats have been developed as a distinct species from a sport which appeared as an offspring of rats with pigmented coats. Albinism is inherited as a recessive trait and is sometimes found in humans as well as other animals. This sudden departure from the ancestral type is called a mutation and the individual itself is a mutant provided the new species

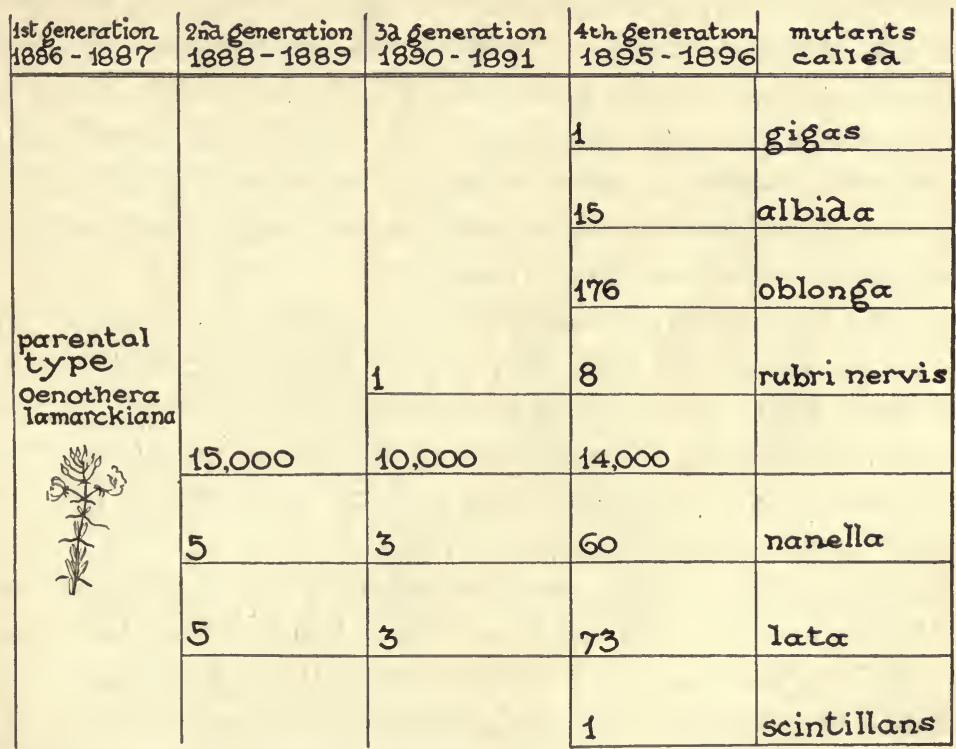

Hugo de Vries bred evening primroses through numerous generations. As his experiment progressed, he observed among them peculiar dwarfed individuals which bred true. He called these nanella. Then he isolated a type with unusually broad leaves which he called lata, and a type with reddish veins, the rubrinervis. In successive generations, he observed other plants with different characteristics. He preserved the new qualities by careful breeding. These were mutant primroses. 


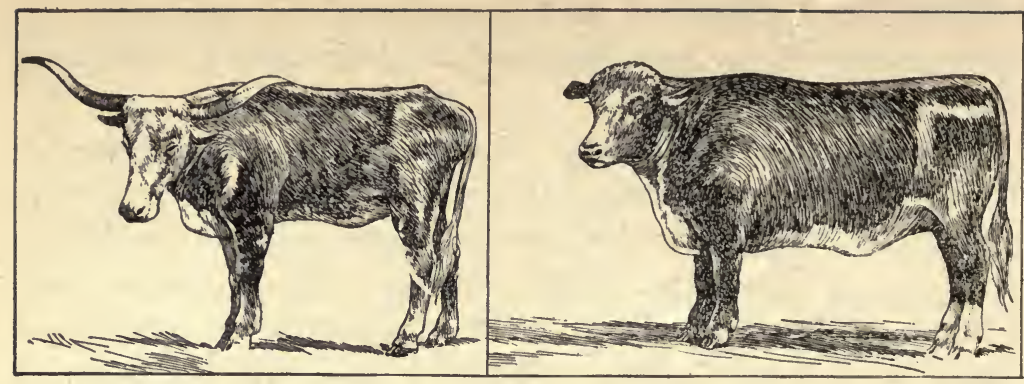

Cattle originally had long horns. There appeared a mutant without horns. This animal became the ancestor of a hornless race of cattle.

breeds true to its type. In Paraguay in 1770, there was born a hornless calf in a herd of ordinary long-horned cattle. Animals without horns are less dangerous to the other animals of the herd and also to their owners. Therefore, this animal was later bred with the ordinary cattle and it was found that the hornlessness was inherited as a dominant factor. Before this, cattle had sometimes been de-horned, polled artificially, but from this one animal an entire race of hornless or naturally polled cattle was developed. The original hornless calf was a mutant.

Short-legged sheep, which cannot jump walls, have been developed from the long-legged Ancon sheep. Six fingers instead of five, two joints in the fingers instead of three, and webbed fingers are a few of the inherited modifications, mutations, that have been found in human beings. The navel orange (seedless orange) originated as a mutant in Brazil.

The first person to use the term mutation was Professor Hugo de Vries of Holland. He was born in 1848 and was, for a long time, a professor at the University of Amsterdam, Holland. He carefully watched the descendants of one primrose plant for several generations and found that it gave rise to seven distinct new species, each of .which bred true. Each mutant differed decidedly from the others in height, shape of leaves, or some other character.

Mutations are inheritable and there is little doubt that they are 
due to some germinal variation. They may be dominant or recessive in character. They are of tremendous economic importance to man since valuable and desirable mutants can be used as a starting point for a new species of plants or animals. De Vries was the first man to advance the theory that many of our present-day plants and animals possibly originated as mutants.

Causes of germinal variations. Our ignorance concerning the causes of germinal variations is profound. We do know that slight variations are exceedingly common; no two living things, even of the same species, are exactly alike. Decided variations such as mutations are more rare, but when we become more observant we find them to be more frequent than, at first, believed. The following are some of the more common explanations of causes of mutations.

(a) Changes in chromosome number. Scientists found that the original primrose had 14 chromosomes, but that the 7 mutants had respectively $15,16,20,22,24,27$, and 28 chromosomes. Other observers have also found at times a different number of chromosomes in other mutants. How or why the number of chromosomes changes is not yet definitely known.
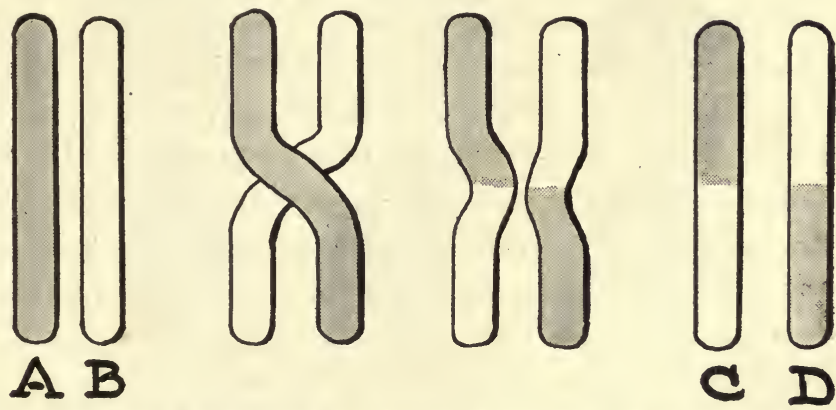

Chromosomes A and B sometimes bend around each other. Before they separate, part of $\mathbf{B}$ becomes joined to $\mathbf{A}$ as in $\mathbf{C}$ and part of $\mathbf{A}$ becomes joined to $\mathbf{B}$ as in $\mathbf{D}$. This is called crossing over. Some of the factors originally in one chromosome are now in the other.

(b) Change in the character of the gene itself. Morgan has found that most of the fruit fly (Drosophila) mutants that he has studied 
had the same number of chromosomes as their parents. Therefore, it may be assumed that when the chromosome number does

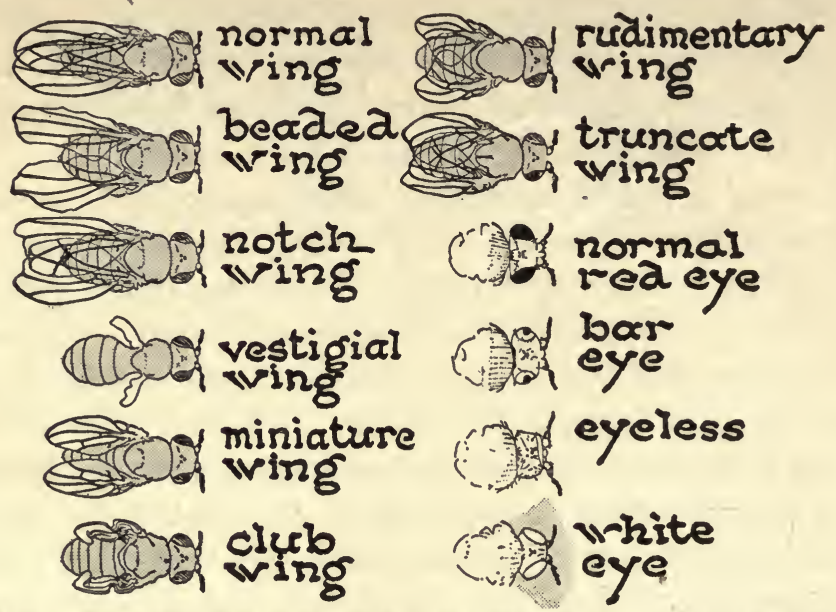

T. H. Morgan and his associates have observed the appearance of hundreds of new characters, mutations, in fruit flies.

change, a mutant is produced, but that germinal variations may also arise in other ways. Morgan has shown that mutations sometimes arise as a result of one gene or more actually changing in character. He has identified over 400 characters which are carried by the eight chromosomes of the fruit fly (four chromosomes in each germ cell). He believes that each character is determined by a gene in a chromosome. Therefore, there are many genes in each chromosome. Experimental work in heredity makes it seem highly probable that these tiny units of inheritance are present in the chromosome, and if they change in character it again seems plausible that a germinal variation would result.

(c) Crossing-over of genes. Several scientists have done experimental work that would seem to show that sometimes the genes actually cross over from one chromosome to another. 'Just before reduction-division, the chromosomes always arrange themselves side by side in pairs. In the fruit fly there would be four 
pairs. Under the microscope it has been seen that the paired chromosomes have occasionally exchanged places. This is not a certainty, because, under the microscope, both members of a pair of chromosomes look exactly alike. But it can be seen that they become twisted. The experimental work makes the possibility seem highly plausible that some of the genes from one chromosome of the fruit fly have crossed over to the other.

(d) Decided changes in environment. Doctor Tower of the University of Chicago collected 40,000 potato beetles, grouped them into colonies, put them in glass cages, and subjected them to varying degrees of heat and moisture. He found that the change in environment had no effect upon that generation, but the eggs laid by these beetles produced offspring of a different color, which continued to

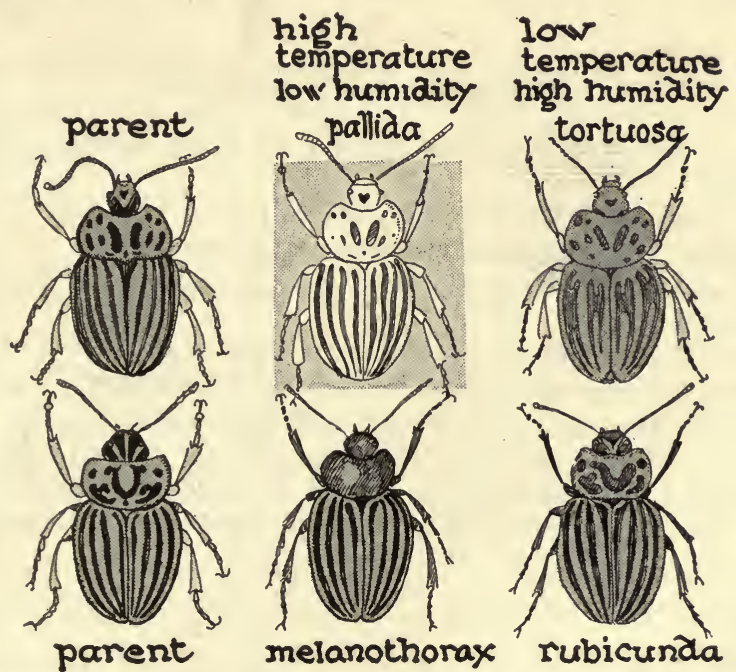

Certain scientists are trying to prove that environmental conditions can produce changed characters in organisms, which will be transmitted to their offspring. Tower subjected parent potato beetles to certain varied conditions of temperature and humidity. The offspring showed new characters which they in turn transmitted. Some of Tower's mutants are shown in the above diagram.

breed true as long as he kept the heat and moisture the same. Tower has been able to produce mutants by environmental 
changes. Other insects, such as butterflies and grasshoppers, have been known to produce mutants by a decided change in temperature, food, and recently by the use of the X-ray. The treatment of parent fruit flies and mice with X-ray and radium rays has caused them to produce offspring with somewhat malformed bodies. These peculiar characters were handed down to the next generations. The germ plasm had been affected. It would seem that in some way the germ plasm is affected by the change in the conditions of the environment, causing mutations to appear in the second generation. It is not conclusively known whether a decided change in environment has ever produced mutants in nature as it has been done under experimental conditions in the laboratory.

\section{Questions And Suggestions}

1. State the doctrine of continuity of germ plasm. How old is germ plasm?

2. What are mutations? Give examples.

3. What is the relation of mutations to heredity?

4. What part did Hugo de Vries play in developing the theory of germ plasm?

5. Give three theories concerning the causes of mutations.

6. Discuss Professor Morgan's work with fruit flies.

7. Discuss Doctor Tower's work with potato beetles.

8. Give a library report on the biography of August Weismann.

9. Look up in a recent science book or magazine and report on the experiments that are now being carried on concerning mutations. 


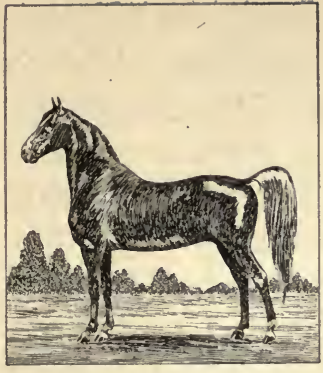

Speed Horse.
CHAPTER XXXV

\section{PLANT \\ AND ANIMAL \\ BREEDING}

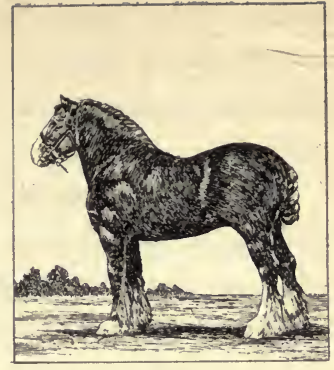

Dray Horse.

What are some of the results of plant and animal breeding? What are some of the methods used by plant and animal breeders to improve the variety?

Importance of plant and animal breeding. It is only a little over one hundred years ago that the English clergyman, Malthus, wrote his famous essay setting forth the principle that population tends to increase much faster than the supply of food. He believed that famines, wars, and plagues were useful, at least, in limiting the population that otherwise would be doomed to slow starvation.

What is the situation to-day? Since the time of Malthus, the population of Europe has nearly trebled, and in the United States it has increased approximately ten times. Yet famines are no longer experienced except in those countries with poor transportation facilities. The world as a whole has at no time been so wellfed nor had so great a variety of foods. Malthus contributed a fact of real value to mankind in calling attention to the rate of increase of the population. Since that time, modern farm machinery, good transportation, and knowledge of food preservation have all aided an increasingly smaller proportion of the people of the world to provide sufficient food from the farms for the needs of all.

Another factor which has greatly increased the production of food, and which is of particular interest to the biologist, is the 
improvement of food-producing plants and animals. The cattle of the Middle Ages were about the size of the average calf of to-day, but through many generations of selective breeding the size of cattle and their yield of milk have been increased to a remarkable degree.

Alfalfa is especially hardy. It withstands droughts, and it may yield as many as nine cuttings a season. Due to careful selection of seeds and cultivation of the ground a field of alfalfa will furnish sufficient food for three to six times the number of cattle which it formerly supported. Plant breeders have been able to increase the amount of sugar in beets so that the number of pounds of beets needed to make one pound of sugar has decreased from eighteen pounds in 1836 to seven pounds in 1904 . Luther Burbank improved the potato by making it resistant to a disease called the potato blight, and by increasing its starch content. It is said, that this species of potato adds seventeen and one half million dollars to the farm incomes each year.

One of the best dairy cows of recent years, gave 20,616 pounds of milk and 1005.9 pounds of butter fat in one year. She gave more than her weight in milk each month. Contrast this cow with a prize cow of 1904, that gave 567 pounds of butter fat in one year.

Every day our agricultural experiment experts and other scientists are improving our foods. These products are not produced in a haphazard way. They are frequently the results of careful experimental breeding. It is, however, only within the last twenty-five years that sufficient knowledge of the laws of heredity has been available to put plant and animal breeding on a scientific basis.

It is the plant breeder's concern to increase the food content of grains and fruits, to produce new species immune to disease, to make certain varieties hardy so that they can be grown in more northern climates, and to hasten maturity. The average yield per acre 
of wheat was between 10 and 15 bushels per acre. By selection and cultivation, the yield per acre has been increased to over 40 bushels. Wheat has been bred so that several species now combine the desirable qualities of large yield per acre, good quality for bread making, hardiness, resistance to rust, and resistance to drought. The ordinary corn stalk usually produces but two or three ears. But through experimentation, corn has been produced with stalks 16 feet high and which bears 32 ears to the stalk.

The particular aims of plant and animal breeding are to establish varieties immune to disease, to produce new species, to breed for desirable characters, to improve quality by proper selection and to make both old and new forms more productive.

Immunity to disease. In some of the Southern States, the cattle had long been subject to Texas fever which was easily spread and caused the loss of many cattle. It was learned that the wild Brahmin cattle of India were immune to the disease. Their flesh, however, was not valuable as beef. Two strains, our southern cattle and the wild Brahmin, were crossed. Immunity to Texas fever was found to be dominant in the $F_{1}$ generation. These hybrids were mated again and from the $\mathrm{F}_{2}$ generation only the immune animals that seemed to show the best beef tendencies were selected and bred. By careful selection
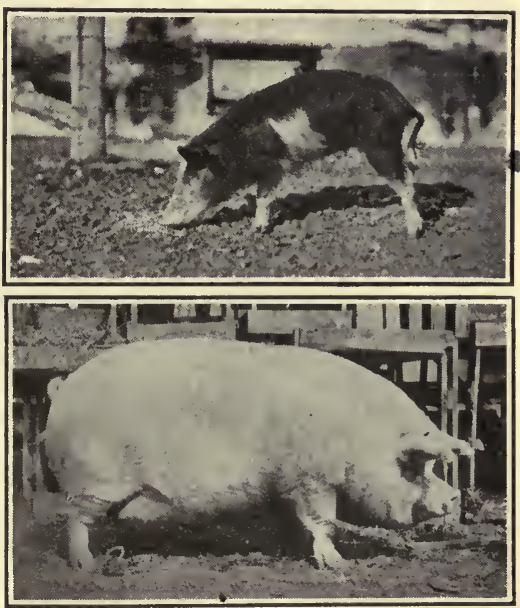

U.S. Dept. of Agric.

Animal breeders have produced from the original wild strain of pig shown above the fine specimen shown below. Compare the food value of the two specimens.

a species of cattle combining both immunity and good beef qualities was thus obtained.

WH. FITZ. AD. BIO. -23 

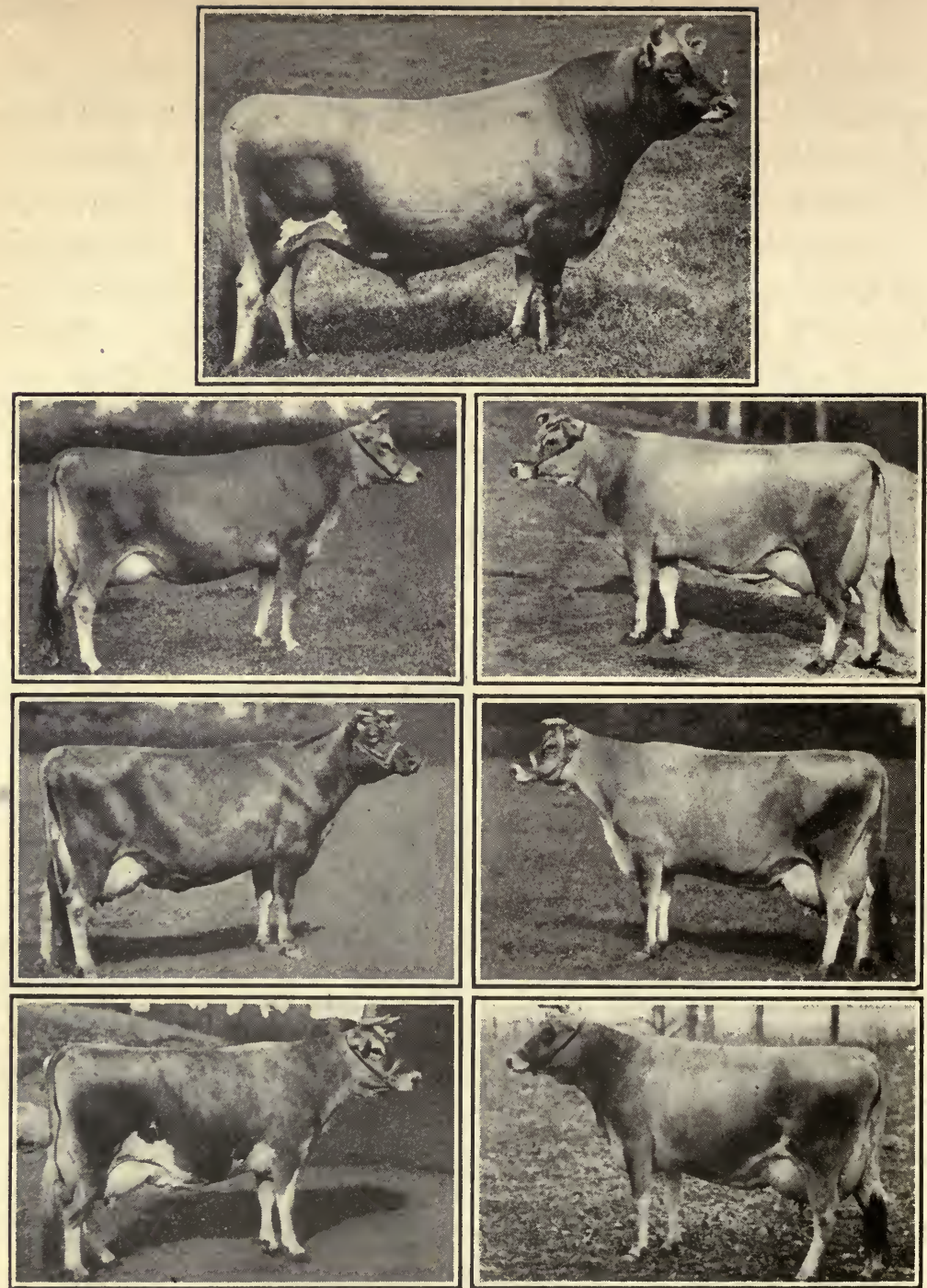

An average dairy cow gives approximately $8304 \mathrm{lbs}$. of milk per year containing from 130 to $300 \mathrm{lbs}$. of butter fat. The daughters of a famous sire, Fauvic's Prince, averaged 17,135 lbs. of milk per year. Their average yield of butter fat is $\mathbf{8 0 1} \mathrm{lbs}$. per year. Fauvic Star, the middle cow on the right broke all records with $30,616 \mathrm{lbs}$. of milk and $1005.9 \mathrm{lbs}$. of butter fat. 
A certain fungus disease in wheat is recessive. In 1906, from a field of badly infected wheat in Kansas, a lone plant was discovered that showed no signs of rust. This single stalk was probably a mutant. It was tested, propagated, and the offspring were carefully selected for immunity as well as for the desirable. qualities of the parent wheat. An immune species that also gave an increased average yield of four and one half bushels per acre was finally produced. In 1917, this strain of wheat, called Kanred (a combination of Kansas and its color-red), was distributed for commercial use and has already increased the wheat income in Kansas by many million dollars per year. The same procedure was followed in combating a blight that often affected corn.

An immense amount of damage is annually done by corn smut. Some

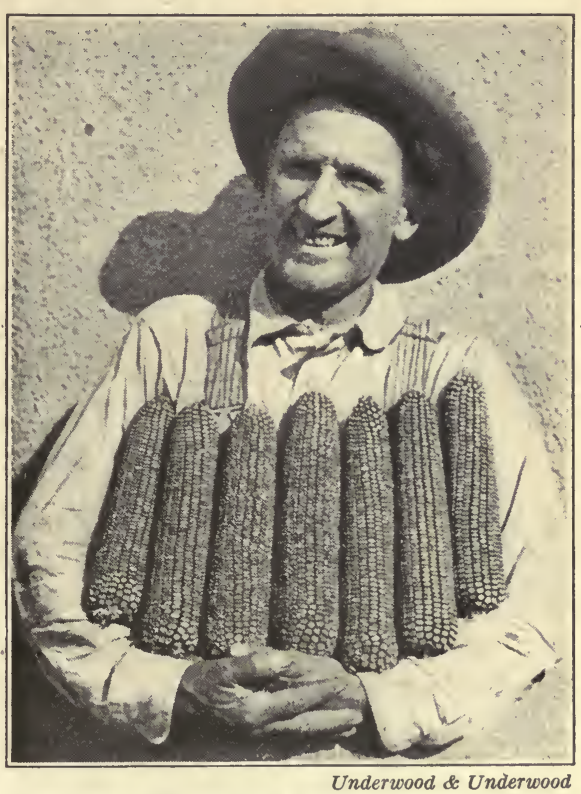

Corn has been improved through careful breeding. Desirable characters such as number of kernels, food content of kernels, regularity of formation of kernels and size of ear have been combined in the same corn plant. A prize red corn of Kansas is shown in the picture.

sound ears were found in fields of corn that were badly infested with corn smut. The sound ears were selected and bred until an immune variety was obtained.

Production of new species. A white blackberry sounds like a paradox, but we use the term in referring to a new blackberry developed by Luther Burbank. He noticed a wild fruit, bitter, 
small, and really a light yellow rather than white. It was, without a doubt, a member of the blackberry family. He hybridized this by crossing it with the Lawton blackberry which was black, large, juicy, and of pleasing flavor. He found that black was dominant over the light yellow. In the $\mathrm{F}_{2}$ generation the light color again appeared, and by selecting for several generations only the plants for breeding that combined the lack of color with the desirable flavor and size, he obtained a large tasty berry that is now known as a white blackberry.

Some varieties of plants have been improved in many different ways. Luther Burbank studied various kinds of plums obtainable here and in other countries. He crossed American, Japanese, and European kinds. Then he selected the best for further experimentation. As a result, he obtained different species: (1) of many varieties in the coloring of skin and the texture of flesh; (2) of a wide variety of shapes; (3) of a wide variety of new flavors and aromas; (4) of sizes that are increased to three inches in length and two and one half in diameter; (5) that produced either early or late species. (Some ripen a month before the earliest of the old varieties; others ripen as late as December.) (6) that are not affected by the frost or cold weather. Some plums have been developed that can be shipped long distances without spoiling. A species has been produced that can remain on the tree a month or two in hot weather without decaying, unlike the old varieties which had to be picked as soon as they were ripe. Some trees bearing these new varieties will begin to bear abundantly the third year if cuttings from young trees are grafted upon trees of ordinary size.

Some of these new plums grow in climates and under conditions where the plum has hitherto been a failure. The Beach plum is a wild species growing along the coast. It is a low, spreading shrub with a small bitter fruit. It is very prolific and is resistant to cold and frost. This was crossed with an American plum. The result- 
ing species, called the Improved Beach plum, bears very abundantly. It is a delicious plum of very fine flavor and with a small stone. It is indifferent to frost, and bears under the most trying conditions of soil and climate.

A cross between a plum and apricot has been produced by Luther Burbank. It is called the plumcot. He also produced a stoneless plum.

Breeding for points. Seedlessness has sometimes developed as a mutation. The seedless navel orange originated as a mutant in Brazil. Twigs from it were grafted on to ordinary orange seedlings. Two of these tiny plants thrived, and from them, propagated by grafting, all of the navel oranges have been produced. The value of vegetative reproduction as a means of making a new species breed true to type is very great. Since this makes use of the body plasm, it will continue to produce other plants like

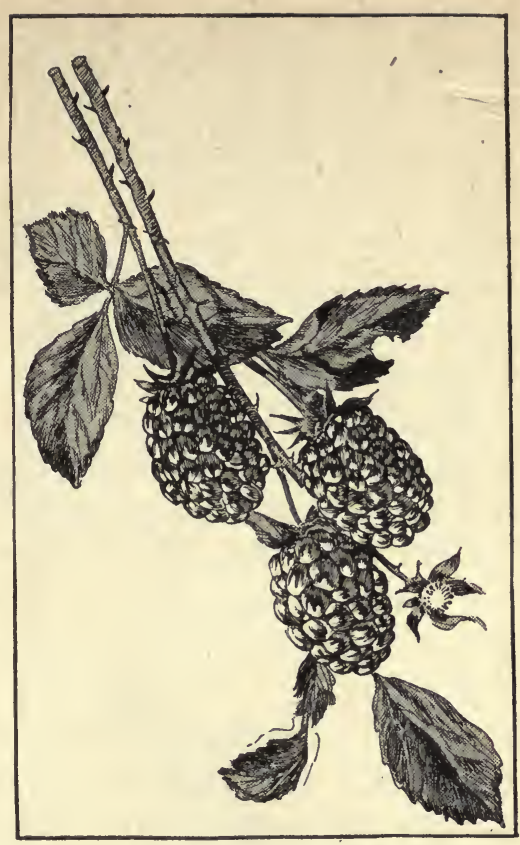

The Lawton blackberry.

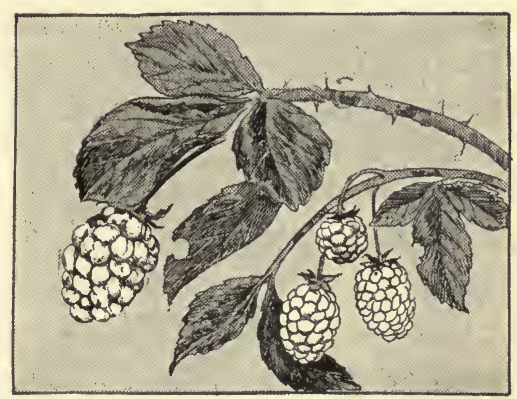

The white blackberry that was crossed with the Lawton blackberry. itself. For instance, if a breeder has produced a large, doublepetaled, red-flowered dahlia, he may be sure that the bulbs of this 
dahlia will give rise to large double blossoms colored red. If he plants the seeds, however, he will probably find outcroppings of the plant's ancestry in the way of small flowers, some single rather than double, some with white petals, and so on. The potato at present rarely bears seeds. When such seeds appear and are planted, different kinds of potato plants may result. Many of these plants may bear potatoes that are small, irregular in shape, and low in food content. They closely resemble their wild ancestors. Therefore, the potato is propagated vegetatively by using parts of the tuber containing the "eyes." Tomatoes were formerly small, tasteless, and full of seeds. But through selection and cultivation, many splendid varieties have been developed, within the last generation. Other details that have been brought out through breeding are: increased egg laying in hens, increased milk supply from cows, and increased starch and protein production in corn.

Improved quality. The southeastern region of the state of Washington was an excellent locality for growing wheat. But the climate is so severe that every three or four years, the entire crop was killed by frost. The problem of producing a hardy variety of wheat was turned over to Mr. Spillman of the United States Department of Agriculture. He selected as a beginning, the Little Club wheat, named for the short, clublike appearance of the head. It was desirable because it had a stem strong enough to resist storms and a head that remained closed long after ripening. Thus the ripe grain was protected and was not likely to be lost before harvesting. This species was crossed with other varieties, including a hardy winter variety that would resist frosts. After ten years of careful selection and propagation, an improved frost-resistant variety of Club Wheat was obtained, that is now grown in the extreme Northwest. Winter character was found to be dominant over spring character and the club head to dominate over long head. 
Increased production. In almost all plants and food animals, a great increase in production has been brought about and there is still room for much improvement. Careful selection of the best types for breeding is a very important factor here. Burbank increased the food content of the potato by 25 per cent; Kanred wheat, męrely through vigilant selection of the most productive plants for seed, surpassed its parent wheat by an increased yield per acre. The average cow's milk will produce 125 pounds of butter per year, but a Jersey cow will usually produce over 1000 pounds of butter in a year. A 300 -acre field in southern Illinois was planted with improved corn and yielded 30 bushels more per acre than the fields planted with the ordinary seed.

Methods of breeding. Hybridization is the crossing of two individuals carrying unlike characters. This has the ad-

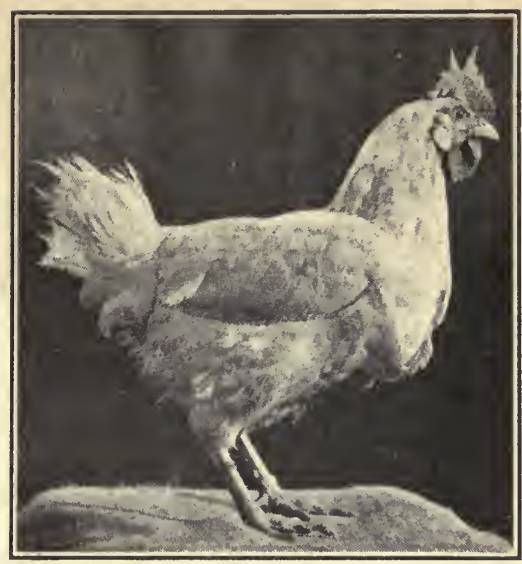

This white leghorn hen established a record by laying 321 eggs in 51 weeks.

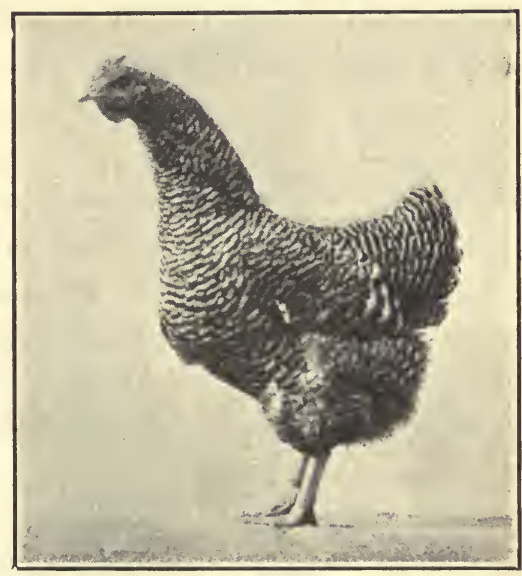

This barred Plymouth Rock hen laid 287 eggs in a year. The American egg record for three consecutive years was 282 eggs and 281 eggs made by two Barred Plymouth Rocks, and 303 eggs from a hen which was a cross between a white leghorn and a Barred Plymouth Rock.

vantage of combining the desirable characters found in the two individuals into one individual. 
Artificial selection. Many experiments in breeding start with hybridization, but they would be of little value unless the individuals showing undesirable traits were disregarded and only those showing the desirable characters kept for breeding. This is called artificial selection. Even so, the organisms showing the useful traits will oftentimes not breed true as they are hybrids. To be successful and establish the desirable qualities, large numbers of matings must be tried out and only those that breed true should be retained. As has been said before, when vegetative propagation is possible, it is a certain means of breeding the plant true to type. Improved kinds of fruit trees are propagated only by grafting.

Method of plant propagation. The typical method of plant propagation was that used by Burbank. The flowers which are to furnish the pollen are carefully gathered a day or so beforehand. The pollen is sifted from the flowers and kept in a cool place. The tree to which the pollen is to be applied is deprived of most of its blossoms in order that the remainder may be sure to develop and that there may not be too many to be properly looked after. The buds of the remaining blossoms are prepared for artificial pollination by cutting away the petals and the attached stamens. The pistils and stigmas are left uninjured and are protected from insects. Since there are no brightly colored petals to attract them, or anything for them to hold on to when entering, bees seldom approach these flowers. If there is any danger of insects visiting

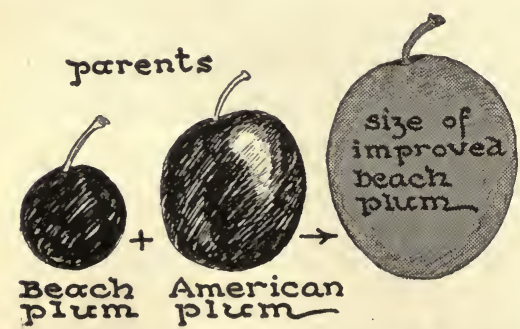

The size, flavor, and quality of plums have been improved through selection and cultivation. the flowers, a paper bag is frequently tied over each individual flower. When the pistil ripens, the pollen is applied by dipping a brush or finger into this yellow powder and touching the stigmas. All of the best seeds that develop from the fruit of the pistil thus 
treated, are saved and planted. When these new plants produce fruit, only the best seeds are selected. Frequently, the young plants are grafted upon other trees to hasten the growth. $\mathrm{Hy}-$ bridization is but the beginning of breeding. Careful selection must be continued, so that a pure type is established.

Methods of animal propagation. If a mutant with a desirable character is found, it is bred. In case the new character is dominant, it will appear in the $\mathrm{F}_{1}$ generation, but the animal of this generation will be hybrid. This hybrid is then mated with an animal which shows the same desired character. Sufficient matings must be made to get the character in a pure form. This sometimes necessitates close breeding. By close breeding is meant mating individuals closely related, as grandparent with offspring of the second, third, or succeeding generations. The fact

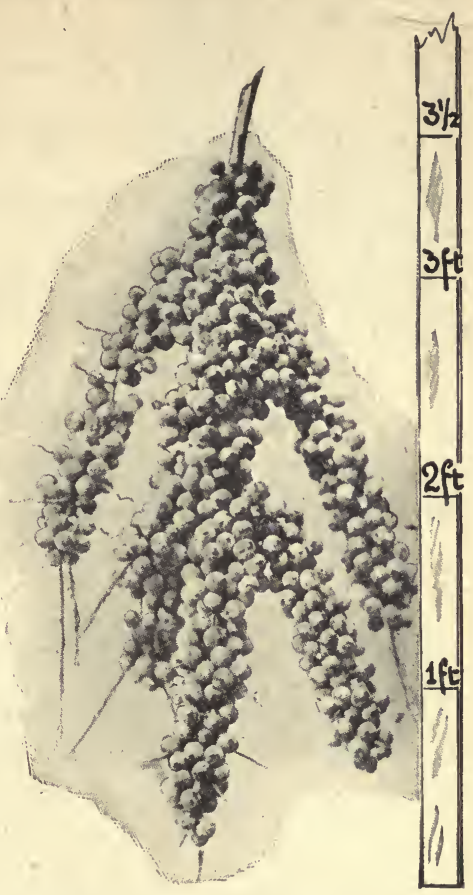

The Beach plum grows in clusters. It is a small plum, smaller than the American plum. When these two plums are crossed, an improved plum, much larger than either parent, is produced growing in bunches like the parent Beach plum. that animals frequently take a number of years to mature is a serious difficulty met with in animal breeding. Close breeding does not necessarily reduce the vigor, but, if there are undesirable characters present, they may be doubled and the offspring, then, cannot be used for breeding purposes. In case the new character is recessive it will not appear until the $\mathrm{F}_{2}$ generation. Then, if two individuals of different sex of this generation pos- 
sess the desired characters, and are mated, their offspring will usually be a pure type.

\section{Questions And Suggestions}

1. What bearing has plant and animal breeding on the food situation of the world?

2. Why is scientific plant and animal breeding a comparatively recent development?

3. State five aims of plant and animal breeders.

4. Describe in detail an example of each aim mentioned in question 3.

5. Describe in detail how a particular trait may be established.

6 . What two biological principles are involved in breeding experiments?

7. State three difficulties animal breeders encounter that are not encountered by plant breeders.

8. Look up and report on the life and work of Luther Burbank.

\section{SuPPLEMENTARY REAdings}

Osterhout, W. S. V., Experiments with Plants (The Macmillan Co.). Sinnott \& Dunn, Principles of Genetics (McGraw-Hill Book Co.). 


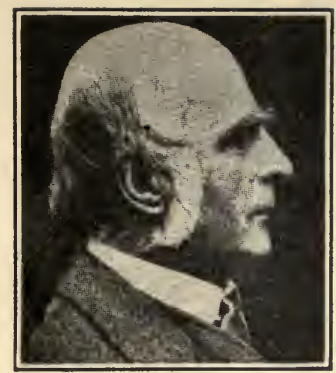

Sir Francis Galton.
CHAPTER XXXVI EUGENICS

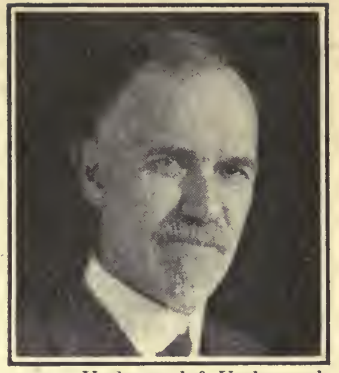

Underwood \& Underwood. Charles Davenport.

What is the importance of eugenics? What is the difference between eugenics and euthenics? How can eugenics and euthenics be applied practically?

Eugenics is the division of biology that deals with the improvement of the human race. It is a new science built upon an application of what we know concerning the laws of heredity. It also includes the study of how to improve the environment, although the effect of environmental conditions on people is frequently referred to as euthenics.

History of the eugenics movement. In 1883, Sir Francis Galton, an Englishman, became interested in the science concerned with the improvement of the human race, to which he gave the name eugenics. He had in mind the improvement of human characteristics through heredity. In 1921, the Second International Congress of Eugenics stated that eugenics was the self-direction of human evolution. This means that individuals through good or poor marriages, can improve or impair the mental or physical heritage of future generations. The science of heredity has contributed much to eugenics. Many family histories have been investigated and different physical and mental traits have been traced through generations, with the utmost care. These investiga- 
tions have given data for the practical applications of eugenics. An Eugenics Laboratory is located at Cold Spring Harbor, Long Island, under the supervision of Dr. Charles B. Davenport. Research in the science is being carried on there by the most careful specialists.

Methods of investigation. - Families of superior ability. Human heredity has not been studied by the experimental method as successfully as animal and plant heredity have been studied. There are three reasons for this failure: (1) The length of life is so long that no investigator can live long enough to study, personally, the heredity of more than two, three, or possibly four generations of a family ; (2) the number of offspring is small and it is difficult to draw valid conclusions from the limited data offered; and (3) our

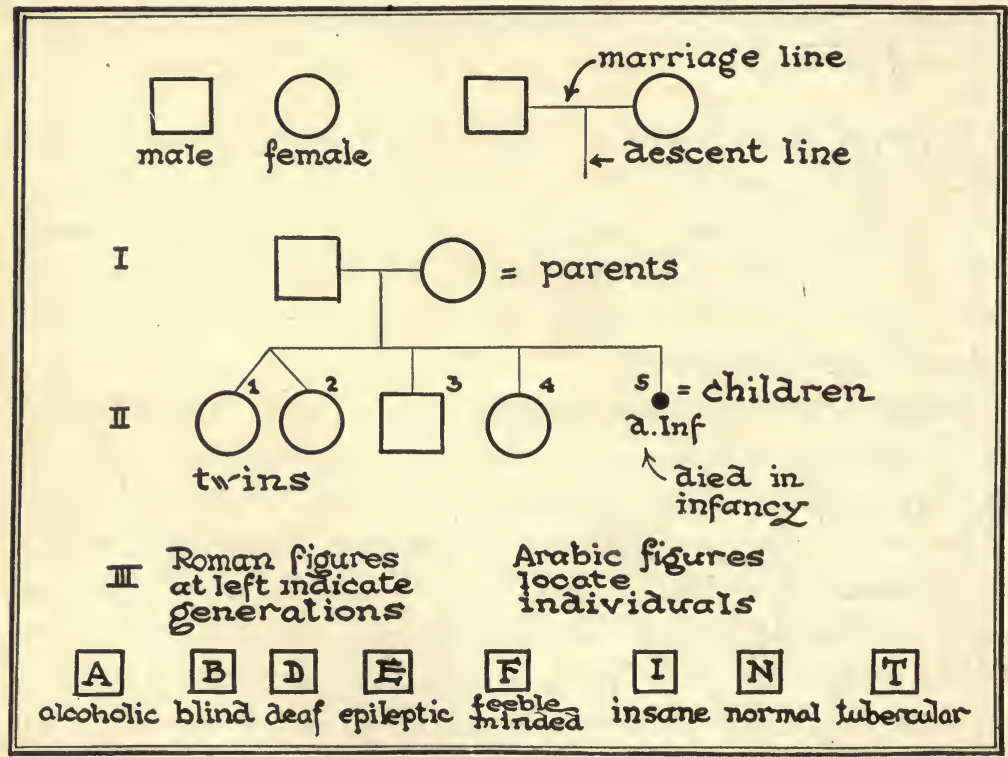

The Eugenics Record Office at Cold Spring Harbor has adopted a standard set of symbols used in chart making.

social customs handicap the work in this research, as people consider family affairs private and will not give information freely. 
Sir Francis Galton applied the statistical method to the study of heredity in human beings. By this system, the pedigrees of many families have been traced for generations and studied as a means of determining what traits seem to occur most frequently in a majority of the descendants. Such a study has been made of the family of Jonathan Edwards. He was born in 1703 and was noted for his strength of character and for his high mental ability. In 1900, 1394 of the descendants of Jonathan Edwards had been traced and the life work of many of them discovered. They included many college presidents, professors, lawyers, doctors, able business men, and state officials. The group as a whole was highly intelligent and capable, with a high sense of civic responsibility. Their historian states that it is not known that any of them were criminals. The Edward family indicates that high mental ability is inheritable.

An investigator recently tested a number of children for intelligence. Forty-one showed superior intelligence. What of their immediate ancestors? Of the forty-one, the investigators found that only two lacked a near relative with a superior intelligence rating. Definite talent in some form was found in different members of the families of these children. A study has been published concerning the intelligence of some students of Oxford University, England. The records of boys who secured marks of "honor" and "pass" were compared with the records made by their fathers. Of the "pass" students only twenty per cent of the fathers had taken first or second honors. That means they, too, were only in the "pass" class in their school days. On the other hand, of the students taking first honors, nearly forty-two per cent of the fathers had taken first or second honors.

Woods has made. a study of several royal families, in which a comparison is made between parents and offspring in mental and moral qualities. There is a very marked similarity and uniformity shown in the results of this study. A resemblance between 
grandparents and'grandchildren is evident though not as noticeable as closer relationships. Outstanding groups of individuals have descended from Peter the Great of Russia, William the Silent of Holland, Isabella of Spain, and Gustavus Adolphus of Sweden. These studies have shown that men of the type of the Sidneys, Balfours, Cecils, and Churchills in England, and the Lowells, Eliots, and Dwights in America are not the result of chance or accident, but that their superior abilities are inheritable traits. When an exceptional man chooses an exceptional wife, the majority, if not all, of their children will probably be exceptional. All members of royal families are not superior. There are some groups that show insanity, imbecility, and other mental defects, because these traits have been introduced through marriages with mental defectives.

There seems little doubt that a certain stock or family will produce men of great ability more frequently than will another stock or family. It is almost certain that the quality of mind and body of the parents will affect the offspring. Parents, sound in mind and healthy in body, tend to produce normal healthy children.

Families of inferior ability. Parents physically weak and of a low-grade mentality will give to the world undesirable progeny, probably physically unfit and with mediocre intelligence. An American family which has been given the fictitious name of "Juke" has been carefully traced through a large number of generations. This family record starts with a shiftless vagabond born in New York state in 1720. In 1915, students had traced 2094 members of this family: 1600 were feeble-minded or epileptic, 310 were paupers, 140 were convicted criminals, and large numbers of the remaining group were traced through the records kept in workhouses and other public institutions. This family by 1915 , had already cost the state of New York more than $\$ 2,500,000$ and the expense still goes on. Their descendants will continue to bear the same traits. The mental ability of this family is very 
low. Only twenty have been known to learn a trade and ten of these did so while in state prisons.

The story of the Juke family indicates that weakness of character and low mental ability will give rise to offspring with little moral or mental stability. Although this study does not prove that a tendency toward wrong-doing is inheritable, it certainly does show, at least, that weak character and low mental ability give rise to a person who often becomes a criminal. The Jukes lack the judgment, memory, and will power that enable people to fulfill responsibilities toward their fellow men.

The Kallikak family (the name is fictitious) throws still more light on human heredity. Martin Kallikak, a normal, healthy

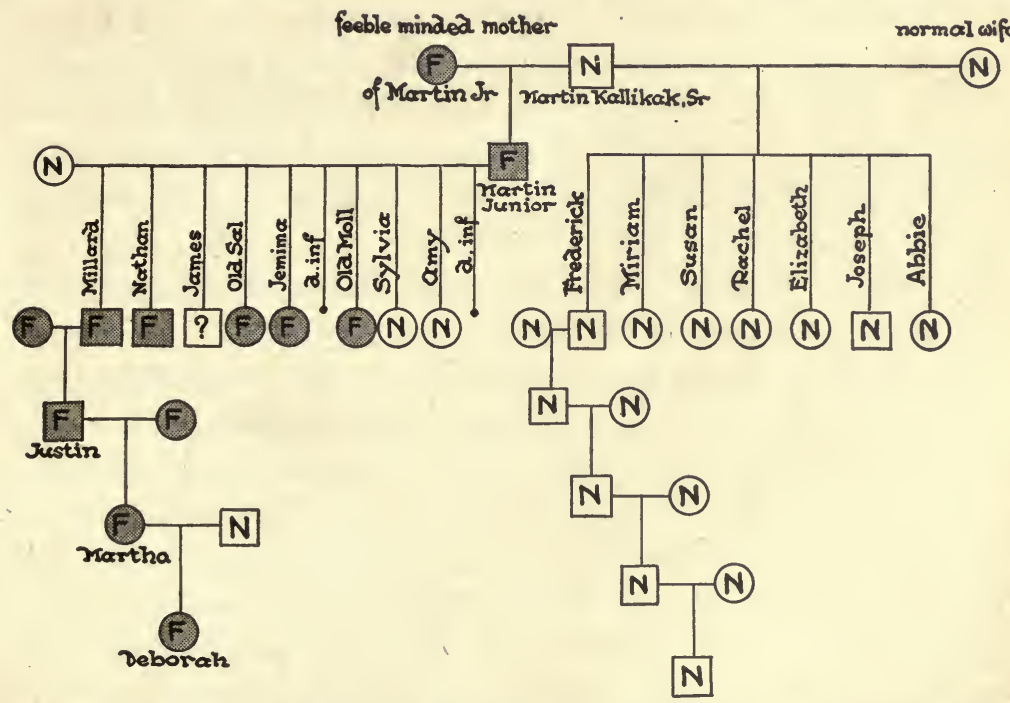

Intelligence and lack of intelligence seem to be inherited characters. One of the lines of descent of Martin Kallikak shows feeble-mindedness inherited from a feeble-minded mother. Of 480 descendants studied, only 46 were known to be normal. The other line of 496 descendants from Martin Kallikak and a normal wife was practically all normal.

young soldier who fought in the American Revolution, had a son by a feeble-minded girl. This son had a family of ten children from 
whom there are 480 known descendants. Of these, only 46 are known to have been normal while 143 were feeble-minded. Knowledge concerning the others is missing or doubtful. After the war, this same soldier, Martin Kallikak, married a normal woman. From this union there have been 496 descendants; none of whom were feeble-minded. The history of the Kallikak family indicates that feeble-mindedness is inheritable. The defective descendants were incapable of earning a living and some could not provide for themselves nor care for their physical comfort. It is necessary that these defectives be fed and sheltered, but it is more important that they be segregated in institutions where they cannot produce more offspring like themselves.

The number of mental defectives in England and Ireland has been estimated to be about five per cent of the population. In 1923, in the United States there about 267,600 mental defectives. The annual cost of caring for these persons was nearly $\$ 80,000,000$, to which should be added three hundred millions of dollars which was the corresponding loss in industrial activity of these dependent people. In some states, one eighth of the total state expenditures is for the care of the insane.

The cost of crime in the United States is ten billion dollars annually. Over twenty per cent of the inmates of jails, almshouses, and other institutions are foreign-born, although only fourteen per cent of the total population is foreign-born. There seems to be twice the number of foreign-born as native-born among the defective group which includes the feeble-minded, insane, epileptics, criminals, blind, deaf, paupers, and other dependents on a community. This is probably due to the fact that many foreigners of poor stock were formerly admitted to this country, but such undesirable aliens are now excluded. Therefore, the above figures include the people admitted before our immigration laws were so strict. They also show the necessity of a careful physical and mental examination of all immigrants to this country. 
A study of the records of 447 criminals showed that among their parents, 10 per cent were criminals, 6 per cent were victims

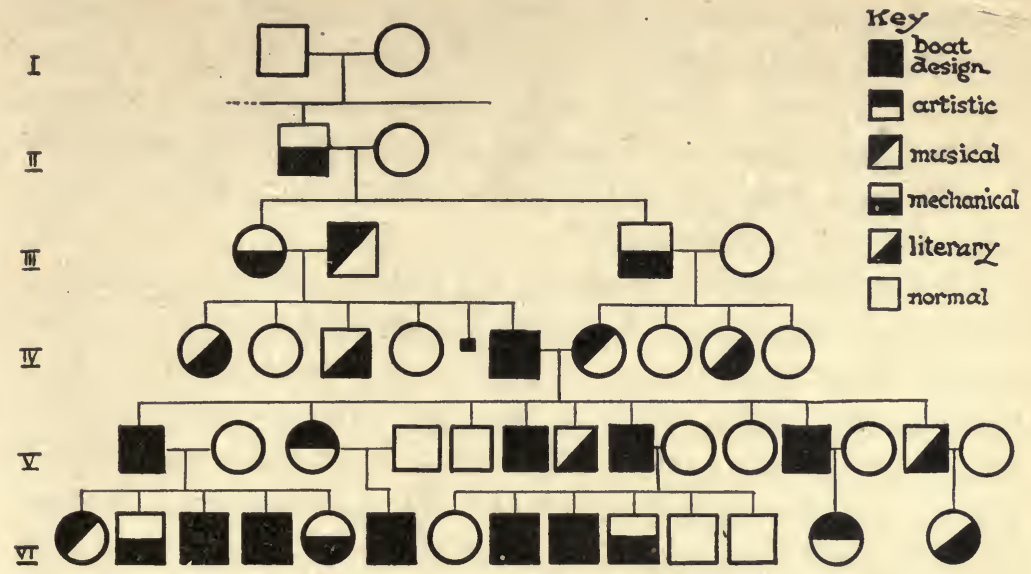

The Herreshoff family is noted for special skill in boat designing and building. Many cup winners have come from their yards, some of which have competed with Sir Thomas Lipton, challenger. The above chart includes one line of descent of the family. The entire genealogy has been traced and is recorded in the Eugenics Record Office at Cold Spring Harbor.

of hysteria, 4 per cent of epilepsy, 15 per cent of alcoholism, and 9 per cent of insanity. In a study of the inmates of certain penal institutions, 20 to 30 per cent were found to be mentally subnormal. Some, however, were normal and some very keen. Yet crime and mental deficiency are usually closely related. A eriminal type, as such, does not exist. When people of low intelligence are trying to fill positions which put too great a demand on their mental ability, they frequently lose their positions. Not understanding the cause, and, in some cases, being unable to get work, they remain idle. This makes them desperate and frequently leads them into bad and vicious company. When low intelligence is combined with emotional instability, the unfortunate persons frequently become wayward and turn to crime.

From these and similar investigations it has been ascertained that desirable traits in man, such as high intelligence, without . WH. FITZ, AD. BIO. -24 
which we cannot develop will power, self-control, or a high moral responsibility, are inherited. Other studies indicate that special aptitudes such as musical or artistic ability as well as mathematical and inventive aptitudes, literary ability, and retentive memory are inherited. On the other hand, a startling number of feeble-minded people and those of low mentality are responsible for the large criminal and defective groups that fill our charitable and public institutions.

Race improvement. There are two ways of improving mankind. This improvement may be called "human conservation." One method of bettering the race consists in giving every child the best possible inheritance. This is eugenics. This requires thoughtfulness in selecting a mate so that the children will have desirable traits. Another method is to improve the environment of every individual and to give him the best possible opportunity to develop his capacities. It is true that anything which safeguards the health of the present generation also helps to safeguard the next. This second method is euthenics. It might be called the science of learning to live well. It consists of an endeavor to improve the individual through improving his environment and his training. If all people understood and practiced eugenics - being well born - and euthenics - living well - the world would be greatly improved.

The Eugenics Laboratory has collected many valuable statistics and data on human heredity. Research work is continually going on. If a man and woman contemplate marriage and question the possibility of some undesirable trait in one or both of the families being transmitted, the Eugenics Laboratory will give an opinion on the desirability of the marriage. For instance, if there is a tendency to deafness in one of the families, with perhaps some members being deaf mutes, there would be a double possibility of the children inheriting deafness if the family of the other prospective parent had the same trait, even though in a milder 
form. In some states marriages of first cousins are prohibited by law. The biological reason for this is made clear by the example above. If an undesirable trait runs in a family, both parents would be likely to carry it and there would be a double possibility of their children inheriting it.

Suggestions for race improvement.

(1) Segregation of the feeble-minded and prevention of their marrying are obvious methods for eliminating defectives and increasing the mentality of society as a group. Most states support such people in institutions. This is a very costly method. A method that shows great promise is the colony system used in some parts of New Jersey. The mental defectives are kept in colonies, the men separate from the women. They are all taught trades, and may travel in groups through the state under the strict supervision of guards. They do some road building, farming, and simple tasks which are parts of other industries and require little intelligence. In this way they earn money and help to maintain themselves.

(2) As far as possible, the insane, criminal, and diseased should also be prevented from marrying. If they could be kept in institutions, and have no offspring, the number of defectives in the succeeding generations would be gradually diminished.

(3) All people should have an understanding of the value of eugenics. This is made possible by teaching pupils in high schools and colleges, the part played by inheritance in the life of every one, and to what extent certain characteristics or tendencies may be inherited. An enlightened public consciousness must be developed all through the world, if racial progress is to be made.

(4) Certain laws in relation to marriage might be enacted and enforced. Each person desiring to marry should be compelled by law to pass a physical examination to determine whether he is physically fit, or whether he possesses certain defects that would make it unwise for him, from an eugenic point of view, to marry and probably pass on these disabilities. Some states already have such 
laws in operation. Wisconsin has enforced such a law since 1914. In this state the people show an intelligent interest in their health. Before any man may receive a license to marry, he must have a thorough health examination. If an applicant for a marriage license is told that he has a disease which makes it unwise for the marriage to take place, in most cases such a person is interested in learning how to overcome the handicap so that he may marry at some future time. The enforcement of this law is having a beneficial effect on the health of the people, as a whole, and it certainly will mean better health for the next generation. When such laws are in operation, only the fittest are selected for parenthood.

(5) The environment may be improved by enacting and enforcing laws concerning:

(a) The eight-hour day

(b) Better tenement and housing conditions

(c) Public playgrounds for city children

(d) Compulsory education

(e) Laborers' compensation laws

(f) Widows' pensions

(g) Child Labor laws

(h) Vocational guidance and training

The eight-hour day permits a man to live a normal life with sufficient opportunity for education, recreation, and physical relaxation. Better tenement and housing conditions will give all children opportunities to grow up in a clean, wholesome environment. All houses should be built so that fresh air, sunlight, and good sanitation will be available for every person, no matter how rich or poor he may be. Public playgrounds for children will prevent accidents that kill and maim so many children who have no place to play except the streets.

The compulsory education laws vary in the United States. Most 
of them insist that all boys and girls remain in school until they are at least fourteen years old or have the equivalent of six years of schooling. But, in some sections of the country, these laws are not enforced at all, while in other sections boys and girls are required to remain in school until they are seventeen years of age or have graduated from a secondary school. The schools are the medium through which the home and the community are educated. They are a direct means of raising the standards of living all through the country. Schools are also the most efficient means of ridding people of unhygienic racial and social customs.

Many state compensation laws guarantee that a man's family will be cared for in case he meets with an accident. Even though this compensation may be small, it is of some value in helping to

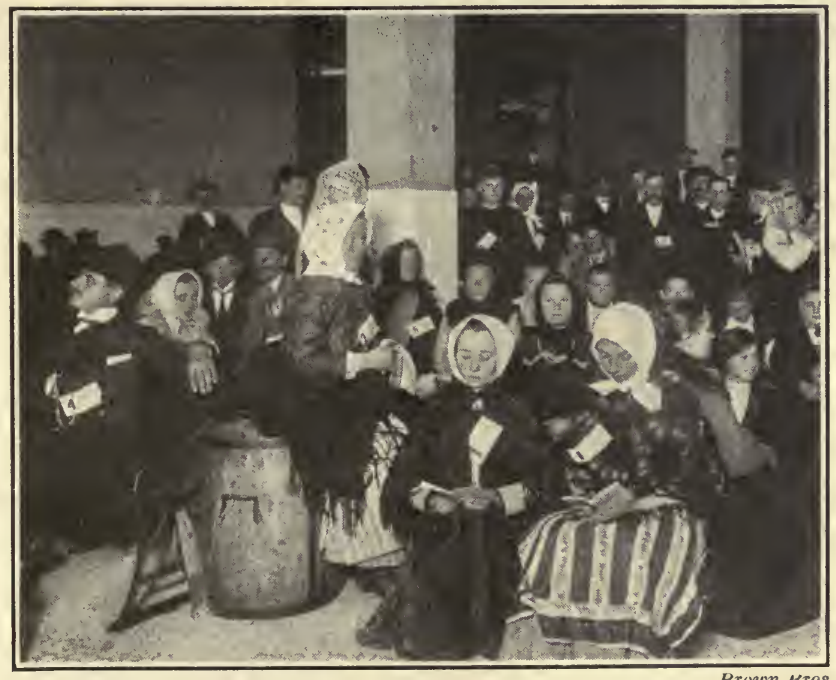

A typical group of immigrants at Ellis Island awaiting admission to the United States.

maintain a good or at least a decent environment for the family of such a person.

In various states, laws have been enacted which provide pensions 
for widows who are in need. Some of these laws even provide for orphans up to the age of fourteen or of eighteen years of age. The average grant usually varies from one hundred to two hundred

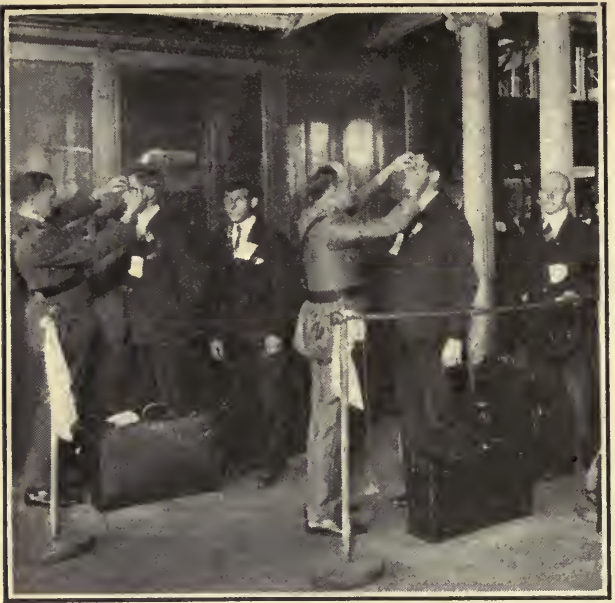

Brown Bros.

Immigrants are examined at Ellis Island to make sure that they are physically and mentally fit. All those suffering from mental disorders or from contagious diseases or who are, otherwise, physically unfit to make responsible citizens are excluded from the country. dollars per year for each child. In many cases, the law demands that the mother be a fit person physically, mentally, and morally, if she is to receive the pension which enables her to keep her children with her.

Previous to the introduction of the modern factory system in the eighteenth century, children were commonly employed in factories. Authorities of pauper institutions in Great Britain permitted pauper children to work as much as sixteen hours a day. In 1847, the hours for child laborers were reduced to ten hours a day. To-day, in most states of the United States a boy or girl may leave school at the age of fourteen and obtain working papers. In many states this is conditioned on the passing of a health examination. Five states of the United States are said to furnish one third of the total child laborers and one half of all child illiterates in the country. Congress has twice tried to pass laws regulating child labor but each time the law has been declared unconstitutional. In order for children to develop physically and mentally to the fullest extent, it would be wise to keep them out of factories and in school with suitable childhood recreation until, at least, the age of 
fourteen. In some European countries, children under eighteen are not permitted to work at night or at hazardous trades. In many of our states the minimal age for hazardous trades such as quarrying and mining is sixteen. There is agitation in many states to keep boys and girls in school until the age of seventeen. They will then stand a better chance to enter industry with a fairly good physical and mental equipment.

Vocational guidance and training are invaluable in directing children into vocations for which their intelligence seem to fit them. When properly directed, people with superior intelligence should be able to get established earlier than they usually do. Less gifted children should be advised to do that type of work which they can do rather successfully. This will give them a feeling of satisfaction in their work, which will make for contentment and happiness When people are continually losing their jobs, due to their inability to perform the tasks given, idle and vicious habits usually result. Continuation and trade schools are invaluable aids in teaching children trades. Such trained children, naturally, tend to be better citizens and better tradespeople. Special classes have been designed for children who are disinterested and unable to keep up with the work of the regular classes. Another type of school called the juvenile vocational school, designed to meet the needs of such children, is now being established in certain sections of the country. Not only will children who cannot get along well in the average public school attend these schools, but those who, due to economic pressure, must earn a living as soon as possible, will find a place to learn a trade.

(6) Only the mentally, physically, and morally fit immigrants should be permitted to enter other countries. At present there are immigration laws restricting certain undesirable persons. Immigrants are examined when they leave their own country, and at Ellis Island before entering the United States. Care is taken to exclude all those with objectionable hereditary traits or with 
contagious diseases. But the effective enforcement of these laws is still a serious problem.

Eugenics versus euthenics. Environment and heredity must go hand in hand if real racial progress is to be made. Bad environment may harmfully affect good germ plasm. Frequency of crime is sometimes laid to environment rather than heredity. This is true to some extent, but heredity is usually responsible for choosing the environment. Intelligent people, as a rule, wish to live in good surroundings where they will meet people like themselves. They try to meet and solve their problems. Subnormal people are frequently content with poor conditions of living. Lacking normal will power and the ability to solve their problems intelligently, they are dominated by the wrong influences. It is very difficult to dissociate, absolutely, heredity and environment. There is no question about the fact that eye color, hair color, and other structural characteristics are inherited, but there is little known about the inheritance of emotional characteristics.

Inheritance of disease. It is a fairly well-established fact that no germ disease is inherited. In order to be inherited, a microörganism would have to become a part of a gene in the chromosome. Since this is impossible, germ diseases are not really inherited although infection may occur at birth, so that the effect on the new individual is the same as direct inheritance. In all probability, organic diseases, like malformations of glands, deafness due to structural defects in the ears, and organic heart disorders due to structural defects in the heart, do run through families. Weaknesses in various organs may be inherited and result in tendencies or predispositions toward disease.

Any disease of the mother, that gives off poisons that will circulate in the blood may affect the germ cells and result in some abnormal development of the unborn offspring. Such children may be born crippled, blind, deaf, or mute. Any disease of the mother that interferes with the nutrition of the unborn child may also 
cause developmental defects. For example, the offspring of a tubercular mother is likely to be delicate, lack resistance to disease, or may show definite defects, because of faulty nutrition. The toxic and nutritional effects on the offspring are due to its environment. In these cases the environment is the mother's body. The relation is called intramaternal environment in contrast with the environment of the child after it is born. This is called extramaternal environment.

The relation of alcoholism to the offspring is not conclusively established. Experiments are constantly being conducted to determine whether alcohol may modify the germ cells or not. It is a fact that children of drunkards are frequently defective. This defectiveness may be (1) due to the fact that alcoholism is merely a symptom of a degenerate stock. In this case the children will be defective, not because their parents drank, but because their parents were defective. The parents' drinking is merely one of the symptoms of their defectiveness. (2) It may be that alcohol directly poisons the germ plasm. In this case parents of sound stock, who become addicted to alcoholism, will have defective offspring. (3) It may be that the intemperate parents do not take adequate care of their children and this leads to the defects of the children. Whether the tendency for drinking alcoholic liquors is inherited, has not yet been definitely proved.

Environment versus Heredity. One of the most interesting studies that has been conducted upon the relation of environment to heredity in the life of a child is the investigation of identical twins. Twins are thought to originate in the following way. When a fertilized egg divides into two cells, some mechanical or chemical difficulty may separate one cell from the other. Then each cell develops separately. The development of the two cells is exactly alike in every particular because each cell resulted from the division of the original cell. Such a development results in twins identical in sex, height, coloring, and almost every other particular. 
Investigators have found a pair of identical twins that had been separated at infancy and had been brought up in different envi-

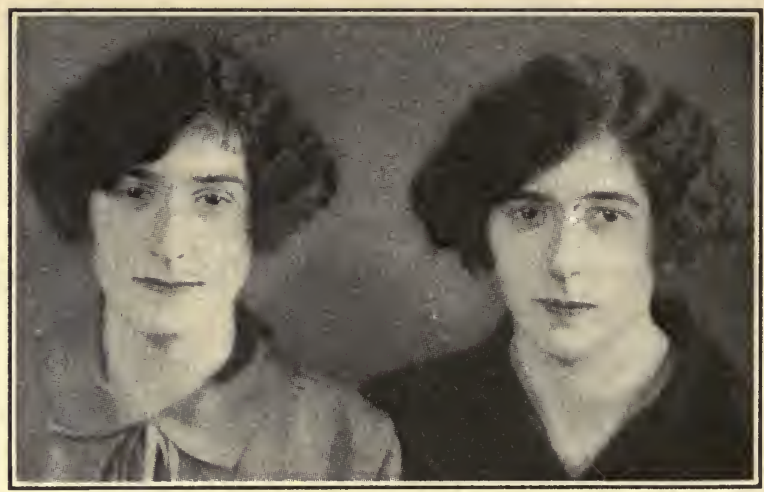

Journal of Heredity

These identical twins were separated in their infancy. They were tested, after seventeen years of separation, and found to be very similar in physical and mental characteristics.

ronments in different families in, approximately, the same social conditions. When they became adults, they were given intelligence tests and both were found to have practically the same intelligence quotients. They were successfully occupying professional positions requiring about the same intelligence. The different environments had produced little change in the inherited mental abilities.

\section{Questions And Suggestions}

1. What is eugenics? Give the history of the science of eugenics.

2. What are three difficulties in investigating human heredity?

3. Discuss how histories were obtained from families of superior intelligence; from families of inferior intelligence.

4. What is the relation of crime to heredity?

5. State two general methods of race improvement.

6. Name seven euthenical measures that may be enforced; discuss their importance.

7. Discuss the topic "Environment versus Heredity." 


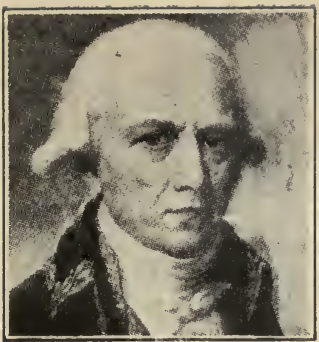

Jean Lamarck.
CHAPTER XXXVII

PROGRESSIVE DEVELOPMENT

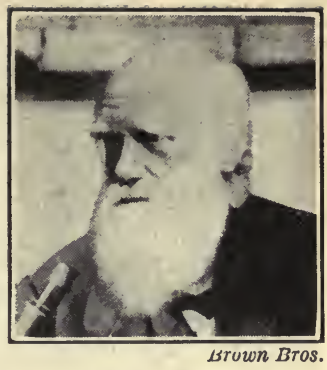

Charles Darwin.

How has organic evolution of plants and animals taken place? How old is the earth? How old is man? What are some of the evidences of evolution?

All plants and animals of to-day are thought to be descendants of earlier and more primitive types. Organic evolution is the science that deals with the origins of species and the changes in them from generation to generation. Evolutionists believe two things: (1) that individuals of the same species always vary; and, (2) that many of these new characteristics or variations are transmitted to succeeding generations. Heredity is, therefore, one of the cornerstones of evolution.

History of evolution. Aristotle had an idea that there had been a gradual succession of living things from the simplest animal to man, but this supposition was unsupported by evidence or fact. He had seen the remains or parts of plants and animals in rock. To-day these are called fossils. He thought they were examples of spontaneous generation taking place in the depths of the earth and that such forms never had a chance to live on the surface. Nearly two thousand years later (1510), Leonardo da Vinci stated that fossils were the remains of former living animals.

The Lamarckian theory of evolution. Jean de Lamarck (1744- 


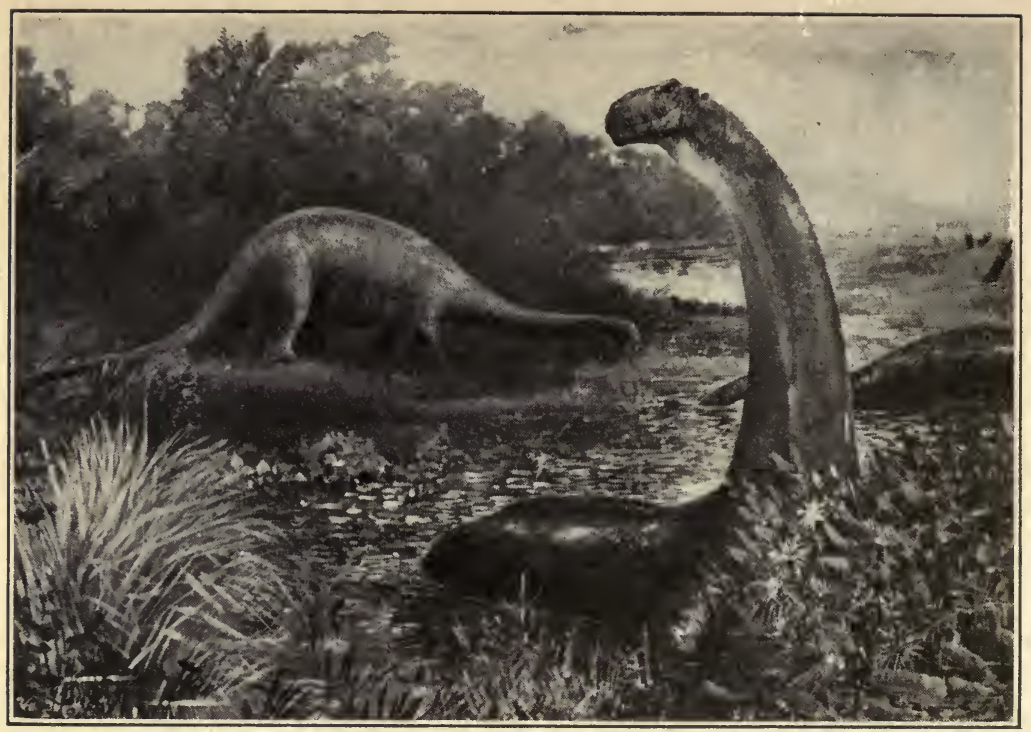

Am. Museum of Nat. History

The skeleton of an amphibian dinosaur, the Brontosaurus, was unearthed in Wyoming in 1898. The animals shown above have been restored by placing muscles and skin over the assembled skeletons. These animals probably ate soft plants and lived in shallow waters and are thought to have crawled out upon land to lay eggs.

1829), a Frenchman, set forth his ideas on evolution in his book, La Philosophie Zoölogique. He coined the expression use and disuse to summarize his ideas of the causes of variation in species. He thought that organisms could adapt themselves to fit the environment. He supposed individuals acquired the characters they needed and these acquired characters were transmitted to their offspring. For example, he said that the original giraffe had a short neck. For some reason, it began to eat leaves from trees and was constantly stretching its neck to reach them. Consequently, the neck started to grow longer and the offspring were born with longer necks. According to Lamarck's theory, if a man developed his mind very carefully, his children would start with better minds. His theory has been generally discarded. Our present knowledge 
of heredity does not show that characters acquired in the life time of an individual organism are inherited.

Recently, there has been a revival of interest on the part of certain modern scientists in Lamarck's theory. They base their ideas on the supposition that over- or under-development of an organ produces hormones in different amounts, which may affect a gene in the germ plasm and cause variations.

The Darwinian theory of evolution. The theory of natural selection was set forth in the book, Origin of Species, by Charles Darwin (1809-1882), but it was also formulated independently by Alfred Russel Wallace (1823-1913). Herbert Spencer (18201903) has exerted a profound influence upon thinking people through his philosophical writings on evolution. Darwin's theory is based largely upon deductions made from observations that he
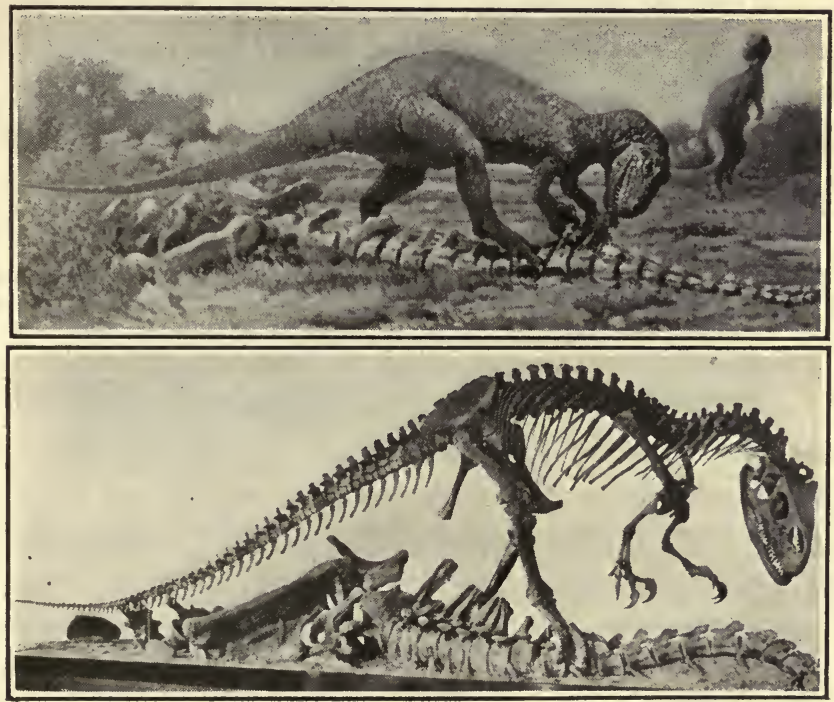

Am. Museum of Natural History

Restoration is shown of a prehistoric reptile, Allosaurus, one of the largest carnivorous animals that ever lived. It was forty-seven feet long with its head twenty feet above the ground when it stood upright. Below is the skeleton shown as unearthed. From its position, scientists think that it was feeding on one of the giant herbivorous dinosaurs just before its death. 
made after reading Malthus' Essay on Population and from material gathered during his trip around the world on a ship commissioned for scientific exploration. It consists of the following principles. (1) Overproduction. More individuals are produced than can possibly reach maturity and reproduce themselves. A plant commonly called the shepherd's purse may produce upon a single stalk as many as 64,000 seeds, and a tobacco plant may produce 360,000 seeds annually. A single fern plant produces about fifty million spores a year. If all these spores matured; the United States would be covered with ferns in two years. A single salmon may lay 2,000,000 eggs, a female codfish 9,000,000 eggs, and a Virginia oyster not less than 15,000,000 eggs. The sea would be a mass of writhing, struggling fish in three years if all survived. This overproduction leads to (2) a struggle for existence. Whenever there are abundant offspring, there must be ample food and room for life and development. Crowding makes the existence of individuals a real contest or fight. A struggle ensues for obtaining food, for finding a mate, and for producing more young. All cannot survive. Those that do are better adapted to meet the conditions of their environment than the rest. It is a well-known fact that all the individuals of a species are not the same. Some are swift and some skillful, which help them to survive. These slight differences are known as (3) variations. These variations play a most important part in the struggle for existence. Each one that is swift of foot, strong, skillful, or protectively colored possesses a variation that fits him to his environment better than those that do not possess such a variation. Variations make animals unequal in the contest of life. There is a struggle for existence: some are killed off; others, the fittest animals or plants, survive. Thus overproduction intensifies the struggle for existence; this struggle results in (4) the survival of the fittest. Darwin's theory of selective survival is often called "natural selection." (5) Variations are usually inherited. Those slight 
variations which tend to fit a species into its environment are passed on to the offspring and thus a new species will eventually

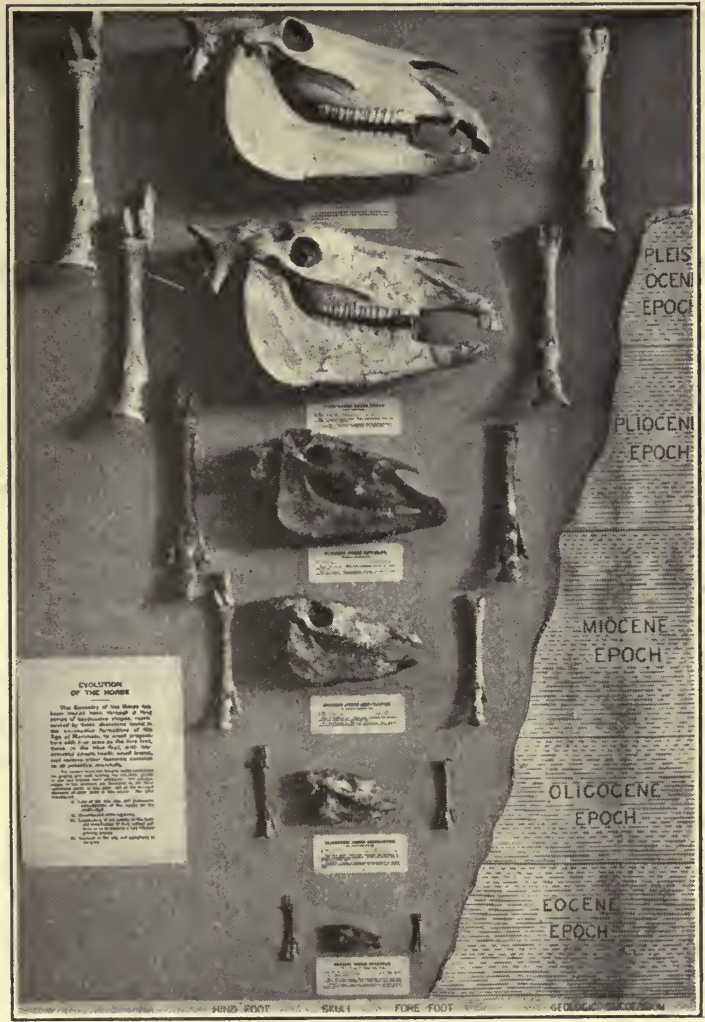

Am. M useum of Nat. History

The earliest horse, the Eohippus, had four toes and the splint of the fifth toe on the forefoot. The Mesohippus had three toes with a rudimentary fourth toe. In the Merychippus, only one toe reached the ground in walking. In the Hipparion one toe became greatly developed and the other toes became more rudimentary. The Pleistocene horse, Equus, and the recent horse show this same characteristic. There were corresponding changes in the fore-feet, skulls and sizes of the various horses.

arise. Since these desirable variations are preserved by heredity, each generation is better fitted to the environment than the preceding one. And so Darwin believed that through the passing 


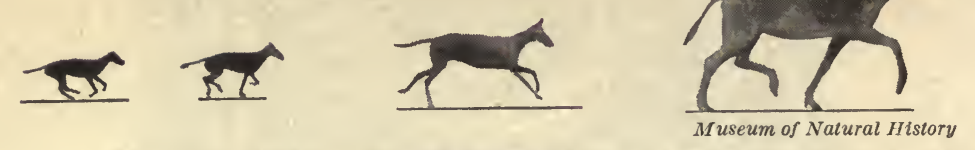

From fossil evidences, the evolution of the horse has been reconstructed. There

of years this slow accumulation of variations will lead to new species as well as to a great diversity in one species.

There are a great many facts to support Darwin's theory of natural selection, although some objections are made to it. Variations are frequently not inheritable. Darwin did not distinguish between somatic and germinal variations. (2) The variations frequently do not have anything to do with the fitness of the organism for its environment. (3) The struggle for existence does not necessarily weed out the unfit organisms. Frequently, the weeding out occurs before the organisms have grown sufficiently to have their characteristic differences appear. Plants and animals are frequently killed off by agencies, forces, and accidents that do not discriminate between the fit and unfit.

The de Vries theory of evolution. The theory of mutation was formulated by Hugo de Vries, a Dutch botanist, in 1904. He published his ideas in a book called Species and Varieties. Charles Darwin had collected many examples of organisms strikingly different from the other members in the species. These he called sports. He did not attach very much importance to them. Hugo de Vries based his theory of evolution on sports which he called mutants. Weismann's conclusions in regard to germ plasm had disproved the inheritance of acquired characters. He had claimed that modifications of the body or somatoplasm did not affect the germ plasm and therefore could not be inherited. This fitted in with the ideas of Hugo de Vries who had made direct observations 

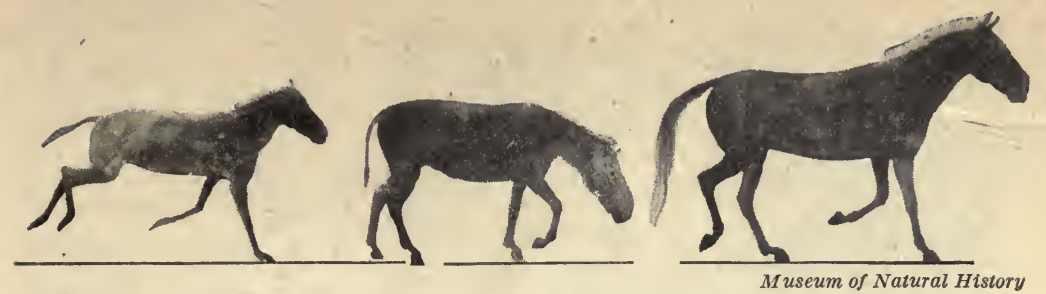

has been a gradual increase in size from the most ancient to the present day horse.

on the appearance of mutations among the plants in his gardens and greenhouses. He observed that mutants usually transmitted their peculiarities to their offspring.

The theory of mutation differs from Darwin's theory of natural selection, in the following facts. (1) Among individuals of a species, different forms arise suddenly and independently, not gradually. These forms differ widely from their parents. The forms showing wide departures are mutations. In many cases these mutations are inherited. (2) Mutations may take place in any direction. They may or may not fit in with environment. They may or may not be favorable. The main thing is that some are capable of establishing, themselves and some are not. (3) In general, the unfit mutants are likely to be eliminated through natural selection. (4) The fit mutants are likely to survive by natural selection. The mutationist does not believe that natural selection really starts the species. He believes that it controls the persistence or disappearance of the mutant. The keynote to the theory of mutations is that organisms must first appear with distinctive qualities that are inheritable in order to start a new species.

There is practically no disagreement among scientists concerning evidences that organic evolution has taken place. But, there is still much disagreement in determining which of the theories thus far formulated most nearly fits the facts. The de Vries theory of mutation is one that is generally accepted by many scientists.

WH. FITZ. AD. BIO. -25 


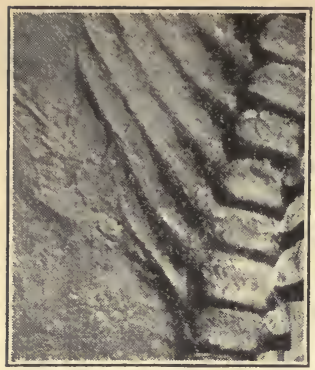

Part of a fossil backbone.

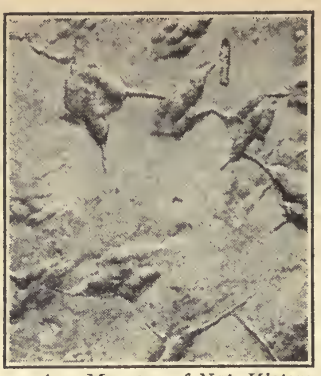

Am. Museum of Nat. History

Fossil footprints.

The age of the earth. Before examining the evidences of evolution of present living things, it is necessary to understand something about Paleontology, a science which deals with the life of past geological periods. Geological time includes all the time since the earth first started to be formed. Geologists have estimated the age of the earth by the relative age of various layers of rocks and metals. For example, the presence of a form of lead has been found in a Norwegian mineral composed chiefly of uranium. Assuming that the production of uranium ceased as soon as the earth and sun separated, geologists have agreed that this lead is about three billion years old. This was determined by estimating the length of time necessary for a small amount of metal uranium to break up into this form of lead. From this time and amount of metal, the age of the lead and, therefore, the earth was calculated.

The cliffs in a valley will show that rocks are laid in strata or layers. The successive layers of the rock suggest that they were deposited one after another from the bottom upward. The top stratum is the most recent one deposited. Each individual layer of rock

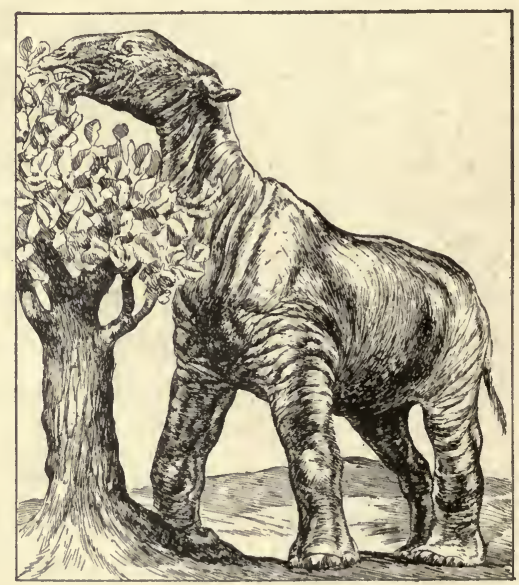

From drawing M useum Nat. Hist.

A colossal, hornless rhinoceros of prehistoric days. 
constitutes a record of the time when it was deposited. The thickness of the stratified rocks now exposed upon the earth surfaces of the continents is very great. Knowing how slowly sediments accumulate upon the sea floor, the age of the earth must measure approximately hundreds of millions of years. Determining the arrangement in which the strata were deposited is difficult, because, in different areas, different strata may be omitted for

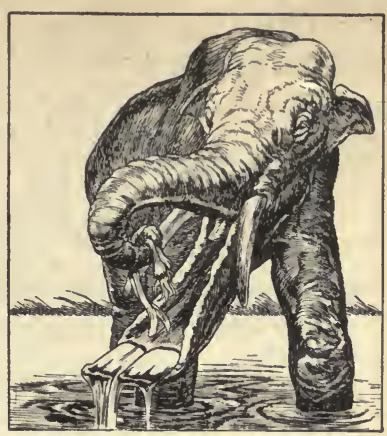

From drawing Museum Nat. Hist.

The shovel-tusked mastodon used its scooplike jaw to gather up vegetation from the water. one reason or another. Consequently, the sequence is not always alike. In some areas a more recent rock deposit is exposed than in others. Sometimes the rock deposit

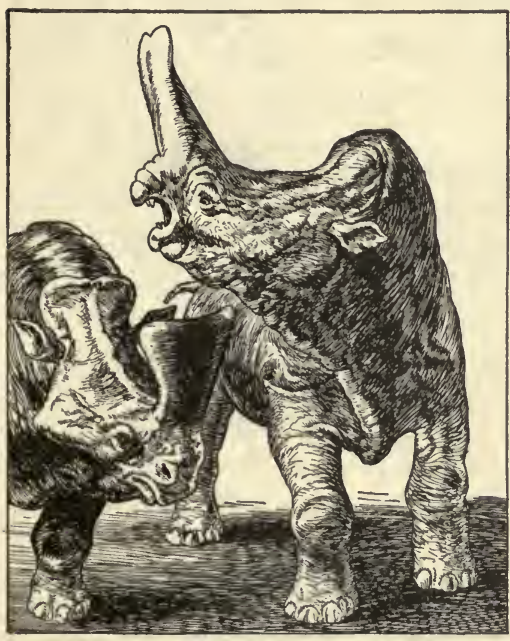

From drawing Museum Nat. Hist.

Fossils of Titanotheres, prehistoric mammals, have been found in both Asia and America. At the time they lived, Asia and America were probably one continent joined where Behring Strait is now located. may have been completely weathered or worn away. The formation of rocks differs in various parts of the country. Some rock was elevated from the sea and forms the land of to-day. This is known because it contains fossils of shells of animals that once lived in the sea. The flatness of the beds of rock seems to show that their movement from the sea was so uniform and gentle that their original formation could not have been broken.

The oldest historical records of Egypt or Babylon date back perhaps six or seven thousand 


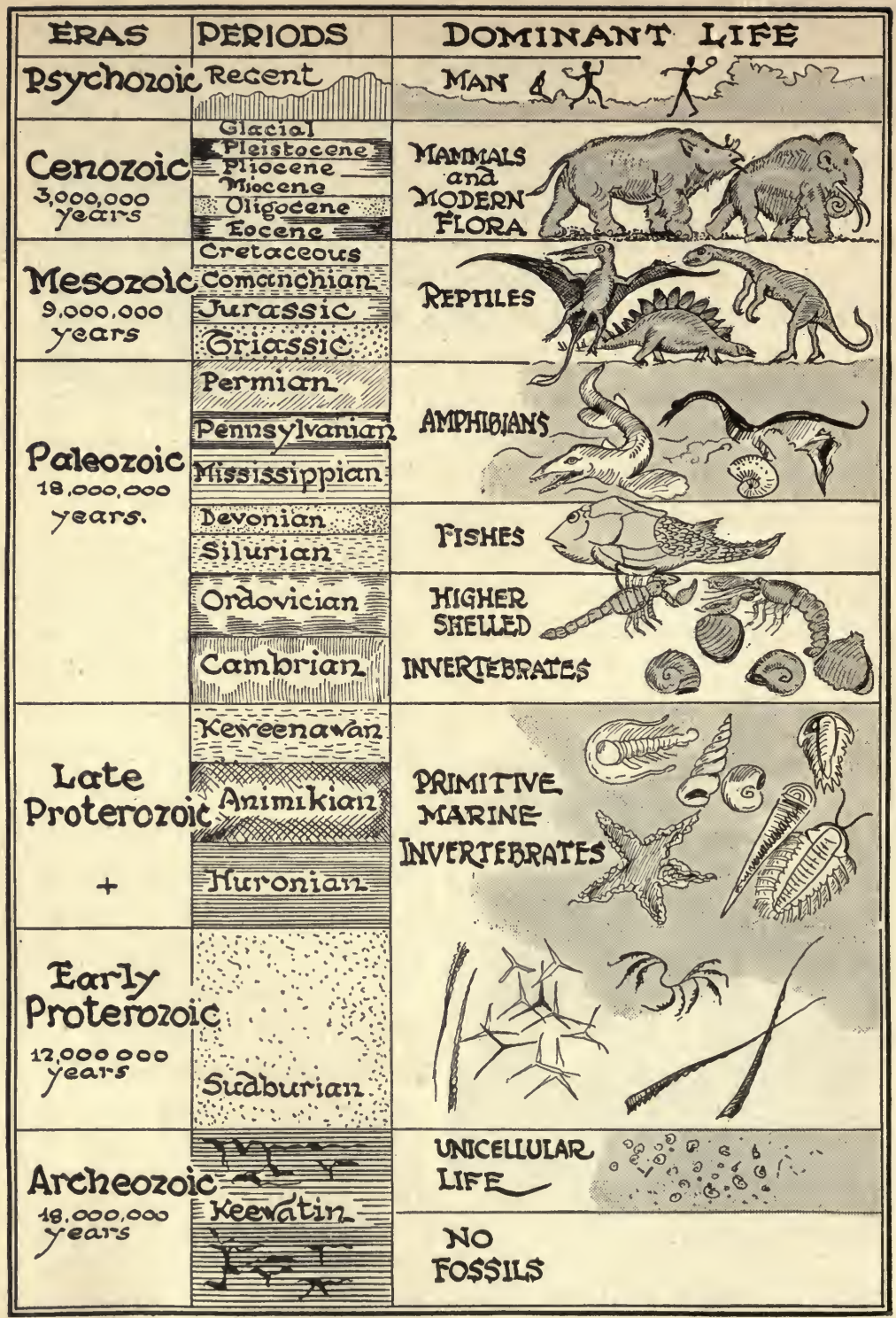


years. Recently, an earlier Egyptian civilization has been unearthed that dates back more than ten thousand years. The carvings left by prehistoric man in the caves of the Pyrenees are perhaps twice as old as the Egyptian culture just mentioned. According to the evidence that is now available, man has probably existed only a few hundred thousand years. Beyond man stretches a chain of varied forms which can be arranged in progression from the highly complex ones of to-day through simpler and simpler ones back to the simplest of all the one-celled plants and animals.

Fossils. In the strata of rock are found fossils in all degrees of their original state of perfection, varying from trails, tracks, or imprints to perfectly preserved shells, wood, bones, and complete skeletons. As a rule, it is only the hard parts of the animals and plants which have been preserved from decomposition. Sometimes the original organic substance is preserved, but more often it has been replaced by mineral matter. This slow process of replacement is known as petrification and the organism or the part of the organism is said to be petrified. In a skeleton of a prehistoric animal, particle by particle the lime was replaced by indestructible silica, the element found in stone, sand, and glass. Probably the most common forms of fossils are molds or casts. The dead animal or plant fell into soft mud in which it made an impression which was preserved long after the organic matter had been destroyed. Sometimes these molds harden or became filled with a substance which formed stone.

Evidences of evolution. The organized data that support the development of the higher organisms from the lower ones are based on the following evidences: (1) geological evidences, (2) geographical evidences, (3) morphological evidences, (4) vestigial evidences, and (5) embryological evidences.

Geological evidences. The oldest known rocks appear to have no fossils in them. The next layer shows traces of life of the 
simplest character. The fossils of the most highly developed animal, so far discovered, found in the oldest rock (Paleozoic)

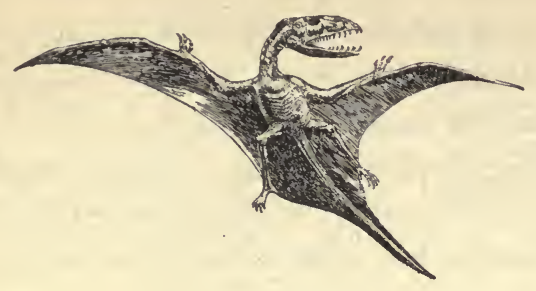

Flying reptiles, the pterodactyls, were probably the ancestors of our modern birds.

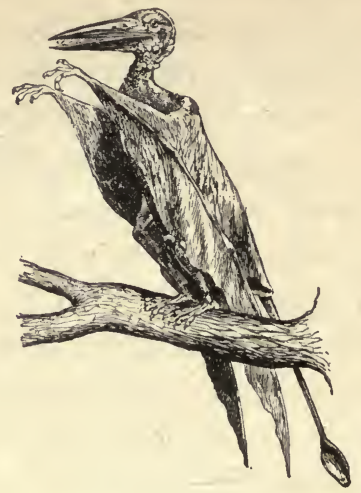

are those of an animal related to a crab, called the trilobite. As higher layers of rock are reached, fossils of more complex animals are found. These are different, but obviously related to the forms in the strata below.

According to the present-day evidences, all life at an early period was in the water. The history of the development of life has been formulated from records made in rocks. It is thought that the most primitive forms probably appeared in the warm shallow waters of the flats between tides. First, there appeared plant forms, algae; later, animal forms, protozoa. There was no life on the land. The rocks and coarse soil were quite bare. Later, countless minute creatures appeared in the water. Their shells made up the great cliffs of chalk.

After centuries had passed an organism appeared that had an organ for breathing air. It is thought that this form developed from the more primitive types. The first traces of the land vertebrates are the footprints of the amphibia. These are preserved in mud molds which have solidified into rock. 
Some amphibia are thought to have developed into animals, the reptiles, that breathed with lungs all their lives. The reptiles of the past are called dinosaurs. Those of the earlier Mesozoic period were small, but in the latter part of the period, they became more numerous and gigantic in size. The Brontosaurus was nearly seventy feet long. (See page 372.) The Allosaurus was a trifle smaller. It was a dragon-like creature that preyed on its larger but clumsier relatives.

During this period, the pterodactyl, a huge bat-like animal, appeared. It glided through the air by means of folds of scaly skin extending from the fore-limbs to the side of the body. It had a large bill and a long tail. Its wing-spread was thirty feet. This is probably the animal that was transitional between birds and reptiles. The Archaeopterix was another ancient birdlike form. It has long, grill-like, true feathers on its wings, a small jaw with teeth, and a vertebrated tail with feathers attached. There were claws on its wings. No transitional animal has been found showing how the scales of the reptiles developed into the feathers of the birds.

While the birds were developing, a small, very inconspicuous group of animals made their appearance. They had hair instead of scales, and had

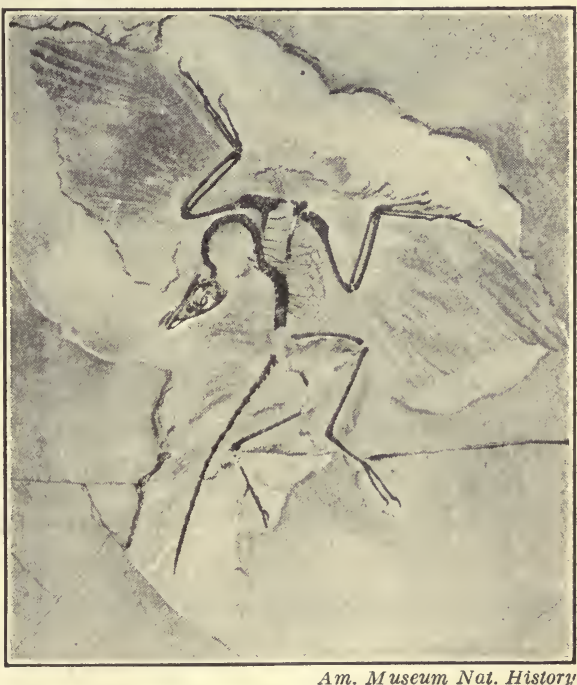

The Archaeopteryx is the earliest known bird. Two fossil skeletons were found in Bavaria. The creature was about the size of a crow, covered with feathers as in modern birds. Unlike present-day birds, it had uniform teeth in both jaws. It probably used all four limbs in climbing trees. The clawed digits were adapted for this. It is probably an intermediate form between reptiles and birds. 
a nourishing fluid for their young in certain glands of their skin. Their rate of metabolism was much higher than the reptiles.

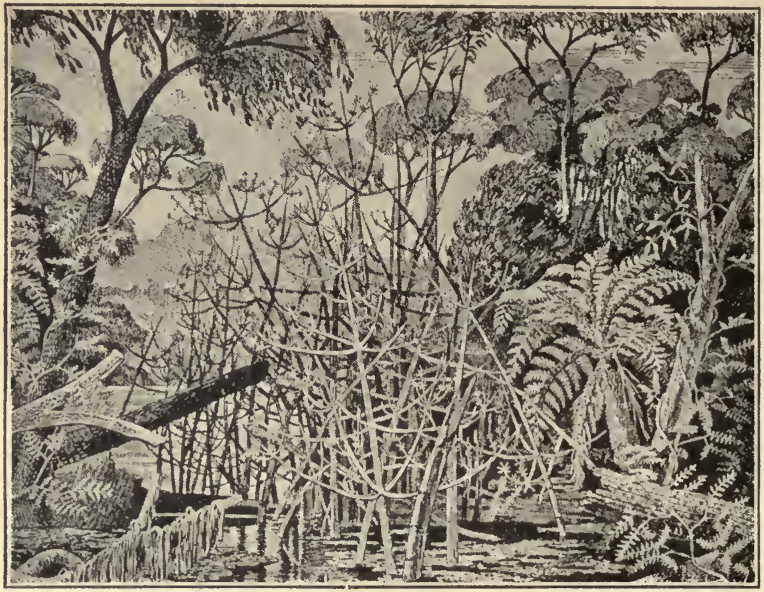

Am. Museum Nat. History

The Paleozoic period is noted for its luxuriance of plant growth. Ferns, club mosses, and ferns related to the horsetails attained the size of trees and formed dense forests. Many cone-bearing trees are found, but none of the higher types of flowering plants. As the plants died and became part of the swamps, some unusual tremendous pressure transformed them into coal beds.

Hence they are called warm-blooded. They retained the eggs inside their bodies until the young were almost fully developed, and supplied them with nourishment during development. These were the mammals. The platypus or duckbill of Australia is thought to be a transitional form between the egg-laying birds and the mammals. It is furry, has a bill like a duck, lays eggs, and feeds, its young with milk (page 513). The marsupials, such as the kangaroo, are somewhat transitional. The young are born before fully developed. They climb into a pouch in the abdomen of the mother and complete their development there. From the early mammals of the Cenozoic era have developed the many species which dominate the earth to-day. Man belongs in this great group of mammals. 
Geographical evidence. It has been found that every group of organisms expands its range just as far as conditions permit. Regions in every way similar, so far as climate, soil, and other conditions are concerned, are inhabited by totally different plants and animals. Thus, the climate of Australia is not very different from some of North America, but the animals and plants living there naturally are not like those of North America. The same is true about other' similar regions.

Regions that are very different are occupied by forms of plants and animals that are sufficiently similar to be considered of the same families. For example, goats and sheep, obviously related to each other, are found in tropical, temperate, arctic, and antarctic circles. They are thus living in varied surroundings.

Darwin pointed out that where similar regions are occupied by different flora and fauna, these regions were always separated from each other by impassable barriers such as oceans, mountain ranges, and deserts. Thus Australia and America were always separated by the ocean, and land animals could not migrate from Australia to America. On the other hand, where similar plants and animals inhabit regions that are markedly different in their climate and soil, these regions are either connected directly, or show evidence of having been connected in the past. For example, the plants and animals found in oceanic islands are frequently related to the inhabitants of the nearest mainland. There is some evidence that tends to show that the islands were a part of the mainland at one time.

The Mongolian Expeditions. It has been known for some time that Behring Strait formerly existed as a land bridge connecting America with Asia. It is thought that a similar connection, by way of Greenland, connected America with Europe. It was observed that the animals of North America, Europe, and Asia north of the Himalaya Mountains were somewhat similar and it was thought that animals must have crossed these areas 
and mingled. For example, American camels and horses may have migrated to Asia or vice versa.
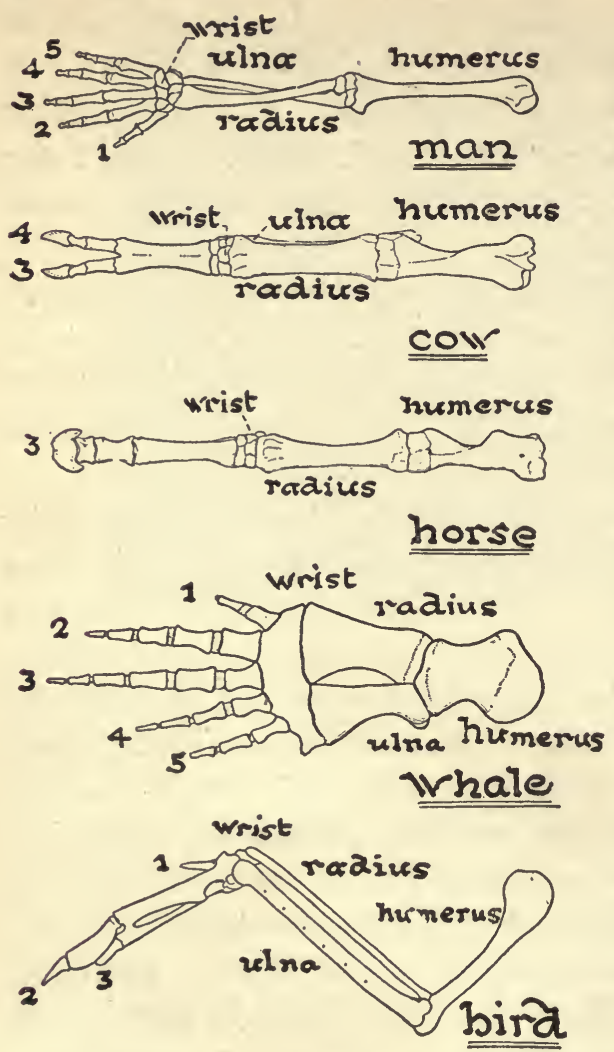

There seems to be a common plan of structure in the limbs of various vertebrates which points to a common ancestor.
It was also known by geologists that the Gobi Desert, Mongolia, in Asia was elevated from the sea during the period of time when reptiles were abundant. It became dry land at that time and has remained dry ever since. The American $\mathrm{Mu}$ seum of Natural History financed expeditions, headed by Roy Chapman Andrews (see frontispiece), to Mongolia to investigate the desert for remains of reptiles. Since reptiles were the predecessors of man, it was hoped that a transitional form between the reptiles and man, or between the early mammals and man, would be unearthed. Because of climatic conditions, deserts are peculiarly fitted to preserve fossils. Few people have penetrated and lived in the desert, so fossils are more or less undisturbed. Asia was thought to be a dispersal center to Europe on one side and America on the other. These expeditions have met with the greatest success in unearthing remains of reptiles and early 
mammals. Dinosaur eggs and dinosaur bones are there in abundance. The largest mammal ever found has just been unearthed. Many of these fossils are on exhibition in the American Museum of Natural History in New York.

Morphological evidences. There is great similarity in the structure of animals, particularly the vertebrates. It is thought that the bones which form the shoulder and hip girdle of man are analogous to the bones which support the front and rear fins of the fish. The bones of the arm and leg are variations of the bones in the fin. Between the bones of the higher apes and man there seems to be a difference in proportion only. There is also a muscle for muscle correspondence.

Vestigial evidence. There are traces or rudiments of organs found in certain higher animals. These vestiges are no longer used. Probably the animal passed through a stage when it used such an organ. The horses of to-day have splints or useless bones high upon either side of their hoofs. These are vestiges of toes. This is one of the evidences that the present one-toed horse developed or evolved from the ancient four-toed horse.

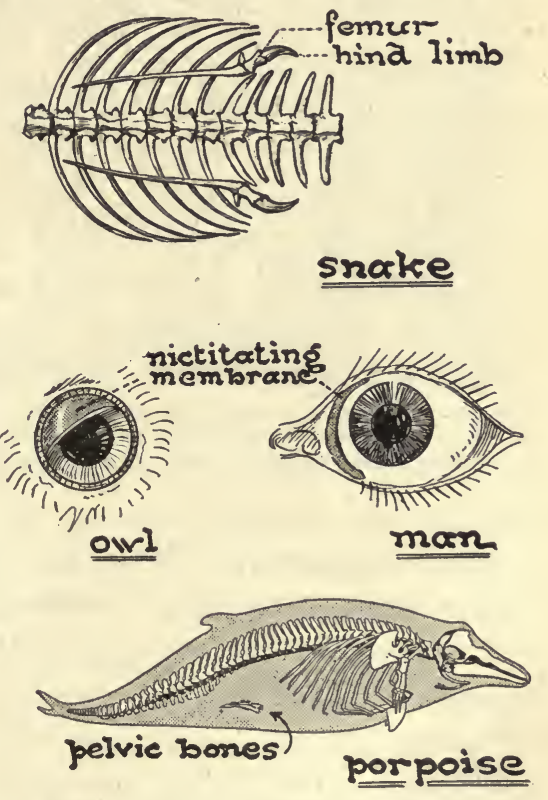

snake

A striking evidence of descent is the rudimentary organs in higher animals. The snake shows a rudimentary pelvis and hind limbs. The nictitating membrane is still found among birds and reptiles. The porpoise shows vestigial pelvic bones. There are numerous other rudimentary organs in various animals.

Human beings have a great many vestiges. The appendix is large and performs an important digestive function in some ani- 
mals ; in man, it is a vestige. The muscles of the ears of human beings are quite functionless although they are of value in aiding lower animals to hear.

Embryological evidence. All animal embryos go through the

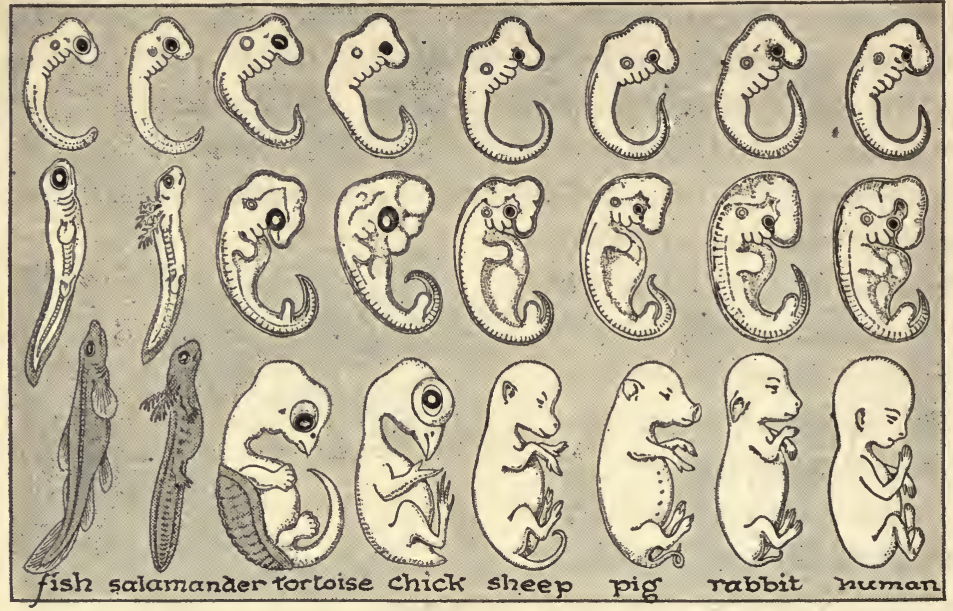

Embryos of various animals appear to go through very similar stages. Before differentiation has progressed very far, it would be very difficult to recognize a particular embryo. In the later stages of embryonic development, species take on special characteristics.

blastula and gastrula stages. The most primitive many-celled animals we know are the sponges. They are simple gastrulae.

The embryos of many vertebrates show similarities that are not noticed in the fully developed organisms. They all go through a similar early development. The nearer alike the adult organisms are the longer they will show similarities in their development. The higher vertebrates, the mammals, in their early stage of development, have gill openings similar to those found in fish. During embryological development, the diaphragm, which in the fishes shuts off the gill chamber from the rest of the body, in the human embryo moves well down into the body cavity.

While the human embryo is at one stage in its development, it has a tail-like structure. The generalization of the development 
of embryos is known as the doctrine of recapitulation or the biogenetic law. It states that the organism, in the course of its growth as an embryo, goes through stages similar to those through which the whole race has developed. This theory was first expressed by Professor Haeckel, a German embryologist. Remember three words - ontogeny recapitulates phylogeny. Ontogeny is the history of an individual; recapitulates means repeats; phylogeny, the history of the entire race or group.

Evolution of plants. It is thought that plants, too, have gone through an evolutionary series, but because they have little in the way of skeletons to fossilize, less is known about their early history than about animals. Early vegetation consisted of luxurious growths of mosses and ferns. One descendant of these primitive fern groups is called the giant Equisetum or horsetail. It is commonly found growing along railroad embankments. This vegetation died, decayed, and collected to a depth many feet in thickness. It became buried beneath newly deposited soil and was later pressed and heated by volcanic action. Ancient forms of bog mosses formed peat. Prehistoric ferns and allied plants formed the basis of our present-day coal deposits. Some fossils of the forms of vegetation then growing are preserved in the coal. The earlier plant forms had certain primitive forms of fibrovascular bundles. Many modern plants, though they have developed a more complex form, grow the primitive form first. Later came the seed-bearing plants.

\section{Questions And Suggestions}

1. What is evolution?

2. What are some of the ideas on evolution held by ancient scientists?

3. Discuss the use and disuse theory of the origin of species.

4. Discuss the natural selection theory of the origin of species.

5. Discuss the mutation theory of the origin of species.

6. What practical difference does it make which theory of evolution proves to be true? 
7. How would Lamarck, Darwin, and de Vries explain the fact that horses in the north have longer hair than horses in the south?

8. How would Lamarck, Darwin, and de Vries explain the fact that the ancestors of giraffes had short necks and present-day giraffes have long necks?

9. Discuss the formation of the earth.

10. How have scientists been able to determine, even approximately, the age of the earth?

11. What is one of the estimates given of the age of the earth?

12. Discuss the formation of different types of fossils.

13. Name five lines of evidence given to support the theory of organic evolution.

14. Discuss the relation of the age and formation of strata of rocks to the fossils found in them.

15. In outline form give the sequence of fossils found in rocks beginning with the fossils first found and going through those found in youngest rocks. What relation does this sequence bear to the simplicity and complexity of the animals?

16. Name three different types of mammals and give the distinguishing characters of each type.

17. Discuss the geographical evidences of evolution.

18. What is the importance of the expeditions to the Mongolian desert?

19. Give examples of morphological evidences of evolution?

20. What are vestigial evidences of evolution? Give examples.

21. What are embryological evidences of evolution? Give examples.

22. Discuss the evolution of plants.

23. Discuss coal formation.

24. Give a report on the life and work of Lamarck, Charles Darwin, and de Vries.

25. Look up and report on one of the Mongolian expeditions.

\section{Supplementary Readings}

Haupt, Arthur W., Fundamentals of Biology (McGraw-Hill Book Co.).

Holmes, S. J., General Biology (Harcourt, Brace \& Co.).

Jewett, F. G., The Next Generation (Ginn \& Co.).

Osborn, Henry Fairfield, From the Greeks to Darwin (Charles Scribner's Sons) 


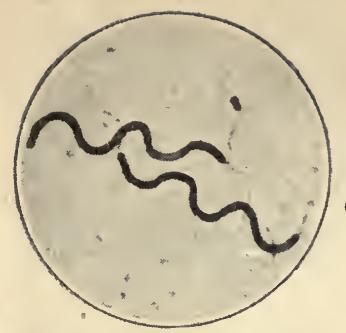

Photomicrograph of spirilla.
CHAPTER XX̃XVIII

BACTERIA

What are parasitic bacteria? How can bacteria be studied? What conditions favor the grouth of bacteria? What is the relation of bacteria to food preservation?

Bacteria possess no chlorophyll. They cannot make their own food ; they are dependent upon other organisms for their nourishment. They, with many other plants lacking chlorophyll, belong to a subdivision of Thallophyta. All bacteria are not harmful. Life on earth would probably cease were it not for the activities of certain bacteria. The disease-producing bacteria are known as pathogenic types.

Structure of bacteria. When unstained, bacteria are colorless, transparent cells with comparatively thick cell walls. The nucleus is not organized, but nuclear material is scattered through the cytoplasm. Sometimes the cell wall absorbs water, becomes swollen, and forms a mucilaginous mass. The " mother of vinegar" represents a mass of cells of this type. Another example is the mass of mucilaginous material that sometimes clogs the drainpipe of the ice box. In other types, the wall becomes gelatinous and thick, and forms a capsule around the individual bacterium. In certain bacteria, small granules are found, which are probably reserve supplies of food. These particles absorb dyes readily and hold the stain very effectively. Some bacteria have one or more whiplike projections of protoplasm called flagella. They are the 

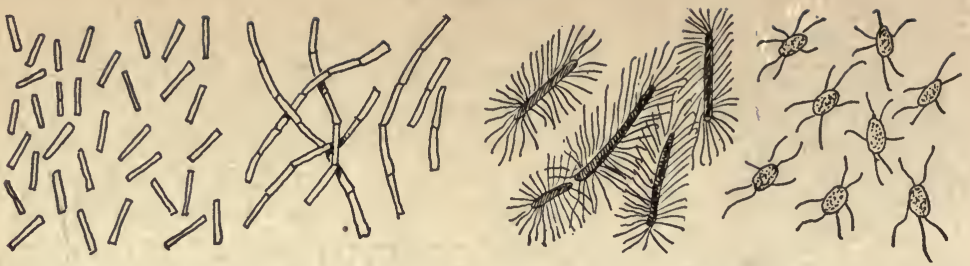

Rod-shaped bacteria, bacilli, sometimes occur singly. They may form strings and are then known as streptobacilli. They sometimes have projections of protoplasm, somewhat similar to cilia, called flagella.

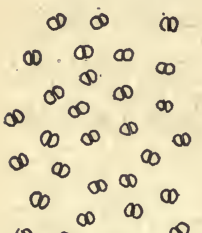

$\infty \infty_{\infty}^{\infty} \infty^{\infty} \infty$
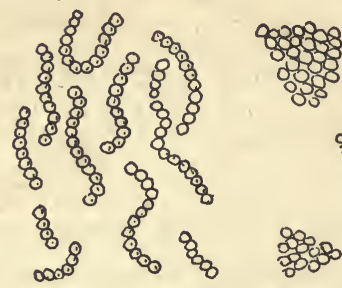

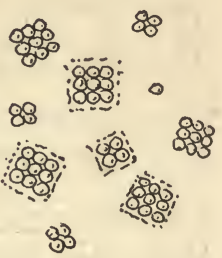

Spherical bacteria, cocci, may occur in pairs, diplococci; or in chains, streptococci; or in clusters, staphylococci.

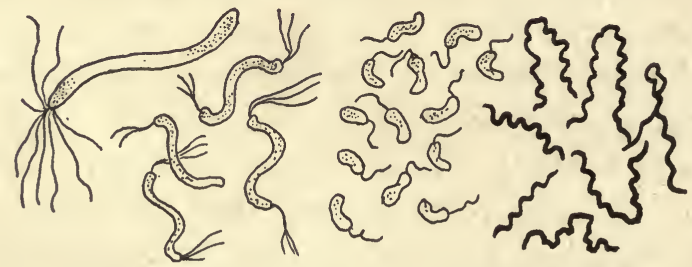

The spiral bacteria, spirilla, may not have flagella, or they may have them. These bacteria are always recognized by their twisted shape.

motile bacteria and are able to move through the water by means of these structures.

There are many different species of bacteria. Most of them may be grouped as one of three forms. These are the bacilli or rod-shaped bacteria, the cocci or spherical, and the spirilla or spiral. Bacteria vary greatly in size and shape within the same group, although they are all microscopic. The micron is the unit of measurement in microscopic work. It is about one-thousandth 
of a millimeter in length or about one twenty-five thousandth of an inch. Bacteria usually vary from 0.5 to 5 microns in length.

There is probably a very large group of disease-producing agents, which may or may not be bacteria, that have never been seen with the microscope. They can pass through ordinary bacteria-proof filters, and are known as filter passers or a filterable virus. These infectious microörganisms may be found in the secretion, the excretion, or blood of the body, and may be passed directly from one person

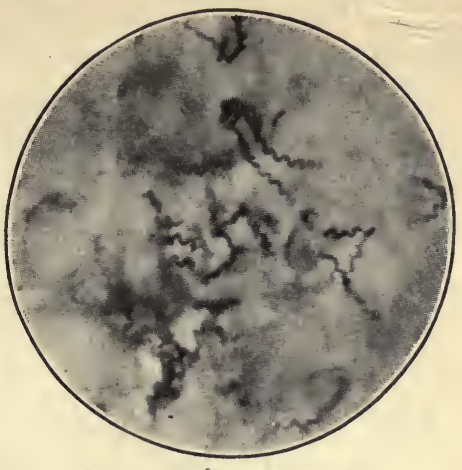

Photomicrograph of spirilla. to another, thus causing a disease. For example, the pus in the abscesses on the skin of a smallpox patient is a filterable virus. This material will generally produce smallpox if it is brought in contact with a well person.

Bacteria are sometimes classified according to their relation to oxygen. Those that need free oxygen in order to live are the aerrobic bacteria, and those that live only in the absence of free

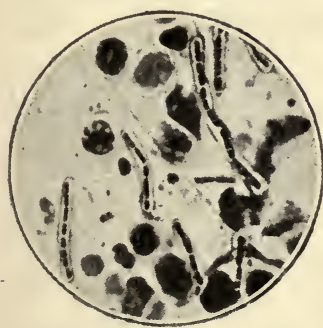

Photomicrograph of streptobacilli enveloped in a mucilaginous sheath. oxygen and perish if exposed to it are the anaërobic bacteria. Certain forms of bacteria of decay thrive without air. They obtain their oxygen by breaking down oxygen-containing compounds.

Physiological functions of bacteria. No bacteria are completely independent. They lack chlorophyll and are, therefore, unable to make their own food, but have to depend upon other organisms for it. Those bacteria that inhabit and obtain their food from living organisms, sometimes causing damage to those organisms, are the parasites. WH. FITZ. AD. BIO. -26 
They are not all necessarily harmful. Certain bacterial parasites are found in the intestinal tracts of animals, but they do not seem to produce any serious effects. The pathogenic bacteria are all parasites. All of these are harmful.

Bacteria that inhabit and obtain their food from nonliving organic material are saprophytes. The bacteria that decay dead organisms are saprophytes. The bacteria that change alcohol to vinegar, sour milk, and ripen cheese are saprophytes. Practically all of these are useful to man.

When a parasite and its host both flourish, and each one promotes the growth of the other, their relation is one of mutual helpfulness and is known as symbiosis. Each member, the bacterium and its host, is known as a symbiont. For example, certain bacteria, known as nitrogen-fixing bacteria, live in little nodules or swellings on the roots of clover plants. These bacteria take free nitrogen from the air and build it into nitrates which the plant can use. The clover plant uses some of these nitrates for making protein. At the same time the bacteria absorb sugar from the clover plant and use it for food. Neither organism suffers from this relation and each one benefits from it.

Nutrition. All types of bacteria give off digestive juices or enzymes which digest the food upon which they are living. This food may be mineral nutrients, dead plant and animal tissue, or even living tissue. Digestion is external to the cell, not within the cell, as it is in the amoeba. For example, the tuberculosis bacilli digest certain cells of the body, then absorb the digested material. Practically all bacteria absorb protein from other organisms. The absorbed protein is either assimilated into new protoplasm or oxidized for the release of energy. As a result of assimilation, the bacteria grow, divide, and form groups or colonies.

Reproduction. After the bacterium reaches its maximum size, it builds a cell wall across the middle of the cell and thus divides in half by fission. If conditions are favorable, certain bacteria 
may divide every twenty minutes. A single cell may produce millions of cells within twenty-four hours. As bacteria divide and cling together, the mass of similar cells is known as a colony.

If conditions are unfavorable, many bacteria will give off moisture, thus lessening their size, and surround themselves with a thick wall. In this form, the cell remains dormant until conditions are again favorable for development. This is known as a spore. All bacteria cannot form spores. Spore-formation in bacteria is not a type of reproduction because no new cells are

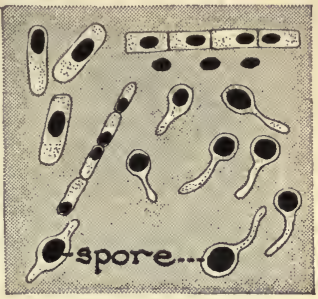

Different types of spores are found among bacteria. Sometimes the spore forms at one end of the cell and sometimes in the center. developed. It is a method by which the cell exists during unfavorable conditions. Spores may be dried without injury. Some spores may be heated to a high temperature, and the organism will still remain uninjured.

Methods of identifying bacteria. Bacteria are so small that other methods of identification must sometimes be used besides their shape, size, and flagella. Some are recognized by their ability to hold an acid stain, or to give particular color reactions with certain stains. Others are recognized by the changes they bring about in or on various substances on which they are grown. These substances are called media. The type of colony formed is also a means of identification. Some produce colonies with smooth, scalloped, or fringed edges. In some, the colonies are opaque; in others, they glisten. Different colonies of bacteria are characterized by different pigments. Some of the pigments are gray, yellow, pink, or brown. Some colonies develop on the surface of the media, others beneath the surface. The place and character of the spore formed are other means of identification. In some, the spore forms at one end of the cell ; in others, in the middle. A tetanus bacillus forms a spore at one end of the cell. 
It swells out and the bacillus looks like a drumstick. The diphtheria bacillus is characterized by granular particles which turn red with a certain stain and will not lose this stain.
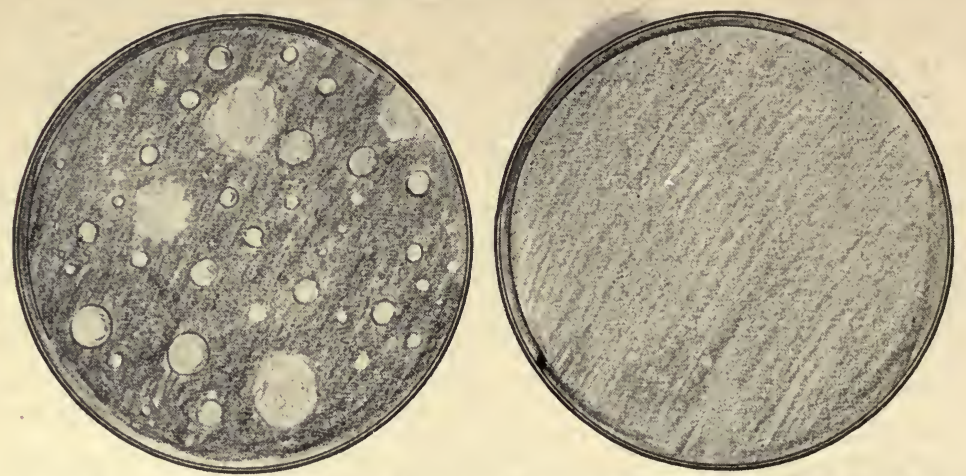

A method of identifying bacteria is through recognition of the types of colonies they form. Some colonies are translucent, others are opaque; some have regular margins, others have irregular margins. One of the above plates has been exposed to bacteria, the other plate has not been opened and is sterile. This is called the control.

Media for cultivation of bacteria. Beef broth is the principal medium used for the cultivation of bacteria. If a small quantity of the seaweed, agar, is added to this, a solid medium results. Sometimes gelatin is used in place of agar, and prune juice, sugar solution, or blood instead of beef broth. The nutrient medium which will furnish the best nourishment for the particular bacterium is selected.

Bacteria are usually grown in Petri dishes or on agar slants in test tubes. The Petri dish was originally devised in 1887 by R. J. Petri in a Berlin laboratory. It really consists of two dishes, each one being a shallow glass saucer with a perpendicular rim. One fits rather snugly as a dust-proof cover over the other.

First, the agar medium is boiled and filtered two or three times. The Petri dishes are thoroughly cleaned and sterilized. The hot sterile agar is then poured into the dishes. Great care is taken, in order to prevent any bacteria in the air from entering during the pouring or plating of the agar. 
In preparing agar slants, the test tubes must be plugged with sterile cotton and sterilized thoroughly. Then, the plug is removed, the sterile agar quickly poured in, and the plug put back in the mouth of the test tube. The test tubes are usually set obliquely for the agar to cool, so that, when firm, the surface of the media is slanting. Hence the term agar slant. This method gives more surface for the growth of bacteria.

In order to introduce bacteria for cultivation into a Petri dish, the dish is uncovered and exposed to the air. After the exposure, the dish is kept closed. Bacteria may also be introduced with water, food, dust, or other foreign material. In introducing bacteria into the agar slant, the cotton plug is removed carefully so as not to get bacteria on it. The agar slant is then streaked or stuck with a needle infected with bacteria.

Occurrence of bacteria. Bacteria are so extremely minute that they float in the air with the particles of dust. It is almost impossible to find any air that does not contain them. Since this

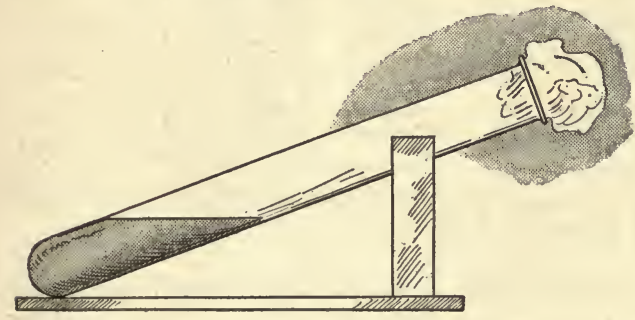

One way of growing colonies of bacteria is on an agar slant preparation. The surface of the agar is streaked with the material containing the bacteria. The long slant gives considerable surface on which growth may take place.

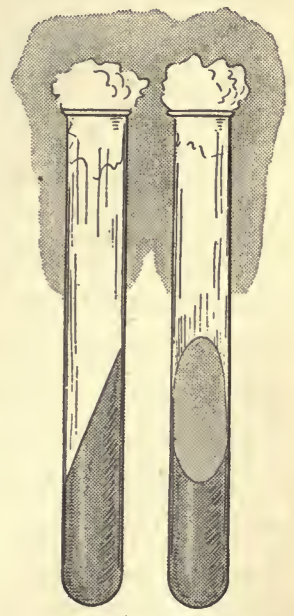

is the case, it is quite impossible for any material exposed to the air, for even a short time, to escape contamination from bacteria. 
Practically all bodies of water on the surface of the earth contain great numbers of bacteria. The numbers in different kinds of water

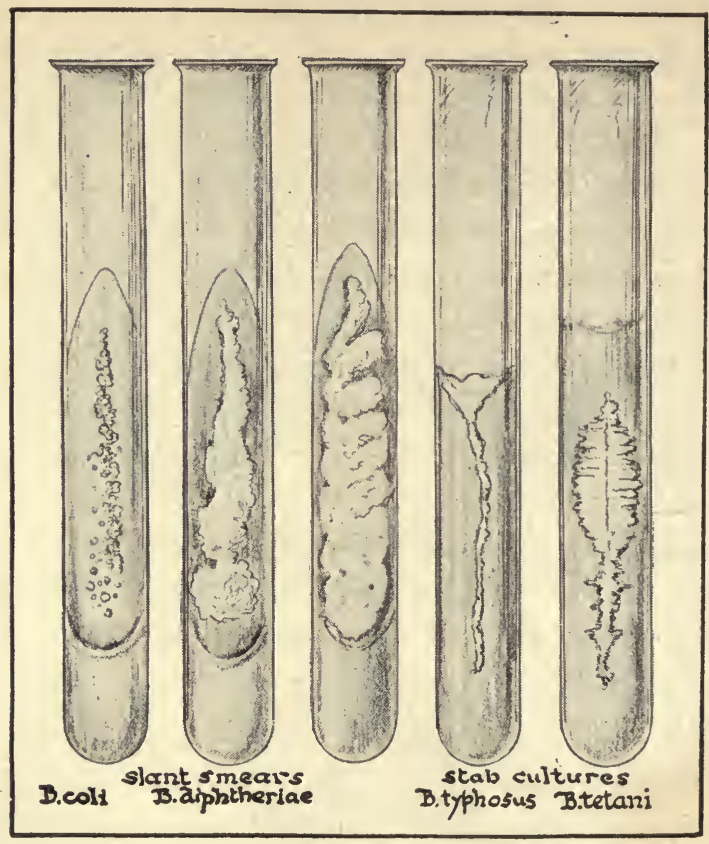

Two types of inoculations, smears and stabs, are made on slant cultures. After the media are incubated, the germs grow into colonies, each having a characteristic form and shape.

vary according to the location of the water. In spring water, the number of bacteria is relatively small, but in water into which sewage from cities drain, the number is extremely large. Sometimes bacteria in water contaminated with sewage are disease producing.

The soil that has been well cultivated is usually rich in bacteria. The deeper layers of soil contain few or no bacteria. Where the soil is dry and sandy, there are relatively few bacteria; where it is moist and loamy, they are abundant. They are found in great numbers around the bodies of dead animals or in soil that contains decaying roots of plants. 
Bacteria are probably found on all cooked foods which have. been exposed to the air, as well as on the surfaces of all fruits and vegetables.

The relation of various conditions to the growth of bacteria. Most bacteria thrive best in darkness. Direct sunlight kills them after a few hours of exposure. Even spore forms may be killed by direct sunlight. The food of bacteria must be organic and must be slightly moist. The best temperature for bacterial growth on culture media is between $20^{\circ}$ and $40^{\circ} \mathrm{C}$, although bacterial life is possible at absolute temperature $\left(-273^{\circ} \mathrm{C}\right.$. $)$ and at $160^{\circ} \mathrm{C}$. The temperature varies with the particular type of organism. For example, the human tuberculosis bacillus grows best at blood heat, $37.5^{\circ} \mathrm{C}$; ; the bird tuberculosis bacillus grows best at about $42^{\circ} \mathrm{C}$. Sudden changes in temperature are more or less detrimental to bacteria. If changes are slow, the spore formers will have a chance to form their spores. Cold checks the growth of bacteria and continued freezing or alternate freezing and thawing may sometimes kill them. Different bacteria are killed at different temperatures. Active typhoid bacteria are killed when subjected to a temperature of $60^{\circ} \mathrm{C}$. for thirty minutes. Ordinarily, all bacteria are killed at $100^{\circ} \mathrm{C}$. Moist heat is found more effective for killing bacteria than dry heat.

Methods of food preservation. Substances may be freed from bacteria, or the action of the bacteria on the substance may be materially decreased by depriving the organisms of some condition that is necessary for their life or growth. Some of the methods of preserving foods against the action of bacteria are dehydration, refrigeration, canning, smoking, and addition of preservatives.

If a food is thoroughly dried, particularly by the sun, the bacteria cannot thrive. The sun, in all probability, will kill them. Dryness is also an unfavorable condition for the growth of bacteria. Refrigeration preserves foods because cold checks the 


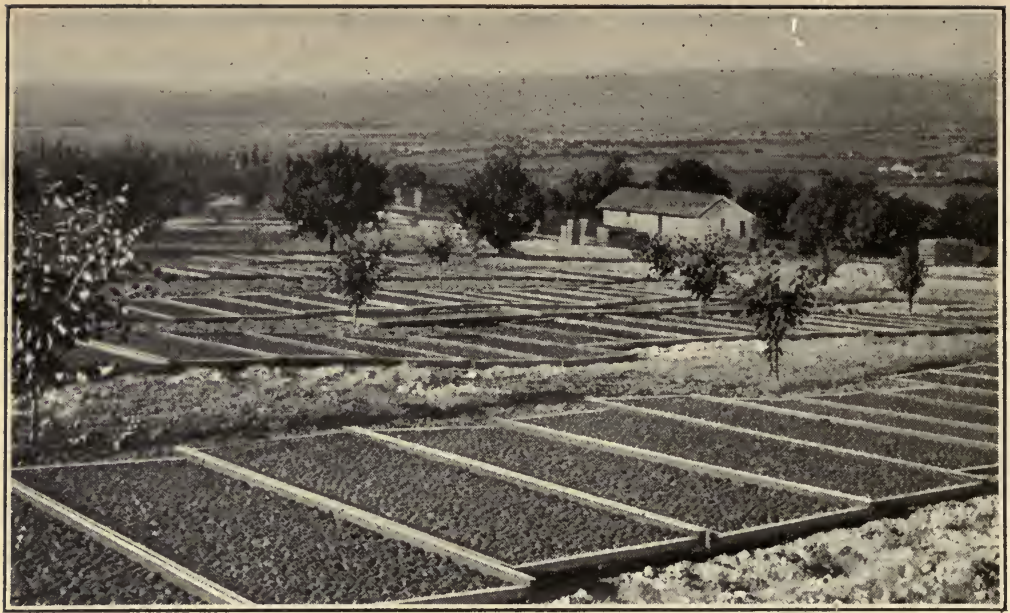

A method of preserving foods is by sun-drying. The picture shows plums exposed to sunlight until dry. This changes them to prunes and safeguards them from the attacks of bacteria.

growth of most bacteria. In the refrigeration process, the flavor is not likely to be spoiled. It is an expensive method, however. Canning or preserving makes use of extreme heat to sterilize, then by placing the food in air-tight containers no new bacteria can enter. Smoke contains creosote, which is poisonous to bacteria, but, in small amounts, is not poisonous to people. Hence, smoking is a method of preserving food. Bacteria in an alkaline or acid solution are more easily killed than those in a neutral solution. Housekeepers have found that canned fruits seldom spoil, but canned vegetables frequently cause trouble. Sometimes sugar, salt, and vinegar in quantity act as poisons to bacteria. They are not injurious to people. Producers frequently add preservatives such as benzoate of soda or mild acids to help kill the bacteria. Many of these may be injurious.

Problem. What is the number of bacteria found in the air in various places in the environment?

Prepare seven or eight Petri dishes of sterile agar media. 
I. Keep one Petri dish closed throughout the experiment. This is the control and will serve as a comparison with the exposed dishes.

II. Expose one dish of the agar media in a classroom at head level; expose one in the hallway through which many pupils pass; one in the lunch room, one in the gymnasium, one in the street, and one in a theater. Cover all dishes immediately after exposure.

A. Incubate the dishes between $30^{\circ}$ to $40^{\circ} \mathrm{C}$. until colonies appear on the surfaces.

B. Compare the dishes with the control.

$C$. Which dishes show the greater number of colonies?

$D$. Which dishes show the greater number of different kinds of colonies?

$E$. What seems to be the relation between dust and bacteria?

Problem. What is the bacterial content of different kinds of milk? *

I Put a couple of drops of certified milk in a Petri dish containing sterile agar. Cover the dish immediately and permit the milk to completely cover the agar.

II. Repeat the experiment for grade A and grade B milk.

III. Incubate the dishes for twenty-four hours at between $30^{\circ}$ and $40^{\circ} \mathrm{C}$.

IV. Which milk shows the greater number of colonies?

V. Which milk shows the greater number of kinds of colonies?

VI. What is the danger of having a great number of kinds of colonies?

VII. In selecting milk, state anything else, besides bacterial content, that must be considered.

VIII. State one possible reason for using pasteurized milk and one reason for using certified milk.

Problem. What is the effect of various antiseptics on the growth of bacteria?

Expose each of five dishes of sterile agar media to dusty air in order to inoculate them or introduce germs into them.

I. Cover one and keep it closed throughout the experiment.

II. Pour a little boric acid into dish number 2. Cover it and tip the dish repeatedly until the boric acid completely covers the surface of the agar. Be sure not to use any more than just enough to cover the agar.

III. Repeat the experiment, using iodine, salt solution, and mercurochrome in each of the other three dishes.

* All cities do not grade milk as A, B, and certified. If different forms of grading milk are used in your community, use those in performing this experiment. 
IV. Incubate the six dishes for twenty-four hours.

V. Make repeated daily observations.

$A$. Which material used seems to be of least use in checking the growth of bacteria?

1. Compare the growth of colonies in this dish with your control.

$B$. Give the effects of each of the other solutions on the growth of bacteria.

$C$. Which antiseptics might be unwise to use on delicate lining membranes such as those in the throat, nose, and eye?

VI. The experiment may be repeated to test the results of various mouth washes.

VII. Antiseptics should be used with great discretion. Some of them kill bacteria rather than check their growth. They are called germicides. Those that check the growth of bacteria are the true antiseptics. Germicides may kill the tissues as well as the bacteria. This, in many cases, will check healing.

\section{Questions And Suggestions}

1. Describe the structure of bacteria.

2. Classify bacteria according to their shape. Give some individual variations of each type.

3. What are aërobic and anaërobic bacteria?

4. Discuss three different types of nutrition found among bacteria. Give an example of each type.

5. Discuss reproduction and spore-formation.

6. Name five methods used in the identification of bacteria.

7. What is the importance of agar?

8. Discuss the sterilization of agar and of Petri dishes. Discuss the methods of inoculation, and conditions for the cultivation of bacteria.

9. Discuss favorable conditions for the growth of bacteria. Which of these conditions are found in the human body?

10. Under what conditions will food spoil?

11. Name some conditions unfavorable for the growth of bacteria.

12. Give in the form of an outline the method of preserving certain foods from the activities of bacteria, and the resulting conditions that are unfavorable for their growth.

13. Discuss the relative number of bacteria in different types of air.

14. Discuss the bacterial content of different types of milk.

15. What is the relation of different antiseptics to the growth of bacteria? 


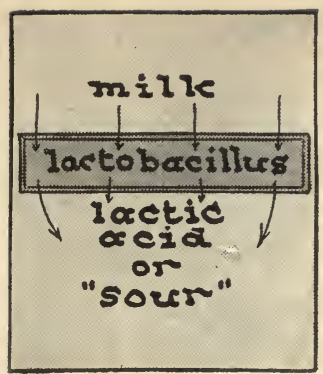

Curdling of milk.
CHAPTER XXXIX

\section{BENEFICIAL}

ACTIVITIES OF

BACTERIA

Why does milk turn sour? What makes sweet cider turn into vinegar? Why will the dead body of an animal putrefy? What is the importance of the rotation of crops?

Bacteria which are of value to man may be grouped into three classes: (1) those necessary for the preparation of certain foods; (2) those aiding industries; and (3) those useful in agriculture.

Bacteria in food preparation. Certain bacteria in milk change the milk sugar, lactose, to lactic acid. The production of this acid coagulates or curdles the protein in milk. This process of acid-formation and protein-coagulation is called souring. When the acid reaches a certain concentration, the process ceases. The presence of lactic acid in cream increases the yield of butter and improves the flavor. Lactic-acid bacteria are necessary for the production of sour-milk cheeses such as Swiss, Edam, and Camembert. The flavor of these cheeses is partly due to the lacticacid fermentation and partly to mold activity.

Acetic-acid bacteria convert alcohol into vinegar. Yeasts first attack the sugar of fruits or grains and convert it into alcohol, then the acetic-acid bacteria attack the alcohol and change it to vinegar. This vinegar is useful in preserving foods because bacteria of decay cannot work in a strong acid medium. 
If cabbage is finely chopped, salted, and packed tightly in jars or barrels, it will undergo a chemical change. The salt will
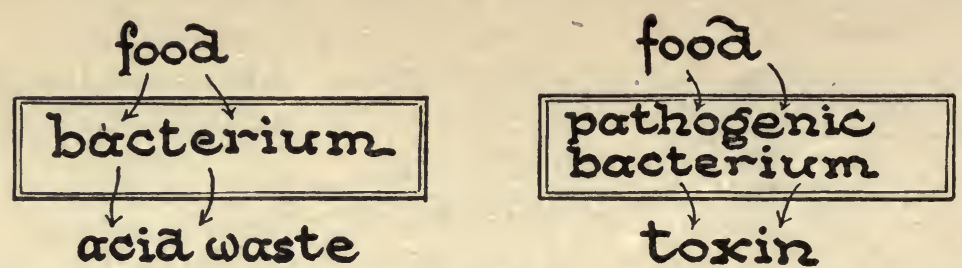

When bacteria absorb and use food, they give off acid wastes. The wastes of diseaseproducing bacteria are called toxins.

extract from the cabbage the juice which is a good medium for the growth of lactic-acid bacteria. This will attack the tissues of the cabbage, changing it to sauerkraut. Meat is made tender by the action of bacteria. When meat is fresh, that is, soon after the animal is killed, it is tough and more or less tasteless. Bacteria attack the muscles and connective tissue, loosen the fibers, and give the meat a taste. Meat is usually kept for some time to permit this bacterial attack. This is the beginning of the decay process. The process must not go too far or products that are objectionable are formed, and the meat is said to be tainted.

Ensilage is prepared for stock by packing finely chopped fodder in a silo. Bacteria and enzymes will attack the tissues of the fodder, causing it to change in appearance, flavor, and nutritive value.

Bacteria in other industries. Sponges are animals. The fairly hard commercial sponge is really the skeleton of the animal. This skeleton is composed of substances secreted by the cells of the animal. The sponges of commerce are prepared by cutting their attachment to the sea floor. The sponges are spread out on the seashore and bacteria destroy the soft organic parts, leaving the fibrous, horny framework or skeleton. This process is known as curing. The exoskeletons are then thoroughly washed and cleaned and sold for commercial uses. 
A similar process takes place in preparing linen fibers. Linen comes from the flax plant. If the flax is cut and thrown into pits and kept damp, certain bacteria will decay the cementing materials which hold the tough, strong fibers together. These fibers, when separated, are used for the manufacture of linen. This process of obtaining the fibers of flax is known as retting or rotting. There is an artificial process of retting which is not as successful as the natural water process. The natural process is used in Ireland and produces a fine grade of linen.

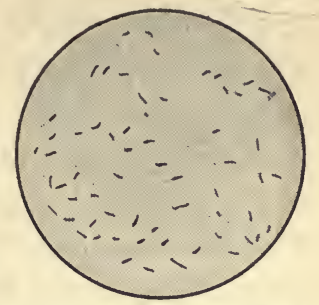

There are many different bacteria which grow in milk. A group attacks milk sugar and produces lactic acid, which results in. souring.

The artificial process, used in the United States, consists of an acid treatment for loosening the fibers. It is much quicker than the natural method, but less perfect, since the acid may roughen the flax fibers.

Bacteria are valuable in curing tobacco. The tobacco stalks with the leaves are piled into great heaps or hung from racks and allowed to sweat and then ferment at a fairly low temperature. This gives to tobacco its special flavor.

In tanning leather, the hides are soaked, scraped, and limed or treated with acids to remove the hairs. The lime is removed,

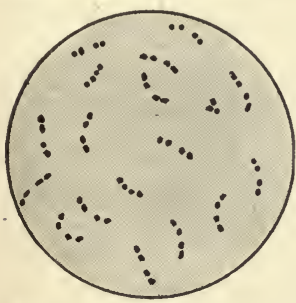

In fruit juices bacteria change alcohol to acetic acid.

protein and protoplasm. The nitrogen of the soil would be exhausted in a comparatively short time if there were no ways and the hides are put into solutions of tanbark. During these processes, certain bacterial fermentations take place, which make the leather soft and pliable.

Bacteria in agriculture. Large quantities of the nitrates from the soil are built into proteins and protoplasm by the plants. When these plants are removed, they take with them the nitrogen in the form of 
of replacing or renewing it. The same is true of sulphur and potassium.

Bacteria of decay are essential in farming. They bring about the decomposition of complex organic compounds into simpler ones. When this is accompanied by offensive odors, the process is known as putrefaction. The complex proteins and protoplasm of the plant or animal contain nitrogen. When a plant or animal dies, the bacteria of decay attack these nitrogen-containing compounds and break them down into simpler substances. One of these is a nitrogenous material, ammonia ; another substance contains sulphur. Phosphorus and the other elements present may be liberated, similarly. The decay process also sets free carbon dioxide and water. Nitrogenous wastes of animals are usually changed into ammonia by the action of the bacteria of decay.

Nitrification. Certain bacteria called the nitrifying bacteria take the ammonia formed by the bacteria of decay and change it into nitrates which the plants can utilize for further proteinmaking. This process is called nitrification. Ammonia is a gas, and would escape into the atmosphere were it not for the nitrifying bacteria. Nitrite bacteria first convert ammonia into nitrites, compounds formed by the union of ammonia with oxygen. Then other organisms cause the nitrites to take up more oxygen and form nitrates which are stable compounds and can dissolve in the soil water. Nitrite and nitrate bacteria are always found together. They work best in alkaline soils. If a sample of soil is heated and thus sterilized, the action does not take place. This indicates clearly that the process is a bacterial one and not simply a chemical one. Nitrification takes place only in soil that is well aërated and well drained. The drainage carries off the acids that might interfere with the process. Aëration is necessary because there must be enough oxygen to combine with the ammonia to form the nitrites and the nitrates. Earth worms aid in this process by constantly working through the soil. This permits the 
entrance of air. Lime is sometimes added to soil to neutralize its acidity so that the nitrifying activities may be increased.

Denitrification. If soil is poorly drained, poorly aërated, and contains fresh organic matter, denitrifying bacteria thrive. They convert the ammonia, formed in the decay process, into free nitrogen. The free nitrogen then escapes into the air and is lost to the

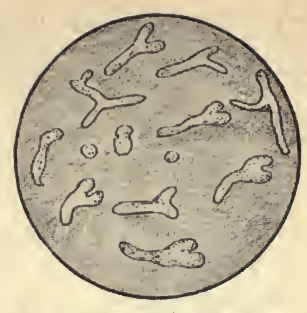

The soil bacteria are irregular-shaped organisms. plants. The denitrifying bacteria can also break down nitrates into

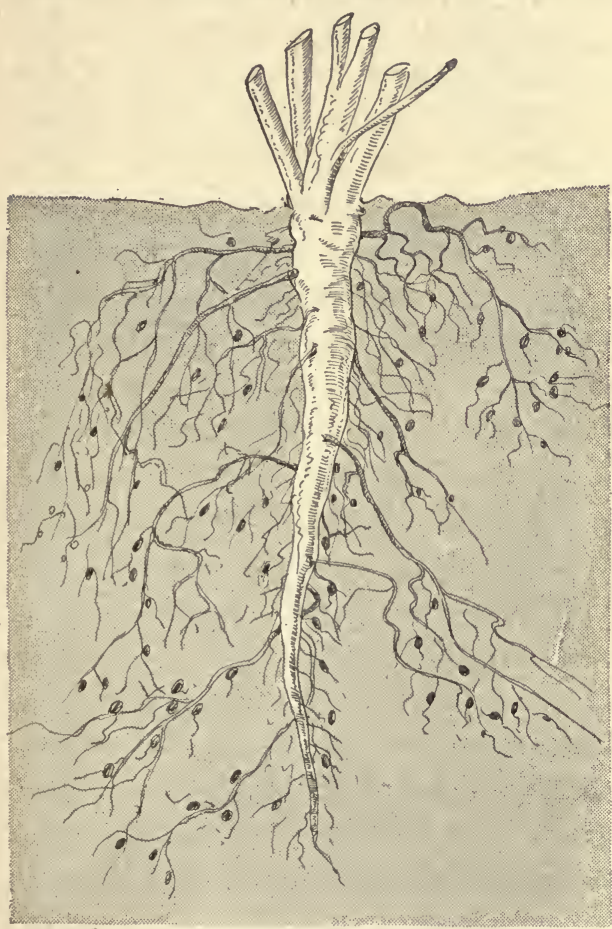

Nitrogen-fixing bacteria form nodules or small swellings on the roots of pod-bearing plants. The countless bacteria in these nodules fix free nitrogen of the air into a usable form, nitrates. nitrites, then into ammonia, and finally into free nitrogen. Soil should not be covered thickly with unrotted manure as the activity of these bacteria are then promoted. From the point of view of conserving soil fertility, the denitrifying bacteria are undesirable. Certain other bacteria are able, however, to take free nitrogen from the air and again change it into a form in which it can be used. These are called the nitrogen-fixing bacteria.

Nitrogen-fixation. The nitrogen-fixing bacteria have already been discussed in the previous 
chapter. When a pod-bearing plant, such as the clover, alfalfa, bean, or pea, is young, its roots are attacked by the nitrogen-fixing

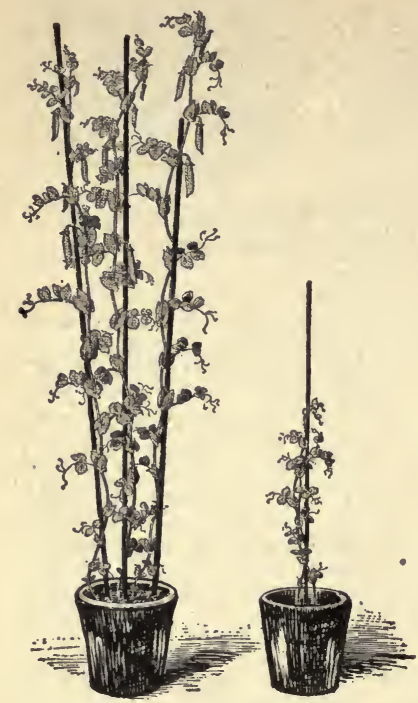

The value of nitrogen-fixing bacteria was demonstrated in the above experiment. When the roots of the plants were examined, those on the left had numerous well-developed tubercles; those on the right had no tubercles. They were both watered with a nutrient solution containing all the necessary nutrients but nitrogen. Explain the relation of the tubercles to the growth of the plant. bacteria found in the soil. They penetrate the roots through the delicate roothairs and establish themselves in the outer layer of the root cells. The root accommodates them by building more cells in that region, forming little nodules or tubercles. In these nodules, the nitrogen-fixing bacteria multiply. They take the free nitrogen from the air which permeates the soil, and build it into nitrites which are later converted to nitrates. When the roots of these plants are plowed under, they decay, and the nitrates are liberated into the soil. Soil may be inoculated with cultures of nodule bacteria. A crop of clover or alfalfa plowed under supplies the soil with about 100 pounds of nitrogen to the acre. Such a crop is as valuable as many loads of manure. A good crop of corn or wheat will take from 50 to 75 pounds of nitrogen per acre from soil. There are certain freeliving soil bacteria which build nitrogen into nitrates when conditions are favorable. The result of the activity of these organisms is not unlike the nitrates formed by nitrogen-fixing bacteria. But they differ from the nitrogen-fixing bacteria in that they do not need roots of plants for their homes.

Lightning and similar electric discharges in the air unite some free nitrogen with oxygen to form nitrates. These are washed from the 
air by rain and thus reach the soil. Exceedingly small quantities of nitrates are formed in nature in this way. Commercial processes. of building nitrogen into nitrates is now being conducted in several sections of the country. Commercial nitrates are generally used for fertilizers. One of these plants is at Muscle Shoals, Alabama. The excellent water-power facilities of that location supply the necessary energy for the project.

Rotation of crops. Crops are rotated, that is, one crop succeeds a

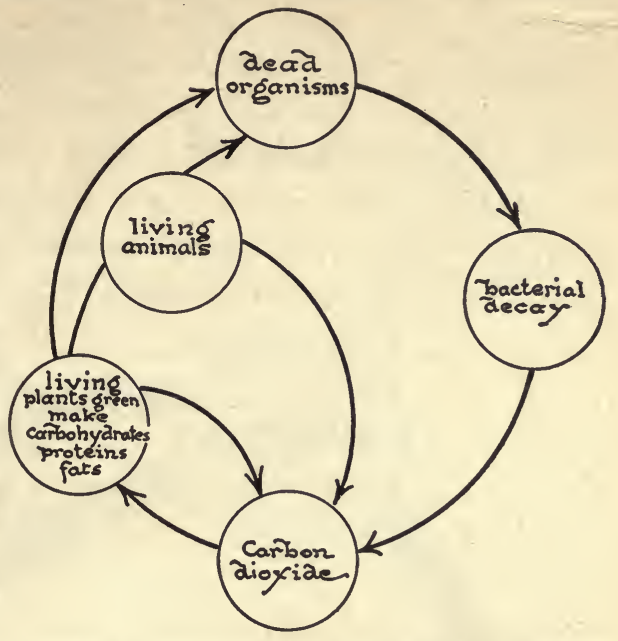

There is a continual building and destruction of carbon compounds. This is described diagrammatically in the carbon cycle. different kind in the same field year after year. The reasons for crop rotation are as follows: (1) Plants use up varying amounts of the different mineral matters in the soil. One crop might need a quantity of calcium salts but not much iron. Another crop will require considerable iron but fewer sulphates. If one crop is planted year after year in the same plot of land, that land will grow poor in one or more mineral salts and the quality of the crops will be greatly impaired unless commercial fertilizers are used. Where rotation of crops is practiced, the same soil can be used for years without adding fertilizer. (2) Plants are subject to diseases caused by bacteria and other fungi. If only one crop is planted, the soil may become infected with spores of disease-producing organisms. Another crop probably would not be affected by these spores. For example, corn smut will not injure potatoes; poWH. FITZ. AD. BIO. -27 
tato blight will not affect wheat. Rotation of crops also kills insect pests that live only on a certain plant. (3) The ungathered roots and stalks of crops remain in the ground, decay, and produce organic acids. These acids are sometimes injurious to the plant producing them, but not to other plants. Hence, the crops should be changed. Bone meal or lime is added to neutralize these acids. Each commercial fertilizer used, however, adds that much to the farmer's expense. (4) Almost all crops remove great quantities of nitrates from the soil. These may

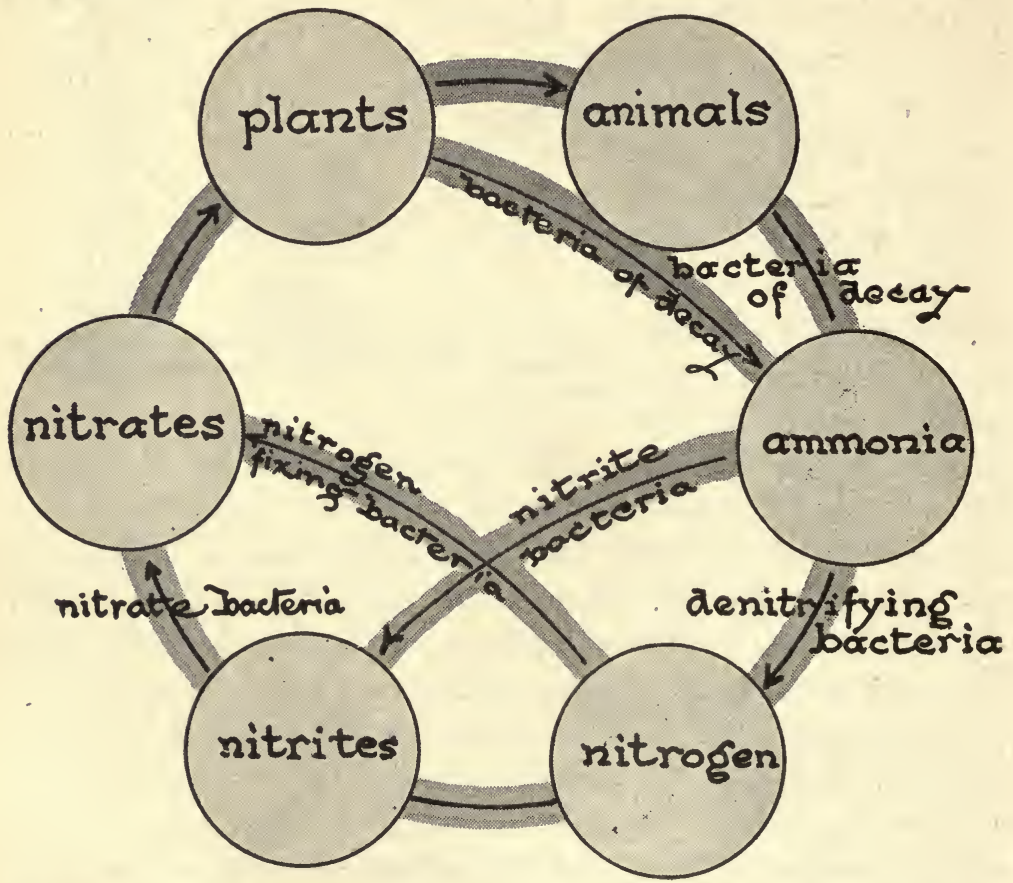

A number of different bacteria aid in transforming nitrogen into a form which plants can use. There is a constant renewal of nitrogen in the soil.

be restored in a variety of ways. Animal waste, manure, may be spread over soil and allowed to decay. This increases the nitrogen 
content. Any organic material which decays will accomplish the same thing. Many commercial fertilizers are made from guano,

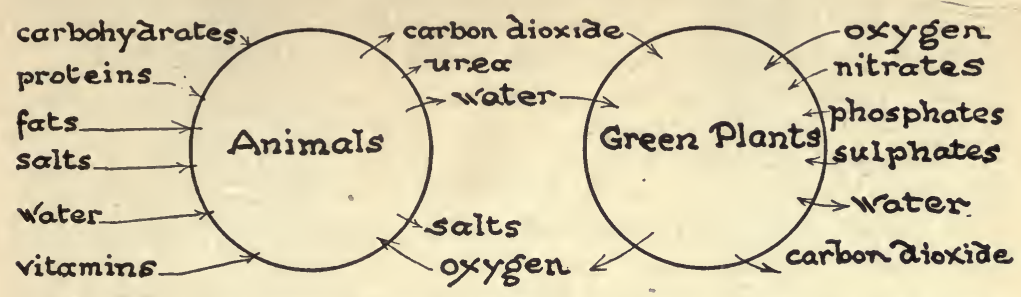

There is an interdependence between green plants and animals. Animals can make use of the oxygen excreted by green plants. These in turn, can make use of carbon dioxide, water, and possibly urea excreted by animals.

bird waste. Refuse from abattoirs, and meat and fish canneries, or specially prepared mixtures of chemical compounds, may be purchased and plowed into the soil. The land may be allowed to lie fallow, that is, unused for a season or so. Certain minerals in the ground may break down in fallow fields and thus restore the usable mineral elements. A leguminous crop, such as peas, beans, or alfalfa, may be planted, allowed to grow, and marketed. At the same time, nitrates are restored to the soil in which they grow.

Soil conservation. If the soil is kept in good condition, nitrates, sulphates, and phosphates are constantly renewed as they are removed. The passage, use, and return of these materials constitute a cycle as shown in the diagram. Were it not for the action of bacteria in the soil, all vegetation on the earth would cease. Soil fertility is primarily a bacterial activity. Bacteria are commonly thought of as being particularly injurious. In reality, the beneficial effects of bacteria far outweigh the injurious effects in many ways.

\section{Questions AND Suggestions}

1. Using the following topics, make out a tabular outline of the relation of bacteria to food preparation.

$A$. Name of bacterium.

B. Material attacked. 
C. Material produced.

$D$. Commercial use of material produced.

2. Make a library report on the tanning of leather.

- 3. What is meant by soil exhaustion and soil renewal?

4. What is the importance of the decay process to agriculture?

5. Explain nitrification in detail.

6. Discuss denitrification.

7. Discuss nitrogen-fixation. Give three different ways of fixing nitrogen.

8. What is the importance of rotating crops? Give at least four reasons for rotating crops.

9. Mention four different facts that must be considered in cultivating soil.

10. Make out an original carbon and nitrogen cycle.

11. Do farmers have scientific knowledge about bacteria or do they depend upon their experience or on hearsay in caring for the soil?

12. Does the government make any effort to give farmers scientific instruction in soil and crop improvement?

13. Discuss a balanced aquarium.

\section{Supplementary Readings}

Gager, C. S., General Botany (P. Blakiston's Son \& Co.).

Greaves, J. E. \& E. O., Elementary Bacteriology (W. B. Saunders Co.).

Holman, R. M., \& Robbins, W. W. A Textbook of General Botany (John Wiley \& Sons, Inc.). 


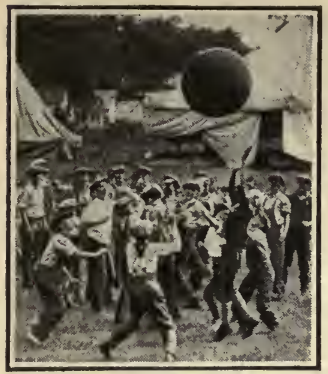

Ewing Galloway

Push ball.
CHAPTER XL

HEALTH

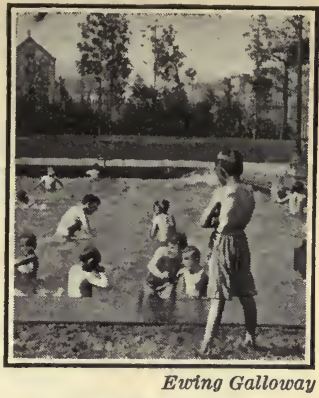

Outdoor swimming pool.

Should people learn about diseases? Is longevity more or less controllable? Has community or individual control of disease been more successful?

Some people object to studying disease because they worry about each disease studied, fearing that they have it or may contract it. Education in health and disease should enable people to exercise an intelligent attitude toward disease, to practice ways of preventing disease, and to learn how to conquer and control disease.

Disease and health. The best way of preventing disease is to stay in good health. Some people feel that they are in a healthy condition if no disease is present. Even though there is no actual impairment of bodily functions, certain organs may not be in normal condition, consequently, health is not necessarily a lack of disease. Nor is the feeling of being well an infallible measure of the degree of health. Frequently people feel very ill when the actual illness is slight; on the other hand, people may have only a slight feeling of discomfort and yet have a serious organic disorder. An adequate measure of health is an annual physical examination. If people are examined thoroughly every year, beginning symptoms may often be detected before a disease has advanced to a serious degree. Health examinations are just as important for well as for sick people. In disease-control, prevention is far more important 


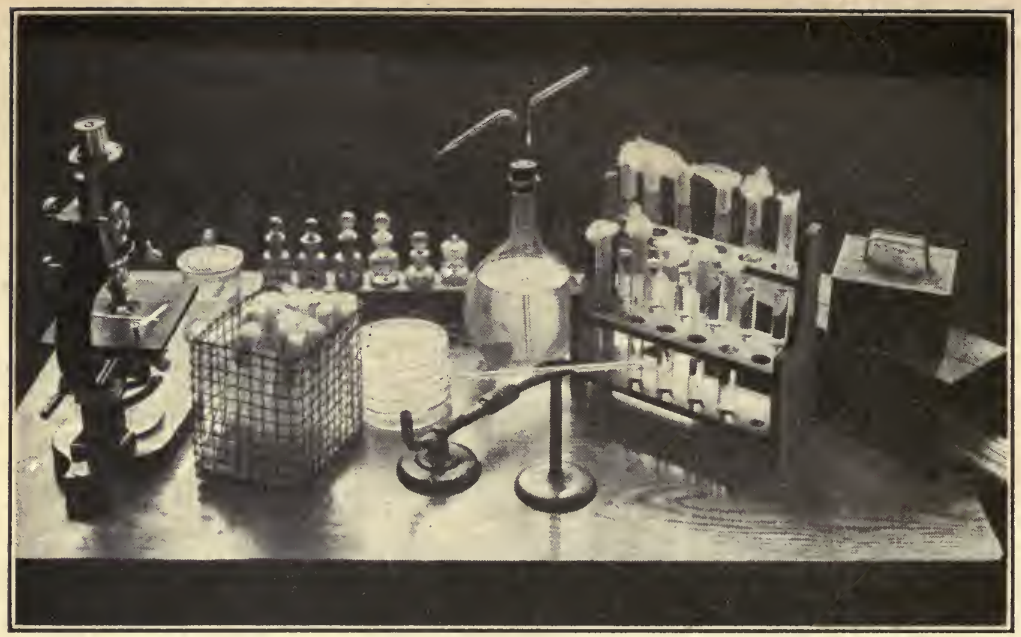

Am. Museum Nat. Hist.

Bacteriological laboratories contain elaborate equipment. Can you identify the microscope, Petri dishes, agar slants in test tubes, staining fluids for bacteria, distilled water in flask for washing off excess stain, and the Bunsen burner? Can you give the use of each article in working with bacteria?

than attempts to cure disease. Each illness, no matter how slight, is likely to lower the resistance of the person just that much.

Health and longevity. Another misconception of health is that it is identified by length of life or longevity. This; too, is probably a fallacy. Some delicate people receive medical treatment all their lives, and, through care, live a long time, although they never really have good health. On the other hand, people in perfect condition may meet with accidents which will cut short the span of their lives.

Health and adaptability. Positively speaking, health is merely the adaptability of the organism to meet a variety of situations. To be healthy, an organism must be able to make rapid and proper adjustment to every situation. For example, normal heart muscles thicken to accommodate the heart to unusual conditions. A diseased heart will not do this, consequently, discomfort results. Acquiring resistance to certain diseases is a process of adaptation. All 
people do not have an equal resistance. Those who have not must learn how to acquire it. Therefore, education along these lines is necessary. People must be taught hygienic living so that they may adapt themselves properly to changes of temperature, work, and food. Many diseases may be avoided by $レ$ correcting improper habits of living and certain physical defects.

Health and education. Health is one of the main objectives of education. Unless the body can adequately adapt itself to changing conditions in life, the person is inefficient. In the past, undue emphasis has probably been placed on defects. Open-air classes were established for cardiac, anaemic, and tubercular children. Nutrition classes were established for malnutrition cases. Each year the health of some children was built up in these classes to such an extent that the children were able to continue in regular classes. Each year new cases would appear in regular classes which would have to be sent to these special classes. Nutrition classes are

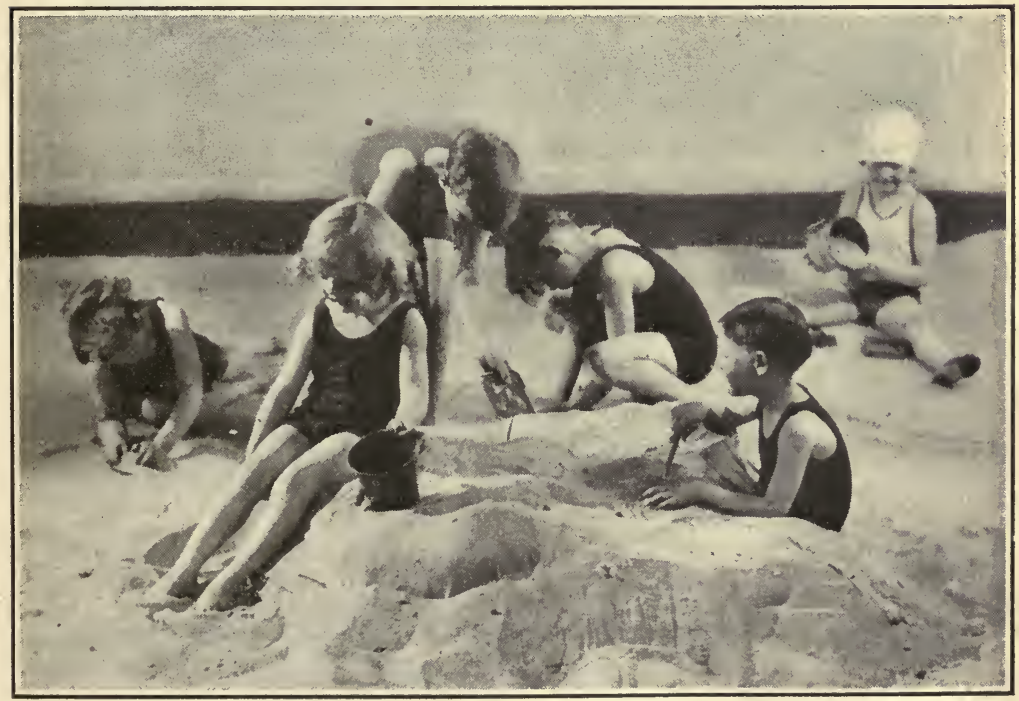

Underwood and Underwood

Play in the sunlight is the heritage of childhood. It leads to a proper physical development and builds a healthy nervous system. 
necessary, but if the entire school were properly educated in health habits, and if there, were a check to see that these habits,

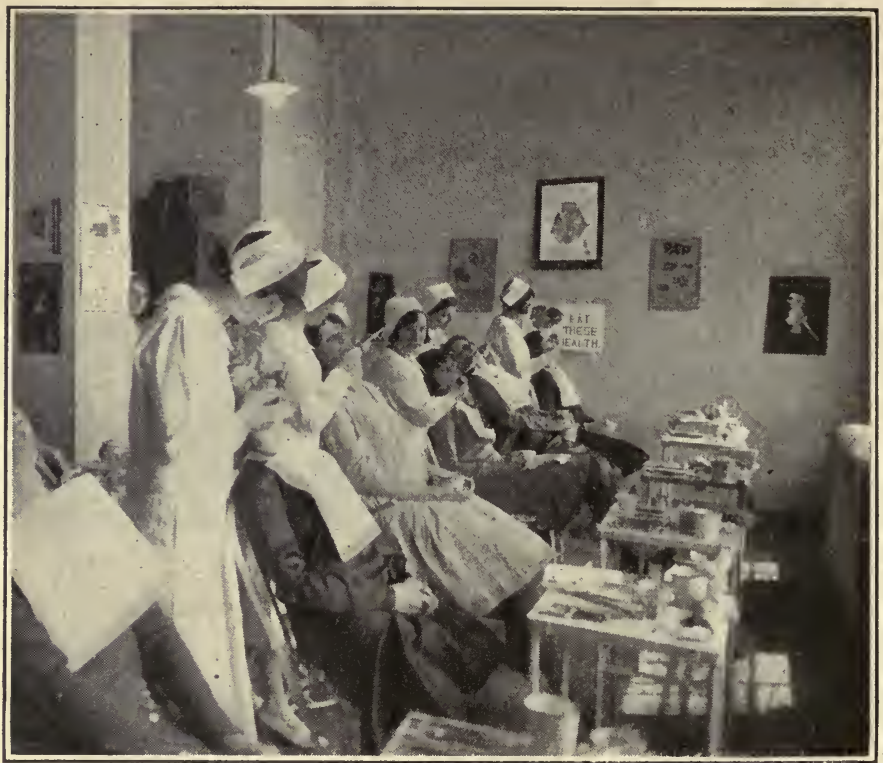

A properly cared for set of teeth is the result of frequent expert inspection. In certain schools, oral hygienists make surveys of mouths and suggest dental treatment when necessary.

once taught, were enforced in the home and community, there would not be so many anaemic, cardiac, and tubercular cases. In order to show how much education could accomplish in disease, the Metropolitan Life Insurance Company and the National Tuberculosis Association conducted an experiment at Framingham, Massachusetts. A campaign of education was carried on so successfully that tuberculosis was reduced 69 per cent in that community within ten years.

Effective education in health is evidently needed. This is particularly pertinent when we consider that the statistics, prepared by General Crowder of the United States Army, showed that 33 per cent of the United States soldiers in the World War were 
unfit for front-line duty. They had been out of school a very short time, but, evidently, had received very little, if any, instruction in health.

Science and health. In the Report on National Vitality, issued by President Roosevelt's Conservation Commission on National Vitality, it was claimed that the reasonable application of scientific knowledge would add at least fifteen years to the average lifetime. It stated that at least 40 per cent of American mortality was preventable or postponable. There are probably always three million people ill in this country at one time. About 50 per cent of this illness is probably preventable. This report was issued in 1909. The expectation of life has gone up since that time from, approximately, forty-five years to fifty-five years, according to the figures of the Life Extension Institute.

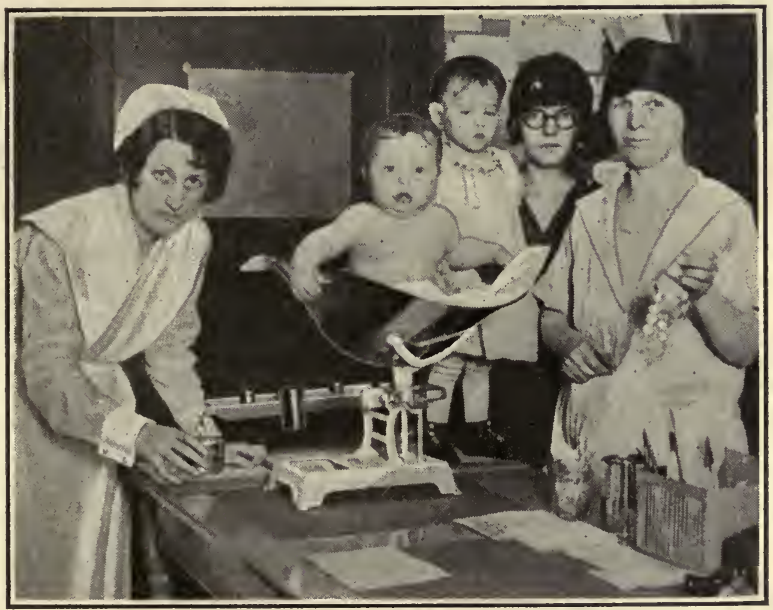

Underwood \& Underwood

Health is more often a matter of prevention than cure. In baby clinics, nurses weigh, measure, and make various examinations of babies to make sure that they are in good health.

Along with the diseases that have been partially or entirely controlled through scientific investigation and health education, there has been a big drop in mortality. The deaths from typhoid fever 
have been reduced 75 per cent, and typhoid is generally termed a vanishing disease. The death rate of tuberculosis has been reduced

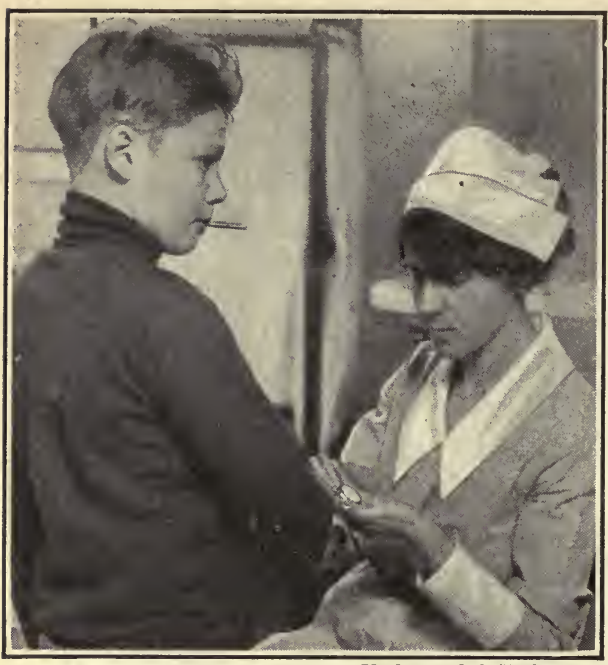

Underwood \& Underwood

A physical examination includes the taking of one's pulse rate and temperature. about 50 per cent. Diphtheria is now preventable and is becoming infrequent. In states where prophylactic measures are used, the Board of Health expects to have the disease under complete control by 1930 . The diseases that may be considered as largely under individual control show increasing death rates. Heart conditions, diabetes; and kidney diseases are examples of these. They are, to some extent, dependent upon a person's habits of living.

It is an accepted fact that the general life expectancy span at birth is increased. Believing that every one has the right to live, science has enabled us to bolster up the weakling at birth and all along the way - but eventually he drops out, the fight is too great. Science has decreased the infant mortality rate only to have the weaker ones die at a younger age than those with a stronger physical start. If life is to be lengthened at the other end, every individual must take more interest in, and insist on living hygienically. Much is still to be done. Ten years could be added to the expectation of life if all sections of the population could live under the same favorable conditions that are enjoyed by groups and communities where the death rates are unusually low. Individuals must be educated to have an intelli- 
gent knowledge of disease and understand its prevention. There are still too many defects such as mouth infection, tonsil infection, overweight, underweight, foot defects, and visual defects. If all people had an annual health examination, many diseases would be prevented. Disease prevention should be an incentive for all people since it will reflect in the substantial gains in the vitality of the nation. In the previous chapters, suggestions concerning diets, sunlight, exercise, rest, and mental relaxation have been given. In subsequent chapters methods of avoiding actual diseases will be discussed.

The measurement of health. A test to show whether one's personal habits are hygienic is the Payne "Habits and Practices in Accident Prevention and Health" given in the Appendix. A high school student should score at least 355 under A, 75 in the items under B, and 70 in the items under C. The points total 500 .

\section{Questions and Suggestions}

1. What is a popular objection to the study of disease?

2. Name three popular misconceptions of health. Discuss the fallacy in each of them.

3. Give a scientific definition of health.

4. How may health be attained?

5. What can be accomplished by education in health?

6 . Why do we find a larger reduction in the diseases that a community can control, than in those that an individual must control? Why is this true?

7. Discuss your ideas on an annual health examination.

8. Give some examples of adaptations of the organism to changing conditions.

9. Discuss your ideas on the scoring of health practices.

10. Score your habits and practices, as suggested in the test in the Appendix, on a separate piece of paper. Add the score and see how closely you approximate hygienic living. 


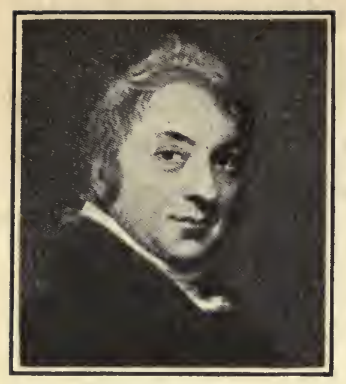

Edwar 1 Jenner.
CHAPTER XLI

SMALLPOX AND

ITS CONTROL

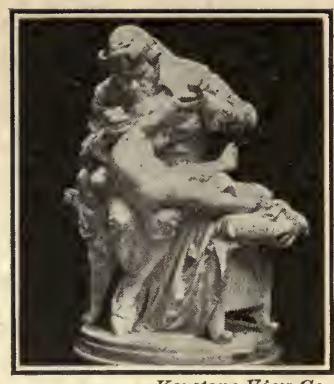

Keystone $\mathrm{View}$ Co.

Tribute to Jenner and vaccination.

Has smallpox ever been epidemic? Is inoculation for smallpox a new method of treatment? How did vaccination first start? What effect has vaccination had on the mortality from smallpox?

History of smallpox. Smallpox was known in China many centuries before the Christian era. It also existed in India, Arabia, Ethiopia, and neighboring countries. It spread through Europe during the Crusades. The first epidemic of smallpox in Europe took place in the latter part of the sixteenth century. For centuries, it was estimated that ten out of every hundred deaths were due to smallpox. More than half the people in Europe were scarred and disfigured by the disease. It is probable that at least $60,000,000$ people died from smallpox during the eighteenth century.

The disease was first brought into America by the Spaniards about 1515 . Within a short period, three and a half million people in Mexico died of it. Probably half of the American Indians died during various epidemics of smallpox. Boston has had six epidemics; in the last one, 1752 , there were 5989 smallpox victims out of a population of over 15,000; of these, 894 died.

History of inoculation against smallpox. Inoculation first originated in the Orient, hundreds of years ago. The people would introduce into a cut or scratch of a well person, the pus or scab 
from smallpox patients. This, in many cases, brought about protection against the disease. The practice of inoculation was carried into Europe by way of Asia, Africa, and Constantinople. In Turkey, certain old women took pus from the pustules of persons suffering from smallpox and inoculated it into the veins of well people.

In 1717, Lady Mary Montague, while traveling in Turkey, had her little son inoculated with smallpox, and he did not contract the disease. Through this incident, the idea of inoculation as a preventative was introduced into England, although many people were afraid of its results. Several years later, authorities offered a number of criminals confined in the Newgate Prison, England, their freedom, if they would submit to an inoculation against smallpox. They did so, the results were satisfactory, and they received their freedom. After this, the practice gained steadily in England. When a person was to be inoculated, he was usually kept on a light diet for about six weeks. He was then purged and bled to make sure that his body was in good condition. Then he was inoculated with smallpox. Pus was taken from the pustules of a person with a mild case of smallpox and placed in several scratches made on the body of the patient. This usually resulted in a mild form of smallpox to the person treated, but was supposed to protect him against a very severe form. This method of inoculation was somewhat dangerous to a community, in as much as new cases of smallpox, however mild, were likely to develop.

History of vaccination. It had been a belief, for an unknown length of time, in rural districts, that people who had contracted cowpox, a disease of cows, did not take smallpox. A farmer named Benjamin Jesty, in 1774, took some pus from the sore of a cow with cowpox and inoculated his wife and children. This made them immune to smallpox infection. But Jesty's experiment never became generally known. It was Edward Jenner 
(1749-1823) of England, who first made known the idea of vaccination. The germ theory of disease had not yet been pre-

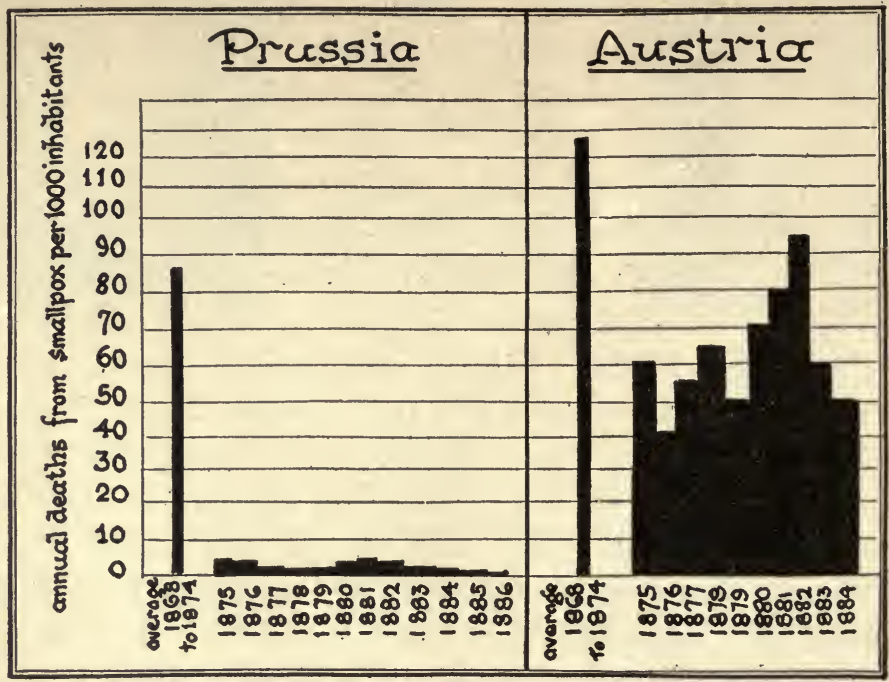

In 1874 vaccination was introduced into Prussia. Note the decrease in smallpox in the following year. Vaccination is not compulsory in Austria. The above graphs indicate a direct relation between the number of smallpox cases and compulsory vaccination.

sented by Pasteur. Jenner, however, collected data from the people who had had cowpox, and afterward had resisted smallpox infections. He began the scientific investigation of inoculation. A dairy maid on a certain farm contracted cowpox. Jenner took some pus from a sore on her hand and inoculated a little boy with it. The boy became slightly ill, but soon recovered. Later the boy was exposed to smallpox and even inoculated with smallpox virus, but he did not contract the disease. Evidently the cowpox infection had given him a protection against smallpox. Jenner made many such experiments and published his discoveries in his celebrated pamphlet An Inquiry into the Causes and effects of the Variolae Vaccinae.

The inoculation with cowpox was called vaccination (vacca- 
cow). There was a storm of protest against this method of inoculation. Newspapers printed bitter attacks and said people would show the characters of cows if they had this filthy material from a cow introduced in them. The results, however, were so successful that vaccination was gradually accepted as the only means of controlling smallpox, and the old method of inoculation was soon forbidden. Napoleon had all his soldiers who had never had smallpox vaccinated. The Empress of Russia urged its practice in Russia. Spain and Sicily also introduced vaccination.

About 1800, at a meeting of the American Academy of Arts and Sciences, presided over by the President of the United States, John Adams, the introduction of vaccination into America was first considered. A supply of vaccine material was secured, and a Dr. Waterhouse vaccinated his five-year-old boy. He afterward vaccinated other members of his family. They became immune to smallpox infection. Later Thomas Jefferson became interested and he had the members of his family vaccinated. From that time on the practice of vaccination spread rapidly through the country. The vaccine virus tends to stimulate certain cells of the body to produce substances known as anti-bodies which remain in the blood as a protection against disease. When a vaccinated person is exposed to smallpox, he is already fortified by the anti-bodies which will act against the smallpox bacteria.

Nature and symptoms of smallpox. Smallpox mortality is 30 per cent greater among unvaccinated than among vaccinated persons. Children are particularly susceptible. In the Montreal epidemic of $1885-1886,2717$ of the 3164 deaths were children under ten years of age.

Smallpox is caused by a filterable virus. About three days after becoming ill with smallpox, little abscesses or pustules form. If the pustules are deep, affecting the dermis seriously, pits or pock-marks will always show on the skin. The scales and crusts from the healing pustules are highly infectious, as are 
Number of Cases and Deaths from Smallpox in the United States

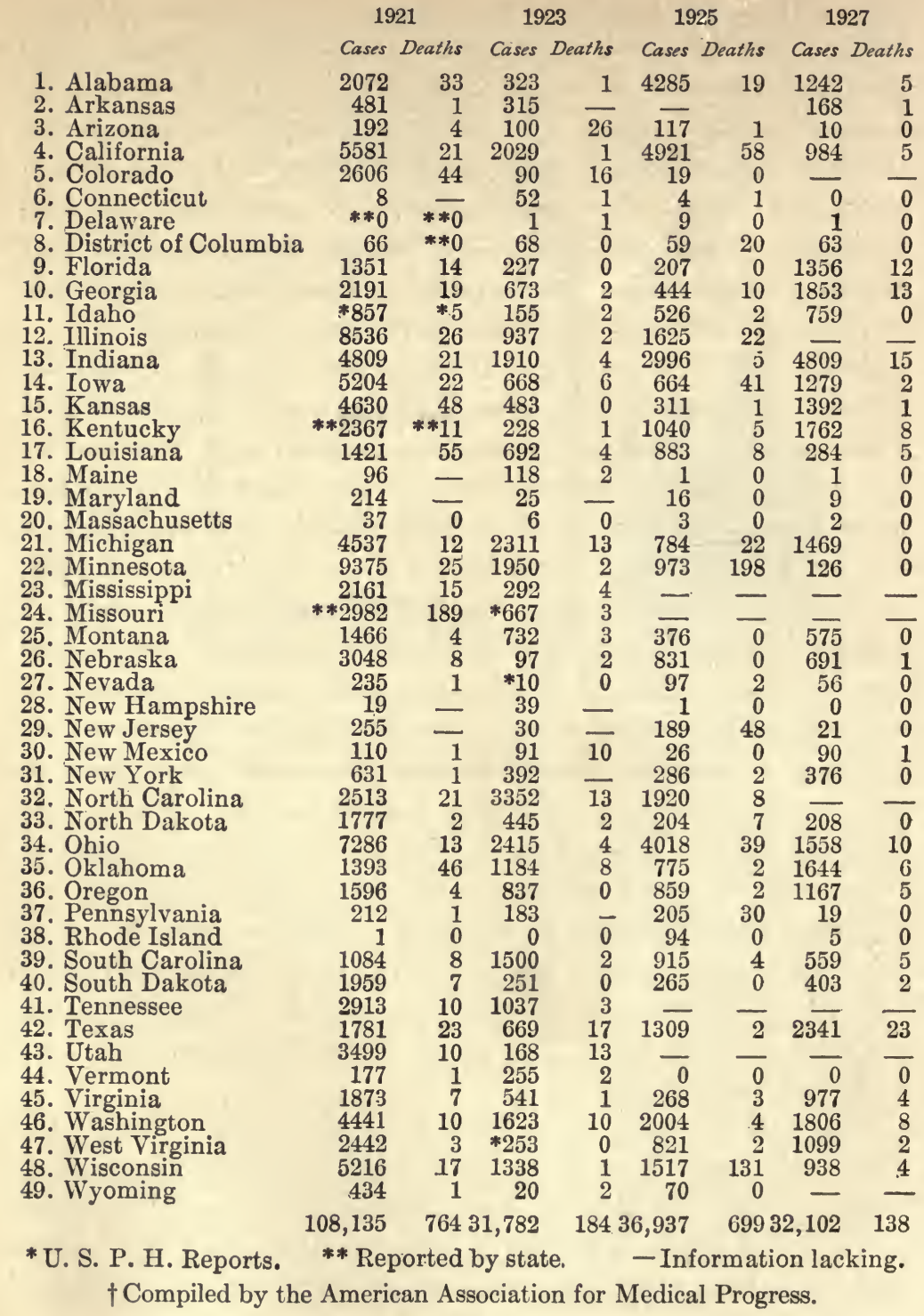


the contents of the pustules. If the disease is severe, the eyes, ears, and kidneys may be irreparably damaged. Recovery from one attack of smallpox usually leaves the individual immune to all subsequent attacks.

Spread. Smallpox is very communicable, that is, it is readily spread by contact. The smallpox virus may enter the body through the respiratory system, or through the digestive system. It is carried by discharges from the mouth and nose, or by the pus and scabs, which may get in food, or on articles used by patients.

Prevention of smallpox. Because of the extremely communicable nature of smallpox, isolation or quarantine of all patients is essential. Sanitary precautions of all kinds should be taken in handling the patients and all the articles used by them. Ex-

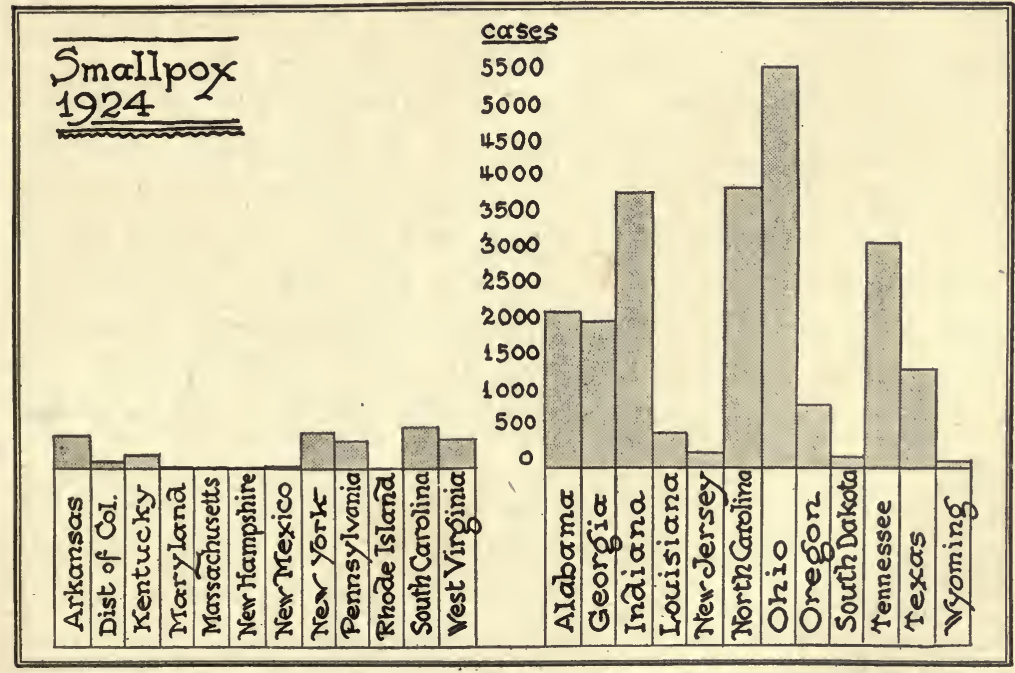

Two groups of states have been compared. The chart on the left indicates those states having compulsory vaccination; the one on the right indicates local enforcement of the law.

tensive education and widespread publicity as to the value of enforcing vaccination are necessary in order to keep the disease in WH. FITZ. AD. BIO. -28 . 
check. A vaccination against smallpox lasts from five to seven years. After that time there should be a second vaccination. The second vaccination may produce immunity for life. By immunity is meant the ability to resist disease. All children should be vaccinated before they are nine months old. Vaccination during epidemic periods is also wise.

The vaccine for smallpox is prepared by first developing the causative organisms in the body of young female calves. After determining that the calves are perfectly healthy, they are thoroughly cleaned and inoculated with the crusts collected from the vaccinations of healthy children. Cysts or vesicles are formed in about five or six days and the contents of these vesicles are scraped off and ground into dilute glycerine in order to destroy all harmful bacteria. This product is then tested several times, in many ways, for purity as well as for effectiveness. The material is then put up in small tubes ready for use.

Value of vaccination. The gradual disappearance of smallpox is due largely to the widespread use of vaccination. In countries where vaccination is not required, smallpox is still causing the deaths of thousands of people each year. It is still one of the main causes of death in China and India. Between the years 1918 and 1922, India reported 63,553 deaths from this disease alone. In Russia, between the years 1902 and 1914, over a million persons became affected with smallpox and over half a million died of it.

In the Philippine Islands, prior to the occupation by the American Army, it was estimated that more than 40,000 smallpox deaths occurred annually. Vaccination was introduced by the Americans in 1905, and has been continued ever since. There were, in 1903, 18,989 smallpox deaths, but this number has decreased rapidly until in 1916 only 239 deaths occurred from this disease. Then the effects of the first vaccinations wore off and people became careless about being revaccinated. This neglect resulted in a 
serious epidemic in 1918. During this year, 60,447 deaths occurred. At this time, only one case developed among the 5422 vaccinated United States troops who were stationed on the Islands.

There are still about 70,000 cases of smallpox in the United States annually, because vaccination is not universally enforced. In two states where vaccination is not required, there are many cases of smallpox. In one of these states there were over 2000 cases and 230 deaths reported in 1926; in the other, 2413 cases and 31 deaths were reported in 1926. Contrasted with these states, a state where vaccination is rigidly enforced had, in the same year, only 56 cases and no deaths.

Objections to vaccination not valid. When there is a specific prevention for a disease, as there is in vaccination against smallpox, it seems unbelievable that there are so many cases each year. The objections raised to vaccination are not well founded. One of them is that it causes lockjaw. There is no evidence to support this belief. Since there is a scratch made in the vaccination process, there is probably the same danger of getting lockjaw as there would be from any scratch. There is no danger of lockjaw peculiar to the vaccination process. Strict government inspection of the virus used in vaccination precludes the possibility of contamination. Ordinary precautions of a vaccination must be taken, however, to prevent infection. Some people think the vaccine is taken from a person who has smallpox and that this material may contain germs of the disease. The material is taken from calves and not people. The calves are examined carefully so as to eliminate the possibility of any disease being transmitted to people through the yaccine. The vaccine virus is always very carefully purified before being used.

\section{Questions AND Suggestions}

1. Trace the early history of smallpox, including its introduction into America.

2. Trace the history of inoculation to the time of Edward Jenner. 
3. Discuss the investigation and acceptance of vaccination for the prevention of smallpox.

4. Give a report on the life and works of Edward Jenner.

5. Discuss the nature of smallpox. How is smallpox spread?

6. What are the chief preventive measures against smallpox?

7. Discuss some of the methods of inoculation used in the past.

8. Has smallpox been checked to the fullest possible extent? Give several reasons for your answer.

\section{SuPplementary Readings}

Broadhurst, Jean, How We Resist Disease (J. B. Lippincott Co.). De Kruif, P. H., Microbe Hunters (Harcourt, Brace \& Co.). Haggard, H. W., The Science of Health and Disease (Harper \& Bros.). Pamphlets published by the Metropolitan Life Insurance Co., New York City.

Zinsser, Hans, A Textbook of Bacteriology (D. Appleton \& Co.). 


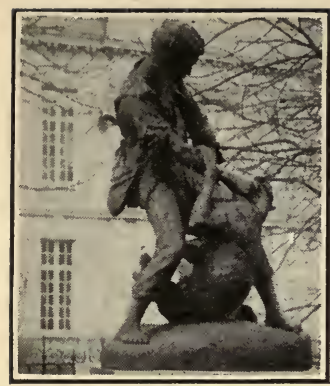

Keystone Vieno Co.

Jupille battling with a mad dog.
CHAPTER XLII

RABIES AND ITS CONTROL

How did Louis Pasteur control rabies? Why does rabies still exist? What advice did Louis Pasteur give to medical students?

History of rabies. Rabies or hydrophobia is a very ancient disease. People termed it hydrophobia because a person bitten by a mad dog develops a fever and a thirst; yet the attempt to drink water produces such painful convulsions that he develops a dread of water. Aristotle thought that man was not subject to rabies. Pliny the Elder recommended the livers of mad dogs as a cure. Galen advised a compound of crayfish eyes. Sea bathing was thought to exert a curative power. In 1780, in France, a prize was offered to the person who could give the best method for treating rabies. It was won by a surgeon-major who recommended cauterization or the burning of the infected area with redhot irons.

Rabies was one of the dread diseases of the past. People were so terrified of victims that had been bitten by mad dogs or wolves that they frequently strangled or suffocated them. They were afraid of contagion in nursing a case of rabies, since all knew that rabies meant certain death. A law was passed in France in 1810 prohibiting the murdering of people suffering from rabies. 
Pasteur (1822-1895). About 1882, Louis Pasteur, a French scientist, realizing that Jenner and others had successfflly immunized persons by vaccination, decided to apply the same principle to rabies. He had learned several things about the disease: (1) that the virus of rabies was contained in the saliva of mad animals; (2) that it was communicated through bites, and that the period of incubation varied from a few days to several months. He thought that there must be some way of preventing the development of the disease during this long period of incubation.

Pasteur had examined certain microörganisms in the saliva of a child that had died of rabies. He thought these were the causative organisms. When he injected them in animals, however, they failed to produce rabies. Pasteur inoculated rabbits with saliva from rabid dogs. Hydrophobia took months to develop and sometimes did not develop at all. Then he introduced blood from rabid dogs into rabbits and again was unsuccessful. Pasteur suspected the disease was in the nervous system and believed that explained the long period of incubation. He took particles of the brain of an animal that had died of hydrophobia and injected them into a number of animals. They all developed and died of hydrophobia. Evidently, the particles of the brain were more potent or virulent than the saliva in causing rabies. Pasteur was unable to use his usual method of investigating disease. He could not isolate the germ and cultivate it in an artificial medium, because he could not detect the germ.

Next, he suspended in a sterilized vial a fragment of the brain of a rabbit that had died of hydrophobia. As the fragment gradually became dry, its virulence or strength decreased until, at the end of fourteen days, it proved to be harmless when crushed, mixed with pure water, and injected under the skin of some dogs. The next day the dogs were inoculated with brain which had dried for thirteen days. The inoculations were continued, using fragments 
of brain of increasing virulence until the brain of a rabbit that had died the same day was used. It was found that the treated dogs were immune from hydrophobia.

Pasteur invited a commission of scientists to investigate his work. Rabid dogs were permitted to bite healthy dogs that had been treated with Pasteur's inoculations and some that had not been treated. The former did not contract rabies, the latter did. The scientists were enthusiastic over this scientific triumph. Pasteur then showed that the development of rabies in dogs that had been bitten by a rabid dog could be prevented by means of similar inoculations.

In 1885, a little Alsatian boy, Joseph Meister, was brought by his mother into Pasteur's laboratory. He had been horribly bitten by a mad dog. Pasteur, after consultation with other scientists, decided to give him inoculations of brain and spinal cord material that he had prepared. His first inoculation was material that had dried fourteen days. This was followed by further inoculations, of

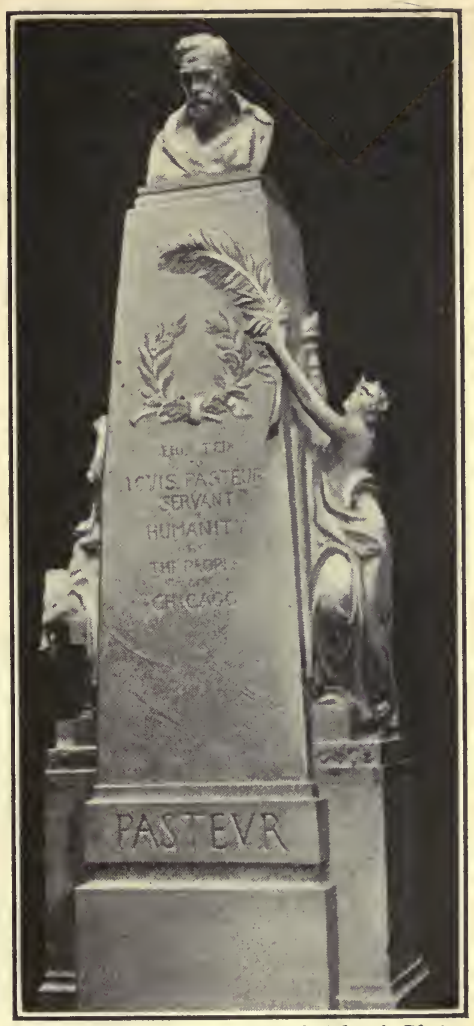

Pacific \& Allantic Photos

The people of Chicago have erected a monument in recognition of the inestimable service of Louis Pasteur to humanity. Millions of people passing the monument give occasional thought to the nobility of character and devoted life of the great scientist. increasing strengths. The treatment lasted ten days and included twelve inoculations. It was successful in preventing rabies and was the first successful treatment for rabies given to a human being. 
Foreign scientists flocked to Paris to learn more about the treatment. Letters came from all over the world for information. Children were brought from far and near for treatment. From America, four small children, who had been bitten by mad dogs, were sent over to Pasteur for treatment. A public subscription was conducted by a New York newspaper in order to supply the funds for their treatments. The inoculations given these children by Pasteur were successful. Nineteen Russians who had been bitten by a mad wolf were brought to Paris by a Russian doctor. The only French word they knew was "Pasteur.". Two weeks had elapsed between the time they received their wounds and their

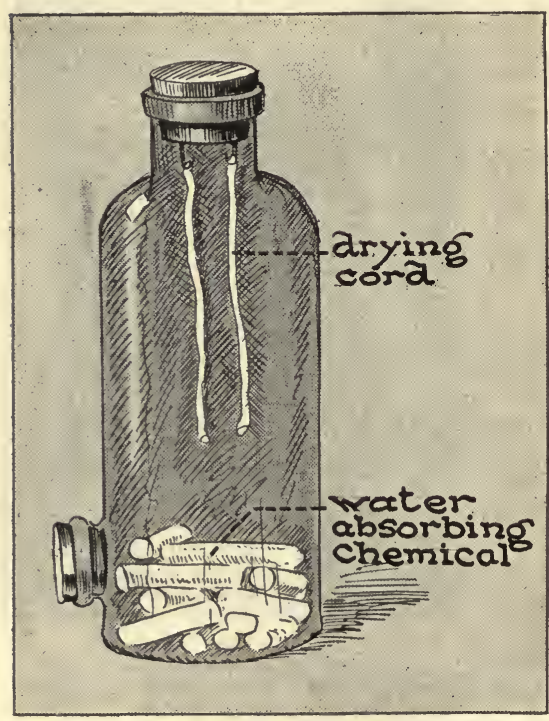

Vaccines for the treatment of hydrophobia are prepared from the spinal cords of animals that have died from the disease. The cords are suspended in a bottle which has a water-absorbing material in the bottom of it. As the cords dry, they become attenuated or weakened in virulence. arrival in Paris. Although they were horribly bitten, all but three of them were saved.

Pasteur was one of the first scientists to develop a method of weakening or attenuating organisms by laboratory procedures for use as a vaccine. To-day, all our large cities have Pasteur Institutes, or similar divisions of the Health Department for giving treatment for rabies.

The nature of rabies. Rabies is an infectious disease of dogs, wolves, cats, and sometimes of horses, cows, rabbits, and other animals. It may be communicated to human beings. The infection is from an organism not yet cultivated or positively identified. 
It is a filterable virus which attacks the central nervous system. This virus can be found in the salivary glands and in the saliva of infected animals.

If some of the nervous tissue of an animal that has died from the disease or been killed during the course of the disease be examined microscopically, characteristic spherical inclusions will be found. These structures are called Negri bodies and the appearance of them in the brain cells is the principal method of determining whether or not a suspected animal is rabid. If they are found in the brain of an animal which has bitten a human being, the Pasteur treatment is immediately prescribed for the person or animal bitten. The relation of these Negri bodies to the causative organism is not yet known. To confirm the diagnosis, a bit of the brain of the dead animal is sometimes injected into another animal. If the disease is rabies, the second animal will become sick and die. Because the rabies organism attacks the central nervous system, the muscles controlled by that system are secondarily affected and, consequently, paralysis is a characteristic of the disease.

The effect of rabies on animals. When a dog becomes sick with rabies, only an expert would suspect what the trouble is. Many cases do not show violent symptoms, although most cases begin with a characteristic change in the animal's disposition. In general, the course of the disease is marked by a change in disposition, irritability and excitement, which is usually followed by depression, paralysis, and death.

Transmission. Rabies is transmitted to man by the bite of an infected animal, because the germs or virus of the disease are in the saliva. The bite breaks the skin and introduces the causative organism. There have been cases in which persons contracted the malady from being scratched by the animals or from allowing the dogs to lick a hand upon which some scratch or wound existed. 
Cure. There is no known cure for rabies once it definitely develops. The only possible help a patient may secure is the preventive treatment which should be given early.

Prevention. One should always be careful in handling sick animals, especially dogs and cats. If a dog, suspected of having rabies, is running loose, it should be penned up for ten days, and if it does not develop rabies in that time it is safe to let it out.

Most departments of health examine and keep under observation all dogs suspected of having rabies. They will also take care of dogs that have been bitten by other animals which are suspected of having the disease. In order to prevent rabies, the health departments of many states require that all dogs be muzzled.

To prevent the development of rabies in a person bitten by a rabid animal, the wound must be washed at once. Under the care of a competent physician, it should be treated with a strong antiseptic or be cauterized. Then the preventive vaccination treatment must be begun at once. This builds up the person's resistance to rabies. Practically no one has ever been harmed by this treatment. In all cases, it is of utmost importance to give the Pasteur treatment immediately.

Large deep bite-wounds are the most dangerous, especially those about the face, head, back, or any part where nerves and lymphatics are abundant. A bite through clothing is usually less dangerous than one on the bare surface.

The eradication of rabies in man depends upon its prevention in domesticated animals. This problem of prevention still exists in certain sections of the world, as the disease is very prevalent among wild animals which transmit it to dogs and other domesticated animals. In 1923, approximately 22,000 persons in the United States applied for and received the Pasteur treatment.

In England, the enforcement of the law requiring the muzzling of dogs and a quarantine on all animals imported from other countries eliminated the disease entirely from 1903 to 1918 . 
It is now possible to give prophylactic or preventive treatment to dogs, which render them immune to rabies. In 1924, approximately one hundred thousand dogs in Japan were immunized, and the number of cases of the disease was reduced to forty-one. Before that time there were approximately 1700 cases of rabies each year among the dogs.

Pasteur's ideals. In closing a chapter that brings in a part of the work of a remarkable man, no more fitting tribute can be paid him than to repeat a part of a speech made to Pasteur on his seventieth birthday. The great theater of the Sorbonne was filled by committees from Denmark, Sweden, and Norway. The members of the French Institute and the Professors of the Faculties were there. Students of

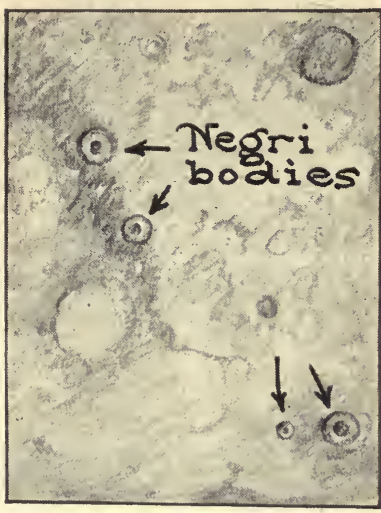

A sketch of a brain smear preparation of a suspected "mad" dog. The presence of nucleated Negri bodies led to a diagnosis - "rabid animal." The boy bitten by this animal was given the Pasteur treatment and did not develop hydrophobia. medicine had crowded into every available place. Lister was there, representing the Royal Societies of London and Edinburgh. Many offerings were tendered Pasteur, many tributes paid, and the last was made by the President of the Students' Association, who said, "You have been very great and very good; you have given a beautiful example to students."

Pasteur's voice, weakened by his emotion, could not have been heard over the large theater. His reply was read by his son:

“... do not let yourselves become tainted by a deprecating and barren skepticism, do not let yourselves be discouraged by the sadness of certain hours which pass over nations. Live in the serene peace of laboratories and libraries. Say to yourselves first: 'What have I done for my instruction?' and, as you gradually advance, 'What have I done for my country?' until the time 
comes when you may have the immense happiness of thinking that you have contributed in some way to the progress and good of humanity. . ..."

\section{Questions AND Suggestions}

1. State some of the ancient ideas on the treatment of rabies.

2. Describe Pasteur's experiments with saliva and blood.

3. Discuss Pasteur's experiments with dried brain and spinal cords.

4. Discuss the first vaccination of animals against rabies.

5. Discuss the first vaccination of a person against rabies.

6. What effect did the discovery of vaccination against rabies have on the world?

7. What stimulus did Pasteur give later scientists by his work on vaccination?

8. Discuss the cause, method of invasion, and symptoms of rabies.

9. Discuss methods of preventing and controlling rabies.

10. What is the importance of vaccination against rabies?

11. Is there anything in Pasteur's speech that indicates why he has been called one of the finest scientists the world has ever produced?

12. Give a report on the life and work of Pasteur.

\section{Supplementary Readings}

Broadhurst, Jean, How We Resist Disease (J. B. Lippincott \& Co.). De Kruif, P. H., The Microbe Hunters (Harcourt, Brace \& Co.). Haggard, H. W., The Science of Health and Disease (Harper and Bros.). Vallery-Radot, René, The Life of Pasteur (Doubleday, Page, and Co.). 


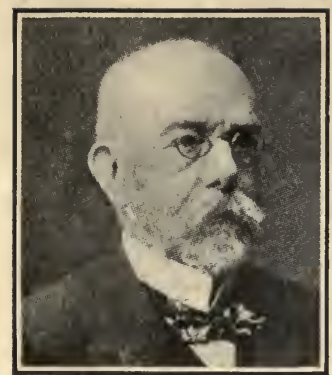

Robert Koch.
CHAPTER XLIII

TUBERCULOSIS

AND ITS

PREVENTION

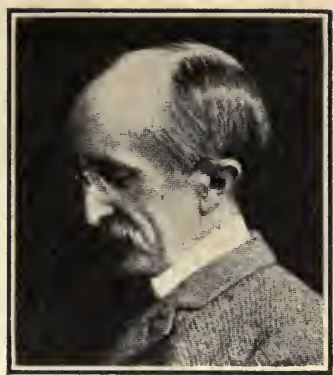

Edward Livingston Trudeau.

How was the germ of tuberculosis discovered? Was the discovery of the tuberculosis germ due to careful scientific investigation? Is tuberculosis a curable disease?

Pasteur presented his germ theory of disease to the world in 1860. At that time many people did not have much faith in his work, but he cleared the way for much of the work done by other scientists of his own day and later. The investigation of tuberculosis would probably have been impossible had it not been for Pasteur's work.

Dr. J. A. Villemin. In the nineteenth century, medicine was not based on scientific research. Pasteur's work on microörganisms was not accepted generally until the latter part of the century. In 1864, Dr. J. A. Villemin, one of the few real scientists, by means of experimental research, brought out the idea that tuberculosis was a disease which reproduced itself and could be reproduced only by itself. In brief, he said it was caused by a specific organism, and could be communicated from one person to another. In order to demonstrate the fact that tuberculosis was communicable, he experimented on animals. He took some sputum of a tubercular patient, spread it on cotton wool which he dried and made into a bed for guinea pigs. The pigs, in time, became tuber- 
cular. People did not accept Villemin's findings. They still clung to the idea of spontaneous generation of this and other diseases. In fact, he was treated as a disturber of medical order and beliefs.

Professor J. Cohnheim, 1839-1884, at Breslau, found that he could give tuberculosis to rabbits by putting a bit of the tubercular patient's diseased lung into the front chamber of a rabbit's eye. Here he could watch the little island of tissue spread and do its deadly work of building tubercles.

Koch discovers the cause of tuberculosis. Robert Koch (18431910) built on Cohnheim's work. As long as animals could be inoculated with the disease, he could experiment with them. At this time, probably, one out of every seven people was dying of tuberculosis. Koch took tubercles from a man who had died of tuberculosis and injected them into the eyes of guinea pigs and rabbits. While he was waiting for them to develop signs of the disease, he examined tissues of people who had died of tuberculosis. No microörganisms showed. He stained the tissues with different dyes, in order to see the germs. (As he worked, he kept dipping his hands into bichloride of mercury, for Lister, the English surgeon, had demonstrated the importance of antiseptics in checking infections.) Finally, Koch smeared some material from tubercles of a tubercular person on a glass slide, dipped it in a certain dye, and mounted it under his microscope. He saw slender rod-shaped organisms very minute in size, about $\frac{1}{15000}$ of an inch long. The last stain had been successful.

In the meantime, the guinea pigs and rabbits, which he had inoculated became sick and died. He examined their bodies, and found tubercles which he stained and examined microscopically. In every case he found the always slender, curved rods. He examined the bodies of many people who had died of tuberculosis and always found tubercles. . Then he injected (inoculated) tubercles into guinea pigs, rabbits, dogs, cats, and many other animals. Invariably all the animals inoculated with the tubercles died of 
tuberculosis. As each animal died, Koch examined its tissues and always found tubercles and the slender rods. Then he examined tissue after tissue of healthy animals and found no tubercles.

He was not yet positive that he had discovered the tubercle bacilli. He decided that he must first cultivate them outside the body of animals and then introduce the cultivated organisms into healthy animals. If the inoculated animals became tubercular, it would indicate the presence of the organisms. He made every kind of broth then known for the cultivation of germs. He kept some of his tubes and bottles'at the temperature of the room, some at the temperature of a man's body, and others at fever temperature. He infected his broths with portions of the infected lungs of guinea pigs. No tubercular organism grew in any of the broths, regardless of surrounding temperatures.

Undaunted, he thought he would try tissue extracts for his media. Possibly tissues had peculiar materials that were essential for the growth of the tubercle bacillus. He obtained serum from blood, mixed it with agar, heated it to make it set, and placed it on slants in test tubes in order to get long, flat surfaces on which to grow the bacilli. He streaked the serum-agar with a bit of the infected lung of a guinea pig that had just died of tuberculosis. Then he placed his tubes in an incubator at the same temperature as that of the guinea pig's body. On the fifteenth day the serum jelly was covered with tiny glistening specks. When magnified with a hand lens, they appeared as dry, tiny scales. He stained and mounted one of the scales under the microscope and found the same bacillus which he had discovered in the lung

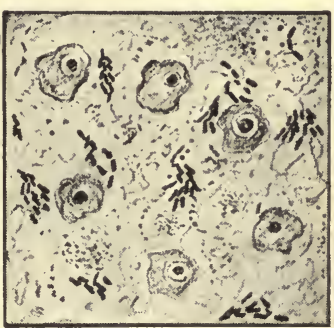

Bacterium triberculosis

The rod-shaped structures, a brilliant red when stained, are the bacilli that cause tuberculosis. of tubercular victims. He had grown tubercle bacilli outside the body of an organism. He then inoculated animals with the bacilli 
grown on his serum-agar; the inoculated animals contracted tuberculosis and died.

He decided to try one more experiment.' He wondered how people contracted tuberculosis, and thought that they had probably inhaled some of the dust particles of which Pasteur had spoken, or possibly were infected by droplets which tubercular people scattered in the air when they coughed. He sprayed bacilli into the air breathed by certain animals. The animals became tubercular and died.

The method of investigating an unknown disease that Koch used in 1882, is still being used to-day. His method is commonly known as Koch's postulates which are: (1) isolate the probable germ from the diseased organism; (2) grow the germs in an artificial media; (3) transfer the germs from the culture to an organism and notice whether they will produce the disease; (4) obtain some of the germs from the second organism and identify them. From Koch's investigation, dates the beginning of the triumph of bacteriology.

Koch reported his findings before the Physiological Society, in Berlin, in March, 1882. The most brilliant men of science in Germany were there. There was no word of criticism against his work; it was too thorough and convincing. The news of his discovery spread rapidly, and the entire world soon learned of his work and his methods of investigating diseases.

Edward Livingston Trudeau. While Koch was investigating the cause of tuberculosis, there was a man named Trudeau living in the United States. He was taking care of a tubercular brother whom he bathed, fed, and even slept with. His doctor warned him not to open the windows as it was bad for the brother's cough. The brother finally died.

Trudeau then studied medicine, became a doctor, but did not practice long, before he realized that he had tuberculosis. Like all people of his day, he thought tuberculosis meant certain death. 
When he realized that he had tuberculosis, he went to end his days in the Adirondack Mountains which he loved. Strangely enough, his health began to improve. 'Two or three doctors, hearing of his improvement, sent other tubercular patients to him and a small sanitarium was thus started in the northern part of New York State. Trudeau heard of Koch's work and went back to New York city to learn the laboratory methods of isolating the tubercle germs. Then, returning to the mountains, he started experimenting on rabbits. $\mathrm{He}$ took three sets of rabbits. He inoculated two of them with tubercle bacilli. One of these sets he placed in a dark camp box that was poorly ventilated, and gave them an insuffi-

\section{TUBERCULOSIS - ALL FORMS \\ Prospective deathrates, 1928 to 1937} Original Registration States and District of Columbia

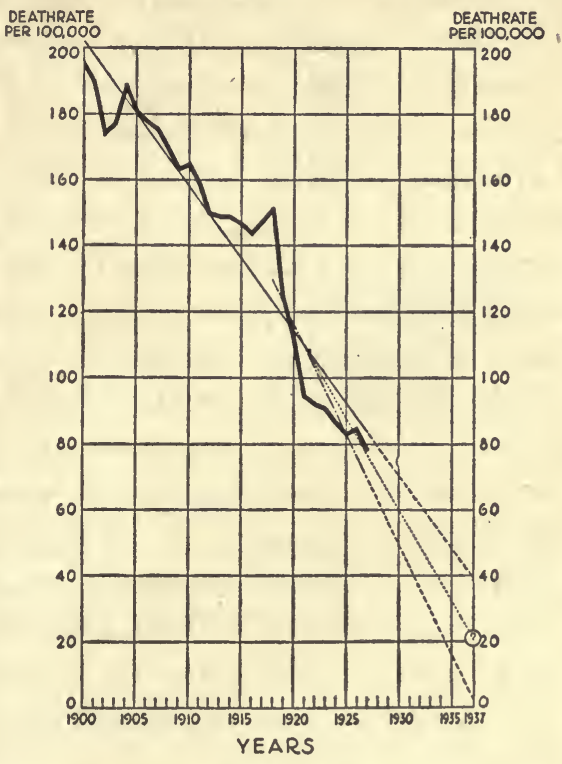

If the trends between 1900 and 1922 are averaged, the expectation of the tuberculosis death rate for 1937 is about 40 per 100,000 . If the decrease of 1926 and 1927 continues, the death rate in 1937 would be 20 per 100,000 . A greater decrease than has yet been shown is necessary to bring the death rate to 0 . (From Health and Wealth by Dublin, Harper \& Bros.)

cient diet. The other that had been inoculated he let roam on an island in the Adirondacks where there was plenty of air, sunlight, and food. The third set, that had not been inoculated, was also put in very unfavorable surroundings. The results were WH. FITZ. AD. BIO. -29 
that the rabbits living in the good environment recovered from the effects of the inoculation. The inoculated rabbits living in poor conditions died within three months from tuberculosis. The rabbits that had not been inoculated but were in the poor environment did not show tuberculosis, although they were not in good physical condition. Repeating similar experiments, he proved that unsanitary conditions, alone, will not cause tuberculosis; the germs must be there. He also showed that unfavorable environments favor the development of tuberculosis and good living conditions help to check the disease.

Trudeau, with the help of another doctor, started his sanitarium at Saranac Lake. He built a number of small cottages and thus started the Cottage Plan of Tuberculosis Sanitariums. He enforced a routine which included plenty of fresh air, rest, good food, and sunlight. This method was revolutionary. Trudeau was the first physician in the United States to teach the importance of fresh air in the treatment of tuberculosis. Since his time numerous sanitariums have been built over the entire world.

Causative organism. A rod-shaped bacillus is the cause of tuberculosis. There are many types of these bacilli. They attack practically all warm-blooded animals. Most of the tuberculosis among children under five years of age is of the bovine type. It is the type that attacks cattle and usually is taken in by the child with milk.

The bacilli of tuberculosis may attack any part of the body but once present the germs are very likely to eventually attack the lungs. They actually feed on and destroy lung tissue. They form little nodules or tubercles filled with a cheesy material and produce poisons or toxins. There is always a toxin called an endotoxin produced within the bacillus and probably an exotoxin, an excretion, is given off. A slight tubercular infection almost always occurs in childhood. The normal resistance of the child's body overcomes the disease by either killing the bacilli or keeping 
them from growing. In the latter case the germs are still alive and will become active again, whenever the resistance is weakened

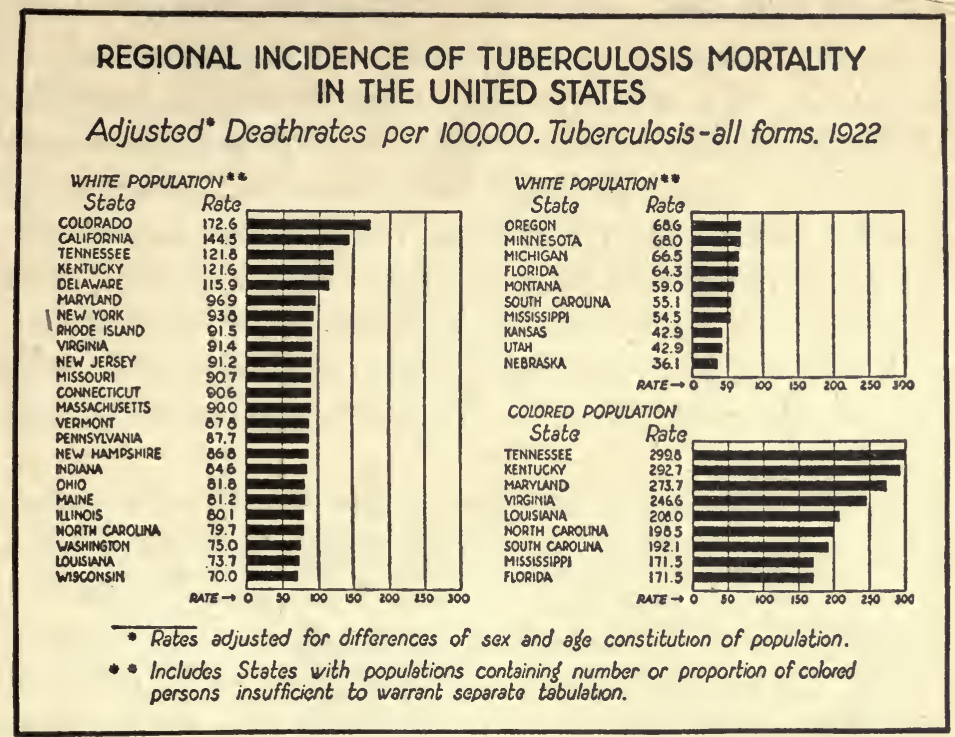

Make a graph from the latest figures of your own and neighboring states and compare it with this chart. Give the possible reasons for a high mortality among the colored population. (From Health and Wealth by Dublin, Harper \& Bros.)

by infection, malnutrition, overwork, and fatigue. Data concerning the prevalence of tuberculosis in childhood and the subsequent recovery of the children from it is known, because investigation proves that the lungs in practically every dead body examined and the X-rays of living lungs show old scar tissue. The scar tissue is characteristic of tuberculosis attacks. Bacilli that get into the blood are frequently carried to a lymph gland, where white corpuscles in great numbers attack and destroy them. The body, meanwhile, builds a hard wall of lime salts around the infected gland, so that the infection is thus removed from the circulation. Sometimes infected glands are cut out to prevent the spread of the germs. Tuberculosis is not hereditary, but susceptibility to 
the disease is probably inherited. People who know there is a history of tuberculosis in the family should be especially careful to live very hygienically. When this is done, they are not likely to contract the disease. Poor environment is a predisposing cause of tuberculosis. If a mother has contracted the disease, her child is likely to live in the same environment and will probably contract the disease. For the good of the child, it should be taken from its parents in such cases, so that it can build up a strong resistance.

Symptoms of tuberculosis. The symptoms are several. Fever, especially in the afternoon, even in earlier stages before coughing has started, habitual coughing and spitting, poor appetite, faulty digestion, and steady loss of weight are usually indicative of an infection.

Diagnosis. Diagnosis consists of a microscopic examination of the sputum for the bacilli. A careful examination of the general physical condition including an X-ray examination of the lungs is made. The X-ray photograph will show any scar tissue. A tuberculin test is sometimes made. This is given by putting toxins, squeezed from tuberculosis germs, under the skin of the suspected patient. If the disease is present in the patient, there will be an increase in fever and pulse, an increase of inflammation of the affected part, and a redness at the point of infection. A negative test is conclusive proof of the absence of the disease. A positive test may indicate a past as well as a present infection. Since a positive reaction may be obtained from nearly every adult, the test is chiefly valuable. for young children.

The tuberculin test for cattle has been used for years. According to laws in some states, state inspectors test the dairy cows. When the test shows the presence of the disease, the cow is killed and the farmer is partially reimbursed for the loss. All states do not have this law. Some states have such laws, but let the townships decide whether or not they will be enforced.

Spread of tuberculosis. Tuberculosis is spread in innumerable 
ways. Two modes of entrance of the germs into the body are through inhalation to the lungs and through ingestion to the alimentary tract, and thence to the lungs. The disease may be actually spread from person to person by: (1) spray thrown out in coughing and sneezing, a droplet method of infection ; (2) contaminated objects, such as drinking cups and pencils, handled or used by a tubercular person; (3) personal contact, such as kissing and handshaking, with an infected person; (4) food infected by being coughed on or handled by a tubercular person; (5) dust from street or car, containing dried sputum of a tubercular patient; (6) houseflies carrying tubercular germs on their bodies may crawl over and infect food; (7) milk and butter of tuber-

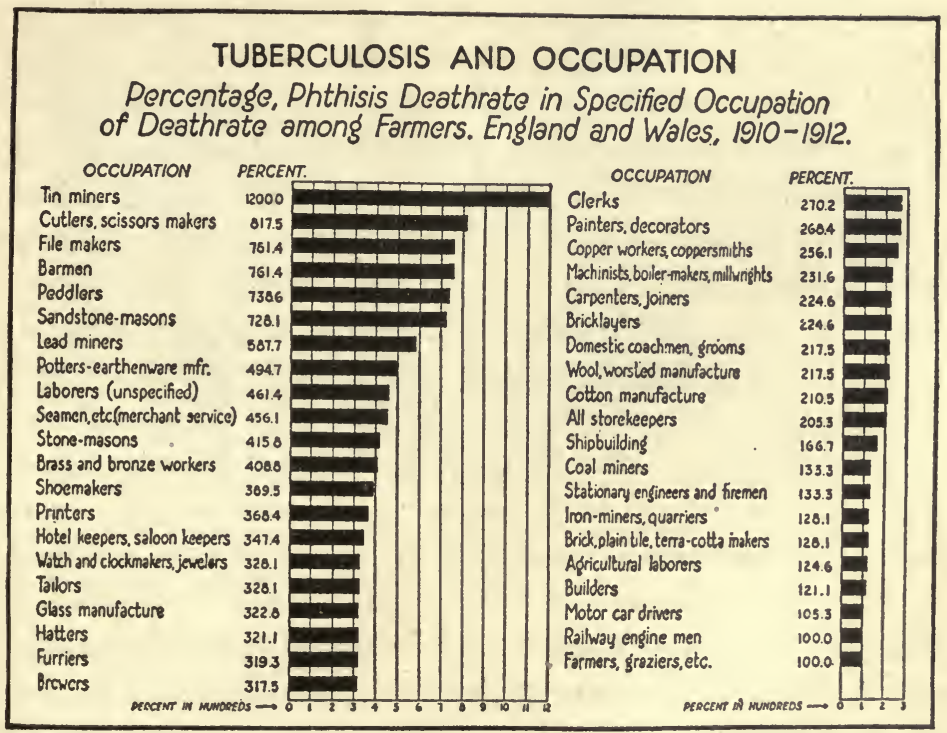

Compile figures for your own country, state, or city and compare them with this table. (From Health and Wealth by Dublin, Harper \& Bros.)

cular cows. (The tuberculin test shows that 15 per cent of the cows of to-day are infected with tuberculosis.) 
Treatment. Since the body itself must fight this disease, it should be given the best possible environment under which to work. Giving the patient the most healthful conditions is the most we can do for him. Fresh air, sunlight, plenty of sleep, freedom from worry or work, and an abundance of easily digested food such as milk and eggs are the main factors in the treatment. Outdoor treatment is usually best if the climate is mild. It is best and most always necessary to have expert treatment at a sanitarium under the supervision of a doctor, for at least six months.

Prevention. . There are a number of measures to be observed by individuals in order to prevent infection: keep the resistance of the body high by proper living habits; have a general physical examination once a year; teach or compel all tubercular persons to cover their mouths with handkerchiefs when coughing; use paper cups and paper towels in public places and use only sanitary drinking fountains; keep the dust down, by using a vacuum cleaner and damp cloth in the home, and by sprinkling and flushing the streets ; keep flies out of the house by screening the doors and windows, and off the food by covering it; report all cases of tuberculosis not under proper medical care to the Board of Health. The communities can also help to control the spread of tuberculosis. They should require the inspection and pasteurization of all milk. Only raw milk from tuberculin-tested cows should be sold. They can also aid by enforcing the existing laws against spitting in public places; requiring Board of Health certificates from the persons who handle foods; providing for healthful living conditions in tenement houses; and providing for more hospitals by fostering the sale of Christmas seals. Probably one of the most important ways of controlling the disease, is to educate the people against the dangers of tuberculosis and the best methods of preventing it.

The tuberculin test was first devised by Koch in 1890 . People kept insisting that he find a cure for the disease, since he already 
knew the cause of it. He was working on a tuberculin inoculation which could be introduced into people as a means of working up a

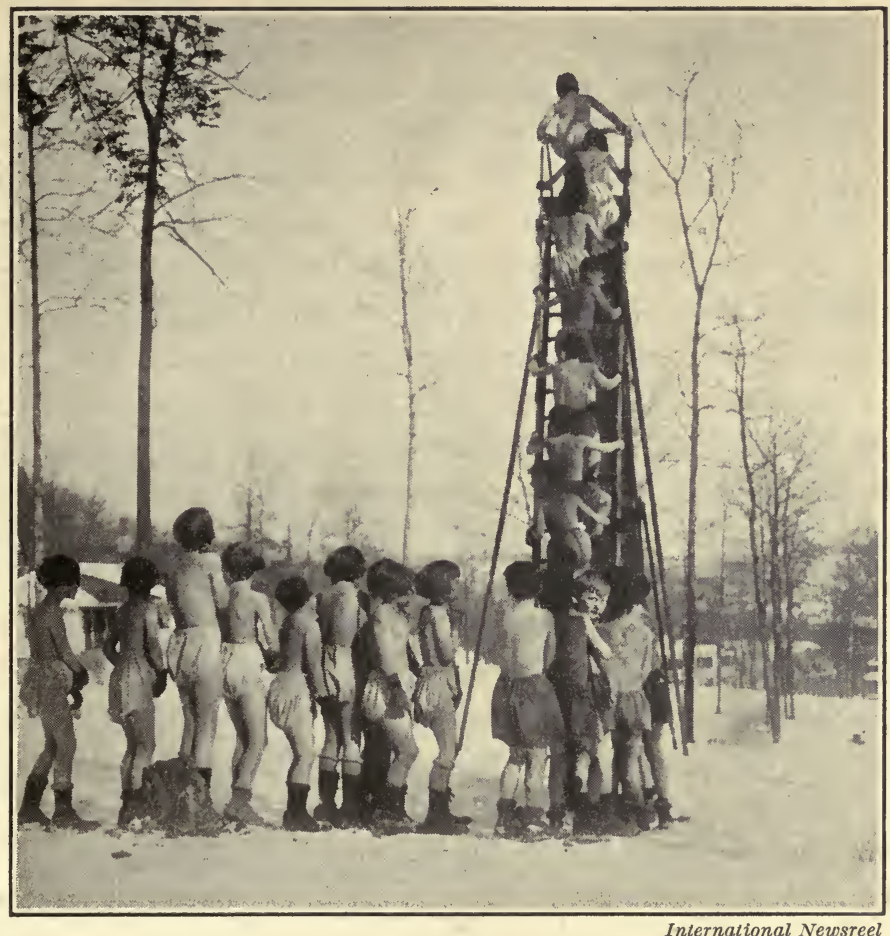

Fresh air is invaluable in treating tuberculosis. Some sanitariums require patients to dress as lightly as possible, even in winter. The exposure of large areas of the body to air and sunlight is helpful in the treatment of tuberculosis.

resistance or an immunity. He was literally forced into publishing his work before he had tested it sufficiently. Doctors began to inoculate people with the tuberculin test devised by Koch. In many cases the results were fatal. Tuberculin inoculation then fell into disrepute.

At present, a group of doctors is working on a tuberculin vaccine at the Pasteur Institute in Paris. The vaccine consists of 
greatly weakened germ material. It is hoped that this will stimulate the body to make protective materials which will stay in the blood. If the vaccinated person then comes in contact with the active disease, he is already fortified and protected and should not contract the disease. In France, the average mortality among infants from tuberculosis is about 20 per cent. In a recent scientific experiment 969 infants who were born of tubercular mothers or were in direct contact with the disease were inoculated. Among the vaccinated infants, for the two years following vaccination, the mortality for this disease was about one per cent; the mortality was nil after two years. The resistance lasted more than four years. Hence the infants were immunized or protected for the period when tubercular infection is most dangerous.

Prevalence and economic importance. It is estimated that tuberculosis causes one tenth of all the deaths in civilized countries; until recent years it was the principal cause of all deaths. There are one million, five hundred thousand cases of active tuberculosis in the United States; one hundred and fifty thousand deaths occur every year. The mortality, therefore, is approximately ten per cent. There are probably twice as many deaths every year from the tubercle bacilli as there were among our military forces during the World War.

The disease is estimated to cost the United States $\$ 225,000,000$ a year. The mortality was reduced from 224 for every 100,000 people in 1911 to 114.2 in 1922 , a reduction of 49.2 per cent. In 1928 the deaths from tuberculosis were the lowest on record.

\section{Questions And Suggestions}

1. What was the contribution of Dr. Villemin to the investigation of tuberculosis?

2. Discuss the experiments of Koch.

3. Name Koch's postulates.

4. Look up and give a report on the life and work of Koch. 
5. What was 'Trudeau's contribution to the investigation of tuberculosis?

6. Give a report on the life of Edward Livingston Trudeau.

7. Discuss the cause, method of entering the body, part of organism attacked, and effect on the body of the tubercle bacilli.

8. Discuss the diagnosis of tuberculosis in people and in cattle.

9. Name all the ways by which tuberculosis may be spread.

10. What is the treatment for tuberculosis?

11. What precautions should be observed in order to eradicate tuberculosis.

12. What is the status of tuberculin vaccinations at present?

13. Is tuberculosis increasing or decreasing? Is it sufficiently under control?

\section{SupPlementary Readings}

Broadhurst, J., How We Resist Disease (J. B. Lippincott \& Co.). De Kruif, P., Microbe Hunters (Harcourt, Brace \& Co.).

Greaves, J. E. \& E. O., Elementary Bacteriology (W. B. Saunders Co.). Haggard, H. W., The Science of Health and Disease (Harper \& Bros.). Zinsser, Hans, A Textbook of Bacteriology (D. Appleton \& Co.). 


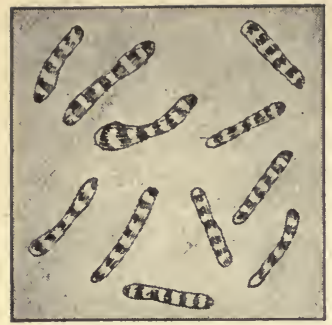

Diphtheria bacilli.
CHAPTER XLIV

DIPHTHERIA, SCARLET FEVER, AND TETANUS

Can diphtheria be completely eradicated? Is there any protection against scarlet fever? What is the relation of tetanus to wounds?

Three diseases that are somewhat similar in their methods of attacking the body are diphtheria, scarlet fever, and tetanus.

History of diphtheria. Fairly accurate descriptions of diphtheria have been given by certain ancient Greeks. There is evidence that the disease has been known for many centuries. In 1826, a scientist in France was the first to look upon diphtheria as a specific and infectious disease that was frequently spread by the use of a common drinking cup. He said that croup was a type of diphtheria, and he differentiated the disease from the sore throat of scarlet fever.

Isolation of organism. Klebs, in Germany, in 1883, discovered bacilli, striped with bars or bands, in the throat of children sick with diphtheria. In 1884, some banded bacilli were isolated and stained with methylene blue by Emil Loeffler, who thought that they could not be the causative organisms of diphtheria. $\mathrm{He}$ was confused by the fact that some perfectly well children had these bacilli in their throats and some children who were ill with what seemed to be diphtheria did not have the germs. Loeffler grew the banded bacilli on broth and injected them under the skin of guinea pigs. The pigs died, although the bacilli did not spread, but stayed at the point of entrance below the skin. 
Isolation of toxin. In 1888, Roux and Yersin showed that the diphtheria bacillus produces a toxin. Roux had realized that the germs might secrete a deadly toxin which he attempted-to separate from the germs. He grew the bacilli on broth and used a porcelain filter that held back the germs, but permitted the soluble material to pass through. The filtered liquid was then inoculated into guinea pigs which developed the symptoms of diphtheria. Thus Roux showed that the diphtheria bacilli kill through a toxin rather than by the spread of the germs. Earler investigators of this disease were unsuccessful because they did not realize that the germs stayed in the throat and sent out toxins (exotoxins) which affected the body. The healthy people who had diphtheria germs were probably diphtheria carriers and the sick people who did not have the bacilli in their throats probably did not have diphtheria.

The discovery of antitoxin. The toxic effects of the diphtheria bacillus led Von Behring to believe that blood contained certain chemical substances which would kill invading microbes without injuring the person or animal. After a series of experiments, he finally inoculated diphtheria toxins under the skin of guinea pigs that had recovered from diphtheria. They were not affected by the toxins, but when he inoculated toxins under the skin of guinea pigs that had not had diphtheria, they became ill.

Von Behring obtained blood from guinea pigs that had recovered from diphtheria, separated the serum, and mixed it with diphtheria toxin. He injected this mixture of serum and toxin into healthy guinea pigs and they were not injured by

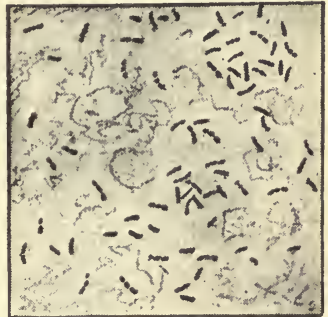

Bacterium aiphtheriae

The beaded appearance, due to the staining, of these germs may be noted in any carefully prepared culture.

it. But, when he mixed blood serum from guinea pigs that had never had diphtheria with diphtheria toxin, and inoculated the 
mixture into healthy guinea pigs, they died. From these experiments, he concluded that guinea pigs which had contracted and recovered from diphtheria had something in the serum that safeguarded them from subsequent attacks of the disease. He called this protective material antitoxin. His experiments showed that the guinea pig's body made protective antitoxins to combat the toxins of bacilli. These antitoxins remained in the blood of animals that recovered from diphtheria.

He then decided to prepare an immune serum which would protect babies. He injected small amounts of diphtheria toxins into sheep and continued to give them doses of increasing strength. Ultimately, the sheep were immune to the most powerful diphtheria bacilli. Then he injected serum from the immune sheep into guinea pigs and later he inoculated diphtheria bacilli into these pigs. They were not affected by the bacilli. Evidently, the antitoxins made by the sheep's body were effective in protecting the guinea pigs against diphtheria bacilli. One serious difficulty was that this immunity did not last. In 1891, Von Behring discussed the use of serum on babies, and in 1893 it was first used in the Children's Hospital in Berlin with some degree of success.

The discovery of active immunity. The fact that sheep, goats, and horses used in the production of antitoxin seemed to develop a permanent immunity to the diphtheria toxin gave the basis for the next step in this work. Persons who had once been sick with diphtheria seemed to be free from further attacks. This gave support to the idea that the presence of toxins stimulates the human body to produce its own antitoxins. The action of the body in producing its own protective material is known as active immunization, and the resulting condition is active immunity.

Investigators mixed antitoxin with toxin and found that the toxin was rendered harmless. Von Behring was the first to attempt to give a permanent immunity to children by means of 
this mixture, but his work was interrupted by the World War. Later, Dr. William H. Park of the New York City Laboratories, inoculated children with the antitoxin and toxin, known as toxinantitoxin, and was successful in having them develop an active immunity which was permanent.

A test for immunity. In 1913, Dr. Bela Schick devised the Schick test of susceptibility. When small quantities of toxin are put under the skin of children, the reaction will indicate whether the child is naturally immune or not. If naturally immune, the toxin will be neutralized by antitoxins in the blood and no effect is produced. If not immune, a slight local irritation results in the form of a red spot which appears and disappears within a definite time period. It indicates that there are not sufficient antitoxins in the blood to neutralize the small amount of toxin injected.

Cause of diphtheria. Diphtheria (sometimes called "membranous croup ") is justly regarded as one of the most dreaded of the diseases of childhood. It is a disease of temperate climates, occurring most frequently in the colder months. In cities, the disease is always more or less prevalent; in rural communities, it is more likely to occur in epidemics.

Deaths from diphtheria occur chiefly among children less than five years old. There is a definite increase in susceptibility to the disease during the first two years of life, and a gradual development of immunity thereafter, especially if the individual lives in a well-

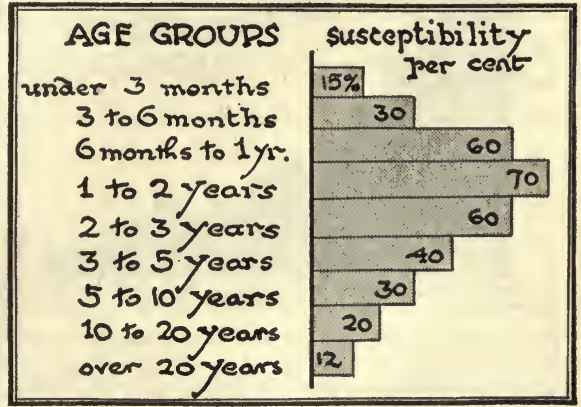

Am. Ass. for Med. Prog.

Note that the ages of greatest susceptibility to diphtheria is among the pre-school children group. This is where the greatest prevention work should be done. populated district and is more or less exposed to the disease. 
Diphtheria is caused by the growth of the diphtheria bacillus, usually in the throat, nose, or larynx. This germ is a slender, slightly curved, club-shaped rod, which does not form spores, and which, when stained, shows characteristic staining particles. These particles make the stained organism easily recognized.

Effect on the body. The diphtheria bacilli grow in the affected part (throat or trachea usually) and first cause inflammation and swelling, and later form a grayish membrane. The bacilli multiply in the membrane and at the same time throw off virulent toxins which will cause death when absorbed by the body in sufficient quantities. If the membrane grows down sufficiently into the trachea, death may occur from suffocation. The action of the toxin on the heart is particularly severe and sometimes brings about heart defects, even after the patient has become convalescent.

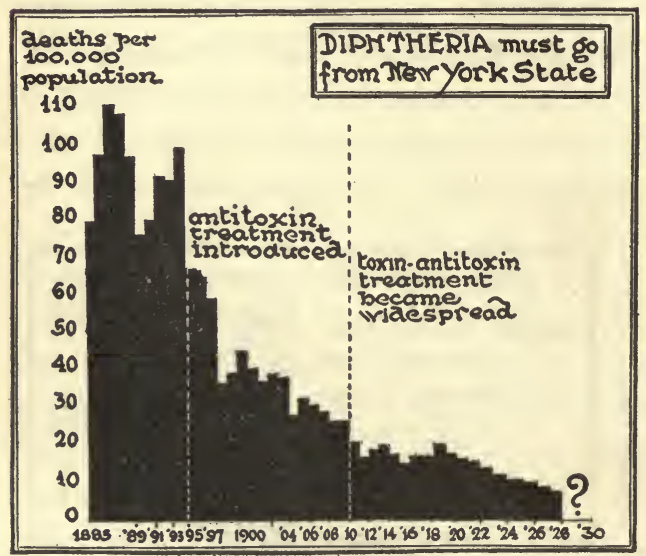

Diphtheria is a disease that can be completely controlled through scientific measures. It has been estimated that the cost of treating one case of diphtheria will protect two hundred children from the disease. Note the effect of antitoxin and toxin-antitoxin on the death rate in New York State.

How diphtheria is carried. Each new case of diphtheria is derived from a previous case of diphtheria or from a diphtheria carrier. The disease may be spread from infected to well persons by direct contact, as by kissing or by mouth spray given off in sneezing or coughing. The germ-laden droplets of such mouth sprays may enter the mouths of others or be breathed in with the air, or they may be carried to the mouth from the hands in eating. Indirectly, the bacilli may be trans- 
mitted through the agency of various objects such as pencils, apples, candy, eating utensils, drinking cups, or the like, which have been handled or used by infected persons. Diphtheria carriers are seemingly well individuals who harbor the bacilli in their bodies. Persons who have been in contact with those suffering from diphtheria are especially likely to be carriers, yet a certain percentage of the population of any community may be found harboring the diphtheria germs, although unaware of having been

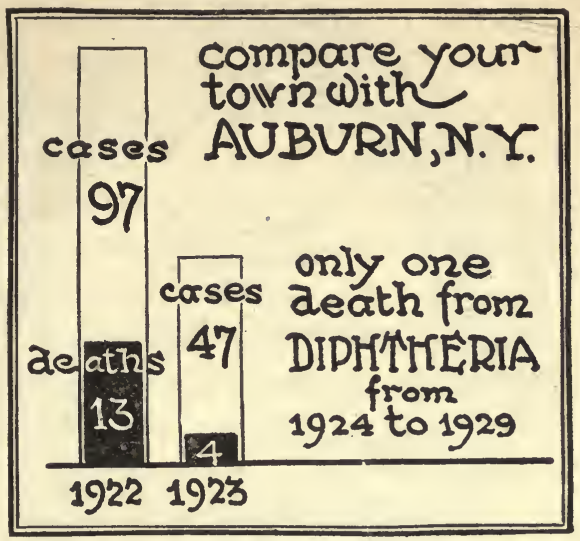

The record of Auburn, New York, is one that can be attained in all towns. Diphtheria can be eradicated if proper prophylactic measures are taken. exposed to any case of diphtheria. The germ of this disease grows freely in milk. As this food undergoes so much handling during production, the germs of diphtheria often have an opportunity to get into milk unless great care is taken.

The diphtheria germ is easily killed by ordinary disinfectant solutions and is rather easily killed by drying. When it is contained in pieces of membrane, it may live for some time. Heat quickly destroys the germ, but temperature as low as freezing is not fatal to it.

Treatment. The communicability of diphtheria renders imperative the strict isolation of patients. Unnecessary furniture should be removed from the room, and that which is left should be of a kind easily cleaned. Separate linen and utensils of every kind should be provided for the exclusive use of the patient. Such materials should be boiled, or, better, treated with a powerful germicide after use. The attendant nurse and the physician 
should be the only persons in contact with the patient. After handling the patient, which should be done as infrequently as

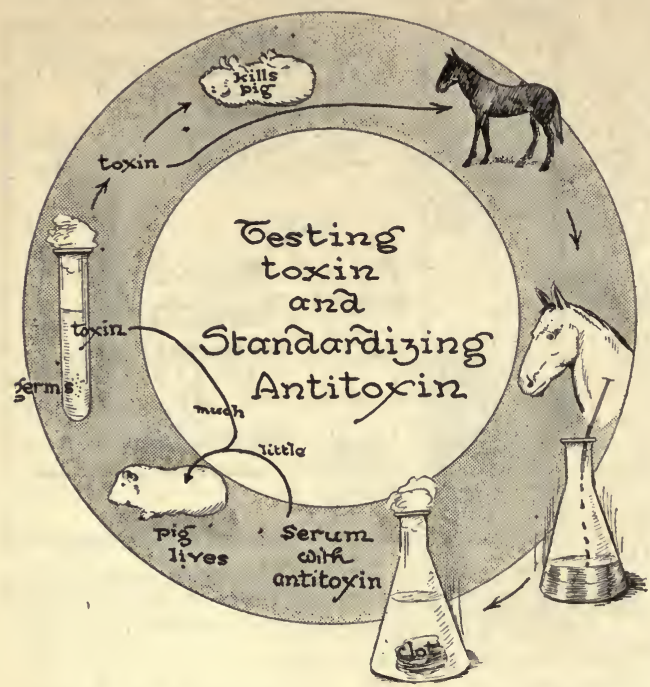

The strength of the toxin is tested by injecting a very small volume into a 250-gm. guinea pig. If the pig dies within four days, it is toxic enough to inject into a horse to produce antitoxin. After a given time, blood is drawn from the horse, the serum with its antitoxin is separated from the blood and again tested. This time, a little serum is mixed with some toxin and the mixture injected into a guinea pig. If the pig lives, the serum is shown to contain antitoxin and will be effective. Look up the exact amounts of material and time involved in this standardization process. possible, the hands of the attendants should be immediately cleansed in a germicidal solution and then washed with soap and water.

Diphtheria antitoxin is usually administered early to help the patient get control of the disease. This antitoxin produces immunity with little or no work on the part of the cells of the patient's body. Such immunity is known as passive immunity. When the antitoxin neutralizes the toxins of the invading germs, the patient's body develops its own antitoxins. This results in an active, permanent immunity. The administering of a sufficient quantity of antitoxin is the primary remedy for the cure of diphtheria. When a physician is not called early enough, the case may advance so far that the administration of antitoxin is valueless. Too much toxin has then been produced by the invading germs for the antitoxin to neutralize. If other members of the family are in contact with the patient and have not been immunized by the toxin-antitox: $n$ 
mixture, it is always advisable to give them an inoculation of antitoxin, which will produce an inmediate immunity for a short time.

The application of the Schick test. By means of the Schick tests it is possible to determine which individuals possess immunity to diphtheria and which individuals are susceptible, that is, are likely to contract diphtheria if exposed to the germs. If a child is found to be susceptible, he is rendered immune by injections of toxin-antitoxin. This stimulates the body to produce its own antitoxins and thus establish an active immunity.

The Schick test and the subsequent inoculations are invaluable in checking diphtheria. The reason for the presence of diphtheria to-day is probably because the inoculations are given to school children instead of children of pre-school age. The susceptibility to diphtheria is very low at birth, but it increases gradually until the individual is two or three years old, and then it starts to decrease. Probably not more than 12 per cent of adults are susceptible to the disease. Since the Schick test indicates the individuals who are not immune, preventive treatment, in the form of toxin-antitoxin, may be given to them.

Diagnosis. The correct diagnosis of diphtheria plays a very important part in its control. Not only does the safety of the community depend on the detection and isolation of cases of diphtheria, but the early recognition of the disease diminishes the mortality because treatment is also earlier. The only dependable means of diagnosis is the microscopic examination of cultures obtained from the throat and nose.

Prevention. Diphtheria has been responsible for the deaths of so many children that health authorities are trying to prevent the disease by completely eradicating it. If all school children should receive the Schick test and be immunized by the toxin-antitoxin method, very few diphtheria cases would be found. This can only be made possible through an educational campaign, by means of which the people will understand the danger and char-

WH. FITZ. AD. BIO. -30 
acteristics of the disease, and the best methods of controlling it. If a physician is consulted as soon as suspicious symptoms appear, he can administer antitoxin immediately and probably prevent a serious case of the disease. Children should be taught to keep pencils and all articles handled by other children out of their mouths. Since diphtheria is a droplet infection, the same rules for prevention apply here as in other diseases spread by this means. Patients who are convalescing from diphtheria should be kept away from well persons until all danger of spreading the infection is eliminated.

\section{Scarlet Fever}

Scarlet fever is a disease similar to diphtheria, in that it works through toxins. The causative organism has not been definitely isolated. A chain form of spherical bacteria called streptococci is always found in the nose and throat of scarlet fever patients, but there is considerable doubt as to whether this is really the causative organism. Seventy per cent of the deaths from scarlet fever are among children under ten years of age.

A test, similar to the Schick test, has recently been prepared to determine the presence or absence of scarlet fever immunity. It was devised by two doctors, G. F. and G. H. Dick, and is called the Dick test. It consists of putting small quantities of toxin prepared from the streptococcus bacillus under the skin of the child to be tested. There is a reaction similar to that in the Schick test.

If a child is not immune, subsequent doses of toxin are inoculated. It is not necessary to neutralize the toxin with antitoxin as in the case of diphtheria, because the scarlet fever toxins are not as powerful as the diphtheria toxins. This inoculation creates an active immunity in the child by stimulating its body to make its own antitoxins. The Dick test and its subsequent inoculations are not used to the extent of the Schick test and inoc- 
ulations, but its use seems to be growing constantly. There is also an antitoxin for scarlet fever. It is prepared from the horse's blood in a way similar to the preparation of diphtheria antitoxin. It has been used with considerable success to produce an immediate immunity in case the child is in the initial stages of scarlet fever.

The method of transmission of scarlet fever is through droplet infection similar to that of diphtheria.

\section{Tetanus (LockJaw)}

Tetanus, a disease that develops from the germs entering a deep injury or wound, is due to the action of the toxins of a particular microörganism. These toxins affect the brain and spinal cord. No noticeable effect is produced in the wound through which the germ enters. Fortunately, tetanus is rare in this country, but when it does occur the death rate is very high. The extensive prevalence of tetanus among the soldiers wounded in the late World War was ascribed to the contamination of their wounds with soil from a highly cultivated territory which had been regularly and frequently fertilized with manure. This soil contained the germs of the disease.

Cause. This disease is caused by the tetanus bacillus, a rodshaped microörganism which grows in long, slender threads. These threads break up into shorter motile rods surrounded by flagella. These bacilli eventually be-

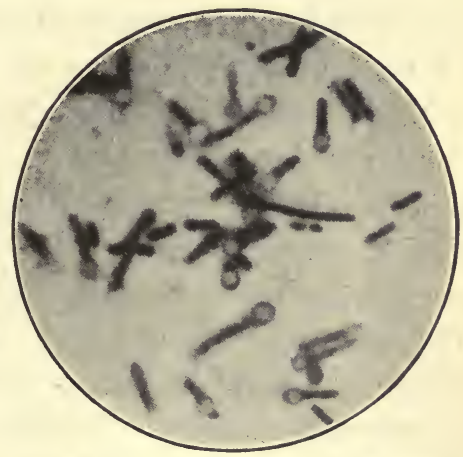

Certain bacteria roll themselves up inside a thickened wall to form a spore. In the photomicrograph of the tetanus organisms the spore appears at one end of the bacillus. come non-motile, lose their flagella, and each forms a spore at one end. This gives to them their characteristic drumstick appear- 
ance. Under favorable conditions these spores remain virulent for years. The tetanus bacillus was first discovered by Nicolaier in 1884 and cultivated by Kitasato in 1889 . Shortly after this, Von Behring succeeded in producing an effective tetanus antitoxin.

Occurrence. Tetanus bacilli are found in damp soil and in dust, especially around stables, in manure, and in dust of the house and street. They are so plentiful in the intestinal contents of horses and cows, whose wastes are commonly employed to fertilize gardens, that tetanus is sometimes regarded as a disease contracted indirectly from these animals. Other herbivorous animals also harbor the germ. The bacilli are likely to be found on rusty nails or implements which have been in contact with the ground, on dirty splinters, and on gunshot.

Tetanus bacilli are anaërobic (cannot grow when exposed to the air) ; therefore, deep wounds are most favorable for their development. They are unusual in that they do not grow on healthy living tissues, but on cells which have been torn and killed. Badly lacerated wounds present a more favorable surface for the growth of tetanus than do those made by very keen clean instruments.

How tetanus enters the body. The bacilli gain entrance to the body through wounds varying in size from a needle prick to an operation wound. Being anaërobic, the organisms infect deep lacerated wounds such as those made on the hand by the accidental explosion of a toy pistol or wounds on the feet made by the deep puncture of a rusty nail. Rusty nails themselves never produce lockjaw, but the bacteria are frequently held in the rough spots of the rust and consequently enter the body if the nail happens to puncture the flesh. Occasionally, the disease occurs without any evident wound. In cases like this, the bacteria have made an entrance through some unnoticed abrasion of the skin or the mucous membrane.

Tetanus has been observed not only in man, but in domesticated animals such as the horse, sheep, dog, cow, and pig. 
The nature of the disease. The effects of the disease are due to the action of toxins produced by the bacilli upon the central nervous system. The bacilli themselves apparently do not move from the deep seated area of entrance. The symptoms of tetanus are usually noticeable any time from two to nine days after a wound has been received. The bacillus which entered the wound as a spore may require some time to become active again.

Treatment. When the organisms have once started to produce their toxins, hope of controlling the disease is only slight. The toxins, even though present in the minutest amount, are so very poisonous to certain parts of the brain and spinal cord that all efforts to neutralize or counteract the activity are usually of no avail.

Whatever treatment is given must be early. Wounds likely to be contaminated with tetanus, as those into which soil may have entered, or gunshot wounds, should be opened and washed with a strong antiseptic. If the danger of infection is considerable, the wound should be cauterized. In addition to this, a dose of tetanus antitoxin (antitetanic serum) ought to be administered. When precautions have not been taken and "lockjaw" sets in, the serum injected into the spinal canal sometimes brings about the desired result.

Tetanus antitoxin. Certain State Departments of Health prepare antitoxin to be used both in the prevention and in the treatment of the disease. Tetanus bacilli are grown in broth, away from the air. The resulting liquid, loaded with tetanus toxin, is filtered gradually and injected at intervals, in increasing amounts, into the veins of a horse. Later, a large amount of blood is drawn from the animal and the serum is separated. This serum contains antitoxin which has been produced in the horse to neutralize the introduced toxin. The serum is known as the antitetanic serum. The antitoxin has a high preventative but a low curative value. Its production is in many ways similar to that of diphtheria antitoxin. 
Prevention. Care should be taken to avoid cuts and wounds of all kinds, especially from objects soiled with manure or fertilized soil. The restriction of the use of fireworks during the past few years has very markedly reduced the number of cases of tetanus occurring over the country at large. All gunshot and "Fourth of July" wounds, any extensive or deep wounds, and every form of punctured wounds should receive care from a physician. Where such wounds have had dirt driven into them, the desirability of an injection of tetanus antitoxin is very great. The antitoxin must be given early as it is preventative and not a cure of tetanus. Wounds suspected of containing tetanus organisms should be opened and thoroughly cleaned. Gauze bandages, which are porous, should be used, never air-tight bandages.

\section{Questions AND Suggestions}

1. Discuss the discovery of the causative organism of diphtheria.

2. Discuss the work of Emile Roux.

3. Review and report on the work leading to the discovery of antitoxin.

4. Contrast active and passive immunity.

5. Discuss the Schick test and its importance.

6. How do diphtheria bacilli gain entrance to the body?

7. How do diphtheria bacilli attack the body?

8. What educational propaganda for parents is still necessary before diphtheria can be eradicated?

9. Contrast and compare scarlet fever with diphtheria in cause, prevention, and treatment.

10. Explain why the prevention of a disease is more to be desired than the cure.

11. What is the similarity between tetanus and diphtheria?

12. Where are the tetanus bacilli found?

13. What is the relation of tetanus to different types of wounds?

14. Is there any truth in the belief that one will surely get tetanus if he cuts himself between the thumb and first finger?

15. Why are tetanus organisms found in dirty places more commonly than diphtheria or scarlet fever organisms?

16. What has caused the reduction in the number of deaths from tetanus? 
17. How should a wound be treated if there is any possibility of tetanus infection?

18. Look up and report on the life and work of Loeffler and of Von Behring.

19. Look up the health bulletin of your village, city, or state and plot a curve of the number of cases of tetanus reported annually for the last ten years. Were any of the cases fatal?

\section{Supplementary Readings}

Broadhurst, J., How We Resist Disease (J. B. Lippincott Co.). De Kruif, P. H., Microbe Hunters (Harcourt, Brace \& Co.). Greaves, J. E. \& E. O., Elementary Bacteriology (W. B. Saunders Co.). Rosenau, M. J., Preventive Medicine and Hygiene (D. Appleton \& Co.). 


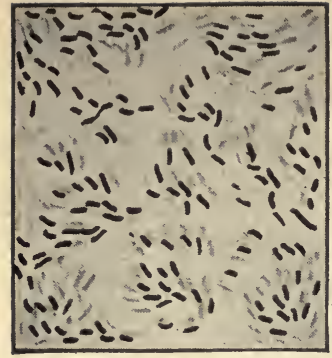

Bacterium typhosum

\section{CHAPTER XIV}

\section{TYPHOID FEVER}

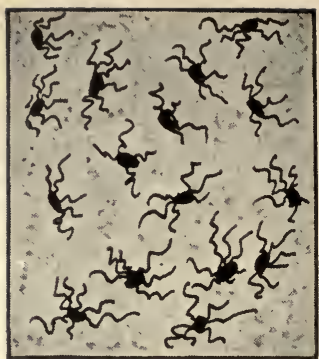

Some have flagella

What is the danger of typhoid infection in the United States? What are some of the measures of preventing typhoid infection? Why are oysters a greater source of contamination than milk, in some cities?

Prevalence of typhoid fever. During the Spanish-American War typhoid spread rapidly through the embarkation camp in Florida. More men died of fever than were killed in battle. During the South African War, the British army lost 7582 men from wounds and 8225 from typhoid. To-day, all soldiers in our army are required to be vaccinated against typhoid fever; and typhoid is probably rarer in military camps than in the most healthful cities and towns. Formerly, the death rate of typhoid in the United States was forty per 100,000. In the last few years this number has been reduced to four per 100,000.

Since the World War, the death rate from typhoid has been reduced most among men between 29 and 45 years of age. This is largely due to the education and practice in disease prevention by vaccination given to the millions of men who were in the army.

Cause. Typhoid is due to a small, motile, rod-shaped bacterium, the typhoid bacillus. It is short, probably about one half the length of the diphtheria bacillus.

Nature and symptoms of typhoid fever. The disease is located primarily in the lining of the intestine. The germs then pene- 
trate the lining and enter the blood stream. The increased activity of tissue cells in combating the germs and their toxins causes fever and the whole body suffers. Convalescence sometimes requires many weeks. Mortality from typhoid fever is about 10 per cent. From two to four per cent of all persons who have had typhoid are typhoid carriers for some time after recovery. This condition may become chronic and remain for years.

One typhoid carrier is reported to have been responsible for several outbreaks of the disease. He infected 30 persons, 5 of whom died. Another carrier was a cook who had prepared a large dish of spaghetti for a dinner. Subsequently, 93 people who attended the dinner became ill with typhoid. There is a similar historic case in New York city. "Typhoid Mary" was a cook and had worked in various families. She had never had the disease but carried the germs. Fifty cases of typhoid were traced to her. Since her entire history is not known, she may have been the cause of many more cases of typhoid. She has finally been confined and her personal habits supervised very carefully to prevent any further contagion. Because of the danger from carriers, the Health Department of various cities and states requires a thorough physical examination of all people who handle foods. This law has resulted in discovering several typhoid carriers and placing them under strict supervision.

Protection of the body against typhoid. The germs of typhoid produce powerful endotoxins, toxins within the cell. The body fortifies itself by producing various protective materials, some of which dissolve the invading germs and are known as bacteriolysin. The body cells also produce chemical substances, called agglutinins, which cause the germs to be surrounded by a gluelike substance. This results in a clumping or agglutinating of the once motile germs of typhoid. When these bacteria are stationary and in masses instead of moving around, the white corpuscles can more readily devour them. The presence of agglutinins can be 
determined by adding blood of a patient who has typhoid, or has recently had it, to some typhoid bacilli on a glass slide.

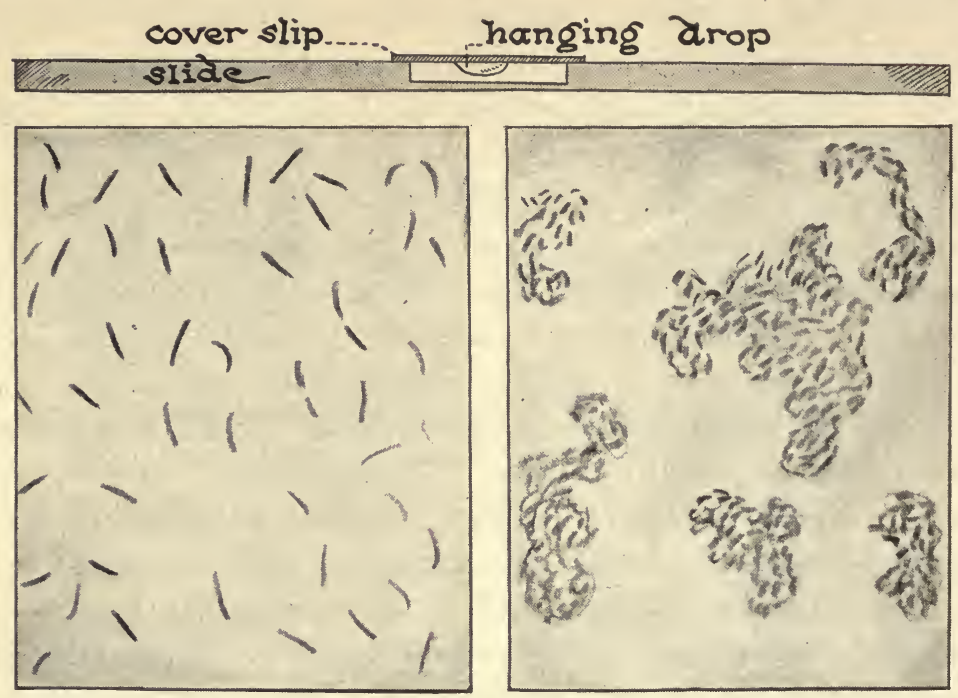

The diagram on the left, shows free swimming typhoid bacilli. The one on the right, shows typhoid bacilli clumped in masses by the presence of agglutinins from the blood of a typhoid patient. The agglutination test is usually made in a hanging drop on a glass slide as shown above.

When this drop is viewed under the microscope, the germs are seen clumped together in masses.

One attack of typhoid produces immunity. This is probably due to the fact that so much protective material is developed to combat the powerful typhoid toxin that much is left over and stays in the blood for life. Since the toxin is not an exotoxin as in diphtheria, antitoxin would be valueless and probably is not produced. The body combats a disease germ which produces an endotoxin by fighting the actual germs with their inclosed toxins.

Diagnosis. As early a diagnosis as possible must be made if the patient is to get the best possible treatment. An early quarantine must be established to prevent the spread of the disease to other people. 
One method of diagnosis is the examination of cultures, made from the feces (excreta) of the patient, for the presence of typhoid germs. Doctors take samples of fecal material from the patient and send it to the Board of Health laboratories. There the material is mixed with media and the developing bacterial colonies are examined for typhoid bacilli.

Another method of diagnosis is the Widal test. This consists of separating serum from the patient's blood and mixing it with a culture of known typhoid germs. If the patient has typhoid, the blood serum will cause the germs to become agglutinated. Agglutinins for typhoid are present in the blood only when typhoid bacilli are in the body or if a person has recently recovered from typhoid. The Widal test is a means of differentiating typhoid fever from other diseases that produce fever.

Method of Spread. Typhoid germs are spread largely through materials contaminated by the excreta of typhoid patients. This may be, and most frequently is, water which has been polluted by sewage and milk which has become infected probably by being kept in containers which have been washed in polluted water. Raw foods such as oysters, if they are grown where they come in contact with sewage, may cause typhoid fever. Raw foods such as celery and lettuce, which may have been watered or washed with contaminat-

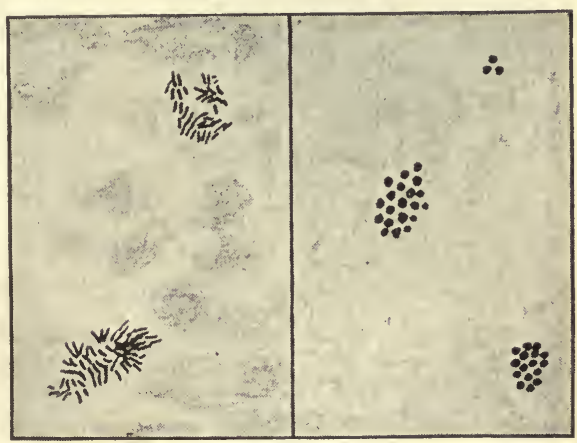

Agglutinins of various types may be present in the blood. The picture on the left shows agglutinated typhoid bacilli, on the right agglutinated pneumonia cocci. ing water, are frequently carriers of the disease. Insects, principally the house flies, which travel readily from filth to exposed 
foods, are notorious carriers of typhoid germs. Human beings may also carry and transmit the germs.

Prevention. Typhoid fever can be prevented both by keeping the typhoid bacilli from entering the body, and by destroying the bacilli. Since typhoid germs are spread by materials polluted by human excretions, or by the housefly, or a human carrier, some of the methods of preventing a typhoid outbreak are the following:

1. Disinfect all excreta of typhoid patients or carriers with chloride of lime. All clothing and bed linen of a patient should be disinfected by being boiled or soaked in carbolic acid or bichloride of mercury solution.

2. Provide a good sewage system.

3. Provide a good water supply. Chlorinate the water. (Boil the water if an infection is suspected.)

4. Pasteurize all milk, or require such sanitary milking conditions that pasteurization is unnecessary. Following the introduction of pasteurization of milk in several cities in 1914, there was a marked decrease in typhoid.

5. There should be proper handling of foods in the grocery, in the market, and in the home. Food should be properly covered and protected from flies. Foods which have been exposed should be thoroughly washed.

6. Health certificates should be required from persons who handle foods in order to eliminate the danger of typhoid carriers.

7. There should be a proper control of the house fly. The destruction of their breeding places, keeping the premises clean and garbage covered, screening the houses, and screening the sickrooms will help exterminate the house fly.

8. Vacationists, nurses, doctors, and any other people who are likely to be exposed to typhoid infection or unsanitary conditions, should be vaccinated. People may be immunized at any clinic, providing they cannot afford to have their own doctors immunize them. The immunity usually lasts from two to four years. 
Vaccination. Immunity to typhoid may be gained artificially through vaccination. In the case of smallpox vaccine, the germ material was weakened through cultivating it in animals. In preparing typhoid vaccine, the bacilli are first grown on agar, then killed by heat, and a little carbolic acid is added to the vaccine for a preservative. When this material is used as a vaccine, the presence of the dead bacilli stimulates the body to make bacteriolysins and agglutinins. Thus a vaccinated person is protected against the invasion of living germs. Usually three inoculations of vaccine are given, each being seven days apart.

Very few people are made ill by typhoid vaccination. If the person is likely to come in contact with paratyphoid, which is a disease somewhat similar to typhoid, he is given a combination vaccine of typhoid and paratyphoid. For most people living in the United States or visiting here, the typhoid vaccine is sufficient, because there are few cases of paratyphoid in the United States.

The investigation of a typical epidemic. During November, 1924, there was a noticeable rise in the number of typhoid fever cases in New York city. This continued through January, 1925. Of the 914 cases recorded in this outbreak, 116 of the residents, and 59 nonresidents who were included in the 914 cases, gave a history of having been out-of-town during the period immediately before their illness.

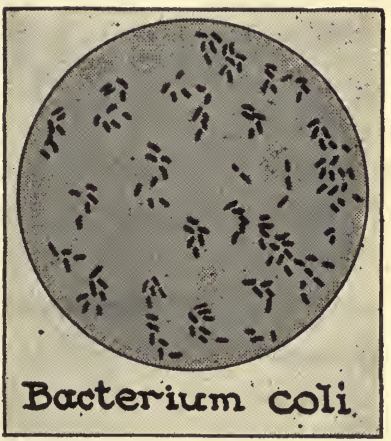

These organisms are commonly present in the intestine of man. Their presence in swimming pools indicates the possibility of contamination by human excreta. This might also indicate the possible presence of typhoid bacilli. Bacteriological examinations of water in swimming pools are frequently made to determine the presence of bacterium coli. Their presence would make the closing of the pool imperative until proper prophylactic measures were taken.

The majority of these had eaten oysters while out-of-town. About 18 per cent of the cases probably acquired their infections in 
this way, but that did not explain the cause of the other cases, so the investigation continued.

The water supply of most cities is constantly tested for bacillus coli, a microörganism found in all human intestines. If the
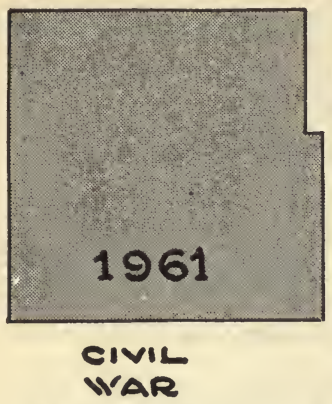

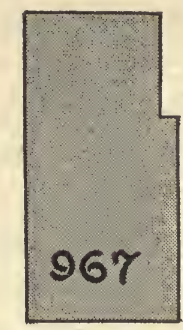

\section{SPANISH - AMERICAN}

bacilli coli are present, there is the likelihood of typhoid also being present. The water of many cities is chlorinated and this results in purification. The bacilli coli are practically never present in water A surgeon general of the U.S. Army has compared the death rates of men treated in hospitals for typhoid, during the first two years of three different wars. The diagram indicates the number of deaths in every 10,000 cases.

which is thoroughly chlorinated. Since the water supply was chlorinated, it could not have been responsible for this epidemic.

The milk supply was investigated and nothing definite was found. The investigators paid particular attention to milk, because in 1913, before pasteurization, an infected milk supply was responsible for 521 cases and 61 deaths from typhoid. Ice cream, water ices, and bottled water supplies were investigated. Again there was no evidence of infection. Uncooked foods, particularly lettuce and celery, were scrutinized with care. No worthwhile evidence was revealed there.

The first bit of evidence was that a great number of the remaining cases gave a history of having eaten oysters approximately two weeks before the onset of the symptoms. Fifty-five per cent or 506 cases gave a definite history of having eaten oysters. In April, 1915, a similar condition had existed. In an outbreak of 150 cases, 80 per cent seemed to be due to the use of raw oysters. Even when oysters are grown in clean, unin- 
fected water, they are frequently contaminated by the people who gather, ship, or otherwise handle them. The same safeguards which have been established to protect milk from pollution at each and every stage of its handling must be exercised in the handling of oysters.

A number of the remedies have been suggested in order to prevent similar epidemics of typhoid fever. The shores and water that have been set apart for oyster beds should be constantly guarded and examined. Sewage and the contents of cesspools should not be emptied near them. Boats, both pleasure and commercial, should be prohibited in such districts. Oysters from a polluted stream should not be transplanted. The gathering, packing and shipping of all shellfish should be efficiently supervised, and the people who handle them in any way should receive frequent and thorough examinations.

In the summer of 1928 , tests were made of the waters of various bathing beaches near different cities and in many cases the water was found to be polluted. The Commissioners of Health suggested that bathing be prohibited in these places. This was not done as certain authorities claimed that the value of the sunshine and bathing was so great, and that it was so possible and

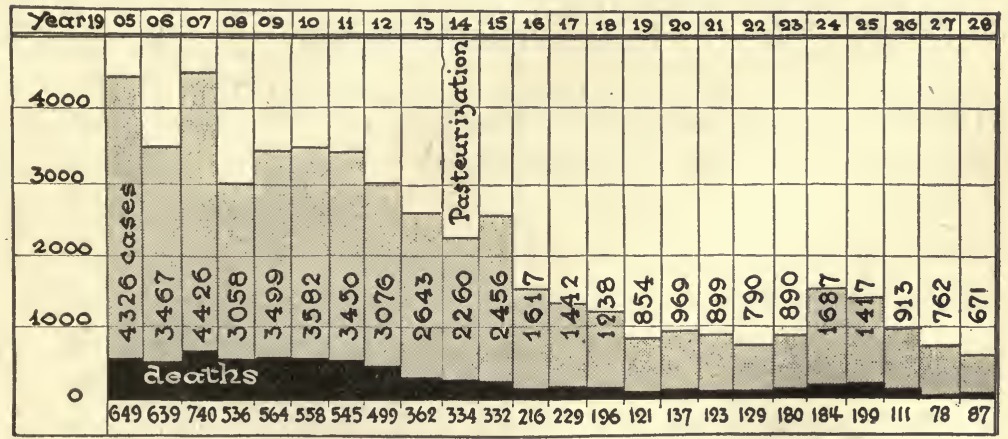

In New York city there has been a steady decrease of deaths from typhoid fever since pasteurization of milk has been required. 
easy to secure immunity from typhoid fever by vaccination, that all bathers should be immunized and thus be protected. Many followed this suggestion. It is always advisable for bathers using water that may become contaminated to be vaccinated. In this way such recreation will be healthful as well as pleasurable.

\section{Questions And Suggestions}

1. Compare typhoid fever statistics in the Spanish-American War and the. World War.

2. Discuss the dangers of typhoid carriers to a community.

3. Name two antibodies produced by the body as protection against typhoid. Discuss the importance of each.

4. Discuss the relation of white corpuscles to combating typhoid in the body.

5. What is the value of an early diagnosis of typhoid fever?

6. Describe two methods used in accurately diagnosing typhoid.

7. How is typhoid fever spread? How can it be prevented?

8. Explain the preparation and use of typhoid vaccine.

9. Discuss how an investigation of a typhoid epidemic is carried on.

10. Why is it unwise to drink water from springs within city limits?

11. Why is the water of swimming pools constantly tested? What does the presence of bacilli coli indicate?

12. Why is it more important for a traveler to have typhoid vaccination than for one staying at home?

13. Discuss any epidemic of typhoid that has been in your home town or city.

\section{Supplementary Readings}

Broadhurst, J., How We Resist Disease (J. B. Lippincott Co.).

Dublin, L. I., Health and Wealth (Harper \& Bros.).

Meredith, F. L., Hygiene (P. Blakiston's Son \& Co.). 


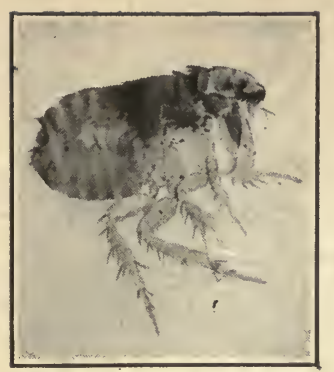

Fleas may carry bubonic plague.
CHAPTER XLVI

CERTAIN OTHER BACTERIA

How may colds be prevented? Have colds any serious effects on the body? Is there any relation of asthma to food? Is it possible or desirable for children to avoid the so-called children's diseases?

Causes of colds. It is generally conceded that there are two types of inflammation called colds. The first type is caused by bacteria and is a real infection. The germs may attack a certain local area, thus causing a cold in the head, a sore throat, laryngitis, or tonsillitis, or they may have general widespread effects as in grippe. The second type of cold is caused by physical agents such as irritant gases or dust. Colds and even pneumonia have been caused by breathing in talcum powder. Dust inhaled in working at trades such as diamond polishing, metal polishing, or marble quarrying may cause colds, followed by pneumonia, or tuberculosis. It is the physical material which starts the irritation, then bacteria enter the irritated tissues and set up infections. Other factors that may cause colds are dry heated air, drafts, sudden changes of temperature, exposure to cold and wet, improper food, and constipation.

Effect of colds on the body. Bacteria causing colds are usually present in the mucous membrane of the nose, mouth, and throat. When the resistance of the body is lowered, it is thought that WH. FITZ. AD. вIO. -31 473 
these bacteria gain a foothold and produce the condition known as a cold. Blood congests in the mucous membrane which becomes swollen and causes a profuse flow of mucus. The mucous membrane of the tear ducts may also swell and the tears will flow continuously. If the inflammation spreads to the Eustachian tubes, they may become closed and hearing is temporarily impaired. If the infection extends to the middle ear, earache usually results. Sometimes infection may extend to the cavities in the bones of the front of the skull called the sinuses and cause inflammation or sinusitis. If the infected material is retained in the sinus, the condition usually becomes chronic.

A chronic cold is known as catarrh. If the disease extends down into the bronchial tubes, the condition is known as bronchitis; the inflammation of the finer bronchi may cause bronchopneumonia. People who work indoors and at sedentary occupations are more likely to suffer from colds than persons who live an outdoor life, as dust, dry air, and noxious gases are continually irritating the respiratory tract and making it susceptible to infection. These same predisposing factors increase the likelihood of the disease extending into broncho-pneumonia. This type of pneumonia is unlike lobar pneumonia. The latter is caused by a specific microörganism, the pneumococcus. Broncho-pneumonia may be caused by any bacteria that infect the bronchial tubes and air sacs.

Colds are frequently neglected and considered of trivial importance. Each cold lowers the resistance and vitality of the body to such an extent that the sufferer is likely to contract other infections. This may hasten the progress of a serious disease like tuberculosis. Every effort should be make to prevent colds. Once a cold is contracted, the best possible care should be taken to avoid any secondary infections.

Method of spread. Even with many of the predisposing factors present, a cold does not develop unless there is an exposure 
to infection from another person who has or is just recovering from a cold. Sometimes the infection already exists in the individual in a chronic but extremely mild state, and it may become acute when the vitality or resistance is lowered. Eskimos and Arctic explorers, in spite of the severe weather conditions, do not have colds. When such explorers return to civilization they may be infected by droplets sprayed by the coughing and sneezing of people whom they meet, and they soon experience a severe cold.

Prevention of colds. Since a cold is a droplet infection, it generally travels through the sputum. Crowds in ill ventilated places should be avoided. The germs can live only for a very short time in air. Their culture medium is the air passages of human beings. In crowds, it is impossible to avoid the sneezing or coughing of other people. That is the way colds are spread and it is the reason for the frequency of colds. Spitting spreads sputumladen bacteria into the air. If a person talks directly into the face of another person, droplets of sputum are given off and inhaled.

An experiment was made in England to show that droplets from the mouth are spread. A certain member of Parliament was asked to wash out his mouth with a culture of harmless bacteria which, when plated, produce red colonies. He then made a loud and eloquent speech. Petri dishes had been distributed in all parts of the House of Parliament, even to the rear of the gallery. When gathered, incubated, and the plates examined, all the exposed dishes contained red colonies. The closed controls showed none. The speaker had showered the entire house with microscopic droplets containing the harmless bacteria.

Coughs and sneezes must be covered in order to prevent the spread of infections. When a person has a cold, he should use gauze instead of handkerchiefs and the used gauze should be burned. Some people seem to be more susceptible to colds than others. This may be due to abnormalities in the nose or throat. Adenoids and infected or enlarged tonsils are excellent locations for 
the harboring and growth of germs, and should be removed. Teeth should be straightened so that correct breathing will be possible. Nasal douches should not be used regularly, as the solutions used in them may be irritating to the delicate mucous membranes, and may cause an infection. If the nasal passages are in healthy condition, strict observance of the rules of individual hygiene is likely to prevent colds. Living and sleeping outof-doors will keep fresh air in the lungs and will prevent colds. Children with colds should be kept home from school in order to keep the infection from spreading.

Remedial measures. A cold is very likely to run a regular course, although proper treatment may relieve some of its unpleasant features. The patient should avoid drafts and keep warm. If it is possible to stay in bed for a day, many of the symptoms can be mitigated. A hot foot-bath, hot drink, and massaging the neck and chest well just before going to bed are often very beneficial. These measures help in stimulating circulation and in breaking up the congestion. If the weather is mild and sunny, the patient should spend as much time as possible out-of-doors. There are special vaccines prepared against colds and used with some slight degree of success. They are sometimes successful in stimulating a person's body to work up an immunity.

Influenza. Epidemics of influenza have spread all over the world, therefore, it is frequently called a pandemic disease. Influenza of the respiratory tract is the most common form of the disease, although there are other forms. It usually starts with a cold, followed by a high temperature and extreme weakness. It may extend into the lungs and cause bronchitis or pneumonia. The disease is spread by direct contact. The secretions of the mouth and nose carry the infectious agents. Droplet infection is generally considered to be the method of spreading influenza. Unlike many other diseases, one attack does not usually establish an immunity. 
Asthma. Any condition of difficulty in breathing is popularly knowin as asthma. Some conditions of asthma are due to lung infections, others to food poisoning. If certain foods disagree with a person, the reaction may be shown by an asthmatic condition. Frequently, rashes are caused by a sensitivity to certain foods. In order to discover what food is causing the reaction, different foods are injected below the skin. If the skin shows irritation, the food causing it is taken from the diet and the asthma often clears up.

When a plant pollen causes asthma, it is known as hay fever. Vaccinations of pollen are inoculated into the skin to see which of the pollens cause the sensitivity. The pollen from the ragweed is responsible for many cases of hay fever. When the right pollen is determined, small quantities of it are inoculated into the sensitive person until he works up an immunity. Up to the present time these vaccinations have not always proved successful.

Focal infections. Bacteria at some point or focus in the body frequently. multiply and produce toxins which are absorbed into the body and affect other parts. When people tire easily, lack normal energy, and are subject to pains in the joints and muscles, they may have a focal infection. Other evidences of focal infections are inflammatory

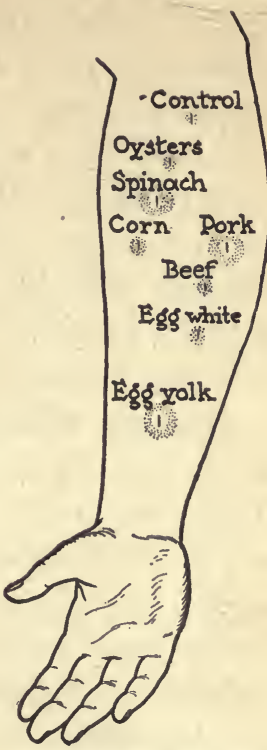

Skin tests may be made to determine an individual's sensitivity to different foods. Small volumes of different foods are injected under the skin or small amounts are rubbed into tiny gashes made on the arm. One cut, the control, is not treated with food. Little or no redness or soreness is present near the control. Varying amounts of inflammation appear about the infected areas. Redness and soreness denote a positive reaction and indicate sensitivity. rheumatism, heart disease, kidney disease, lumbago, and nervous conditions such as neuritis and neuralgia. When any of these conditions occurs, the doctor usually looks for a focal infection. 
Common foci of infection in the body are diseased tonsils, chronic infections of the ear and nasal cavities, which may spread to the sinuses, pockets of pus about the roots of the teeth, and a diseased appendix. When the focus of infection is removed, the symptoms tend to disappear. Regular and systematic care of the teeth by a competent dentist, with the use of X-rays whenever possible, will bring to light pus pockets in teeth. Avoidance of, and proper care of colds will prevent sinus and ear infections. Regular periodic health examinations will usually detect these foci of pus before they cause disease in the body. Once a focal infection is started, it may be very difficult to cure it.

Measles, whooping cough, and chicken pox. These diseases are common among children because they are spread through the unhygienic habits that are prevalent among all children. When a little girl hugs another one, takes a bite of her apple, borrows her pencil, or performs endless other acts that result in personal contact, disease germs may be passed from one child to the other. In some households, if one child contracts one of these diseases, all the children of the family are purposely exposed to it. It is easier to take care of all at the same time. This is a very wrong procedure. These diseases, in themselves, are not very serious and seldom fatal. But they are frequently followed by secondary infections such as pneumonia, deafness, rheumatism, heart disease, or kidney disease. The after-effects are far more serious than the original disease. When one child gets a disease from another, the second child may get it in a more severe form than the first. In growing in the first child's body, the disease germ seems to acquire greater virulence and, consequently, affects the second child more seriously. Either this is the case or weaker strains of bacteria are killed in the first child's body and only those virulent organisms that are resistant to the defenses of the child's body are passed on. Children who show any signs of illness should remain home from school until they are again perfectly well. Because 
they can stay out in the sunny open air, if well enough, or relax in bed, their recovery will be hastened, and they will not spread the infection through the school.

\section{Questions And Suggestions}

1. Discuss the causes of different kinds of colds.

2. What effects have colds on the body?

3. How are colds spread?

4. How can the vitality of the body be kept high so that colds may be prevented?

5. What unhygienic practices have you noticed among students which may account for epidemics of colds in a school?

6. How can epidemics of colds in schools be prevented?

7. Compare influenza to an ordinary cold.

8. Discuss the common causes of asthma.

9. What do asthma vaccinations consist of ?

10. Discuss focal infections and their relation to the health of the body.

11. What is the relation of so-called children's diseases to the vitality of a child?

12. Discuss some after-effects that may result from measles, whooping cough, and chicken pox.

\section{Supplementary Readings}

Broadhurst, How We Resist Disease (J. B. Lippincott Co.).

Haggard, H. W., The Science of Health and Disease (Harper \& Bros.). Rosenau, M. J., Preventive Medicine and Hygiene (D. Appleton \& Co.). 


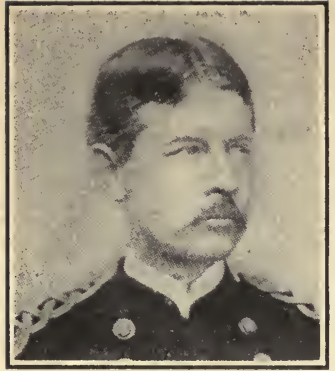

Walter Reed.
CHAPTER XLVII

THE CONTROL OF MALARIA AND YELLOW FEVER

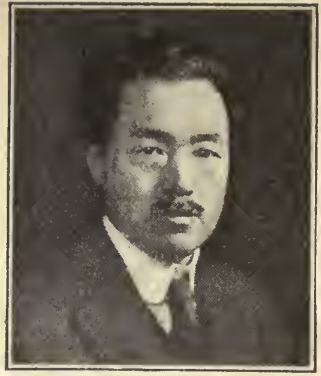

Underwood \& Underwood Hideyo Noguchi.

How has malaria been controlled? Why were the French unable to build the Panama Canal? Who was Hideyo Noguchi?

Malaria is caused by a microscopic protozoan, known as a plasmodium. There is some discussion as to whether the organism that produces yellow fever has really been isolated.

Prevalence of malaria. In 1908 there were more than three million deaths from malaria in India. Along the rivers and coasts of tropical countries, malaria is the white man's greatest obstacle to settlement. In temperate climates such as the United States malaria is not usually fatal, but in the tropical countries it occurs in most severe forms and the death rate is very high.

The use of the drug, quinine, is most effective in treating malaria, since it kills the malaria protozoans in the blood. In 1902, the Italian government began the sale of quinine at low prices to certain communities. This drug was distributed free to those unable to purchase it. In 1904, the Italian towns gave it to all working people. In consequence, there has been a progressive reduction in the amount of malaria in Italy. During the ten years previous to 1902, Italy averaged 14,048 deaths per year from malaria. In the nine years following 1902, the average fell to 3853 . 
Fifty years ago malaria was so common in our Middle Western States that it was a serious problem. But this malady has gradually been reduced by scientific control.

Malaria is still fairly common in the tropical countries. It was recently estimated that, approximately, 90 per cent of the people of Calcutta are suffering from this disease.

History of malaria. The ancient Greeks thought that malaria was due to bad air arising from the marshes. Hence they called the disease malaria, which means bad air. Malaria always prevailed near swamps and was thought to be caused by some kind of emanation from decaying matter. In 1880, a French army surgeon, Charles Laveran, noted and described the malarial parasites in the red corpuscles of the blood of persons suffering from malaria. But he was not able to ascertain how they entered the blood. This was not learned until 1895, by Major Ronald Ross, an English army surgeon, who started investigating malaria in India, where malaria was prevalent and existed in its worst form. Discovering that birds were susceptible to malaria, he first studied the organisms in the blood of the birds. He suspected that this disease was not contagious but was transmitted by the bite of a mosquito, and he permitted mosquitoes of a certain species to bite infected birds. He killed the mosquitoes, and found little swellings in the walls of their stomachs. Then he let similar mosqui-

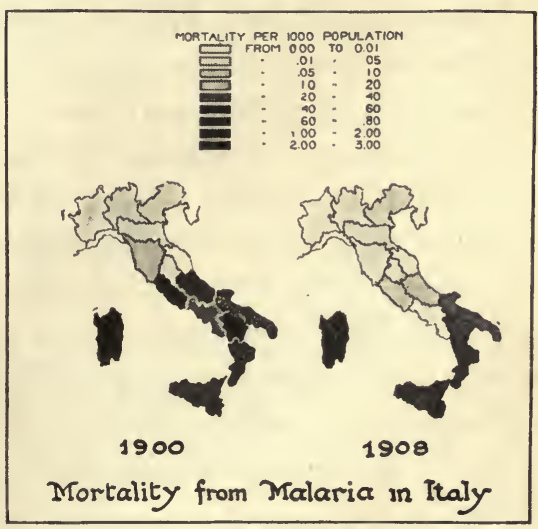

Am. Museum of Nat. Hist.

The Italian government has steadily decreased the cases and deaths from malaria. toes bite birds that were not infected with malaria, and no such swellings appeared in the stomach walls. He continued examin- 
ing many of these small swellings in the stomach of the mosquitoes and found that they contained numerous parasites. When the swellings reached a certain size they would burst and the parasites were scattered through the mosquito's body. Some entered the salivary glands. Ross concluded that these parasites in the saliva of the mosquito would pass into the blood of the bird when the mosquito bit the bird.

Cause. Malaria is caused by a protozoan parasite, Plasmodium malariae, somewhat like the amoeba. It has two hosts: the female mosquito of the genus Anopheles, and man. There are really three different types of malaria, caused by three different but related microörganisms. Malaria occurring in our latitude is a mild form of the disease.

Spread. The germ of the disease is spread by the bite of the female Anopheles which has previously bitten a malarial patient. (The male feeds on plant juices.) The common mosquito, of the genus Culex, does not transmit malaria or yellow fever.

Life history of the malaria parasite, Plasmodium malariae. The amoeba-like organism of malaria cannot complete its life history in the blood stream of man alone; it requires two hosts, the mosquito and man. Germs are injected into man through the bite of an infected mosquito. They enter the red blood corpuscles and multiply there, finally forming from six to sixteen spores. At intervals of 24,48 , or 72 hours, depending on the type of malaria, the spores, having destroyed the corpuscle, escape into the blood stream. The sudden release of these poisons, and the subsequent rallying of the body in an effort to counteract them, are thought to cause the chills and then the fever which are characteristic of malaria.

Each escaped parasite now fastens itself to another red corpuscle, enlarges, forms spores, and having used up and disintegrated the corpuscle, again is set free, not as one but as many parasites. The cycle is repeated over and over again, until the 
patient recovers or succumbs. Some of the parasites undergo certain changes which differentiate them into sex cells. Since all the parasites were injected into the blood at approximately the same time, and since it takes each one just so long to grow, form spores, excrete wastes, and escape from the corpuscle, all, with their wastes, are ejected into the blood stream at one time. Therefore, the chills and fever occur at intermittent periods.

When a mosquito bites a patient, it takes in the malarial patient's blood with the plasmodia which developed into two types of sex cells. These undergo divisions and changes. Study the diagram (p. 484) and note that some cells take different forms. Some of them become male cells, others, female cells. Fertiliza-

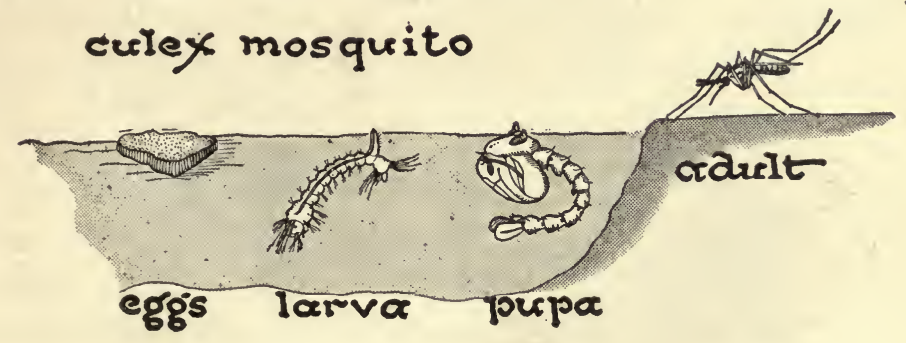

\section{aropheles mosquito}

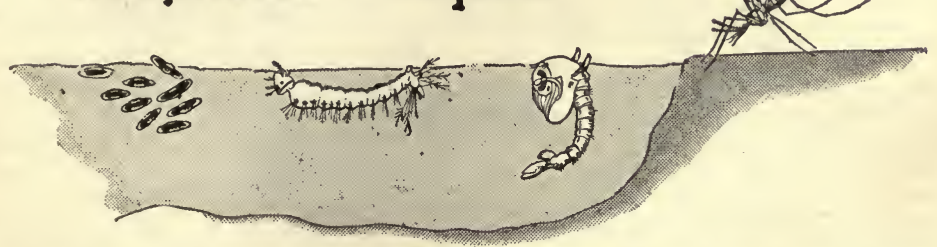

Culex, the house mosquito, differs in appearance from Anopheles, the malarial mosquito. Compare the different stages of growth in the life of these two insects.

tion occurs in the stomach of the mosquito by the union of the male and female plasmodia. The fertilized cells bore into the 
wall of the stomach and form cysts on the outer stomach wall. In these cysts, the parasites break into thousands of needle-shaped

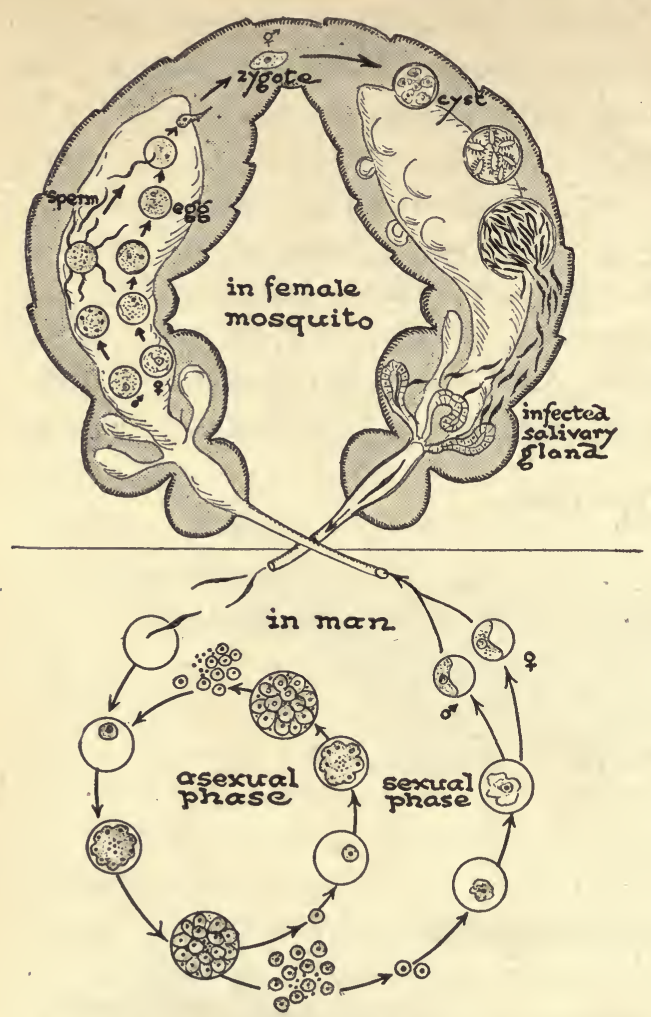

The malarial parasite undergoes a cycle in its life history from mosquito to man and back again to the mosquito. Trace the complete history of the organism in the diagram above. spores, which finally escape from the cysts, enter the blood of the mosquito, make their way around the body, and especially infect the salivary glands. When the mosquito now bites another person, malarial organisms enter the victim's body with the mosquito's saliva. The malarial parasite seems to produce no ill effects on the mosquito. In the human host the malarial parasite reproduces asexually and differentiates into sex cells which may be called a sexual phase; in the body of the mosquito it reproduces both sexually and asexually.

Nature. The poison produced by the germs causes a marked chill, followed by fever, then profuse perspiration, and a fall of temperature. This is followed by a period of well-being until the onset of the next chill, usually 48 hours later in our type of malaria. Destruction of the red corpuscles may cause anaemia 
and general weakness, which is particularly serious in that it often renders the body"more susceptible to the attack of other diseases. Common malaria is rarely fatal, but it does weaken the body's resistance. . Tropical malaria is often fatal.

Diagnosis. The presence of malarial parasites may be ascertained by a microscopic examination of a blood sample. Chills followed by fever often come with other diseases such as typhoid and appendicitis, which are sometimes diagnosed as malaria. A microscopic examination gives a conclusive test for malaria as the plasmodia will be found' present in the suspect's blood.

Treatment. Frequent doses of quinine bring about the destruction of the malarial parasite, but because of the peculiar effect

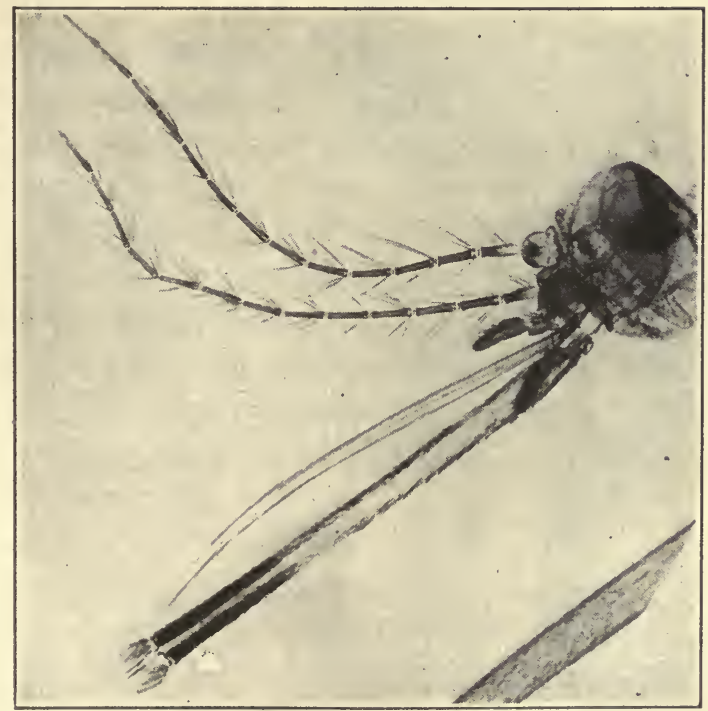

Photomicrograph of a mosquito's head.

The female Anopheles is the carrier of the malarial germ. The male does not feed on human blood.

of quinine on the nerves of the ears, and on the salivary glands, it must be used with care and discretion. 


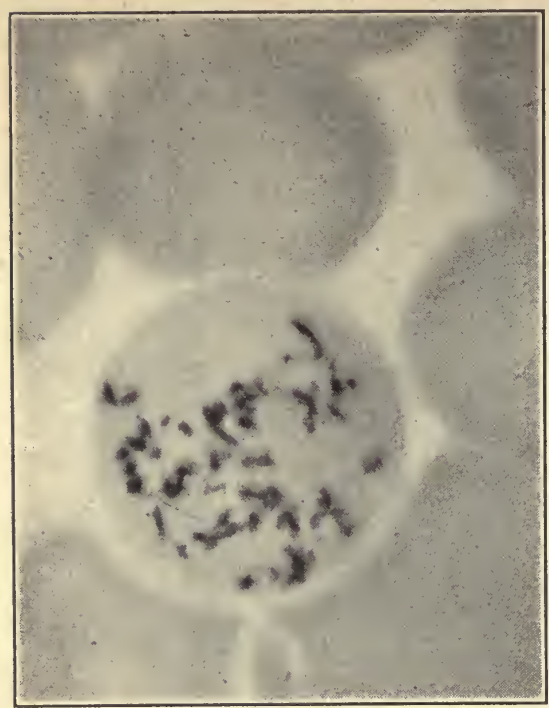

Photomicrograph of malarial parasites in a red blood corpuscle.

Prevention. The control of malaria depends upon the destruetion of malaria germs in patients by the use of quinine and the destruction of the mosquitoes (Anopheles). The frequent use of quinine in malarial regions by those not sick will kill the parasite before it has a chance to multiply. The rooms occupied by malarial patients should be thoroughly screened, so that the disease may not be spread through the infection of more mosquitoes. In fact, all dwellings in any malarial district should be well screened. Since the mosquitoes are in search of food in the evening, it is unwise for people to go beyond screen protection after dusk. Probably the best method of eradicating malaria is to destroy the breeding places of the mosquitoes by draining the swamps and filling in the low places.

\section{YeLLOW FeVER}

Prevalence of yellow fever. Yellow fever is a much more severe disease than malaria. The mortality in various epidemics has ranged from 15 per cent to 85 per cent of the population. It is also a disease which is more prevalent in tropical and subtropical countries than in cooler climates. Formerly, epidemics occurred in the Southern States, and the disease was very common in Cuba and the Canal Zone. Much has been accomplished in the last thirty years in eradicating this disease. In 1878, there were one 
hundred and twenty-five thousand cases and twelve deaths from yellow fever in the United States. Since 1905 not a single case has been reported. Havana and Rio de Janeiro used to be centers of infection. To-day, due to the control of yellow fever, they are health and vacation resorts. There is still one very bad district in western Africa. Efforts are now being made to control the fever there.

History of the control of yellow fever. It is claimed that yellow fever was the diseáse that nearly annihilated the second expedition of Columbus in Santo Domingo in 1495.

Yellow fever was so bad in certain parts of Cuba that no one could live there safely. In 1900, after the Spanish-American War, a commission was appointed to make an investigation of yellow fever in Havana. The commission was composed of Major Walter Reed, a bacteriologist, and Dr. James Carroll, Dr. Jesse W. Lazear, and Dr. Aristides Agramonte. Dr. Reed could not find a microörganism in the blood of the infected people. $\mathrm{He}$ decided that if the fever were caused by a bacterium, the nurses handling the patients would contract the disease. This did not seem to be true. He also observed that members of the same family did not seem to get the disease from each other. At the end of two or three weeks, people in the neighborhood of the original cases would contract the disease. This

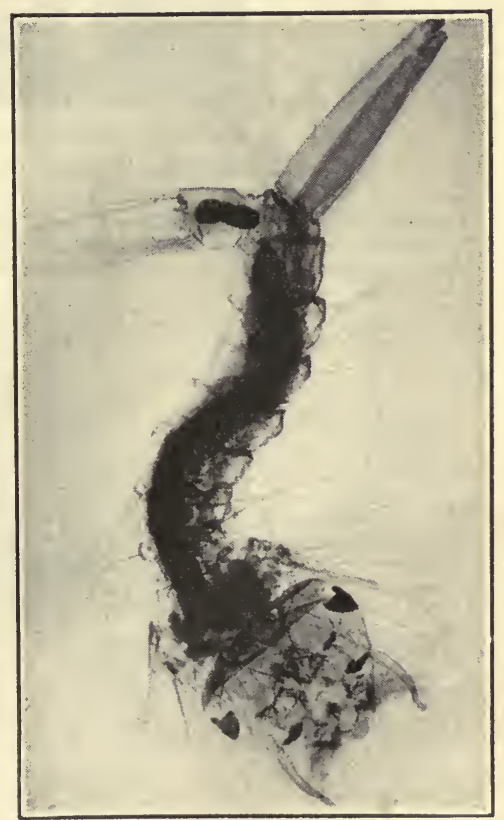

Photomicrograph of a mosquito larva. 
seemed to indicate that the germ was transmitted by a carrier, possibly an insect, and it took that length of time to grow in the

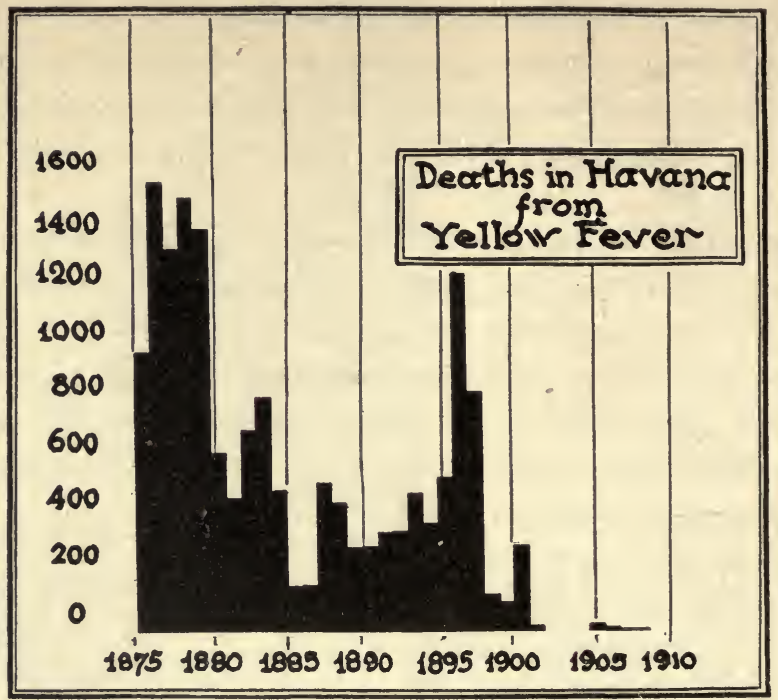

Am. Museum of Nat. Hist.

The numerous swamps of Havana were the breeding places for mosquitoes, and yellow fever was very prevalent. By 1900 , the effect of the extermination of mosquitoes began to be evident in a lower death rate of yellow fever.

insect's body. Probably the knowledge of the cause of malaria gave these investigators clues on which they based these theories.

People had formerly thought that yellow fever was transmitted by fomites, substances such as garments and bedding, which had been in contact with yellow fever patients and had absorbed the germs. To test the fomes theory, an experimental hut was filled with articles from a hospital for yellow fever at Havana. Volunteers agreed to sleep in this hut. They did not contract the disease. The experiment was repeated with a number of persons and always with the same results, which definitely proved that fomites did not transmit the disease.

The theory that the disease was transmitted by mosquitoes 
was then investigater. No animal was susceptible to yellow fever, so Major Reed could not experiment on animals. Dr. Carroll permitted himself to be bitten by a mosquito known as the Aëdes or Stegomyia mosquito which was suspected of transmitting the fever. He contracted yellow fever but recovered. A mosquito accidentally alighted on Dr. Lazear's hand and he permitted it to bite him. He died September 25, 1900, one of the first martyrs to the yellow fever investigation.

Major Reed then decided to set up controlled experiments to prove definitely whether or not the mosquito carried the disease. He wanted to segregate a group of men for a number of weeks from all contact with yellow fever to make sure they had not already contracted it before mosquitoes bit them. He asked for

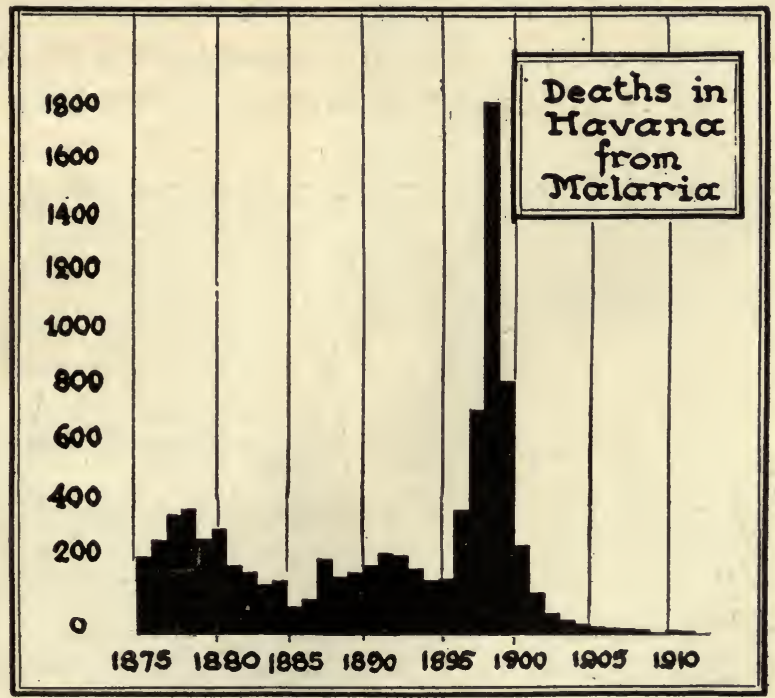

Am. Museum of Nat. Hist.

Due to the control of the mosquito, malaria has practically disappeared from Havana.

volunteers for the experiment. Private Kissinger of Ohio and John J. Moran, a civilian clerk, offered themselves. Major Reed WH. FITZ. AD. BIO. -32 
carefully explained the dangers of the experiment. The volunteers said they understood the ravages of the disease but "we volunteer solely for the cause of humanity and in the interest of science." They asked for no compensation for their services. It was a tense, dramatic moment when the Major raised his hand in salute to the private, saying, "Gentlemen, I salute you!" His further comment was, "In my opinion this exhibition of moral courage has never been surpassed in the annals of the army of the United States." Kissinger and Moran were bitten by mosquitoes. They both contracted yellow fever but both recovered. After studying many other cases, the investigators concluded that the only way to contract yellow fever is through the bite of an infected mosquito. It takes about twelve days for the parasite to complete its cycle in the mosquito's body. Therefore, an infected mosquito does not transmit the disease until about twelve days after taking in the parasite from the body of an infected person.

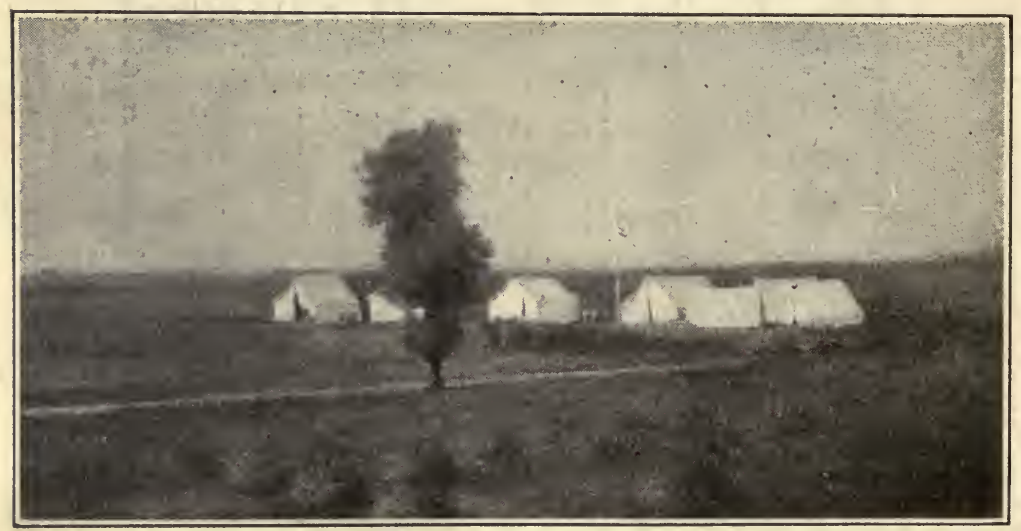

Am. Museum of Nat. Hist.

Camp Lazear, Cuba, was the experimental camp in which the yellow fever investigation was conducted. It was named for Dr. Jesse W. Lazear, who died a martyr to the investigation.

For 130 years Havana had been continuously infested with yellow fever. The average death rate from it was about 750 deaths 
per year. After the yellow fever investigation, Major Gorgas was sent by the United States government to rid Havana of mosquitoes. Within ninety days there was not a single new case of yellow fever.

The Isthmus of Panama was considered a plague spot for yellow fever and malaria. About 1880, the French started building a canal across the Isthmus, but had to give it up, because of the prevalence of the diseases which caused the death of thousands of the workers. Twenty years later, the United States bought the Isthmus from the French. Since Gorgas had helped to rid Havana of yellow fever, the government sent him to eradicate yellow fever from Panama. There were

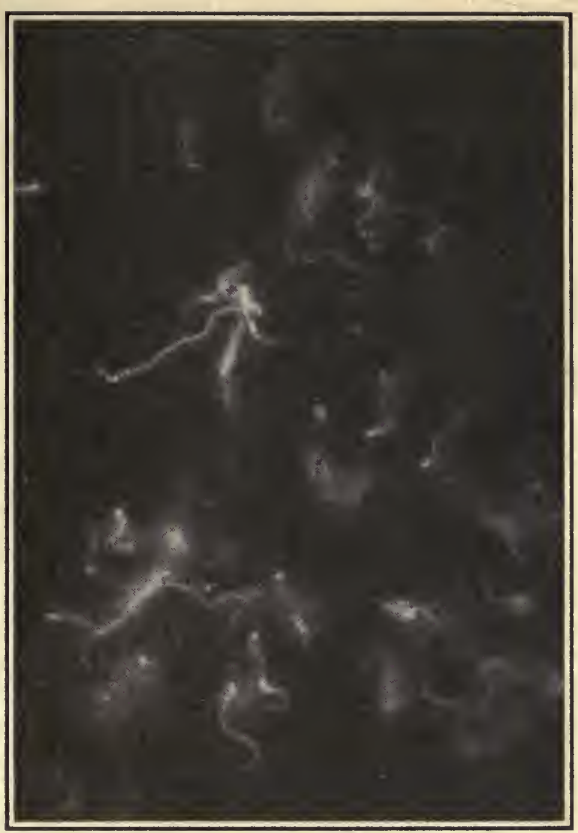

Courtesy Rockefeller Institute

The structure isolated by Dr. Noguchi which is believed to be the cause of yellow fever. In the photo, the spirochaete is magnified $\mathbf{3 0 0 0}$ times.

neither suitable drains nor water supply in the cities, so Gorgas had a system of drainage constructed, the streets paved to eliminate water-filled ruts, the water supply of the cities improved, and the windows and doors screened against mosquitoes. Pools were either drained or oiled. Endless care, thought, and time were devoted to this work. In about two years yellow fever was eradicated from the Isthmus and the building of the canal was made possible. This was as great a hygienic feat as the canal was an engineering feat. 
Later Investigations. Hideyo Noguchi of the Rockefeller Institute isolated a microörganism, called a spirochaete, from yellow fever victims in South America, which has been, until recently, a focus for yellow fever. It is more like a protozoan than a bacterium. He made a vaccine from this organism, which is administered early in the disease and is very effective. The disease has been practically exterminated in the Western Hemisphere, due to his efforts. But experimental workers in Africa were unable to isolate the spirochaete from the blood of victims of yellow fever in that country. They were unable to inoculate monkeys and guinea pigs with the disease as Noguchi had done. Apparently, the germ causing yellow fever in Africa was different from that causing the disease in South America. Noguchi went to Africa to investigate the disease and he succeeded in inoculating one species of monkey with the disease. Unfortunately Noguchi became infected with yellow fever in Lagos, Nigeria, and died in the spring of 1928. After he contracted the fever, he insisted that samples of his own blood be inoculated into monkeys. His associates carried on his experiments with the cultures which he had started. It has not yet been decided whether there are one or two types of yellow fever. 'From data left by Noguchi, the Rockefeller Institute for Medical Research is inclined to believe that there are two distinct forms of the disease. Scientists are most anxious to clear up the infection in western Africa before the transcontinental railroad is opened. If the disease spreads to the East Coast, it may pass over into India and southern China, which are full of the Aëdes mosquito.

Cause. A spiral protozoan, spirochaete, was discovered in 1918 by Noguchi and designated by him as the cause of yellow fever. Experiments are being performed, at the present time, to determine whether or not the spirochaete is really the specific organism causing yellow fever. The vaccine prepared by Noguchi has not yet been accepted as a definite preventive. 


\section{Questions AND Suggestions}

1. Discuss the prevalence of malaria in the world.

2. How did the disease receive its name?

3. Discuss the experiments of Major Ross.

4. Discuss the cause and spread of malaria.

5. Discuss the life history of the malarial parasite, including both the asexual and sexual phases.

6. What effect has malaria on the body?

7. How is malaria diagnosed; how is it prevented?

8. Discuss the prevalence of yellow fever.

9. Discuss the investigation conducted by Major Reed.

10. What biological and sociological effects did the investigation of yellow fever have on Havana?

11. Why is the building of the Panama Canal considered as great a hygienic as an engineering feat?

12. Discuss the contribution of Hideyo Noguchi to the eradication of yellow fever.

13. Discuss the cause and the method of spread of yellow fever.

14. Discuss the prevention of yellow fever.

15. Which of Koch's postulates was Noguchi unable to carry out in his investigation of yellow fever?

16. Does the government give pensions or aid in any form to persons (or their dependents) who have risked their lives in scientific investigations?

\section{Supplementary Readings,}

De Kruif, P., Microbe Hunters (Harcourt, Brace \& Co.).

Greaves, J. E. \& E. O., Elementary Bacteriology (W. B. Saunders Co.). Haggard, H. W., The Science of Health and Disease (Harper \& Bros.).

Zinsser, Hans, A Textbook of Bacteriology (D. Appleton \& Co.). 


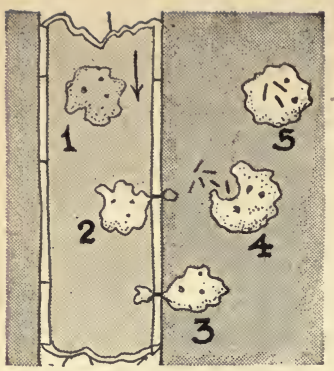

The scavengers of the body.
CHAPTER XLVIII

DEFENSES

AGAINST DISEASE

How is the body protected against bacteria? Are the protections adequate? Under what conditions are the defenses inadequate?

Diseases of the body may be due to glandular disturbances such as cretinism; inadequate diets, as rickets; constitutional tendencies or disturbances; or to infections from microörganisms. Parasitic microörganisms exist in great numbers, but most of them are either kept out of the body or destroyed when they enter the body. There are comparatively few diseases caused by bacteria when compared with the existing number of parasitic bacteria.

Behavior of bacteria in the body. Bacteria have different modes of attack and different ways of breaking through the defenses of the body. Some bacteria actually destroy tissue. For example, the tuberculosis bacillus devours various tissue cells, and thus destroys them. It is thought that certain parasitic bacteria which cause boils and abscesses send out enzymes which dissolve the white blood cells so that they can be absorbed by the bacteria. The pus formed in boils and abscesses is dead white corpuscles. Other bacteria act on the body chiefly through the toxins or exotoxins they produce. These pass into the tissues surrounding the bacteria, get into the blood, and circulate through 
the body. For example, diphtheria toxins produce soreness in a throat on which the diphtheria bacilli are multiplying, and the blood carries the exotoxins around the body. The heart, kidney, or some other remote organ may be affected. The tetanus bacilli develop their exotoxin at the place where they enter the body. This toxin then goes to the various tissues, especially the nerves.

Bacterial poisons or endotoxins result from the breaking down or the disintegrating of certain bacteria. It is thought by some scientists that these endotoxins are never produced, as are the exotoxins, by the bacteria themselves, but are only set free during the breaking down of the bacterial body. The typhoid germs contain a very powerful endotoxin; tuberculosis, too, has a strong endotoxin. These are probably due to the breaking down of the proteins in the bacteria cell and the consequent formation of substances that are poisonous to the tissues. They are more correctly called poisonous split-proteins. There is still a fourth way in which bacteria are related to disease. Protein foods are sometimes attacked by bacteria and are only partly digested or broken down to a group of products called ptomaines, some of which are injurious to the body. Ptomaines differ from toxins in that they are products of food decomposition, while the toxins are products of bacterial manufacture. The ptomaines are usually formed in foods under storage conditions in the shop or house, and not in the body. For example, if protein foods are not properly preserved, bacteria may attack them, causing disintegration. Partially disintegrated fish, crabs, cheese, oysters, or milk often contain injurious ptomaines and, when eaten, will have a poisonous effect on the body.

How bacteria enter the body. One of the common avenues of invasion for bacteria is the alimentary canal. Bacteria of typhoid fever and tuberculosis are frequently taken in with milk or other food through the mouth. Pencils, finger nails, and drinking cups are often responsible for an attack of diphtheria or scarlet fever. 
The respiratory tract furnishes a means for the entrance of germs. Spray or droplets of sputum sneezed or coughed out may be breathed in by other people. Germs of pneumonia, diphtheria, scarlet fever, and colds often enter the body in this way.

Certain other organisms enter the body through wounds or skin abrasions. These skin openings may be of various kinds. Cuts, scratches, torn hangnails, and cracked skin are responsible for the entrance of dirt which may carry not only tetanus germs but, when present, ringworm (a parasitic mold), hookworms, or other pathogenic organisms. Insect bites form openings through which germs may be introduced. The bite of an Aëdes mosquito may transmit yellow fever; the Anopheles mosquito, malaria; the rat flea, bubonic plague; the body louse, typhus fever; and the tsetse fly, African sleeping sickness. The bites of rabid dogs, cats, wolves, and other animals are responsible for hydrophobia. Some organisms enter the body through the eyes. If the eyes are rubbed with an infected hand or dried with an unclean towel, such diseases as trachoma and pink eye may possibly result.

Safeguards of the body against disease. The best defense of the body is the strong natural resistance that accompanies good health. As long as rules of health are followed, the vitality is likely to be high. Even though bacteria then invade the body the natural
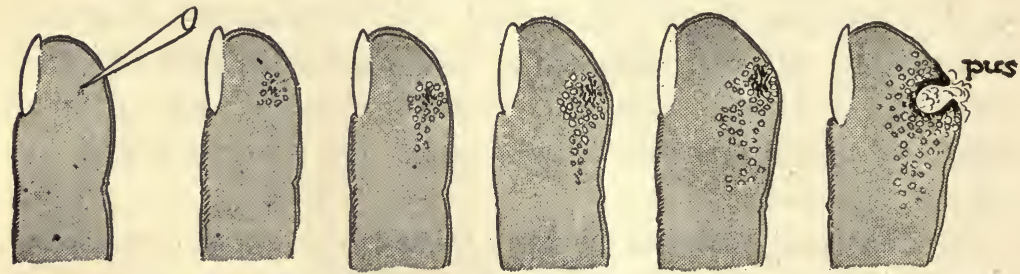

An unbroken skin is a protection against invading bacteria. If the skin is pricked or otherwise broken, bacteria may enter and cause an infection.

protective substances and cells tend to control them with success. If resistancé is low, due to insufficient air or sunlight, inadequate 
diet, lack of proper exercise or rest, the body cannot use its natural protective agencies to the fullest extent, and invading bacteria gain a foothold in the body, multiply, and bring about a condition of disorder known as disease.

As long as the skin is unbroken it forms an effective barrier against the entrance of germs. There is, however, some doubt whether a bacterium or a Protozoa can gain an entrance only through unbroken skin. A few parasites such as hookworms are known to penetrate unbroken skin and may cause a disease which undermines the vitality, and results in great lethargy and consequent inefficiency. Just as soon as skin is abraded or broken all kinds of parasites may enter. Broken skin should always be treated with antiseptics to inhibit the growth of any germs that attempt to enter. Some antiseptics have an additional value of promoting healing.

The tears which constantly wash the eye will remove any bacteria and drain them into the nose, from which they may be removed. (If bacilli prodigiosus, bacteria that are pigmented red, be dropped in the eye, they will shortly disappear from the eye and appear in the nose.) The tears are slightly antiseptic in action so that they exert a chemical as well as a physical protection.

The mucous membrane in the nose, mouth, and throat catch the germs on its sticky surface and prevent them from traveling further into the body. This mucus is slightly antiseptic in action and is responsible for the destruction of some germs. When the membrane has caught germs in its secretion, an irritation is set up which stimulates sneezing, coughing, or blowing of the nose. Phagocytes probably destroy some of these bacteria. There are usually some bacteria found in the nose, throat, and mouth. As long as the resistance of the body is high and there is no break in the membrane, these germs do not attack the body. The body in good condition seems to acquire a certain immunity 
to the germs which it constantly harbors. Saliva as well as mucus is weakly antiseptic. Hairs grow on the lining of the nasal passages and act as a coarse filter which strains dust particles from inhaled air. This dust may then be expelled from the nose. There are specialized epithelial cells in the windpipe and bronchial tubes with numerous cilia on their free surfaces, which wave and fan bacteria or very fine dust particles up and out. The foreign particles are then coughed out of the throat.

The gastric juice in the stomach probably digests many bacteria with the food. The acid of gastric juice sometimes destroys or inhibits the growth of many germs. Such bacteria then pass on through the canal with the food. The high acidity in a dog's stomach will kill bacteria that will cause intestinal infections in man. Tuberculosis, typhoid fever, and dysentery germs are not affected by the action of gastric juice and will pass into the small intestine. Bacteria of putrefaction are always abundant in the large intestine. If they are too active, intestinal disturbances. result. Most bacteria can work only in an alkaline medium; therefore to control and inhibit their activity, efforts are made to introduce acids into the large intestine. The acids in most foods are absorbed or neutralized before they reach the large in-
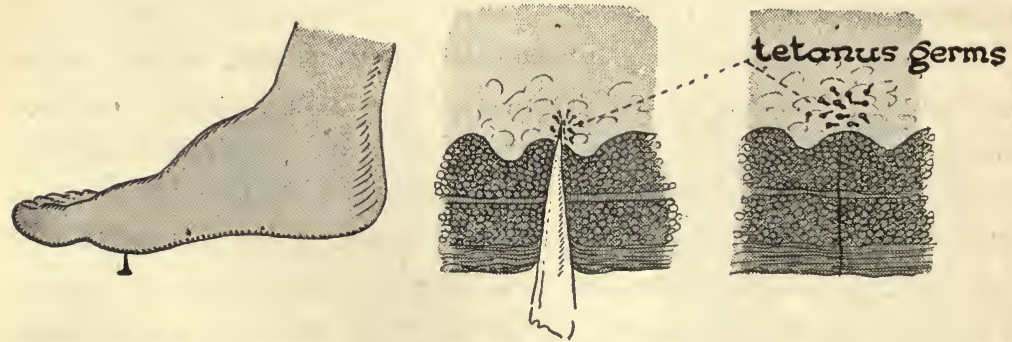

Stepping on a tack, rusty nail, or other sharp objects may set up an infection. Tetanus has been known to get into the body in this way and cause blood poisoning.

testine. A certain bacillus, called the Lactobacillus bulgaricus, is one of the organisms causing the souring of milk. When this 
bacillus is taken into the body, it is thought to go into the large intestine, where it creates an acid medium. Some scientists think this aids the work of the bacteria of putrefaction and, consequently, clears up auto-intoxication. Recently another strain of bacteria that sours milk has been found that seems to be more effective. It is the Lactobacillus acidophilus. These bacteria will live longer in the intestine than do the Lactobacillus bulgaricus.

There are a number of other conditions that protect the body against the invasion of germs. Even after germs enter, very few can cause a disease unless they multiply in great numbers. The body may be thought of as a great living culture medium. The inside is dark, moist, warm, and supplies food in the form of digested foods or tissue cells for invading saprophytes or parasites. In reality, each specific germ needs a definite combination of conditions. For example, the temperature of the body is not high enough for the bird type of tuberculosis. Therefore, this type cannot attack man. The human type grows only between $37^{\circ} \mathrm{C}$. and $40^{\circ}$ to $41^{\circ} \mathrm{C}$. A bird's temperature is much higher than man's, so that human tuberculosis cannot grow in birds. Bacteria that ordinarily attack warm-blooded animals are not likely to affect cold-blooded ones. If, however, the temperature of the cold-blooded animals is raised, they become susceptible to the invading germs. Frogs are naturally immune to tetanus, but if their temperature is raised, they become susceptible to the disease. Bacteria such as tetanus are anaërobic and grow only in the absence of air. If tetanus enters a surface wound exposed to air, it does not multiply. It is only when it enters deep wounds, where there is no air present, that it sets up an infection. Most tissue cells offer a high natural resistance to the entrance of germs. It is only when they are torn or lacerated that conditions are favorable for the growth of germs in them. Many bacteria taken in with food are of the saprophytic type. They make no attempt to attack the tissues but simply feed on food in the alimentary canal. 
When bacteria actually enter the body and get into the blood or lymph stream, there are many methods of protection. The

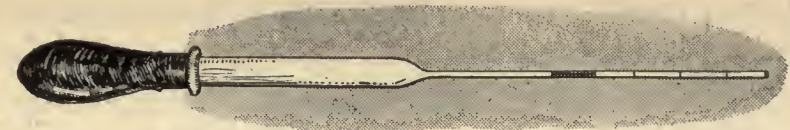

\section{Opsonic Index Determination}
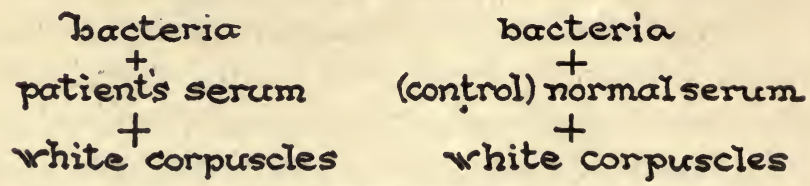

$$
\begin{gathered}
48 \leftarrow \begin{array}{l}
\text { percentage corpuscles } \\
\text { ingesting bacteria }
\end{array} \\
\frac{48}{24}=2 \text { patient's opsonic index }
\end{gathered}
$$

Opsonins affect bacteria so that white corpuscles can more easily engulf them. Definite opsonic indexes may be calculated from microscopic examinations. A pipette, marked as shown in the diagram, is used to measure the amounts of bacteria in the patient's serum and white corpuscles used in the test.

white corpuscles devour bacteria and digest them. Thus, the body is rid of them. If numbers of bacteria get into the lymph system, they are frequently carried to a lymph node or gland, where the bacteria are filtered out. Then a collection of corpuscles attack the invading germs and destroy them. When a number of tuberculosis germs are combating white corpuscles in a lymph gland, the body sometimes deposits a wall of calcium around the infected center. This removes the whole mass of infected material from the circulation. Infected lymph glands are sometimes cut out of the body to prevent a possible reinfection from the tuberculosis germs inclosed.

The type of white corpuscle that devours bacteria is the phagocyte. When germs invade the body, there is an increase in the number of phagocytes produced in order to check the infection. If blood is examined, and the number of white corpuscles is very great, this may indicate an infection in the body. 
Antibodies. There are many protective substances produced in the blood in response to the entrance of disease germs or their poisons. These substances are known as antibodies. Both toxins and antibodies are specific for each disease. The toxin produced by a typhoid bacillus will cause only typhoid and will never cause diphtheria or any other disease, and the antitoxin made by the body to fight diphtheria will have no effect on any other disease but diphtheria. This is true of all antibodies. Antitoxin is one type of antibody produced in the blood for the neutralizing of exotoxins. There may be antitoxins in the blood for diphtheria, others for scarlet fever, and still others for tetanus. The antitoxins neutralize the toxins produced by the bacteria and at the same time the phagocytes destroy the actual bacteria.

Other protective bodies called lysins are present in normal blood, which actually dissolve the bacteria. The lysins which dissolve the bacteria are known as bacteriolysins. There are specific bacteriolysins produced to dissolve diphtheria germs. Others dissolve typhoid bacilli and still others dissolve meningitis germs. There are other kinds of lysins besides bacteriolysins. One kind may dissolve foreign red blood corpuscles and is called hemolysins. In blood transfusions, if one group of blood is introduced into another group of blood, the hemolysins of the first blood may dissolve the corpuscles of the second type. Therefore, transfusions are made only among bloods belonging to similar groups.

Certain protective substances are produced in blood to assist the white corpuscles. For example, agglutinins cause the germs to stop moving and to gather in clusters or clumps. Then the phagocytes and lysins can destroy them more quickly. Agglutinins are produced in the body in combating typhoid.

There are also precipitins known to the biologist. Their action is similar to that of agglutinins. They are specific for different types of foreign proteins, bacterial and otherwise. Precipitins harden 
or precipitate foreign proteins out of the blood. They are used as a test for specific bloods, human and other animals.

Opsonins prepare the germs for ingestion by the white corpuscles. Opsonins seem to combine in some way with the bacteria and so alter them that the white corpuscles can better engulf them. There are different opsonins produced by the blood in combating tuberculosis, boils caused by a staphylococcus, and meningitis. When opsonins are present, the white corpuscles are better able to destroy the bacteria. The test for the amount of opsonins in a patient's blood is a very interesting one. For example, blood is taken from a patient suffering from tuberculosis, and the serum is separated from it. The amount of opsonins in this serum is to be measured. The serum is mixed with white corpuscles from a healthy animal or person. The white corpuscles are well washed to make sure that no opsonins are in the mixture before the serum is added. The serum and white corpuscles are then added to some tuberculosis bacilli. A little of this mixture is put on a glass slide, stained, and examined under the microscope. Then a count is taken, either of the number of bacteria ingested by the first hundred corpuscles seen, or an estimate of the per cent of the first hundred white corpuscles seen to have ingested bacteria. The second estimate is easier than the first because the white corpuscles devour so many bacteria that in some cases it is impossible to count them. The estimate is then compared to normal blood in order to see the increase of opsonins present, and thus determine the increase in the activity of the white corpuscles. Any difference in the white corpuscle activity is attributed to extra opsonins developed in the patient's blood.

The relation of mental poise to disease. In his farewell address as the retiring dean of the college of physicians and surgeons of one of the large universities, a famous doctor stated that the sugar pill was the outstanding discovery of his generation. He stated in very decided terms that his long years of experience had con- 
vinced him that it was not so much the medicine as the fight that the patient made, which augmented and strengthened the defenses of the body. A sense of humor, joy, courage, optimism, and faith are true defenses against disease. A healthy and contented mind in a properly functioning and intelligently cared for body is the goal that each reader should strive to achieve.

\section{Questions and Suggestions}

1. Discuss three ways in which bacteria attack the body. Illustrate each with a specific disease.

2. Discuss the different ways in which bacteria enter the body.

3. Discuss the natural defenses of the body against the invasion of bacteria.

4. Discuss the protective substances found in the blood, which prevent the activity of the bacteria in the body.

\section{SupPlementary Readings}

Broadhurst, J., How We Resist Disease (J. B. Lippincott \& Co.).

Greaves, J. E. \& E. O., Elementary Bacteriology (W. B. Saunders Co.). Zinsser, Hans, A Textbook of Bacteriology (D. Appleton \& Co.). 


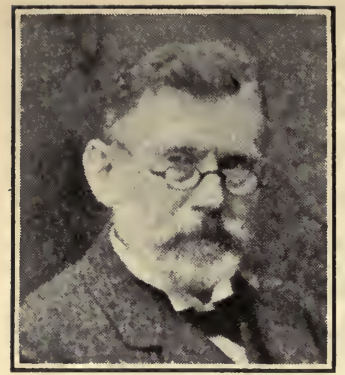

Paul Ehrlich

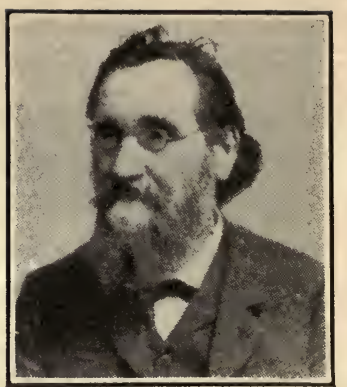

Eli Metchnikoff

Why can some people resist disease successfully and others have very little resistance? What theory of immunity is most generally accepted? Can all people be made immune to disease?

The ability of the body to resist disease is known as immunity. To-day, when the emphasis is placed on preventive medicine rather than curative, immunity is one of the most important phases of biology. It is a comparatively new science. Two men largely responsible for founding the science of immunity were Eli Metchnikoff and Paul Ehrlich.

Eli Metchnikoff. By 1883, Pasteur and Koch had succeeded in arousing in many scientists an interest in microbes. Metchnikoff, a Russian naturalist working in Sicily, studied the way sponges and starfish digest their food. In investigating these animals he noticed certain cells moving in their bodies. These wandering cells acted and looked like amoebas. He fed particles of powdered carmine to the transparent larvae of starfish and the wandering cells ingested the particles. Metchnikoff wondered whether these wandering cells would engulf microbes. He stuck some thorns from a rose bush into the transparent starfish. Masses of the wandering cells crowded around the slivers. He concluded that these cells killed invading germs, and 
he gave to them the name phagocytes. He published many articles and gave lectures concerning his discovery.

Metchnikoff went to Paris to continue his work in Pasteur's laboratory. Pasteur believed in Metchnikoff's theory of phagocytosis, but Von Behring did not. The latter had already demonstrated that if tiny quantities of the poisons of tetanus and diphtheria were injected into rabbits, the rabbits became used to the toxins and did not become ill. Von Behring thought that chemical substances in the blood were responsible for this protection. This experiment was done before the germ of tetanus had actually been discovered. Von Behring felt certain that the plasma of the blood and not the phagocytes killed the germs and their poisons. We now know that both Von Behring and Metchnikoff were right. Not only do phagocytes devour germs, but blood produces protective substances, lysins, that dissolve bacteria, and antitoxins that neutralize bacterial toxins. Metchnikoff formulated the phagocytosis theory of immunity, in which he stated that phagocytes alone were responsible for immunity. Later he agreed with Von Behring, that the blood contained other antibodies.

Paul Ehrlich. A German medical student, Paul Ehrlich, was working on the staining of tissues. He believed that the reaction of certain bacteria to special drugs or stains might be a method of killing these bacteria without injuring the organism they were invading. If he could find stains or dyes with the ability to attach themselves to certain bacteria, he might introduce a poison with the dye and thus kill the dyed bacteria. In his staining experiments he had seen tuberculosis bacilli before Koch had, but had not recognized them nor described them as such. When Koch isolated the tuberculosis bacilli, Ehrlich showed him a simple method of staining them. Ehrlich inoculated mice with different dyes to see whether he could not make them immune to a certain disease caused by a spirochaete. In 1893, after over six hundred attempts, he discovered an arsenic WH. FITZ. AD. BIO. -33 
compound called 606, which gave immunity to the mice. Ehrlich tried his 606 on a certain disease in human beings caused by spi-
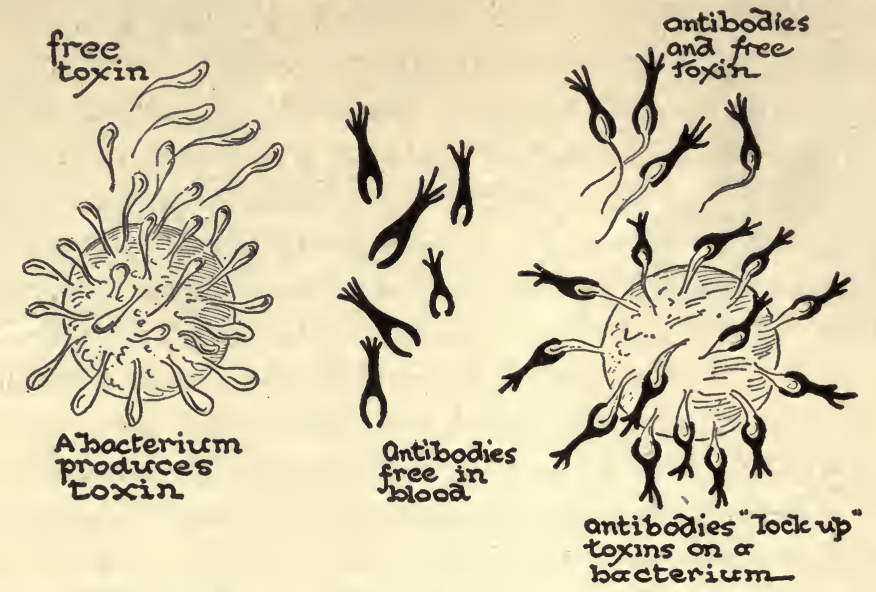

Diagrammatic representation of toxins uniting with antitoxins. The toxin has stimulated the cell to develop an antitoxin which locks or neutralizes the toxin. This is according to the sidechain theory of Paul Ehrlich.

rochaetes and found that it cured them, also. He called the arsenic compound salvarsan. He thought the salvarsan united with the cells of the body and helped to produce animmunity. Instead, it was later proved that the dye united with the spirochaete and killed it.

Ehrlich was the founder of the humoral or side-chain theory of immunity, which is still generally accepted by biologists of to-day. The humoral theory states that protective substances are produced within the body, usually in the blood, which counteract the effects of bacteria. Metchnikoff and Ehrlich stimulated research in the study of immunity. Since then there has been much progress. To-day, it is one of the big features in preventive treatment. Many of the cures and discoveries of to-morrow will be built on the foundations laid by these men.

Types of immunity. Natural immunity is the immunity one has at birth. It stays with the individual always and, therefore, there 
is no need of an inoculation, nor any danger of an attack of the disease against which there is the immunity. That there is a natural immunity of species is shown by the innumerable diseases of animals to which humans are not susceptible. Similarly, animals contract very few of the human diseases. A natural immunity is, also, evidenced by various groups of the same species, although this is somewhat relative. The natives of South America and Africa are more immune to yellow fever than are the white people. Perhaps this is due to a weeding-out process during which the fittest has acquired an immunity while the unfit died. This does not always explain natural immunity, however. Measles is a mild disease with white people, but fatal to natives of certain South Sea islands. Jews are more immune to tuberculosis than Irish and English people. Eskimos, Indians, and Negroes are highly susceptible to tuberculosis. The North American Indian seems to be immune to scarlet fever. The colored people in the southern part of the United States seem to have a natural resistance to diphtheria. This type of natural immunity is often called racial immunity. Certain members of the same race show a natural immunity to disease while others do not. For example, some children seem naturally immune to diphtheria as seen in their reactions to the Schick test. Whether natural immunity is the result of natural selection, or whether it can be explained by environmental conditions, is still not decided.

Acquired immunity differs from natural immunity in that it is developed during the lifetime of the individual. There are two types of acquired immunity, active and passive. Active acquired immunity is produced by the body itself as a result of having the germs or the toxins of a disease enter the body. There are three ways in which this may be accomplished. (1) By an actual attack of the disease. If this method produces immunity for any appreciable length of time, it is likely to last for life. For example, once a person has had typhoid, smallpox, or diphtheria he is likely to 
develop an active immunity which lasts for life. (2) By vaccination. The introduction of dead or attenuated (weakened) germs of the disease, in small doses, stimulates the body to produce its own antibodies. In vaccinating against typhoid and yellow fever, the dead organisms are used. In smallpox, germs weakened by passing them through a cow are used to make up the vaccine. This is known as animal passage. In rabies, the germs are attenuated by drying them. Germs may also be weakened by the application of slow heat or by growing them on media that are not quite favorable. In each of these cases the body cells respond to the inoculation by producing antibodies. (3) By the injection of toxin from which the bacteria have been filtered. Sometimes the toxin has some antitoxin mixed with it to dilute and make it safer, as in diphtheria toxin-antitoxin. Sometimes small quantities of the toxin itself are used, as in scarlet fever immunization. Here, again, the body produces antibodies. All actively acquired immunity is usually lasting in its effects. Active immunity takes some time to produce because the cells require time to make their reactions.

Passive acquired immunity is obtained by the injection of antitoxins or immune serums from the body of another person or an animal. Such immunity is immediate in its effects, but it does not last for very long. The antitoxin is already prepared. It sets to work promptly neutralizing the toxin present. The injection of such material does not stimulate the body to produce its own antibodies and hence this immunity lasts for only a short time. It is used in the actual treatment of the disease or to protect some one who has been exposed. For example, diphtheria patients and their families are given antitoxin. An immune serum is frequently given for pneumonia and infantile paralysis.

The immunity of to-morrow. A very recent investigator, d'Herelle, has demonstrated the presence of what he has called a bacteriophage, a kind of super bacteria that destroy other bacteria 
and produce an immunity. Experiments have been made in France and are now being conducted at Harvard and Yale universities with this material. Watch for results of the work. The problem of immunity is still far from its solution. Scientists are just beginning to solve many of the questions. You who are to be scientists of to-morrow will have opportunity for research in this and many other fields of biology.

\section{Questions And Suggestions}

1. Discuss Metchnikoff's experiments with phagocytes.

2. Give a report on the life and work of Metchnikoff.

3. Discuss Ehrlich's experiments with dyes and chemicals on bacteria.

4. What theory of immunity was founded by Ehrlich?

5. Give some interesting facts about the life and work of Ehrlich.

6. Discuss natural immunity; acquired immunity.

7. What test may be used to see whether a child is or is not naturally immune to a certain disease?

8. What specific inoculations should be given all children to make them actively immune to diseases with which they come in contact?

9. What specific inoculations should be given a person who is traveling to make him actively immune to diseases with which he might come in contact?

10. State two differences between active and passive immunity.

11. Under what conditions will a person be actively and passively immunized against diphtheria?

12. Answer some objections that may be currently raised against immunization. 


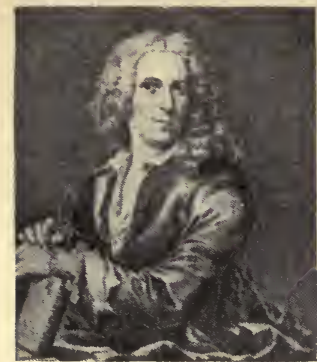

Linnaeus
CHAPTER L

TAXONOMY

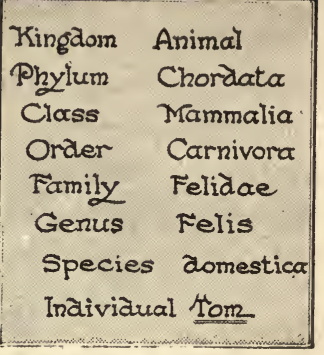

A cat named Tom

How can the many plants and animals of the world be identified? What is the purpose of identification? What is taxonomy? What contributions made by scientists have helped to systematize the classifications of plants and animals?

It is said that Alexander the Great was a pupil of Aristotle, the Father of Biology. Alexander held his teacher in the highest esteem, and, during his campaign and conquests, kept a group of couriers to carry unusual plant and animal forms back to his friend and teacher. These specimens came in such large numbers that Aristotle had to devise a means of caring for them in an orderly manner. He used a system of classification that was largely based on the habitat of the organisms. For his animals he had eight groups, four of which were blood-containing, and known as mammals, birds, egg-laying quadrupeds, and fishes; while four were bloodless, namely, squid-like animals, crustacea, insects, and animals with shells.

The binomial system of Linnaeus. Various other scientists changed this system, but Carolus Linnaeus, a Swedish naturalist, devised the system upon which the modern method of classification is based. Before his time, in order to describe a kind of grass and not confuse it with other forms more or less 
like it, a descriptive name similar to the following was used: Gramen Xerampelino, Miliacea, praetenius ramosaque sparsa panicula, siva Xerampelino cogener, arvense, aestivum. Carolus Linnaeus was an assistant to the Professor of Botany in the University of Upsala, Sweden. While cataloguing plants, he had come across many descriptions such as the one just given. He realized that such a name was too long and inconvenient and he initiated a number of reforms in the existing scheme of classification. He gave the name "Pea bulbosa" to this plant. In his system which he called the binomial nomenclature, two names sufficed to distinguish this organism from all others. One name designates the genus of the plant or animal, the other the species. All organisms that have similar characters are grouped together and this group is called a genus. The name given them is the generic name. The genus is subdivided into groups with varying characters called a species and the name given is the specific name. The generic name for all the members of the cat tribe is Felis. The genus Felis is subdivided into the species such as leo (lion), pardus (leopard), domestica (house cat). Therefore, the lion is known as Felis leo, the leopard as Felis pardus, and the house cat as Felis domestica. The initial letters of the name of the genus is always capitalized and that of the specific name is written with a small letter.

What a task Linnaeus undertook! He published his most important work, Systema Naturae, in twelve editions and like all scientists of his time, wrote the descriptions in Latin. This practice continued until very recently. Exchanges of scientific papers on classifications could be made throughout the world in a common language. Recently, scientists have employed their mother tongue for the description of any new creature. When the discovery is an important one, the paper is translated by those who need the data. Linnaeus died in 1778, but his work lives on and, with a few modifications, is still used to-day. 


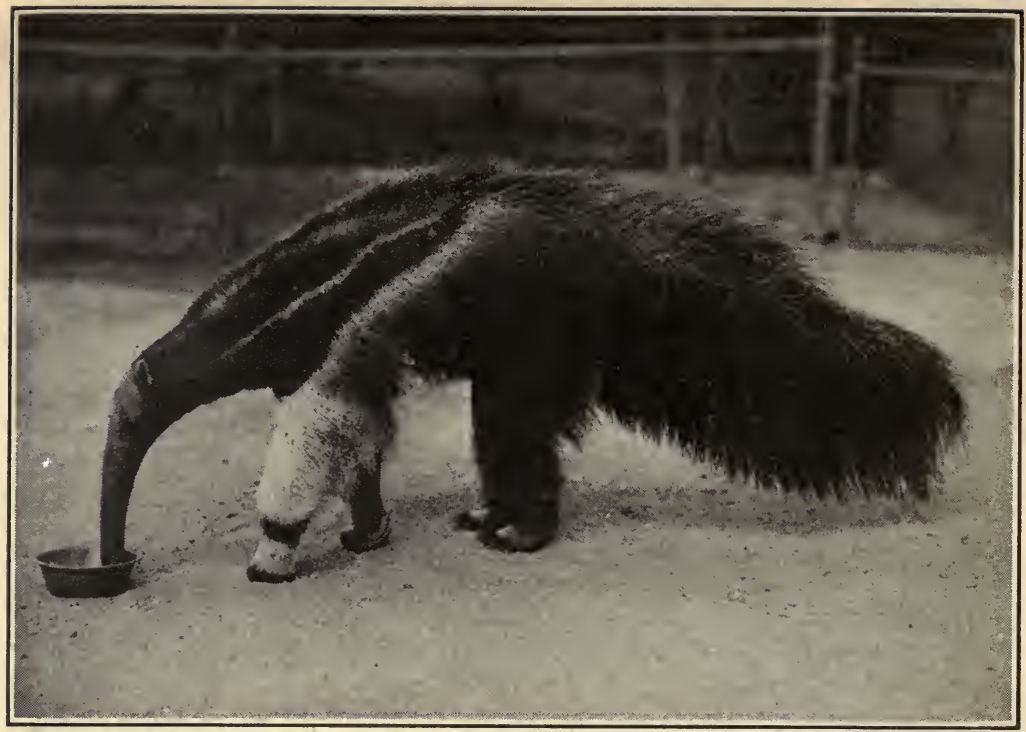

N. Y. Zoölogical Society

There are marked differences in size, shape, and habits of present day mammals. The giant anteater, Myrmecophaga jubata, is a native of tropical America. Its coarse hair is almost bristle-like in structure. The long narrow snout and a long tongue enable it to lick up insects.

The basis of the classification of to-day. The scientist of to-day knows many more animals than Aristotle or Linnaeus knew. There are probably more than a million different kinds of animals now known, and nearly as many plants. With the method of identifying and classifying animals and plants in an orderly and scientific manner there has grown a division of biology, taxonomy, that is concerned with this classification. Taxonomy (from taxis - arrangement; nomos - law) is the division of biology that has to do with the classification of animals and plants on the basis of fundamental similarities. The modern method of classification of plants and animals does more than catalog animals for the convenience of the scientists. It expresses a kinship and relationship. The relationship of plants and animals has been de- 
termined from their structure, embryology, habits, habitat, ability to crossbreed with related forms, and from certain other facts.

By structure is meant the shape and arrangement of the parts of a plant or animal. Certain plants are grouped together because they have chlorophyll; certain animals are classified together because they have six legs. The embryology of a plant or animal refers to its development from the time it is first formed until it is a fullgrown adult. A caterpillar looks somewhat like a worm which is really one of the stages in the life history of an insect. Plants and animals that have similar embryological developments are closely related; those with dissimilar embryological developments are not closely related. Animals and plants which are closely related usually have some similar habits. In general, most water plants

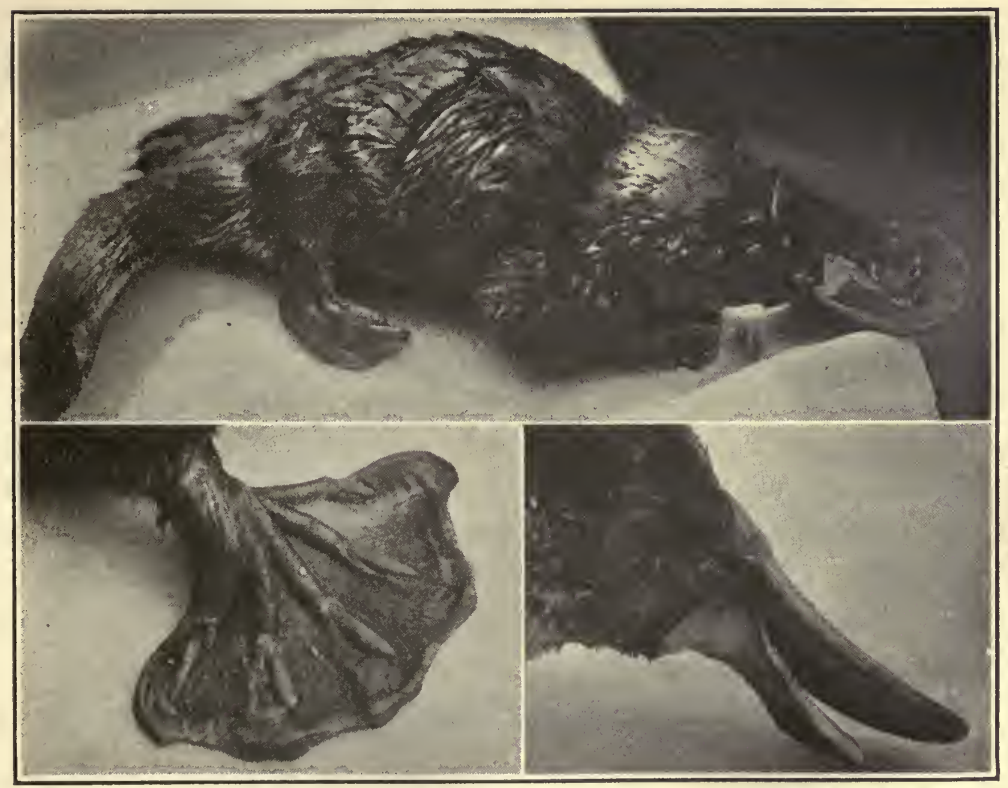

N. Y. Zoological Society

The duckbill (Ornithorhynchus anatinus) is an egg-laying mammal, native of Australia. It is covered with dark fur, and has a bill and five-toed webbed feet. 
are closely related. Animals that carry their young in pouches are classified together. Birds that have wading habits belong to the

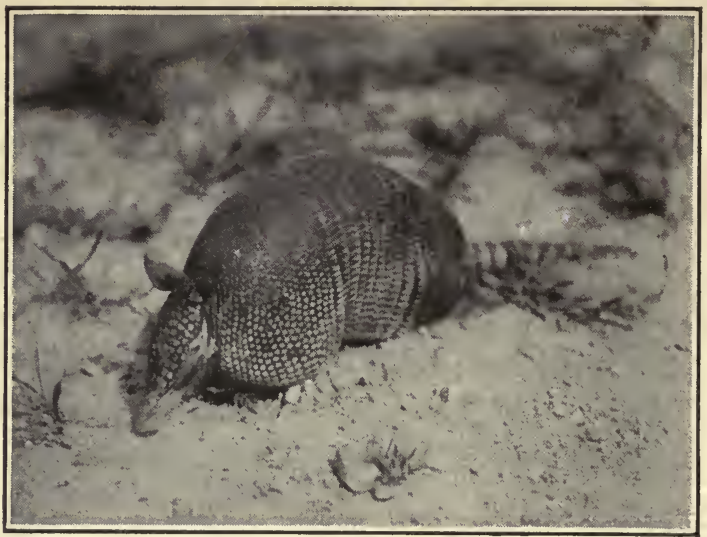

N. Y. Zoological Society

The Armadillo (Tatu novencinctum) is covered with a bony shell. Some species can curl up into a ball, presenting the armor on all sides.

same group. Habits alone do not determine classification; other facts must be considered. The whale is frequently thought of as a fish because it swims and lives in the water. Actually it is a mammal because it suckles its young. When animals or plants crossbreed with each other, they are usually found to be closely related. For this reason successful crossbreeding is only possible with those forms that are closely related. For example, the horse and donkey will crossbreed and produce a mule. The mule is probably always sterile, and will not produce offspring. Dogs and wolves will crossbreed and the hybrid offspring will reproduce. Consequently, dogs and wolves are thought to be more closely related than are horses and donkeys.

The Law of Priority. So many modifications of Linnaeus' system of classification arose that confusion resulted. An International Commission on Classification was founded in 1895. Its purpose was to draw up a set of rules which would be accepted and 
adopted by the scientists of the entire world. One of the basic rules formulated by this commission is the Law of Priority. This has to do with the scientific name each animal and plant is to bear. Suppose an unfamiliar microscopic animal is observed. The scientist draws it, photographs it, perhaps he kills it, and studies it as stained and sectioned material. He then consults the descriptive classification organized since 1895 and seeks the proper place to catalog his specimen. If the specimen is not similar to any known organism, the scientist gives it a generic and specific name, writes a description, and publishes his findings. If no other scientist reading his paper has ever come across it or seen a description of it elsewhere, this scientist is thereafter credited with the discovery. He has added a new creature to the list of known organisms.

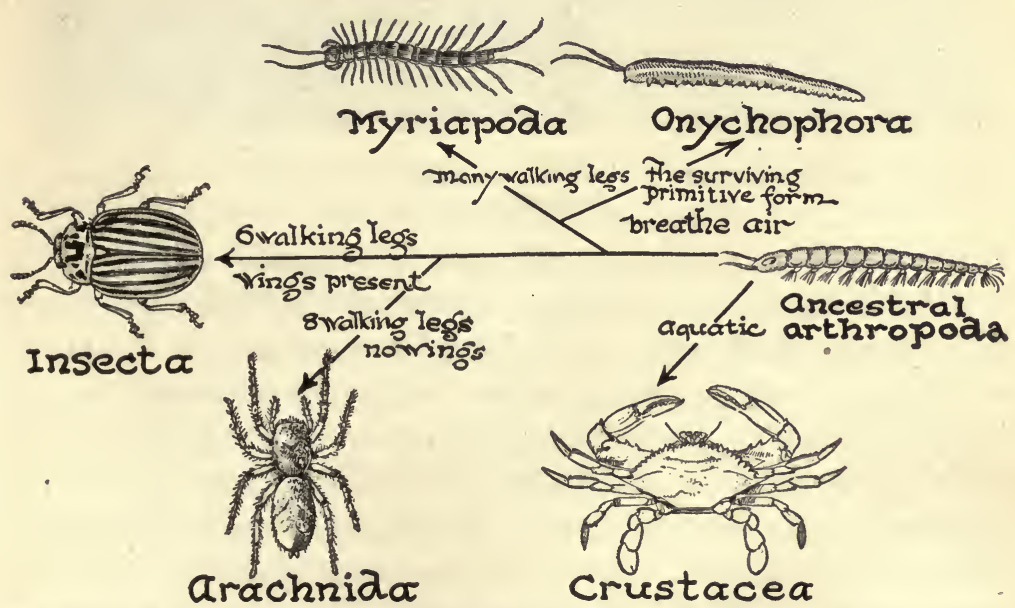

The phylum Arthropoda is subdivided into five present-day classes. There are many fossil arthropods, some of which are quite unlike present-day forms.

The method of the classification of to-day. All living things are placed into one of two kingdoms, the plant kingdom or the ani- 
mal kingdom. These two kingdoms are further divided. Certain outstanding likenesses enable a scientist to divide the plant

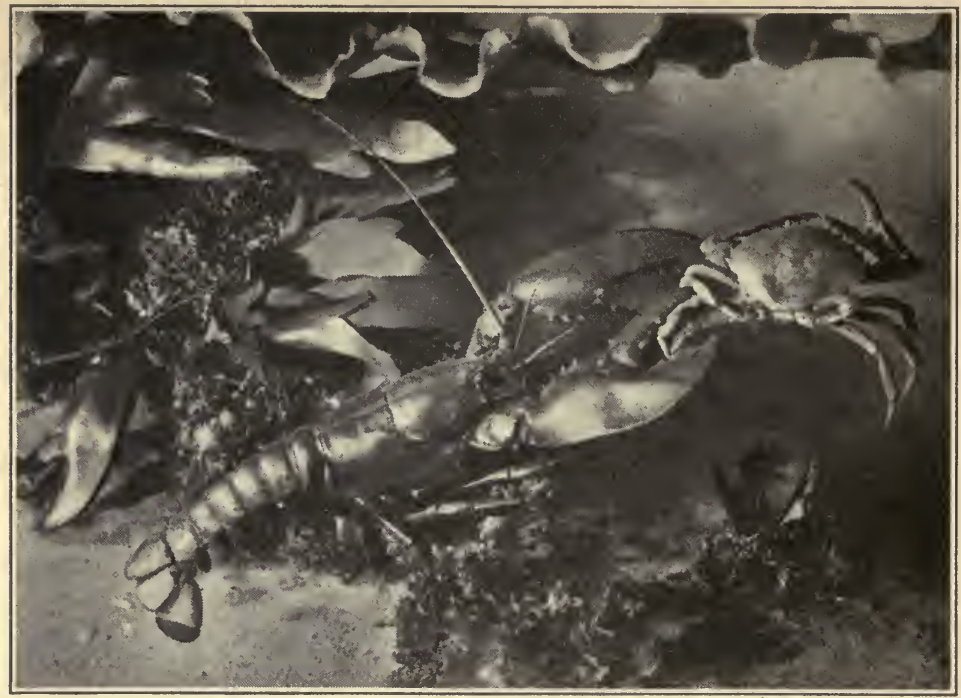

Museum of Natural History

The crustacea vary in size and shape from microscopic representatives to lobsters weighing nearly fifty pounds.

kingdom into four divisions called phyla (singular, phylum). In this book the animal kingdom will be divided into ten phyla. The various phyla have been carefully analyzed and compared, and finer groupings, called classes, made. Again a finer sorting is made and the classes are split into orders. Certain characters again permit a subdivision of the orders into genera. The genera are separated into groups showing very close relationship. These are the species. They are very much alike, but may still differ slightly in form, habitat, or distribution.

One phylum of animals, the arthropoda, is distinguished from the other ten phyla in that all of its members are segmented, have an exoskeleton and jointed legs. One of the classes of the phylum arthropoda has a characteristic exoskeleton impregnated 
with lime. The organisms with a lime skeleton are put in the class called the Crustacea. All the arthropods with lime skeletons and ten legs are then grouped into an order, the Decapoda. In this order are found the crabs, lobsters, crayfishes, and the like. The different members of the order Decapoda are then separated into various genera and species. The sea-living lobster belongs to the genus Homarus, while the land-living and fresh-water crayfish belongs to another genus, Cambarus. There are different species of lobsters in the genus Homarus. The North American lobster belongs to the species americanus which is slightly different from the European lobster, vulgaris. The farther down the scale of classification the closer is the relationship. Species are more closely related than genera and, in turn, genera are more closely related than orders, etc.

Phylum Arthropoda.

Class Crustacea.

Order Decapoda.

Genus Homarus.

Species americanus (American Lobster).

The Paramecium may be similarly classified.

Phylum Protozoa (one-celled animal).

Class Infusoria (numerous hairlike processes used for locomotion and feeding, presence of fixed openings for food ingestion and the extrusion of solid wastes).

Order Holotrichia - animals with cilia of equal length distributed over the entire body.

\section{Genus Paramecium.}

Species caudautum.

Using this modified Linnaeus method, let us consider a buttercup. It belongs to the great branch of phylum of the plant kingdom known as the Spermatophyta. It is placed in the class Angiospermae. It is of the genus Ranunculus, and there are many species, 
The classification of man is another example.

Phylum Chordata (most members have backbones and have central nerve cords).

Class Mammalia (presence of hair; the young are fed on milk; a muscular diaphragm separates the thorax from the abdomen).

Order Primates (erect or nearly so).

Genus Homo.

Species sapiens.

Classification of plants.

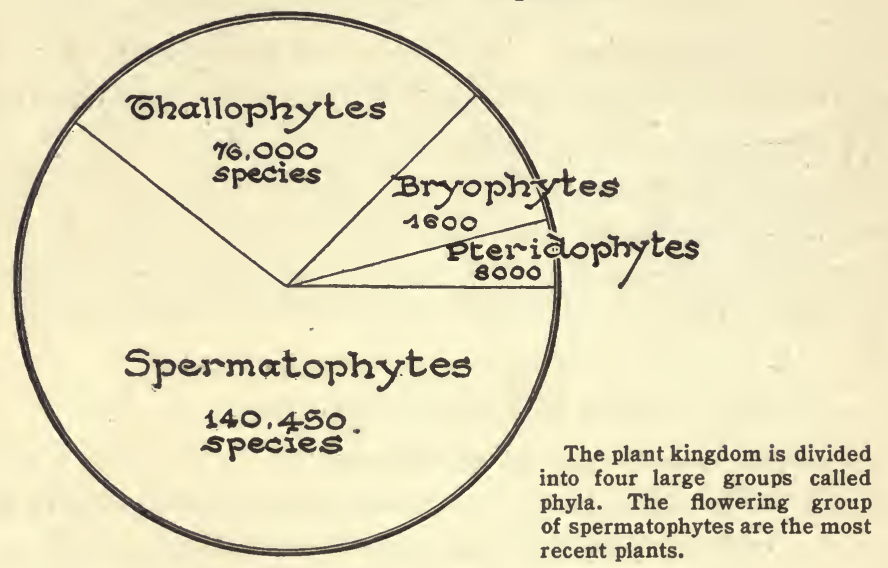

I. Phylum THALLOPHYTA (thallus - young branch; phyton - plants). Includes very simple plants, sometimes single-celled, but more often many-celled; some have chlorophyll, others are without this green material; none have roots, stems, or leaves. There are two subphyla, Fungi and Algae. The Fungi are non-green plants of very great economical importance. There are four classes found in the subphylum fungi.

Class I - Fission fungi. Bacteria.

Class II - Tube fungi. Have tubular bodies. Example is bread mold. 
Class III - Sac fungi. Produce spores in a sac. Examples are the yeasts, powdery mildews, and many others.

Class IV - Club fungi are so-called because their spores are produced upon a club-shaped structure. Mushrooms, puffballs, smuts, and rusts belong to this group.

The subphylum Algae includes the chlorophyll-bearing thallophytes. In some forms the chlorophyll is masked by some other coloring matter. They range from single-celled forms to filamentous colonies or even long ribbon or rope-like masses many feet in length. They are nearly all aquatic. The subphylum Algae is subdivided as follows:

Class I - Blue-green algae contain a blue pigment in the cells in addition to the green color. Examples are Nostoc pruniforme and Oscillatori violacea.

Class II - Green algae are of countless forms, unicellular, filamentous, platelike, and in irregular masses of cells. There are both fresh-water and salt-water forms, and others live on land. One form will grow on snow patches. Pleurococcus vulgaris and Vaucheria terrestris are examples.

Class III - Brown algae are nearly all marine plants. They are the commonly known seaweeds.

Class IV - Red algae are mostly marine. Our most delicate and beautiful seaweeds belong to this main class.

II. Phylum - BRYOPHYTA (Gr., bryon-moss; phytonplant). Contains only two classes, the liverworts and the mosses. These plants are small and live mostly on land. They show a greater development of tissues than the algae and may be either thallus-like (liverworts) or have stems with rootlike projections and very simple leaves. They reproduce by forming spores.

III. Phylum - PTERIDOPHYTA (Gr., pteris - fern). This includes a group which, when the world was younger, played a very important part in the vegetation on the earth. Most coal is 
made from ferns of the past. They have true roots, stems, and leaves, but reproduce like the mosses, by forming spores. The Pteridophyta include three classes: the true ferns, the horsetails (Equisetum), and the lycopods or club mosses.

IV. Phylum - SPERMATOPHYTA (Gr., sperma - seed). The seed-bearing plants are grouped into two subphyla. The Gymnospermae (Gr., gymnos - naked), or naked-seeded plants, include a small group related to the ferns on one side and the flowering plants on the other. Two classes are found in this subphylum: the Cycads, of which the so-called sago palm is an example, and the Conifers or evergreens, as pines, spruces, firs, hemlocks, cypress, and others. The evergreens include the sequoias, the largest and oldest trees. The subphylum Angiospermae (Gr., angeion - case or vessel), or true flowering plants, include the common grasses and grains, flowering trees and shrubs, and flowering plants. It is divided into two sub-classes, Monocotyledones and Dicotyledones.

The classification of animals.

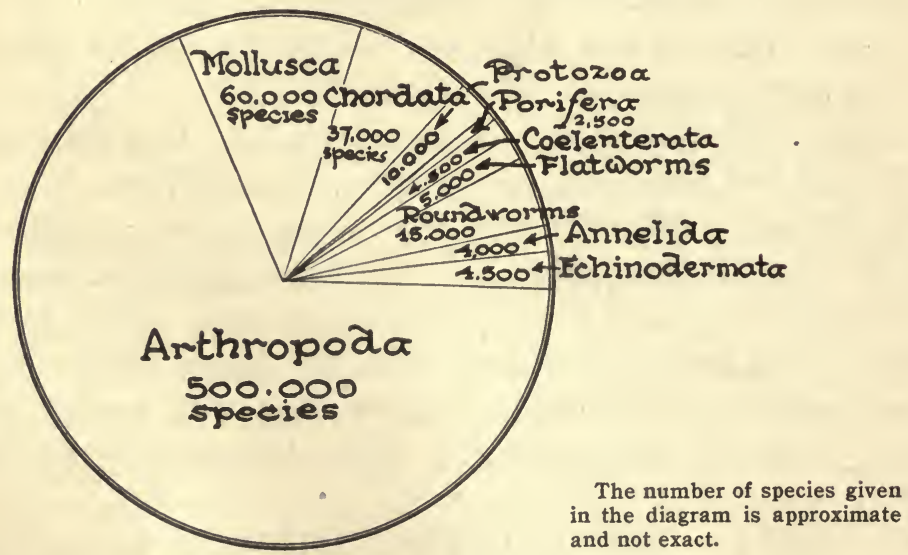

Phylum I-PROTOZOA (Gr., protos - first; zoon - animal). Single-celled animals without true organs, or tissues. Occasionally 
protozoa are colonial, in which case the unit cells of the colony are all potentially alike. There are four classes of protozoa.

Class I - Rhizopoda (root-footed). These cells have no fixed form. The Amoeba proteus is one of the best known animals in this class. The Amoeba histolitica causes a disease of the mouth. Amoeba dysentericus causes summer complaint in children.

Class II - Mastigophora. Move by one or more long, whiplike threads of cytoplasm called flagella. Euglena viridis is an example.

Class III - Sporozoa. Parasitic Protozoa, usually lacking motile organs or mouth. They reproduce by spores. Example: Plasmodium malariae.

Class IV - Infusoria. Animals which have many vibratile processes (cilia), a cuticle, and fixed mouths and anal spots. Paramecium caudatum, Vorticella.

Phylum II - PORIFERA (Lat., porus - pore; ferre - bear). Many-celled animals, so arranged as to form two layers of cells. Their bodies are usually penetrated by numerous pores. The cells of the body are supported by a skeleton of "spicules" or material called spongii.- There are three classes.

Class I - Calcarea. Sponges with spicules composed of calcium carbonate. Example: Grantia.

Class II - Hexactinellida. Sponges with spicules of silica triaxon in form. Glass sponges. Venus flower basket.

Class III-Demospongia. Sponges with skeletons of spicules of spongin or a combination of spongin and silicon. The bath sponge is an example.

Phylum III - COELENTERATA (Gr. koilus - hollow ; enteron - intestine). Composed of animals made of two layers of cells invaginated to form a gastrula or internal cavity; they have a mouth surrounded by tentacles and no anus. They are protected by stinging cells which also aid in killing prey.

Class I - Hydrozoa. Single animals like Hydra fusca or coloWH. FITZ. AD. BIO. -34 
nial animals, as Obelia weismania. These animals reproduce by buds, and by eggs and sperms. In the colonial types, certain buds of the original colony are free-swimming jellyfish. These produce the eggs and sperms.

Class II - Scyphozoa. Marine jellyfish, large in size. Examples are Aurelia flavidula and Portuguese man-of-war.

Class III - Anthozoa. Large hydra-like animals, single or colonial, usually attached, with many tentacles arranged in circles of multiples of five. The sea anemones and corals are the best known examples.

Phylum IV - PLATYHELMINTHES (Gr., platys - flat; helminthos - worm), or flatworms. Three-layered animals, bilaterally symmetrical, usually small, ribbon- or leaf-like, flat, and live in water. Most flatworms are parasitic. Examples are tapeworm and liver fluke.

Phylum V - NEMATHELMINTHES (Gr., nematos - a thread), or round worms. Three-layered, elongated, thread-like animals, often parasitic. Vinegar eels, the horsehair worm, the pork worm or trichina, the threadworm, and the hookworm are examples.

Phylum VI-ECHINODERMATA (Gr., echinos-hedge hog; derma - skin). Radially symmetrical, spiny-skinned animals which live in salt water, more complicated in structure than the worms. Five classes:

Class I-Asteroidea. Starfishes.

Class II - Ophiuroidea. The brittle stars or snake stars.

Class III - Echinoidea. Sea urchins.

Class IV - Holothuroidea. Includes the sea cucumbers.

Class V - Crinoidea. Stone-like, deep-sea forms, now almost extinct. Sea lilies and sea feathers are examples.

Phylum VII - ANNELIDA (Lat., anellus - a ring). Bilateral, segmented worms; composed of body rings or segments. The digestive tract is a tube within a tubelike body. No jointed appendages. There are two classes: 
Class I - Archiannelida. Primitive marine worms without parapodia or setae. Example: Polygordius.

Class II - Chaetopoda. Bristles along the body. Examples are the earthworm or sandworm.

Class III - Hirudinea. Without bristles and having suckers at both ends of the body. Examples are the leeches.

Phylum VIII. MOLLUSCA (Lat., mollis - soft). Soft-bodied unsegmented animals, often provided with a shell which is secreted by a part of the body.

Class I - Gastropoda (belly-footed). With or without shells, which, when present, are of one piece and coiled. Snails, whelks, and slugs.

Class II - Pelecypoda (hatchet-footed). Shells in two valves or parts. Clams, oysters, scallops, and mussels.

Class III - Cephalopoda (head-footed). Foot partly surrounds head and bears tentacles or grasping organs. Squids, octopuses, and cuttlefishes.

Phylum IX - ARTHROPODA (Gr., arthros - joint; pous foot). Animals are segmented, and have chitinous exoskeletons and jointed appendages. They live in water, on land, in the air,

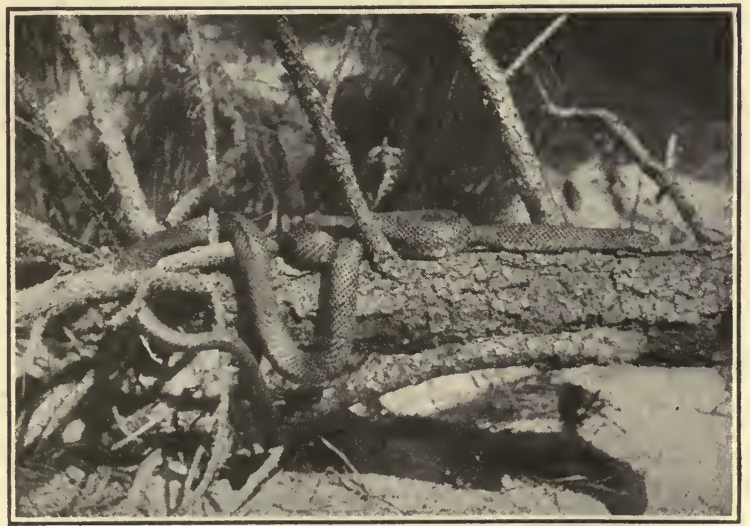

The reptiles are legless vertebrates with a skin covering made of scale-like plates. 
or in all three places. Most of them undergo a metamorphosis. There are five classes:

Class I- Onychophora. Primitive air-breathing arthropods with tracheae and nephridia. Peripatus capensia.

Class II - Crustacea. Breathe by means of gills. The head and thorax are fused; two pairs of antennae. They have a "crusty" exoskeleton, strengthened with lime. Examples, crabs and lobsters.

Class III - Myriapoda (numberless legs). Have long bodies with many segments and many paired jointed appendages; breathe by tracheae. Centipedes and millipeds are examples.

Class IV - Arachnida (Gr., arachne - spider). This group has no antennae, four pairs of legs, and a pair of clawlike appendages on each side of the mouth. Head and thorax combined as in crustacea. Breathe by book-gills, book-lungs, or tracheae. The spiders, daddy-long-legs, scorpions, mites, and ticks are in this class.

Class V - Insecta. The largest class of animals. Body segmented; three body regions, head, thorax, and abdomen; three pairs of jointed legs; usually compound eyes; breathe through tracheae or air tubes. Insects.

Phylum X. - CHORDATA (Lat., chorda - cord). Animals having a skeletal axis, gill slits in embryo or adult, and a nerve cord dorsal to the alimentary canal. This phylum is divided into four subphyla, one of which is the Vertebrata in which a nerve cord is protected by a segmented, bony spinal column. These vertebrates are divided into seven classes.

Class I - Cyclostomata. Eel-like vertebrates with round mouths and without functional jaws, without scales and fins. Lampreys and hagfishes.

Class II - Elasmobranchii. Fishlike vertebrates without a bladder, with jaws, and with a cartilaginous skeleton. Sharks, rays, and skates. 


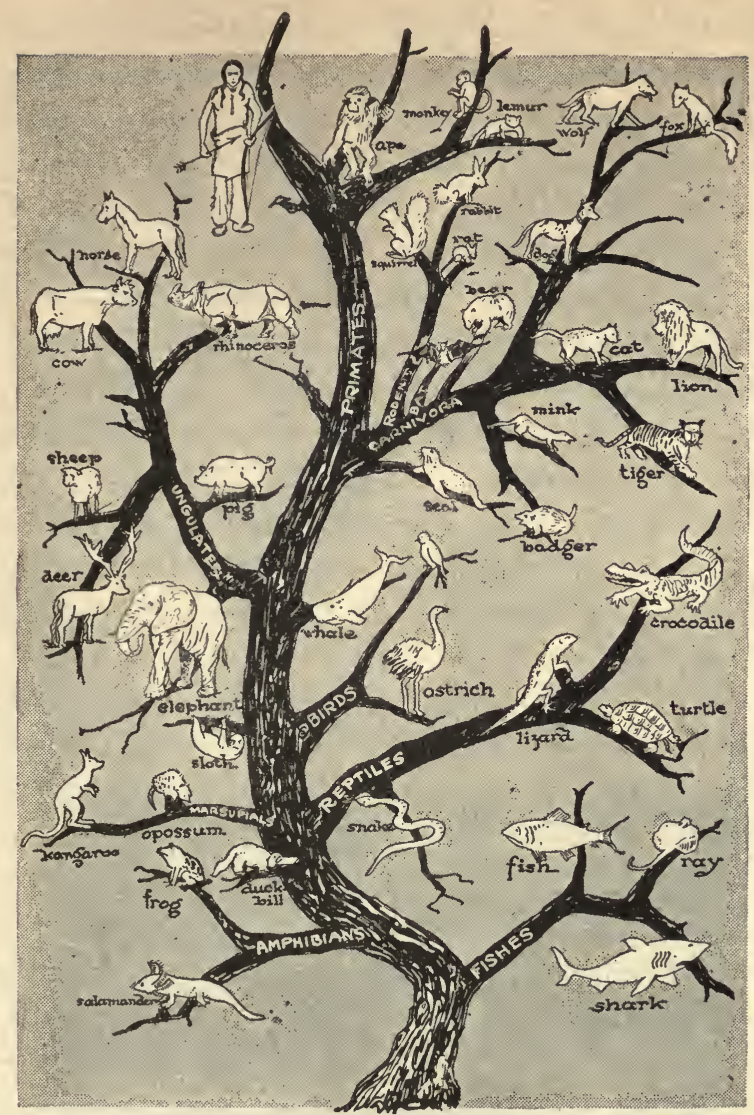

The relationships of the backboned animals is often shown by a tree. Animals supposed to have appeared early are on lower branches. The very recent animals and man are on the tips of the higher branches. Related animals are found on the same or nearby branches.

Class III - Pisces. The fishes. Aquatic, cold-blooded vertebrates breathing by means of gills, having an air bladder, a two-chambered heart, and a skinlike exoskeleton of scales.

Class IV - Amphibia. Cold-blooded vertebrates breathing by means of gills in some stage of their life history. Skin is not covered with scales, the heart is three-chambered. Most 
amphibians undergo a complete metamorphosis. The larvae form comes from the egg and live in the water and possess gills. Frogs.

Class V - Reptilia. Cold-blooded vertebrates usually covered with scales, breathing throughout life by means of lungs. The heart is three-chambered. Lizards, snakes, and turtles.

Class VI-Aves. The birds. Warm-blooded vertebrates covered with feathers. Front limbs are wings ; they have air spaces in the bones, no diaphragm, and a four-chambered heart. Birds lay eggs with a shell of lime, and usually care for their young.

Class VII - Mammalia. Warm-blooded animals covered with hair, at some stage. Usually have mammary glands and suckle the young. They have a diaphragm between the thorax and abdomen. This class may be divided into eleven orders:

Order 1 - моnotremata. Egg-laying mammals. Duckbill, spiny anteater.

Order 2-marsupialia. Carry immature young in a special abdominal pouch. Kangaroo, wombat, opossum.

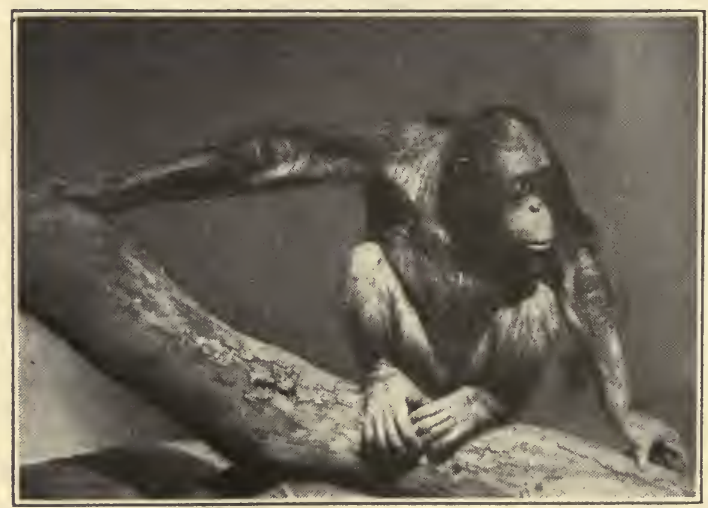

N. Y. Zoölogical Soctety

Darwin did not say that man came from monkeys. He and other evolutionists believe that there is a common ancestor of man and certain of the apes.

Order 3 - edentata. Toothless or with very simple teeth. Hairy anteater, sloth, armadillo. 
Order 4 - CÉtacea. Adapted to marine life. Whale, porpoise, dolphins.

Order 5-sirenia. Fishlike in form; pectoral limbs paddle-like. Examples: manatee, dugong, sea cow.

Order 6-Rodentia. Incisor teeth chisel-shaped, usually two above and two below. Examples: beaver, rat, porcupine, rabbit, squirrel.

Order 7 - ungulata. Hoofs; teeth adapted for grinding.

a. Odd-toe. Horses, zebras, rhinoceros.

b. Even-toe. Ox, sheep, antelope, camel, giraffe, deer, pig, hippopotamus.

c. Proboscidea. Elephants.

Order 8 - insectivora. Small, insect-eating, furry or spiny covered; long snout. Moles, shrews, hedgehog.

Order 9 - Chiroptera. Fore limbs adapted to flight, teeth pointed. Example: bat.

Order 10 - CARNIVora. Long canine teeth, sharp and long claws. Examples : dog, cat, lion, bear, seal, and sea lion.

Order 11 - PRIMATES. This order of animals includes man. Erect or nearly so, fore appendages provided with a hand. Lemurs, monkeys, baboons, mandrills, apes, gibbons, orangutans, chimpanzees, gorillas are in this group.

$a$. Lemuroidea. Small squirrel-like animals living in trees and bushes. The lemurs and marmosets.

b. Cebidae. The New World monkeys. Grasping tails and flat noses. Howling monkey, spider monkey, capuchin.

c. Cercopithecidae. The Old World monkey. Tail not grasping, or short; nostrils pointing downward. Distinct, opposable thumb. Baboon, mandrill.

d. Simiidae. The anthropoid (man-like) apes. No distinct tail; arms longer than legs. Gibbon, orangutan, chimpanzee, and gorilla.

e. Hominidae. The human race. 



\section{APPENDIX}

\section{HOW TO PREPARE CULTURES OF PRO'TOZOA}

Mixed cultures. Ordinary clear pond water may be used. Water taken from an aquarium, in which plants have been growing, frequently contain Protozoa. If tap water is to be used, let it stand in open vessels for at least a week in order to let the chlorine or other antiseptic gases escape.

Cultivation of Paramecia. Prepare a hay infusion by cutting timothy hay stems into short lengths. Fill a six-inch by eight-inch sterilized battery jar one half to two thirds full of water and add a small handful of the cut hay stems. Set the jar on a table in medium light and do not cover. After a few days a scum will form on the surface of the water. First bacteria and then Protozoa including Paramecia, will appear. The Protozoa will feed on the bacteria. A succession of forms will appear within a space of three to four weeks.

Pure culture of Paramecia. Cut timothy hay stems into short lengths. Boil these with plenty of distilled water until the water turns brown. Three different concentrations of culture media should be prepared. Sterilize all glassware by boiling.

Solution 1-Put two heaping tablespoonfuls of boiled hay in a large beaker containing two thirds of the undiluted hay water.

Solution 2-Put one tablespoonful of boiled hay in a large beaker containing diluted hay water (half hay water, half boiled water, plain).

Solution 3-Put a heaping teaspoonful of boiled hay in a large beaker containing two thirds of diluted hay water.

Let the solutions ripen, exposed to the air for about a week. Then inoculate with a pure culture of Paramecia. ${ }^{1}$ Check your solutions carefully so that you will know which one gives the best results.

Cultivation of amoeba. Water weed method. - Boil some fresh water plants, Elodea or Ceratophyllum. Place a very few of these 
dead, though still green, water plants in the bottom of a 4 by 6 -inch battery jar which has been carefully sterilized, and pour on aërated distilled water to a depth of $2 \frac{1}{2}$ to 3 inches. It is very important not to use too much plant material. In general, sufficient weed material should be added to cover the bottom with a greenish layer. In addition, place in each culture 8 or 10 wheat grains which have been thoroughly boiled. After the culture has stood for one week, add a pure culture of amoebas. If you have no amoebas, look for them in their natural habitat, e.g., pond or aquarium. Only a very small variety of amoebas are usually found in such places. If a greater variety is preferred, they may be purchased from a biological supply house. ${ }^{1}$ By re-culturing from time to time amoebas can be kept for the entire school year.

The amoeba culture should be kept in medium light and in a cool room where the temperature does not vary greatly. An optimum of $20^{\circ} \mathrm{C}$. $\left(68^{\circ} \mathrm{F}\right.$.) is satisfactory. It is difficult to keep amoeba cultures during the summer because of the high temperature. However, if the cultures are stored in a cool, fairly well-lighted basement, the animals will usually survive.

Upon the bottom of the properly prepared amoeba culture, there forms a greenish layer of loose material. Microscopic examination will show that this layer is rich in diatoms, desmids, and other plant cells and it is on and in this bottom layer of greenish material that the amoebas feed and multiply. Within a week or, at most, two weeks after inoculation the culture should be rich in amoebas which will generally live and reproduce for some time.

The main point to be emphasized in connection with this type of culture is that the water must remain clear. That is, it should not become cloudy or show evidence of fermentation in the formation of a surface film or scum. If the culture develops either of these characteristics, too much plant material is present and such a culture should be thrown out and a new one started.

${ }^{1}$ Satisfactory cultures of Paramecia and amoebas may be obtained either from the New York Biological Supply House or J. D. Dawson, College of the City of New York, New York. 
Fuel Value of Certain Foods ${ }^{1}$

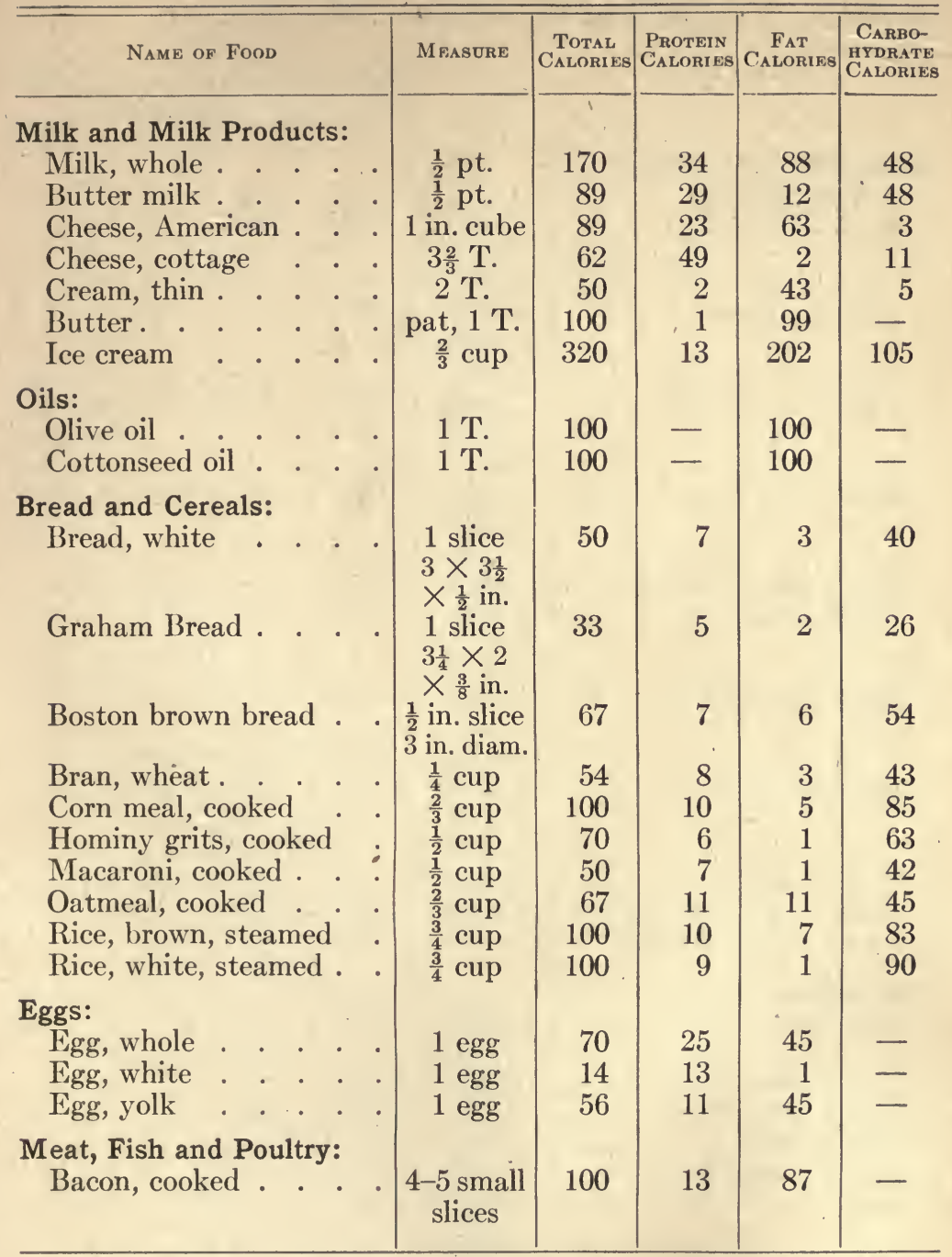

${ }^{1}$ Adapted from Charts in Amer. Red Cross pamphlet, "Food. Why? What? How?" 
Fuel Value of Foods (Continued)

Name of Food

Continued

Beef, lean, broiled

Chicken, roast . . . .

Fish, lean, broiled . . . 1 slice,

$4 \times 2 \frac{1}{4}$

$\times 1$ in.

Ham, boiled . . . . . 1 slice,

$4_{4}^{3} \times 6$

$\times \frac{1}{8}$ in.

Lamb, chop, broiled . . 1 chop

Lamb, roast . . . . 1 slice,

$3 \frac{1}{2} \times 4 \frac{1}{2}$

$\times \frac{1}{8}$ in.

Liver, calf, broiled . . medium size

serving

Mutton, roast . . . . 1 slice,

$3 \times 3 \frac{3}{4}$

$\times \frac{1}{8}$ in.

Oysters, raw . . . . 4

Pork chop, lean, broiled

Veal leg, lean, broiled .

\section{Vegetables:}

Asparagus . . . . 4 stalks

Beans, lima, dried

Beans, lima, fresh . . .

Beans, dried . . . . .

Beans, string, fresh . .

Beets . . . . . .

Cabbage, chopped .
1 chop

1 serving

4 in. long

$\frac{1}{4}$ cup

$\frac{1}{2}$ cup

$\frac{1}{4}$ cup

$\frac{1}{3}$ cup

$\frac{1}{3}$ cup

in cubes

$\frac{1}{2}$ cup

\begin{tabular}{|c|c|c|c|}
\hline $\begin{array}{c}\text { Total } \\
\text { CaLories }\end{array}$ & \begin{tabular}{|l} 
Protein \\
CaLORIES
\end{tabular} & $\underset{\text { CALORIES }}{\text { Fat }}$ & $\begin{array}{l}\text { CARBO- } \\
\text { HYDRATE } \\
\text { CALORIES }\end{array}$ \\
\hline & & & t \\
\hline 200 & 96 & 104 & - \\
\hline 100 & 51 & 49 & - \\
\hline 133 & 81 & 52 & - \\
\hline 150 & 44 & $106^{\circ}$ & - \\
\hline 100 & 40 & 60 & - \\
\hline 100 & 41 & 59 & - \\
\hline 100 & 62 & 38 & - \\
\hline 100 & 33 & 67 & - \\
\hline 75 & 37 & 18 & 20 \\
\hline 200 & 64 & 136 & - \\
\hline 150 & 105 & 45 & - \\
\hline 10 & 3 & 1 & 6 \\
\hline 200 & 42 & 8 & 150 \\
\hline 100 & 23 & 5 & 72 \\
\hline 171 & 45 & 8 & 118 \\
\hline 15 & 3 & 1 & 11 \\
\hline 25 & 3 & 1 & 21 \\
\hline 10 & 2 & 1 & 7 \\
\hline
\end{tabular}




\begin{tabular}{|c|c|c|c|c|c|c|c|c|c|}
\hline Name of Foo & & & & & Measure & $\mid \begin{array}{c}\text { Total } \\
\text { CaLORIES }\end{array}$ & $\mid \begin{array}{l}\text { Protein } \\
\text { CALORIEs }\end{array}$ & $\underset{\text { CaLORIES }}{\text { Fat }}$ & $\mid \begin{array}{l}\text { CARBO- } \\
\text { HYDRATE } \\
\text { CALORIES }\end{array}$ \\
\hline \multicolumn{10}{|c|}{ Vegetables - Continued } \\
\hline Cauliflower . & & & & & $1 \frac{1}{5}$ small & & & & $0 T$ \\
\hline & & & & & head & 20 & 5 & 3 & 12 \\
\hline Celery . . & & & & & $\frac{1}{4}$ cup. & 6 & 2 & - & 4 \\
\hline Corn, fresh & & & & & 1 ear, 6 in. & 50 & 6 & 4 & 40 \\
\hline Corn, canned & & & 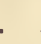 & & $\frac{1}{3}$ cup & 100 & 11 & 11 & 78 \\
\hline Cucumber . & & & • & & $\begin{array}{c}\frac{2}{5} \text { cucum- } \\
\text { ber }\end{array}$ & 16 & 3 & 2 & 11 \\
\hline Dandelion green: & & & & & $\frac{1}{2}$ cup & 75 & 12 & 11 & 52 \\
\hline Lentils, dried . & & & & & $3 \frac{3}{4} \mathrm{~T}$ & 150 & 44 & 4 & 102 \\
\hline Lettuce . . & & & & & $\frac{1}{4}$ head & 12 & 3 & 2 & 7 \\
\hline Okra . . . . & & & & & 5 to 6 pods & 20 & 3 & 1 & 16 \\
\hline Onions $\cdot$ & & 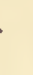 & • & & $\begin{array}{c}3 \text { to } 4 \\
\text { medium }\end{array}$ & 100 & 13 & 6 & 81 \\
\hline Parsnips . . & . & • & . & & $\begin{array}{l}\frac{1}{2} \text { cup } \\
\text { in slices }\end{array}$ & 50 & 5 & 3 & 42 \\
\hline Peas, dried . & & & & & $\frac{3}{8}$ cup & 253 & 70 & 6 & 177 \\
\hline Peas, fresh & & & & & $\frac{3}{8}$ cup & 50 & 14 & 2 & 34 \\
\hline $\begin{array}{c}\text { Potatoes, white } \\
15 \mathrm{~min} .\end{array}$ & & & ilec & & 1 medium & 100 & 11 & 1 & 88 \\
\hline Potatoes, sweet, & ba & ke & & & 1 medium & 200 & 12 & 10 & 178 \\
\hline Rutabaga, raw & & & - & & $\frac{2}{5}$ cup & 17 & 2 & 1 & 14 \\
\hline Spinach, cooked & & & & & $\frac{5}{8}$ cup & 25 & 3 & 2 & 20 \\
\hline Squash, cooked, & su & $\mathrm{Im}$ & me & & $\frac{1}{2}$ cup & 55 & 4 & 5 & 46 \\
\hline Tomatoes, fresh & & & & & 1 small & 25 & 4 & 4 & 17 \\
\hline Turnips, cubes, & $\mathrm{rav}$ & & • & & $\frac{1}{2} \operatorname{cup}$ & 25 & 3 & 1 & 21 \\
\hline \multicolumn{10}{|l|}{ Fruits, Fresh: } \\
\hline Blackberries & & & & & $\frac{1}{2}$ cup & 100 & 9 & 16 & 75 \\
\hline Cantaloupe . & & & & & $\frac{1}{2}$ melon & 50 & 3 & - & 47 \\
\hline Cherries, stoned & & & & & $\frac{1}{4} \operatorname{cup}$ & 25 & 1 & 2 & 22 \\
\hline Cranberries . & & & & & $\frac{1}{2}$ cup & 25 & 1 & 3 & 21 \\
\hline Grape fruit . & & & & & $\frac{1}{2}$ large & 100 & 7 & 4 & 89 \\
\hline Grapes, white & & & & & 22 & 100 & 5 & 15 & 80 \\
\hline Huckleberries & & & & & $\frac{1}{2} \operatorname{cup}$ & 50 & 2 & 4 & 44 \\
\hline Lemon juice . & & & & & $1 \mathrm{~T}$. & 5 & - & - & 5 \\
\hline Olives, green & & & . & & 4 medium & 50 & 1 & 41 & 8 \\
\hline Oranges . & • & • & . & & 1 medium & 75 & 5 & 2 & 68 \\
\hline
\end{tabular}


Fuel Value of Foods (Continued)

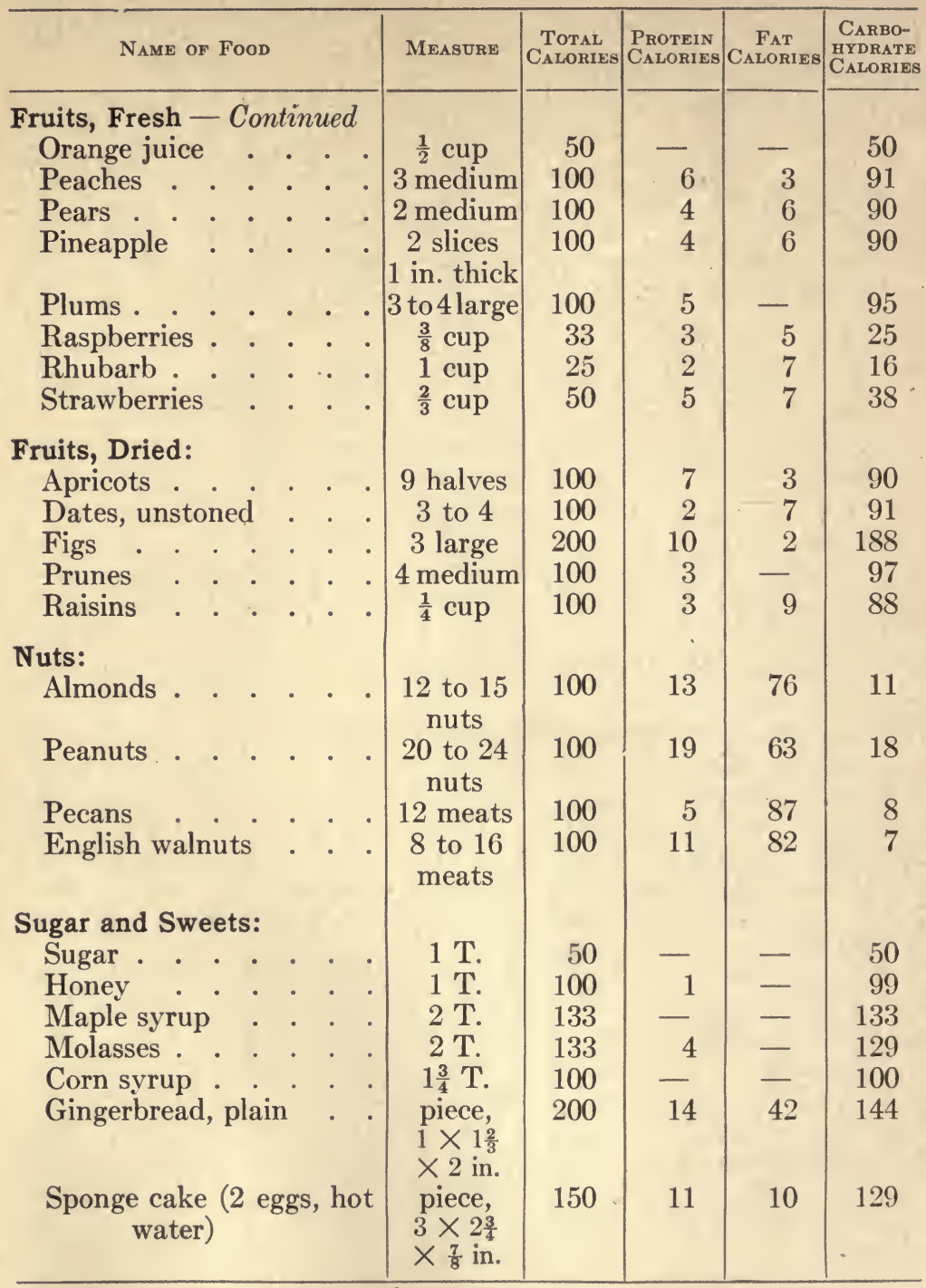




\section{HEALTH HABIT SCORE SHEET}

Adapted, by permission, from An Analysis of Instruction for Habits and Practices in Health and Accident Prevention prepared by E. George Payne (Lyons \& Carnahan, publishers).

Directions. The teacher should go over the outline point by point and explain any items upon which the students wish help. The habits and practices may be checked at any time, near the beginning and the end of each semester. The scoring should be recorded in the biology note book. Each student should aim to make his report as accurate as possible.

\section{Directions for Scoring.}

1. Allow full value or nothing for each item.

2. Practice in any item does not mean that there can never be an exception. For instance, if a boy or girl is kept up one night a week beyond his regular. hour of retiring to attend a moving picture show, nothing should be allowed for the first item under regularity. On the other hand, should there be an imperative reason for keeping him up later than the regular hour on an occasion of special nature, he may receive full credit. But if such occasions occur often or regularly, he should be given no credit.

3. In scoring $\mathrm{X}$ and $\mathrm{XI}$ the boy or girl should be given full credit for items with which he has had no experience. For instance, some children would have no incentive to play on railroad tracks, because there would be none in their vicinity.

4. Add the scores and compare with the maximum of 500 .

SCALE FOR MEASURING INDIVIDUAL AND SOCIAL BEHAVIOR - HABITS AND PRACTICES IN ACCIDENT PREVENTION AND HEALTH

\section{Variety}

\section{Food}

Drink from a pint to a quart of milk every day . . . . . . 3

Eat bread and butter every meal . . . . . . . . . . . 5

Eat some fruit every day (fresh, dried, or preserved) . . . . 5 
Eat some green, leafy vegetable every day (spinach, lettuce, kale, etc.) . . . . . . . . . . . . . . . . . 5

Eat some starchy vegetable every day (as potato) . . . . 3

Eat a cooked cereal for breakfast daily . . . . . . . . 2

Eat meats but once daily . . . . . . . . . . . . . . 2

Eat candies, cakes, etc., only as dessert. . . . . . . . 4

Quantity - Check with results determined in personal dietary work 20

Food Requirements in Calories - Age - Sex

\begin{tabular}{c|c|c|c||c|c|c}
\hline \hline \multicolumn{1}{c|}{ Bors } & \multicolumn{3}{c}{ Grrus } \\
\cline { 2 - 7 } Age & Total Cal. & Protein Cal. & Energy Cal. & Total Cal. & Protein Cal. & Energy Cal. \\
\cline { 2 - 7 } $12-13$ & $2300-2700$ & $276-324$ & $2024-2376$ & $1850-2150$ & $222-258$ & $1628-1892$ \\
$13-14$ & $2500-2900$ & $300-348$ & $2200-2552$ & $1950-2250$ & $234-270$ & $1716-1980$ \\
$14-15$ & $2600-3100$ & $312-372$ & $2288-2728$ & $2050-2350$ & $246-282$ & $1804-2068$ \\
$15-16$ & $2700-3300$ & $324-396$ & $2376-3204$ & $2150-2450$ & $258-294$ & $1892-2156$ \\
\hline \hline
\end{tabular}

\section{Regularity}

Eat a warm breakfast every morning . . . . . . . 2

Eat something warm for lunch (as soup) . . . . . . . . 3

Eat meals every day at the regular hour and in regular amounts . 3

Do not eat candies, cakes, ice-cream, etc., between meals . . 3 .

If hungry eat some bread and butter. Do not eat within two hours of another meal

\section{Manner of eating}

Eat slowly in a calm, unexcited frame of mind . . . . . 5

Chew all foods thoroughly . . . . . . . . . 5

Engage in pleasant conversation with the family . . . . 5

Tell a story or anecdote or interesting incident of the day . . 5

\section{Breathing}

II. AIR

Breathe deeply - take ten deep breaths before open window night and morning

Breathe always through the nostrils, not tnrough the mouth . 
Bedroom air

Sleep with windows well open every night $. \quad . \quad . \quad . \quad$. 5

Do not sleep in draft - use window boards if necessary . . 3

Air bedroom every day . . . . . . . . . . 4

Schoolroom and study room

See that room where you live or study is properly supplied with fresh air . . . . . . . . . . . . 5

Time in open air

Spend from two to three hours daily in exercise in the open air . 4

Do Not Smoke .

Amount

III. DRINK

Drink four to six glasses of water every day $\ldots . . . \quad$. 2

Regularity

Drink a glass of water on rising in the morning . . . . 1

Drink two glasses of water every forenoon at regular times . 1

Drink two glasses of water every afternoon at regular times . 1

Sanitation

Do not drink out of a cup used by some one else . . . . . 5

Drink only pure water from the fountain or out of a clean cup 4

Do not drink cold water while overheated from play or work . 3

Do not drink water containing cracked ice . . . . . . 2

Tea and coffee

Do not drink tea or coffee . . . . . . . . . . . . . . 9

\section{Variety}

\section{Exercise}

Two hours of out-door exercise daily. Run, skate, hike, swim, or play tennis, baseball, basket ball, volley ball, hockey, or any other game . . . . . . . . . . . . 20

Only light exercise should be taken one half hour before each meal or one hour after each meal. wH. FITZ. - AD. BIO. -35 


\section{Relaxation and Rest}

Amount of Sleep .

Sleep needed (Sleep alone)

\begin{tabular}{|c|c|c|c|c|c|c|c|c|c|c|c|c|}
\hline & & & & & & Hours & & & & 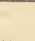 & & Houns \\
\hline 13 years & • & • & • & . & • & $8 \frac{1}{2}-10$ & 16 years & • & • & • & • & $8 \frac{1}{2}-9$ \\
\hline 14 years & - & . & . & . & $\cdot$ & $8 \frac{1}{2}-10$ & 17 years & . & . & . & . . & $8 \frac{1}{2}-9$ \\
\hline 15 years & . & . & . . & . & . & $8 \frac{1}{2}-9 \frac{1}{2}$ & 18 years & . . & . & . & . & $8 \frac{1}{2}-9$ \\
\hline
\end{tabular}

Regularity of sleep

Go to bed at same hour every night . . . . . . . . . 5

Get up at same hour every morning . . . . . . . . 5

Manner of sleep

Sleep on the side, mainly the right side . . . . . . . . 3

Relaxation

Use small pillow . . . . . . . . . . . . . . . . 1

Cultivate a hobby that will be of lasting benefit . . . . . 10

Read good books and magazines regularly . . . . . . . 5

Be punctual to all engagements . . . . . . . . . . . 5

Sitting

\section{Posture}

Sit erect while conversing . . . . . . . . . . . . 3

Sit erect while studying and writing . . . . . . . . . 3

Standing

Stand erect with chest forward, head high . . . . . . . 3

Walking

Walk with erect carriage, feet pointing directly forward . . . 3

Carry books in hands, with arms straight . . . . . . . 1

Hands and nails

\section{Cleanliness}

Wash hands before each meal . . . . . . . . . 10

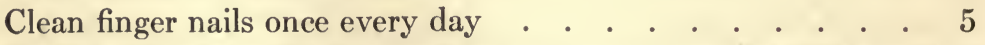

Keep hands and nails clean and cuticle pushed back at all times $\quad 9$

Keep nails out of mouth - do not bite the nails . . . . . 5 
Teeth, mouth, head

Clean teeth, mouth, and tongue morning and night . . . . 5

Do not put fingers, pencils, etc., in the mouth . . . . . 3

Do not dampen fingers in the mouth to turn pages of a book . 3

Do not lick postage stamps or envelopes . . . . . . . 3

\section{Bathing}

Take a tub bath twice every week : . . . . . . . . 10

Take a morning shower . . . . . . . . . . . 5

Always use an individual towel . . . . . . . . . . . 5

\section{Bowel movement}

Have a bowel movement regularly every day . . . . . . 10

Do not take drugs or medicine for this. Depend solely on food, water, exercise, and habit . . . . . . . . . 10

\section{Clothing}

Cleanliness

Keep clothing well dusted and properly cleaned . . . . . 5

Keep dresses and hose properly mended . . . . . . . . 4

Wear clean hose every day . . . . . . . . . . . . 4

\section{Suitability}

Wear warm, porous clothing in winter . . . . . . . 3

Wear light, porous clothing in summer . . . . . . 3

Wear shoes with broad heels, and of sufficient length . . . . 4

\section{Miscellaneous}

Put on a wrap when sitting down after exercises . . . . . 3

Keep clothing properly aired . . . . . . . . . . . . 3

Do not sleep in clothing worn during the day . . . . . . 5

Always have a clean handkerchief . . . . . . . . . 3

Regularity

\section{Home Study}

In general, study at the same time each day, preferably early evening . 


\section{Light}

Use a steady and sufficient artificial light. Avoid a glaring light

Quiet

Have a quiet room for study. Avoid family conversations, radio, etc.

System

Avoid movies and parties during the school week

\section{Safety Habits}

In the streets

Look in both directions before crossing the streets . . . . 3

Go straight across the street and at the crossings only . . . 3

Do not tarry in the street but cross promptly . . . . . 3

Help younger children to cross the street safely . . . . 3

Do not play on railroad tracks . . . . . . . . . . 3

Do not handle dangling wires or come in contact with electric wires . . . . . . . . . . . . . . . . 5

Do not ride on the outside of street cars . . . . . . . 3

Do not beg rides on autos . . . . . . . . . . 5

Do not climb on trucks and wagons . . . . . . . . 3

At home

Be careful about the use of matches; keep them in a safe place 2

Be careful about the use of kerosene and other inflammable materials; keep them in a safe place; do not start a fire with them

Be careful always in using the gas range . . . . . . . 3

Be sure electric wires are disconnected before touching them . 3

Be careful about the stairways and fire escapes . . . . . 3

Do not climb on chairs, tables, and step-ladders unless necessary, and then only after examining them . . . . .

Do not place heavy objects or sharp instruments where they may fall upon some one .

Do not leave chairs or other objects where some one may stumble over them in the dark 
Do not start your automobile engine in a closed garage

Have an annual health examination and have remediable defects corrected . . . . . . . . . . . . . . 10

Visit the dentist every six months . . . . . . . . . 5 At school

Do not hurry down the stairways . . . . . . . . 3

Do not run in the halls . . . . . . . . . . . . 3

Look before going in and out of doors, and do not rush . . . 3

Take one step at a time on stairways . . . . . . . 3 At play

Do not run on busy traffic streets in play . . . . . . . 3

Do not play near high places or on rough grounds . . . . 3

\section{Service - Social and Civic Habits and Practices}

Service at home

Give some help to your mother or father every day . . . . 5

Keep shoes shined, clothes brushed . . . . . . . . 5

Go on errands cheerfully . . . . . . . . . . . 5

Service at school

Serve on Health or Safety Committees . . . . . . . . 5

Call attention in every case to children who violate health or safety practices . . . . . . . . . . . . 5

Service to the community

Notify the Police Department of any obvious violations of ordinances . . . . . . . . . . . . . . . 3

Notify the Fire Department in case of fire . . . . . . . 3

Notify the Health Department of menaces to health in the neighborhood . . . . . . . . . . . . . 5

Notify the Street Department of holes in the street, obstructions, unclean alley in neighborhood, etc. . . . . . . 


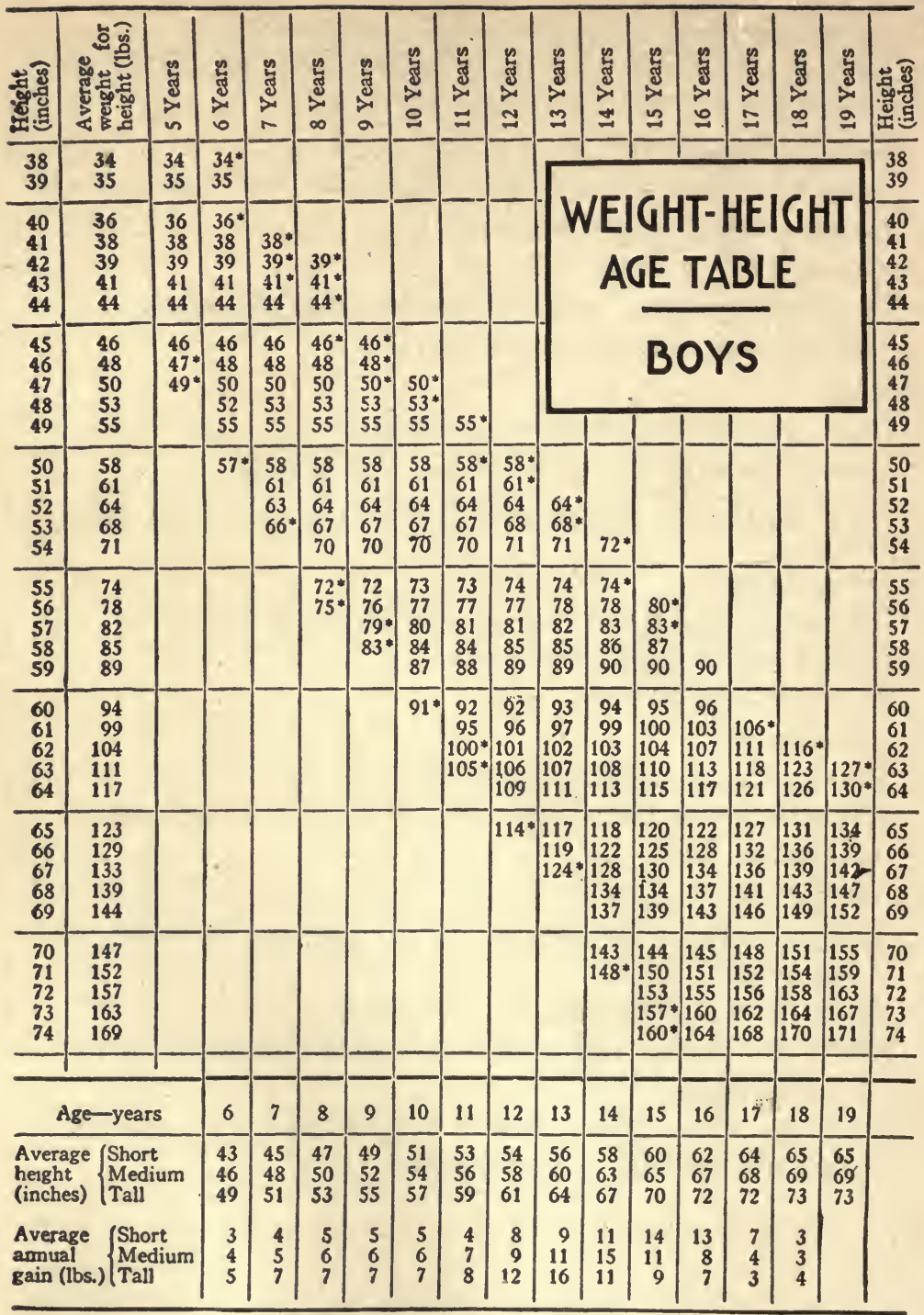

Baldwin, B. T., and Wood, T. D., Weight-Height-Age Tables. American Child Health Association, New York, N. Y. 


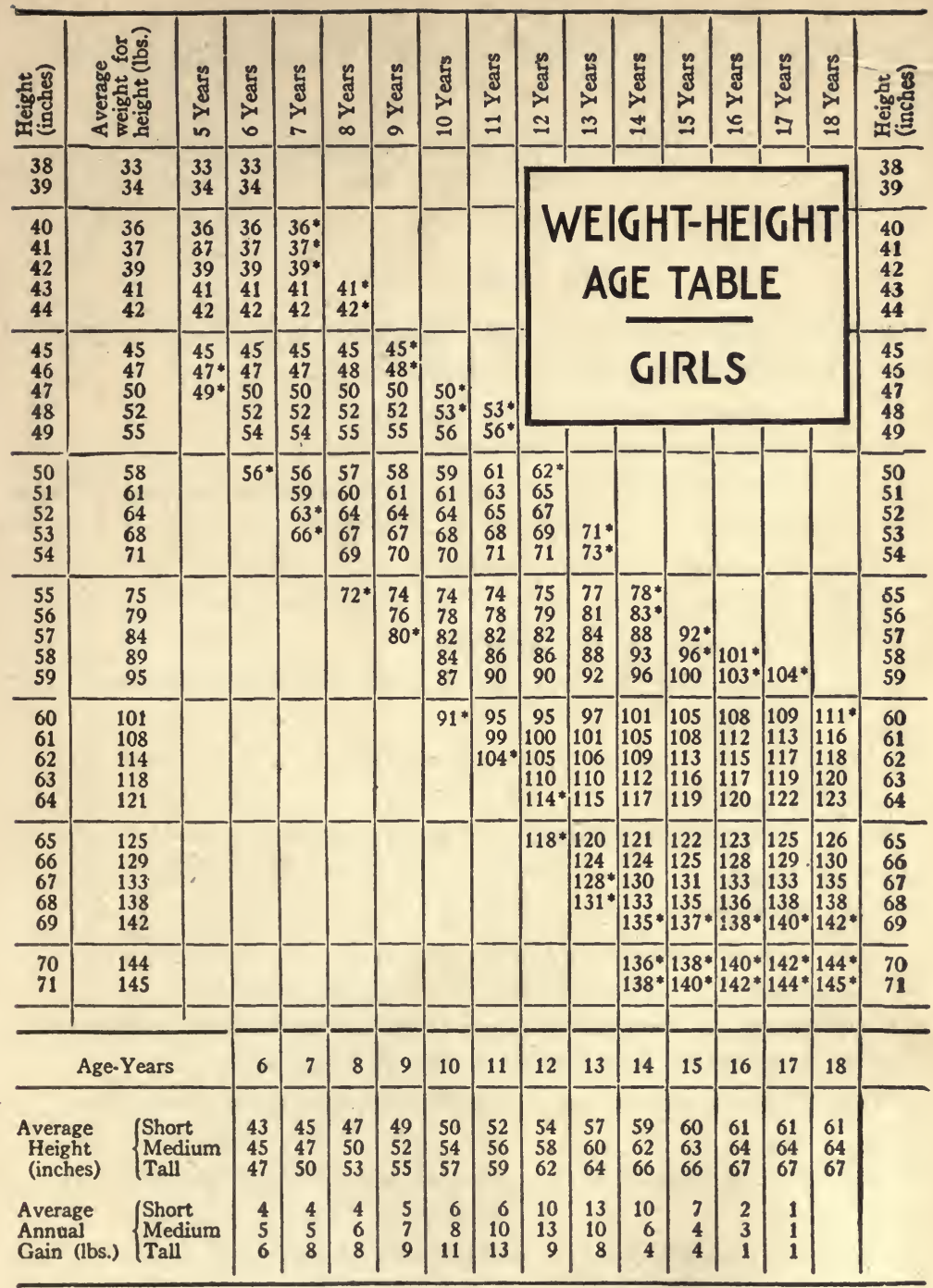

Baldwin, B. T., and Wood, T. D., Weight-Height-Age Tables. American Child Health Association, New York, N. Y. 


\section{A SUGGESTED COURSE OF STUDY IN ADVANCED} BIOLOGY

(Now being tried in 30 schools)

\section{TERM I}

\section{Cell Studies}

1. Study of the living green cell ; illustrated in Spirogyra or Elodea.

(a) Structure; microscopic study.

(b) Functions; emphasis on food-manufacture.

(c) Adaptations to environment.

2. Study of the living animal cell; illustrated in amoeba or Paramecium.

(a) Strúcture; microscopic study.

(b) Functions.

(c) Adaptations to environment.

(d) Comparison of plant and animal cells as to structure and functions.

3. Study of the typical or generalized cell.

(a) Structure.

(b) Comparison and nature of protoplasm.

4. Cell division.

(a) Amitosis.

(b) Mitosis ; names of phases optional.

5. Association of cells in tissues and organs.

(a) Study of human tissues; four of the following required: epithelial, muscle, bone, connective, nerve.

(b) Differentiation, specialization, physiological division of labor.

(c) Comparison of tissue cell with independent plant or animal cell.

6. History of cell theory ; contributions of any three of the following: Hooke, Malpighi, Leeuwenhoek, Schleiden, Schwann, Max Schultze. 


\section{Nutrition}

1. (Optional) Study of the frog as an introduction to human nutrition.

(a) Identification of systems of organs.

(b) Appearance and location of organs.

2. Study of metabolism in man.

(a) Digestion.

1. Purpose.

2. Foods; uses of different nutrients; include vitamins and deficiency diseases; omit planning of diets and food tests.

3. Alimentary canal and digestive glands.

4. Enzymes, intermediate products, end products.

5. Experiments showing digestion.

6. Peristalsis.

7. Hygiene of digestion.

(b) Absorption.

1. Purpose.

2. Adaptations.

(c) Circulation.

1. Purpose.

2. Composition of blood; function of each part; clotting.

3. Organs of circulatory system.

4. Course of blood; changes in composition in various organs.

5. Lymph and lymphatic system.

6. Hygiene of circulation.

7. Discoveries of Harvey, Malpighi, and Leeuwenhoek on the blood and circulation.

(d) Assimilation.

(e) Respiration.

1. Brief study of air passages.

2. Emphasize cell respiration.

(f) Secretion.

1. Duct glands. 
2. Endocrine glands and hormones; secretin, pancreatic hormone; secretions of thyroid, adrenal, and pituitary glands.

(g) Excretion.

1. Formation of principal waste products ; carbon dioxide, water, urea, uric acid.

2. Organs of excretion.

3. Hygiene of excretion.

(h) Summary of metabolism of carbohydrates, fats, and proteins.

3. Parasitism, saprophytism, and symbiosis.

(a) Definitions and examples; omit all detailed study, except as included under bacteria and malarial parasite.

(b) Life history and importance to mankind of one parasite; malarial organism suggested.

(c) Effect of parasitism on host and on parasite.

4. Comparative study of different types of nutrition.

\section{Irritability}

1. A function of protoplasm ; illustrated in protozoa.

2. Tropisms in plants; geotropism, hydrotropism, phototropism; demonstrate, unless covered in elementary biology.

3. Irritability in man.

(a) General structure and functions of the nervous system; emphasis on function.

1. Central nervous system.

a. Brain; including cerebrum, cerebellum, medulla, and their general functions.

$b$. Spinal cord and its general functions.

c. Nerves, cranial and spinal ; omit names of individual nerves.

2. Autonomic system.

3. Neurons, cranial and spinal; omit names of individual nerves.

(b) The types of nervous reactions.

1. Inborn automatic activities; reflex actions and the reflex arc. 
3. Bacteria as useful organisms.

(a) In food preparation.

1. Ripening and flavoring of dairy products.

2. Making of vinegar.

(b) In agriculture.

1. Decay.

2. Nitrification; comparison with dentrification.

3. Nitrogen; fixation. Nitrogen cycle.

4. Rotation of crops.

(c) In other industries and arts.

1. Retting of flax.

2. Tanning of leather.

4. Bacteria as harmful organisms.

(a) In decay of foods (optional) ; methods of food preservation.

(b) In causing disease.

5. Pathogenic microörganisms; study of the following diseases: diphtheria, tuberculosis, colds, typhoid fever, smallpox, tetanus, hydrophobia, malaria, yellow fever, focal infections; to bring out the following principles:

(a) Sources of infection; contact, food, and drink, air, human carriers, insect carriers.

(b) Bacterial poisons ; toxins, ptomaines.

(c) Methods of protections; skin, adaptations of respiratory tract, white corpuscles. Antibodies (antitoxins, lysins, agglutinins, and opsonnis).

(d) Methods of immunity and immunization.

1. Active; vaccination, toxin-antitoxin innoculation; Pasteur treatment.

2. Passive; antitoxin treatment.

3. Tests for immunity; Schick test.

(e) Description of the causative organism, general character of disease, symptoms, diagnosis, prevention, and treatment (where discussion is feasible) for each of the diseases.

(f) Work of the Department of Health.

(g) Biography; scientific contributions of Jenner, Lister, Pasteur, Koch, Metchnikoff, Ehrlich. 


\section{INDEX}

Ability, families of inferior, 358-362 ; families of superior, 356-358

Abrasion, meaning of, 168

Absorption, digestion and, 125-141; from large intestine, 138 ; from small intestine, 136-138; in organism, 34; in green cell, 34-36; stomach, 136

Acidopholus milk, 140

Acne, definition of, 175

Acquired reactions, $220-221$

Acromegaly, 197

Active immunity, acquiring, 507-508

Activities, acquired automatic, 220-221 ; inborn automatic, 215-217; kind of mental, 215 ; reflex, 215-216; voluntary, 219-220. See also Nervous activities

Adam's apple, 179

Adaptability, relation to health, 414-415

Adaptations, of tissues, 88-89; of higher plants, 69-72

Adaptive processes, in organisms, purpose of, 34

Addison's disease, causes of, 198-199 characteristics of, 198

Adenoids, 179-180 ; colds result of, 475476

Adipose tissue, use of, $81-82$

Adolescence, table of, 302

Adrenal glands, effect of secretion on body, 197-198; location of, 197; relation to arterial pressure, 197-198. See Addison's disease

Adrenaline, 197; use of, 199

Adrenin, effect on body, 197-198

Afferent axon, 209

Agar slant, inoculation of, 398 ; preparation of, 396-397

Age, chronological, 229 ; mental, 228 ; of earth, 378,381 ; of science, 1-3

Agglutination test, 465-466, 467

Agglutinins, 465, 467, 469; in typhoid, 467 ; production of, 501 ; to determine presence of, $465-466$; use of, 501

Agriculture, bacteria in, 405-411

Air, composition of expired, 183 ; composition of inspired, 183 ; tidal, 183

Air sacs, 179

Air tubes, $179-180$

Alcohol, effect on circulation, 168-169; effect on nervous system, 237
Algae, characteristics of, 33 ; classified, 519

Alimentary canal, laboratory study of, in frog, 116; in man, 117 ; description of man's, 118, 119-120

Alkali, laboratory problem, effect on a fat, 131-132

Allosaurus, prehistoric reptile, description of, 383 ; restoration of, 372

Alveolar theory, of nature of protoplasm, 54

Amitosis, meaning of, 60 ; process of, 60

Amoeba, assimilation in, 44 ; behavior of, 44 ; digestion of, 43 ; ingestion of, 43 ; irritability of, 44-46; laboratory problem on study of, $42-43$; life functions of, 43 ; locomotion of, 46 ; regeneration of, 56 ; reproduction of, 44,248 ; respiration of, 44

Amphibia, 525-526

Amylase, an enzyme, 38, 131 ; amylopin, 131

Anabolism, in cell, activities in, 60 ; meaning of, 59,187

Anaemia, causes, 146, 167 ; symptoms, 147

Anaphase, stage in cell division, 61,62

Ancestry, influence in development, 310 313

Andalusian fowls, heredity in, 318-320

Andrews, Roy Chapman, on Mongolian expeditions, 385-387. See frontispiece

Angiospermae, meaning of, 520, classified, 520

Animal, and plant breeding, 343-354

Animal cell, typical, 42-52

Animal passage, meaning of, 508

Annelida, classified, 522-523; meaning of, 522

Anopheles, spread of malaria by, 482-484

Ant eater, giant, 512

Anthozoa, 522

Antibodies, agglutinins, 501; antitoxin, 501 ; lysins, 501; opsonins, 500, 502; precipitins, 501-502; production of, 146,501 ; use of, 146

Antiseptics, laboratory problem to show effect on growth of bacteria, 401-402; use of, 4

Antitoxin, discovery of diphtheria, 451452 ; kinds of, 501; preparation of 
diphtheria, 451-452, 456 ; preparation of tetanus, 461; produced in body, 501 ; scarlet fever, 459 ; standardizing, 456 ; use of, 501

Aorta, 153, 159

Aphids, 271

Appendicitis, 135

Appendix, vermiform, 135

Arachnida, 524

Archaeopteryx, earliest known bird, 383

Archiannelida, 523

Aristotle, classification by, 510; Father of Biology, 10; idea of evolution, 371 ; work of, 11

Arterial blood, 144

Arteries, direction of flow of blood in, 154,156 ; functions of, 154,156 ; hardening of, 168; meaning of name, 153,156 ; regulation of size of, 156 ; rupture of, 168; structure of walls of, 156

Arterioles, 159

Arteriosclerosis, causes of, 168

Artery, pulmonary, 153

Arthropoda, classified, 517; 523-524; meaning of, 523

Artificial selection, importance in animals and plants, 352

Ascaris, diagram of mitosis of egg of, 61

Asexual reproduction, by vegetative propagation, 255-263; of algae, 247 248 ; of bacteria, 247 ; of hydra, 248 , $249-250$; of protozoans, 248 ; of yeast, 250-251

Assimilation, a life process, in amoeba, 44 ; in green plants, $39-40$

Asteroidea, 522

Asthma, cause of, 477 ; treatment of, 477 ; vaccination for, 477

Auricles, of heart. See Heart

Autonomic nervous system, description, 210 ; diagram of, 210 ; functions of, 210-211; importance in nervous activities, 221-222 ; relation to central nervous system, 210-211

Aves, animals of class, 526

Axon, of nerve cell, 86,87

Bacilli, form of bacteria, 392

Bacillus, acidopholus, 140; bulgaricus, 140 ; typhoid, 465

Bacteria, 391-411; action on body, 494-495; aërobic, 393; anaërobic, 393; beneficial activities, 403-411; cause of pus, 494 ; cause of disease, $494-$ 495 ; carried through lymphatics, 500 ; colony of, 395 ; conditions necessary for growth, 399-400; cultures of, 396397 , 398; denitrifying, 407 ; destruction of white corpuscles by, 500, 504-505; effect of temperature on,
399,499 ; forms of, 392-393 ; functions of, 393-395; growth in milk, 140 ; growth of, 398-399; in agriculture, 405-411; in our body, 494500 ; in air, $495-496$; 475 ; in dust, 473 ; in eyes, 496 ; in food preparation, 403-404, 405; in industries, 403-404; in lymph and lymph nodes, 166-167, 500 ; laboratory problem to determine bacterial content of milk, 401 ; laboratory problem to determine number of bacteria in air, 400-401; laboratory problem to show effect of antiseptics on growth of, 401-402; media for cultivation, 396-397; methods of entering body, 495-496; methods of identifying, 395-396 ; nitrifying, 406 ; nitrogen-fixing, 394, 407-409; nutrition of, 394 ; occurrence of, 397-399; of decay, 406; parasitic, 393; pathogenic, 394 ; physiological functions of, 393-395 ; relation to disease, 494-495; saprophytic, 391, 394; soil, 405-411; structure of, 391-393

Bacterial content, laboratory problem, in air in various places, 400 ; in milk, 401

Bacteriolysin, antibody, 501; function of, 465,469

Bacteriology, definition of, 17

Bacteriophage, 508

Banting, F. G., work with insulin, 199

Beach plum, improved, 348-349, 352, 353

Beetle, potato, effect of environmental conditions on, 341

Behring. See Von Behring

Beriberi, cause of, $98-99,100$; prevention of, 99,100

Bile, basis for manufacture of, 161 ; function of, 132

Binet, Alfred, tests by, 228

Binet scale, 228

Binet-Simon Scale, Terman's revision, 228

Binomial system, of classification, 510511

Biogenetic law, 388-389

Biology, affected by work in other sciences, 19 ; divisions of, 17 ; meaning of, 15 ; of to-morrow, $1-9$; purpose of, $6-7$; relation to chemistry, 16 ; relation to health, $7-8$; relation to other sciences, 16 ; relation to physics, $15-16$; relation to vocations, 8-9

Birds, egg-laying of, 299 ; food of, 299 ; nest-building of, 298; infancy among, 298-299; reproduction of, 288-289

Bishop, on vitamin $\mathrm{E}, 103-104$

Blackberry, production of white, 347348,349

Bladder, urinary, function of, 175-176 ; structure of, 175 
Blastula, 286

Bleeders. See Haemophilia

Blood, arterial, 144; a tissue, 87, 146; circulation of, 143, 153-161; clotting of, 148 ; composition of, 145 ; corpuscles of, 87-88, 147-148; count, 147 ; course of, 159-161 ; early drawing of circulation of, 151 ; functions of, 145-146, 150,162 ; grouping of, $148-149$; heat of, 150 ; importance of, 143-150 ; laboratory problems on study of, 87 , 143-144; laboratory study of corpuscles of, 144-145; laboratory study of serum of, 144 ; necessity of circulation of, 149-150; need of water to, 167 ; of frog, 89 ; of man, 88 ; oxygenated in lungs, 179 ; properties of, 145146 ; serum of, 144 ; transfusion of, 149 ; venous, 144

Blood plasma, composition of, 145

Blood platelets, function of, 148 ; structure of, 148

Blood serum, laboratory study of, 144

Blood vessels, 143 ; laboratory study of, 151. See also Arteries, Capillaries, and Veins

Bone, formation of, 79; laboratory problems on microscopic structure of, 78 ; laboratory study of cross structure of, 77-78; proper growth of, 80

Bone cell, diagram of, 80

Brain, parts of, 206-207 ; structure of, 206-207

Breathing, control by carbon dioxide, 183 ; control by oxygen, 183 ; laboratory problem of modifications of, 183 ; laboratory problem on mechanics of, 181; movements of, 181-182

Breeding, aims of, 345-351; artificial selection in, 352 ; for improved quality, 350 ; for increased production, 351 ; for points, 349-350; hybridization, 351 ; importance of animal, 344-345 ; 353 ; importance of plant, 343-345 ; Mendel's experiments in, $322 ;$ methods of animal, 353-354; methods of plant propagation in, $351-353$; results of close, 353-354

Breeding experiments, of plants, by Mendel, 322

Bronchi, 178, 179

Bronchial tubes, 179

Bronchitis, 474

Broncho-pneumonia, predisposing factors, 474

Brontosaurus, restoration of, 372. See Dinosaur

Brown, Robert, described movement of molecules, 35-36; observed nucleus of cells, 27-28

Brownian motion, 36

WH. FITZ. AD. BIO. -36
Bruise, cause of, 168

Bryophyta, 519

Budding, laboratory problem in yeast cells, 248-249 ; method of reproduction, 248-250; of hydra, 249-250; of sponges, 253

Bulbs, propagation by, 262-263

Burbank, Luther, experimental methods of, 14 ; fruit improved by, 347-349; on potato blight, 344

Calcarea, 521

Calcium, need for, 111

Calkins, Dr., experiments on regeneration, 56

Calorie, defined, 93 ; needs, 105

Calorimeter, measurement of human energy by, 189

Calyx, of flower, function of, 274

Canning, 400

Capillaries, broken, 168; function of, 157 ; in lungs, 179; pressure in, 157; structure of, 157

Carbohydrates, manufacture of, 36-37 ; metabolism of, 188; constituents of, 36-37

Carbon cycle, 409

Cardiac opening. See Stomach, parts of

Carnivora, animals of Order, 527

Carrell, Alexis, cultivation of cells, 5455 ; preparation of Dakin solution, 55

Cartilage, functions of, 79-80; kinds, 79

Catalytic agents, in digestion, 38

Catarrh, cause of, 474

Cebidae, 527

Cell, absorption in green, 34-35; amitosis in, 60 ; assimilation in, $39-40$; cultivation of, 54-55; digestion in green, 38 ; direct division of, 41,60 ; discovery of nucleus of, 27-28; division of, 41; excretion in green, 40 ; food manufacture of studies of, $36-38$; functions of green, 34-40; history of, 27-33 ; growth and division, 40, 41, 53-64; inclusions in, 37 ; indirect division of, 60-63; irritability in green, 40-41; key to all biological problems, 29-30; laboratory study of Elodea, 28-29; laboratory study of onion, 25-26; laboratory study of narcissus leaf, 3031 ; laboratory study of Spirogyra, 31-33; mitosis of, 60-63; named and described by Hooke, 27-28; nature and composition of, 53-55; primary, 269,285 ; relation to heredity, 55 ; respiration in green, $38-39$; resting and dividing, 53-64; sex, 269 ; somatic, 269 ; structure of, 30 ; theory, 28,30 , 52,64 ; typical animal, $42-52$; unit of function of organism, 30 ; unit of structure of organism, 30,$53 ; 73$; 
$57-58$; wall of, 52,58 ; work of all, 162

Cellulose, of plant cells, 52

Central nervous system, divisions of, 205 ; functions of, 206-210; protection of, 205-206

Centrosome, function of, 59 ; in cell division, 61-62

Cephalopoda, 523

Cercopithecidae, 527

Cerebellum, function of, 207; level of reaction in, $218,219,220$; nature of, 207

Cerebro-spinal nervous system. See Central nervous system

Cerebrum, function of, 206-207; level of reaction in, 218,220 ; nature of, 206 ; position of, 207 ; size in various animals, 207 ; structure of, 206

Cetacea, animals of Order, 527

Chaetopoda, 523

Chambers, Prof. R. H., dissections under microscope by, 23,45 ; on heredity, 55

Chance, law of, laboratory problem to demonstrate, 317

Character, dominant, 324,333 ; of offspring, 306-317; recessive, 325,333

Character-determiners, in chromosomes, 55,319

Characters, acquired, 336-337 ; dominant, 324,333 ; inherited, 336 ; 318' 333 ; law of unit, 327-333 ; recessive, 325,333 ; secondary sexual, 281-282

Chemistry, and its relation to biology, 16

Chemotropism, shown by amoeba, 46 . See Tropisms

Chicken pox, effects of, 478 ; method of spread, 478

Child-labor laws and eugenics, 366-367

Chiroptera, animals of Order, 527

Chlorophyll, in plants, function of, 36-37, 39,59

Chloroplasts, first seen, 28 ; function of, 59 ; structure of, 28

Chordata, classified, 525-527 ; meaning of, 525

Chromatin granules, function of, 57 ; structure of, 55

Chromosome, changes in number, 339 ; number in sex cells, 270-271

Chromosome theory, of inheritance, 331333

Chromosomes, 55; behavior in cell division, 62 ; behavior in maturation, $268-269,284,316$; continuity of, 306 , 307 ; laboratory problem to show chance combinations of genes in, 317 ; laboratory problem to show possible combination of genes in, 326-327; location of genes in fruit fly, 312 ; locus of genes, 331 ; number in sex cells and body cells of various animals, 307 ; $270-271$; relation to heredity, 315 $317,324-326,331-333$

Chronological age, 229

\section{Chyme, 129}

Cilia, in Paramecium, 48, 49, 50; in respiratory tract, 75,179

Circulation, of blood, 143; coronary, 154 ; early investigations of the, 163 165 ; effect of violent emotions on, 167 ; necessity of, 149-150 ; organs of, 153-154; portal, 160-161; pulmonary, 160 ; systemic, 159-160; time for complete, 161. See Blood

Circulatory system, 151-162; diagram of, 155; functions of, 168; hygiene of, 167-169

Classification, basis of, 512-514; difficulties in, 511-514; evolution in, 517518; geographical distribution and, 573; habits and, 513-514; International Commission on, 514-515; morphology in, 513 ; of plants and animals, 518-527 ; present method of, 515-518 Cloaca, in frog, 115

Close-breeding, reasons for and results of, 353-354

Clotting of blood, 148

Cocci, form of bacteria, 392

Coelenterata, classified, 521-522 ; meaning of, 521

Cohnheim, J., experiment on transmission of tuberculosis, 438

Cold, a common disease, causes of, 473475; chronic, 474; effects on body, 473-474; predisposing factors, 474 ; prevention of, $475-476$; remedial measures, 476 ; transmission of, 474-475

Collip, experiment with parathyroid extracts, 196

Conjugation, 264 ; of Paramecium, 4950, 267-268

Connective tissue, $81-82$

Conservation, of soil, 411

Constipation, causes of, 139 ; prevention of, 139,140

Continuity, of germ plasm, 336

Contractile tissue, 83

Contractile vacuole, of amoeba, 42 ; or Paramecium, 47

Corolla, of flower, function of, 274

Coronary circulation, 154

Corpuscles, white, description of, 88: functions of, 88, 147-148; in infection, 148; number of, 88, 147; red. destruction of, 146; formation of, 146 ; function of, 88,146 ; number of, 88,147 ; size of, 88 ; structure of, 88

Cortex, of brain, areas of, 206-207, 213 ; functions of, 207, 213; structure of, 206

Cotyledons, 278-279 
Cowpox, inoculations with, 421-423

Cows, increased products from, 344

Cretinism, 194

Cretins, cause of, 194

Criminals, heredity of, 361

Crinoidea, 522

Crops, rotation of, 409-411

Crossing-over, of genes, 339, 340-341

Crustacean, 524 ; the young of, 296

Culex, house mosquito, 483

Cuts, treatment of, 168

Cuttings, method of vegetative propagation, 255-256

Cyclostomata, animals of Order, 524

Cytoplasm, 58 ; structures found in, 57

Cyton, of nerve, 208, 209

\section{Dakin solution, 55}

Darwin, Charles, 371 ; on natural selection, 373-376

Darwinian theory, of evolution, 373376

Darwin's, theory of, evolution, 373-376 ; over-production, 374 ; struggle for existence, 374 ; variations, 374-376 ; survival of the fittest, 374 ; inheritance, 374-375

Davenport, Charles, 355 ; work in eugenics, 356

da Vinci, Leonardo, 163-164; on fossils, 371

Defecation, 135

Demospongia, animals of class, 521

Dendrites, $86-87$; axon, 208

Dentine, 107

Dentrification, process of, in soil, 407

Dermis, structure of, 172

Development, progressive. See Organic evolution

Development, influenced by ancestry, 310-313; influenced by environment, 313-315

Development of species, use and disuse, 372

de Vries, Hugo, experiments with evening primroses, 337 ; mutation theory of, 376-377; portrait of, 335 ; theory of evolution, 376-377; mutants, 376-377, 338-339

d'Herelle, on immunity, 508-509

Diabetes, insulin in treatment of, 199

Dick test, for scarlet fever, 458

Diet, complete, 93 ; essential, 104 ; insufficient, 93; insufficient in minerals, 95-96; insufficient in proteins, 93, 95 ; investigation by McCollum on, 93,95 ; laboratory problem on personal, 105 ; relation of teeth to, 111112

Digestion, and absorption, 125-141; chart of chemical, 134; hygiene of,
138-139; in mouth, $120-121$; in plants, 38 ; in small intestines, 132133,135 ; laboratory problem of relation of size of protein food to time of, 126 ; laboratory problem of relation of different animal proteins to, 127 ; life function of amoeba, 43; meaning of, 117-118; process of, $117-119$; salivary, 121-122, 130; time necessary for, 133,140

Digestive organs, of frog, 114-116, 117 ; of $\operatorname{man}, 116-117,119$

Digestive system, 114-123 ; function of, 117 ; laboratory study of the frog's, 114-116; laboratory study of man's, 117

Dinosaur, amphibian, 383; eggs of, 306 ; restoration of, 372

Diphtheria, ages most susceptible to, 453 ; causes of, 453-454; diagnosis, 457 ; discovery of active immunity to, 452453 ; discovery of antitoxin of, 451452 ; effect on the body, 454 ; history of, 450 ; isolation of organism of, 450 ; isolation of toxin of, 451 ; prevention of, 457-458; Shick test of susceptibility, 453; transmission of, 454-455; treatment of, 455-457

Disease, and health, 413-414; and mental poise, 502-503; causes of, 494; due to bacteria, $445,455,459,482$, 492 ; due to physical agents, 473 ; immunity of animals to, 345 ; immunity of plants to, 347 ; inheritance of, 368369 ; safeguards of the body against, 496-503 ; caused by toxin, 450-451, 458

Division, cell, process of, 41 ; purpose of, 40

Division of labor, physiological, 66-67

Division, reduction. See Maturation

Dominance, complete, 323-324 ; incomplete, 318-321; meaning of, 323 ; Mendel's or Mendelian law of, 322-324

Dominant characters, 323-324; list of, 333

Dogs, developed from common ancestor, 310,311

Duck bill, 384, 513

Duct, thoracic, 165

Ductless glands, 191-201 ; adrenal, 197199 ; pancreas, 199 ; parathyroid, 196 ; pineal, 200 ; pituitary, 196-197; reproductive, 200-201; secretions of, 191-192 ; spleen, 200 ; thymus, 199200 ; thyroid, 192-195

Dujardin, protoplasm named by, 28 Dwarfism, 197

Earth, age of, basis for estimate of, 378381 ; calculations of, 378 ; evidences of, $378-379,380$ 
Echinodermata, classified, 522 ; meaning of, 522

Echinoidea, 522

Ectoderm, development of, 286 ; of embryo, 286 ; systems formed from, 286

Edentata, 526 ; ant eater, 512

Education, and eugenics, 365 ; health an objective of, 415-417

Edwards, Jonathan, family of, 357

Effector, response activity of, 209

Egg cell, characteristics of, 268

Eggs, differ from seeds, 281; of insects, 281 ; of birds, 281 ; production by frog, 284-285

Ehrlich, portrait, 504; theory of immunity, 505-506; work of, 14

Elasmobranchii, animals of Order, 524

Elastic tissue, use of, 81

Elodea, diagram of, 30 ; laboratory study of cells of, 28-29

Embryo, development of seed, 278-279; protection of, in lower animals, 295296 ; 288-289 ; in insects, 297-298; in mammals, 299-301; in man, 301

\section{Embryo sac, 274, 276, 277}

Embryological evidence, of evolution, 388-389 ; similarities in development of embryos of vertebrate animals, 388-389

Emotions, dangers of violent, 167

Enamel, of teeth, 107

Encystment, of protozoans, meaning of, 253; why formed, 253

Endocrine glands, 191

Endoderm, of embryo, 286

End-organs, in skin, functions of, 173

Endosperm, 278, 279; in grains, 279; systems formed from, in embryo, 286

Endotoxins, meaning of, 495 ; of tuberculosis, 442 ; of typhoid, 465-466

Energy, human, measured by calorimeter, 189; kinetic in plant cells, 39

Enterokinase, use in digestion, 133

Environment; effect on ancestral traits, 313-315; influence on development of animals, 308-310; influence on development of plants, 308, 309 ; methods of improving, 364-367; versus heredity, 369-370

Evironments, effect of different, 308

Enzymes, digestive action of, 128,131 ; function in plants, 71 ; in gastric juice, 128; in intestine, 132-133; in plant cells, 38 ; in saliva, 121 ; of pancreatic juice, 131 ; specific action of, 128-129, 131

Epidermis, laboratory problems on study of leaf, $30-31,67$; laboratory problem on cross section of leaf, 6768 ; outer layer of skin, 171,172

Epiglottis, use of, 122
Epithelial cells, laboratory problem on 75 ; shape of, 75

Epithelium, 75-77

Erepsin, 132

Esophagus, 122, 123

Essay on Population, Malthus, 343

Eugenics, 355-370; child labor laws and, 366-367; compulsory education and, 365; immigration and, 367-368; laborer compensation laws and, 365 ; movement founded and named, 355 ; need for understanding, 363 ; relation of marriage to, 355-356; 363-364; Second International Congress of, 355 ; versus euthenics, 368 ; vocational guidance and, 367 ; widows' pensions and, 365-366

Eugenics Laboratory, at Cold Spring Harbor, 356; symbols used in chart making at, 356 ; work done by, 362363

Eustachian tubes, 122

Euthenics, consists of, 365-368 ; meaning of, 355,362 ; versus eugenics, 368

Evans, on vitamin E, 103-104

Evening primroses, de Vries' experiments with mutants of, 337

Evolution, organic, Aristotle's idea of, 371 ; Darwin's natural selection theory of, 373-376 ; de Vries' mutation theory of, 376-377 ; embryological evidences of, 388-389 ; geographical evidences of, $385-386$; history of, 371 ; geological evidences of, 381-384; in plants, 389 ; Lamark's use and disuse theory of, 371-373 ; meaning of, 371 ; morphological evidences of, 386,387 ; vestigial evidences, $387-388$

Evolutionists, beliefs of, 371

Excretion, definition of, 170 ; hygiene of, 174-175, 176; importance of, 170 ; of man, 170-176; process in a green cell, 40

Excretory organs, 170-176

Exotoxins, 495; in diphtheria, 451 ; in scarlet fever, 458 ; in tetanus, 459 ; in tuberculosis, 442

Experimentation, modern methods of, 15

Expiration, meaning of, 182; process of in man, 182

$\mathbf{F}_{1}$, defined, 319

$\mathbf{F}_{2}$, defined, 319

Families, of inferior ability ; $358-362$; of superior ability, 356-358

Fatigue, cause of, 232; effect of, on individual, 232-233; how to overcome, 233 ; test of muscles of frog for, 230

Fats, laboratory problem of effect of alkali on, 131-132; metabolism of, 190 
Fermentation, during reproduction of yeast plants, $250-251$

Fertilization, adaptations for, in plants, 293 ; double, 278; external and internal, 283, 288; in frog, 270-271, $283-284,285$; in higher plants and animals, 268,269 ; in plants, 277-278 ; significance of process, 285

Fever. See Yellow fever, Typhoid

Fibrinogen, in blood, 143, 145, 148

Fibrous tissue, functions of, $80-81$

Fibrovascular bundles, structure of, 70

Filament, of Spirogyra, 32

Filter passers, 393

Filterable virus, 393

Fish, care of young, 296; spawning of, 283-284

Fission, binary, a form of cell division, $44,247-248$

Fixation, of nitrogen, by bacteria, 407408

Flanders, on intelligence testing, 239-231

Flatworms. See Platyhelminthes

Flax, useless parts removed by bacteria, 405

Flowers, calyx of, 274 ; corolla of, 274 ; essential organs of, 274 ; production of female gamete, 276-277; production of male gamete, $275-276$

Fluid, tissue, 165

Foam theory, of nature of protoplasm, 54

Focal infection, common, 478; defined, $174-175,477$; effect of, 477 ; prevention, 478

Focusing, miscroscope, rules for, 24

Folds, in small intestine, 136

Follicle, hair, 173

Food, bacteria in preparation of, 403404 ; definition of, 91 ; improvement in quantity and quality of, 344-345; manufacture in green cells, 36-38; nutrients, $91-104$; of plant cells, 36 ; preservation of, $399-400$; storage of, 71

Food manufacture, in green cells, 36-38; process of, 36-37; the products formed in, $37-38$

Food nutrients, 91-104; table of, 94

Foods, containing mineral, 96 ; containing proteins, 95 ; energy value of, 92 ; fuel value of, 92 ; importance of, 91

Formation of fruit, 279

Fossils, formation of, 378,381 ; state of perfection, 381

Four-o'clocks, heredity of, $320-322$

Freak. See Mutants

Frog, breeding habits of, 282-283; development of, 285-286; laboratory study of alimentary canal of, 116 ; laboratory study of internal organs, 114116; life history of, 287 ; produced parthenogenetically, 271-272 ; produc- tion of eggs, 284-285; production of sperms, 285

Fruit, function of, 279

Fruit fly, location of genes in chromosomes of, 312,332

Fuel, in plants, 37

Functions, of green cells, 34-41

Fungi, characteristics of, 33 ; classified, $518-519$; meaning of, 33

Galen, idea of sirculation, 163,164

Galton, Sir Francis, application of statistical method to study of human heredity, 357; interest in eugenics, 355

Galvanotropism, shown by amoeba, 46

Gametes, female, 266 ; formation of, 264 ; male, 266 ; production in flowering plants, 275-277

Ganglion, 210, 211

Gastric juice, cause of flow of, 120 ; composition of, $125-126$; effect on protein, 126 ; enzymes in, 128 ; functions of, 128 ; laboratory problem, how protein is affected by, 126 ; secretion of, $127-130$

Gastropoda, 523

Gastrula, 286

Generation. See Spontaneous generation

Genes, change in character of, 339-340 ; character of, 316 ; combination in sex cells, 332-333; crossing-over of, 339, 340-341 ; definition of, 306 ; double, 331 ; functions of, 306 ; laboratory problem to show possible combinations in cells, 336-337; location in chromosomes of fruit fly, 312,332

Geographical evidences, of evolution, habitats of animals, 385 ; Mongolian Expeditions, 385-387

Geological evidences, of evolution, 381384 ; fossils, $379,381-382$

Geotropism, 203

Germ carriers, 397-399, 454-455, 482

Germ plasm, causes of variation, 336$337,339-342$; continuity of, 336 ; effect of environment on, 341-342 ; theory of, 335-336

Germicide, 402

Germinal variations, transmitted, 312 , 339-342

Germination, 279

Gigantism, cause of, 197

Gilbert, W., influence on Harvey, 163

Gills, tadpole, 287

Gland, oil, 173 ; sweat, 171, 173

Glands, ductloss, 191-201; endocrine, 191; experiment of grafting, 201; meaning of, 118; location in man, 192 ; location in a rat, 193 ; position 
of gastric, 125 ; rate of secretion of, 127 ; regulation of secretion, $127-128$; relation to digestion, 118,119 . See Adrenal, Intestinal, Liver, Pancreas, Parathyroid, Pineal Body, Pituitary, Reproductive, Salivary

Granular theory, of nature of protoplasm, 54

Glottis, 122

Glycogen, in liver, 161

Goiter, and iodine, 194-195 ; districts, 194-195; endemic, 194; exophthalmic, 195

Goldberger, Dr. Joseph, work on vitamin P-P, 104

Gonads, 200

"Goose flesh," cause of, 172

Gorgas, eradicating yellow fever, 491

Grafting, bud, 258; effect of, 258-259 ; in surgery, 259-260; process of, 257259 ; propagation by, 257-260; stem, 257-258; tongue, 259

Greeks, contributions to science, 10-12

Growth, of science, 10-17; mental, 225

Guard cells, of stomata, 67

Guinea pigs, in breeding experiments, 329

Gymnospermae, classified, 520 ; meaning of, 520

Habit, breaking a, 224-225; formation of, 220-221 ; meaning of, 221

Haemoglobin, 182; and oxygen, 146 ; function of, 88, 146-147 ; in corpuscles, 88

Haemophilia, 148

Hair follicles, origin of, 173

Harvey, William, discoverer of circulation of blood, 163, 164-165; influence of Gilbert on, 163; portrait of, 143 ; theory confirmed by Leeuwenhoek, 19-20

Health, and adaptability, 414-415; and education, 415-417; and longevity, 414; disease and, 413-414; measurement of, 419 ; science and, $417-419$

Health education, need of, $7,415-417$

Heart, auricles of, $152,154,159-160$; laboratory study of, 152-153; properties of muscles of, $85,152,154$; structure of, 152, 154; valves of, 152, 154 ; ventricles of, 152,154

Hemolysin, antibody, 501

Hepatic vein, 161

Heredity, in Andalusian fowls, 318-320 ; in four-o'clocks, $320-322$; in organisms, 333 ; methods of investigating human, 356-362 ; rise of knowledge of, 318-333; versus environment, 369370

Herreshoff family, inheritance of, 361
Hexactinellida, 521

Hippocrates,.Father of Medicine, 10-11

Hirudinea, 523

Holothuroidea, 522

Hominidae, 527

Hooke, Robert, first observer of cells, 27 ; microscope of, 27

Hormone, definition of, 133 ; secretin, 133,191

Horse, evolution of the, $375,376,377$

Human heredity, difficulty in studying, 357 ; methods of investigating, 356362

Humoral theory, of immunity, 506

Hybrid, appearance of, 319,324 ; descendants of, 319-322; meaning of, 319,321 ; proportion in different generations, 319-322

Hybridization, method of breeding, 351

Hydra, reproduction of, 249-250

Hydrochloric acid, in gastric juice, effect of, 129-130

Hydrogen, a chemical element, test for, 16

Hydrophobia. See Rabies

Hydrotropism, 204, 205

Hydrozoa, 521

Hygiene, defects revealed by examination, 416-417; mental, 222-237 : of circulatory system, 167-169; of digestion, 138-141; of respiration, 185; of skin, 174-175

Hyphae, of mold, 264, 265

Hypocotyl, 278

Immigration, and eugenics, 367-368

Immunity, 504-509; active, 452, 456, 458; acquired, 501; antitoxins in, 505; Ehrlich's side-chain theory of, 506 ; establishing active, 458-459; establishing passive, 508; lysins in, 505 ; meaning of active, 452 ; meaning of passive, 456 ; meaning of permanent, 456 ; natural, 506-507; of animals, 345 ; of plants, 347 ; of tomorrow, 508-509; phagocytic theory of, 504-505; racial, 507

Immunization active, 452

Improved Beach plum, development of, 348-349, 352, 353

Impulse, nature of nerve, 222-223

Impulses, types of, 215

Incomplete dominance, meaning of, 319

Indigestion, causes of, 138-141, 168

Ingestion, a life function, of amoeba, 43

Infancy, among birds, 298-299; among insects, 297-298; among lower animals, 295-296; among mammals, 299-300 ; among seed plants, 293-295; in $\operatorname{man}, 301-303$

Infection. See Focal infection 
Inferiority, conflict, nature of, 236 ; prevention of, 236

Influenza, symptoms of, 476 ; transmission of, 476

Infusoria, 521

Inheritance, chromosome theory of, 331333 ; of disease, $368-369$; of variations, 374-375

Inoculation, smallpox, in the Orient, 420421 ; introduction into England, 421 ; method of, 421 ; origin, 421

Insecta, 524

Insectivora, classified, 527

Insects, the young of, 297 ; wasps, 297298

Inspiration, definition of, 182

Insulin, in treatment of diabetes, 199

Intelligence quotient, definition of, 228229

Intelligence, definition of, 227 ; measurement of, 228-229; of students of Oxford University, 357 ; relation of progress in school to, 229-230, relation of vocations to, $230-231$

Intercellular materials, 74

Intestine. See Large intestine, Small intestine

Intestinal gland, in relation to digestion, 118

Intestinal juice, 131, 132

Introspection, advantages of, 236 ; disadvantages of, 236

I.Q., 228-229

Irritability, cause of, 202-203; in amoeba, 44-46; in animals, 202-203; in green cell, 41 ; in Paramecium, 50 51 ; in plants, 202 ; in man, 205

Islands of Langerhans, 198; location of, 199 ; value of, 199

Isogametes, defined, 267, 268

Jenner, Edward, discovery of vaccination by, 421-422

Juke family, history of, 358-359

Kallikak family, history of 359-360

Kanred wheat, how produced, 347 ; increased yield of, 351

Katabolism, in cell, activities, 60 ; meaning of, 59

Kidneys, elimination of waste through, 170 ; excretory function of, 175-176; position, 175; secretion of urine by, 170,175 ; structure of, 175

Kitasabo, cultivation of tetanus bacilli by, 460

Klebs, discovery of diphtheria bacilli by, 450

Koch, discovered cause of tuberculosis, 438-440; effect of his discovery, 440 ; experiments with guinea pigs, 439-
440 ; portrait of, 437 ; postulates of, 440 ; tuberculin test prepared by, 446-447

Labor, physiological division of, $66-67$

Lacteals, 136-137, 166

Lamarck, Jean de, theory of evolution, 371-373

Lamarckian theory, of evolution, 371-373

Langerhans, islands of. See Islands of Langerhans

Large intestine, 135; absorption from, 138; bacteria of, 135,140 ; bacterial action in, 135; function of, 133, 134 ; divisions of, 119,135 ; relation to constipation, 135, 139

Larynx, 178,179

Laveran, discoverer of malarial parasites, 481

Laws, of inheritance (Mendel's), 322-330

Lawton blackberry, berries produced from, 348,349

Laxatives, kinds, 139-140 ; use, 139

Layering, method of vegetative propagation, 260

Lazear, work on yellow fever, 487-491

Leaf, functions of, 70

Learning, laws of, 223-224

Leeuwenhoek, contribution to controversy on origin of life, 240-241 ; improved microscope, $19-20$

Lemuroidea, 527

Levels of reactions, 216, 217 ; diagrams to show, $218,219,220$

Life, origin of, 239-245 ; ancient idea, 239 ; a present-day theory of, 245

Ligaments, functions, $80-81$

Lindbergh, contribution to science, 1

Linnaean system, of classification, 510511

Linnaeus, Carolus, system of classification by, $510-511$

Linin, in nucleus, 57

Lipase, an enzyme, 38 ; steapsin, 131

Lister, Sir Joseph, contribution to surgery, 4

Little Club wheat, improvement of, 350

Liver, circulation through, 160-161; function of, 132, 161 ; relation to digestion, 118; secretion of, 132 ; storage of sugar in, 161

Locomotion, of amoeba, 46 ; of Paramecium, 49

Lockjaw. See Tetanus

Loeb, J., on artificial parthenogenesis, $271-272$

Loeffler, isolation of diphtheria bacilli by, 450

Lungs, flow of blood through, 160 ; infection of. See Pneumonia and Tuberculosis; laboratory problem on struc- 
ture and function of, $180-181$; movement of, 182 ; protection of, 179 ; red corpuscles, 179 ; size, 182 ; structure of, 179 ; volume of air in, 183

Lymph, circulation of, 165-166; fluid, 137 ; pressure of, 166 ; source of, 165 ; spaces, 166 ; vessels, $165-166$

Lymph gland, function of, 167

Lymph nodes, bacteria in, 167 ; functions of, 167 ; position of, 166-167; structure of, 167

Lymphatic system, 163-169

Lymphatics, 137 ; functions of, 166-167 ; valves in, 166

Lysins, use of, 501 ; kinds of, 501

M. A., 228

Macronucleus, in Paramecium, 49-50

Malaria, 480-486 ; cause, 482 ; diagnosis of, 485 ; history of, 481-482 ; investigation of cause, 15 ; method of spread, 482 ; nature of, $484-485$; prevalence of, $480-481$; prevention of, 486 ; treatment of, 485

Malarial parasite, life cycle of, $482-484$; multiplication of, 483-484

Malnutrition, cause of, 104 ; effect on embryo, 313

Malpighi, drawing of circulation by, 151; movement of blood in capillaries first seen by, 164

Malpighian layer, of skin, 172

Malthus, effect of essay on Darwin, 373; Essay on Population, 343

Mammalia, classified, 526-527

Mammals, development of embryo, 299300 ; food of, 300 ; infancy, 299-301 ; years of dependence, 300-301, 302

Man, infancy in, 301-303; protection of young, 30

Manufacture, of food, by green plants, 36-38

Marconi, Guglielmo, portrait of, 1

Marsupialia, animals of Order, 526

Mastication, importance of, 123 ; process of, 120

Mastigophora, 521

Mastodon, restoration of, 379

Maturation, of egg, 264; of sex cells of fruit fly, 269; of sperm, 264; process of, $268-270$; variation due to, 285

McCollum, investigation on diets, 9395

Measles, after-effects, 478; method of spread, 478; prevention of, 478

Measurement, of health, 419

Media, definition of bacterial, 395 ; for cultivation of bacteria, 396-397

Medicine, early practices of, 3, 10-13

Medulla, oblongata, description of, 207 ; function of, 207
Membrane, meaning of, 75 ; mucous, 75 ; serous, 76

Mendel Gregor, experiments with peas, $322-326$, 327-329; portrait of, 318 ; rediscovery of writings, 330

Mendelian laws, of dominance, 322-324; of segregation, 324-326; of unit characters, $327-330$; of heredity, summarized, 331

Mental age, 228

Mental hygiene, 227-237

Mental poise, cause of lack of, 233-235; need of, 227, 233; relation to disease, 502-503

Mesentery, function of, 77

Mesoderm, of embryo, 286 ; development of, 286 ; systems formed from, 286

Metabolism, basal, 193 ; in cell, $59-60$; kinds, 187 ; meaning of, 59-60; of carbohydrates, 188; of fats, 190; of proteins, 188-190

Metamorphosis, of frog, 287

Metaphase, stage in cell division, 62

Metchnikoff, on bacteria of putrefaction, 140 ; theory of immunity, 504-505

Method, modern scientific, 13-15

Micron, unit of measurement, microscopic work, 392-393

Micronucleus, in Paramecium, 47-50, 248

Micropyle, of egg, 270, 276

Microscope, 19-25; care of, 23-24; compound, 20; history of development, 19-20; Hooke's, 27 ; laboratory problem on use of, $24-26$; parts of, 21-23; rules for focusing, 24

Milk, acidopholus, 140 ; increase in products of, 344 ; influence on typhoid, 467; laboratory problem to determine bacterial content, 401

Minerals, foods containing, 96 ; insufficiency, 95 ; need of, 95

Mitosis, in animal cell, 61-63; in plant cell, 63, 64; laboratory study of, 63 ; process of, $60-63$

Mitral valve, of heart, 160

Mold, laboratory problem, on spore formation, 251-252; hyphae, 251; mycelium, 257 ; rhizoid, 251 ; sexual reproduction in, 264, 265 ; sporangium of, 251-252, 253

Molecular theory, 35

Molecules, laboratory problem to show movement of, 35

Mollusca, classified, 523; meaning of, 523

Mollusks, the young of, 296

Mongolian expeditions, $385-387$

Monotremata, 526; duckbill, 513

Morgan, T. H., on heredity, 55 ; portrait of, 318 ; work on heredity, 14; work on mutations in fruit flies, 312 
Morphological evidences, of evolution, in structure of animals, 387-388

Morphology, meaning of, 17

Morula, 285

"Mother of vinegar," 391

Mouth, of man, $120^{-}-121$; structure of, 120 ; use of, in digestion, $120-121$

Mucous, lining of nasal passages, function of, 178

Muscle tissue, 85-86; cardiac, 85; cross-striated, 85 ; function of, $85-86$; structure of, $83-85$; unstriated, 85

Muscle, laboratory problems on structure of skeletal, 83-84; laboratory problem on structure of smooth or involuntary, 84

Muscles, antagonistic, 86

Muscular action, antagonistic, 85-86

Mutants, causes of, 377 ; examples of occurrence, 337-339; importance in breeding, 353-354; meaning of, 337

Mutation theory, of evolution, 376 ; and natural selection, 377

Mycelium, in mold, 251

Myriapoda, 524

Myxedema, 194

Narcissus, laboratory study of epidermis of leaf of, 30-31

Nasal passages, removal of dust by, 178 ; warming and moistening air by, 178

Natural immunity, meaning of, 506 ; of races and individuals, 507

Natural selection, 373-375; and mutation theory, 377 ; Darwin's theory of, $373-376$; objections to theory of, 376

Navel orange, development of, 349

Needham, experiments to prove spontaneous generation, 241

Negri bodies, in rabies, 433,435

Nemathelminthes, 522

Nerve centers, 208

Nerve tissue, in man, 86 ; unit of structure, $86-87$; work of, 86

Nerves, function of, 208-209; motor, 210 ; pathway of, 209-211 ; sensory, 209

Nervous reactions, importance of, 223 ; kinds of, 215, 216-221; laboratory study of, 225 ; nature of, 216-217

Nervous system, 202-214; autonomic portion of, $210-211$; cellular structure of, 208-210 ; cerebro-spinal or central portion of, 205-210; effect of tobacco, 236-237 ; effect of alcohol, 237 ; main subdivision of, 205; principle of operation of, 205 ; structure of, 208210 ; studies of, man and other vertebrates, 211-212; the methods of studying, 212-213

Neuron, associative, 209 ; description of, 208 ; diagram of, 209
Nicolaier, discovery of tetanus bacillus, 460

Nitrification, process of, in soil, 406-407

Nitrogen, cycle, 406,410 ; fixed by bacteria, 406, 407-409

Nitrogen, fixation, 407-409

Nodes. See Lymph nodes

Nodules, use of root, to soil, 408

Noguchi, Hidego, 2-3; work on yellow fever, 491,492

Nostrils, 178

Nuclei, polar, 276, 277, 278; sperm, 275

Nucleoplasm, structures found in, 57

Nucleus, of cell, 27-28; endosperm, 278 ; egg, 277 ; generative, 275 ; tube, 275 ; work of, $57-58$

Nutrients, food, 91-104; definition of, 91-92; table of, 94

Nutrition, of Paramecium, 48-49

Nutritive processes in organisms, purpose of, 34

Objections, to vaccination, 423,427

Offspring, cause of differences among, 315 ; character of, 306-317

Oil glands, 173

Onion cells, laboratory study of, 25-26

Ontogeny, history of individual, 388389

Ony chophora, 523

Oögenesis, meaning of, 270

Ophiuroidea, 522

Opsonins, effect on bacteria, 500, 502; test for amount of, 502

Orange, navel, 349

Organ, definition of, 73

Organic evolution, 371-389; Darwinian theory . of, 373-376; embryological evidences of, 388-389; de Vries' theory of, 376-377; factors in, 371; geographical evidences, 385-386 ; geological evidences, 381-384; history of, 371 ; Lamarckian theory of, 371373 ; morphological evidences of, 386387 : vestigial evidences of, $387-388$

Organism, offspring of simple, 292-293

Organisms, heredity in, 333 ; purpose of life processes in, 34

Organs, ' of digestion, 118-119; 125-135

Origin of Species, by Darwin, 373

Osmosis, in absorption of food, 136, 137 ; in root hair, 69 ; in photosynthesis, meaning of, 34 ; process of, 34-35

Osterhout, on heredity, 55

Ova, of frog, 283

Overproduction, of species, 373

Ovules, in flower, 274, 276-277, 279

Oxygen, a chemical element, test for, 16; importance to life, 241-242

Oxyhaemoglobin, 147 
Palate of man, 120

Paleontology; 378

Palisade cells, of leaf, 67

Pancreas, digestive secretions of, 131 ; internal secretion of, 199 ; relation to digestion, 118. See Diabetes, Insulin

Pancreatic juice, enzymes of, 131

Papillae, of tongue, 120 ; functions of, 173

Parasite, definition of, 252; group of bacteria, 393-394

Paramecium, conjugation of, 49-50, 248, 267-268; effect of alcohol on, 50 ; irritability of, $50-51$; laboratory study of, 46-47; locomotion of, 49 ; mode of defense, 50-51; nutrition of 48-49; reproduction of, $49-50$

Parathyroid glands, location of, 196; relation to metabolism, 196; secretion of, 196. See Tetany

Parental care, shown by, birds, 298-299; fishes and frogs, 296 ; insects, 297-298; mammals, 299-301; man, 301-304

Parenthood, development of, 303-304

Park, Dr. W. H., inoculations with toxinantitoxin, 452-453

Parotid gland, in mouth, 121

Parthenogenesis, 271-272

Passive immunity, acquiring, 508

Pasteur, Louis, 3 ; ideals of, 435-436 ; treatment for rabies, 430-432; work to disprove spontaneous generation, 242-244

Pavlov, experiments on digestion, 138-139

Payne, "Habits and Practices in Accident Prevention and Health," 419, the appendix

Peas, sweet, Mendel's experiments on heredity of, $322-326 ; 327-330$

Pelecypoda, 523

Pellagra, deficiency disease, 104

Pepsin, 128

Pepsinogen, 128

Peptic gland, relation to digestion, 118

Peptone, form of protein, 128

Pericardium, of man, 76

Periosteum, of bone, 78

Peristalis, described, 123

Peritoneum, of man, 76

Perspiration, 170

Petri, R. J., devised Petri dish, 396

Phagocytes, function of, 146-147, 500 504-505

Phagocytic theory, of immunity, 504-505

Phagocytosis, 147

Pharynx, 122

Photosynthesis, meaning of, 37 ; process of, 36,37

Phototropism, laboratory problem to show, 203; shown by amoeba, 46

Phrenology, 5, 214
Phylogeny, history of entire race or group, 388-389

Phylum, in taxonomy, 516

Physics, and its relation to biology, 15-16

Physiological functions, of organism, 34

Physiology, meaning of, 17

Pigment, in skin, 172

Pineal body, effect of injury to, 200 ; location of, 200

Pisces, animals of class, 525

Pituitary gland, location of, 196 ; relation to growth, 196-197. See Acromegaly, Dwarfism, Gigantism

Placenta, animals, 289, 291; of plants, 274,278

Plant, and animal breeding, 343-353; experiments by Mendel, 322 ; organs of flowering, 273

Plants, cellular nature of, 30-31; growth of seed, 293-295; manufacture of food, 36-38; reproduction of higher, 273-279; specialization in higher, 6972 ; storage of food in, 70-71

Plasma, composition of blood, 145

Plasma membrane, function of, 58 ; of cell, 32

Plasmodium malariae. See Malarial parasite

Platelets. See Blood platelets

Platyhelminthes, 522

Pleurae, of man, 76

Pleurococcus, diagram of, 33 ; laboratory problem on, 31 ; reproduction of, 247

Plexus, 210

Plum, development of new species of, 348-349

Plumcot, how produced, 349

Plumule, 278

Points, breeding for, 349-350

Poise, mental. See Mental poise

Polar, nuclei, 276, 277, 278; bodies, 270

Pollen, agencies to scatter, 276 ; effect of, 277-278; grains, 275, 276, 277; laboratory study of effect of sugar solution on, 275 ; nuclear division in, 276 ; production of, 275 ; tube, $275,276,277$

Pollination, agents 'of, 293; artificial method of, $352 ;$ process of, 276

Polyneuritis, cause of, 100 ; cure for, 100

Population, Malthus' Essay on, 343

Pore, of skin, 173

Porifera, classified, 521 ; meaning of, 521

Portal, circulation, $160-161$; vein, 161

Postulates, of Koch, 440

Potatoes, propagation of, 350

Pouchet, on spontaneous generation, 242-244

Precipitins, function of, 501-502

Preservation of food, discussed, 399

Primates, classified, 527; animals of order of, 527 
Primroses. See Evening Primrose

Priority, law of, 514-515

Production, breeding for increased, 351 ; of new species, 347-349

Progress, in school, relation of intelligence to, 229-230

Propagation, methods of animal, 353354 ; methods of plant, $352-353$; of dahlia, 349-350; of potato, 350 ; vegetative. See Vegetative propagation

Prophase, stage in cell division, 62

Protease, an enzyme, 38; erepsin, 132 ; trypsinogen, 131

Protection, of young, 292-304

Protein, foods containing, 95 ; formation of, 38 ; insufficiency of, 93,95 ; laboratory problem on effect of gastric juice on, 126 ; laboratory problem on relation of time of digestion to size of, 126 ; laboratory problem on rate of digestion of different animal, 127 ; manufacture of, 37-38 ; metabolism of, 188-190; process of synthesis, 37-38; product of protein-synthesis, 37-38

Proteose, form of protein, 128

Protoplasm, cause of variety in, 306307; cause of likeness in, 307-308; characteristics of, $29-30,55-57$; discovery of, 28 ; functions of different parts of, 57-60; effect of alcohol on, 50 ; named, 28 ; specialization of, 5 !52 ; structure of, 53-55; reticular theory of, 53-54; alveolar or foam theory of, 54 ; granular theory of, 54 ; variety in, 306-308

Protozoa, classified, 520-521

Pteridophyta, classified, 519-520

Pterodactyl, prehistoric flying reptile, 382-383

Ptomaines, 495

Ptyalin, enzyme of saliva, 121

Pulmonary, artery, 153; circulation, 160

Pulse, cause of, 156; laboratory study on rate of, $156-157$

Punnett squares, $328,330,332$

Purkinje, discoverer of protoplasm, 28

Pustules, of smallpox, 423, 425

Pyloric valve, of stomach, 125

Pyorrhea, cause of, 110-111

Pyrenoids, structures in Spirogyra, 32

Quality, breeding to improve, 330

Rabies, cure of, 434 ; discovery of treatment by Pasteur, 430-432; effect on animals, 433; eradication of, 434 ; nature of, 432-433; preparation of vaccine for, 432 ; prophylatic or preventive treatment of, 434-435 ; transmission of, 433
Race improvement, necessity of, 362363 ; suggestions for, 363-368

Reactions, conscious, 219; glandular, 221-222; in learning, 219 ; levels of, $216-217,218,219,220$; types of, 216217. See Nervous reactions

Recapitulation, doctrine of, 388-389

Recessive character, defined, 324 ; list of, 333

Red corpuscles, in lungs, 179

Redi, Francesco, experiments on spontaneous generation, 239-240

Reduction division. See Maturation

Reed, Major W., work on yellow fever, 487-491

Reflex, conditioned, 217-219; development by conditioned response, 218; nature of, 217-219; simple, 215

Refrigeration of foods, 399

Regeneration, in amoeba, 56 ; in higher animals, 257; in lower animals, 256, 257 ; similarity to vegetative propagation, 255-256; in Stentor, 56; in Stylonychia, 56 ; in Uronychia, 56

Rennin, 128, 129

Reproduction, asexual. See Asexual reproduction

Reproduction, by binary fission, 247248 ; by budding, 248-250; by spore formation, 250-251; by vegetative propagation, 255-263; by two special cells, 264; necessity in cells, 246-247; of amoeba, 44,248 ; of animals, 281 291 ; of bacteria, 247 ; of birds, 288 ; of frog, 282-287; of higher plants, 273-279; of mammals, 288-291; of mold, 264, 265; of Paramecium, $49-50 ; 248 ; 267-268$; of Pleuroccus, 247 ; of Spirogyra, 247-248, 265-267

Reproduction, sexual. See Sexual Reproduction

Reproductive glands, relation to normal development, 200-201; secretion of, 200

Reproductive processes, in organisms, purpose of, 34

Reptile, flying, 382

Reptilia, animals of order, 526

Respiration, 177-185; definition of, 182 ; external, 183; hygiene of, 185; internal, 183 ; in plants, $36,38-39$; of amoeba, 44 ; of fish, 177-178; of insects, 177 ; of higher animals, 178 ; products of, $182-183,184$; rate of, 183

Respiratory, movements, 181-182 ; passages, irritation of, 179-180

Respiratory tract, diseases of, adenoids, 179; tonsillitis, 180

Response, laboratory prohlems, of plants to gravity, 202-204; to sunlight, 203 ; to water, 204 
Reticular theory, of nature of protoplasm, 54

Rhinoceros, prehistoric, 378

Rhizoda, classified, 521

Rhizoid, of mold, 251

Rhizomes, plants with, 261; propagation by, 261

Rhythmic segmentation, of small intestine, 130

Rickets, cause, 102-103; prevention and cure of, 100,103

Rodentia, classified, 527

Rootstock, plants with, 261 ; propagation by, 261

Root hairs, of plants, 69

Ross, Major R., investigation of malaria, 481-482

Rotation of crops, need for, 409-411

Round worms. See Nematheliminthes

Roux, isolation of diphtheria toxin, 451

Runners, use of, in propagating, 260-261

.Saliva, composition of, 121-122 ; digestive action of, 121-122; enzymes of, 121; factors influencing secretion of, 121 ; functions of, 120-121 ; secretions of, 121

Salivary glands, position of, 120 ; funetion of, 120-121

Saponification, in the digestive process, 131

Saprophytes, group of bacteria, 251, 394, 499

Scarlet fever, probable cause of, 458 ; test to determine susceptibility to, 458 ; transmission of, 459

Schleiden, knowledge of cells, 28

Schultze, contribution to cell theory, 29

Schwann, Theodore, work on cells, 28

Science, age of, $1-3$; aim of, 6 ; and health, 417-419; contributions by Greeks, 10-12 ; growth of, 10-11 ; of primitive man, 10

Scientific method, modern, 13-15

Scurvy, 97 ; cause of, 100,102 ; treatment for, 100, 102

Scyphozoa, 522

Secretagogues, 128

Secretin, 133

Secretions, internal, 191-192; of green cell, 40

Seed, appendages, 294 ; differ from eggs, 281 ; dispersal of, 294-295; food reserve of, 294-295; formation of, 278279 ; protection of, 294

Segmentation, rhythmic, of small intestine, 130

Segregation, of genes during maturation, 325,328 ; Mendelian law of, 324-326

Selection, artificial, importance in plants, 352
Self-acting nervous system. See Autonomic nervous system

Self-pity, as trait of personality, effect of, 236

Serum of blood. See Blood

Sexual characters, secondary, 281-282

Sexual reproduction, in higher plants and animals, 264, 268, 273-291; in mold, 264-265 ; meaning of, 264 ; in Paramecium, 267-268 ; in Spirogyra, 265-267

Shick test, application of, 457 ; of susceptibility to diphtheria, 453

Side-chain theory, of immunity, 506

Sieve tubes, of plants function of, 68

Simiidae, 527

Simon, Theodore, tests by, 228

Sirenia, animals of Order, 527

Skin, functions of, 173-174; hygiene of, 174-175; laboratory study of the, $170-$ 171 ; organ of elimination, 170,173 174 ; sense organ, 173 ; structure of, 172-173; thickness of, 172

Slant, agar, 397

Slips, method of vegetative propagation, $255-256$

Small intestine, absorption from, 136137 ; adaptations of, $136-138$; digestion in, $130-131,132-133,135$; digestive juices of, 132-133; folds in, 136 ; glands of, 131; movements of, 130 ; villi of, 136, 137

Smallpox, cases and deaths in United States, 424 ; epidemics of, 420 ; history of, 420 ; history of inoculations against, 420-421; nature and symptoms of, 423,425 ; preparation of vaccine for, 426; prevention, 425-426; spread, 425 ; value of vaccination, 426-427. See Vaccination

Soil, bacteria of, 405-411; conservation of, 411

Somatic cells, 269

Somatic variations, not transmitted, 310

Spallanzani, on spontaneous generation, 241

Spawning, of fish, 283-284; of frogs, 282-283

Species, production of new, 347-349; in taxonomy, 517

Species andVarieties, by De Vries, 376-377

Species, Origin of, by Darwin, 373

Specialization, in higher plants, $69-72$

Sperm cell, characteristics of, 268

Spermatogenesis, meaning of, 269

Spermatophyta, 520

Sperms, production of frog, 285

Sphincters, 125

Spillman, production of hardy wheat by, 350

Spinal cord, description of, 207-208; diagram of cross section, 208; func- 
tion of, 208; level of reactions of, 218, 219,220 ; relation to reflexes, 208; structure of, 207-208

Spireme, formation of, in cell division, 61-62

Spirilla, form of bacteria, 392

Spirochaete, causative organism of yellow fever, 492

Spirogyra, description of, 31 ; laboratory problem on the cells of, 31-33; meaning of, 32 ; reproduction of, $247-$ 248 ; 265-267; specialized structures of, 32 ; typical green cell, $31-37$

Spleen, activity of, 200 ; location of, 200

Split-proteins, poisonous, formation of, 495

Sponges, use of bacteria in preparation of, 404

Spontaneous generation, controversy on, 242-244; experimental evidence on, $239-241 ; 242-243$; theory of, 239

Sporangia, laboratory problem, in various molds, 252

Spore, of bacteria, defined, 395

Spore-formation, cause of, 250 ; of yeast cells, 250; reproduction by, 250

Sport. See Mutants

Sporozoa, 521

Stanford Revision of Binet-Simon scale, 228

Starch, digestion of, 130

Steapsin, 131

Stentor, regeneration of, 56

Stomach, function of, 129 ; glands of, 125-126; of frog, 115 ; parts of, 152 ; shape of, 129 ; valves of, 125

Stomata, of leaf, 67,69 ; guard cells of, 67 ; position of, 67

Storage, of food in plants, 70-71

Structure, of amoeba, 42 ; of Paramecium, $46-47$; of cell, $53-55$; of protoplasm, $55-57$; of higher plants, $66-72$

Struggle for existence, 373

Stimulation, of glands, chemical, 121, 128; mechanical, 121 ; psychical, 121, 127

Stimulus, meaning of, 40 ; of glands. See Stimulation

Stylonychia, regeneration of, 56

Sublingual gland, in mouth, 121

Submaxillary glands, in mouth, 121

Succus entericus, 131, 132

Sunlight, in relation to rickets, 103

Supporting tissues, in man, 77 ; plants, 71 . 80

Surgery, antiseptic, 4; aseptic, 45; early, $3,11-13$; grafting in. 259-260 modern methods in, $13-15$

Survival of the fittest, 374

Sweat glands, 171,173
Sweet peas, Mendel's experiments in heredity of, 322-326; $327-330$

Symbiosis, defined, 394

Sympathetic nervous system. See Autonomic nervous system

Synapse, properties of, 208, 209, 210

System, circulatory, 151-162; diagram of circulation, 155 ; nervous system, 202-214; lymphatic, 163-169

System development in embryo, 286

Systema Naturae (Linnaeus), 511

Systemic circulation, of blood, 159-160

Table, of caloric needs, 105; of foods containing minerals, 96 ; of food nutrients, 94 ; of vitamins, 101

Tartar, on teeth, 109

Taste-buds, importance of, 120

Taxonomy, meaning of, 512 ; difficulties, 513-514

Teeth, arrangement of, 108-109; causes of decay, 109-112; causes of malformation, 180 ; dental care, 110 111; kinds of, 107-108; individual care of, 109-111; laboratory problem on condition of teeth, 112; number of, 107-108; of adults, 108 ; of children, 108,109 ; relation of diet to, 111-112; structure of, 107, 108; tartar on, 109

Telophase, stage in cell division, 63

Temper, bad effect of, 236 ; reason for, 235-236

Terman, revision of Binet-Simon scale by, 228

Tendons, use of, 81

Tetanus, antitoxin, 461 ; bacilli, 460 ; cause of, 459-460; discovery of bacillus of, 460; entrance into body, 460 ; nature of, 461; occurrence, 460 ; prevalence, 459 ; prevention, 462; treatment, 461

Tetany, cause of, 196

Thallophyta, classified, 518-519; meaning of, 33 ; plants of, 33

Thermotropism, shown by amoeba, 46

Thigmotropism, shown by amoeba, 46

Thoracic duct, 165

Thymus gland, location of, 199 ; relation to growth, 199

Thyroid gland, disturbances of secretion, 194-195; grafting of, 193-194; influence on growth, 194; position of, 192-193; secretion of, 193. See also Cretinism, Cretins, Goiter, Myxodema

Thyroxin, secretion of thyroid, 194

Tidal air, 183

Tissue, blood, $87,145-148$; contractile, 83 ; use of epithelial, $75-77$; functions of, 88-89; muscular, 85 ; nerve, 86 87 ; of leaf, 68-69

Tissue fluid, 165 
Titanotheres, prehistoric mammal, restoration of, 379

Tobacco, effect on nervous system, 236237,169 ; use of bacteria in curing, 405

Tongue, a sense organ, 120

Tonsillitis. See Tonsils

Tonsils, enlargement of, 180 ; infection of, $180 ;$ colds result from, 475-476

Tourniquet, when to use, 168

Tower, experiment of effect of environment of germ plasm, 341-342

Toxin-antitoxin, discovery of value of, $452-453$; isolation of diphtheria, 471 ;

of tuberculosis, 442

Trachea, 179,178

Tracheids, in a leaf, 68,70

Traits, ancestral, effect of environment on, 313-315

Transfusion, of blood, 149

Trichocysts, of Paramecium, function of, $47,50-51$

Tripe, meaning of, 126

Tropism, in amoeba, 45-46; meaning of, 45 ; kinds of, 45-46, laboratory problems to show plant, 203-204; use of, 204

Trudeau, 440-442; investigations of tuberculosis, 441-442; method of treatment for tuberculosis, 442 ; portrait of, 437 ; sanitarium of, 441, 442

Trypsinogen, 131

Tubercle bacilli, discovery of, 438-439; effect of, 442,443 ; staining of, 438 ; when found, 439

Tuberculin test, devised by Koch, 446447 ; for cows, 444,445

Tuberculosis, acquired from milk, 445 ; bacilli of, 438,439 ; causative organ=ism of, 442 ; conditions favoring development, 443-444; diagnosis, 444; discovery of cause of, $438-440$; economic importance of, 448 ; eradication of, 444 ; experiments on spread of, $437-438$; immunity to, 442-443; in relation to heredity, 443,448 ; mortality in England and Wales, 445; mortality in United States, 443; of animals, 444 ; prevalence, 448 ; prevention, 446-448; spread of, 444-445; symptoms of, 444 ; treatment of, 446 ; types of, 442 ; vaccination for prevention of, $447-448$

Tubers, propagation by, 261-262

Tubules, of kidneys, 175

Tulip, laboratory study of parts of, 273-274

Twins, causes of identical, 314,370

Tyndall, John, apparatus and experiment to disprove spontaneous generation, 244-245 ; importance of experiment, 245
Typhoid, 464-472; bacilli, 464 ; carriers of, 465 ; cause of, 464 ; diagnosis of, 466-467; epidemic, 469-472; food infection, 467; immunity to, 469 ; "Mary," 465 ; milk infection, 467, nature of, 464-465; prevalence, 464; prevention of, 468 ; protection of body against, 465 ; routes of infection, $467-468$; symptoms of, 464 ; transmission of, 467-468; vaccination and, 469

Ultra-violet rays. See sunlight

Ungulata, classified, 527; animals of order, 527

Unit characters, $318,327-330$

Urea, 170, 172-173; formation of, 161

Ureters, position of, 175 ; function of, 175

Urine, 170

Uronychia, regeneration of, 56

Uvula, 120

Vaccination, small pox, decrease of smallpox in Prussia and Austria due to, 422; history of, 421-422; introduction into America, 423; objections to, 423, 427; results of compulsory, 425-426; typhoid, 469 ; value of, $426-427,508$

Vaccine, for rabies, 432 ; for smallpox, 426 ; preparation of, 426,508

Vacuole, function of contractile, 59 ; function of food, 59 ; structure of food, 58-59

Valve, mitral, 160 ; in veins, 158

Variations, germinal, $312,339-342$; in species, 374 ; inheritance of, $374-375$; somatic, 308-310

Varicose veins, cause of, 168

Varieties, species and, of de Vries, 376377

Vascular, and supporting tissues of leaf, laboratory problem, 68-69

Vegetative propagation, Artificial methods of, by cuttings, 255-256; by grafting, 257, 260; by regeneration, 256257; Natural methods of, by bulbs, 262-263; by layering, 260 ; by rhisomes, 261 ; by runners, 260-261; by tubers, 261-262

Veins, direction of blood flow in, 157160 ; hepatic, 161; portal, 161; pressure in, 158-159; valves in, 158; varicose, 168

Vena cava, lower or inferior, 153,160 ; upper or superior, 153,160

Venous blood, 144

Ventilation, methods of, 185

Ventricles. See Heart

Vermiform appendix, 134-135

Vesalius, contributions to surgery, 12 , 13 ; knowledge of circulation, 164 
Vessels, blood, laboratory study of, 151 Vestigial evidence, of evolution, traces or rudiments of organs in higher animals, 387

Villemin, J. A., experimental research in tuberculosis, 437-438

Villi, described; 136, 137

Vinci, Leonardo da, scientific work of, 163-164

Virus, filterable, 393 ; of smallpox, 423424

Vitamins, diseases caused by deficiency of, 97-99; names of, 96-99, 101; list of foods containing, 101 ; presentday knowledge of, 99-100, 102-104; sources of, 101

Vocations, relation of intelligence to, 230 231

Voice-box, 178

Von Behring, discovery of diphtheria antitoxin, 451-452

Von Haeckel, on recapitulation, 388-389

Von Leeuwenhoek, improved microscope, 19-20; contribution to controversy on origin of species, 240-241

Von Mohl, Hugo, description of cell by, 28

Vries; Hugo de. See de Vries

Wasp, care of their young, 297-298

Water, need of drinking, 167-168

Waugh, C. W., intelligence test to motormen, 231
Weismann, portrait of, 335 ; theory of germ plasm, 335-336

Wheat, Little Club, 350

Whooping cough, after-effects, 478; method of spread, 478

Widal test, in typhoid fever, 467

Wilson, E. B., on heredity, 55

Windpipe, 122

Woodruff, L. L., experiments with Paramecia, 50

Woods, study of royal families by, 357358

Woody fibers, of plants, use of, 71

Worry, causes of, 234-235; effect of, 233-234

Wounds, disease through, 497

Xerophthalmia, cause of, 99 ; treatment for, 98,99

Xylem, conductors of water, 69

Yeast cells, formation of spores in, 250 ; laboratory study of budding in, 248249

Yellow fever, 486-492; cause of, 492 ; commission, 487; history of control, $487-491$; history of, $486-487$; later investigations, 492; prevalence of, 486-487

Young, protection of, 292-304

Zygopore, defined, 264-265; 266

Zygote, defined, 264, 268 



